it

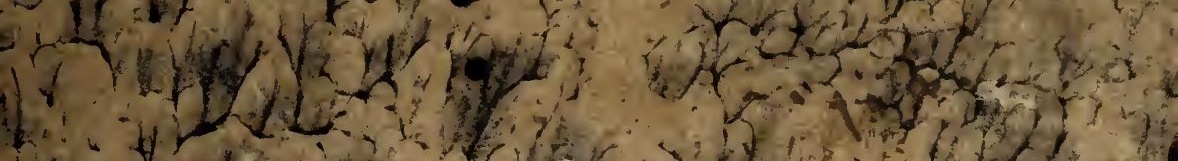
- ther 7 (x)

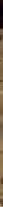

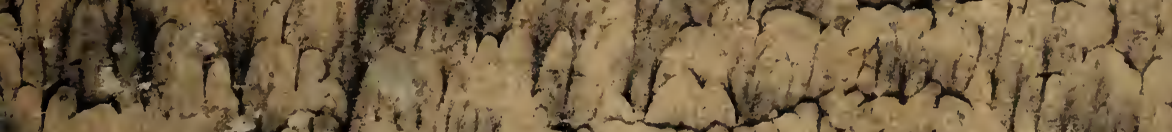

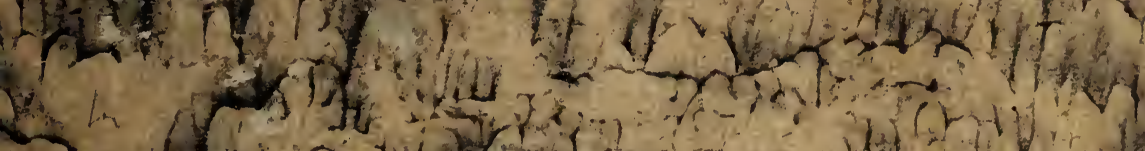

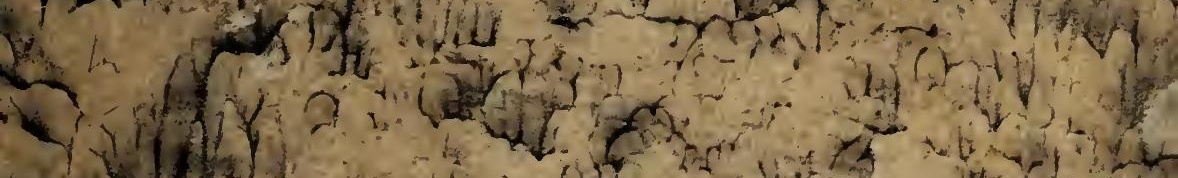
(i)

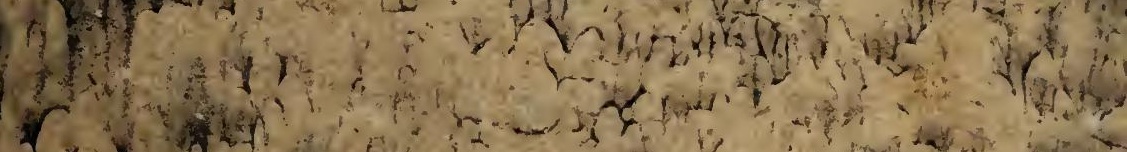

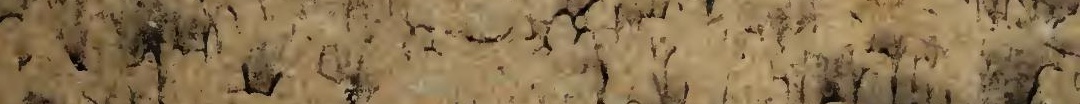

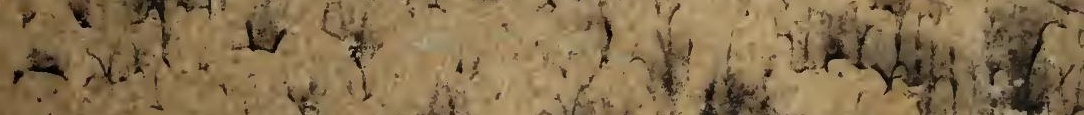

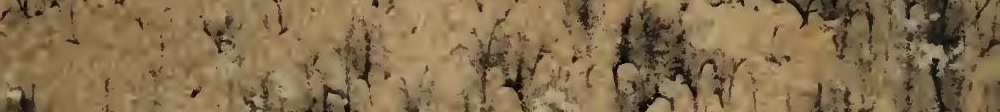

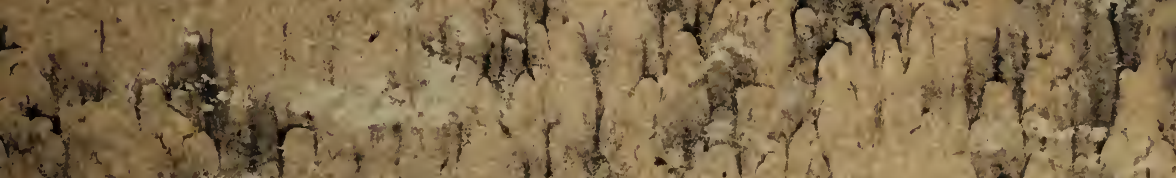
(5) 11 the

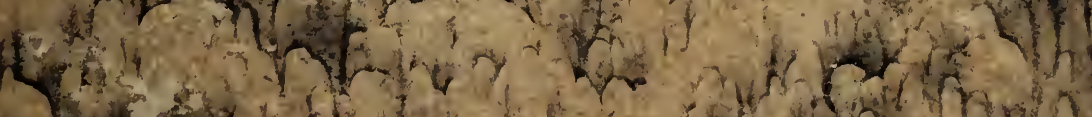

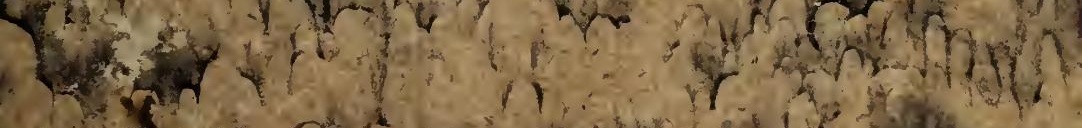

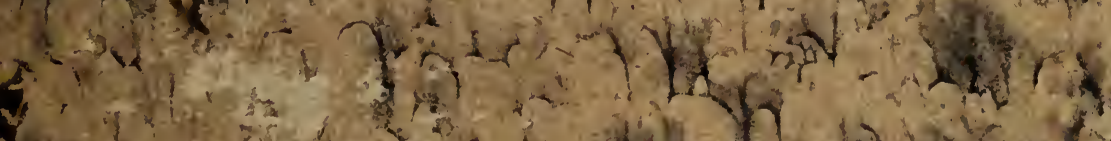

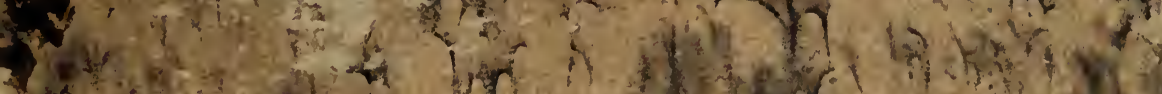

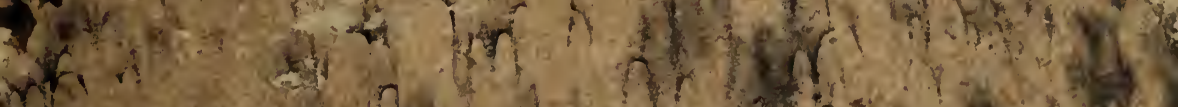
man $x+4$

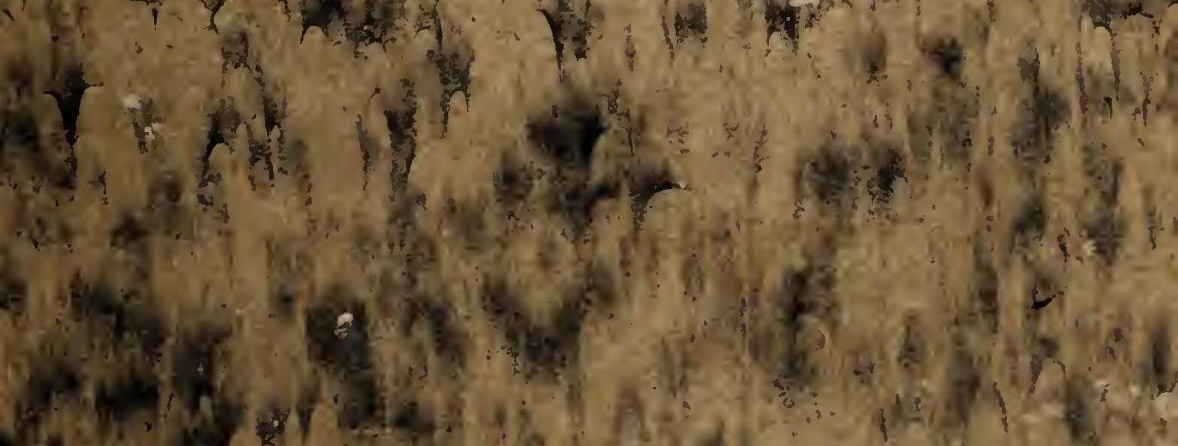

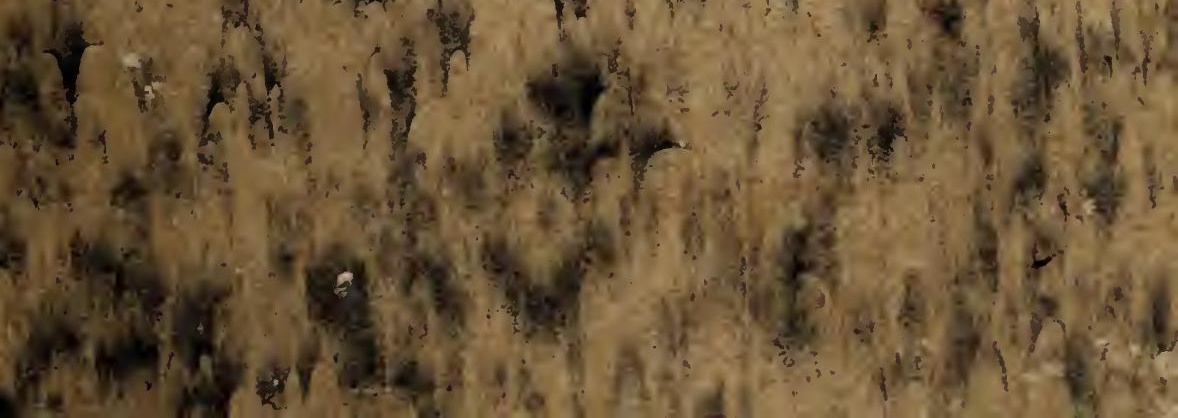


.82

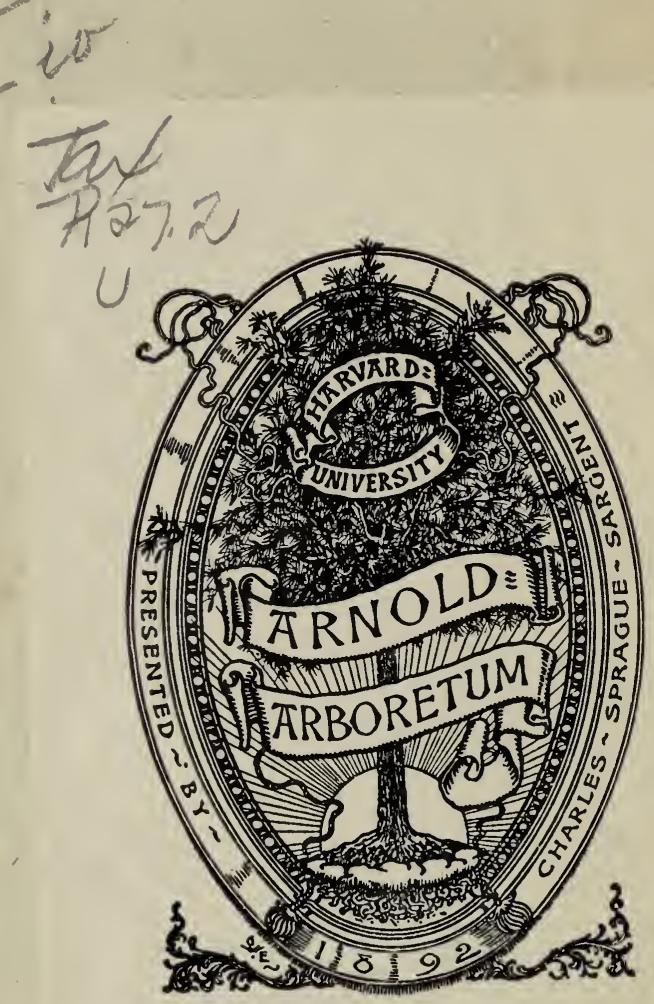





\section{U E B E R S I C H T}

$$
\text { DES }
$$

\section{GEWAECHS - REICHS.}

E R S T E R T H E I $\dot{L}_{\text {. }}$ 
Eine grosse Anzahl von Gattungen, so wie kritische und neue Arten, finden sich in folgendem, in monatlichen Heften fortlanfenden Doppel-Werke von dem Verfasser des gegenwärtigen. Es erscheint bei Friedrich Hofmeister in Leipzig und ist durch alle gute Buchhandlungen zu erhalten.

Tcones plantarum rariorum et minus rite cognitarum Florae Europacae (Iconographia botanica s. Plantae criticae); oder: Kupfersammlung kritischer Gewächse, Abbildungen seltener und weniger genan bekannter Gewächse der Flora Europa's, als Tínpfersammlung und Supplement, vorzüglich zu den Werken von Willdenow, Schkuhr, Persoon u. s. w. gezeichnet und nebst kurzer Erläuterung herausgegeben.

Davon sind sechs Bände, jeder mit 100 Kupfertafeln erschienen, nämlich :

Erste Centurie, 1823, enthält no. 1-210.

Zweite Centurie, 1824, enthält no. 211-342.

Dritte Centurie, 1825, enthält no. $343-473$.

Vierte Centurie, 1826, enthält no. 474-586.

Fïnfte Centurie, 1827, enthält no. 587-693.

Sechste Centurie, 1828, enthält no. 694-919.

Siebente Centurie fiir 1829, ist bereits unter der Presse, wird ausser den Gattungen Orobanche, Chara, Biscutella, mehrere Saxifragen und Orchideen, und manche neue Gewächse, sowohl der portugiesischen als auch vorzïglich der deutschen Flora enthalten.

Iconographia botanica cxotica s. Hortus botanicus, oder: Kupfersammlung der neuesten, oder bisher weniger genau bekannten und verwechselten a us1 ä n dis ch en G e wä chs e, (nebst Angabe ihrer Cultur fïr Gartenfreunde.)

Davon sind zwei Bände erschienen.

Erste Centurie, 1824-27, enthält no. 1-100.

Zweite Centurie, 1828, enthält no. 100-200.

Dritte Centurie für 1829 ist unter der Presse und wird rorziiglich noch nicht abgebildete $G$ e ne ra enthalten. 


\section{\# U E B E R S I C H T}

DES

\section{GEWA E CHS - REICHS \\ IN}

SEINEN NATUERLICHEN ENTWICKELUNGSSTUFEN.

\section{E I N V E R S U C H}

\section{ron \\ H. G. JUDWIG RIICHEIBACH, KOENIGL. SAECHS. HOFRATHE,}

DR. D. PHILOS. MEDIC, U, CHIRURG. PROF, D. NATURGESCH. UND DIRECT. D. BOT. GART. AN D. KOENIGL. MEDIC. CHIRURG. ACAD. IN DRESDEN ; ERSTEM INSPECTOR DES KOENIGL. SAECHS. ZOOLOG. UND MINERALOG. IUUSEUMS; D. KAIS. LEOP. CAROLIN. ACAD. DER NATURFORSCHER, D. KAIS. RUSS. SOC. D. NATURT. ZU MOSKAU, D. ACADEMIA TRUENTINA ZU ASCOLI, D. NATURFORSCH. GESELLSCHAFT ZU LEIPZIG, HALLE, PARIS UND FRANKFURT, DER DES OSTERLANDES, D. WETTERAUISCHEN GESELLSCHAFT FÜR DIE GESAMTTE NATURKUNDE, DER GES, ZUR BEFOERD. D. GESAMMT. NATURWISSENSCHAFTEN ZU MARBURG, DER PHYSIOGRAPHISCHEN GESELLSCHAFT IN LUND, DER KOENIGL, BAYERSCHEN BOTANISCHEN GESELLSCHAFT ZU REGENSBURG, DER GROSSHERZOGL. WEIMARISCHEN MINERALOG. GESELLSCHAFT ZU JENA, DES KOENIGL. BAYER. PHARMACEUT. VEREINS, DRS APOTHEKERVEREINS IM NÖRDL. DEUTSCHLANDE, DER WBRNERISCHEN NAT. HIST. SOC. IN EDINBURGH, DER GESELLSCH. FUER NATUR- UND HEILKUNDE IN DRESDEN, DER OEKONOMISCHEN GESELLSCHATT IM KORNIGREICHE SACHSEN, MITGLIEDE ODER EHRENMITGLIEDE; DER FLORA ODER GESELLSCHAFT FÜR BOTANIK UND GARTENBAU IN DRESDEN D. Z, SECRBTAIRE.

\section{ERSTER THEIL. \\ S C H L U E S S E L FUER \\ HERBARIEN UND GAERTEN ODER \\ ANORDNUNG DES GEWAECHSREICHS

\author{
CLASSEN, \\ ORDNUNGEN, \\ FORMATIONSREIHEN, \\ UND UNTERGATTUNGEN, \\ FAMILIEN, \\ GRUPPEN, \\ GATTUNGEN \\ MIT REICHHALTIGEM REGISTER DER GATTUNGEN, UNTERGATTUNGEN, SY- \\ NONYMEN UND FRANZOESISCHEN NAMEN.
} N ACH}

\section{I I $\mathrm{I}$ I G,}

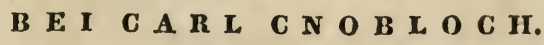

1828. 


$$
\begin{gathered}
34429 \\
\text { mar } 3,1923
\end{gathered}
$$

a) 
Natürlichkeit in die Betrachtung der Natur zu bringen, bleibt für die Wissenschaft immer das letzte Bestreben! So tönt uns der Nachhall aus dem thätigen Leben des grossen LinnéE. -

Er hatte sein Sexualsystem einem Zeitalter als Schliissel zu einem Chaos iibergeben, welches er selbst auf andere Weise aufzuräumen, damals noch nicht vermochte, aber er selbst fiihlte nur zu gut, dass in ihm sich ein Streben nach etwas Wichtigerem regte, und minus doctos, weniger Verständige, nennt er ausdrücklich diejenigen, welche die natürliche Methode gering schätzten.

Jussieu's grosser Geist brach die Bahn, von welcher Linnée zu früh abgerufen, auf ihr aber vielleicht mit grüsserem Ruhme als noch geschehen, zu wandeln verdient hätte. So entspann sich freier die nene, die geistreichere Art und Weise, die Natur zu erforschen, die passive Methode der Erkenntniss, die reine Hingebung an die Iebendige Schöpfung.

Während wir den blinden Eifer gewisser Linneaner, welche LinnéE's wahres Streben gänzlich verkannten, beklagen, während die in diesem verkannten Geiste der Zeit geschaffenen Gattungen uns quälen, erfreuen wir uns der bessern Erzeugnisse Anderer, welche, Lrnsée's höheren Andeutungen folgend, sich zu Jussieu's Forschungsweise bekannten.

Aber dennoch liegt hent zu Tage ein neues Chaos uns vor, und abermals ist der Faden gänzlich verloren, an wel- 
chen die Formen sich reihen sollen, der Ueberblick, der uns leiten kann, durch eine Welt voll Mannigfaltigkeiten und Räthsel in Bedeutung und Leben.

Was künnt' es uns nützen, wollte Jemand die seit Jussieu aufgestellten Familien des Pflanzenreichs, oder Ordnungen, wie man gewöhnlich das nennt, zusammenraffen, und in gehaltlosbeliebiger Unordnung unter einseitig aufgefasster Beziehung uns vorlegen?

„Zur Nachahmung würd' es doch führen!‘6 hör' ich erwiedern, und leugne das nicht, denn es giebt nichts so verkehrtes unter der Sonne, was nicht Nachahmung fände, aber eben darum will das auch sehr wenig bedeuten.

Manche haben doch schon den Gedanken ausgesprochen, es müsse auch in der Betrachtung der Natur ein gewisser nothwendiger Zusammenhang vorhanden seyn, und es dürfe möglich seyn, demselben anch die Betrachtung von ihr näher zu fiihren.

Auch ich bewahre diesen Glauben, und möchte ohne ihn dem Studium der Naturkunde niemals mein Leben geweiht haben.

Gewiss giebt es einen Faden, welcher durch das Labyrinth der Formen hindurch leitet, Beziehungen, welche das Studium der ganzen Natur beleuchten, die Entwickelungsstufen der organischen Körper nach ihrer Bedentung erklären, uns davon Kunde geben, welche Formen absolut und relativ höher, welche niedriger stehen, alle zu ihren einfachen Typen zuriickfiihrend, endlich uns selbst von der Chimäre der Anomalieen und Abnormitäten befreiend, uns erst durch ihre Allgemeinheit über das Wesen und die gegenseitigen Verhältnisse in Werth und Bedeutung der einzelnen, bisher immer losgerissen betrachteten Stufen belehren, und so uns endlich zu einer freudigen und befriedigenden Anschauung einer höhern Harmonie treu und sicher dahinleiten. 
Aber nur die gewichtige, aus Deutschland entsprungene, vom Auslande vielfach nach seiner Weise gemissdeutete Lehre von der Metamorphose, ist solchem Geschäfte gewachsen. Sie allein kann mit Erfolg das Streben beginnen, jenen Faden zu finden.

Wohl erkennt man hier und da die Nothwendigkeit, unter solchen Ansichten die Natur zu bearbeiten; aber wer soll das Ungeheure wagen, wer an das Werk gehen, die heut zu Tage so unübersehbar gewordenen Massen zu ordnen, sie alle vereint einem einzigen, grossen Blicke zu bieten?

So fragte wohl mancher, die Wichtigkeit und Grösse dieses Problems erkennend; aber wo wäre dann ferner die Masse von Pflanzen und Büchern beisammen, vereint mit nüthiger Sorgfalt und Ausdauer im Arbeiten, mit von den Fesseln der Gewohnheit befreietem Urtheil, mit reiner Hingebung und brennendem Eifer fïr die Forschung, ja was mir noch besonders wichtig scheint, mit gleichförmiger Kenntniss der ganzen Naturwelt im Einklange?

Auch ich kann natürlich nur sagen, dass es mir nicht gänzlich an einer von diesen Anforderungen gebrach, dass ich keine derselben durchaus vermisste; und so begann ich also den schwachen Versuch, so dass ich schon vor einem Iahrzehend eine Uebersicht des Gewächsreichs, meinen damaligen Hülfsmitteln und Kräften entsprechend, entwarf.

Die Art und Weise, wie diese Uebersicht aus Beobachtung der Entwickelung des Pflanzenlebens abstrahirt war, und das Schema der Anordnung, legte ich späterhin der im Iahre 1822 in meiner Vaterstadt Leipzig zum ersten Male vereinten Gesellschaft deutscher Naturforscher und Aerzte zur Beurtheilung vor. Manches gab ich seitdem über diesen Gegenstand heraus, ein vollständigeres Werk aber beschäftigt mich noch, und sein gegenwärtiger Band müge 
nur als Vorläufer dienen, müge mir die Hoffnung gewinnen, dass man nicht ein alltäglich Raisonnement, aus Halbwisserei oder Nichtkenntniss der wirklich offenbarten Natur entsprossen, erwarte.

Der Verständige wird einsehen, welche Masse Facta geprüft und durch eigene Erfahrung erlernt werden musste, um schon gegenwärtige Aufzählung zu schreiben.

Seit jener Zeit des ersten Entwurfes hat mich ein rastlos fortgesetztes Studium, eine durch eigene Untersuchung einer grossen Menge ron Formen bereicherte Kenntniss, in sofern befriedigt, dass ich grösstentheils die Wahrheit der Entwickelungsfolge, den Zusammenhang der Glieder, und nicht weniger die fortschreitende Verwandtschaft als anch die seitlichen Beziehungen der Familien, welche bei meiner Anordnung gleichfalls berücksichtigt sind, bestätigt fand.

Keineswegs will ich aber das kleine Buch für ein abgeschlossenes Werk ausgeben; bei so schwieriger Aufgabe kann alle Lösung nur schwacher Versuch seyn, und ich fühle selbst, dass einige Reihen noch nicht vollkommen natürlich, einige Gattungen, ihrem Platze zu entsprechen, noch nicht genugsam bekannt sind. Letztere sind meistens durch Fragzeichen ausgezeichnet, und überhaupt darf ich versichern, dass ich nichts hinschrieb, was ich nicht so gut, als ich konnte, geprïf hatte. Wo mir nicht Exemplare zur Hand waren, verglich ich die Abbildungen, welche existiren, und so blieben vorzüglich solche Gattungen im $Z$ weifel, für welche keine oder schlechte Abbildungen da sind. Leider schafft man aber auch heut zu Tage noch Gattungen ohne Prüfung ihrer Verwandtschaft, eine Erscheinung, die fast unbegreiflich wird, und der Wissenschaft mehr schadet als nützt.

Gelesen habe ich, was zu lesen war. Schon ror drei Ialiren war ich im Begriff, die zerstreuten Abhandlungen 
der Franzosen und Engländer, die ich mit eigner Hand ïbersetzt habe, herauszugeben, als die deutsche Sammlung von Roвеnт Bкоwrs Schriften erschien, und ich betrachtete dann die von mir übernommene Arbeit um so lieber als fïr eigene Belehrung veranstaltet, je leichter die ïbrigen Botaniker mit mir in den Stand gesetzt waren, jene so zweckmässig eingerichtete Ausgabe zu benutzen. Ich wïnschte, dass diese fortgesetzt würde, und werde aus der meinigen nur eine Auswahl der Abhandlungen der übrigen Engländer und der der Franzosen, zum Theil ganz, zum Theil im Auszuge geben.

In gegenwärtigem Buche ordnete ich die Pflanzengattungen, welche mir bis zu seiner Herausgabe bekannt wurden. Das Manuscript gab ich im Ianuar des laufenden Iahres an die Druckerei, reiste aber während des Sommers nach Holland und Frankreich, wodurch der Druck unterbrochen wurde. Ich lernte die trefflichen holländischen Botaniker kennen, und besuchte die schon in der Vorzeit so berühmten Gärten der reichen Batavia. In Frankreich genoss ich den Umgang der Männer, welche in der Wissenschaft seit lange voranleuchten; auch meine höchsten Wünsche wurden erfïllt, ich sahe Trianons Gärten, die Wiege der natiirlichen Ordnungen des Gewächsreichs; und Jussiev selbst, der ehrwürdigste der Veteranen, empfing mich mit einer Heiterkeit und Liebe, die ihn allein schon als wahrhaft grossen Geist und wahren Gelehrten beurkundet.

Aber auch mehrere der übrigen trefflichen Männer, welche Frankreichs Residenz zieren, erfrenten mich mit unerwarteten Beweisen ihres Wohlwollens, und verehrten mir ihre gelehrten, neuesten Werke. Vorzuiglich schätzte ich mich glücklich, nach langer Trennung von unserer gemeinschaftlichen Vaterstadt, Kuхти wieder zu sehen, und 
die in frühester Jugend geschlossene Freundschaft von ihm auch mir mit der Treue bewahrt za sehen, die stets diesen ausgezeichneten Gelehrten seinen Freunden so liebenswürdig gezeigt hat.

Durch so angenehm lehrreichen Umgang mit so gewichtigen Männern wahrhaft neu belebt, kehrte ich in das freundliche Vaterland zurïck, und fand Gelegenheit, bei fortgesetzter Correctur der Bogen so manches zu ändern und zu verbessern. So leiteten mich z. B. bei Anordnung der Rhamneen, Ternstroemieen und Guttiferen die ganz neuen vorzüglichen Bearbeitungen von Ad. Brongeniart und Cambessedes, deren freundschaftlicher Güte ich sie verdankte.

Raspail's Anordnung der Gräser, so wie W. Mexers System der Flechten, konnte ich deshalb nicht unbedingt folgen, weil ich nicht im Stande war, alle von mir aufgezählten Gattungen auf dieselben zu reduziren, und weil noch keine entsprechenden Reductionen der übrigen Familien vorhanden sind, so dass die Gleichförmigkeit für das Ganze der Ergebnisse heutiger Ansichten verloren gegangen seyn wïrde.

Von A. St. Hiraire's Flora des mittägigen Brasilien, welche Ad. de Jussiev und Jac. Cambessedes, allen Wünschen entsprechend, fortsetzen, fand ich bei meiner Rückkehr das siebente bis neunte Heft vor; von den Plantes usuelles desselben Verfassers, durch die genannten beiden Gelehrten gleichfalls fortgresetzt, brachte ich vierzehn Hefte mit.

Das Botanical Magazane von Curris und Sims besass ich bis dahin vollständig bis Tab. 2861, also von der durch Ноокеr zum grüssten Vortheil für das prächtige Werk besorgten New Series vom 54sten und 55sten Bande XXII. Hefte; von dem ähnlichen Prachtwerke The Botanical Re- 
gister, welches jetzt unter Lrnduex's Hand wahrhaft bliht, hatte ich zuletzt Tab. 1187 erhalten, und das Botanical Cabinet, worin so manche seltene Gattung gut und treu dargestellt ist, bis zu Tab. 1380. Hooken's Exotic Flora besitze ich, als Zeichen des freundlichen Wohlwollens ihres Verfassers, vollständig. Aus Press's Monographieen in den Reliquiis Haenkeanis, bedauerte ich, einige Gattungen dieses kenntnissreichen Autors in die Nachträge verweisen zu müssen, und von BLuME's schon mit grossen Merkwürdigkeiten beginnender Flora Javanica, lernte ich nur das erste Heft durch die Güte des freundlichen Verfassers kennen. Von Gaudichauds schönem Atlas zu seiner und FrexciNETs Reise benutzte ich neun Hefte; von allen grossen und wichtigen älteren Werken, vermisste ich kaum eines.

Von H. Cassini's Gattungen der Syngenesisten konnte ich einen Theil, als noch nicht beschrieben, für jetzt nur in der Reihenfolge auffiihren, in welcher dieser grosse Kenner und unermüdete, aber nicht genug von andern studierte Forscher sie selbst gegeben; einige Bände des Dictionnaire des scienses naturelles empfing ich auch zu spät, um sie noch zu benutzen, arbeite aber an einer fasslichen Uebersicht der ganzen Synanthereologie.

Die Autoren habe ich gewissenhaft angezcigt, die Rechte der Anciennität gewürdigt, sehr oft entrissenes Eigenthum zurückgegeben, die wichtigeren Synonyme beigesetzt, Untergattungen vorsichtig unterschieden, kleine Gruppen aus den am meisten verwandten Gattungen zusammengesetzt, diese zu höheren Gruppen versammelt, aus diesen Sectionen gebildet, welche sich zu Familien gliedern, Familien folgen in Parallelreihen ihren vou der Natur vorgeschriebenen Formationen, und diese erscheinen wieder durch ihre Ordnungen nothwendig verknüpft. Aus den 
Ordnungen bilden sich die natïrlichen Classen, und das einzige wahre Urprinzip, den in der Natur der Sache begründeten Unterschied zwischen einem künstlichen Systeme und einer natïrlichen Anordnung, habe ich anderwärts schon erläutert. ${ }^{* *}$ )

Das Register ist wohl unstreitig, so wie die Aufzählung selbst, ungeahtet mancher Mängel, das vollständigstc. Ich habe es mit Sorgfalt gefertigt, und anch die Nachweisung der französischen Namen darum nicht für überflüssig geha!ten, sondern dieselben sind aus den vielen Bänden der classischen Encyclopédic méthodique mühsam herausgesucht werden, weil die Franzosen in ihren lexicographischen Werken sie (leider!) voransetzen, auch besonders die Reisebeschreiber sich derselben bedienen.

Ob ich übrigens die Formen gekannt habe, dic ich verzeichnete, oder nicht, wird gewiss der am besten beurtheilen, der sie selbst kennt. Derselbe wird auch entscheiden, ob ich die vorhandenen Quellen gुehörig benutzte, und ihnen oder eigenen Ansichten zu folgen vorzog. Nie mochte ich blind einer fremden Meinung anhängen, und nie schien mir etwas verächtlicher und beklagenswerther, als das unbedingte Abschreiben der Schriften Anderer.

Wohl nur sehr wenige Gruppen, und kaum solche möchten übrig seyn, die ich nicht aus natürlichen Exemplaren erkannt habe. Ich freute mich nicht wenig, als ich nach der Rückkehr von meiner Reise, im Vaterlande noch die, auf der ganzen Reise vergeblich gesuchten $\mathrm{G}$ il liesiace n vorfand, vom Herrn Dr. Pöprig, meinem

*) In meiner Ausgabe von: Hemprichs G r undriss der Naturgeschichte. Berlin 1828. 
ehemaligen trefllichen Zuhörer in Leipzig, aus dem durch ihn der Wissenschaft wieder aufgeschlossenem Chili gesendet.

Ich eröffne überhaupt hiermit eine Uebersicht des Gewächsreichs, wie seit Jussieu Niemand gegeben. Wir vermissen gänzlich ein Buch, welches mit gleicher Liebe durch alle Stufen durchgeführt, nach gleichförmigen Ansichten von den Stufen zu Ordnungen und Classen erhoben wäre.

Ruhig und vertranensvoll erwarte ich die Urtheile derjenigen, denen ein Urtheil über diesen Gegenstand zukömmt. Und wenn das Beduirfniss unserer Zeit und die glückliche Richtung des Zeitgeistes, eine Menge ähnlicher Leistungen hervorrufen sollte, so kann doch immer nicht dasselbe wieder erscheinen, und viele Bahnen führen zur Wahrheit! Meine Nachfolger finden aber das schun gethan, was ich mühsam gesammelt.

In der That wünsche und hoffe ich indessen, dass schon durch dies Büchlein nicht wenige veranlasst werden möchten, dem wahren natürlichen Zusammenhange der Pllanzenwelt nachzuforschen, und so sich zu der Einheit im Anschauen zu erheben, welche einzig und allein erfreuliche Resultate für die Wissenschaft zu geben vermag.

Nur der Mangel eines Leitfadens hielt so manchen davon ab, dieser Richtung und Neigung zu folgen, er sahe sich genöthigt, seine Sammlungen im Widerspruche mit der Natur zu ordnen und zu studiren.

Schreibt man im Herbario die Nummern aus gegenwärtiger Aufzählung auf die Umschlagsbögen der Gattung. oder auf deren Namenschilder im Garten, so findet sich augenblicklich, mit Hülfe des Registers, jede beliebige Pflanze, und man ist aller Abänderung für immer ïber- 
hoben, da sich die später erlangten $Z$ wischenglieder leicht eintragen lassen.

Jede Verbesserung und gründliche Berichtigung des Buches werde ich dankbar erkennen und von Zeit zu Zeit nachtragen; und wünsche, dass dasselbe seinen beabsichtigten $Z_{w e c k}$ einigermassen zu erreichen im Stande seyn möge.

\author{
Dresden, \\ den 1sten Dezember 1828.
}

Der Verfasser. 


\section{GLASSISI. \\ F U N G I. \\ P I L Z E. \\ ORDO I. \\ GY M N O M Y C E T E S. HUELLENLOSE PILZE.}

Formatio $I$.

BLASTOMYCETES.

KEIMPILZE.

Fum. I. Uredinei.

Brandpitze.

Fam. II. Tubercularini.

Warzenpilze.
Formatio II. H YPHO M Y CE T ES.

FADENPILZE.

Fam. III. Byssacei.

Faserpilze.

Fam. IV. Mucedinei.

Schimmelpilze.

\section{O R D O II. \\ DERM T T MY CETES. \\ HUELL - PILZE.}

Formatio I.

GASTEROMYCETES.

BALGPILZE.

Fam. V. Sclerotiacei. Knorpelpilze.

Fam. VI. Lycoperdacei. Haarflechtpilze.

Fam. VII. Sphaeriacei. Schlauchpilze.
Formatio II. HYMENOMYCETES.

HAUTPILZE.

Fam. VIII. Tremellini. Gallertpilze.

Fam. Ix. Morchellini. Morchelpilze.

Fam. x. Hymenini.

Hutpilze.

CT Vorlänfige deutxche Erklärung dazu in meinem Buche: Botanik. Leipzig, 1828. von p. 154-198. 
Quoique les familles naturelles aient déjà fixé l'attention particulière d'un grand nombre de botanistes, leurs genres ne sont encore fondés ni sur des bases assez solides, ni sur des rapports assez naturels. C'est cependant la découverte de ces rapports qui est le but principal :que l'on doit se proposer aujourd'hui dans l'etude de la botanique. La philosophie de la science suppose non seulement des connoissances générales, mais aussi cette connoissance précise des espéces de chaque famille, qui seule peut guider dans la revision des geures déjà établis. Cette revision à la fois si necessaire et si difficile supposeroit un botaniste qui embrasse l'ensemble des espèces, et leurs deviations plus ou moins prononcées d'un type commun.

CH. KUNTH.

Mém. Mus. II. p. 62. 


\section{CIASSIS I. \\ $\begin{array}{lllll}\mathbf{F} & \mathbf{U} & \mathbf{N} & \mathbf{G} & \mathbf{I} \text {. }\end{array}$ \\ P I L Z E. \\ ORDO I. \\ GYM N O M Y CE T E S. \\ IUUELLENLOSE PILZE. \\ FORMATIO 1. \\ BLA T O M Y C E T S. \\ KEIMPILZE.}

FAMILIA I.

UREDINEI, BRANDPILZE。

A. Procformativi.

1. Mycomater. Fries.

2. Nosophloea. FR.

3. Phloeoconis. Fr.
4. Strumella. Fin.

5. Spermoedia. Fr. Sphucelia. ILeveill. (j, Naevia. Fr.

B. Entophyti.

a. Hypodermii.

\%. Spilocoea. $F_{R}$.

8. Ure do. Pers.

a. Ustilago. $\mathrm{L}$ k.

b. Sphaerotheca. Des v.

9. A e cidium. Pers.

u. Caeoma, Lk. diss. b. Hypodermium. L k, diss.

c. Roestelia. L k.

10. D ic a eoma. N. v. E.

11. Triphragium. Lr.

12. Phragmidium. Lk.

13. Xen odoclens. Schchlto.

14. Puccinia. Pers.

\section{b. Naemasporei.}

15. Phyllo edium. Fr.

16. Papularia. Fr.

17. Bullaria. DEC.

18. Septoria. Fr.

Septaria. Fr. non Z oolog. $^{\circ}$
19. N a cmaspora. Pers. Myxosporium. L k.

20. Blennoria. Movg.

21. Schizoderma. Kz. Itypodermium $\mathrm{L}$ k. sp. non diff.

\section{c. Stilbosporei.}

22. Cylindrosporium. GK. 24. Me lanconium. Lк. 23. Cryptosporium. Kz. 25. Didymosporium. N.r.l. 
26. Stilbospora. Pers.

2\%. Cheirospora. Moug.
28. Astrosporium. Kz.

29. Seiridium. N. v. E. Siridium Spr.

d. Sporodesmii.

30. Coryneum. N. v. E.

32. Podisoma. Lx.

31. Phragmotrichum. Kz. 33. Gymnosporangium.DEC.

Ambigui

34. Exosporium. Lk.

35. Sporidesmium. LK.
35 b. Corynodesmium. WahLR. Cephataeodium. Kz. 01.

FAMILIA II.

TUBERCULARINI. WARZENPILZE.

a. Tubercularii.

36. Fus a ri um. Lx.

88. Tubercularia. Ton.

37. Atractinm. LK.

b. Dermosporii.

39. Epicoccum. Lк.

41. A egerita. Pers.

40. Sclerococcum. Fr.

42. Dermosporium. Lx.

c. Scoriadei.

43. Dacrina. FE.

45. Ceratium. Arb. Scrwz.

44. Epichysium. Tod.

46. Scoras. Fr.

d. Cephalotrichii.

47. Sporocybe. Fr.

50. Antzina. Fr.

43. Amphichorda. Fr.

51. Isaria. P.

49. Cephalotrichum. Lк.

52. Sarcopodium. Ehrnb. 


\section{FORMATIO II. H Y P H O M Y C E T ES. FADENPILZE.}

FAMILIA III.

BYSSACEI. FASERPILZE.

A. Spurii.

a. Metcorici.

53. Hy pha. Pers.

54. Lanosa. Fr.

b. Germinatorii.

55. Herpotrichum.

56. Tophora. FH.

c. Cellularii.

57. Taphrina. Fr. Taphria Fr. non Zoolog. 58. Erineum. Pers.
59. Phyllerium. Fr.

60. Phlegmatiun. Fr.

\section{d. Destructorii.}

61. Lepraria. Acr. excl. sp. 62. Mycinema. FR.

\section{B. Genuini.}

a. Byssei.

63. Tetracolium. $K z$. 63 b. Torula. Pers. 64. O idium. LK. 65. Trentepohlia. Mant. 66. ? Sporendonema. Desmaz. 6\%. Byssus. L. Amphiconium. N.v. E.
Chroolepus. Ag.

68. Monilia. Pers. 69 Alternaria. N. v. E. 70. Amphitrichum. N. r.E. 71. Antennularia. Rchb. Antennaria. Lk. non Gärt.

b. Racodiucei.

72. Haplotrichum. Eschw. 79. Racodium. Pers.

73. Gliotrichum. Escrw. 80. Myxotrichum. Kz. 74. Trichosporum. Fr. 80 b. Ophiotrichum. Kz. 75. Oedemium. Lk. 81. Colletosporium. Lк. 76. Gonytrichum. N. v. E. 82. Нуросһиus. Енкмв. 7\%. Helicosporium? N. v.E. 83.? Clasterisporium. Schwz. 78. Helmisporium. Lk.

\section{Coenogenei.}

84. Ozonium. Pers.excl.e. 8\%. Coenogonium. Eнhno. 85. Gausapia. Fr. Dichonema. N. v. E. 86. Thermutis. Fr, 
88. Cilicia. Fi. 90. Lichina. Ag.

89. Cora. Fr.

\title{
d. Rhizomorphei.
}

91. Periconia. Tod.

95. Coenocarpus. Rebent.

92. Ascidiophora. Renp. 96. Synalissa. FR. Ascophora. Schwz. non Tod. 9\%. Thamnomyces. Ehnis. 93. Phycomyces. K\%. 94. Melidium. Eschw. 98. Hhizomorpha. Rти,

\author{
D $u$ b i i. \\ Dictyonema. AG. \\ Dematium Theleph. SPr. etc.
}

FAMILIA IV.

HIUCEDINEI. SCHMMELPLZE.

A. $G$ ? $n u i n i$.

a. Sporomyci.

99. Sporisorium. Lk. 100. Dematium. Pers.

Acladium. Lk.

Cladosporium. Lk. excl. c. 101. Polythrincium. Kz. 102. Sporophloeиm. LK.
103. Goniosporim. Lk.

104. Camptoum. LK. 105. Arthrinium. Kz. 106. Conoplea. Pers. 10\%. Psilunia. Fr.

\section{b. Trichomyci.}

108. Dendrina. Fr. 109. Fus isporium. LK. 110. Épochnium. LK.
111. Sepedonium. Iкк.

112. Thrichothecium. Lк. 113. Sporotrichum. Lк.

\section{c. Botrytidei.}

114. Acrosporium. N. v. E.

115. Botrytis. Mich.

«. Spicularia Pers, ex part. Polyactis. Lk. sp.

b. Cladobotryon. N. vo k.

c. Virgaria. N. v. E.

d. Botrytis. N. v, E. e. p.

c. Stachylidium. N. v.E. Spicularia, P. ex p.

$f$. Polyactis, Lk. diss. g. Cephalacladium. R.

h. Haplaria. Lk.

i. Haplotrichum. Lk. non Esehw. 116. Macrotrichum, Ginev. 11\%. Dimera. Fr. Diplosporium, Lk. 118. Aspergillus. Mсн. 119. Penicillium. LK. 120. Coremium. Lк. 


\section{d. Trichothecii.}

121. Circinotrichum. N. v.E. 123. Scolicotrichum. Kz.

Gyrotrichum. Spr.

122. Macrosporium. $F_{R}$.

Menispora. Lk.

Cumptosporium. Lk. S p r.
124. Chloridium. Lx. 125. Actinocladium. Enrxb. 126. Camps o trichum. Ehrse. 12\%. Chatopis. Grer.

B. Mucorini.

128. Hydronema. Carus.
a. Hydronematei.

b. Syzygitei.

129. Bactridium. Kz.

131. Azygites. Moug. 130. Coccotrichum. Lk. Alcurisma granulos. Mart.
132. Syzygites. Eнаты.

\section{c. Acremonii.}

133. Mусоgоne. Lк. 134. Acremonium. LK.
135. Verticillinm. N. r. E. 136. Stachylidium. Lr. Spondylocladium. Mart.

d. Mucorei.

13\%. Eurotium. L/k. 138. Thamnidium. Lk. 139. Mucor. Мгсн.
a. Rhizopus. Ehrnb.
b. Ascophora. Tod.
c. IIycirophora. Tod.

d. Mucor. Tod. 140. Didymocrater. MAkT. 141. Diam phora. Makt. 142. Aerophyton. Esciw. 143. Cephaleuros. $K_{z}$.

e. Stilbini.

144. Stilbum. Tod.

145. Chordostylium. Ton. 


\section{ORDO II. \\ DERMATOMYCETES. \\ HUELL - PILZE.}

\section{FORMATIO I. GASTEROMYCETES. \\ BALGPILZE.}

FAMILIA V.

SCLEROTIACEI. KNORPELPILZE.

BRÖCKLINGE

a. Perisporii.

146. Perisporium. Fr.

147. Lasiobotrys. Kz.

148. Podosphaeria. Kz.
149. Erysibe. Rebrt Erysiphe. Hdg. DC. Alphitomorpha. Wallr.

b. Sclerotii.

1óo. Neuroedium. Kz. 152. Acinula. Fr.

150 b. Sclerotium. Tod. 153. Periola. Fr.

151. Acrospermum. Tod.

c. Apiosporii.

154. Illosporinm. Mart. 15\%. Apiosporium. Kz. 155. Coniosporium. LK. 158. Podostrombium. Kz. 155 b. Collacystis. Kz. 156. Chaetomium. Kz. Fung. guiın.

d. Rhizogonei.

159. Rhizoctonia. DEC. 162. Mylitta. Fr. 160. Anixia. Fr. 161. Cenococcum. Movg. 163. Pachyma. Fr. Gemmularia. Rafin?

FAMILIA VI.

LYCOPERDACEI. HAARFLGHTPILZE. STREULINGE.

A. Trichophacelli.

AA. Trichodermacei.

a. BIyrothecii.

164. Trichoderma. Pens. 166. Dichosporium, N.v. E. 165. Myrothecium. Tod. 


\section{b. Pilacrini.}

16\%. Pilacre. Fr. 168. Onygena. Pens.
169. Hypochaena. Fr.

170. Asterophora. Dita.

c. Trichodermei.

171. Synconis. Fr. 172. Institiale. Fr. 173. Ostracoderma. FR.

d. Aethalini.
176. Lachnobolus. FH. 17\%. Reticularia. Bunt. Strongylium. Ditm. Diphtherium. E hrab. Lignidium Lk.
174. Amphisporium. Lr. 175. Hyphelia. Fr.

BB. Trichospermi.

a. Trichiacei.

182. Cirrolus. Mart.

183. Cribraria. Schrad. 184. Dicty dium. Schrad. 185. St temonitis. GLED.
178. Pittocarpium. Lk. 179. A ethalium. Lk. 180. Enteridium. Ehrnb. 181. Spumaria. Pers.

\section{b. Physarei.}

189. Licea. Schrad. 190. Perichaena. Fr. 191. Craterium. Trentep. 192. Leangium. Lk.
186. Diachea. Fr. 18\%. Arcyria. PEKS. 188. Trichia. Hath.
193. Phys arum. Pers.

194. Didy mi um. Śrнad. Diderma. Lk. Didymium. Lk. 195. L ycogala, Mсн.

c. Lycoperdinei.

196. Rimella. Rafin. 197? Acinophora. Rafin. 198. Tulostoma. Pers 199. Podaxis. Desv. 200. Lycoperdon. Mich. 201. Bovista. Pens.
202. Geastrun. Mich.
a. Geastrum, Desv.
b. Myriostoma. Desv.
c. Plecostoma. Desv.

203. Actinodermins. N. v.E. sterrebeckia. Lk.

204. Stemastrum Panin.

d. Sclerodermei.

205. Mitremyces. N. v. E. 208. Scleroderma. Pens. 206. Cauloglossum. Grev. 209. Pisocarpium. Lк. $20 \%$ Diploderma. Lк.

Polysuccum. DeC. 
Pisomyces, Fr.

Pisolithus. Alb. S c hw.

Polyperce. P.
210. Uperrhiza. Bosc. Hyperhiza. S p r.

211. Elaphomyces. N.v. E.

\section{B. Angiogastres. \\ a. Carpoboli.}

212. Pilobolus. Tod.

214. Thelebolus. Tod.

213. Sphaerobolus. Tod. 215. Atractobolus. Tod.

\section{b. Nidulariacei.}

116. Polyangium. LK. 218. Arachnion. Schwz. 21\%. Myriococcum. Fr. 219. Nidularia. Bull.

c. Tuberacei.

220. Endogone. LK. 221. Polygaster. Fr.
222. Rhizopogon. FH. 223. T u ber. Мich.

\section{d. Platloidei.}

224. Clathrus. Micri. 22\%. B a t arrea. Pens. a. Latemea. Tu op. Colouna- 228. P h a 11 us. Mich. ria Rafiu?

b. Clethroa. I. Br.

225. A s c r o ̈̈. Labili. 226. Lysurus. Fr. a. Phallns, Mich.

b. Dictyophora. Desr. Hymenophullus, N. v. E. Diclyopeplos. v. H ass.

FAMILI VII.

SPHAERLACLI. SCHLAUCHPILE.

SGHLAUCHLINGE.

A. Cytis $p \circ r$ : $i$.

a. Xylomacei.

229. Depazea. Fr. 230. Asteroma. DEC. 231. Ectostroma. Fr. 23\%. X y loma. Pens, 333. Bryocladium. K\%. 234. Sacidium. F. N.v. E. 235. Leptostroma. FR 236. Actinothyrium. Kz.
23\%. Pilidium. Kz. 238. Labrella. Fr. 239. Prosthemium. Kz. 240. Schizoxylon. Pens. 241. Sphinctrina. Fr. 242.? Labrella. Desmaz. 243.? Sphinctosporium. Kz. guian. 
b. Phomacei.

244. Phoma. Fr.

Cryptosphacria. Gre v.

245. Ceuthospora. Fr. 247 b. Cyrtocnon. Lx. 246. Hercospora. Fr. 24\%. Cytispora. Ehris. Bostrychia. Fr. 248. Sphaeronema. Fr. Splicieromyxu. Spr. 249. Zythia. Fh.

\section{B. Phacidiacei.}

a. Phrecidiei.

250. Excipula. Fr. 253. Phacidium. Fn.

251. Hysterium. Fr. 254. Rhytisma. Fu. 252. Graphiola. Port.

b. Cliostomei.

255. Cliostomum. Fr. 257. Lophium. Fr. 256. Actidium. FR.

258. Glonium. MühLxB. Solenarium. Spr.

c. I)ermei.

259. Heterosphaeria. Grev. 260. Cenangium. Fr.

a. Scleroderris. Fr. b. Triblidium. Rebent.

c. Clithris. Fr. 261. Dermea. Fr.

d. Pretellarii.

262. Tympanis. ToD. 263. Patellaria. Fr.
364. Stegilla. Rcub. slegia. Fr. nо и DeG.

$$
\text { C. } S p \text { h а e r } i \text { ace } i \text {. }
$$

a. Dothidini.

265. Ascospora. Fr. 266. Dothidea. FR.
Polystigma. D e C. 26\%. Vermicelaria. ToD.

b. Strigulini.

268. Myxothecium. Kz. 270. Strigula. Fr. 269. Meliola. Fr. 271 Corynelia. Fr.

Amphitrichum. Spr.

$$
\text { c. Dichaenei. }
$$

272. Gibbera. FR. 273. Ostropa. Fr.
2\%4. Hypospila. Hn. 275. Dichaena. FR. Phlacoseorict. Wallr. 
d. Sphaerini.

276. Sphaeria HaLL.

a. Epiphericae. Fr.

b. Subinnatae. Fr.

27\%. Valsa. Fr.

a. Heterostomum. Fr.

b. Valsa. Fr.

c. Perigrapba. Fr.

278. Hypoxylon. Bux.

a. Xylaria. Pers. b. Poronia. W.

c. Iypoxylon. Fr.

d. Diatrypa, Fr.

a. Nectria, Fr.

b. Hypomyce. Fr.

c. Hypocrea. Fr.

d. Cordyceps.Fr.

FORMATIO II. HYMENOMYCETES. HAUTPILZE.

FAMILIA TIIY.

TREMELLINI. GALLERTPILZE.

a. Hydrotremellini.

280. Actinomyces. Mexer.

b. Hymenuli.

281. Hy menula. Fr. 283. Phyllopta. Fr.

282. Agyrium. Fr. 284? Amphiphytum. Schivz.

c. Tremellei.

285. Pyrenium. Tod. 28\%. Naematelia. Fr. 286. Dacryomyces. N. r. E. 288. Tremella. DilL.

d. Cupulares.

289. Exidia. Fr. 291. Hirneola. $F_{R}$. 290. Lemalis. Fr.

e. Pileolares.

292. Guepinia. $F_{K}$. 293. Cyphella. Fk 294. Helotium. Tov. Peronci. P.

FAMILIA IX.

MORCHELLINI. MORCHELPILZE.

A. $\quad C \quad l a v a r i$.

a. Pistillarii.

295. Pištillaria. Fr. 29\%. Calocera. Fr.

296. Grinula. Fr. 
b. Clavulati.

298. Pterula. Fr. 300. Clavaria. VArLI. 299. Typhula. Fr. 301. Gomphus. P.

c. Mitrulati.

302. Geoglossum. Pers. 304. Spathularia. Pers. 303. Mitrula. Fn. Spatt:ulea. Fr.

\section{d. Pileolati.}

305. Sparassis. Fr. 30\%. Gomphora. Fir. 306. Hericium. Fr.

\section{B. Helvellarii.}

a. Stictei.

308. Solenia. Hofrm. non Ag. 309. Stictis. Pres.

b. Claviculcuè.

310. Ditiola. Fr.

311. Volutella. Tod.
312. Sarea. Fr.

313. Vibrissea. Fr.

c. Cupulati.

314. Bulgaria. Fr. 315. Ascobolus. Pers.
316. Peziza. Druc. 31\%. Rhizina. Fr.

P. Mitrati.

318. Le otia. Hru.

Hypolyssus. P.

Leotia. P.
319. Verpa. Sw. 320. Helvella. L. 321. Morchella. Drut.

FAMILIA $X$.

HYMENINI. HUTPILZT

A. Hydnei.

a. Auricularii.

322. Midotis. Fr.

323. Stereum. Lx.

324. Auricularia. Buhl.

a. Resupinatus.

b. Apus.

c. Pleuropus.

d. Mesopus.

325. Phlebia. Fr.

Richnophora. P.
326. Coniophora. Drc. 327. Thelephora. Eurr.

Zonaria. Rouss.

a. Phylacteria.

b. Apus.

c. Merisma.

d. Pleuropus.

c Mesopus. 


\section{b. Raduluvii.}

328. Gyrolophium. Kz. 332. Cyclomyces. Kz. ined. ined. exot.

329. Radulum. Fr.

330. Irpex. Fr.

331. Sistotrema. Pers. exot.
333. Hydnum. L.

$\alpha$. Resupinatus etc.

334. Fistulina. Bunt

E. Polyporei.

335. Porothelium. Fn.

336. Merulius. Haru.

Xylomycon. P.

33\%. Daedalea. Pers.

338. Polyporus. Mrch.

a. Resupinatus. b. Apus.

e. Merisiua.

d. Pleuropus.

e. Mesopus.

f. Polysticta.

339. Bolefus. Drut.

C. Agraicini.

340. Schizophyllum. Fr.

341. Nyctális. Fr.

342. Cantharellus. Ao.

Craterellus. $\mathbf{P}$.

Merulius, $\mathbf{P}$.

343. Xerotinus. Rснв.

Xerotes. Fr, non R. Br.

344. Lentinus. Fr.

345. Favolus. P. B.

346. Russula. Pers.

347. Galorrhoeus. Fr.

448. Coprinus. Pers.

349. Agaricus. L.

A. Coprinarius.

B. Pratellus.

a. Psathyra.

b. Psilocybe.

c. Hypholoma.

d. Gomphus.

c. Psalliota.

f. Volvaria.

C. Phaeotus.

“. Pratellarius.

b. Derminus.

a. Crepidotus.

b. Tapinia. c. Galena.

d. Naucoria.

e. Flammella.

$f$. Hebeloma.

$g$. Pholiota.

$\boldsymbol{E}$. Inocybe.

a. Inocybe.

F. Cortinarins.

a. Dermocybe.

b. Inoloma.

c. Phlegmacium.

d. Telamonia.

G. Hy porrhodius.

a. Nolanea.

b. Leptonia.

c. Eccilia.

d. Clitopilus.

$\boldsymbol{H}$ Leucosporus.

a. Pleurotus.

b. Mycena.

c. Collybia.

d. Omphalia.

e. Clitocybe.

$f$. Tricholoma.

g. Limacium.

h. Lepiota.

i. Amanita. 


\section{Fungorum genera:}

\section{a. Spuria.}

Mycoderma. P.

Xylostroma. Ton.

Himantia. P.
Phlebomorpha. P.

Fibrillaria. P.

Acrothamuium. N.v.E. Capillaria. P.

Athelia. P.

Ceratonema. P.

b. ulterius inquirenda.

Serratia bergamasch. osserv. Mycastrum. Rafin.

(Gasteromyc.?)

Dicarphus Rafin.

(Hydno affin.)

Priapus. Rafin.

(Iydno affin.)

Perisperma. Rafin.

(Lycoperdac.)

Phorima. Rafin.

(Boletac.)

Leptostroma. Rafin.

\section{(Boletuc.)}

Eriosperma. Rafin.

(Lycoperdac.)

Xylissus. Rafix.

(Mucoroid?)

Astrycum. Rafin.

(Lycoperd.)

Piesmyces. Rarin.

(Lycoperd.) (affin praec.)

Dysticia. Rafin.

(Cluthrus. absq. volva.)

Cerophora. Rafis.

(affin. Hydno.)

Calostoma. Dest.

(Scleroderma calost. P.)

Hepataria. Rafin.

(Tremellac.)

Hydrom yces. Rafir.

(Tremelluc.)

Hypolepia. Rarin.

(Boletac.)

Chaos. B. St. V.

(Theleph? sungu. Pers.)

(Desmaz. crypt. I.)

Inoconia. A. Lrb.

(bryogena, Racodiucea?) 



\section{CLASSIS II. \\ L I C H E N E S. P S O RA E. \\ $F \boldsymbol{L} C \boldsymbol{C} \boldsymbol{H} \boldsymbol{E}$. \\ ORDO I. \\ G $\quad$ Y $M$ M $N$ O $P$ S O R A E. NACKTKEIM - FLECHTEN.}

Formatio I.

BLAST OPSO RAE.

KEIMFLECHTEN.

Fam. XI. Pulverariae.

Staubflechten.

Fam. X11. Coniocarpicae.

Staubfruchtflechten.
Formatio II. HY PHOP SORAE.

FADENFLECHTEN.
Fam. XIII. Crateroideae. Kelchflechten.

Fam. XIV Sphaerophoreae. Siaubkugelflechten.

\section{ORDO II.

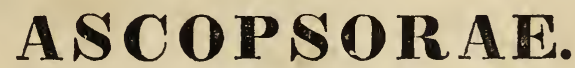

\section{SCHLAUCHKEIM - FLECHTEN.}

\section{Formatio I. \\ GASTEROPSORAE.}

KERNFLGHTEN.
Formatio II. HYMENOPSORAE. HAUTFLECHTEN.
Fam. XV. Porineae.

Warzenkernflechten.

Fam.XVI. Trypetheliaceae. Balgkernflechten.

Fam. XVII. Endocarpicae.

Lederkernflechten.
Fam.XVIII. Graphideae. Schriftllechten. Fam. XIX. Parmeliaceae. Schiisselflechten. Fam. XX. Cladoniaceae. Kopfflechten.

DF Vorläufige deutsche Erklärung dazu in meinem Buche: Botanik. Leipzig, 1828. von pag. $198-227$. 
Hinc ordines naturales minime in series disponi possunt, gradibus fere aeque distantibus, uti solent distribuere. In series parallelas potius disponendi, cum eaedem formae repetantur, tum inter ordines naturales genera agminaque intermedia admittenda, ita ut ordines vere naturales reficulo quasi junganfur. Hocce reticulum e legibus supra dictis intelligitur.

I. L I N K.

Elem. philos, bot. p. 430 . 


\section{CLASSIS II. \\ L $C \mathbb{E} \mathbb{N} \mathbb{E}$. F L E C H T E N. \\ ORDO I. \\ G Y M N O P S O R A R. NACKTKEIM - FLECHTEN.}

\section{FORMATIO $\mathbf{r}$ BLA S T O P S ORAE. KEIMFLECHTEN.}

FAMILIA $\mathbf{X I}$.

PULVERARIAE. STAUBFLECHTEN.

350. Pulveraria. Ach. Fuk. Lepraria. Ach. excl. excl.

Lepra. H a l 1. M e y.

FAMILIA XII.

CONIOCARPICAE. STAUBFRUCHTFLEGTEN.

a. genuinae.

351. Coniocarpon. DEC. 352. Trachylia. FH. Spilom. sp. A ch.

b. spuriac, in gemmultes solutae.

353. Arthonaria. Fr. 357. Spiloma. Acr. exp.

354. Incillaria. Fr. 358. Variolaria. Press.

355. Naevia. Fr. 359. Isidium. Acr. 356. Arthonia. Acr.

FORMATIO II.

\section{H Y P H O P S O R A E.} FADEN FLECHTEN.

FAMILIA XII.

CRATEROIDEAE. KeLChFLechten.

360. Calicium. Pens. a.Calicium Ach. b. Cyphelium Ach. 361. Coniocybe. Acrr. 
20 CLASS. II. LICH. OID.II. ASCOP. FAM. XV. PORIN. TR YPETH.

FAMILIA XIV.

SPHAEROPHOREAE. STAUBKUGELFLECHTEN.

362. Spha erophoron. Pers.

\section{ORDO II. \\ ASCOPSORAE. \\ SCHLAUCHKEIM-FLECHTEN.}

\section{FORMATIO 1. \\ GASTEROPSORAE. KERNFLECHTEN.}

FAMILIA XV.

PORINEAE. WARZENKERNFLECHTEN.

363. Porina. Acr.

Porophora. Mey.

364. Chiodecton. Acr.

365. Por othelium. Escrw.

Mycoporum. Mey.
366. Stigmatidium. Mer.

a. Nematora. F e e.

b. Phyllocharis. F e e.

c. Craspedon. Fee.

d. Melanophthalmum. F e e.

FAMILIA XVI.

TRYPETHELIACEAE. BALGKERNFLCHTEN.

a. Limborince.

36\%. Limboria. AcH. 368. Gyrostomum. Fr. 369. Urceolaria. Acr.
370. Thelotrema. AcH. Ocellularia Mey.

b. Vermucarinae.

371. Pyrenothea Fr.

372. Pyrenastrum Eschw. Parmentaria Fee.
373. Verrucaria Pras.

a. Pyrenula Ach.

b. Verrucaria, Ach.

c. Trypethelince.

374. Sphaeromphale Rchi. 366. Astrothelium. Eschw. Segestria Fr. non Zoolog. 375. Popodothion Fr. 37\%. Trypethelium. SPR. 
CLASS.II, LICH. ORD. II. ASCOP. FAM. XVII. END. PARM. 21

FAMILTA XVII.

ENDOCARPICAE. LEDERKERfLechten.

378. Sagedia. Acr. 380. Findocarpon. Hedw. 379. Dermatocarpon. Eschw. 381. Antrocarpon. Mer.

FORMATIO II.

HYMENOPSORAE.

\section{HAUTFLECHTEN.}

FAMILIA XVIIT.

\section{GRAPHIDEAE. SchriftFLech TEN.}

a. Genuincee.

382. Conangium. Fr.

383. Ustalia. Fr.

384. Sclerophyton. Eschw. 385. Lecanotis. Eschw. 386. Opegrapha Pers. a. Eruptoria. b. Scaphis:

c. Oxystoma.

d. Hysterina A ch. 38\%. Graphis. Acr. 388. Leucogramma. Mer. 389. Platygramma. Mex.

b. Glyphidene.

390. Coniol oma. Fu.

Asterisca. Mey.

391. Medusula. Eschw. 392. Glyphis. Acr.

c. Gyrophoreae.

393. Pyxine. Fr. 394. Umbilicaria. Hofrm. Gyrophora. Ach.

FAMILIA XIX.

PARMELIACEAE. Schüsselflechten.

a. Collemacere.

395. Micarea. Fr. 396. Ephebe. Fr.
397. Collema. Hoffm. 398. Leptogium. Fr. 
b. Parmeliacene.

399. Gyalecta. Acr. 400. Z e ora. $F_{K}$.

a. Tapelia. R.

b. Rinodina. F r.

c. Imbricaria. II o $\int \mathrm{fm}$.

401. Dirina. Fr. 402. Parmelia. Ach. adnatue:

a. Rinodina. Fr.

b. Psoroma. A c h.

c. Placodium. $\mathrm{H}$ o f $\mathrm{f} \mathrm{m}$. d. Amphiloma. Fr. liberae:

e. Xanthoria. Fr.

f. Physcia. A ch.

g. Imbricaria. II of $\mathrm{f} \mathrm{m}$.

h. Lobaria. II of $\mathrm{fm}$. 403. Sticta. Schreb. 404. Peltigera. Hoffm.

a. Saccolina. R.

b. Solorina. A c h.

c. Peltidea. A c h.

d. Nephroma. A c h.

c. Usneacene.

405. Cetraria. Acr.

a. Squamaria. II of $f$.

b. Physcia. Ach.

c. Cornicularia. A c h.

d. Alectoria. Ach.

406. Siphula. Fr.

Dufourea. A c h.

40\%. Roccella' Déc.

408. Ramalina. Acr.

409. Evernia. Acr. a. Thamnium, Vent.

b. Physcia. A ch.

c. Cornicularia. A c h.

d. Alectoria. A c h.

410. Us n e a. Dilk.

a. Thamuium. Vent.

b. Physcia A ch.

c. Connicularia. A ch.

d. Alectoria. Ach.

\section{Familia $\mathrm{xx}$.}

\section{CLADONIACEAE. Kopfflechten.}

\section{a. Thecidencene.}

411. Lecidea. Acr.

a. Rinodina. F r.

b. Psoroma. A ch.

c. Placodium. H o $f \mathrm{fm}$.

d. Imbricaria. H of $\mathrm{m}$.
412. Patellaria. Hoffm.

Bialora. Fr.

a. Rinodina, Fr.

b. Psoroma. Ach.

c. Placodium. Hoffm.

b. Capitulariaceae.

413. Bacomyces. Pers.

414. Cladonia. Hofrm.

Cenomyce. Ach.

a. Pycnothelia. Ach.

b. Scyphophora. Ach.

Capitularia. Flk. c. Schasmaria. Ach.

d. Helopodia. Ach.

e. Cladonia. Ach.

f. Cerania. Ach.

415. St e re oc a ulon. Schreb.

Thamnium. Vent. e. p. 


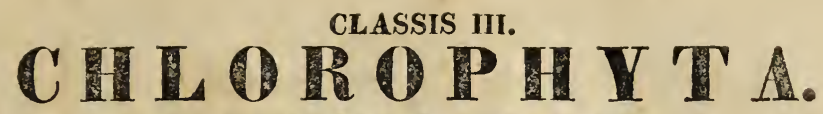 SAUGPFLANZEN.

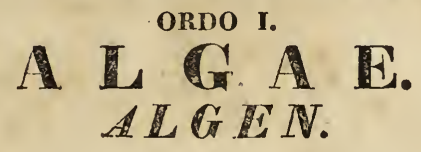

Formatio I.

GONG YLOPH YCAE.

KNOSPENALGEN,
Fam. XXI. Nostochinae. Gallertalgen.

Fam. XXII. Conferveae. Fadenalgen.
Fonnatio 1 .

ASCOPHYCAE.

BALGALGEN.

Iram. Ххй. Florideac. Haulalgen.

Fam. XXIV. Fucoicieae. Tangalgen.

\section{ORDO II. \\ I U $\triangle \mathrm{C}$. MOOSE.}

Formatio I. GONGYLOBRYA.

KN OSPENMOOSE.

Fum. XXV. Ricciea. Riccieen.

Fam. XXVI. Salviniacea. Sulviniaccen.
Fornatio I. SPORANGIOBPYA.

KAPSELMOOSE.

Fum. XXVII. Jungermanniea. Lebermoose.

Fam. XXVII. Bryoidea.

\section{ORDO III.}

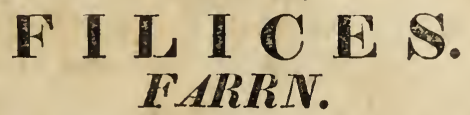

Formatio I.

THRYPTOPTERIDES. RISSFARR.

Fum. XXIX, Marsiliaceae. Wurzelfurrn. F(ım. XXX. Polypodiaceae. Wedelfarrn.
Formatio II. ANOEGOP'ERIDES. SPALTFARRN. Fam. XXXI. Osmindaceae Trualienfarrn. Fum. XXXn. Cycadeae. Pcalmenfurrn.

LF Vorläufige deutsche Erklärung dazu in meinem Huche: Botanik Leipzig, 1828. 
Nondum adest systema plantarum naturale, quod est unicum physiologicum.

F. C. L. FISCHR.

Certe affirmandum est, pancas naturae partes illum observasse, qui ubique utrumque sexum perfecte formatum adesse contendit.

F. C. L. FISCHR,

De Filic. propag. p. 19. 


\title{
CLASSISIIT. CHLOROPH YTA. SAUGPFL ANZEN.
}

\author{
ORDO I. \\ $A L G A$ E. \\ $A L G E N$.

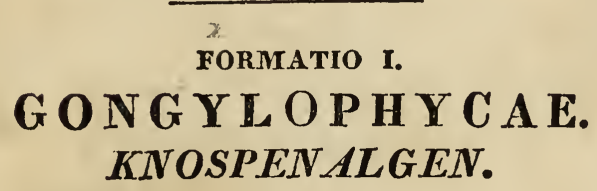

FAMILIA XXI.

NOSTOCHINAE. GallertalgeN.

A. Cha od i $n a e_{\text {. }}$

416. Hygrocrocis. Ag.

417. Phycomater. Fr.

418. Protococcus. Ag.

sphaerella. S o m m erf.
Chlorococcum. F r.

Coccochloris. Sp r. 419. Catoptridium. Brib.

\section{B. Undinea $e$.}

420. Nostoc. Adant.

421. Undina. Fr. Nost. sp. a uct.

422. Le athesia. Grat.

Corynephora. Ag.

Hydrocoryne Schwab.

423. Rivularia. RrH.

Linkia. Lgb.
424. Cha etophora. Schrk. Myriodactylon. Desv. 425. Schizonema. AG. 426. Hydrurus. Ag. 427. Palmella. Lgb. 428. Gloionema. AG. 429? Scythymenia. Ag.

C. Batrachospermeae.

a. Chordariaceae.

430. Chordaria. Lk. 431. Liagora. Ag.
432. Helminthora. Fr. Mesogl. sp. rubr. Ag.

b. Mesogloiariae.

433. Polyides. Ag. 434. Mesogloia. AG.
435. Aegira. Fr.

Link. Zosterae. Lgb. 
c. Thoreacerc.

436. Thorea. B. St. V. 438. Draparnaldia. B. St. V. 43\%. Batrachospermum. Rth. 439. Myxonema. Fr. Batrachosperma. B. St. V.

\section{d. Juptomitere.}

440. Pythium. N: v. E. 442. Saprolegnia. N. v. E. 441. Achlya. N. v. E. 443. Leptomitus. Ag.-gen.?

FAMILIA XXI'。

\section{CONFERVACEAE. Fadenalgen.}

A. Echinelleae.

444. Frustulia. Ag. 44\%. Meridium. Ag. 445. Gomphonema. Ag. 448. Echinella. Ag. 446. Achnanthes. B. St. V. 449. Acetabulum. Lamk*).

\section{B. Fragilarinae * $^{\text {*⿰氵 }}$ ).}

450. Di atoma. Dec.

451. Fragilaria. Lgb. Gaillonella B. St. V.
452. Meloseira. Ag. 453. Desm idium. Ag.

\section{Oscillatorinae.}

454. L y $n g$ by a. Ag. Priestleya. Ni eyen non Dec. 45\%. Stigonema. AG. 455. Oscillatoria. Vauch. 458. Protonema. Ag.

a. Anabaena. B. St. V.

b. Vaginaria. B. St. V. non Rich. IIicrocoleus. D e ma $z$.

c. Oscillatoria. B. St. V.

d. Dillwynella. B. St. V. Calothrix. Ag.
456. Scytonema. Ag. 459. Sphaeroplea. Ag. 460. B a $\mathrm{n}$ i a. Lgb. 461. L e m an ea. B. St. V. Gonycladon. Lk. Nodularia. Mert. Schrd.

*) planta! teste Schwergorr, Deurlus aliisque, plerumque incrustata tantum ad nos renit. :

**) Badiaga Buxr. Schwar. ap. Spreng. syst. veg. Nobis est Spongilla JaA r. Tupha Ok. Ephydatia Lanx. $c f r$. nostram Enumerationem animalium in opusculo: Zoologie, nach eignen Ansichten bearbeitet. Dresden b. Illscher. 1828. 


\section{U $l v a c e a c$.}

a. Zygnemere.

462. Tiresias. B. St. V.

Prolifera. Vauch. Oedogonium. L k.

463. Hydrodictyon. Rith.

464. Leda. B. St. V.

465. Mougeautia. Ag.
466. Zygnema. AG.

Conjugula. Vauch.

a. Globulina. Lk. Salmacis. B. St. V.

b. Spirogyra. Lk. Tenturidea. B. St. V.

c. Conjugata. Lk.

b. Conferveae.

46\%. Conferva. L.

a. Conferva. Gaillon.

b. Chloronitum. Gaillon.
468. Bulbochaete. AG. 469. Chantransia. DEC. 4\%0. Ectocarpus. Lgb.

c. Vaucherieae.

471. Codium. Stackh. Flabellaria. L a m k.

472. Ectosperma. VAUCH.

a. Botrydium. Wallr.

b. Vaucheria. Dec.
473. Bryopsis. Lamx.

474. Alysium. Ag.

375. Coccoderma. KNz. guian. ined.

\section{d. $U l v \in a e$.}

476. Valonia. GINN. 479? Scinaia. Brvon.

478. Ilea. Fr.

Solenia Ag. non $\mathrm{H}$ o f $\mathrm{fm}$.

479. UIva. L.
a. Porphyra Ag.
b. Phylloma. Lk.
c. Tetraspora. Lk.
d. Prasiola. Lk.

\section{FORMATIO II. \\ A S C O P H Y C A E. \\ BALGALGEN.}

FAMILIA XXIII.

FLorideaE. Hautalgen.

A. Ceramiaceae.

481. Digenea. AG.

482. Wrangelia. Ag.

483. Callithamnion. Lgb. a. Ceramium. Bonnem, Ag.

b. Boryna. Gaillon.

c. Gaillonia. Bonnem. Desmaz. Grateloupia Bonnem non $\mathbf{A g}$. 
484. Ceramium. RTh.

Grammita Bonnem.

Hutchinsia Ag. non RBr.

485. Poly siphonia. Grev.
486. Griffitsia. Ag.

48\%. Champia. Ag.

488. Gigartina. LaMx. Mesogl. capill. Ag.

\section{B. Sphaerooocceac.}

489. Rhytiphloea. AG. 490. Rhodomela. Ag.

a. Gigartina. Lgb.

b. Odonthalia. I.gb.

c. Volubilaria. Lamx.

491. Ptilota. Ag.

492. Liagora. Lamx.

493. Chondria. AG.

494. Dasia. AG.

495. Thamnophora. Ag.
496. Plocaria. N. V. E.

Gratcloupia Ag. non Bonnem. 497. Sph a erococcus. Stackr.

a. Chondrus. Lamx.

b. Gelidium. Lamx.

c. Hypnea. Lamx.

d. Plocamium. Lamx.

e. Gigartina. Lamx. sp.

$f$. Erinacea. Lamx. Delisea. Lam.

\section{Halymeniaceae.}

499. Amansia. Lamx.

500. Halymenia. Ag.

501. Wormskioldia. Spk.

Delesseria Lamx. falso, nec

Lessertia DeC.
Dawsonia. Lamx. non R. Br. 502. Claudea. Lamx.

Oneillia Ag.

FAMILIA XXIV.

\section{FUCOIDEAE. Tangalgen.}

A. Sporochneae.

503. Cladostephus. Ag. Myrsidium. Rafin.

504. Sphacelaria. Lgb. 505. Scytosiphon. AG.

Chordaria. Lk.
Chorda. Lgb.

506. Sporochnus. Ag.

Desmarestia. Lamx.

Desmia. Lgb.

\section{B. Dictyoteae.}

50\%. Encoelium. AG. Asperococcus. Lamx. 508. Dictyota. Lumx. a. Zonaria. Drap.

Padina. . I a m x.

b. Haliseris, Ag. 
CLASS. III. CHLOROPH. ORD. I. ALGAE. FAM. XXIV. FUCOID. 29

C. Laminariaceae.

509. Laminaria. LAmx.

510. Macrocystis. Ag.
511. Polyphacum. Ag. Osmundaria. L a m x. 611b. Eklonia. Hornem.

D. F $u$ c e $a$ c.

512. Furcellaria. Lanx. 514. Sargassnm. R.

513. Fucus. L. e. e.

a. Cystoseira, Ag.

a. Himanthalia. Sqb.

b. Sargassum. Ag.

b. Halidrys, Lgb. 


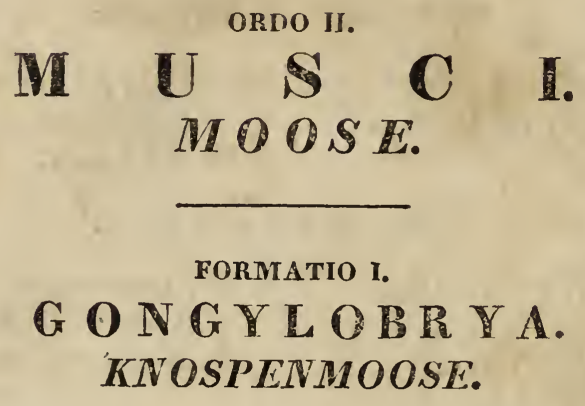

FAMILIA XX甲.

RICCIEA. Ricciees.

515. Riccia*). Mrch.

FAMILIA XXVI.

SALVINIACEA. SALVINTACEEN.

516. Azolla. Lam. 51\%. Salvinia. Guetr.

\section{FORMTATIO II. SPORANGIOBRYA. KAPSELMOOSE.}

FAMILIA XXVII.

IUNGERMLNNIEA. LEBERMOOSE.

A. Hypophyllina.

518. Targionia. Mrch.

519. Rupinia. L. Aitonia. Forst.
520. Grimaldia. Rado. non Schrk.

*) Ricciellas cl. Braurri specinina esse aliarum, aqua tantmm extensarnm, exinde steriliom, quia in aqua, sicnt Lemnae, (cf. libellnm; Botanik Leipz. 1828. p. 255.) maceratione iam solvuntur atque e parenchymate soluto gemmascunt, ex earum transitu anno praeterlapso in loco innndatn

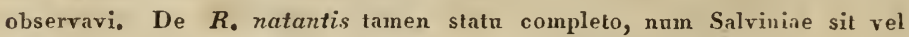
Marchantiae, quamquam copiosissimam vidi, nondum certus snm. 


\section{B. Epiphyllina.}

521. Sedgwickia. Bown.

522. Sphaerocarpes. Mrer.

523. Corsinia. Raddi.

Günthera. Trev.

524. Mo noclea. Hоок.

525. Anthoceros. L.

526. Blandovia. W.

527. Fimbriaria. N. v. E.

528. Marchantia. Mrch. a. Duvalia, N, ง, E.

b. Dumortiera. N. v. E.

c. Fegatella. Raddi.

d. Rebonillia. Raddi.

529. Lunularia. Mich.

Staurophizora. W.

530. Merkia. Borkн.

a. Roemeria. Radd.

b. Pellia. Radd.

c. Metzgeria. Radd.

C. Entophyllina.

531. Lejeunia. A. Lrв.

Carpolepis. P. B. e.e.

532. Jungermannia. Mrcr.

a. Carpolepis. P. B.

b. Conianthus. P. B.

a. Bellinginia. Radd.

b. Antoiria.- Radd. c. Frullania, Radd.

d. Candollea. Radd, non al.

e. Jungermannia. Radd.

f. Fossombronia. Radd.

g. Calypogeia. Radd.

h.? Porella. Dill.

D. Eleutherophyllina.

a. Sphagnea.

533. Sph agnum. L.

b. Andreacacea.

534. Andreaea. Lhrh.

FAMILIA XXVIII.

\section{BRYOIDEA. LAUBMoOSE.}

A. Hypophyllocarpia.

(IUNGERMANNIACEA).

535. Helicophyllum. Brid. 53\%. Cyathophorum. P. B. Aniclangium. $\mathbf{H}$ o o k. Arn. . Anoeclangium. Hdg. Schistidinn?. Brid.

536. Hypopterygium. Brin.

Hookerice. sp. Sm.

538. Racopilum. P. B. Aubertia. P. B. msc. non B.

St. $\mathbf{V}$. 


\section{B. Entophyllocarpia.} (FILICOIDEA.)

539. Schistostega. W. M. Skitophyllum. La P y 1 . Dicksonia. Ehrh, non Herit. 542. Phyllogonium. Brid. 540. Drepan ophyll um. Rich. non Wib. a. Phyllogonium. Brid.

b. Eustichia, Brid.

541. Octodiceras. Brid.

Harrisonia. Ad a ns. c. al. Cecalyphum. P. B. 543. Fissidens. Hdg. Skitophyllum. L a P y l. 544. Rhizogonium. Brid.

\section{Maschalocarpia.}

\section{a. Fontinaloidea.}

545. Hookeria. SM. 549. Es enbeckia. Brid. Pterygophyllum. B ri d. Hypopterygium. B rid. 550. Cryphaea. Web. Pilotrichum. P. B.

Chactophora. Brid. nou al. 551. Pilotrichum. P. B.

546. Distichia. BRID. Neckerae sp. dist. Hdg. a. Pilotrichum. P. B. Pilotrichum. P. B.

54\%. Erpodium. Brid. Anoectangii sp. Spr.

548. Leptodon. WEB. Lasia. B ri d.

b. NIetorium. Brid. 552. Lepidopilum. Brid. Trachypodium. Brid. 553. Fontinalis. L. 554. Spiridens. N. v. E. Leskea. S p x. Rec.

b. Hypnoidea.

a. Pleuridia.

555. Pleuridium. Brid.

bb. Climacioidea.

556. Trachyloma. Brid. 55\%. Climacium. W. M. a. Zygotrichia. Brid.

b. Porotrichum. Brid.

\section{cc. Leucodontina.}

558. Rhegmatodon. Brid. 563. Astrodontium. Schwg. 559. Schlerodontium. Schwr. 564. Antitrichia. Brid. 560. Leucodon. Schwgr. 561. Lasia. P. B. Anomodon. Hook. et Tayl. 562. Dicnemon. Schwgr. 565. Trichomitrium. BRID. (I. $p . \mathrm{XLV}$. nomen.)

Eucnemis. Brid, non Zoolog. 
566. Anoectangium. Hog. 568. Orthodontium. Schwg. 56\%. Neckera. Hog. Neckera. Sp r. Rec.

a. Neckera. Röhl.

b. Cyrtopodia. Röhl. 569. Actin od on tium. Schwg. Hookeria. S p r. Rec.

Cyrtopus. Brid. Eleutheria. P. B.

5\%. Daltonia. Houk. et Tayx.

\section{ee. Leskeacea.}

5\%1. Fabronia. Raddr.

573. Anacamptod on. Brid. Pterophyllum. Brid. msc. 5\%4. Pylaisaea. Desv.

572. Maschalanthus. Hdg. Fir. 575. L es k ea. Hedw.

Pterigynandrum. Hdg.

Pterogonium. Sw.

Maschalocarpus. Spr. a. Leskia. Brid.

b. Omalia, Brid.

576. J sothecium. BrId.

ff. $\quad$ Hyp $n \in \boldsymbol{a}$.

57\%. Hуриит. L. 5\%8. Stereodon. Brid.

D. Acrocarpia.

a. Splachnoidea.

aa. Phascea.

5\%9. Archidium. Brid.

580. Phascuin. L.

581. Physedium. Brid.
582. Bruchia. Schwg. Saproma. B rid.

583. Voitia. Hornsch.

bb. Gymnostomea.

584. Gymnostomum. Hdg. 585. Hymenostylium. Brid.

a. Pottia. Ehrh. Anodontium, Brid.

b. Physcomitrium. Brid

c. Hyophila. Brid.

Rottleria. Brid, non Rxb. 586. Pyramidium. BRID.

Pyramidula. Brid.

58\%. En tost h y m eni um.Brid.

cc. Grimmiacea.

588. Harrisonia. Adans. e. e. 591. Dryptodon. Brm. Schistidium. Brid.

Campylopus. P. B.

Luida. Ad ans.

589. Grim mi a. HDg. 592. Racomitrium. Bro 590. II y d r o p o go n. Brid. Dryptodon. B rid. 593. Olomitrium. BrIn. 
594. Orth ot he ca. Brid.

595. Cinclidotus. P. B. Sekra. Ad ans.
596. T etraphis. Hog.

a. Tetrodontium. Schwg.

b. Tetracmis. Brid.

dd. Campylopodia.

597. Campylopus. Brid. 598. T h y a n o m it ri um.

Scrwg.

ee. Splachnea.

599. O edip od i um. Schwg. 600. Orthodon. B. St. V. 601. Aplodon. BBr.

a. Dissodon Grew. et Aru.

Eremodon. Brid.

b. Auchenangium. Brid. 602. Splachnum. L. $a$. Pycnapophysium. R.

b. Cystapophysium. R.

c. Discapophysium. R. 603. Sys ty li u m. Hornsch. 604. Tayl oria. Hook. Hookeria. S ch 1. Phrissolrichia, Bxid. msc.

b. Dicranoidea.

aa. Weissiacea.

605. Weissia. Hog. Afzclia. Ehrh. non al. Cavonillea. B o rk h.

606. Discelium. BrId.

60\%. Catoscopium. Brid. 612. Calymperes. Sw. Melania. B rd. ol.

608. Coscinodon. Spr. Anacalypta. Röhl.

609. O reas. Brid. non Cham. 614. Le ucophanes. Brid. Auchenangium. Brid. Msc.
610. Sy r rhopodon. Schwg.

a. Cleistostoma, Brid.

b. Syrrhopodon. Schwg.
611. Tr a chym itri um. Brid.

613. O ctoble pha rum. Hog. Octoblcpharis. Schreb.

Luida. A d a n s.

bb. Dicranea.

615. Oncophorus. Brid. 618. Ceratodon. Brid.

616. Trem a todon. Brid. Acgicerus, Green non Gärt. 61\%. Dicranu m. Hog.

$$
\text { cc. Orthotrichea. }
$$

619. Brachystel e um. Rchв. Brachypodium. B ri d.nonP. B.

620. Gl y phom itrium. Brid. Griffithia. R. B r. non A g.

621. Orth ot richum. Hog. a. Brachytrichum. Röhl.

b. Orthotrichum. Röhl. 622. Ulota. W, M.

a. Dorcadion. Adans, non Zoolog.

b. Blankana. Ad. 
623. L e i o the ca. Brid.

624. Macromitrinm. Brid.

625. Schlotheimia. Brid.

$$
\text { non Strine. }
$$

626. Zygodon. Ноoк. et TAYL.
Amphidium. N. v. F.

Gayea. $R$ a d di non $K$ e r r. 62\%. Codonoble pharum.

Schwg.

\section{dd. Trichostomea.}

628. Trichostomum. Hog. 632. Desmatodon. Brib.

629. Didy modon. HDG. Ditrichum. Tim m.

630. Pilopogon. Brid.

631. Pla u belia. Brid.
633. Le u colo in a. Brid.

634. Z y gotrichia. Brid.

cf. Climacium. W. M.

ee. Barbulacea.

635. Barbula. Hog. Mollia. S ch r k. non a 1. Streblotrichum. P. B.
636. Syntrichia. Mohr. Tortula. S chwg. non W. 63\%. Encalypta. Hog.

\section{c. $B a r t r a m i a c e a$.}

aa. Bryacea.

638. Cynodon. Brid.

Cynodontium. Hdg.

Swarzia. H d g. n o n al.

Didymodon. S chreb.

Afzelia. Ehrh.

639. P t y ch os to m u m.

\section{HoRnsch.}

Brya incompl. S p r. Rec. c. Polla. Adans.

643. Cynclidium. Sw. Amblyodon. P. B.

644. Leptostomum. RBr. Orthopyxis. P. B.

645. Brachymenium. Hoor. Brya incompl. S p r. Rec.

646. Le p totheca. Schwg.

640. Hemisynapsium. Brid. 647. Macrauchenium. Brrd. 641. Cladodium. BrID. 642. Bryum, L.

648. Macrothecium. BRID. Acidodontium. $\mathbf{S c h}$.

a. Webera. Hdg. non Schreb. 649. Pohlia. Hdg.

b. Bryum. Dill.

Amphirrhinum. Green.

bb. Mniacea.

650. Paludella. Ehrh. Orthopyxis. P. B.

651. Mnium. Dill. 652. Aulacomnion. Schwr.
Gymnocephulus. $8 \mathrm{chwg}$. non $\mathrm{Z} \circ 010 \mathrm{~g}$.

653. Arrhenopterum. Hog. 
36 CLASS. III. CHLOR. ORD.II. MUSCI. FAM. XXVIII. BRYOYD.

\section{cc. Conostomea.}

654. Bartramia. Hog.

655. Philonotis. Brid. 656. Gly phocarpa. RBr.

Glyphocurpus. Brid.
65\%. Cryptopodium. Brid. 658. Plagiopus. Brid. 659. Conostomum. Sw. 660. T immia. HDG.

\section{dd. Funariacea.}

661. Enthostodon. Schwg. 662. Funaria. Hog. 663. Meesia. Hog.
Amblyodon. P. B.

664. Diplocomium. W. M. Tristichis. Ehrh.

$$
\text { d. Polytrichoidea. }
$$

665. Diphyscinm. W. M. Hymenopogon. P. B. 666. Dawsonia. RBr. Triphocoma. La P y 1 . Stylocomium. Brid. msc. 667. Lyellia. RBr.
Gomphoplorus. Brid. msc. 668. Psilopilum. Brid. 669. Catharinea. Eнrн. 670. Pogon atum. P. B. 671. Polytrichum. L. 


\section{OR D O III. \\ F $\quad$ I $\quad \mathbf{L} \quad I \quad C \quad \mathbb{S}$ S. $\begin{array}{llllll}F & A & R & R & N\end{array}$ \\ FORMATIO 1. THRYPTOPTERIDES.}

RISSTARRI.

FAMILIA XXIX.

MARSILIACEAE. WURZELFARN.

672. Pilularia. L.

6\%3. Marsilea. L.

FAMILIA $\mathrm{x} \times \mathbf{x}$.

POLYPODLACEAE. W FDFLARN.

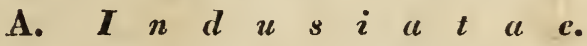

a. Hymenophylleae.

(Jungermanniaceae.)

674. Hymenophyllum. Sin. a. Feea: B. St. V. 6\%5. Trichomanes. L. b. Hymenostachys.

b. Cyutherceue.

676. Alsophila. RBr. 6\%8. Hemitelia. Br. 67. Chnoophora. Kaulf. 679. Cyathea. Sm. Trichopteris. Prs1.

c. Aspidiuceue.

681. As pidium. Swr. Nephrodium. MIchx.

b. Ophiopteris, Reinw.

c. Kumohra. Radd.

a. Neuronia. Don.

d. Davalliaceac.

682. Davallia. Sm.

683. Peranema. Don. 684. Saccoloma. KaUeF. 685. Dicksonia. Henit. 686. Bal an ti um. KAULF.
68\%. Cibotium. Kaulf.

Pinonia. G a ud i ch. 688. Lecanopteris. Rernw. a. Ouychium. Reiuw. b. Lecauopteris, Reiuw. 
38 CLASS. III. CHLOROPH. ORD. III. FILIC. FAM. XXX. POLYPOD.

e. Adiantheac.

689. Adiantlinm. L.

690. Cheilanthes. Sw.

691. Cassebeera. KaUlf.
692. Lindsaya. Drx.

Schizoloma. Gaudich.

\section{f. Pteroideac.}

693. Pteris. L.

694. Vittaria. SM.

695. Leptostegia. Don.
696. Lonchitis. L.

69\%. Monogramma. Schk.

698. Anthrop hy m. Kaulf.

g. Asplenioideae.

699. Asplenium. L. 703. Diplazium. Sw. 700. Allantodia. RBr. 701. D area. W. 704. Didymochlaena. Dest. 702. Scolopendrium. SM.

h. Blechnoideae.

705. Allos orus. Bernh. Cryptogramma. $\mathrm{RBr}$.
Tegularia. Reinw.

706. Ony chinm. KaUlf. 70\%. Hymenolepis. KaUlf. 711. Blechnum. L: 708. Leptochilus. Kaulf. 712. Sadleria. Kaulf. 709. Ellebocarpus. Kaulf. 713. Woodwardia. SM. Ceratopteris. Gaudich. 714. Doodia. R. Br.

\section{i. Onocleaceae.}

715. Onoclea. L. 716. Struthiopteris. W.

k. Woodsi e a $e$.

(subindusiatae.)

71\%. Woodsia. RBr. 718. Niphobolus. KaULF. 719. Pleopeltis. K. H. B.
720. N othochlaena. R. Br. Cincinalis. Desv, cum al.

B. $N \quad \boldsymbol{u} \quad d \quad a \quad e$. (GIMNOPTERIDES.)

721. Taenitis. Sw. 722. Marginaria. 723. Polypodium. L. a. Dipteris, Reinw.

b. Drymaria B. St. V.

c. Lastrea. B. St. V. 
CLASS. III. CHLOR. ORD. IIT. FILIC. FAM. XXX. POLYPOD. 39

724. Cochlidium. Kaulf. 730. Gymnogramma. Desv. 725. Ceterach. W.

731. Hemionitis. L.

726. Xiphopteris. Kaulr. 732? Adenophorus. Gaudre. 727. Selliguea. B. St. V. 733. Acrostichum. L. 728. Grammitis. Sw. 729. Meniscium. Schri.

734. Polybotrya. K. H. B. Olfersia. Radd.

FORMATIO II.

ANOEGOPTERIDES.

SPALTFARRN.

FAMILIA XXXI.

OSMUNDACEAE. Traubenfarkn.

A. Schizaeacea e.

735. Aneimia. Sw. 736. Schizaea. Sw. Lophidium. Rich.
737. Lyg odium. Sw. Hydroglossum. W. 738. Mohria. Sw.

B. Osmundeae.

739. Todea. W. 740. Osmunda. L.

D. Gleichenieae.

741. Gleichenia. Sw. 743. Platyzoma. RBr. 742. Mertensia. W. non Thb.

et al.

D. Marattiaceae.

744. Marattia. Sw. 746. Angiopteris. Horrm. 745. D a n a a. Sw. 
40 CLASS, HI. CHLOROPH. ORD, HI, FILIC. FAM. XXXII, CYCAD.

FAMILIA XXXIr.

CYCADEACEaE. Palmenfarik.

(FILICES CENTRICAE.)

A. Ophioglosseac.

747. Ophioglossum. L.

Botryopteris. Prsi.

748. Helm inthostachys. 749. Botrychium. Sw.

KAULF.

B. Zamieae.

750. Z a in ia. L. 751. Arthrozamia. Rchb.

C. Cycadeae.

752. Cycas. L. 


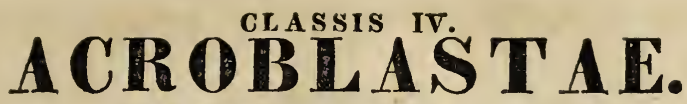 SPITZKEIMER. ORDO I. \\ RHIZO - ACROBLASTAE. WURZEL - SPITZKEIMER.}

Formatio I.

L I M N O B I A E.

TAUCHERGEWAECHSE.

Fam. XXXIII. Isoeteae.

Brachsenkrïuter.
Formatio II. HELOBIA E. SCHLAMMWURZLER.

Fam. XXXYI. Typhaceae. Rolvkolben.

Fam. XXXIV. Potamogetoneae. Fam. XXXYI. Alismaceae. Laichkviuter.

Fam. Xxxy. Aroideae. Arone.

Wasserliesche.

Fam. XXXIII. Hydrocharideae. Nixblumen.

\section{CAULO - ACROBBLASTAE. ST AMM - SPITZKEIMER. \\ Formatio I. \\ GLUMACEAE. \\ SPELZENGEWAECHSE. \\ Formatio II. \\ E NSA T A E. \\ SCHWERTELGEWAECHSE.}

Fam. XXXIX. Gramineae.

Grïser.

Fam. XL. Cyperoideae. Cypergrïser.

Fum. XLI. Commelinaceae. Liliengräser.

Fam. XLII. Irideae.

Iris-Schwertel.

Fam.XLIII. Narcisseae.

Narzissen - Schwertel.

Fam. XLIV. Bromeliaceae. Ananas - S'chwertel.

\section{O R D O III. \\ PHYLLO - ACROBLASTAE. BLATT - SPITZKEIMER.}

Formatio $\mathbf{I}$.

LILIA C EA E.

LILIENGEWAECHSE.

Fum. XLV. Juncaceae.

Simsenlilien.

Fam. XLVI. Sarmentaceae.

Zaukenlilien.

Fam. XlVII. Coronariae. Kronlilien.
Formatio II.

P A L M A C E A E.

PALMENGETAECHSE.

Fam. XLVIII. Orchideae.

Orchideen.

Fam. XLIX. Scitamineae.

Bananen.

Fam. L. Palmae.

Palmen.

L Vorläufige deutsche Erklärung dazu in meinem

Buche: Botanik. Leipzig,, 1828, pag. $294-370$.

warum sie nicht Monocotyledoneen heissen können : p. 371. 
Il est evident, que pour classer les plantes selon les rapports naturels, on ne doit pas àvoir égard à l'absence, à la présence et an nombre des Cotyledons. MIRB. Ann. d. Mus. XVI. p. 420.

Les cotyledons sont jugés indispensables par les botanistes, parceque de leur absence et de leur nombre, on a fait le caractère des trois grandes séries du règne végétal: mais d'autres caractères plus généraux, plus évidens, moins sujets à anomalies, commencent déja à paroitre. Corkea de Serra Annal. de Mus. XIV. p. 81. 


\title{
CLASSIS IV. \\ ACROBLASTAE. SPIT TZKEIMER.
}

\section{ORDO I. \\ RHIZO - ACROBLASTAE. \\ WURZEL - SPITZKEIMER.}

\author{
FORMATIO I. \\ $\begin{array}{lllllllll}\text { L } & \text { I } & \mathbf{M} & \mathbf{N} & \mathbf{O} & \mathbf{B} & \mathbf{I} & \boldsymbol{A} & \mathbf{E} .\end{array}$ \\ TAUCHER GEWAECHSE.
}

FAMILIA XXXIIr.

ISOETEAE. Brachenkraeuter.

753. Is oetes. L.

FAMILIA XXXIV.

POTAMOGETONEAE. FLUTHKRAEUTER.

A. Zostereac.

754. Zostera. L.

755. Thalassia. Banks.

Zost. sp. F orsk.
Kernera. W. -Caulinia. DC. 758. Halophila. A. P. Th. 756? Phucagrostis. Caror. 759. Barkania. Ehrnb. 757. Posidonia. Caul. et Koen.

\section{B. Potamogeae.}

760. Z annichellia. L. 761. Ruppia. L.

762. Graumiillera. Rchb. Rupp. antarct. LaB.
763. Potamogeton. L.

a. Peltopsis. R afin.

b. Potamogeton. Rafin. 
44 CLASS.IV.ACROBL. ORD. I. RHIZO-ACR. FAM. XXXV.AROID.

FAMILIA XXXV.

AROIDEAE. AROIDEEN.

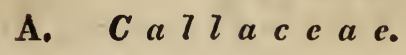

a. Lemneae.

764. Lemna. L.

765. Horkelia. Rchb.

Lenticula. T. Wolfia. Hork non Spr.

b. Pistiaceae.

z66. Pistia. L.

76\%. Ambrosinia. L.

c. Calleae.

768. Cryptocoryne. Frsch., 772. Calla. L.

6. Clociasis Xink

Ictodes. Big e 1.

769. Arisarum. T.

7\%0. Arum. L.

a. Arum. A uct.

b. Candarum. Rchb.

Arum Canda Rxb. non Canda.

$L$ a m x. Z o ol.

7\%3. Richardia. Knth.

Calla. $\mathbf{L}$.

Zantedeschia. \$p pr.

774. Caladium. Vent.

a. Caladium. A uct.

b. Culcasia. P. B e a u v

7\%1. Symplocarpus. Salisb.

c. Baursea. Angl. Sputhyema. Rafin.

d. Pothoinae.

7\%5. Dracontium. L. 7\%6. Pothos. L.

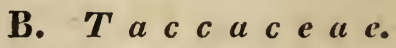

a. Gymnostachyeae.

7\%. Gymnostachys. RBr. 778. Acorus. L.

b. Tacceae.

799. Orontium. L.

780. Rohdea. Rth.

781. Tup istra. KER.

782. Aspidistra. Ker,

783. Sanseviella. Rchb.
Sanseviera. A $\mathbf{u} \mathbf{d} \mathbf{r}$.

784. Eriospermum. Jace. 785. Tacca. Forst.

786. Roxburghia. W. 
CLASS. IV. ACR. ORD. I. RHIZO-ACR. FAM. XXXV. AROID. 45

$$
\text { C. Nepenthea e. }
$$

78\%. Peliosanthes. ANdr. 788. Nepenthes. L. phyllamphora. Lou r.
Cantharifera. Ru mph. 789. Sarracenia. L.

FORMATIO II.

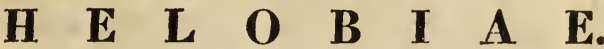
SCHLAMMTURZLER.

FAMILIA XXXVI.

TYPHACEAE. ROHRKOLHEN.

790. T yph a. L.

FAMILIA XXXVII.

ALISMACEAE. Wasserhiesche.

A. Saurureae.

791. Sparganium. L.

792. S a ururus. L.

793. Aponogeton. Thrb.
Spatium. L o u r. 794. H o ut u y i a. Thub. non Houtr.

B. Alismeae.

795. Alisma. L.

796. Actinocarpus. RBr. Damasanium. Juss. 79\%. Hydrocle is. Rich. 798? Hydromystria. W.Mex. 801? L e wisia. Prsh.
799. Limnocharis. Hb. Bpl. non Limnochares Latr. Zoolog. 800. H y d rog e t o n. Pers. Ouvirandra. A. P. Th. 
46 CLASS. IV.ACR. ORD. I. RHIZO - ACR. FAM. XXXVIII. HYDH.

808. Dam a sonium. Schreb. 809. Limuobium. Rrch. Ottelia. Pers.

B. Hydrochareac.

810. Hydrocharis. L. 811. Cabomba. Aubl. Nectris. Schreb.
812. Hydropeltis. Michx. Brasenia. Prsh.

813. F lo e rk ea. WiLLD.

$$
\text { C. } N y m p h a \text { e a c e a e. }
$$

a. Nymphaeac.

814. Ancslea. Andr. Nenuphar. Hayne. Euryale. Salisb. non Zoolog. 816. N y in p h a a. L. 815. Nuphar. SM. 817. Barclaya. WALL.

b. Nelumbere.

818. Nelumbo. T. Cyamus. Salisb. Nelumbium. Juss. 


\section{onpon \\ CAULO - ACROBLASTAE. \\ STAMM - SPITZKEIMER.}

\section{FORMATIO I. \\ $\begin{array}{lllllllll}G & \mathbf{L} & \mathbf{U} & \mathbf{M} & \mathbf{A} & \mathbf{C} & \mathbf{E} & \mathbf{A} & \mathbf{E} \text {. }\end{array}$ SPELZENGEW AECHSE.}

FAMILIA XXXIX.

GramineaE. Graeser.

I. S C H I Z O G YNA E.

A. Spicatac.

I. SPICA TERMINALT.

A. Spicularum bractea solitaria.

a. Ophiureae: spiculis immersis.

a. Rachis articulata.

819. Psilurus. Trin. Asprella. Hst.

820. Rottbo ella. L. Fir.

B. Rachis continua.
Stegosia. Lour. 821. Hemarthria. R. Br. 
48 CLASS, IV. ACROBL. ORD.II. CAUL. ACR, FAM. XXXIX. GRAI.

836. Trachyozus. Rchb.

Echinatysium, Trin.

Truchys. Pers. non Zoolog. 841. Aeluropus. This.

83\%. Hilaria. K. H. B. $>$ 842. Elymus. L.

838. Pterium. Desv. a. Cuviera. Koel.

839. Cynosurus. L. B. Elymus. Koel.

840. Elytrophorus. P. B. 843. Spinifex. L.

\section{Spicularumbracteis nullis.}

d. Hordeacea e: gluma a glumella sejuncta, rarius mulla.

844. Gy mostichum. Schreb. 845. Hordenm. L. Asprella. Gav. a. Zeocriton. P. B.

Hystrix. II n ch. $\quad$ b. Hordeum. P. B.

e. Secalinae: gluma glumellam utrinque amplectens.

846. O reochloa. Lk. Brom. distuch, et rigid. Rth. Sesler. disticha. P. 852. Brachypodium. P. B.

84\%. Wangenheimia. Trin. 853. A gropyrum. Gaert.

848. Ca tapodium. Lk. a. Elytrigia. Desv. Brachyp. loliac. P. B. $\quad$ b. Agropyrum. G.

849. Brizopyrum. Lk. 854. Triticum. L. Poce sicula et Uniol. spic. W. 855. Aegilops. L.

850. Gaudinia. P. B. 856. Secale. L.

851. Trachynia. Lk.

\section{SPICIS LATERALIBUS.}

\section{f. Chlorideac: glumis corinatis.}

c. Uniflorae.

85\%. Stenotaphrum. Trin.

858. Spartina. Schreb.

a. Trachynotea. MIch $x$.

Limnetis. Rich.

B. Cum alterius floris pedicillo. b. Ponceletia. A.P. Th. non RBr. 859. H y men a chne. P. B. 860. Lodicularia. P. B. 861? Cy m b a ch ne. "Retz.
862. Cynodon. Ricir. Capriola. Ad.

Fibigia. Koel. non MI u ch.
Digituria. Schrad.

Cabrera. La g. 


$$
\begin{gathered}
\text { भ. } \quad \text { i }-m u l t i-f l o r a c . \\
\text { ace. glumarum nulla multiaristata. }
\end{gathered}
$$

863. Campulos us. Drsv. 864. Dineba. Des.

865. Leptochloa. P. B. Leptostachys. MI e y.

a. Oxydenia. Nutt.

b. Rhabdochloa. P. B. 866. Chloris. Sw.

a. Chloris Dsv. Tetrapogon. Dsf.

b. Eustachys. Dsv.
Schultesia. Spr.

867. Campuloa. P. B.

Ctenium. Pz. MTonathera. Rafin.

Monocera. E11.

869. Dactyloctenium. W. 8\%0. Beckmannia. Host. Joachimia. Ten.

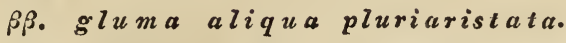

871. Choudrosium. Desv. Actinochloa. W. Bouteloua. L a g.

8\%2. Heterostega.

873. Atheropogon. Mhlnb. Eutriana. Trin.
Triathera. D esv. 874. Pentar rha phis. K.H.P. 875. Poly odon. K.H. B. 8\%6. Triaena. K. H. B. 877. A e gopogon. P. B. 878. Pleuraphis. Torr. Hymenothecium. 5-setum. LaG :

g. Paspalaceae: glumis eccrinatis.

$$
\text { c. uniflorue. }
$$

879. Reimaria. FlgG.

880. Epiphystis. Trin.

881. Zoysia. W.

Zoydia W. et P. erron?

Osterdamia. N e ck.

Matrella. P.

882. Mibora. An.

Knappia. S w.

Chamagrostis. B o r k h.

Sturmia. II p pon $\mathrm{Rch}$.
886. Helopus. Trin.

Oedipuchne. Lk. 88\%. Paspalum. L.

a. Paspalus. Flgg.

b. Axonopus. P. B.

c. Ceresia. P.

888. Streptostachys. Desv.

889. Oplismenus. P. B.

Orthopogon. RBr.

890. Echinola ena. K. H. B.

883. Echinolaena. K. H. B. 891. Echinochloa. P. B.

884. Microchloa. RBr.

a. Urochloa. F. B.

885. Eriochloa. K. H. B.

B. subbiflorae, flosculo altero masculov. neutro.

892. D igita ria. HALL. Syntherisma. Walt. 893. Thrasya K. H. B.
894. Arthraxon. P. B.

895. Thu a rea. P.

Microthonarea. A. Y. Th. 
50 CLASS.IV. ACROBL. ORD. II. CAULO-ACR. FAM. XXXIX. GRAM.

896. M a nisuris. L. FIL.

b. Peltophorus, P. B.

a. Manisuris. P. B.

\%. subtriflorae, flosculo intermedio neutro.

- 89\%. Lappago. Schreb.

Tragus. Hall.

$$
\text { B. P } a \text { u ic ul } l \text { at } a \text { e. }
$$

I. UNI - BI - FLORAE.

h. Agrostidea e: glumae et glumellae consistentia subaequales. a. Cinneue.

895. La gurus. L. 899. Ch a et urus. Lk.

900. Polypogon. Desv. Santia. S a v.

901. Grastridium. P. B. non $\mathbf{L} \mathbf{g} \mathbf{b}$.

902. Agrostis. L.

a. Agrostis. P. B.

b. Trichodium. Mrchx.

c. Agraulos. P. B.
905. Trichochloa. DC.

a. Stenocladium, Trin.

b. Acroxis Trin.

Tosagris. P. B.

906. P od as a emum. Dest.

90\%. Apera. Adons. Anemagrostis. Trin. 908. Ca l am agrostis. Rтн. a. Calamagrostis. Rth.

b. Deyeuxia. Clar.

903. L achnagrostis. Trin. 909. A mmophila. Host.

904. Miihlenbergi i. Schreb. Psamma. P. B.

910. Remirea. Aubl.

b. Iriliaceae.

911. Cole anthus. Serde. 913. Cleomena. P. B. Schmidtia. Tratt. non Mnch. 914. Milium. L.

912. Vilfa. Adans. Mritiarium. M $\mathbf{n} \mathbf{c h}$.

a. Colpodium. Trin.

Plispsia. Trin.

b. Sporobolus. $\mathbf{R B r}$.

915. Urachne. P. B.

a. Achnatherum. P. B. e. e.

b. Piptatherum. P. B. e. e.

c. Stipaceate.

916. Brachyelytrum. P. B. Dilepyrum. M chx.

91\%. Oryzopsis. Mchx.

918. Gy m n o gon. P. B. Anthopogon. N ut t.

919. Olyra. L.

a. Lithachne. P. B.

b. Olyra. P. B.

920. Stipa. L.

2. Jarava. Rz. Pav.

c. Eriocòma. Nutt, non K.H. B.

921. Aristida. L.

a. Aristida. P. B.

b. Curtopogon. P. B.

c. Chaetaria. P. B.

d. Arthratherum. P. B.

922. Streptachne. K. H. B.

923. Pentapogon. RBr.

a. Aristella. Trin.

20,6. dasiagrestis ar 
i. Paniceae: glumae et glumellae consistentia distinctae, ecarinatac.

$$
\begin{gathered}
\text { a. Pennisetea : } \\
\text { Thyrsoideae. }
\end{gathered}
$$

924. Diplopogon. RBr. Diplogon P о ir. exrou. Dipogonia. P. B.

925. Setaria. P. B. Pennisetum. RBr.

926. Pennisetum. P. a. Pennisetum. P. B. b. Gymnothrix. P. B. 92\%. Penicillaria. W. 928. L у си rus. K. H. B. 929. Amphipogon. RBr. 930. P a p poph orum. Schreb. Énneapogon. D esv.

$$
\begin{gathered}
\text { B. P a } \\
\text { (effusae. })
\end{gathered}
$$

931. Anthenantia. P. B. Tristegis. N. v. E. Aulaxanthus. E11. Aulaxis. - $\mathbf{N}$ u t t. 936. Panicum. L. a. Milium. Mnch, non L.

932. Trirrhaphis." RBr. b. Paractaenum. P. B. 933. Ectrosia. RBr. c. Bambusella, R. 934. Plenroplitis. Trin. 935. Melinis. P. B. 93\%. Is achne. R. Br. 938. Neurachne. RBr. Suardia. S chrk. 939. Chamaerrhaphis. RBr.

y. Coiceae: spiculis masculis e gluma feminea effusis.

940. Coix. L.

Lithagrostis. Gärt.

k. Oryzeae: glumac et glumellae consistentia distinctue, carinatae.

a. Phalarideae: triandrae.

941. Cornucopiae. L. 942. Pommereulla. L. 943. Crypsis. Art. a. Heleochloa. Host.

b. Antitragus. Gärt. 944. Tozzettia. SAv. 945. A lopecurus. L. 946. Echinopogon. P. B. 94\%. Colobachue. P. B. 948. Phleum. L. a. Phleum. P. B.

b. Chilochloa. P. B. 949. Achnodonton. P. B. 950. Poarion. Rchв. Aegialitis. Trin. non RBr. 951. Pha laris. L. 952. Baldingera. G. M. S. Typhoides. Mnch. Digraphis. T r i n. 953. Leersia. Sw. non Hedw. 4 * 
52 CLASS.IV. ACROBL. ORD.II. CAULO-ACR. FAM. XXXIX, GRAM.

Asprella. S c h $\mathbf{x} \mathbf{e b}$.

Homalocenchrus. Mi e g.

954? Caryochloa. Spr.

b. Genuinae: 4-10 andrae.

c. Terandrae.

955. Tetrarrhena. RBr. 956. Microlaena. RBr.

B. Hexandrae.

95\%. Potamophila. RBr. non 959. Zizania. L.

Schrk. 960. Ehrharta. Thnв.

958. Hydrochloa. RBr. non 961. Trochera. Ricн.

Harta. 962. Oryza. L.

r. Decandrae.

963. Luzio Ia. Juss.

1. Saccharinae: rachis articulata, spiculae geminae, altera sessili, alterc pedicellatc.

a. Triandrac.

964. Elyon urus. W. Elionurus. W. erron.

965. Diectomis. K. H. B.

966. Meoschium. P. B.

96\%. Lepeocercis. Trin.

968. Pogonatheru m. Homoplitis. Trin.

969. Heteropogon. P.

9\%. Schizopogon. Rchb.

971. Rhaphis. Lour. . Centrophorum. Trin.

972, Cymbopogon. Spr.

9\%3. Andropogon. L.

a. Dichantium. Willem.

b. Andropogon. P. B.

974. Pollinia. Spr.

Chrysopogon. Trin.

975. Thelepogon. Rтн.

976. Dimeria. RBr.

9\%. Xerochloa. RBr.
978. Apluda. L.

979. Z Zugites. Schreb.

950. Spodiopogon. Trin.

981. Anthestiria. L. Anthistiria. La m.

P. B. 982. Calamina. P. B.

Themedu. Forsk?

983. Calam ochloë. Rchr. Goldbachia. Trin. non DC.

984. Sorgh um. P.

Blumenbachia. Ko el.

985. Perotis. Art.

Xystidium. Trin.

986. Diplachyrium. N. v. E.

98\%. Imperata. CXr.

988. Erianthus. Mchx.

Ripidium. Trin. non Zoolog.

989. Sa ccharum. I.

a. Eriochrysis, P. B.

b. Saccharum, P. B. 


\section{B. Hexandrae.}

990. Pharus. L.

991. Leptaspis. RBr.

\section{BI - MULTI - FLORAE. \\ 1. Disepalae.}

a. Rachis excisa, excisuris solitariis vel geminatis.

in. Festucuceae: spiculae extrorsum angustatae, termino emerso.

a. Thyrsoideae, subsecundae.

992. Echinaria. Dsf. Panicastrella Mnch.

Roram. Ad.

993. Rostraria. Trin.

994. Trichaeta. P. B.

995. Psilathera. LK.
Sest. tenella. H os t.

996. Sesleria. Hard. 99\%. Chrysurus. P.

Lamarkia. Mnch. 998. Sclerochloa. P. B. 999. Dactylis. L.

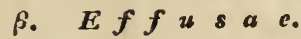

1000. Vulpia. GM.

Mygalurus. L k.

1001. Festuca. L.

1002. Diplachne. P. B.

1003. Ceratochloa. P. B.

1004. Michelaria. Dumort. 1010. Chasmanthium. Lr.

Libertia. $\mathbf{L}$ e $\mathbf{j}$. non $\mathbf{S} \mathbf{p} \mathbf{r}$. 1005. Triplasis. P. B. 1006. Cento theca. Desv.
100\%. Calotheca. Desv. 1008. Uniola. L.

1009. Corycarpus. ZEA.

Diarina. Rafin.

Diarrhenu. Rafin.

Uniola gracilis. Mchx.

n. Melicaceae: spiculae extrorsum dilatatae, termino incluso.

1012. Sphenopus. Trin.

1013. Schismus. P. B.

Electra. Pz.

1014. Melica. L.

Beckeria. B r nh.

Mrelica. B ruh.

1015. Uralepis. Nutr.
Uralepsis. Nutt. exrou.

Diplocoea. Rafin.

1016. Coelachne. RBr.

101\%. Triodia. RBr.

a. Graphephorum. Dsv.

b. Sieglingia. Brnh.

1018. Streptogyna. P. B. 
b. Rachis sulcata vel striata.

o. Porcea e: spiculce extrorsum angustatce.

1019. Airochloa. LK.

Ischnanthus. R. S. e rron.

-1020. Koeleria. Pers. e. e. 102\%. Glyceria. RBr. non 1021.' Falimiria. Bess.

Arthrostachya. L k.

1022 Bromus. L.

a. Micranthera. Bess.

b. Bromus. Bess.

1023. Tripogon. Rтн.

1024. Schedonorus. P. B. 1030. Poa.'L.

Schoenodorus. Auct. erron. 1031. Eragrostis. P. B.

1025. Tricus pis. P. B. non P.

Tridens. R. S.

Windsoria. Nutt.

a. Eragrostis. P. B.

b. Megastachya. P. B.

1032. Briza. L.

1026. Ichnanthus. P. B,

p. Avenaceae: spiculce extrorsum dilatate.

1033. Anthoxanthum. L.

1034. Airopsis. Desv.

1035. Periballia. Tren.

1036. Aira. L.

a. Corynephorus. P. B.

b. Deschampsia. P. B.

Campella. $\mathbf{r}$.

103\%. Dupontia. RBr.

1038. Toresia. Rz. PAv.

Disarrhenum. LaB.

1039. Hierochloa. Gm.

Savastana. Schrk.

1040. Holcus. L.

1041. Arrhenatherum. P. B.

1042. An isopogon. RBr.

1043. Avena. L. a. Avenaira, R.

b. Ventenata. Koel.

c. Avenaria. R.

1044. Trisetaria. Fonsk.

Triselum. $\mathbf{P}$.

1045. Danthonia. RBr.

Triodia. P. B. in ind.

1046. Pentameris. P. B.

104\%. Arundo. L.

Donax, P. B.

Scolochloa. M. K.

1048. Phragmites. Trin.

Czernya. Prs1.

1049. Ampelodesmos. LK.

1050. Gynerium. KHB.

2. Trisepalae.

q. $\boldsymbol{B} a$ mbusaceae.

a. Triandrae.

1051. Ludolfia. Wirlo. Amendinaria. M chx. Miegicl. Pers.
Triglossum, F is ch.

Mocronax. Rafin. 
B. Hexandrae.

1052. B ambusa. Schreb. 1055. N astus. Juss.

Bumbos. Rtz.

1053. Guadua. KNтн.

1054. Chusquea. Knтн.
Stemmutospermum. P. B. 1056. Melocanna. Trin. Beeshu. R h e d.

II. HOL O G Y N A E.

A. Spicatae.

r. Nardecie: uniflorae.

105\%. Nardus. L.

s. Spartecue: biflorcue.

1058. Ly geum. L.

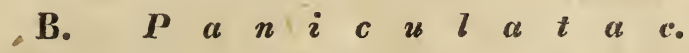

t. Zzecene.

1059. Zea. L.

Gramineae dubiae.

1060. Agrosticnla. RAdd. 1063. Navicularia. Radd. 1061. Acicarpa, Radd. 1064. Rettbergia. RadD. 1062. Aruudinella. Radd.

\section{FAMILIA XL.}

CyperoideaE. Cypergraeser.

A. $C a r i c i n a c$.

a. Elynaceac.

1065. Elyna. Schrad. 1066. Kobresia. W.
106\%. Catagy a. RB. 1068? Diaphora. Lour.

b. Cariceae.

1069. U n c i in i a. Pers. Caric. sp. L. S c h k. Raspail.

1070, Viguea. P. B.
10\%1. Carex. L. e. e.

a. Triplima. Rafin.

b. Triodex, Rafin. 
56 CLASS.IV.ACROBL, ORD. II.CAULO-ACR. FAM.XL.CYPFR.

c. Chrysithriceac.

10\%2. Lepironia. Rich. ap. 1074. Chorizandra. RBr. Pers. 1075. Chrysithrix. L. FiL.

10\%3. Chondrachne. RBr.

\section{d. Scleriaceue.}

1076. Opetiola. Gaertiv. 10\%\%. Diplacrum. RBr. 10\%8. Scleria. BERG. a. Sclerella, R.

b. Lilhocarpella. R.

B. $\begin{array}{lllllllll}\boldsymbol{y} & \boldsymbol{y} & \boldsymbol{p} & \boldsymbol{e} & \boldsymbol{r} & \boldsymbol{i} & \boldsymbol{n} & \boldsymbol{a} & \boldsymbol{e} \text {. }\end{array}$

a. Cypereae: calyce nullo.

a. Stigmata bina.

1079. Spermodon. P. B. 1083. S chel h a m e ria. 1080. Z osterospermum. P.B.

1081. Pycreus, P. B.

1082. K yllingia. L. f.

Kyllinga. Rott b.

Thryocephatus. Forst.
Moench.

non Schelhammera. RBr.

Carex cyperoides. $\mathbf{L}$.

1084. M elanacranis. VAHL.

b. Stigmata terna.

1085. Hypoëlytrum. Rich. 1094. Elynanthus. P. B.

Hypölyptum. Vahl. errou. 1095. Arthrostylis. RBr.

1086. Mapania. Aubl.

ex herbb. Rich. 1096. Hypolepis. P. B.

105\%. Remirea. Aubr. Miregia. S c hreb.

1088. Cyperus. L.

1089. Ma ris cus. Halu. Adur $u_{i}$. Bosc.

1090. Pa py rus. Bruce. 1091. Abildgardia. VaHL.

Iria. Ric ic b. 109\%. Schoenus. L.

1098. Schoenopsis. Lestib. 1099. La mpocarya. RBr. 1100. Baumea. Gaudich. 1101. Gahnia. Forst. 1102 Cládium. P. Brwne. 1103. Caustis. RBr. 1104. Tetraria. P. B. 1092. Hemichlaena. Schrad. 1105? Evandra. RBr. 1093. Trasi. P. B.

1106? To rulini m. Hamint.

b. Duli c $h i$ e a e. calyce setoso.

a. Stigm. bina.

110\%. Nomochloa. P. B. 1109. Rhynchospora. VAhL. 1108. Dulichium. Rrch. 


\section{b. Stigm. terna.}

1110. Carpha. RBr. e. e. 1112. Machaerina. Rich. et 1111. Chatospora. $\mathrm{RBr}$. VAhL.

$$
\text { C. S } c \text { ir } \boldsymbol{p} \text { in a e. }
$$

a. Finbristyleae: calyce nullo.

\section{a. Stigm. bina.}

1113. Fimbristyles. Rrcr. Fimbristylis. Vahl.
Dichostyles. P. B. 1115. Dichromena. Rich.

1114. Echinolytrum. Dest.

$$
\text { b. Stigm. tria. }
$$

1116. Trichelostylis.Lestrib. 111\%. Is o lepis. RBr.

IIr. G. Holosenuming $\alpha x$

b. Scirpeae: calyce setoso.

a. Stlgm. bina.

1118. Hele ocharis. Lestrb. 1119. Hele o g i o n. Lestib. Eleocharis. RBr. Heleophila. P. B.

Bulbostylis. St e . 6. Blysinues Sany

$$
\text { b. Stigm. tria. }
$$

1120. Limnochloa. P. B.

1123. Eriophorum. L.

1121. Scirpus. L.

1124. Hymenochaete. P. B.

1122. Trich ophorum. Rich.

c. Fuireneae: calyce membranaceo.

a. Stigm. binu.

1125. B eera. P. B.

1126. Diplasia. Rich.

b. Stigm. tria.

112\%. Fuirena. Rotrb.

1129. Oreobolus. RBr.

1128. Lepidosperma. LaB.

FAMILIA XLI.

COMMELINACEAE.

a. Restioneac.

1130. Centrolepis. LaB. 1131. Alepyrum. RBr. Desucuxiu. RBr. 1132. Aphelia. RBr. 
1133. El egia. Thnв.

1134. Le p y rodia. RBr.

1135. An arthria. RBr.

1136. Restia. L.

113\%. Loxoc arya. RBr.

1138. Leptocarpus. RBr.

1139. T ha m n o hortus. 1145. Rapatea. Aubl. *)
1140. Willd enowia. Thunb.

1142. Lyginia. RBr.

1143. Hypolaena. RBr.

1144. Cha et an thus. RBr.
1141. An arthria. RBr.

Berg.

Inasium. S chreb.

b. Xyrideae.

1146. T o nina. Aubr. Hyphydra. S c hreb. 114\%. Eriocaulon. L.

a. Nasmythia. Huds.

'b. Schoenocaulon. R. 1148. Abolboda. K. H. B. 1149. Xy ris. L.

a. Xyris. A. P. Th.

b. Xyroides. A. P. Th.

1150. Aphyllanthes. L.

1151. L axmannia. RBr. 1152. Sowerbaea. SM.

1153. Iohnsonia. RBr. 1154. B aumgartenia. Spr. Borya. L a b. non W. 1155. Xantorrhoea. SM.

c. Commelineae.

a. Phitydrinae.

1156. Philydrum. Banks. 115\%? Cartonema. RBr. Garciana. L o u r.

\section{b. Xiphidieae.}

1158. Maуaca. Aubr.

Syena. S chreb.

1159. X iphidium. Loefre.
1161. If agenbachia. N. v. E. 1162. Pollia. Thunb.

1160. Wachendorfia. L. 1163. Flagellaria. L.

$$
\begin{aligned}
& \text { c. } \quad \text { t } p \text { t } \boldsymbol{x} \text { ereue. } \\
& \text { aa. Spathaceac. }
\end{aligned}
$$

164. Le ptanthus. Mchx. a. Pontederia. Rafin.

1165. Het e rant he ra. P. B. b. Umsema. Rafin.

1166. Pontederia. L.

*) Plantam vere glumacean esse, docuit natura. 
bb. Bracteatae.'

116\%. Commelina. L. Commelyna. If $\mathrm{f} \mathrm{gg}$.

a. Anisanthina. R.

b. Isanthina. R.

1168? An an thopus. Rafin.

1169. Aclisia. E. Mex.
11\%. C a pelia. Rrcr. Zanonia. Pln m. non $\mathbf{L}$. 11\%. Tradescantia. L.

a. Craterostegia. R. b. Phyllostegia, R.

11\%1b. Cyanotis. Don.

cc. Nudiflorae.

1172. Callisia. L. . Hapalanthus. J c q. em.

1173. Aneilema. RBr.
1174. Palisot a. Rerb. Commel. ambigua. P. B.

\section{FORMATIO II. \\ E N S A T A E. \\ SCHWERTEL - GEWAECHSE.}

FAMILIA XLIr.

IRIDEAE. IRIS - SCHWERTEL.

a. Ferrariece.

1176. Cipura. Aubl.

1184. Paters onia. RBr.

Marica. Schreb.

117\%. Sis y rinchium. L. 1185. Moraea. L. Homeria. $\mathbf{V}$ e $\mathbf{n}$ t.

Syorrhynchium. IIffg g. e m. 1186. V i e us s e u xi a. LA

Bermudiana. Grärt.

1178. Bobartia. L.

1179. Libertia. SPr. non al.

118\%. Iris. L.

Renealmia. RBr, non $\mathbf{L}$.

Rосн.

1180. Tigridia. Juss.

a. Pogonirion. R.

b. Lophiris. Tsch.

1181. Cypella. Ноок.

c. Limniris. Tsch.

1182. Ferraria. L.

d. Xyridion. Tsch.

1183. Witsenia. L.

e. Spathula. Tsch.

f. Xiphium. Mill.

\section{b. $\quad G l a d i o l$ e $a$.}

1188. Dias ia. Del.

Aglaea. P ers.

Melasphaerula. K e r.
1189. D ip l ar r he na. Lab.

1190. Gladiolus. L. 
60 CLASS.IV. ACROBL. ORD. II. CAULO - ACR. FAMT, XLIII. NARC.

a. Gladiolus, I.

b. Hebea. Pers.

c. Lemonia. Pour.

1191. Montbretia. DeC.

1192. Wa tsonia. Ker.

a. Micranthus. $\mathbf{P}$.

b. Callanthus. R. Houttuynia. Hout t. non Th n b.
1194. Ovieda. Spr.

1195. Anomatheca. Ker.

Lapeyrousia. Pou r. non

Thn b.

1196. Wa itzia. Rchв.

Tritonia. Ker. non Cuv. Zool.

119\%. S paraxis. Ker.

1198. B a b i a na. Ker.

1193. Antholyza. L.

c. I $x$ i $e$ a $e$.

1199. Galaxia. L. 1200. Crocus. L.

1201. Tapeinia. Juss.

1202. Trichon e ma. Ker.

Romulea. M ar at t.

1203. G e i s s o r rhiza. Ker.

1204. Ixia. L.

1205. Hesperantha. KER.
1206. Aristea. L.

1207. Genlisia. Rchb. Wits. corymb. $\mathbf{S} \mathbf{m}$.

Nivenia. V ent. non RBr.

1208. B el em c a u d a. Moench. Pardanthus. K e r. 1209? Ixiolirion. Herb.

FAMILIA XLIII.

NARCISSEAE. NARZISSEN - SCHWERTEL.

a. $B u r m a n n i e a e$.

1210. Tripterella. Mchx. 1212. Maburnia. A. P. Th. Anonymos. Walt.

Burmannia. Lo u r

Vogelia. Gm. s yst. non al. 1213. Burmannia. L.

1211. Sonerila. Roxb.

b. Haemadoraceae.

a. Hypoxideae.

1214. Gethyllis. L.

1215. Hypoxis. L.
121\%. Compsoa. Don.

Compsanthus. Sp r.

Fubricia. Thub. non Gärt. 1218. Curculigo. Gaert.

1216. Campynema. Lab.

\section{B. Hacmodoreac.}

1219. Haemodorum. Sm. 1221. Conostylis. RBr.

1220. Phlebocarya. RBr. 1222. Anoegosanthus. Rchb. 
Anigozanthos. LaB.

Anigosanthus. $\mathrm{R}$ ed. Schwachrigenia. S pr.

1223. Dilatris. L.

1224. Astelia. BNks et Sor.

1225. Lanaria. Art.

Argolasia. Juss.
1226. Lophiola. Ker.

122\%. Lachnanthes. Eux.

1228. Barbacenia. VAND.

1229. X e rophyta. Juss.

1230. Vellosia. Vand.

Cambderia. Knth.

Rudia. A. Rich.

c. Amaryllidede.

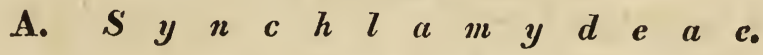

a. Cyrtantheae.

1231. Gastronema. Sims.

1232. Bravoa. Heris.

1233. Cyrtanthus. Arr.

1234. Urceolina. Rснв.
Urceolaria. H e r b. non a 1. 1235. Pyrolirion. Herb. 1236. Vallota. Herb. 1236b. Jimatophyllum. Hook.

\section{b. Amarylleae.}

123\%. Sternbergia. W. K. Sternebergia. Herb. erron.

1238? Oporanthns. Herb.

1239. Strumaria. JAcQ.

1240. Hessea. Berg.

1241. Ne rine. Herb.

1242. Eucrosia. Ker.

1243. Griffinia. Ker.

1244. Ly coris. Herb.

1245. I m h o fia. Herb.

1246. Brunsvigia. Ker.

1247. A m aryllis. L.

1248. Ha e manthus. L.

a. Polystegia. R.

\section{B. Diplarchlaryde a e.}

a. Galantheae, transitoriac. b. Tristegia. $\mathbf{R}$.

1249. B uphone. Herb. 1250. Am mocharis. Herb. 1251? Spha erotele. Prsi. 1252. Crinu m. L. 1253. Lepiedra. LaG.

1254. Z ep h y ran thes. Herb. 1255. Sprekelia. Herb. 1255. P hycella. Linde. 125\%. H ippeastrum. Herb. 1158. Chlid anthus. Linde.

a. Clinanthus. Herb.

b. Chlidanthis. HIerb. 1259. Ga lanthus. L. 1260. L e uс о i m. L.

$$
\text { b. } N \text { arcissoinae. }
$$

1261. Eustephia. Car.

1262. Chry soph i a la. Lamb.

a. Leperiza. Ierb. b. Corpodetes. Herb.

c. Stenomessou. Herb. 1263. H y in en o callis. Herb. 
1264. Pancratium. L. 1265. Proiphy s. Herb. 1266. Calostem ma. RBr. 126\%. Liriopsis. Rerb.

Liviope. Herb. non L our. 1268. Narcissus. L. a. IIermione. Salisb.

b. Narcissus. Salisb.

c. Queltia. Salisb.

d. Aiax. Salisb.

1269. I sm e ne. Herb.

FAMILIA XLIV.

BROMELIACEAE. Ananas - schwertel.

a. $P a n d a n e a c$.

12\%. P a nd an us. L. f. Allhrodactylis. Forst.
Elephanthusia. W.

12\%2. Fre ycine tia. Gaudich. 1271. Phyteleph a s. Rz. PAr.

$$
\text { b. } \quad T i l l a n d s i e \text { e } e \text {. }
$$

12\%3. Guzm annia. Rz. 1276. Bonapartea. Rz. Pav.

$$
\text { Pav. }
$$
Acanthospora. Spr.

12\%4. Caraguata. Piso. 12\%. Pourretia. Rz. Pav. 12\%5. Tillandsia. L.

$$
\text { c. } \quad \mathbb{B} r \text { onelie } a \text { e. }
$$

12\%. A n a nas a. Lrnde. 1283. Doryanthes. RBr.

12\%9. Bromelia. L.

1280. Billbergia. Thns.

1281. Aechmea. Rz. Par.

1282. Pitcairnia. L. Herit.
128\%. Ag are. I.

a. Littaea. Tagliab. Bonupartea. W. Lk.

b. Fourcroya. Vent.

c. Agave. $\mathbf{L}$. 


\title{
onon ont \\ PHYLLO - ACROBLASTAE. \\ BLATT - SPITZKEIMER.
}

\author{
FORMATIO 1.

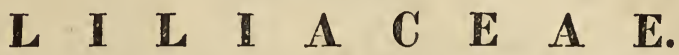 \\ LILIEN GEW AECHSE. \\ Familia xht. \\ JUNCACEAE. Simsenlilien.
}

$$
\text { a. } J u n c e a e \text {. }
$$

1285. Cephaloxys. Desv. 1286. Luzula. Desv. 128\%. I uncus. L.

a. Rostkovia. Desv.

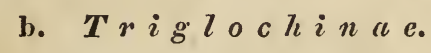

1288. Triglochial L.

$$
\begin{gathered}
\text { c. Me } l a n t h e a e \text {. } \\
\text { a. Veratreac. }
\end{gathered}
$$

b. Marsippospermum. Desv. c. Juncus. Desv. d. Prionoschoenus. R.

1290. Scheuchzeria. L. 1291. Veratrum. L.

$$
\text { B. Helonical. }
$$

1292. Nolina. Mchx. 1293. To fiel dia. Hubs. Toficlda. P. Heritiera. S chrk. Hebelia. G m. Iridrogalva. P. B. 1294. Helonias. L.

a. Chamaelivium. W.

b. Helonias. WV. 1295. Calochortus. Prsh. 1303. Mel anthi um. L. 1296. Wu rmbea. Thns. 1304. Kolbea. Schlecht. 129\%. Anguillaria. RBr. 1298. Zig a d en us. Mchx.
1299. Burchardia. RBr. 1300. Or i t h o g los $\mathrm{s}$ um. Salisb.

Lichtensteinia. W. Cymation. Sp r.

1301. A pdrocy m bium. W. 1302. Ex y th r o stictus. Schlecht. 
64 CLASS.IV.ACROBL. ORD.IIT. PHYLL-ACR. FAIT. XLVI, SARM.

$$
\text { c. Colchiceac. }
$$

130\%. Hermodactylos. BRr. 1310. Monocaryum. RBr. 1308. B ulb oc odi un. L. Hypox, fascicul. $\mathbf{L}$. 1309. M e rendera. MB. 1311. Colchicum. L.

FAMILIA XLVI.

SARMENTACEAE. Z Z AUKENLILIEN.

a. Xerotere.

1312. Xerotes. RBr.

a. Dracaenella. R.

b. Xerotella. R.

c. Lomandra. LaB.

d. Spathandra. R. e. Stachyandra. R. 1313. D a s y o gon. RBr. 1314. Calect as ia. RBr. 1315. Kingia. $\mathrm{RBr}$.

b. $S$ mil a c e a c.

1316. Paris. L.

131\%. Trillium. L.

1318. Drymophila. RBr. 1319. Asparagus. L. 1320. Streptopus. Mchx. 1321. Convallaria. L. 1322. Poly go on at um. Desfo 1323. Ledebouria. R non al.

1324. Oph i opogon. Ker. Flïggea. Rich. non al. Slateria. D e sv.

6. Maianthemum. Wigg.

$$
\text { c. Dioscorea } e \text {. }
$$

1335. T a m us. L. Tamnus. Jus s. Thamnus. $\mathbf{L}$.

1336 Testudinaria. SAlisb. 133\%. Rajania. L. 1338. Dioscorea. L.
1325. Smilacina. Dsf. 1326. Med e ola. L. 132\%. Eustrephus. RBr. 1328. L u z u riaga. Rz. PAv. 1329, Myrsiphyllum. W. 1330? Leptodermis. WaLL. Hamiltonia. Do $\mathbf{n}$. 1331. Ruscus. L. 1332. Ripogonum. Forst. 1333. Smilax. L.

1334. Herreria, Rz. PAv. 1339? Oncus. Lour. oncorrhiza. P e rs. 1340? Aechmea, Rz. PAv. 1341? Floscopa. Lour. 1342? Hollböllia. WALL. 
FAMILIA XLVII.

CORONARIAE. KronliLien.

$$
\text { a. Methonicere. }
$$

1343. Alströmeria. L.

a. Bomarea. Mirb.

b. Alströmeria. L.
134\%. Schelhammera. RBr.

1348. Di is porum. SAlisb.

1349. Urularia. L.
1344. Philesia. Juss. 1346. Callixene. Juss. 1346. Lapageria. Rz. PAV.
1350. Erythronium. L.

1351. Glorios a. L.

Methonica. H e rm.

Mendoni. $\mathbf{R}$ h e ed.

b. $T u l i p a c e a e$.

1352. Rhabdocrinum. Reнв. Antheric. serot, graec. a u ct. 1353. Fritillaria. L. Fritill. imperial. $\mathbf{L}$. 1354. Petili um. L. H. Cliff. 1355. Lilium. L. a. Martagon. Cam. b. Eulirion. R.

$$
\text { c. Asphodel e a e. }
$$

a. Gilliesicae.

1356. Gilliesia. Lindu. 1358. Brodiaea. SM.

135\%. M i e r s i a. Lindo.

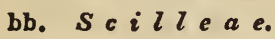

1359. Massonia. L. 1360. Eucom is l'Herit. Basilaca. L a m. 1361. Muscari. Desf. 1362. Hyacinthus. L. 1363. Bellevalia. LAP. 1364. S cilla. L. 1365. D r i in i a. Icq. 1366. B arnardia. Lindu. a. Gagea. K e r. b. Stellaris. Mnch. c. Albucea. R. 1368. Albuca. L. 1369. Lachenalia. JcQ. 13\%. Uropetalum. Ker. Polemannia. Berg?

1371. Puschkinia. An. Adamsia. W.

136\%. Ornithogalum. L.

$$
\begin{aligned}
& \text { d. Antherice } e \text { e. } \\
& \text { aa. Hemerocallideac. }
\end{aligned}
$$

1372. Agapanthus. l'Herit. Abumon. Ad.
Mauhlia, D a h $\mathbf{~}$. 1373. Cyrtanthus. Arr. non

Schreb. 
66 CLASS.IV. ACROBL.ORD.III. PHYLL-ACR. FAM. XLVII. CORON.

Timnia. Gm. S y st. 1374. Hemerocallis. L. 1375. Czackia. Bess.
1376. Funkia. SPR.

$$
\text { Hosta. Tratt. }
$$

\section{bb. Alliaceac.}

137\%. Allium. L.

Ophioscorodon. W a $11 \mathrm{x}$. 1378. Porrum, $T$.
1379. Codonoprasum. Rchr. 1380. Milla. CAf.

1381. Tulbaghia. L.

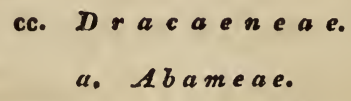

1382. Tricoryne. RBr.

1383. Narthecium. Монн.

\section{Abama. Ad.}

1384. Bulbine. W.

Anthericum. Lam.

1385. Arthropodium.

1386. Anthericum. L.

Phulangium. Lam. non Zoolog.

1387? Monochonia. Prst.

1388. Chlorophytum. Ker.

1389. Asphodelus. L.

1390. Er e m urus. MB.
1391. Caesia. RBr. 1392. Sty pandra. RBr. 1393. Dianella. Lam. 1394. Cordyline. Сомm. 1395. Conanthera. Rz. PAy. Echeandia. Ort.

1396. Cyanella. L.

1397. Thys anotus. RBr. Chlamysporum. Salisb.

1398. Phormium. L.

\section{B. Aletrineae.}

1399. Polianthes. L.

Polyanthes. W. P. 1400. Sanseviera. Thnb. non Andr.

1401. Veltheimia. Gued. 1402. Aletris. L. 1403. Liriope. Lour.
Aletr. fragr. et hyac. $\mathbf{L}$. Sulmea. C a v. Pleomeles. S a 1 i s b. 1404. Blandfordia. SM. 1405. Tritomanthe. IIFGg. Tritoma. Ker. non $\mathrm{Z}$ oolog.

\section{y. Yucceac.}

1406. Apicra. W. 140\%. Haworthia. Duv.
1408. B ow i ea. Haw. 1409. Gasteria. Dur. 
1410. Aloe. L. e. e. Rhipidodendrum. Wr. 1411. Lomatophyllum. W.
1412. Dracaena. I. 1413. Y uсca. L.

\section{FORMATIO II.

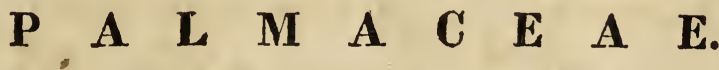 \\ PALMENGEWAECHSE.}

FAMILIA XLVIII.

ORCHIDEAE. OrChIDEE.

a. Neottie a e.

1414. Pelexia, Port. 1426. Ponthieva. R. Br. 1415. Goodyera. RBr. 1416. Physurus. Rich. 141\%. Haemaria. Lindu. 142\%. Neottia. L. 1428. $L$ is te $r$. $R B$. Distomaea. S p e n n. 1418. Thelymitra. Forst. 1419. Diuris. Sm. 1429. Spiranthes. Rich. 1430. Zeuxine. Lindu. 1420. Epiblema. RBr. 1431. S ten orrhy nchus. Ricr. 1421. Cryptostylis. RBr. 1432. Calochilus. RBr. 1422. Orthoceras. RBr. 1433. Synassa. Lindu. 1423. Prasophyllum. RBr? 1434. Cyclopogon. Prsc. 1424. Cranichis. Sw. 1425. Chloraea. Lindu. Gavila. F e uill. 1435. Microchilus. Prse. 1436. Stenoptera. Prsu. 1437. Sarcoglottis. PrsL.

\section{b. Aretbuseae.}

1438. Arethusa. Sw. 1447. Caladenia. RBr. 1439. Limodorum. Tours. 1448. Chiloglot tis. RBr. 1440. Calopogon. RBr. 1449. Cyrtostylis. RBr. 1441. Pog on ia. Juss. 1450. Corys an thes. RBr. 1442. Eriochilus. RBr. 1443. Pterostylis. RBr. 1444. Glossodia. RBr. 1451. C a l a ena. RBr. 1452. Microtis. RBr. 6. Cophatasthera 1445. Het e rotaxis. Linde. 1454. Corallorrhiza. Hale. 1446. Ly peranthis. RBr. 


$$
\text { c. } G a s t r o d i e a e \text {. }
$$

1455. Gastrodia. RBr. 1458? Hysteria. Rerww. 1456. Epipogium. RBr. 1459. Epistephium. Krтн. 145\%. Prescotia. Lrxpl. 1460. Vanilla. Sw.

$$
\begin{aligned}
& \text { d. } \begin{array}{lllllllll} 
& \boldsymbol{O} & \boldsymbol{h} & \boldsymbol{r} & \boldsymbol{y} & d & \boldsymbol{e} & \boldsymbol{a} & \boldsymbol{c} \text {. }
\end{array} \\
& \text { aa. Antheraerecta. }
\end{aligned}
$$

1461. Orchis. L.

1462. Glos sil L. Linde.

Gilossaspis. Spr.

1463. An acamptis. Rich.

1464. Nigritella. Rich.

1465. Diplomeris. Dov.

Paragnathis. $\mathbf{S}$ p r.

1466. Aceras. $\mathrm{RBr}$.

Loroglossum. R ich.

- G. Himantoglossum. Spr.

146\%. Ophrys. L.

1468. Serapias. L.
1469. Altensteinia. KNтh. 1470. D is a. Berh. 1471. Habenaria. W. 1472. Gymnadenia. RBr. 14\%3. B on atea. W. 14\%4. P la tanthera. Rich. 1475. Chamorchis. Ricy. Chamuerepes. Spr.

1476. H e r m i n i um. RBr.

14\%. Holothrix. Rich. 1478. Dryopoeia. A. P. Th.

bb. Anthera resupinata v. horizontali.

14\%9? Bartholina. RBr. Dipera. Spr. 1480. Repandra. Linde. 1481. Pterygodium. Sw. 1483. Satyrium. Srr. 1482. Disperis. Sw. 1484. Corycium. Sw.

$$
\text { e. } V a n d \in a e \text {. }
$$

1485. C alanthe. RBr. 1486? Octomeria. RBr. 148\%. Arpophyll u. LaLrav. 1488. Pinalia. Lindu. 1496. Telipogon. Kктн. 149\%. Or in it ho e p hal us.

Ноок. 1489. Maxillaria. Rz. PAv. 1499. Ps it tacoglos um. 1490. Sophronia. Linde. LaLrav. 1491. Polystachya. Hook. 1500. Alamannia. LALlav. 1492. Camaridium. Linde. 1501. Tipularia. Nutr. 1493. Ornithidium. Salisb. 1502. Gastrochilus. Don. 1494. Pholid ota. Lindu. 1495. Sunipia. Linde.
1503. A ërides. Lour.

1504. Vanda. RBr. 
1505. Zygopetalon. Hook. 1523. Cyrtopodium. RBr. 1506. Sarcanthus. Lindu. 1524. Cyrtochilum. Knth. 150\%. Robiquetia. Grunrch. 1525. Cuitlanzina. Lalatav. 1608. Aëranthes. Linde. 1526. Anguloa. Rz. Pav. 1509. Angraecum. A. P. Th. 152\%. Catasetum. Rich. 1510. Cryptopus. Lindu. 1528. Eulophia. RBr. 1511. Oeonia. Linol. 1512. Jon opsis. Knth. Cybelion. Spr.

1513: Cymbidium. Sw. 1514. Lis sochilus. PiBr. 1515. Geodorum. Jacks. 1516. Sobralia. Rz. Pav. 1517. Gastrochilus. Don. 1518. Dipodium. RBr. 1519. Oncidium. Sw. 1520. Macraden iá. RBr. 1521. Brassia. RBr, 1529. Xylobium. Linda. 1530. Trize u xis. Linde. 1531. Lockhartia. Ноик. 1532. Fernandezia. Rz PAr. 1533. Rodriguezia. Rz. PAv. 1534. Gomeza. RBr. 1535. Cirrhaea. Linve. 1536. Notylia. Linde. 1537. Megaclinium. Lindo. 1538. Trichoceros. Knth. 1539. Masdevallia. Rz. PAv. 1540. Gongora. Rz. Pav. 1522. Odontoglossum. Knth. 1541. Renanthera. Louk.

$$
\text { f. } E p i d e n d r e a e \text {. }
$$

1542. Bletia. Rz. Pav. 1543. Brassavola. RBr. 1544. Epidendrum. L. 1545. Pleione. Don. 1546. Ei cyclia. Ноок. 154\%. Cattleya. Lrind.
1548. Broughtonia. RBr.

1549. Is ochilus. RBr. 1550. Dinema. Linde. 1551. Schismoceras. Prsia. 1552. Elle anthus. Prsio 1553. Acronia. Prst.

g. $M a l a x i d e a e$.

1554. Eria. Linde. 1556. Dendrobi um. Sw. 1555. Acianthus. RBr.

1563. Microstylis. Nutr. 1564. S tu rmia. Reнu. Liparis. Rich, non Zoolog. 155\%. Pachyphyllnm. Knth. 1565. Diena. Linde. 1558. Stenoglossum. Knth. 1566. Empusaria. Rimв. 1559. An isopetalum. Hook. 1560. Restrepia. Knth. 1561. Coelogyne. Lindu. 1562. Malaxis. L.
Empusa, Lindl, non Zoolog. 156\%. Orchidium. Sw. Calypso Salis b. non A. P.'Th. nec $\mathrm{Z} 00 \mathrm{l}$.

Norna. W a $1 \mathrm{n}$ b. 
1568. Pleurothallis. RBr. 1569. Stelis. Sw. 1.70. Ptilocnema. Don. 1571. Tribrachia. Lindu. 1572. B u 1 b o p h y 11 u $\mathrm{m}$.
1573. Pedilea. Lindx. 1574? Zygoglossnm. Rernw. 15\%5. Schoenorchis. Rernw. 15\%6? Ach roanthes. Rafin. Malax. unifolia. M $\mathrm{ch}$.

\section{A. P. Th.}

h. Cy $p$ ripedieae.
157\%. Cypripedium. L.
a. Calceolus.
b. Hypodema. R.
c. Venustum. Wall.

\section{i. Loci incerti.}

1578. Sarcochilus. RBr. 1583. Acriopsis. Rerrw. 15\%9. Cirrhopetalum. Lrnde. 1584. Cryptochilus. Wart. 1580. Renanthera. Lovr.

(cf. 1540b.) forte Vandea. 1585? Is otria. Rafin. 1581. Thrixspermum. Lour. 1586? D iphryllum. Rafiv. 1582. Gal e ol a. Lour. 158\%? Ceraia. Lour.

FAMILIA XLIX.

SCITAMINEAE. BANANEY.

a. $A m o m e a e$.

1588. Mantisia. Curt. 1594. Costus. L.

1589. C e r at an the ra. Horr. 1595. Zingiber. Gärt.

Colebrookia. Do n.

1590. Globb a. L.

Catimbium. Jus s.

Renealmia. $\mathbf{L}$. f.

1591. Alpinia. L.

Elettaria. Maton.

1592. Leptosolena. Prsc. 1593. Hellenia. W.
1596. Hornstedtia. Retz. 1597. A momum. L. 1598. Kolowratia. Prst. 1599. Curcuma. L. 1600. Kämpferia. L. 1601. Roscoea. SM. 1602. Hedychium. Koen.

\section{b. $C a n n a c c a c$.}

1603. Myrosma. L. fil. 1604. Phryuium. W. 1605. Thalia. L. 1606. Maranta. L. 160\%. C al at he a. W. Mer. 1608. Canua. L.

Peronia. DG. Red. 


$$
\text { c. } M \approx \text { s } a \text { c } e \text { a e. }
$$

1609. Heliconia. L. 1610. Strelitzia. Art. 1611. Musa. L.
1612. Ravenala. Anaxs.

Urania. Schreb.

FAMILIA L.

\section{P A L M A E. Palmex.}

a. Cyclantheac,

1613. Cyclanthus. Porr.

1614. Carludovica. Rz. PAv.
Ludovia. Poit.

Salmia. W.

b. Phoeniceae.

1615. Phoenix. L.

$$
\begin{aligned}
& \text { c. } \quad \boldsymbol{C} n u \text { i } n a \text { e. } \\
& \text { 2a. S a goin a e. } \\
& \text { a. Pinnatae. }
\end{aligned}
$$

1616. Calamus. L. Raphia. P. B. 1617. Sagus. Rmpr. Metroxylon. Rot t b. 1618. Nipa. ThNB.

\section{B. Flabellatae.}

1619. Lepidocary um. Mart. 1620. Mauritia. L.

$$
\begin{aligned}
& \text { bb. C } \circ \text { c } 0 \text { i } n \text { a } \\
& \text { a. Simplicifoliae. }
\end{aligned}
$$

1621. Mänicaria. Gaert.

Pilophora. Jc q.

$$
\text { B. Pinnata e. }
$$

$$
\text { a. Florib. immersis. }
$$

1622. Astrocaryum. W.Mer. 1624. Elaeis. JcQ. 1623. Acrocomia. Mart. Alfonsia. K. H. B.

$$
\begin{aligned}
& \text { Bs. Florib. sessilibus. } \\
& \text { a. Drupa trisperma (inermes). }
\end{aligned}
$$

1625. Areng. LaB. Saguerus. $\mathbf{R} \mathbf{m} \mathbf{f}$.
Gomutus. $\mathbf{R} \mathbf{m p f}$. 1626. Attalea. K. H. B. 


$$
\begin{gathered}
\text { bb. } D r u p a \text { mon } 0 \text { s p rma. } \\
\text { aaa. Inermes. }
\end{gathered}
$$

162\%. Martinezia. Rz. PAv. 1629. B actris. JcQ. 1628. Guilielma. Mart. 1630. Desmoncus. Mart.

\section{bbb. Aculeatae.}

1631. Diplotheminm. Mart. 1634. Cocos. L. 1632. Maximilianea. Mart. 1635. Elate. Art. 1633. Juba e. K. H. B. 1636. Syagrus. Mhrt.

$$
\begin{gathered}
\text { cc. Arecaceae. } \\
\text { a. Nudiflorae. }
\end{gathered}
$$

163\%. Le opoldinia. Mart.

$$
\begin{gathered}
\text { B. } s p a t h a c e \text { o. } \\
\text { ac. Fissae. }
\end{gathered}
$$

1638. Hyospathe. Mart.

Gynestum. Poit. 1639. G e on ema. W.

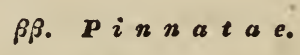

1640. Pt y chospe rma. LaB. 1644. Eut e rpe. Gaert. 1641. Kunthia. Humb. 1642. Areca. L. 1643. Oenocarpus. Mart. 1646. Iriartea. Rz. PAv. 1647. Wallichia. RxB.

\section{yr. Bipinnatae.}

1648. Caryota. L.

$$
\begin{aligned}
& \text { dd. } s \quad \begin{array}{llllllll} 
& b & a & l & i & n & a & e .
\end{array} \\
& \text { a. Fissae. }
\end{aligned}
$$

1649. Cham a edorea. W. 1650? Nunnezia. W. Nunnezharia. Rz. Pav.

f. Flabellatae.

1651. Thrinax. L. Fic. 1653. Licuala. RMpr. 1652. Sabal. Ad. 
CLASS. IV. ACROBL, ORD.II, PHYLL-ACR.FAM.L.PALMAE. 73

$$
\begin{gathered}
\text { ee. Coryphaceae. } \\
\text { a. Fissue. }
\end{gathered}
$$

1654. Morenia. Rz. Pav.

\section{B. Flabellatae.}

1655. Rhapis. Art. 165\%. Livistona. RBr.

1656. Cham a erops. L. 1658. Corypha. L. Chamacriphes. Ponted. 1659. Taliera. MART. Phoenix. C a v. ic. Corypha, $\mathbf{R} \times \mathbf{b}$.

$$
\text { ff. } B \text { or assea } e \text {. }
$$

\section{(Flabellatae.)}

1660. Hyphaene. Gaert. 1662. Lodoicea. LaB. Cuciferu. D e 1. 1661. L at a nia. Com. Cleophora. Gärt. Borcassus. S o nner. 1663. B or as us. L. Lontarus. $\mathbf{R} \mathbf{m} \mathbf{p}$. 



\section{CLASSIS V. \\ SY NCHLAM YDEAE. ZWEIFELBLUMIGE. \\ ORDO I. \\ E N E R $\quad \boldsymbol{V} I$ A E.

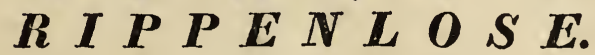

Formatio I.

N A J A D E A E.

NAJADEN.

Fam. LI. Characeae.

Al'mleuchtergevü̈chse.

Fam. LII. Ceratophylleae.

Hornbluttgewüchse.

Fam. LIII. Podostemoneae.

Podostemoneen.

\section{Formatio II.}

I M B R I C A $\mathbf{T}$ A E. SCHUPPENGEW ÄCHSE. Fam. LIV. Lycopodiaceae. Bürlappe.

Fan.LV. Balanophoreae. Kolbenschosser.

Fam. LVI. Cytineae.

Cytineen.

\section{ORDO II. \\ R I G I I F O I A E.}

STEIFBLAETTRIGE.

Formatio I.

I N C O N S P I C U A E.

SCHLECHTBLÜTHIGE.

Fam. LVII. Equisetaceae.

Schachielhaline.

Frun. LYIII. Taxeae.

Eiben.

Fam. LIX. Santaleae.

Santulecn.
Formatio II.

A M B I G U A E. DOPPELDEUTIGE.

Fam. LX. Strobilaceae. Zapfenbäume.

Fam. LXI. Proteaceae. Proteaceen.

Fam. LXII. Thymeleae. Seideln.

ORDO III.

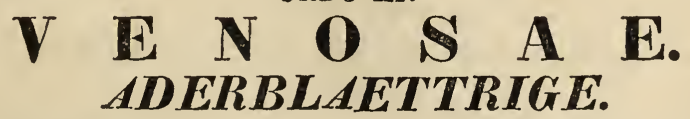

Formatio I.

Formatio II.

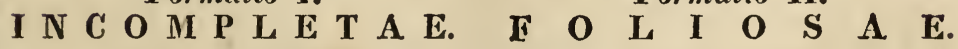

UNVOLLKOMMENE.

Fam.LXIII. Amentaceae.

Käizchenblïthler.

Fam. LXIV. Urticeae.

Nesselgwächse.

Fam. LXV. Nyctagineae. Nyctagineen.
BLATTREICHE.

Fam. LXVI. Piperaceae.

Pfeffergewächse.

Fam. LXVIr. Aristolochieae. Osterluzeigewächse.

Fam.LXVIII. Laurineae.

Lorbeergewächse.

15. Hierüber vergl. Botanik p. 371-404. 
Le but auquel on doit tendre, et dont on approchera sans donte indéfinement, sans l'atteindre peutêtre jamais, c'est que les caractères des classes comparées entre elles, aient une égale valeur; que dans chaque classe ceux des familles aient une égale unTeur entre eux; que de méme dans une famille coux des tribus, des genves, des sections aient une vileur égrale! - - -

DEC. Annal. d. Mus. XVI. p. 140. 


\section{CLASSIS V. SYNCHLAM Y DEAE. ZWEIFELBLUMIGE.}

\section{ORDO I.

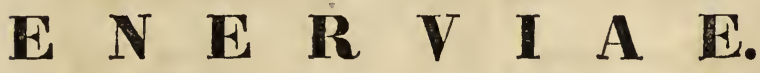 RI P P E NLOSE.}

FORMATIO I.

$\begin{array}{llllllll}\mathbf{N} & \mathbf{A} & \mathbf{J} & \mathbf{A} & \mathbf{D} & \mathbf{E} & \mathbf{A} & \mathbf{E} .\end{array}$ $N A J A D E N$.

FAMILIA LI.

CHARACEAE. Armleuchtergewaeche. 1664. Nitella. AG. 1665. Ch ara. L.

\section{FAMILIA LII.}

CERATOPHYLLEAE. Hornblattgewaechse. 1666. Ceratop hyllum. L. 1668. Naias. L. 166\%. Caulinia. W. Fluvialis. P.

FAMTLIA LIIT.

PODOSTEmoneaE. Podostemonen.

1670. Mniopsis. Mart.

Crenias. Spr.

1671. I a cis. Schreb. 1672. Marathrum. Humb.

BPL.
Dicracia. A. P. Th.

1674. Tristicha. A. P. Th. Dufourca. W.

1673. Podost emon. Mchx, 1675.Hydrostachys.A.P.Th. 
FORMIATO II.

$\begin{array}{lllllllllll}\text { I } & \mathbf{M} & \mathbf{B} & \mathbf{R} & \mathbf{I} & \mathbf{C} & \mathbf{A} & \mathbf{T} & \mathbf{A} & \mathbf{E} \text {. }\end{array}$ SCHUPPENGEWAECHSE.

FAMILIA LIV.

LYCOPODIACEAE. BAERLAPPGEWÄCHSE.

1676. Lycopodium. L.

a. Plananthus. F. B.

b. Stachygynandrum, P. B.

c. Diplostachyum. P. B.

d. Gymnogynum. P. B. e. Lepidotis. P. B.

f. Selaginella. P. B.

167\%. Tmesipteris. Bernh.

16\%8. B e rnhardia. W.

Psilotum. S w.

FAMIIIA LV.

BALANOPHOREAE. KoLBTNSCHOSSER.

16\%9. Cynomorium. L. 1681. Balanophora. Forst. 1680. Langsdorfia. Rrсн. 1682. Helosis. Rich.

\section{FAMILIA INI.}

l. Hypolepis Taz'

\section{CYTineal. Cytineen.}

1683. Aphyteia. L.

1684. Gony anthes. Buum.

1685. $R$ afflesia. $R B r$.
1686. Sarcoph y tum. Sparm. Ichthyosma. S chle cht.
168\%. Cyt in us. L.

1688. A podanthes. BuUme. 


\section{ORDO II. \\ R I G I D I F O L I A E. STEIFBLAETTRIGE.}

\section{FORMATIO I.

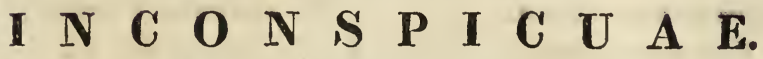 SCHLECHTBLUETHIGE.}

FAMILIA LVII.

EQQUISETACEAE. Schachtelhatme. 1689. Equ is et um. L.

FAMILIA LVIII.

TA X E A E. EIBEN.

1690. Casuarina. L.

$$
\text { a. Casuarineac. }
$$

b. Ephedrece.

1691. Ephedra. L. 1692. Batis. L.

c. Taxinere.

1693. Ta xus. L. 1694. Podocarpus. l'Hentr. 1695. Dacrydium. BNos. 1696. Phyllocladus. Rich.
d. Myriceae. 1696. Phy llocladis.

Thalamia. Spr.

Brownetera. Rich. 169\%. Ginkgo. ThNB. Salisburia. Sm.

1698? Clarisia. Rz PAv. 1700. Myrica. L. 1699. Comptonia. Banks. 1\%00b. Nageia. Gaent.

\section{FAMILIA LIX.}

\section{SANTALEAE. SANTALEN.}

a. Ophireae.
1700. Grubbia. Berg.
1700d. O phira. L. *)

*) Minime cum Grnbbia eadem planta, quod colliges forsan ex ipsa Flora Capensi 'Thunbergiana. Utrinsque plures formas possideo, et huins fructum optine descriptum legi in Encycl. method. IV. p. 566. 
b. Osyrideae.

1701. Anthobolus. RBr. 1708b. Comandra. Nutr.

1702. Osyris. L.

1703. Santalum. L.

1704. Fusanus. L.

170sc. St emonurus. Buume.

1705. Quincham ali um. Juss.

Quinchamati. Feuill.

1\%09. Leptomeria. RBr.

1710. Choretrum. RBr.

1711. Hamiltonia. W. Pyrularia. MIchx.

Calinux. Rafin.

1\%06. Cervantesia. Rz. PAv. 1712. Nyssa. I.

170\%. Exocarpus. LaB.

1708. The sium. L.

a. Thesium.

b. Frisea. R.

c. Thesiosyris. R.

1\%13? Oct arill um. Lour. 1714? Myoschilos. Rz. Par. 1\%14b Laurophyllus.

ThNB.

c. Elaeagneac.

1715. Elaeagnus. L. 1\%1\%. Shepherdia. Nutr. 1716. Hippophä̈. L. 1718. Conuleum. Ricн.

FORMIATIO II.

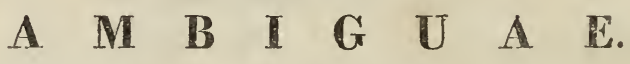

DOPPELDEUTTGE.

\author{
FAMILIA LX. \\ STROBILACEAE. Zapfenbaeume. \\ a. Cupressinue. \\ 1723. S chu bertia. Mrrb.
}

1719. Juniperus. L. 1722. Cupressus. L.

1720. Thuia. L.

1721. Callitris. Vent.

Frenela. Mirb.

b. Abietinae.

1724. Pinus. L.

1725. Abies. T.

1726. L a rix. T.

172\%. Cedrus. T.

1728. Cunninghamia. Ricн. non Schreb.

Bctis. Salisb.
1729. Agathis. SAlisb. Dammara Mirb.

1730. A r a ucari a. Juss.

Dombeya. Lamb.

Eutussa. Salisb.

Colymbea. Salisb.

1731. Altingia. Noronh. 
f FAmilia lixi.

PROTEACEAE. Pruteaceen.

A. Nuciferae.

a. Syu $\boldsymbol{x}$ phe e a e.

1\%32. Syn aphea. RBr. 1\%34. Simsia. RBr.

1733. Conospermum. SM.

b. Protecas.

1735. Adenan thos. LaBilx. 1743. Le eu cospermum. RBr. 1736. Spatalla. RBr. Diastella. Sal. 173\%. Sorocephalus. RBr. 1744. Protea. L. Soranthe. Sal. Erodendron. Sal.

1738. Nivenia. RBr. Paranomus. Sal.

1739. Serruria. Salisb. 1740. Petrophila. RBr. 1741. Isopogon. RBr. 1\%42. Mimetes. Salisb.
1745. Le uc a d e ndro n.Bokrh. Euryspermum. Sal.

Gissonia. Sal. Chasme. Kngt.

1746. Guevin a. Moun. Quadria. Rz. Pav.

c. Persoonieae.

174\%. Symphyonema. RBr. 1751. Cenarrhenes. LaB. 1748. Agastachys. RBr. 1752. Persoonia. Sm.

1\%49. A ulax. Berg. Lintia. Cav. 1753. Brabeium. L.

1750. Frank landia. RBr.

d. Bellendenere.

1754. Bellendena. RBr.

\section{B. Folliculares.}

a. Grevillecae.

1755. Hak ea. Schrad. Conchium. Sin. 1756. Grevillea. RBr.
Lysunthe. Sal. Slylurus. Sal. 175\%. Anadenia. RBr. 


\section{b. Jambertieae.}

1758. Lambertia. SM.

1759. Xylouelum. SM.

1760. Oritis. $\mathrm{RBr}$.
1761. Rhopala. Aubu. Helicia. Lour. Euplassa. Sal.

c. Knightieate.

1762. Knightia.

d. Embothrieae.

1763. Stenocarpus. RBr. 1765. Oreocallis. RBr. Cybele. Sal.

1764. Lom atia. $\mathrm{RBr}$.

Tricondylus. Sal.

1766 Embothrium. Forst. 176\%. Telopea. $\mathrm{RBr}$. Hylogyne. Sal.

e. $B a n k$ siene.

1768. Dryandra. RBr. 1769b? Cylindria. Lour.

Josephic. Sal.

1769. B anksia. L. FIL. 17\%0? Phyla. Lour.

FAMILIA LXII.

\section{THYMELEACEAE. SEIDELN.}

a. Darwinieac.

17\%1? Goniodium. Kz. 1\%1b. Darwinia. Rudg.

b. Daphneae.

17\%2. Thecanthus. Wickstr.

Nectandri. ex p. Berg.

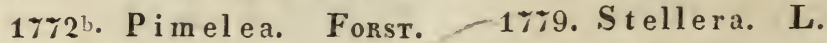

$17 \% 3$ ? Drapetes. LAM. 1780. Passerina. L.

1\%74. Gnidia. L.

Struthia. Roy.

Neclandra. ex p. Berg.

17\%5. Th ymelina. Hrag.

1781. Daphne. L.

1782. Sch o en obiblus. Mart.

1783? Dilobeia. A. P. Th.

1\%т. Lachnaea. L.

Daphnitis. Spr.

17\%. Dais. L.

17\%8. Struthiola. L.

1783b. L a gennla. Lour.

1ร83c? Ca p urá. L.

- jotestion. niels.

c. Aquilurinac.

1784. Aquilaria. Lam. 1786. Gyrinops. Gaent. 1785. Ophiospermum. Lour. 1786 Roydsia. Roxb. 


\section{ORDO III. \\ $\begin{array}{llllllll}V & E & \mathbf{N} & \mathbf{O} & \mathbf{S} & A & \mathbf{E} .\end{array}$ ADERBLAETTRIGE.}

\section{FORMATIO I.

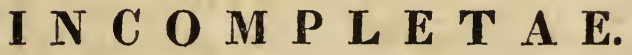 UNVOLLKOMMENE.}

FAMILIA LXIII.

AMENTACEAE. Kaetzchenbluethler.

a. Saliceae.

1787. Salix. L.

(Ascarina. Forst. teste

1788. Morella. Lour.

J us s.)

1789. Populus. L.

b. Betulacene.

1790. B e tula. L.

1791. Aluus. T.

1792. Carpinus. L. 1793. Ostrya. Mici.

c. Plataneae.

1794. P l at anus. L.

d. Fagineae.

-1795. Corylus. L. 1796. F a gus. L.
179\%. Castanea. T. 1798. Quercus. L.

FAMILIA LXIV.

URTICACEAE. Nesselgewaechse.

a. Urticeue.

1799. Pilea. Lindt. 1800. Urtica. L. 1801. Boehmeria. Jce. 1802. Procris. Commers. 1803? Ne rau dia. Gaudich. 180\%. Parietaria. L. 
87 CLASS. V. SYNCHL. ORD. III. VENOSAE. FAM. LXV. NYCT.AG.

a. Helxine. Req.

b. Parietaria. Req. 1808. Forskolea. L.
1809. Clib a dium. Allem.

1810. Hu mulus. L.

b. Dorstenieac.

1811. Dorstenia. L. 1812. Ficus. L. 1813. Olmedia. Rz. Par. 1814. Broussonetia. Vent: 1815. Morus. L.
1816. Antiaris. Lesch. 1817. Artocarpus. L. Sitodium. Gärt. Rademachia. Th $\mathbf{n} \mathbf{b}$. Polyphema. L o u r.

c. Cecropieac.

1818. Gunnera. L. 1819. Cecropia. L.
1820. Cann abis. L.

1821? Sorocea. A. St. H.

d. Mfonimiene.

1822. Mithridatea. Commers. 1825. Laurelia. Juss

1823. A mbora. Juss.

Pavonia. R. P.

Tambourissa. S o n n,

1824. Monimia A. P. Th.

e. Lacistemene.

1826. Lacistema. Sw.

Nematospermum. Rich.

f. Ulmeae.

1827. U 1 mus. L. 1828. Abelicea. SM. 1829. Planera. Мснх.
1830. Cel tis. L.

1831. Didymeles. A. P. Th. Didymomeles. Spr.

FAMILIA LXV.

NYCTAGINEAE. Nrctagineer.

a. Calycantheae.

1832. Calycanthus. L. Büttneria. Duh. non $\mathbf{L}$. Beurreria. E h $\mathbf{x}$.
Basteria. Ad.

1833. Chim on anthus. Lixdu. 
CLASS. V. SYNCHL. ORD. IIT. VENOS. FAM. LXVII. ARISTOL. 85

1834. Citrosma. Rz. Pav. 1836. Hed ycarya. Forst. 1835? Mollinedia. Rz. Par.

\section{b. Atherospermeat.}

1837. Atherosperma. LaB. 1838. Peumus. P.
Boldea. Juss.

Ruizia. Rz. Pav.

c. Allioniacere.

1839. B oerhavia. L.

1840. Oxybaphus. l'Her. Vitmannia. Turr. Calyxhymenia. Cav.

1841. Allionia. L.

1842. Tric ratus. l'Herrt. Abronia. Juss.

1843. Mirabilis. L. Nyctago. Juss.
1844. Reichenbachia. Spr. 1845. Sal piant hu S. HB. BNpL. 1846. Bold o a. Cav. 1847. Torrey a. Spr. 1848. Pis onia. L. 1849. Tricycla. CAv. 1850. A xi a. Lour. 1851. Ne a a. Rz. P. 1852. Buginvillea. Сомm. 1853. Calpidia. A. P. Th.

FORMATIO II.

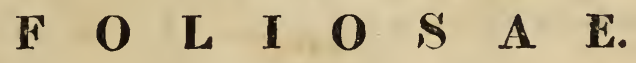

BLATTREICHE.

FAMILIA LXV1.

PIPERACEAE. Pfeffergew Ächse.

1854. Peperomia. Rz. PAv. 1855. Piper. L.

FAMILIA LXVII.

ARISTOLOCHIEAE. Osterluzeigewäche.

a. Genuinae.

1856. Aristolochia. L. 185\%. Bragantia. Lour.

a. Serpentaria. R.

b. Siphorolochia. R.

1858? Ananthopus. Rafin.

c. Pistolochia. R.

d. Cardiolochia. R.

1859. M u n n i ckia. Buume.

Ceramium. B l ume. 
86 CLASS. V. SYNCHL. ORD. III. VENOSAE.FAM. LXVIII. LAURIN.

b. Asarineae.

1860. As a rum. L.

1861. Thot tea. Rorrb.

c. Myristiceae.

1862. Myristica. L.

1864. Hernandia. L.

1863. Kn e ma. Lour.

FAMILIA LXVIII.

LAURINEAE. LoRbERgewächse.

a. Menispermeae.

aa. Lardizabaleac.

1865. Lardizabala. Rz. PAv. 186\%. Burasaia. A. P. Th. 1866. Stautonia. DC.

bb. $G \in \operatorname{c} u$ in a e.

1868. Spirospermum. A. 1882. Pselium. Lour.

P. Th. 1883. Gynostem ma. Buume. 1869. Braunea. W. - 1884. Cissampelos. L. 18\%0. Cocculus. C. Baur. 1885. Clypea. Butme. 18\%1. Chondodendron. Rz. 1886. Menispermum. T. Pav. 188\%. Abuta. Aubl.

1872. C e batha. Forsk. 1873. Leaeba. Forsk. 1874. Wend l andia. W. 18\%5. Androphilax. Wende. 1890. Holboellia. Wall. 18\%6. Fieraurea. Lour. 187\%. Limacia. Lour. 18\%8. Nephroia. Lour. 18\%9. E pibaterium. Forst. 1880. B a g a latta. RxB. 1881. B a u $\mathrm{m}$ a rtia. MNCH.
1888. Trichoa. P.

Batschia. Thn b. 1889? A gdestis. Moc. Sess. 1891. Men iscotia. Buume. 1892. Jo des. Buume. 1893. Co s cinium. Colebr. 1894. An a mirta. Colebr. 1895. Tiliacora. Colebr.

$$
\text { cc. } S \in h i z a n d r e a c \text {. }
$$

1896. S chizandra. Mchx. 1898. Sarcocarpon. Buvme. 189\%. Sphaerostem ma. Blume. 
CLASS. V.SYNCHL. ORD. III.VFNOSAE. FAM. LXVIII. LAURIN. 87

b. Hamamelideae.

1899. Ham amelis. L.

1900. Loropetalum. RBr.

1901. Dicoryphe. A.P. Th. 1903? Fothergilla. L.

c. Laиreae.

1904. Cassytha. L.

1905. Endiandra. RBr.

1906. Galvezia. Rz. PAv. 1907. Laurus. L.

1908. Evosinus. Nutr. 1909. Tetranthera. JAcQ. Tomex, Th nb.

Litsea. L a m.

Sebifera. Lour.

Hexanthus. $\mathrm{L}$ o u r.

Gilabraria. I.
1902. Dahlia. Thus.

1910. Dovera. Ehrnis.

1911. Cryptocarya. RBr.

1912. Persea. GaERT.

Cinnamonum. N. v. E.

1913. Ocotea. K. H. B.

1914, A niba. Aubl.

1915. Polameia. A. P. Th.

1916. A g a th ophy 11 u m.

Commers.

Ravensua. Soun.

Evodia. Gä x t. non Forst.

d. Pterigieae.
1917. Pterigium. Cork. d.
b. Shorea. Bnks. SERR.
c. Dryobalanops. Gärt. f.
a. Dipterocarpus. Gakrt. $f$.
d. Caryolobis. Gärt. 



\section{S Y N P E TA LA E. GANZBLUMIGE. \\ F I S S I F F L O R A E. SPALTBLUMIGE.}

Formatio I.

A G GR E GA T A E. HAEUFELBLUETHLER.

Fam. LXIX. Plumbagineae. Plumbagineen.

Fam. LXX. Caprifoliaceae.

Geisblattgewächse.

Fam. LXXI. Rubiaceae. Rubiaceen.
Formatio II.

C A M P A N A C E A E. GLOCKENBLUETHLER.

Fam. LXXII. Compositae. Scheibenbliithige. Fam.LXXIII. Cucurbitaceae. Kürbisgewächse.

Fam.LXXIY. Campanulaceae. Campanulaceen.

\section{O B I F L O RA E. LAPPIGBLUMIGE.}

Formatio I.

$\begin{array}{lllllllllllllllllll}\mathbf{T} & \mathbf{U} & \mathbf{B} & \mathbf{I} & \mathbf{F} & \mathbf{E} & \mathbf{R} & \mathbf{A} & \mathbf{E} & \mathbf{L} & \mathbf{I} & \mathbf{M} & \mathbf{B} & \mathbf{A} & \mathbf{T} & \mathbf{A} & \mathbf{E}\end{array}$ ROEHRENBLUETHLER.

Fam. LXXY. Labiatae.

$\begin{array}{cc}\text { Lippenbliithler. } & \text { Polygaleen. } \\ \text { Fam.LxxVI. Asperifoliaceae. } & \text { Fam. Lxxix. Personatae. }\end{array}$ Rauhblüttrige.

Larvenbliithler.

Fam. LXXVII. Convolvulaceae. Fam. LXXX. Solanaceae. Windengewächse. Nachtschattengewüchse.

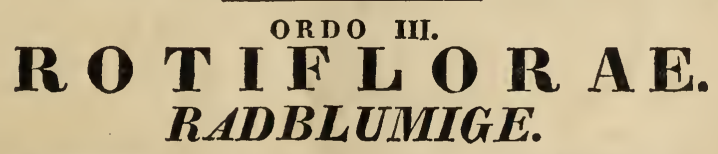

Formatio I.

Formatio II.

C R A T E R I F L O R A E. BECHERBLUETHLER.

STELLIFLORAE.

STERNBLUETHLER.

Fam. LXXXI. Lysimachiaceae. Fan. LXXXIV. Asclepiadeae. Lysimachieen. Asclepiadeen.

Fam. LXXXII. Primulaceae. Fam. LXXXY. Contortae. Primuleen.

Fam. LXXXIII. Ericaceae. Heidegewüchse. Drehblumige. Fam. LXXXVI. Sapotaceae. Supotcen.

I5 Vgl. Botanik. Seite 405-447. 
On est même encore loin d'avoir prouré, qu'il éxiste aucune coupe naturelle intermédiaire entre les classes et les familles: c'est à la fixation de ces sous-classes que les botanistes qui aiment à s'occuper d'ideés geénérales, doivent donner nne sérieuse attention!

DeC. Annal. Mus. XYI. p. 141. 


\section{CLASSIS VI. \\ S I N P E T A L A E. GANZBLUMIGE. \\ ORDO I. \\ F IS S I L L O R A SPALTBLUMIGE.}

\section{FORMATIO 1.

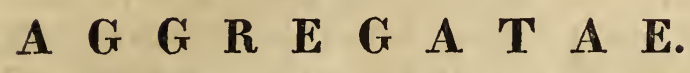 HAEUFELBLUETHLER.}

- FAMILIA LXIX.

Plumbagineae. Plumbagineen.

a. Stylidieae.

1918. Leeuwenhookia. RBr.

Candollea. LaB.

ein.

Levenhookia. R. Br. 1919. Stylidium. Sw. Ventenatia. S m.

1920. Forstera. L. 1921. Phyllachne. Fonst.

b. Brunoniaceae.

1922. Brunonia. $S_{M}$.

c. Staticeae,

1923. Armeria. W. 1924. Statice. L.

a. Statice. RBr. b. Taxanthema, RBr. 1925. Aegialitis. RBr.

d. Dentellariece.

1926. Plumbago. L. 1928? Thela. Lour. 1927. Vogelia. Lam. 
92 CLASS. VI. SYNPET, ORD. I. FISSIFL. FAM. LXX. CAPRIF.

FAMILIA LXX.

CAPRIFOLIACEAE. Geisblattgewächse.

a. Dipsaceae.

a. $O p e r c u l$ a $r i n a c$.

1929. Opercularia. Gaert. 1930. Pomax. Soland.

bb. $s$ c a biosea $e$.

1931. Knautia. L. Cephalaria. Schrad. 1932. Pterocephalus. VArLc. Cerionanthus. Schott. 1933. Asterocephalus. 1935. Scabiosa. L. Varlu.

\section{VAILL.}

a. Sclerostemma. Sch ot t.

b. Spongostemma. Rchb. 1934. Su cisa. VArlt. Trichera. Schrad. 1936. Dipsacus. L. 1937. Triosteum. L. cc. $S c h r a d e r e a c$.

1938. Cephalanthus. L. 1942. Morinda. VArul. 1939. Acrodryon. Spr. 1943. Schradera. V. 1940. Nauclea. L. Urceolaria. Gm.

1941. Adina. Salisb. 1944. Cornus. L.

b. Valerianeae. aa. Genuinae.

1945. Centranthus. DeC.

Valerianella. T. 1946. Valeriana. L. 1948. Patrinia. Juss. 194\%. Fedia. Mrch.

$$
\text { bb. } s a m b u c e a c \text {. }
$$

1949. Sa mbucus. L.

cc. Viburne a $e$.

1950. Vi burnum. L. 1952. L entago. Rafin. 1951. Opulus. Rafin. 1953. Trapaulos. Rafin.

c. Caprifoliaceare, enuinae. aa. Lorantheae.

a. Chlorantheae.

1954. Ascarina. Fonst. 1955. Chloranthus. Sw. Nigrina. Thn b. non $\mathbf{L}$.
1956. Hedyos mum. Sw. Tufalla. Rz. Pav。 195\%. Gn etum. Forsk. 1958. Thoa. AUbL. 


\section{B. Viscinae.}

1959. Viscum. L.

Lonicera. P In m.

1960. Arceuthobium. MB.

a. Holanthus. R.

1961. A u cuba. L.

b. Petalanthus, R.

1962. Loranthus. L.

1963. Tupeia. Cham.

$$
\text { y. M e mecyle a e. }
$$

1964. Memecylon. L. 1965. Scutula, Lour.
1966. Mouriri. Aubl.

Mouriria. Juss.

Petaloma. S w.

\section{S. Rhizophoreae.}

196\%. Olisbea, DeC.

1968. Rhizophora. L. et Bruguiera. L a m. 1969. Ca rallia. Roxb.
1970. Cassipourea. Aubr. Tita. Scop. Legnotis. $\mathrm{S} \mathrm{w}$. Richacia. A. P. Th, non a I. Weihea. Spr.

bb. Vaccineae.

1971. Oxycoccus. Pers. schollera. Rth. 1972. Vaccinium. L. 1973. Ceratostemma. Juss. 1974. Thibaudia. Rz. PAv.
Cavinium, A. P. Th. 1975? Symphysia. Prsi. 1976. Ga ylussacia. K.H.B.

cc. Lonicereac.

197\%. Linnaea. Gron. 1978. Abelia. RBr. 1979. Schöpfia. Schreb. 1980. Symphoria. Prsh. Symphoricarpus. K. H. B. 1981. Aidia. Lour.
1982. Diervilla. T. 1983. Coll a d o nia. Spr. 1984. Xylosteum. T. Lonicera. $\boldsymbol{L}$. 


\section{b. Coffecaceac.}

1993. Knoxia. L. 1994. Plocama. Ait. Placoma. L. G m.
2018. Hydroph y tum. ЈАск. 2019. Tetramerinm. GaERT.f. Potima. P.

1995. Richardsonia. K.H.B. 2020. Froelichia. V. Richardia. L.

1996. Cuncea. Hamit. 199\%. Spermacoce. DiLl.

a. Gruhlmannia. Neck.

b. Covalia. Nerk.

c. Chaenocarpus. Neck. 1998. Bigelovia. Spr. Borreria. II ey non $\mathbf{A} \mathrm{ch}$. 1999. Mithracarpus. Zucc. 2000. St a ëli a. Cham. 2001. Diodia. Gron. 2002. Hy drophylax. L. f. Sarissus. Gärt. Scyphiphora. Gärt. 2003. Ernodea. Sw. 2004. Mitchella. L. Chumueduphne. II itch. 2005. Nertera. Banks. Nerieria. S m. Erythrodamum. A. P. Th. Gomezia. Mut. Gomozia. S chreb. 2006. Si d e ro d e n dro n. Schreb. Sidernxyloides. J c q. a m. 200\%. Polyosus. Lour. 2008. F a r a mea. Aubr. 2009. Baconia. DeC. 2010. Chomelia. Jç. am. 2011. Ixora. L. 2012. Pavet a. Rheed, 2013. Declienxia. K. H. B. 2014. Psyllocarpus. MarT. 2015. Dunali a. Spr. pug. 1815 non K. H. B. 1818. 2016. Las i anthus. JACK. 201\%. H elospora. J JCK. Billarderia. V.

2021. Cous sarea. Aubl. Pecheya. Scop.

2022. Malanea. Aubl. Cunninghamia. S c h reb. Chomelia. Scop.

2023. Antirrhea. Commens. 2024. Scolos anthus. V.

2025. St en os to mum. GAert. f. Sturmia. Gärt. f. Laugeria. $\mathrm{S}$ w. 2026. Rutidea. DeC. 2027? Cattutella. Rreed. 2028. J a cki a. WAll. non SPR. Zuccagnia. S pr. 2029. A rgoste mma. WaLL. 2030. Chiococca. J. Br. 2031. Psych otria. L. Psychotrophum. J. B r. a. Antherura. Lour. Caryophyllaster. $\mathbf{R} \mathbf{m} \mathbf{p h}$.

b. Simira. Aubl.

c. Mapouria. Aubl. d. Myrstiphyllum. J. Br. e. Viscoides. Jacq, am. $f$. Hilacinm. P. B.

2032. Palicourea. Aubl. Stephanium. Schreb. Galvania. Vell. non Spr.

2033. Chas a lli a. Comas,

2034. Coffea. L.

2035. Canthium. Lam.

Gardenia. L. f. Webera. Schreb. Damnacanthus. Gä $\times \mathbf{t}$. $\mathrm{f}$. 2036. Geophila. Don. 
203\%. Ru dgea. S Sulsi. 2038. Ronabea. Aunc. 2039. Ly godis. Rz. PAv. Disodea. Poir.
2040. Chim arrh is. Jce. am. 2041. Machaoni a. HB. BPL. 2042. Alsëis. Schotr.

\section{c. Cinchoneae.}

2043. Hedy o $t$ is. L. 2044. O ldenlandia. PuUM. Heuchera. MI ur. Aeginetia. Cav.

2045. Polypremum. L. 2046. Bouvardia. Salisb. 2047. Carphalea. Juss. 2048. Hoffmannia. Sw. 2049. Na cibea. Aubr. ITanetlia. $\mathbf{M} \mathbf{u}$. Ophiorrhiza. Forsk.

2050. Ro ndeletia. Plum.

Lightfootia. Schreb. 2051. Tula. Ad. 2052. D en tella. Forst. 2053. Danais. Commers. 2054. Hymenopogon. WarL. 2076. Stigmant hus. Lour. 2055. Lecananthus. JАск. 20\%. Po in a ti um. G̈̈rt. 2056. Psilobium. JAck. 205\%. Virecta. L. f. 2058 ? S ipanea. Aubl. 2059. Spermadyction. Rxв. 2060. Belonia. L. 2061. Mus s a e ud a. Herm. Belillie. Phe d. Braclearice. Pö pp. 2062. Pinck nе уа. Michx. 2063. Landia. Сомм. 2078. Oxy anthus. DEx. 2079. Genipa. T. 20ś. Gardenia. EuLis. Ceriscus. Gärt. 2081? Rothman uia. Thb. 2082? Sahlbergia. Neck. 2083. Am a i о u. Aubr. Kihrenbergia. Spr. non M axt. 2084. Randi a. Houst. Euclinia. Salis b. 2064. Macrocue mum. J.Br. 2085. St ylocoryna. Car. 2065. Cinchona. L. Kitlkina. Ad. 2086. Bertiera. Aubl. 2087? Zaluzania. Commers. 2066. Cosmibuena. Rz. Pav. 2088. Hippotis. Rz. Pav. 
2089. Petesia. J. Br.

Lygistum. J. B r.

2090. W e b era. G̈̈Rt. f. non W.

2091. Catesbaea. Gron. 2092. Fernelia. Сомм. 2093. Coccocy p e lum. J:Br. 2094. Tontanea. Aubl.
Bellardia. S $\mathbf{c}$ h r e b. Sicelium. J. Br. 2095. Higginsia. P. O-Higginsia. Rz. P. Nacibea. A u b 1. ? Sabicea. A u bl. 2096. Seris s a. Cumm. Buchozia. l'II e r. Dysoda. Lour.

d. Guettardeae.

209\%. Nonatelia. Aubr.

a. Oribasia. Schreb.

b. Retiuiphyllum. Hb. Bpl. 2098. Ancylanthus. Dsf. 2099. B o b e a. Gaudich. 2100. Erithalis. J. Br.

Herreria. Ad.

Psathura, C o m m.?

2101. Ps athura. Comm. 2102. Cuviera. Den. 2103. V angueria. Juss.
Vavanga. V. 2104. Pyrostria. Сomm. 2105. Myonima. Com. 2106. L a ugeria. Jcọ. am. Edechi. Löffi. 210\%. Guettard a. L. Halesia. P. 13 r. Cadamba. S on. Ravapa. Rhe d. NIatthiola. Blum.

\section{e. Hameliaceae.}

2108. Evosmia. Hв. Bpr. 2109. Hamelia. JcQ. am.

Tangaraca. Ad.

Duhamelia. P.

2110. Leycestera. Warr.

2111. Piringa. Juss.

Caquepiria. G m.

Thunbergia. MI ont.

Berghias. So nn.
2112. Is ertia. Schreb.

2113. Polyphragmon. Dsf. 2114. Sabicea. Aubt. Schwenlifeldia. Schreb.

2115. Gonzalea. P.

Gonzalugunia. Rz. P.

Buena. C a v.

Tepesia. Gärt?

2116. Patima. Aubr.

\section{f. Cephaelideae.}

211\%a. Canephora. Juss. $211 \%$. Patabea. AubL. 211\%c. Burchellia. RBr. 2118. Evea. Aubl. 2119. Cephaëlis. Sw. a. Tapogomea. Aubl.

b. Carapichea. Aubl.

c. Callicocca. Bret.

2120? Matthissonia. RAdD. 
FORMATIO II.

\section{A M P A N A C E A E. GLOCKENBLUETHLER.}

FAMILIA LXXII.

COMPOSITAE. Scheibenblüthige.

(SYNANTHEREAE, SYNGENESISTEN.)

A. $H$

a. L $a c t u c e \boldsymbol{c} e$.

aa. Hyoserideac.

2121. Lapsana. L. 2127. Krigia. Schreb.

Lampsana. T. 2128. H y o seris. Juss.

2122. Rhagodiolus. T. 2129. Aposeris. Neck.

2123. Kölpinia. Pall. 2130. Hedypnois. $T$.

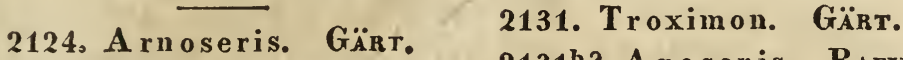

2125. Hispidella. Lam. 2131b? Agoseris. Rafin. Soldevilla. LaG. 2132. Hymenonema. H.CAss.

2126. Mos caria. Rz. PAr. 2133. Catananche. L. Moschifera. Mol.

IIosigia. Spr.

Catanunce. T.

2133b. Cichorium. L.

bb. $C$ repide a $e$.

2134. Thrincia. Rrн.

2135. L e o n t o d on. Juss. Apargia. Schreb.

2136. Picris. L.

213\%. Medicusia. M McH.

2138. Helminthia. G̈̈нт.

2139. Z acyntha. T.

2140. Nemau chenes. H.CAss.

2141. G at y on a. H. CAss. Crepidium. Tsch.

2142. B ark ha us ia. Mrch. Borlihausia. Lk. Spr.

a. Anisoderis. H. Cass. Hostia. Mnch.

b. Barkhausia. H. Cass. Wibetia. G. M. S.
2143. Lagoseris. LK.non MB. a. Lagoseris. R. ap. Mössıl.

b. Aegoseris. R. ib.

c. Lepidoseris. R. ib.

2144. Phaecasium. H. Cass. 2145. Crepis. L.

Wibelic. Hpp.

Beriniu. Brigu.

2146. Brachy derea. H. Cass.

Hapatosteptium.

2148. H o m a locline.H. Cass. em.

Omulocline. II. Cass.

2149. Ge ra сі й. Rснв.

2150. Hiera cium. L.

Aracium. Neck.

Micgia? Neck.

2151. Tolpis. GäkT. 
Sucrticu. Lndw.

Drepania. Juss. 2151b. Schmidtia, MNCH. Polychaetia. Tsch.

\section{cc. Andrya $l$ e a $e$.}

2152. Deloderium. H. CAss.

Myoscris. Lk.

2153. Hy pochaeris. L. Arachnospermum. Berg.

2154. Porcellites. H. Cass. Achyrophorus. Gärt. non Vaill.

2155. Crepinia. Rchв. *) 2158. Rodigia. SPR. ${ }^{* *}$ ) 2159. P i t o pogo n. H.CAss. 2160. Robertia. DeC. 2160b. S eriol a. L. Achyrophorus. Vaill. 2156. Pterotheca. H. CAss. 2160\%. R othia. Schreb. Lagoseris. MT. B. 215\%. Intybellia. H. CAss. 2160d. Andryala. L. dd. S corzonereac.

2161. Willemetia. Neck. Wibclia. Röhl. Calycocorsus. Schm. Peltidiun:. Zollik. Zollikoffera. N. $\mathbf{\text { v. E. }}$ 2162. Ta $\mathrm{r}$ a $\mathrm{x}$ cum. Halt. Leontodon. Schreb. 2162b. A theorrhiza. H. CAss. 2175. S corzonera. L. 2162c. I x e ris. H. CASs. 2163. Lomatolep is. H. Cass. 2164. Rhabdotheca. H. CAss. 2165. Chondrilla. L. 2166. Phoenixopus. H. Cass. 2178. Urospermum. Scop. 216\%. L actuca. L. 2168. Mycelis. H. Cass. Cicerbita. Wallr, ex $p$. 2169. Prenanthes. L. 21\%. N a b a lu s. H. CAss. Prionanthes. Schrk.

2176. Lasiospora. H. CAss. Lasiospermum. Fisch. non LaG. 2176b. Aster othrix. H. CAss. 217\%. Gel as i a. H. CAss. Arnopogon. W. 2179. T ragopogon. T. 2180. Geropogon. L. 2181. Scolymus. L. 2182. Myscolus. H. Cass.

$$
\text { b. } E u p \text { «t }
$$

2183. Corymbium. L.

2188.Spargan oph orus. Gänt. Struchium. P. Br.? cf. 2224.

to 2184. Achyrocoma. H. CASs. 2189. Voigtia. SPr.

2185. V e r n on ia. Schreb. Turpinia. Bnpl.

2186. Ascaricida. H. CAss. 2190. O dontoloma. K. H.B. 2187 Ethulia. L.

*) E.tian ap. Morsserr p. 1415. et in Pl. crit. Cez VII.

${ }^{* *}$ ) Ic. ixol. 126. ibi et alia horum generum, e tim Plantis criticis alia. 
2192. Centrapalus. H. Cass. 2209. Monenteles. LaB. 2193. Centratherum. H. CAss. 2210. Chlaenobolus. H. CAss. 2194. Distreptus. H. Cass. 2211. Pluchea. H. Cass. 2195. Distephanus. H. CAss. 2212. G ym anthe mum. 2196. Dialesta. K. H. B. H. CAss. 219\%. Pollalesta. K. H. B. 2213. Heterocom a. DeC. 2198. Ampherephis. K.H.B. 2214. Hololepis. DeC. Spixia. Schrk. 2215. I s on ema. H. Cass. 2199?? Wickströmia. SPr. 2216. Le pidaploa. H.CAss. non Schrad. 221\% L i a bum. Adans. 2200. Pacourin a. Aubr.

Haynea. W. 2201. P a cour in opsi s. H. Cass. 2202. S t o k e s i a. L. Her. 2203. Ol ig oc a rph a. H. CAss. Brachyluena. RBr. 2204. A rrhe n a chn e. H.CAss. 2205. Ta rchon anthus. L. 2206. Tessaria. Rz. PAv. 220\%. Mo n arrhenus. H. CAss.

2208. P h a 1 a c $r$ o m e s us. 2226? Albertinia. Spr. H. CAss. bb. Archetypae.

222\%. Eupatorium. L. 2236. Sclerolepis. H. CAss. 2228. Mikania. W. 2229. A geratum. L. 2230. Batschia. Mrcir. 2218. L y chnophora. MART. 2219? Munnozia. Rz. PAv. 2220. O ligactis. H. CASs. 2221. Oli ganthes. H. Cass. 2222. Pip to coma. H. CASS. 2223? Shawia, Forst. 2224. Struchium. P. Br. 2225 ? X anthocephalum. W. 2231. Coelestina. H. Cass. 2232. Alomia. K. H. B. 2233. Gypt is. H. Cass. 2234. Pi q u eria. Car. 223\%. Stevia. Cav. Arusteliu. Sp r. 2238. Kuhnia. L. f. 2239. Trilis a. H. CAss. 2240. Li atris. Schneb. 2241? Acilepis. Don. 2242? Leucomeris. Don. 2235. Adenostemma. Forst. 2243 ? Giintheria. Spr.

Lavenia. Soland.

$$
\text { cc. Adenostyleac. }
$$

2244. Homogyne. H. Ciss.

Palcolaria. H. C a s s. 2245. Adenostyles. H. CAss. 2247. Nothites. H. CAss. 2246. Pal a foxia. LaG. 2248. Celmisia. H. Cass. 
100 CLASS. VI. SYNPET. ORD.I. FISSIFL. FAMT. LXXII. COMPOS.

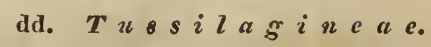

2249. Tussilago. T. Farfara. DeC.
2251. Petasites. CB. 2252. Hop pea. Rchв.

2250. Nardosmia. H. C.Ass.

$$
\text { c. } \quad C \quad y \quad u \quad \ll \quad r \quad e \quad u \quad e \text {. }
$$

aa. Irtisicae.

2253. Onoseris. W.

2254. Is ot y u s. K. H. B. Seris. W.

2255. P a r d is iu m. N. L. Burm.

2256. Trichocline. H. Cars.

225\%. Gerbera. Gron.

Gerberia. H. Cass.

Aphyllocuuton. LaG.

2258. Lasiop us. H. CAss.

2259. Chaptalia. VENT.

2260. Lox odon. H. CAss.

2261. Lieberkiihna. H. Cass.

2262. L e ria. DEc.

Thyrsanihema. Neck?

2263. P e r di cium. L.
Idicium. $\quad \mathrm{Ne} \mathbf{c}$.

2264. Le ib in it ia. H. CAss. Anundria. Si g gesb.

2265. Proustia. LaG. 2266. Cherina. H. CASs. 226\%. Chatanthera. Rz. Par. 2268. Gu a riruma. H. Cass. 2269. A plophyllum. H. CAss. $22 \%$. Mutisia. L. f.

22\%. Dolichlasium. LaG. 22\%2. Lycoseris. H. Cass. 22\%3. Hipposeris. H. Cass. 22\%4? Flotovia. Spr.

bb. $\quad N a s s a u v i c a c$.

22\%5. Dum erilia. LaG.

22\%6. Jungia. L. f.

Trinacle. Gä r $\mathbf{t}$.

22\%. Martrasia! LaG.

22\%8. Lasior hiza. LaG.

Rhinactina. W.

Frageria. Del.

Bertolonia. DeC, sub ic.

Chabraea. DeC.

22\%9. Le ucheria. LaG.

Leucueria. DeC.

2280. Trixis. P. Br.

non $\mathrm{MI}$ it ch. nec $\mathrm{Sw}$.

Tenorea. B erter.
Holecheilus. H. Ca s.

2282. Perezia. LaG.

2283. Clarionea. LaG. Homanthus. K. H. B. 2284. Hom o i a thus. Bonpl. Heteranthus. Bnpl. Homanthis. K. H. B. 2285. Drozia. H. CAsss. Homanthis. K. H. B.

2286. P a up a lea. LaG. Pamphulea. DeC. Ceratolepis. H. C as s.

228\%. Triptilion. Rz. P. 2281. Platycheilus. H. Cass. 2288. Tria chne. H. CAss. 
2289. Nas sauvia. Commers. Nassovia. P. Nassawia. L a G.

2290. M a s tigo phor us. H. CASS.

2291. Caloptilium. LaG. Sphaerocephalus. LaG. 2292. P an argy rus. LaG.
Panargyrum. DeC. 2293. Polyachyrus. LaG. Polyachurus. Dec.

2294. Plazia. Rz. P. 2295. Barnadesia. L. 2296. Bacazia. Rz. P.

$$
\text { cc. } \quad C \text { arline a } e \text {. }
$$

229\%. Cardopatium. Juss.

Brolera. W. 2298. St o b a ea. Thнв. 2299. D icoma. H. CAss. 2300. Xeran the mum. L. GänT. 2301. Staehelina. L.

2302. Chuquir aga. Juss. Johannia. W.
Joannesia. P. 2303. Chardinia. Dsf. 2304. S a ussurea. Dec. 2305. Carlowitzia. MNCH. Alhanus. Neck.

2306. Carlina. L.

230\%. A tracty lis. L. nor Vaill. a. Cirsellium. G. b. Acarna. G.

dd. $\quad C a v d u a c e a c$.

a. Serratuleae. ac. Carthameae.

2308. C a rduncellns (Lobel) - Onozroma. Gärt. Adrs. 2309. Carth a mus. T. Carthamoides. Vaill. 2310. Cestrinus. H. CAss. B.. Rhaponticeac.

2311. R haponticum. VAILL. Rhacoma. A d n s. Rhacomae sp. A dins. Hookia. Ne c k.

2312. Leuzea. DeC.
2313. Fornicium. H. Cass. 2314. Stemuacantha. H. C.ass.

\section{\%. Ge $n u$ u}

2315. J u rinea. H. CAss. 2316. Klasea. H. Ciss. 231\%. Serratula. L.
2318. Mastrucium. H. CAss. 2319. Lappa. T. Arclium. L. Vill. 
102 CLASS. VI. SYNPET, ORD. I. FISSIFL. FAM. LXXII. COMPOS.

$$
\begin{aligned}
& \text { B. Carduinae. } \\
& \text { ua. Silybeac. }
\end{aligned}
$$

2320. Alfredia. H. CASs. 2321. Echenais, H. CAss. Hookia. N e ck? 2322. Sil ybum. VallL.

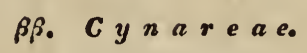

2323. Cynara. T.

2324. Onopordon. Varrix. Acancs. Adans.
2325. Arctium. Lam.non L. Arction. D a l e ch. Villaria. Guet t. Berardia. Vill.

\section{gr. L a myreac.}

2326. Platy raphium.H. CASs. 2328. Ptilostemon. H. CAss.

232\%. L a my r a. H. CAss. Polyacantha. Vaill. Xylanthema. N e ck.
Chamaepeuce. P. Alp. 2329. Notobasis. H. CAss. Polyacantha. Vaill.

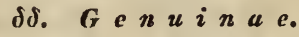

2330. Picnomon. Dalech. 2336. Galactites. MNGh. Acarna. Vill, non W.

2331. Lophiolep is. H. Cass.

2332. Eriolepi s. H. Cass. Eriocephalus. $\mathbf{V}$ a ill 1 , non $\mathbf{L}$. $X$ ylanthema. Neck.

2333. Onotrophe. H. Cass. Cnicus $\mathrm{N}$ eck ?' non $\mathrm{V}$ a ill.

2334. Cirsium. T. Cardui, s p. L.

Cnicus Hoffm. W. non Vaill. 2341? Ptern ix. Rafin.
Erioeephalus, Vaill. non $\mathbf{L}$. Lupsia. $\mathbf{N}$ e c $\mathbf{k}$. 233\%. Tyrimnus. H. C.Ass. 2338. C ard u us. T. Platylepis. H. c. Slenolepis. H. c. Clomium. Ad ans. 2339? Hohenwartha. Vest. 2340? Onopix. Rafin.

2335. Orthocentro n. H. Cass.

$$
\text { d. } \quad E \quad c \quad h \quad i \quad n \text { o }
$$

a. Gymnostyleac.

2342. Soliva. Rz. P. Gymnostyles. Juss.

$$
\text { bb. } \quad \text { a g a s c ine a e. }
$$

2343. Lagascea. CAv. Nocca. Cav. Noccaea. Jcq.

2344. Elephantopus. L. 2345. Spiracantha. K. H.B. 
cc. $R \circ l$ a $n d r \in a e$.

2346. Trichospira. K. H.B. 2348. Guudelia. L. 2347. Rolandra. Roтtв.

$$
\text { dd. } Q \text { e } n \sim \boldsymbol{i} \boldsymbol{n} \text { e. }
$$

2349. Echinops. L.

Echinopus. Plin.

Echinanthus. N e ck.

B. A mphigynanthae.

a. Taget e a e.

2350. Cryptopetalon. H. 2358. Tetranthus. Sw.

CAss. 2359. Clom en ocoma.H.CASs.

2351. Diglossus. H. Cass, 2360? Glyphia. H. CAss. Micria. Lk.? 2361. Hy menatherum. H.

2352. Adenophyllum. P. CASS.

2353. Chthonia. H. CAss. 2362. Kleinia. Juss.

2354. Dyssodia. Cav. 2363. Lebetina. H. CAss.

2355. Enalcida, H. CAss. 2364? Microspermum. LaG. 2356. Pectis. L. 2365. Thymnophylla. LaG. 235\%. Porophyllum. VArLt. 2366. Tagetes. T.

\section{b. $A n t h e m i d e d e$.}

a. Chrysanthemeue. a. Artemisieae.

236\%. Oligo o po rus. H. Cass. 2368. Artemisia. T. 2369. Absinthium. Anss. 2370. H u me a. SM.
Calomeria. V ent. Agathomeris. Delaun. Oxysphaeria.

Razumovia. S p r.

$$
\text { B. Cotul e a e. }
$$

2372. Hippia. L.

2373. Leptinella. H. CASs. 2374. Cenia. Сомm.

Lancisia. Gä $\mathbf{r}$.
2375. Cotula. L. Ananthocyclus. Vaill. Lancisia. P o nt ed. Baldingeria. N eck. 
104. CLASS, VI. SYNPET, ORD. I.FISSIFL. FAMI. LXXII. COMPOS.

$$
\text { \%. Ta u a cete } a \text { e. }
$$

2376. Bals a in ita. Valll. 23\%. Pentzia. Thnb.

Psanacelum. Neck. 23\%s. Tanacetum. T. L.

d. Iratricarieae.

23\%. Gymnocline. H. CAss. 2380. Pyrethrum. Hats. Mijconia. Neck. 2381. Chrysanthe in am. T.I. Bellidioides. $\mathrm{V}$ a i 11. Leucanthemum. Adns. 2382. Centrachena. Scnotr.
Heterunthemis. Schot t. Centrospermum. Spr.non K. H. B. 23\$3. Matricaria. VAlle. Chamaemelum. $\mathbf{T}$. 2384. Li ibeckia. Berg. Lancisiu. P.

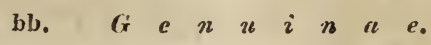

\%. Santolineae.

2385. Hymenolepis. H. Cass. 2391. Ayac yclus. L.

2386. Athanasia. L.

238\%. Lonas. Adrs.

a. Hiortria. Neck.

b. Anaofcius. Neck.

2388. Diotis. DsF.

2389. Santolina. T.

2390. Lasiospermum. LaG.

2392. Mataxa. Spr.

Lanipila. $\mathbf{B} \mathbf{u} \mathbf{r} \mathbf{c h}$.

Lasiospermum. Trer.

non Fisch.

B. Anthemeac.

2393. Anthemis. Mrch. 2400. Osmitopsis. H. Cass.

2394. Chamaemelum. T. 2401. Osmites. L.

2395. Maruta. H. C.sss. 2402? Lepidophorum. Neck.

2396. Ormenis. H. CAss. 2403. Sphenogyne. RBr.

239\%. Cladant hus. H. CAss. 2404. Ursinia. Cärt.

2398. Eriocephalus. Dink. 2405? Zeyheria. Spr.

2399. Achille a. VAILL.

$$
\begin{aligned}
& \text { c. J } n u l e a r \text {. } \\
& \text { aa. Gnaphalieac. }
\end{aligned}
$$

2406. Relhania liHer. Michanxia. Neck. Eclopes. G.
240\%. Rosenia. Thins. 240s. Leysera. L. Cullicomia. Burm. 
2409. I. e p to phy tum. H. CAss. 2410. Longchampia. Willo.
Trichandrum. $\mathrm{N}$ e c k.

Elichrysum. W.

2430. Podolepis. LaB.

2411. Chevreulia. H. CAss. 2431. Antennaria. Gärt. 2412. Lucilia. II. C.Ass. Disynanthus. Rafin. 2413. Faceli s. H. Cass. 2432. Oz othamnus. RBr. 2414. Podosperma. Lab. Podothecu. II. Cass.

2433. Petalolepis. H. CAss. 2434. Metalasia. RBr. 2435. Endoleuca. H. CAss.

2415. Syncarpha. DeC. Roccurdiu. Neck?

2416. Faustula. H. C.dss.

2436. Shawia. Forst.

243\%. Perotriche. H. Cass. 2438. Seriphium. L.

241\%. Phagnalon. H. CAss. 2439. St o ebe. L.

2418. Gnaphalium. L. RBr. 2440. Disparago. G̈̈rt.

2419. L asiopogon. H. CAss.

2420. If $\log$ a. H. CAss. Wigandia. Neck.

2441. O edera. L.

2442. Elytropappus. II. CAss.

2421. Piptocarpha. RBr.

2422. Cassinia. RBr.Bot. Reg. non $\mathrm{H} . \mathrm{K}$ Ev.

2423. Ixodia. $R B$ r.

2443. Siloxerus. LaB. Siylonceriss. S p r.

2444. Hirnellia. H. CAss. 2445. Gnephosis. H. Cass. 2446. Angianthus. Wendu. Cassinia. RBr. H. Kew.

2425. Lepiscline. H. CAss. 2426. An axeton. G̈̈вt. Argyrunthes. N e ck.

244\%. Calocephalus. RBr. 2448. Leucophyta. RBr. 242\%. Edmondia. H. CAss. 2449. Craspedia. Forst. 2428. Argyrocome. Gärt. 2429. Helichrysum. VaIhL.

Cartodium. Sol.

Richea. LaB. non $\mathbf{R B r}$.

2450. Leontop odium. P.

bb. Ge $n u$ i $u a c$.

2451. Filago. W.

Evux, G.

2452. Gifola. H. CASS. 2453. Logfia. H. CAss. 2454. Micropis. L. 2455. Oglifa. H. Ciss.

2456. Conyza. H. CAss.
245\%. Inula. G̈̈вт.

2458. Corvisartia. Merat. 2459. L imbarda. Aons. 2460. Duchesnia. H. Cuss. 2461 Pulicaria. Gärt. 2462. Tubilium. H. CAss. 2463. Jasonia. H. CAss. 2464. My riadenus. H. CAss. 
2465. Carpesium. L. 2466. Denekia. Thns. 246\%. Nestlera. Spr.

Columellea. Jcq. non Rz. Pav.
2470. Rhanterium. DESF. 2471. Cylind rachne. H. Cass. 2472. Telekia. BaUmG. Molpadia. H. Cass.

2468. Pentanema. H. CAss. 2473. Neurolaena. RBr. 2469. Iphiona. H. CAss.

$$
\text { cc. } B u p h t h a l m e a c \text {. }
$$

2475. Buphthalmum. L. Busticu. A da n s.

24\%6. P a l l en is. H. CAss. obeliscotheca. Adns. Athalmum. N e ck.

24\%7. Nauplius. H. Cass.

2478. Ceruana. Forsk.

2479. Egletes. H. Cass. 2480. Grangea. Adns.
2481. Centipeda. Lour.

2482. Sphaeranthus. L. non Scop. Sphaeranthos, Vaill. Polycephalus. Forsk. 2483. G y muarrhena. Desf. 2484? Lio ydia. Neck. 2485? Lachnos permum. W. 2486? D isyna thes. RAmin.

4486.6. Beslidestion- Senecioneae.

- 2487. Doronicnin. L. 2488. Grammart hron. CASs. Aronicum. N e ck.
2489. Cul ci tium. Bonpl. H. 2490. Eriothrix. H. Cass. 2491. Aspelina. H. Cass. 2492. Do ro baea. H. CAss.

\section{B. Ge $n u$ u $n$ as $e$.}

2493? At he ol aena. H. CAss. 2503. F a ujasia. H. CAss. 2494. Carderina. H. Cass. 2504. Ne o ceis. H. Cass. 2495. Senecio. L. e. e. Ptileris, R a fin?

2496. Obaejaca. H. Cass. 2505. Cremocephal um. H. Anecio e. $e . \mathrm{Neck}$. CASS.

249\%. I a co b a e. T. Crassocephalum. H. Cass. 2498. Sc ler o ba is. H. CASs. 2506. Gy u u r a. H. Cass. 2499. S yn arthrum. H. CAss. 250\%. Eu dorus. H. Cass. 2500. Gynoxis. H. Cass. 2508. Pericalia. H. Cass. 2501? Scrobicaria. H. CAss. 2509. Cacalia. L. e. e. 2502. Hubertia. B. St. V.

Klcinia. L. non Jcq. Juss. 2510. Pentacalia. H. CAss. 


$$
\text { r. Othon ne a e. }
$$

2511. Erechtites. Rafin.

Aristotela. A d ns.

2512. Emilia. H. CAss. Oth. et Hertia. Neck.

2513. Pithosillum. H. Cass. 2516? Doria. Thnb. non Adns. 2514. Eu r yops. H. CAss. Werneria. K. H. B. 2515. O thouna. L. 2517. Cineraria. L. e. e. 2518? B r a ch y g 1 ot t is. Forst.

$$
\begin{aligned}
& \text { d. } A s t e r e \text { a } \\
& \text { a. Solidagineas. } \\
& \text { a. Grindeliaceae. }
\end{aligned}
$$

2519. Xanthocoma. K.H.B. 2521. D onia. RBr. 2520. Grindelia. W. Aurelia. H. C a s s.

Demetriae sp. LaG.

$$
\text { B. Psiadiacea } e \text {. }
$$

2522. Elphegea. H. Cass. 2523. Sarcanthe mum. H.CAss. Epilatoria. C o m m. Gilutinaria. C o $\mathbf{m} \mathbf{m}$. 2524. Ps i adia. JcQ. 2525. Nidorella. H. CASS.

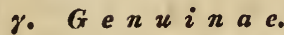

2526. Euthamia: Nutr. 2527. Solidago. L. Virga aureu. Vaill. 2528. Dipl opappus. H. CAss.

$$
\text { S. Lepidophylleac. }
$$

2530? Brachyris. Nutr. 2532. Lepidophyllum. H. 2531. Gutierrezia. LaG. CAss.

$$
\begin{aligned}
& \text { bb. } \quad B \quad a \quad c \quad c \quad h \quad a \quad r \quad i \quad d \quad c \quad a c \\
& \text { a. Chrysocomeac. }
\end{aligned}
$$

2533. P te ronia. Printz. Crinita. Mnch, non Iloutt. Ptcrophorus. Vaill. non Zool. 2536. Linosyris. H. CAss. Pterophora. N e ck. 2534. Scepinia. Neck. 2535. Crinitaria. H. CAss.
Diplogon. Rafin. Chrysopsis. Nutt. 2529. Heter otheca. H. C.sss. 
108 CLASS. VI. SYNPET. ORD. I. FISSIFL. FAMT, LXXIT. COIIPOS.

B. $B a c c h a r e a c$.

2539. Sergilus. G̈̈вt. 2541. Tursenia. H. Cass. 2540. Baccharis. L. non 2542. Fimbrillaria. H. CAss. VAILL. MIursea. Adns.

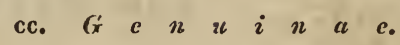$$
\text { c. Erigerea } \text {. }
$$

2543. Eschenbachia. Mönch. Placus. L o u r?

Dimorphantes. II. Cass.

2544. La ennecia. H. CAss.

2545. Trimorphaea. H. CAss. Trimorpha. H. Cass. 2546. Iiriggeron. L.
Conyzella. Dil1.

Cacnotus. Nutt. 254\%. Mf un ychia. H. CAss. Felicia. H. C as s. 2548. Podocoma. H. Cass. 2549. Stenactis. H. CAss.

$$
\text { B. Asterinac. }
$$

2550. Diplostephium. K. 2554. Olearia. Mösch.

$$
\text { H. B. 2555. Printzia. H. CAss. }
$$

Chrysopsis. Nutt. 2551. Aster. T. Asteriphotis. Pont. Amellus. 1 d us. Pinardia. $\mathbf{N} \in \mathbf{c} \mathbf{k}$. 2552. Eu r y bia. H. CAss. 2552. Galatella. H. Cass.
Lioydia. Neck?? 2556. Chiliotrichum. H.CAss. 255\%. A g a tha ea. H. CAss. Detris. Adns. 2558. Kaulfussia. N. v. E. Charieis. H. Cass.

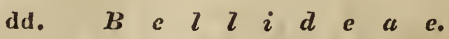

$$
\begin{aligned}
& \text { a. } A m e l l o i d e a c \text {. }
\end{aligned}
$$

2559. Amellus. L. 2560. Felicia. H. Cass. 2561. Henricia. H. CAss. 2562. Kalimeris. H. CAss. 2566. P aquerina. H. CAss. 2563. Callistephus. H. CASS. 2567? The lyth a m nos. Spr. Callistemma. H. C a s s.

$$
\text { f. Gienuina e. }
$$

1568. Lagenophora. H.CAss.

Lagenifera, H, C ass. 
CLASS. VI. SYNPET. ORD. T. FISSIFL. FAM. LXXII. COMTPOS. 109

2569. Bellis. T. L.

B. et Kyberia $\mathrm{N}$ e $\mathrm{ck}$. 25\%1. Bellidistrum. Mrchec. non $\mathrm{V}$ aill. 25\%. B e 11 i u m. L.

\section{Amphicenianthae.}

a. Heliantheae.

aa. Helenieue.

2572. Achyropappus. K. 2585. Florestiua. H. CAss. H. B. 2586. Gaillardia. Fouger

25\%3. A c tinea. Juss. de Bond.

2574. A llocarpus. K.H. B. 258\%. Galinsoga. Cav.

25\%. Bahia. LaG.

258s. Helenium. L.

25\%6. Balbisia. W.

2589. Hy m e иор а p pus. l'Her.

Tridax. L. 2590. Leout ophthalmum. W.

25\%\%. Balduina. Nutr.

2591. Leptopoda. Nutr.

2578. Calea. RBr.

25\%9. Caleacte. RBr.

2592. Marshallia. Schreb.

Persoonia. I I c h $\mathbf{x}$.

2580. Calydermos? LaG. 2593? Hysterionica. W.

2581. Cephalaphora. Csr. 2594. Mocinna. LaG.

258\%. Grah amia. Spr.

Graemia. Hook.

2595. Poly pteris. Nutr.

2596. Ptilosteplitum. K.H.B.

2583. Dimerostemma. II.

CAss.

259\%. Schkuhria. Rтr.

2598. Sog a $\mathrm{g}_{\mathrm{g}}$ in a. H. CAss.

2584. Eriophyllum. LaG. 2599. Trichophyllum. Neck.

\section{bb: $\quad C$ oreopsidea}

2600. Aspilia? A. P. Th. 2608. Espeletia? Bonpt. 2601. Bailleria. Aubr. 2609. Georgina. W.

2602. Bidens. T. L.

2603. Chrysanthellina. Cass.

2604. Chry s anthe 11 um.

Ricir.

Sebastiania. Bertol.

Colluea. S p r.

2605. Calliopsis. Rcub.

2606. Coreopsis. L.

260\%. Cosmos. Cav.
Dahtia. C a v.

Georgia. S p r.

2610. Glos s oc a rdia. H. CAss. 2611. Guardiola? Bonpl. 2612. He teros permum. Car. 2613. Kerneria. MNCH. 2614. Lerchia. H. CAss. 2615? Mnesiteon. Rafin. 2616. Narvalina. H. CAss. Necdhamia. II. Cass. non RBr.

Cosmea. W. 
261\%. Neuractis. H. CAss. 2621. Synedrella. G̈̈нt. 2618. Parthenium. L. 2619? Peramibus. Rafin. 2622. Tetragonotheca. DruL. Gonotheca. Rafin. 2620. Silphium. L.

$$
\text { cc. Ge } n u \text { in } \boldsymbol{a} \text { e. }
$$

2623. Acmella. Rich. 2635. Platypteris. K. H. B. 2624. Blainvillea. H. CAss. 2636. Pterophyton. H. CAss. 2625. Dit r ichum. H. Cass. 263\%. Salm ea. Dec. 2626. Encelia. Adns.

Hopkirkia. S p r.

2627. Hamulium. H. CAss. 2638. Sanvitalia. Lam. 2628. Harpalium. H. Cass. 2639. Simsia. Pers. 2629. Helianthus. L. 2630 ? Is ocarpha. RBr. 2631. Leighia. H. CAss. 2632. Lip otriche. RBr. 2633. Melanthera. Rohr. 2634. P e trobium. RBr. 2640. Spilanthes. Jcp. 2941. Tragoceros. K. H. B 2642. Verbesina. L. 2643. Viguiera. K. H. B. 2644. Ximenesia. Car. 2645. Zinnia. L.

$$
\text { dd. } R \text { u d b e c kie a e. }
$$

2646. Baltimora. L. 2657? Heliophthalmum. 2647. Chatiakella. H. CAss. 2648. Adelmannia. Rchв, Diomedea.

$$
\begin{aligned}
& \text { H. C a s s. non } \\
& \text { Zoolog. }
\end{aligned}
$$
2649. Dracopi is. H. C.Ass. 2650. Echinacea. MNch. 2651. Eclipta. L. 2652. Selloa. K. H. B. Fecuea. S p r.

2653. Ferdinanda. LaG. 2654. Fougeria. Minch. 2655. Gymnol omia. K. H. B. 2656. Helicta. H. CAss.

\section{Rafin.}

2658. Heliopsis. Pens. 2659. Kallias. H. CAss. 2660. Obeliscaria. H. CAss. 2661. Pascalia. Ont. 2662. Podanthus. LaG. 2663. Rudbeckia. L. 2664. Stemmodontia?H. CASs. 2665. Tilesia. MEY. 2666. Tithonia. Desf. 266\%. Wedelia. Jce. 2668. Wulffia? Neck.

\section{b. Arctotideate.}

2669. Heterolepis. H. CAss. 26\%. Cryptostemma. RBr. Heteromorpha. H. C a s s. Anemonospermum. Co mmel. 
26\%1. Arctotheca. Wendi. non $\mathrm{V}$ a ill.

2672. Arctotis. L.
Anemonosperm. $\mathrm{C}$ o m me 1 . Spermophylla. Neck.

2673. Da matria s. H. CAss.

$$
\text { c. Gorteriea e. }
$$

2674. Herpicium. H. CAss. 2675. Gorteria. L. e. e. Personaria. La m. 26\%6. Ictin us. H. CAss. 267\%. Gazania. G̈̈RT. Moehnia. N e ck.? Mrussinia. WV.

2678. Mel anchrysum. H. CASS.

26\%9. Cuspidia. Gärt. Aspidulis. Gärt. 2680. Didelta l'Herrt. Choristea. Thub.
Breteuillia. Buchoz. 2681. F a v o n i m. G̈̈rt. 2682. Cull u mia. RBr. 2683. Berkheya. Eнrн.

Croeodilodes. Adans. non Vaill.

Basteria. Hout t. Agriphylli:m. J $\mathbf{4}$ s. Rohria. Vahl. Thub. Apuleja. Gä r t. Zurabellia. N eck. Gorteria. L a m. 2684. Evopis. H. CASs.

\section{d. Centauree $\boldsymbol{C}_{e}$.}

2685. Crupina. Pers.

2686. Crocodylium. Varle. 2691. Centaurea. L. e. e. 268\%. Centa iurium. Anns. Jucea et Centaurea. Neck. 2688. Calcitrap a. VAILL.

a. Calcitrapa. P.

b. Seridia. P. 2689. Cn icus. VArLe. non W.
Onobroma. G. Lepteranthus. DeC. Cyanus. D e c. 2692. $Z_{\text {o eg ea. L. }}$

2693. Chryseis, H. CAss. 2694. Cyanopsis. H. CAss. 2695. Goniocaulon. H. CAss. 2696. Volut a ria. H. CAss.

2690. Kentroph yllum. Neck. Carduncellus. Adns.

D. Amphicarpanthae. a. Mill e r i e a e.

269\%. Alcina. CAv. 2698. Biotia. H. CAss. 2699. Brotera. SpR. 2700? Caesulia. Rxв.
2701. Centrospermum. Knth. 2702? Chrysogonum. L. 2703. Dys o dium. Rrch. 2704. EIvira. H. C.sss. 
2\%05. Eny dra. Lour.

Meycra. Schreb.

Hingstha. R $0 \times \mathrm{b}$.

Cryphiospermum. P. B.

270\%. Euxenia. Chaла.

2708 Flaveria. Juss.
2\%06. Eriocoma. K. H. B.

2716. Monactis. K. H. B.

271\%. O giera. H. CAss. Eleutheranthera. Poit?

2\%18. Ph a etus a. G̈̈rt.

2719. Polymnia. L.

2720. P o.ly un $\mathrm{n}$ a s $\mathrm{t} x \mathrm{um}$.

Lam.

2709. Hybridella. H. CAss. 2721. Riencurtia. H. CAss. 2710. Jaegeria. Kлтн.

2\%11. Madia. Molrn.

2\%12. Nelampodium. I.

2713? Acanthospermum. Śchrк.

2\%14. Meratia. H. CAss.

2\%15. Milleria. Mart.
2722. S clerocarpus. Jce. $2 \% 23$. Siegesbeckia. L. 2724? Hingstonia. RAfin. 2725. Unxia. L. Fil. 2726. Villanova. LaG. 272\%. Delilia. Spr.

b. $\quad C \backsim l e n d u l \backsim c e \ll e$.

aa. Ge $n u i n a e$.

2\%28. Calendula. L. Callita. T.

2729. Bla xi um. H. CASS.

2\%30. Meteorina. H. CAss.

Cardispermum, Trant.
Gattenhoffia. Neck.

Lestibodea. N e ck. 2731. Arnoldia. H. Cass. 2732. Castalis. Il. Cass. bb. Osteospermeae.

2733. Gibbaria. H. Cass. 2734. Garuleum. H. CAss.
2735. Osteospermum. L. IIonilifera. Vaill. 2736. Eriocline. H, CAss.

$$
\text { c. } C \text { a } l y c e r e a l \text {. }
$$

273\%. Calycera. CAv.

2738. Boopis. Juss.
2\%39. A cicarpha. Juss. Cryptocarpha, Ir. Cass.

\section{d. $A m b$ rosiace $a$ e. \\ aa. J $v a c$ a $a$ e.}

2740. Iv a. L. 
CLASS. VI. SYNPET. ORD, I. FISSIFL. FAM. LXXIII, CUCURB. 113

bb. $G \in \backsim u i n a c$.

2741. Ambrosia. L. 2743?? Cevallia. LaG. 2742. Franseria. Cav.

6c. $X a n t h$ ie $a$ e.

2744. Xanthium. L.

$$
\text { dd? A mbrate a } c \text {. }
$$

2745 ? Liquidambar. L.

\section{FAMILIA LXXIIT.}

\section{CUCURBITACEAE. KÜRBISڤ̈E,Ẅ̈̈HSE.}

$$
\text { a. } C u c u r b i t e a e \text {. }
$$

2746. Sechium. P. Br. 274\%. Sicyos. L. Sicyoides. T.

2748. Elaterium. L. 2749. Bryonia. L. Solena. Lour. 2750. Turia. Forsk. 2751. Erythropalum. BLum. 2752. B enincasa. SAr. 2753. Ecbalium. Rich. Filaterium. $\mathbf{M} \mathbf{n c h}$. 2754. Mo m ordica. L. Anrordicu. N eck. Poppya. Neck. 2755. Neurosperma. Rafin. 2756? Micrampelis. Rafin. 275\%. Mel othria. L. 2758. Trichos anthes. L. 2767? Allasia. Lour. Ceratosanthes. Juss. 2768? ? Myrianthus. P. B.

$$
\text { b. } N h a n d i r o b e a c \text {. }
$$

2769. Kolbea. P. B. 27\%0. Joliffia. Bos. Telfuiria. H o ok. 2771. F evillea. L.
Fenitlea. P.

Nhandirob̈a. Plu m. 27\%2. Z a n o ni a. L. non Plum.

a. Zanouia. Blume.

b. Alsowitra. Blume. 
114 CLASS. YI.SYNPET, ORD. I. FISSIFL. FAM, LXXIV. CAMPAN.

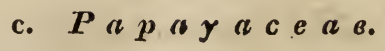

2\%7. Carica. L.

Papaya. T.

FAMILIA LXXIV.

CAMPANULACEAE. GLöckLer.

a. Goodenoviene.

27\%4. Goodenia. SM.

27\%5. Selliera. Car.

2796. Calogyne. RBr.

2797. Euthales. RRr.

2798. Velleia. Sm.

a. Menoceras. RBr.

b. Velleia. RBr.

27\%. Distylis. Gaudreh.
2780. Lechenaultia. RBr. 2781. Anthotium. RBr.

2782. Scaevola. L. 2783. Diaspasis. RBr. 2\%84. D ampi era. RBr. 2785? Moquinia. Spr. 2786? Del isse a. Gaudich.

$$
\text { b. Lob e li ace a e. }
$$

2787. Lobelia, L.

2790. Glossocomia. Don.

2788. Clermontia. Gaudrer. 2791. Ly sip om a. K. Н. B. 2789. Jsotoma. RBr.

$$
\text { c. } P h y t e u m e a e \text {. }
$$

2792. Jasione. L. 2793. Phyteuma. L.

2\%94. Trachelium. L.

$$
\text { d. C } \because m p a n u l e a \theta \text {. }
$$

2795. Ro ёll a. L. 2796. Cervicina. Desil. 279\%. Lightfootia l'Her. 2798. W ahlenbergia. Schrad. 2802. Canarina. L. 2\%99. Adenophora. Fiscir. 2803. Michauxi a l'Her. Flörkea. Spr. non W.
2800. C ampanula. L. 2801. Prismatocarpus l'Her. Legousia. D u ra nd. 2804. Ceratostemma. Juss. 


\section{ORDO II. \\ LO B I F L O R A E. \\ LAPPIGBLUMIGE.}

FORMATIO I.

$\begin{array}{llllllllll}\mathbf{T} & \mathbf{U} & \mathbf{B} & \mathbf{I} & \mathbf{F} & \mathbf{L} & \mathbf{O} & \mathbf{R} & \mathbf{A} & \mathbf{E} .\end{array}$ ROEHRENBLUETHLER.

FA MILIA LXXV.

LABIATAE. Lippenblüthler.

a. S a $l$ vi $e$ a $e$.

2805. S a lvia. L.

a. Horminum. T.

ъ. Sclarea. T.

c. Salvia. T.

2806. Rosm arinus. L.

2807. Hemiandra. RBr.

2808. Collinsonia. L.

2809. Lumn itzera. JAcQ.

2810. O c i m u m. L.

2811. Plectranthus l'Her.

Germaneu. L a m.

2812. A eollanthus. Mart.

2813. Prunella. L.

Brunella. T.

2814. Dracocephalum. L. 2815. Prasium. L.

Guleopsis. T.

2816. Phryma. L.

2817. Cleonia. L. 2818. Trichostemma. L. 2835. Synandra. Nutr.
2819. Thymus. L.

a. Zygis. $\mathbf{P}$.

b. Serpyllum. T. 2820. Acinos. P. 2821. Calamintha. Lk. 2822. Melissa. L. 2823. Horminum. L. 2824. Gardoquia. Rz. P. 2825. Thymbra L. 2826. Lepechinia. W. 282\%. Stenarrhena. Don. 2828. Chlinopodium. L. 2829. Melittis. L. 2830. Scutellaria. L. Cassida. T. non $Z_{0}$ o $10 \mathrm{~g}$. 2831. Chilodia. RBr. 2832. Prost anthera. LaB. 2833. Cryphia. RBr. 2834. Perilomia. K. H. B.

b. Nepetere.

2836. He deoma. P. 283\%. Cunila. L.
2838. Zizlphora. T. 2839. Monạrda. L. 
-2840. Westringia. SM. 28\%0. Glechon. Spr. 3841. Microcorys. RBr. 28\%1. Mentha. L. 2842. Hemigenia. RBr. 2872. Galeobdolon. SM. 2872b. Galeopsis. L. 2843. Amethystea. L. 2844. Lycopus. L.

2845. IIoslundia. L. 2873. Orvala. L. 2874. L a m i um. L.

a. Pollichia, Rth.

b. Lamium, Rth.

2846. A j и g. L.

a. Bujula. T. Juss.

b. Chamaepytis. T. 284\%. Moscharia. Forsk. 2848. Anisomeles. RPr. 2849. Craniotom e. Renb. 2850. Scorodonia. Mnch.

2851. Tе еистіum. L.

a. Chamaedrys. T.

b. Polium. T. 2852. Pheboanthe. Tsch. 2853. Colebrookia. Rxr. 2854. Perilla. W. 2855. Leucosceptrum. SM. 2856. Lavandula. L.

a. Lavandula. T.

b. Stoechas. T. 285\%. Sideritis. L. 2858. Phytoxys. Mour. 2859. Origan um. L. 2860. Pусnа nthemum. Mchx. 2861. Is a thus. Mcrx. 2862. Satureia. L. G. In irromeria 28\%5. Glechoma. L. 28\%6. Nepeta. L.

a. Cararia, T.

b. Stachyura, Rchb. 28\%. Hyptis. JAce. 28\%. Marsupianthes. Mart. 28\%9. St a chys. L.

a. Trixago. Mnch.

b. Chamaesideridis.

c. Friostomum. Hurgg. Lk.

2880. Zi etenia. Gued.

2881. Le on urus. L.

a. Cardiaca. T.

b. Cardiacastrum. R.

c. Pauzeria. Minch. 2882. Cha eturus. Енrн. 28s3. Ballota. L. 28s4. Betonica. L. 2885. Ma rrubium. L.

a. Anisodontium, R.

b. Marrubiastrum.

c. Pseudo - Dictamuus. 2886. Phlomis. L. a. Phlomis. Sabbatia. M n ch. 2863. Bystropogon l'Herit. 2864. Peltodon. Ponl. 2865. Pogostemon. Desa. 2866. Vleckia. Rafin. 286\%. Hyss opus. L. 2868. Elsbolzia. W. 2887. Hemist emma. Ehrnb. 2888. Le ucas. RBr. 2889. Le on otis. RBr. 2890. Moluccella. L. 2891. Rizoa. C $\Delta$ v. 2892. Colquhounia. Wate. 2869. Pycnostacleys. Hook.

2893. Morina. L. 
c. $V e r b$ e $n e r e$.

2894. Verbena. L. Gilandutaria. G m.

2895. Priva. Adans. Pirisma. Forsk. Tortulu. Rxb.

2896. S'tachytarpheta. V 289\%. Pat onia. liafrN.

2398. T amonea. Aubr.

Kämpfera. Houst.

Leptocarpus. W.

Ghinia. WV.

2899. Citharexylon. L. 2900. Taligalea. Aurz. 2901 Melasanthus. Poнr. 2902. Aloysia. Ont. 2903. Vitex. L. 2904 Chloanthes. RBr.

2905. Spielmannia. L. 2906. Casselia. N. v. E. 290\%. Petrea. L. 2908. A saphes. Spr. Morina. D о и. 2909. Duranta. L. 2910. Premna. L. 2911. Congea. Rxв. 2912. Streptium. Rxb.

2913. If olmskioldia. RTz. Hastingia. $\mathrm{S}$ m. Kö $\mathrm{n}$. Plutunium. Juss.

2914. Oxera. LaB. Oncoma. Spr.

2915. R ap utia. Aurr. Sciuris. Schreb. 2916. Cornutia. L. Hostu. J $\mathbf{c} q$.

291\%. Petitia. Jc?. 2918. Buchia. K. H. I3.
2919. Pityrodia. RBr. 2920. Callicarpa. L. Spondylococcus. Mitch. non $\mathrm{W}$.

2921. Porphyra. Lour. 2922. Wallenia. Sw. 2923. Nuxia. Conmers. 2924. Aegiphila. L. 2925. Manabea. Aubr. 2926. Chilianthus. Burch. 292\%. Tectona. L. Thela. Rhe ed.

2928. Ovieda. L. non SpR. Siphonanthus. L.

2929. Clerodendron. L. Volkmunnia. J c q. Agricoluea. Schrk. 2930. Wallrothia. Rтr. 2931. Pyrostoma. Mex. 2932. Hilsenbergia. Tsch. 2933. Volkamera. L. 2934. A masonia. L. 2935. Gmelina. I. 2936. Mendozia. Rz. PAv. Mendoncia. V ellz. V a nd.

293\%. Z apania. Ścop. 2938. P erama. Außr.

Mattuschliea. S c h r e b. 2939. Lippia. L. 2940. L a n t ana. L.

2941? Chrysomallum. A. P. Th.

2942. Avicenuia. L. a. Halorlendrou. A. P. Th. b. Avicennia. A. P. Th. 


\section{FAMILIA LXXVI.}

\section{ASPERIFOLLACEAE. Scharfblättrige.}

\section{a. $E c$ c i e $九 e$.}

2943. Echium. L.

2953. Rind e ra. Palz.

2944. Echiochilon. Desf. 2954. Moltkia. LenM.

2945. Onos modium. Mchx. 2955. On os ma, L.

Osmodium. R a f. 2956. Coldenia. L.

Purshic,. S p r.

2946. Lith os permum. L. 294\%. Pul monaria. L.

Bessera. S chult. 2948. Lycopsis. L. -2949, Nonea. Dec. 2950. Cols mannia. Lrhm. 2960. Preslea. Spr. 2951. Craniospermu m.

LehM.

2952. Dioclea. Spr.

Preslaca. II a $\mathbf{t}$.
2957. Cerinthe. L.

a. rerionanthe. $\mathbf{R}$.

z. Cerinthe. T.

2958. Heliotropium. L. 2959. Tiaridium. LeHM.

2961. Trichodesma. RBr. Pollichia. MI edic

\section{b. Borragine uc.}

2962. Myosotis. L.

2963. Anchusa. L.

2964. Stomatechium. Lehm. 2970, Rochelia. Roнв.

2965. S o l e n a n th u s. Ledeb.

2966. Cynoglossum. L.

296\%. Mattia. Schult.

2968. O m phalodes. T. picotia. Schult.
2969. Echin ospermum. Sir. Rochetia. R. S. non R. S. 29\%1. Asperugo. L. 2972. Exarrhena. RBr. 2973. Borrago. L. 2974. Symphytum. L.

$$
\text { c. } H y d r \circ p l y \text { y } l l e a e \text {. }
$$

29\%5. Rotula. Lour. 2976? R a p ourea. Aubu. Camax. Schreb.

297\%. Hydrophyllum. L. 2978. Nemophila. Bart. 2979. Eutoca. RBr.

2980. Ellisia. L. 2981. Phacelia. Mcux.
Aldeaca. $\mid \mathbf{R}$ z. $\mathbf{P a v}$. 2982. Tournefortia. I. Pitlonia. Plum. 2983. Messerschmidia. L. 2984. B e ureria. Jce. 2985. Ehretia. L. 2986. Patagonula. L. 298\%. Cordia. L. Cerdana. Rz. $\mathbf{P}$ a v. 
29s8. Varronia. L. 2990. R h a bdi a. Mart . 2989. Cordiopsis. Hanilt. 2991? Saccellium. K H. B.

FAMILIA LXXYIr.

CONVOLVULACEAE. WINDENGEẄ̈CHSE.

$$
\text { a. Co } \quad \text { v } v \text { o } l \quad v \text { ul } l \text { e } a \text { e. }
$$

2992. Dichondra. Fonst. 3004. Cortesia. CAv. Steripha. Gärt. 3005. Menais. L. 2993. Falkia. L. 3006. Retzia. Thns.

2994. Polymeria. RBr. 2995. Evolvulus. L. 300\%. Humbertia. Сomm. Endrachium. Juss. 3008. B rewe ria. RBr. 3009. Bonamia. A. P. Th. 2996. Cress a. L. 2997. Wilsonia. RBr. 2998. Prevostea. Chols. Calycobolus. W. Dufourea. K. II. B. Reinwardta. S p r. 2999. Neuropeltis. WaLr. 3010. Porana. Burm. 3011. Convolvulus. L. 3012.' Calystegia. RBr. 3013? Diplocalymma. Spr. 3014. Argyreia. Lour. 3000.Louchostoma. Wickstk. 3015. I p o in e a L. 3001. Sagonea. Aubl. Reicheliu. Schreb. 3002. Cervia. Rodnigv. 3003. Erycibe. Rxi.

Quamoclit. $\mathbf{T}$. 3016. Mouroucoa. Aubl. Mraireria. S c op. 301\%?. Navarretia. Rz. P.

$$
\text { b. } H y d r o l e a c e a c \text {. }
$$

3018. $\mathrm{N}$ a m a. $\mathbf{L}$. 3020. Hydrolea, L. 3019. Wigandia. K, H, B.

○. Polemoni

3021. Phlox. L. 3022. Hoitzia. Juss. 3023. Collomia. Nutt. 3024. Gilia. Rz. P'. Ipomopsis. II c L X. Ipomeria. Nutt. 3025. Polemonium, L. 3026. Heteryta. Rifin. 302\%. Cantua, Juss. Periphragmos. Rz. P. 3028? Lo eselia, L. 
FOPITATIO II.

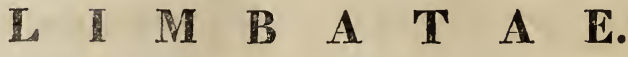 SAUMBLUETHLER.}

FAMILIA LXXVIII.

POLYGalaceali. Porygaracen.

a. Polygal e a $e$.

3029. Polygala. L.

a. Timutia. DC.

b. Senega. DC.

c. Clinclinia. DC.

d. Blepharidium. DC.

e. Polygalor. DC.

f. Chamaebuxus. DC.

g. Psychanthus. Rafin.

3030. Brachytropis. Dec. 3031. Comesperma. LaB. 3032. Salom onia. Lour. 3033. Badiera. Dec. 3034. I a ckia. BLUME.
3035. Soulamea. Lam. 3036. Muraltia. Neck. Heisteria. Berg. 303\%. Mu ndia. K. H. B. 3038. Mon ina. Rz. Pav.

a. Hebeandra, Bonpl.

b. Pterocarya. DeC.

3039. Bredemeyera. W. 3040. Securidaca. L.

$$
\text { b. } M y \circ p \circ r i n \ll e .
$$

3042. Krameria. Löfr.

3043. Stenochilus. RBr. 3044. Pholidia. RBr. 3045. Bontia. L.
3046. Myporum. Banks.

Pogonia. Andr. Andrewsia. Vent. Bertolonia. Del Spig. 304\%. Eremophila. RBr.

$$
\text { c. Pittosporeat. }
$$

3048. Bursaria. CAv. 304Sb? Galvania. VAND. 3049. Bill ardiera. SM.
Labillardiera. R. S. 3050. Pittosporum. BNas.

\section{FAMILIA LXXIX.}

PERSONATAE. LaRVENbLüthLER.

A. $R \boldsymbol{h} i \boldsymbol{n} \boldsymbol{a} \boldsymbol{t} \boldsymbol{h} \boldsymbol{e} \boldsymbol{a} \boldsymbol{e}$.

a. $O$ robanche 0 a

3051. Obolaria. L. 3053. Epiphegus. Nutr. 3052. Schultzia. Rafin. 
3054. L athraea. L. Clandestina. T.

3055. Orobanche. L.

a. Osproleon. Wallr.

b. Trionychon. Wallr. 3056. Phelipaea. T.
305\%. Aeginetia. L. 3058. Cist anche. LK. IIfrgg. 3059. Hyobanche. L. 3060. Alectra. ThNB. 3061. Dodartia. L.

b. $P$ edicular $i$ u $u$ e.

aa. Veroniceae.

3062. Cochlidiosperma.

Rсни.

a. Omphalospora. Bess.

b. Diplophyllum. Lehm.

3063. Veronica. L.

$a$. Aïdelus. Spr. Ver. peregrina. L. etc.

b. Veronicastrum. Vet.

c. Hebe. Juss.

3064. L e p t a n d ra. Nutr. Veronicaistrum. Mnch.
Callistachya, Rafin. 3065. Curanga. Juss. 3066. Paederota. L. 306\%. Wulfenia. JAce. 3068. Sibthorpia. L. 3069. D is andra. L.

30\%0? Hemiphragma. WALL. 3071. Romanzoffia. Cham.

\section{bb. Melampyreae.}

3072. Tozzia. L.

3074? Poarium. Hamilt. 3073. Mel a mp yum. L.

\section{cc. Pedicularinae.}

3075. Castilleia. Mur. 30\%6. Rhinanthus. L. Elephas. T.

30\%\%. Alectorolophus. Hazu. 3078. Orthocarpus. Nutr. 3079. Parentucellia. Viv. 3080. Eup hrasia. L. 3081. Odontites. Pers. Stev. non SPR. 3082. Starbia. A. P. Th. 3083. Lam ourouxia. K.H.B. 3084. Bartsia. L.
Staehelina. Hall, non L. 3085. Euchrouna. Nutr. 3086. Trixago. Stev. non LK. HFFG.

Lasiopera. Lk. IIffgg. 308\%. G y m n andra. PaLL. Lagotis. Gärt. 3088. C a m y leia. A.P.Th.

Campuleia. H o ok. 3089. La fuentea. LaG. 3090. P edicularis. L. 3091. Prosopia. Rchb. 
c. $A$ c $\because n t h \ll c e a e$.

a. Selagineae.

3092. Globularia. L.

a. Globularia. T.

b. Alypum. T.

3093. Selago. L.

3094. Hebenstreitia. L. 3095. Microdon. Chors.

Talea. Gärt, non a l. 3096. Polycenia. Chors.
309\%. D is chimia. Chors. 3098. Agathelepis. Crrors.

3099. Campy lanthus. Rтн.

bb. Buchnereae.

3102. Heteranthia. N.r. E. 3106. Piripea. Aubu. Vrolikia. Spr.

3103. Erinus. L.

3104. Buchnera, L.

3105. Manulea. L.
310\%. Palmstruckia. Rrz.

fil.

3108. Browallia L.

3109. Franciscea. Pohr.

cc. Acantheae.

3110. Elytraria. Mchx.

3111. Nelsonia. RBr.

3112. Justicia. L.

a. Dianthera. $\mathbf{L}$.

b. Justicia. L.

3113. Brillantaisia. P. B. 3114. Dicliptera. Juss. 3115. Hypoëstes. Sol. 3116. Eranthe mum. RBr. 311\%. Sanchezia. Rz. P.

3118. Ac anthodium. DeL. 3119. Diliraria. Juss. 3120. A canthus. L. 3121. B lepharis. Juss. 3122. Ruellia. L.
3123. B 1 e chu m. Juss. 3124. Harrachia. JAce. Crossandra. S al is b.

3125. Aphelandra. RBr.

3126. Aetheilema. RBr. Micranthus. Wendl. Phaylopsis. W.

312\%. Lepidagathis. W.

3128. Barleria. L.

3129. Hygrophila. RBr.

3130. Adenosma. BBr.

3131. Thubergia. L. 3132? S e ptas. Lour. non $\mathrm{L}$. 


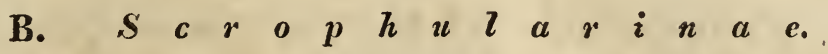
a. L $i n d e v n i a c e a e$.

3133. Bonnaya. LK。 , 313\%. Limosella. L. 3134. Hemiant hus. Nutr. 3138. Lindernia. L. 3135. Microcarpaea. RBr. 3139. Scoparia. L. 3136. Peplidium. Dec. 3140. Sphaerotheca. Cirum.

b. Antivrhineae.

3141. a e a. Commers. 3142. Calce ol aria. L. 3143. Iovell an a. Rz. P. 3144. S chizanthus. Rz. P. 3145. Salpiglossis. Rz. P.

3146. Schwenckia. L.

3147. Gratiola. L.

3148. Hydranthelium. K. 3168. Dichroma. CAr. H. B. 3169. Schwalbea. L.
3163. Sopubia. Dox.

3164. Chirita. Hamilt. 3165. Gerardia. L.

a. Gerardia, Cham. Schlecht.

b. Melasma Berg.

Dargeria. Cham. Schlecht. Nigrina. L. non Thub.

3166. Se y me ria. Punsh. Afzelia. Gm.

316\%. L o x o n i a. JACK.

Willichia. Mut.

3149. Diasci a. Lk.

3150. V andellia. P. Pr.

Matourea. Aubl.
31\%0. Hornemannia. W. 31\%1. Anarrhinum. Dess. Simbuleta. $\mathbf{L}$. 31\%. Nemesia. Vent. 3151. Toren ia. RBr. 3152. Nortenia. A. P. Th. 3173. Chaen arrhinum. DeC' 3153. Tittmannia. Rchb. 3174 Linaria. $T$. 3154. Geochorda. Cham. 3155. Herpestes., G. 3156. Montira. Aubl.

Heintzelmannia. $\mathrm{Neck}$. 315\%. Limnophila. RBr. 3158. Achetaria. Chanr. 3159. B eу richia. Chà. 3160. Glossostylis. Crims. 3178. Chelone. L.
3161. Cybbanthera. Hamirt. 3179. Pentastemon JHerit. 3162. Centranthera. P. Br. 3180. Conobea. Aứb.
31\%. Antirrhinum. L.

a. Asarina. T.

b. Orontium. P. non $\mathbf{L}$. 31\%6. Ma r randia. JAcQ. Usteria. C av.

31\%. Lophospermum. Dox. 317\%b. Elmigera. Rснв. Chel. barbata, Cav. 3179. Pentastemon IHerit.

Gumtcolis, H amilt. 
3181. Cymbaria. L. 3182. Mimulus. L. 3183. Mazus. Lour. 3184. Morgania. RBr. 3185. Uvedalia. RBr. 3186. Es cobedia. Rz. PAV. 318\%. Scrophularia. L. 3188. Angelonia. Hв. Bre. 3189. Hemimeris. L. Alonsoa. Rz, Pav. 3190. Celsia. L. 3191. Verbascum. L.
3192. Collinsia. Nutr. 3193. Stemodia. L. 3194. Russ elia. L. 3195. Weigelia. Thne. 3196. Halleria. L.

3197. Diplanthera. RBr.
3198. Digitalis. L.

a. Digitalis, Lindl.

b. Isoplexis. Lindl.

\section{c. Ca prariaceac.}

3199. Aragoa. K. H. B. 3200. F a biana. Rz. Pav.

3201. Anthocercis. LaB. 3202. D ub o is ia. RBr.

3203. Teedia. Rud. 3204. Buddleia. L. Buddlea. S p r. 3205. C a p raria. L.

a. Cabritta. R.

b. Capraria.

c. Freylinia. II. Ital. 3206. Physocalyx. Poht. 320\%. Xuaresia. Rz. Pav. 3208, Virgularia Rz, PAY. 3209. Esterhazya. Mrк. 3210. Go mara. Rz. PAv. Gomaria. Spr.
3211. Calytriplex. Rz. P. 3212. Ourisia. Commers. 3213. Xenopoma. W. 3214. A ptosimum. Burch. 3215. Leucophyllum.K.H.B. 3216. Tа pura. AubL. Rohria. Schreb. 321\%. Brunsfelsia. L. sp. Houtt. Juss. Lam. Brunfelsia. L. gen. S. N, et W. Lk. Spr.

3218. Cerium. Lour. 3219. Crescentia. L. 3220. Tanaëcium. Sw. 3221. Tripinnaria. $P$. Tripinna, L ou r. 3222. Picria. Lour.

$$
\begin{aligned}
& \text { C. Bignoniace a e. } \\
& \text { a. Gesnereae. }
\end{aligned}
$$

3223. Cyrtandra. Forst. 3224. Henkelia. SPR. 1817. Rotilera. V. Didymocarpus. J a ck. 3225. Lo o onia. JACK.
3226. Lysionotus. Don. 322\%. Trichosporum, Don. Aeschynanthus. J ack.

3228. Sarmienta. Rz. Pav. 
3229. Columellia. Pz. P. 3238. Martynia. L.

3230. Besleria. L. 3231. Tussacia. Renв. 3232. Columnea. L. 3233. Q и о уа. Gaudich. 3234? Razumovia. Spr. 3235. Trevirania. W. Achimenes. P. Br. Cyrilla. l'H e rit. Büchnera. Scop. 3236. A chimenes. VAnL. 323\%. Gloxinia lHerit.
3239. Craniolaria. L. 3240. Eriphia, P. Br. 3241. Sinningia. N. V. E. 3242. Gesnera. L. 3243. Codonophora. Linde. 3244. P e n a r h a p hi a. Linde. 3245? La phia. Hamilt. 3246. Mitraria. CAV. $324 \%$ Fieldia. Cunningh.

\section{b. $S$ e $s$ a me a $e$.}

3248. Ses e a. Rz. Pav. 3249. Ses amum. L. 3250. Rogeria. Gar.
3251. Pretrea. Gax. 3252. Pedalium. L. 3253. Iosephinia. Vent.

$$
\text { c. Bignonieae. }
$$

3254. Schrebera. Roxb,

3255. Caralpa. Juss. 3256. Sickingia. W. 3257. Plat y carpum. Hв.Bpt. 3268. Rhizogu m. Burch. 3258. Friedericia. Mart. 3259. Bignonia. L. 3260. T e com a. Juss. 3261. I a ca r a d d a. Juss. 3262. Spathodea. P. B. 3263. Zeyheria. Mart. 3264. Eccremocarpus. Rz. Pav.
3265. In c a rvill ea. Juss. 3266. Gels e mium. Juss. 326\%. Tourretia. Dumb. 3269. Argylia. Don. 32\%. Chilopsis. Don. 3271. Astianthus. Don. 3272. Delostoma. Don. 32\%3. Stenolobium. Don. 3274. A m p h l op h u m. K. H. B. 3275. Cobaea. CAv.

FAMILIA LXXX.

SOLA NACEA E. Solanaceen.

a. Nol a $n \in e$ a $e$.

3276. Nolana. L. 327\%. Triguera. Cav. 
126 CLASS. VI. SYNPET. ORD. II. LOBIFL, FAM.LXXX.SOLAN.

\section{b. $D a t u r e a$.}

3278. Ramondia. Rrch. Myconia. La p. Chaixia. La p.

2279. Hy о sсуатus. L. 3280. Scopolina. R. S. Scopolia. J c q. non Forst. nec $\mathbf{L}$ a m.

3281. Le hmannia. Spr.
3282. Petunia. Juss. 3283. Nicotiana. L.

a. Nyctagella.

b. Tabacum.

c. Tabacina.

3284. Nierembergia. Rz. P. 3285. D a tura. L. 3286. Bruguania. P.

\section{c. Solanea e.}

3287. Vestia. W. 3288. C e s t r um. L. 3289. Lamarkea. PoIr. Markia. Rich. 3290. Dartus. Loun. 3291? D o ra en a. Thнв. 8292. Aquartia. JAce. 3293. Nycterium. Vent. 3294. S o l a num. L. 3295. Witheringia l'Herit. 3296. Lycopersicon. T. 329\%. Di erbachia. Spr. Dunatia K. H. B. 1818. non S p r. 1815.

3298. Bassovia. Aubl. 3299. Capsicum. L. 3300. Physalis. L. 3301. Herschellia. Bowd.
3302. D n perreya. Gruvich. 3303. I uanull oa. Rz. PAv. Ulloa. $\mathbf{P}$.

3304. Saracha. Rz. Pat. Bellinia. R. S. 3305. Atropa. L. 3306. Witharia. Pauquy. 330\%. Mandragora. T. 2308. Nicandra. Adans. 3309. Anisodus. Lк. 3310. Nectouxia. K. H. B.

3311. Jab or osa. Juss.

3312. Linkia, Pers. non Linkea. Mich. Desfontuinia. R z. Pav. 3313. Lycium. L. 3314. Rapinia. Lour. 3315. So landra. L. 3316. Metternichia. Mrk, 


\section{ORDO III. \\ R O T I F L O R A E. \\ RADBLUMIGE.}

\section{FORMATIO I. \\ C R A T E R I F L O R A E. BECHERBLUETHLER.}

FAMILIA IXXXI.

LYSIMACHIACEAE. LrsimachieEn.
a. Lentibularieac.

331\%. Utricularia. L.

a. Lentibularia, Gesu.

b. Ambulligera. R.

331s. Pinguicula. L. 3319. B r a nd on ia. Rchr. Bot, Reg. 126. Hook. ex Fl. 16.

\section{b. $A$ nas $s l l i d e$ e .}

3320. Centunculus. L. 3321. A nagallis. L. 3322. Micranthe mum.

\section{Мснх.}

3323. Euparea. Banks. 3324. Hemianthus. Nutr. 3325. Hottonia. L. 3326. Ast e rolinum. Lк.
3327. Lysimachia. L.

a. Lerouxia. Merat. Godinella. Lestib.

b. Thyrsauthus. Schrk.

c. Lysimachia.

d. Ephemerum. 3328. Lubinia. Vent. 3228b. Aitonia. L. fil.

$$
\text { c. } \quad E \begin{array}{lllllllll} 
& \boldsymbol{a} & \boldsymbol{c} & \boldsymbol{r} & i & d & e & a & e .
\end{array}
$$

aa. Stypheliaceae.

3329. Oligarrhena. RBr. 3335. Monotoca. RBr. 3330. Needhamia. RBr. 3336. Leucopogon. RBr. 3331. Pentachoudra. RBr. 333\%. Lissanthe. RBr. 3332. Decaspora. RBr. 3338. Cyathodes. Lab.e. e. 3333. Trochocarpa. RBr. 3339. Melichrus. RBr. 3334. Acrotriche. RBr. 3340. Stenanthera. RBr. 
3341. Astroloma. RBr. Ventcnutiu. Cav. 3342. Styphelia. Sm. e. e.

$$
\begin{aligned}
& \text { bb. E } \quad \text { p a c r e } e \text { a } e \text {. } \\
& \text { a. Richeaceae. }
\end{aligned}
$$

3343. Cystanthe. RBr. 3345. Dracophyllum. LaB. 3344. Richea. RBr.

$$
\text { B. sprengeliaceae. }
$$

3346. Cosmelia. RBr. 3348. Ponceletia. RBr. 334\%. Andersonia. RBr. 3349. Sprengelia. SM. Poiretia. C a v.

$$
\text { r. Lysinemaceac. }
$$

3350? Prionotes. RBr. 3352. Epacris. Forst. e. e. 3351. Lysinema. RBr.

FAMILIA LXXXII.

\section{PRimulaceae. Primulaceen.}

$$
\text { a. } P l a n t a g i n e a t \text {. }
$$

3353. Littorella. L. 3355. Psyllium. T. Juss. 3354. Pla ntago. L.

$$
\text { b. Andros a ce a e. }
$$

3356. Cyclamen. L. 3360. Douglassia. Lindu. 335\%. Do decatheon. L. 3361. Primula. L.

3358. Soldanella. L.

a. Aleuritia. Dub. 3359. Androsace. L.

b. Auricula. T.

c. Arthritica. Dub.

a. Aretia. $\mathrm{L}$.

d. Corthusina.

b. Androsace. $L$. 3362. Corth usa. L. Andraspis. Duby. 3359b. Gregori a. Duny.

$$
\text { c. } S a m \text { o } l \text { e } a \text { e. }
$$

3363. Samolus. L. 3364. Sheffieldia. Fonst. 3365. Bacopa. Aubu,
3366. B a obotrys. Forst. Maesa. Forsk. Maasa. R. S. Siburatia. A. P. Th. 
CLASS. VI. SYNPET. ORD. III. ROTIFL. FAM. LXXXII.ERIC. 129

FAMILIA LXXXIII.

\section{ERICACEAE. Ericaceen.}

\section{a. Pyroleac.}

336\%. Monotropa. L. 3368. Schweinitzia. ELL.

Monotropsis. Schwnitz, ined. 3369. Pterospora. Nutr. 33\%. Pyrola. L. 33\%1. Chimophila. Prsh. em. RAD.

Chimaphila. Prsh.
3372. Argophyll um. Forst.

3373. Galax. L.

Erythrorrhiza. Mchx.

Solanandra. Vent.

Blandfordia. Andr.

3374? Lepur opetalon. ELL. Pyxidanthera, Schreb. non Mch x.

33\%5. Befaria. Mut.

b. $E \boldsymbol{r} i c e a e$.

33\%6. Sy mpieza. Lrchtst. 33\%. B lairia. L.

3378. Salaxis. SALISB. 3379. Erica. L. 3380. Calluna. SALISB. 3381. Menziesia. Sm. 3382. Phyllodoce. Salisb. 3383. And rom eda. L. 3384. Ly onia. Nutr.
3385. Arbutus. L.

3386. Arctostaphylos. Adans. Mairania. Neck. 338\%. Pernettia. Gaudich. 3388. Encyanthus. Lour. Ekianthus? Lou r. em. Spr.

3389. B ros s a e a. Plum, 3390. Gaultheria. L.

\section{c. $R$ hodorace a e.}

3391. Sonerila. Rxb.

3392, Azalea. L. e. e. Loiseleria. D e s v. Chamaeledon. L k. 3393. Pyxidanthera. Mchx. 3394. D i ap ensia. I.

Diapenzia. Whlnb. lapp. 3401. Led um. L. 3395. Epigaea. L. text. 3396. Cal od r y u m. Desv.
339\%. Anthodendron. Rerb. Azalea. Auct. 3398. Rh od ora. L. 3399. Rhododendron. L. 3400. Rhodothamnus. Rchв. 
3403. Kalmia. L.

3404. Elli otia. Mühlnb.

3405. Clifton ia. Banks.

Mylocaryum. W.
3406. Clethra. L.

\section{Tinus. L.}

Volkameria. P. Br. 340\%. Cuellaria. Rz. PAf.

FORMATIO II.

\section{S T E L L I F L O R A E.} STERNBLUETHLER.

FAMILIA LXXXIV.

ASCLEPIADEAE. Asclepiadeen.

A. $\quad \begin{array}{llllllllll}A & \boldsymbol{s} & \boldsymbol{c} & \boldsymbol{l} & \boldsymbol{e} & \boldsymbol{p} & \boldsymbol{i} & \boldsymbol{e} & \boldsymbol{a} & \boldsymbol{e} \text {. }\end{array}$

a. Stapelie a e.

3408. Ceropegia. L.

a. Ceropegia.

b. Stephanotis. A. P. Th.

3409. Hu ernia. RBr. Heurnia., S p r.

3410. Piaranthus. RBr.

3411. Stapelia. L.

a. Hewenia. Haw.

b. Caruncularia. Haw.

c. Duvalia. Haw. d. Obesia. Haw.

e. Orbea. Haw.

f. Tromotriche. Haw.

g. Tridentea. Haw.

$h$. Podanthe. Haw.

i. Gonostemon. Haw.

k. Stapelia. Haw.

3412. D esmid o chus. Ehrne. 3413. Carall uma. RBr. 3414. Microstem ma. RBr. 3415. Leptadenia. RBr.

b. Cynancheae.

a. Pergularinae.

3416. H о у a. RBr. Schollic. J c q.

3418. Marsdenia. RBr. 3419. Pergularia. L.

3420. Dischidia. RBr.

3421. G y m e m a. RBr. 3422. Sarcolobus. RBr.

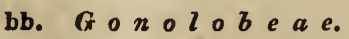

3423. Gonolobus. RBr. 3424. Matelea. Aubr. 
cc. C y

3425. Asclepias. L. 3426. Acerates. ElL. 342\%. Gromp hocarpus. RBr. Sabia. C o lebr. 3428. Enslinia. Nutt. 3429. Oxystelma. RBr. 3430. Xysmalobium. RBr. 3431. Podostigma. Exu. 3432. Calotropis. RBr. 3433. Lachnostoma. K.H.B. 3434. Macroscepis. K.H. B. 3435. Oxypetalum. RBr. Gothofreda. Vent. 3436. K an a h i a. RBr. Canatia. Spr. 3437. Sonninia. Rchb.
Diplolepis. RBr. non Z, 001 . 3438. Seutera. Ronb. Lyonia. E 11. non N it t. 3439. Holoste in ma. RBr. Fischera. DeC. 3440. Cyn an ch um. L.

$a$. Vincetoxicum. P.

b. Schubertia. Mart, non al. 3441. Solen os tem ma. HAYNE. 3442. Metaplexis. RBr. 3443. Ditassa. RBr. 3444. Do emia. RBr. Dimia. S p r.

3445. Philibertia. K. H. B. 3446. Sarcostemma. RBr. 3447. Pentaphragma. Zucc. 3448. Eustegia. RBr.

\section{c. Astephaneae.}

3449. Metastelma. RBr. 3453. Physianthus. Marr. 3450. Microloma. RBr. 3451. Astephanus. RBr. 3452. Arauja. Brot. 3454. Baxtera. Rchв.

Harrisonia. Hook, non

\section{B. $\begin{array}{lllllllllll}\boldsymbol{P} & \boldsymbol{e} & \boldsymbol{r} & \boldsymbol{i} & \boldsymbol{p} & \boldsymbol{l} & \boldsymbol{o} & \boldsymbol{c} & \boldsymbol{e} & \boldsymbol{a} & \boldsymbol{c} \text {. }\end{array}$}

a. Secamoneat.

3455. Secamone. RBr.

$$
\text { b. G e } n u i n u e \text {. }
$$

3456. Hemidesmus. RBr. 3458. Gymnanthera. RBr. 345\%. Periploca. L.

$$
\text { c. Cryptostege a e. }
$$

3459. Cryptostegia. RBr. 


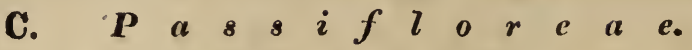 a? Paropsieae.}

3460. Smeathmannia.BANKs.

IIaracanga. A. P. Th. 3461. Paropsia. Noronh. ap. 3462. Astrophea. DeC. A. P. Th.

\section{b. $G r a n a d i l l e a c$.}
3463. Tetrapathaea. DeC.
a. Pentaria. DeC. 3464. V a reca. G̈̈вт.
b. Decaria. DeC. 3465. Deidamia. A. P. Th. 3471. D isemma. LaB. 3466. Thompsonia. RBr. 346\%. M o de c c a. Rheed. 3468. Pas chanthus. Burcir. 3469. Tacsonia. Juss.

a. Psilanthus. DeC.

6. Distephana. Juss. Distephia. S a lis b. c. Aldinia, Rchb. Bracteogama. DeC.

d. Eutacsonia. DC. 34\%0. Murucuia. T. 3472. Passiflora. L.

a. Polyanthea. DeC.

b. Cieca. Medik. Astephananthes. B. St. V. IIonactineirma. B. St. V.

c. Decaloba. DeC. Passiflora. B. St. V.

d. Granadilla. DeC. - Anthactinia. B. St. V. e. Hellmannia. Rchb. Tacsonioides. DeC. f. Dysosmia. DeC.

$$
\text { c. } I \text { a } l \text { esherbie a e. }
$$

3473. Malesherbia. Rz. PAv.

Gynopleura. Cav.

FAMILIA LXXXV.

C O T T O R T E. Drehblüthlek.

$$
\text { A. } G^{\prime} e \quad n \quad t \quad i \quad a \quad n \quad e \quad a \quad \text {. }
$$

$$
\text { a. Menyantheae. }
$$

, 3474. Menyanthes. L. 3475. Villarsia. Vent. Nymphoides. T.
Waldschmidtia. Wigg. Schweykerla. Gm.

\section{b. Chironieae.}

347\%. Exacum. L. Cicendia. Ad.
ITicrocule. Hff $g$. Lk. Hippocentaurea. S chult. 
34\%8. Schiiblera. MART. Curtia. C h a m. 3479. Seb a a. RBr.

3480. Prepusa. Mart.

3481. Schultesia. Mart. 3482. Cout oubea. Aubl.

Picrium. Schreb.

Cutubea. II a rt.

3483. Houstonia. L. Poiretia. G m.

3484. Centa urella. Mchx. Bartonia. W. Andrewsia. Sp r.

3485. Frasera. WALt.

3486. Tachia. Aubr. Myrmecia. Schreb.

348\%. Pl ad e ra. Roxb.

Canscora. R. Br.

3488. Lisianthus. L.

3489. Helia. Mart. 3490. Irlbachia. MArT.

3491. Chlora. L.
3492. Slevogtia. Rorb. Hippion. Spr. non Schm. 3493. Sabbatia. Ad. 3494. Callopisma. Mart. Deianira. Ch a m.

3495. Voyra. Aubl.

Vohiric. La m.

Lita. S chreb. 3496. Erythrae a. Rich. 3497. Chironia. L. 3498. Rochefortia. Św. 3499. Gentiana. L.

a. Hippion. Schm. Gentianella. Brkh. Eurythatia. Brkh. Eriocoila. Brkh.

b. Pneumonanthe. Schm. Ciminatis. $\mathbf{B r k h}$. Dasystephana. B r k h. Coilanthe. Brkh.

c. Gentiana, Schm. Asterias. Bxkh. non $\mathrm{Z} 00 \mathrm{l}$. 3500. Swertia. L. 3501. Halenia. Ввкн.

\section{c. Log a nie a e.}

3502. Logania. RBr.

Evosma. Andr.

3503. Geniostoma. Fonst. Anasser. Ju s s.

3504. Usteria. Lam. non Cav. 3505. P ag a mea. Aubl. 3506. Gärtnera. LAM. non RoxB.
350\%. Potalia. Aubl.

Nicandra. S chreb.

3508. Anthocleista. Afzez. 3509. Fagraea. Thnв.

Willugbeia. Scop. 3510? Fereira. Vaud.

3511? G nmillaea. Rz. PAv.

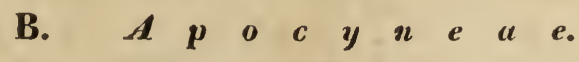

a. $E$ c $\boldsymbol{k} i \boldsymbol{t} \in \boldsymbol{e}$ e.

aa. Ecoronatac.

3512? Alafia. A. P. Th. 3514. H a e m a dic t y o 3513. Echites. L.

Lindx. 
3515. Ichnocarpus. RBr. 3520. Parsonsia. RBr. 3516. Beaumontia. WALL. 3521. Lyonsia. RBr. 351\%. Holarrhena. RBr. 3522. Apocynum. L. 3518. Is o n e m a. RBr. 3523. Cryptolepis. RBr. 3519. Vallaris. RBr. 3524. Thenardia. K. H. B. Peltanthera. R th. Emericia. R. S. 3525. Alstonia. RBr. 3526? Syringosma. Mart.

bb. Coronat a e.

352\%. Prestonia. RBr. 3528. B a If u ria. RBr. 3529. Nerium. L.
3530? Systrepha. Burch. 3531. Strophanthus. DeG. 3532. Wrightia. RBr.

b. Vince $\quad$ \& $e$.

3533. Vinca. L. 3533b. Lochnera. Rchв. V. rosea. $\mathrm{L}$.
3536. Cameraria. L. 353\%. Ams onia. Walt. 3538. Plumeria. L. 3534. Tabernaemontana. L. 3539. Plectaneia. A. P. Th. 3535. Vahe a. LaM. Urceola. Vaud?

$$
\text { c. Allamandeac. }
$$

3541. Alla manda. L. 3543 ? Anabata. W. 3542. Aspidosperma. Mart.

$$
\begin{aligned}
& \begin{array}{llllllllll}
\text { C. } & C & a & \boldsymbol{r} & \boldsymbol{i} & \boldsymbol{s} & \boldsymbol{s} & \boldsymbol{e} & \boldsymbol{a} & \boldsymbol{e} \text {. }
\end{array} \\
& \text { a. Jasminea e. }
\end{aligned}
$$

3544. I a s m in um. L.

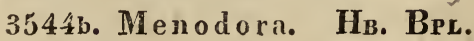
3545. Mogorium. Juss. 3546. Ligustrum. L. 354\%. Syringa. L. Lituc. T. 3548. Forsythia. VAHL. non WaLth.

\section{b. R}

3549. Bolivaria. Cham.

3550. Mitreola. L. Riсн. Iritra. Houst. 3551. Spigelia. L.
Arapabaca. Plum. 3552. Cynoctonum. Gis. 3553. O ch rosia. Juss. Tanghinia. A. P. Th. ophioxylon. P. 
3554. Rauwolfia. L. 3555. Alyxia. Banks. Gynopogon. Forst. 3556. Vallesia. Rz. Pav.
355\%. Theretia. Juss. 3558. Voacanga. A. P. Th. 3559. Ophioxylon. Burm.

$$
\text { c. Cerberea e. }
$$

3560. Cariss a. L. 3561. A rduina. L. 3562. Ambelania. Aubu. 3563. L e u c o n otis. JAck. 3564. Landolphia. P. B. 3565. Hancornia. Gomez. 3566. Monetia. l'Herit.

Azima. La m.

3567? D issolena. Lour. 3568. Rouhamon. Aubr. Lasiostoma. Schreb. Curare. II u m D.

3569. Cerbera. L.
Ahouai. T. 35\%. Maripa. Aubr. 35\%1. Dicaryum. W. hb. 3572. Coprosma. Forst. 3573. Melodinus. Forst. 3574. Urceola. Rxi. 3575. Paederia. L. *) 3576. Pacouria. Aubu. 357\%. Couma. Aubl. 3578. Strychnos. L. 3579. Ignatia. L.

Ignatiana. Lour.

FAMILIA WXXXVI.

S A P O TA C E A E. S A P O T GE

A. S $t$ y $r$ a

a. Fraxineat.

3580. Fraxinus. L. 3581. Ornus. P.
3582. Chionanthus. L. 3583. Linociera. Su.

\section{b. Oleinae.}

3584. Notelaea. VENT. 3585. Phillyrea. L. 3586. Olea. L.
3587. Norouhia. A. P. Th. 3588. May e pea., Aubr.

-) Unc Lygodysodeam Rz. PAv. duxit ill. Sprknger, Rubiacean nobis ex icone Fl, peruv, vel habitu vel characteribus diversan. 


$$
\text { c. Styraceatgenuinae. }
$$

3589. M a b a. Forst.

Pisonia. Rottb. non L.

Ferreola. Rxb.

3590. Pouteria. Aubl.

Labatia. S w.

3591. Phe 11 ine. LaB.

3592. Styrax. L.

3593. Halesia. L.
3594. Diclidanthera. Maft.

3595? Houmiri. Aubl.

Houmiria. Juss.

Houmirium. Rich.

IIyrodendron. Schreb. 3596? C y r ta. Lour.

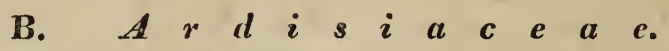
a. Triental $\operatorname{Tea} e$.

359\%. Trientalis. L.

$$
\text { b. Myrsineat. }
$$

3598. Bladhia. Thnb.

3602. Roemeria. Trinb.

3599. Ardisia. Sw.

3600. Myrsine. L.

3603. Embelia. Burm. 3604. Othera. Thns.

3601. Opilia. Rxb. 3605. Aegiceras. L.

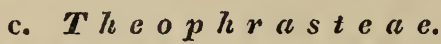

3606. I acquinia. L. 360\%. Theophrasta. L. 360s. Clavija. Rz. PAv. 3609. Le onia. Rz. Par.

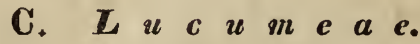

$$
\begin{aligned}
& \text { a. Olacince. } \\
& \text { a. Genuinae. }
\end{aligned}
$$

3610. Olax. L. 3615. Pseudaleia. A. P. Th. 3611. Spermax y rum. Lab. 3616. Pseudaleioides. A. 3612. Fis silia. Commers.

P. Th.

3613. Heisteria. L.

3616b. Vantanea. Avis.

3614. Ximenia. Plum. Heymassoli. Aubl. Gela. L our? Lemniscia. S chreb.

3617? Ic a c in a. A. Juss. 3617b? Millingtonia. Rxb. 


$$
\text { B. Asterantheas. }
$$

3618. Asteranthus. DEsv. 3619. Napoleona. P. B. Belvisia. D es v.

\section{b. $A q u$ if oliaceac.}

3620. Cassine. L. 3621. Hartogia. Thrs. 3622. Curtisia. Art. non Schreb.

Doratium. S ol.

Relhania. Gm. syst.

Junghansia. Grm. syst. 3623. My ginda. JcQ. am. Rhacoma. L. Crossopetulum. P. Br.

3624. Il ex. L. Aquifolium. T.
3625. Botryceras. W. 3626. Prinos. L.

a. Prinoides. DC.

b. Ageria. Ad.

c. Winterlia. Mnch. 362\%. Nem op anthes. RAFrs. Ilicioides. Dum. Cours. 3628. Skimmia. ThNB. 3629. L e pt a. Lour. 3630. Brexia. Noronh. Venuna. Lam.

$$
\text { c. } M i m u s \text { op e a e. }
$$

3631. Montoubea. Avbr. Montabea. A ubl. tab. Cryptostomum. Schreb. 3632. Acosta. Rz, PAv. **) non Lour.

3633? Roussea. Sm.

3634. Sa mara. L. Rapanea. Aubl.
3641. Manglill a. Juss. Duhamelia. Domb. Caballeria. Rz. Pav. Scleroxylon. W. 3642. Lucum a. Juss. 3643. Bonellia. Brot.

3635? Sphenocarya. Waxk. 3645? Luclea. L. 3636. Bumelia. Sw. 3646? Ehrenbergia. SPr. 363\%. Hunteria. Ruxb. 363s. Sideroxy lon. L. 3639. Sersalisia. RBr. 3640. Chrysoph yll u m. L. Cainito. Plum.

364\%. Diospyros. L.

Guaiacuna. T.

Ebenus. Co m mers. 3648. Cargillia. RBr.

*) Ex dissecto flore, Ilici proxima, habitus refert $I$. chinensem D. Mug. 20.43. Fructam non vidi.

**) Frnctu $5 \rightarrow$ loculari et hilo seminis in medie, videtur a praecedente differre. 
138 CLASS. VI. SYNPET, ORD. III. ROTIFL. FAM. LXXXVI. SAPOT.

3638. Imbricaria. Commern. 3654. H op ea. Roxb. 3639. Mimus ops. L. Binectaria. Forsk. 3651. In oc ar pus. Forst. Gajanus. $\mathbf{R} \mathbf{m p h}$. 3652. Rо уеиа. L. 3653. Turaria. MoL. 3655. Bassia. Kös. 3656. Visnea. L. Mocanera. Juss. 3653b? Dëcadia. Lour.

365\%. Omphalocarpus. P. B. 3658. Sy mplocos. L. 


\section{A I Y Y CA A N T T H A E. KELCHBLUETHIGE.

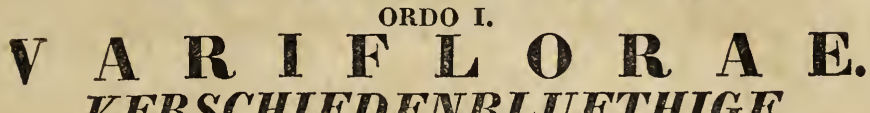 VERSCHIEDENBLUETHIGE.}

Formatio $\mathbf{I}$.

PA R V I L O RA E. LE G U M I N O S A E. KLEINBLÜTHIGE.

HÜLSENFRÜCHTIGE.

Fam. LXXXYII. Umbelliferae. Fam. XC. Papilionaceae.

Doldengewüchse.

Fam. LXXXYII. Rhamneae. Fam. XCI. Cassiaceae.

Kreuzdorne.

Cassiaceen.

Fam.LXXXIX.Terebinthaceae. Fam. XCII. Misnosaceae.

Terebinthaceen. IMimosaceen.

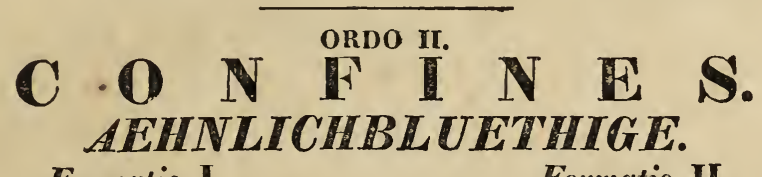

Formatio I.

Formatio II.

S E D I F L O R A E.

SEDUMBLÜthige.

Fam.XCIII. Corniculatae.

Fam. XCIV. Loasaceae.

Gehürnt frïchtige.

Loasacecn.

Fam. XCV. Ribesiåceae. Ribcsiaccen.

R O S I F L O R A E. ROSENBLÜThIGE.

Fam. XCVI. Portulacaceae. Poriulacaceen.

Fam. XCVII. Aizoideae. Aizoideen.

Fam. XCVIII. Rosaceae. Rosaceen.

\section{0 N $\mathrm{C}^{\text {ORDo }} \mathrm{III} N \mathrm{~N}$. GLEICHIOERTIGE.}

Formatio I.

O N A G I F L O R A E NACHTKERZENBLÜTHIGE. Fam.XCIX. Halorageae. Halorageen.

Fam. C. Onagreae. Nachtkerzen.

Fam. CI. Lythreae. Weidricke.
Formatio II.

M Y R I I F L O R A E. MYRTENBLÜTIGE. Fum. CII. Melaleuceae. Melaleucecn. Fam. CIII. Myrtaceae. Myrtacecn.

Fan. CIV. Amygdalaceae. Amygdalaccen.

to Vgl. Botanik. s. $448-485$. 
La science est dans l'ensemble des faits, et non dans de certaines méthodes systematiques, qui n'envisagent les objects que sous un seul point de vue.

\author{
MIRB. Ann. d. Mus.. XVI. p. 420.
}




\title{
CLASSIS VII. \\ C A L Y C A N T H A E。 KELCHBLUETHIGE.
}

\section{ORDO I. \\ V A R I F L O R A E. VERSCHIEDENBLUETHIGE.}

\author{
FORMATIO I. \\ P A R V I F L O R A E. \\ KLEINBLUETHIGE.
}

FAMILIA LXXXVII.

UMBELLIFERAE. DOLDENGEW ÄCHSE.

A. $G e n u$ i $n$ a $a$ e.

$$
\text { a. } A r m a t a e \text {. }
$$

1. Scandicineae (paucijugac campylospermae subinermes):

3659. Anthriscus, Pers, 3662. Spermatura. Rohb.

«. Anthriscus. Riv. Uraspermum. Nutt.

b. Cerefolium. Hall. 3660. Scandix, T. L. 3661. Schultzia, SPR.

B. Caucalineae (multijugae campylospermae armatae):

3665. Ca uca lis, L. 366\%. Torilis, AD. 3666. Turgenia. Hoғвм.

r. Daucineac (multijugae orthospermae armatae): ace Cumineae (contractue):

3668, O liveria。Vent, 3669, Cuminum, L, 


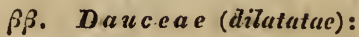

36\%. D a ucus, L.

a. Daucus. G.

b. Visnaga, G.
36\%1. P laty s perm um. Hoffm. 36\%2. Orlay a. Hofrm.

\section{b. $A l$ l $\quad \boldsymbol{t}$ a $e$.}

a. Peucedaneae (paucijugae orthospermac diptcrae):

3673. Anethum. L. 36\%4. Peucedanum. L.

a.Peucedanum. L.

b. Thysselinum. T.

c. Oreo elinum. T. Cervaria. G.

d. Callisace. Fisch.

3675. Im peratoria, T. 36\%. Capnophyllum. G̈̈rr.

Rumia. Lk.

36\%. B u bou, L.

3678. F e rula, L.

36\%9, Ferulago. Косн.

3680. O popanax, Косн.
3681. Pastinaca. L.

a. Pastinaca. Ho $\mathrm{fm}$.

b. Malabaila. Hoffm.

3682. Heracleum. L.

a. Wendia, Hoffm.

b. Heracleum. Hoffm.

c. Sphondylium. T.

3683. Zosimia, Hoffu.

3684. Krubera, Hofrm. Ulospermum. L k.

3685. Condy locarpus. Hofra. 3686. T ord ylium. L. 368\%, Hasselquistia. L.

\section{B. Thapsieae (multijugae orthospermae):}

3688. Palimbia. Ress. 3689. Siler. Scop.

3690. Laserpitium. L. 3691. Prangos. Lindu.
3692. Thapsia. L. 3693. M e l a n o s e lin u m.

$\%$. Angeliceae (paucijugae orthospermae):

3695. S el in um. L. 3696. Levisticum. Косн. 369\%. Ostericum. Hoffm. 3700? Cymopterus. Rafin. HoFrM.

$$
\text { c. Cost at } a \text { e. }
$$

a. Coriandreae (mullijugue orthospermae globosae):

- 3\%01. Coriandrum. T. L. 3702. Biforis. Spr.
Corion. Lk.

3\%03? Exoacantha. LaB. 


\section{B. Ammineac (paucijugae orthospermae):}

$\alpha{ }$. Seselineae (terctiusculae) :

3704. Foeniculum. T. 3712. Mol o p ospermum. 3705. M e um. T. Косн.

3706. Trochiscanthes. Kосн. 3\%13. C nidium. Cuss. 370\%. P a chy pleurum. Ledeb. 3714. S o ranthus. Ledeb.

3708. Ligusticum. T.

a. Ligusticum. Spr.

Gaya. Gra ud.

b. Wallrothia. Spr.

c. Gingidium. Forst.

3\%09. Sila us. BEss.

3710. Ath a in anta. L.

3711. Brignolia. Bertol.
3715. Seseli. L.

a. Seseli. Spr.

b. Bubon. Spr.

c. Libanotis. Hoffm.

3716. Crith un m. T.

371\%. Coenolophium. Косн.

\section{BP. Ammineaegenuinae (contractae):}

3718. Carum. L.

a. Carvi. T.

b. Bunium. L. Bulbocastanum. $\mathbf{T}$.

3719. A m mi. L.

3720. Crit amus. Trag.

Drepanophyllum. Wib. Hoffm. 3721. Conioselinum. Hoffm. 3722. Sison. "L."

3723. Ptychotis. Koch. 3724. T rinia. Hoffm. 3725. Petroselinum. Hofrm. 3726. A p i un. L. 3727. Zizia. Kосн. Thapsium, Nutt.

3728. Ru in ia. Hofrm. 3729. A ethusa. L. Cicuta. T. 3\%30. Cicuta. L.
Cicularia. Riv. Lam.

3\%31. Otto a. K. H. B. 3732. Huan aca. Cav. 3733. Oenanthe. L.

$a$, Oenanthe. $\mathrm{L}$

b. Phellandrium, T. L.

3734. Annesorrhiza. Cham. 3735. Helosciadium. Kосн. 3736. S i u m. L. Sisarum. T. Berula. II o $\mathrm{fm}$.

373\%. Conopodium. Косн. 3738. Pimpinella. L.

Tragoselinum. T.

a. Pimpinella. Sppr.

b. Tragium. Spr.

c. Ledebouria, Lk.

भ. Smyrnicae (paucijugue campylospermac turgidae):

3739. Trachy pleurum.

Rснв.

B. granuloso, glaucum etc.
3740. Bupleurum. L. a. Odontites. Spr. ex p. b. Bupleurum. Hoffm. 
c. Diaphyllum. Hoffm.

d. Isophyllum. Hoffm.

e. Tenoria. Spr.

3\%41. Heteromorpha. Cham.
3744. Smyrnium. L.

3745. Physospermum. Crss. Danak. All.

? Hänselera. LaG.

3746. P l e u r o p e r m n m.

Hoffм.

374\%. Hi ppomarath rum.LK. 3\%48. Cachrys. T. L.

B. $A \begin{array}{llllllllll}A & \boldsymbol{a} & \boldsymbol{a} & \boldsymbol{l} & \boldsymbol{i} & \boldsymbol{a} & \boldsymbol{c} & \boldsymbol{e} & \boldsymbol{a} & \boldsymbol{c} \text {. }\end{array}$

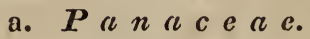

a. Lagocciece.

3749. Lagoecia. L.

B. $E r y n g$ i e a e.

3750. Eryngium. L.

Actinotus. LaB.

3751. Alepidea. LARoch. 3753. Astrantia. L.

3752. Eriocalia. SM. 3754. Arctopus. L.

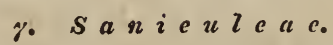

3\%55. S anicula. L.

3\%58. P anax. L.

3756. Petagia. Gouss.

375\%. D ondisia. Spr.

Dondia e sphalm?

3759. Cus s onia. ThNB.

$$
\text { b. } \quad B \quad 0 \quad l \quad a \quad c \quad e \quad a \quad e \text {. }
$$

$$
\text { c. II er ma d e a e. }
$$

3760. Cogswellia. Spr. 3760c. Hiigelia. Rснв. ${ }^{*}$ )

Lomatium. Rafin. 3\%61. Hermas. L.

3760b. Trachymene. Rudg. 3762. Lichtensteinia. Cham non Spr.

$$
\text { B. I y drocotylea } e_{\text {. }}
$$

3\%63. Hydrocotyle. L. 3\%68. Spananthe. Jce.

3\%64. Chondrocarpus. Nutr. 3\%69. Bowlesia. Rz. PAv.

Glyceria. Nutt. non al. 3\%70? Drusa. DeC.

3\%65. Crantzia. Nutr. 3\%1. Micropleura. LaG.

3\%66. Erigenia. Nutr. 3\%2. Pozoa. LaG.

3\%6\%? Tris anthus. Lour. 3\% 3 ? Asteriscus. Cham.

* Hiigelia cyanea Rcris. Jc. exot. 201. planta ex elegantissimis. 
3794. Trachymene. Rudg. Fischera et Trdchym. S p r. uou Rudg. 37\%5. B o l ax. Commers. 3776. Mulinum. Pers.
379\%. Siebera. Rснв.

Chamitis. G. 3798? Fragosa. Rz. PAr. 37\%9. Pectophyllum.K.H.B.

$$
\text { c. Araliacea ge } \operatorname{se} u \text { in a e. }
$$

3780. Maralia. A. P. Th. 3784. Schefflera. Forst. 3781. Aralia. L. 3785. Polyscias. Forst. 3782. Sciadophyllum. RBr. 3786. Gilibertia. Rz. PAv. 3783? Actinophyllum. Rz. 378\%. Phytocrene. WalL. Par.

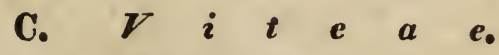

a. Hageniacea $e$ ?

3788? Hagenia. Lam.

$$
\text { b. Cissea } e_{\text {. }}
$$

3789? Cayratia. Juss. 3791. Ampelopsis. Mchx. Columella. Lour, non $\mathrm{Jcq}$. 3792. Vitis. L. 3790. Cissus. L.

$$
\text { c. } H \text { e derace } a \text { e. }
$$

3793. Hedera. L. 3795? Dicophe. WaLL. 3794. Gastonia. Commers.

FAMILIA LXXXVIII.

R H A M E A E. KREUZDORNE.

a. Gou $a$ त $i$ ace $a$ e.

3796. Crumenaria. MArt. 379\%. Gouania. L. Retinaria. G.

b. Ce.anotheae.

3798? Spixia. Leand. non 3801. Pomaderis. Lab. Sсннк.

Pomatoderris. Hffgg. Pomatiderris. Knth.

3\%99. C e a n o thus. L. e. e.

Forrestia, Rafin.

3802. Crypt andra. SM.

Euceanothus (Sect.) DC. 3803. Trich ocephalus.

3800. Willemetia. Brongn.

Brngn.

Ceanothus. Rafin. 3804. Phylica. L.e. e 
3805. Soulangia. Brang. 380\%. Colletia. K. H. B. 3806. Scutia. Сомм. mse. 3808. Hovenia. Thrb. Sentis. C o m m. b b. 3809. Colubrina. Rich.

$$
\text { c. Fva } n g u l \text { a c e } a \text { e. }
$$

3810. Retanilla. Brign. Molinaer. Com m. msc. 3811. Paliurus. T. Dsf. 3812. Sageretia. PnngN. 3813? Eustathes. Lovr. 3814. Olinia. Thмз. 3815. Goup ia. Aubl.

Gupia. I. St. H. Glossopetalum. Schreb. 3516? C a rpodetus. Fonst. 38:7. Zizy phus. T. Dsf.
3818. Condalia. Cir. 3819. Berchemia. Neck. Oenoplea. IIIg. Oenorlia. R. S. 3820. Ventilago. G̈̈rt. 3821. Dulongia. KNTh. 3\$22. Rh a mus. L. e. e. a. Alaternus, $\mathbf{T}$. Marcorella. Neck.

b. Crevispina, Dill.

c. Frangula. T.

FAMILIA ZXXXIX.

TEREBINTHACEAE. TEREMTHACEN.

A. Chaillesiaceac.

2823. Chailletia. DC.

Patrisia. Roh r.

a. Mestotes. Soland.

b. Dichapetalum. DC,

3824. Plappertia. Rcub.
Leucosiu. A. P. Th. non Zoolog.

3825. T'apura. Aubl. Rohria. Sch reb.

$$
\text { B. Connaracear. }
$$

3826. Con narus. L.

Muibrunciu. N e ck.

Omphalobium. G.

382\%. Rourea. Aunl.

Robergia. Schreb.
382 ¿. Eurycoma. JAC. 3829. Cnestis. Juss.

3830? T e tradium. Loun.

$$
\begin{gathered}
\text { C. Tereb. genuinae. } \\
\text { a. Amyridea.e. } \\
\text { ". Inglandeae. }
\end{gathered}
$$

3031. Juglans. L. 3832. Caryi. Nutr.
3833. Pterocarya. Nutt. 3834? Decostea. Rz. PAr. 


$$
\text { B. } B \text { us erea e. }
$$

3835. Elaphryum. JAcQ. 3843. Marignia. Commers. 3836. Boswellia. Rxb. 383\%. Pegia. Colesr. 3838. Bal sam oden dron.

KNTi.

Balsanea. Gled. 3839. Icica. Aubl. 3840. Protium. Burm. 3841. Bursera. JAcQ. 3842. Crypt oc a pa. KNтн. Dammara. Gärt. non al. 3844. Colophonia. Commers. 3845. Canarium. L.

a. Canaria.

b. Pimela. Lour. 3846. Sch wä gr ich en ia. Rснв. Hedwigia. Sw. *) Tetragastris. G. 384\%. Sorindeia. A. P. Th. 3848. Gra rug. Rxi.

$$
\because \text { A myridea ge иu }
$$

3849. Amyris. L. e. e.

$$
\text { b. } S u \text { on } a \in h i n a e \text {. }
$$

3850. Rhus. L.

a. Cotinus. T.

b. Metopium.

c. Rhus. T.

Toxicodendron. T. non al. Pocophorum. Neck.

d. Thezera. DC. e. Lobadiuns. Rafin. Trurpinia. Rafin. non Desv. Schmalzia. Desv. 3851. M auria. K. H. B. 3852. Duvaua. KNTr. 3853. Schinus. L. Molle. $\mathbf{T}$.

$$
\begin{aligned}
& \text { c. } G a \text { s s } u \text { v } \quad \text { i e re e. } \\
& \text { a. } S p \circ u d i a c e a c \text {. }
\end{aligned}
$$

3854. Spondias. L. Mombin. Plum.
Cylheraca. DC. 3855. Po oupartia. Cомменs.

$$
\text { B. Lentisceate. }
$$

3S56. Picramnia. Sw. ? Turiti. A ubl. 385\%. Comocladia. P. Br. Dodonata. Plum. non L. 3858. Astronium. Jcq.

$$
\text { \%. Anacardieae. }
$$

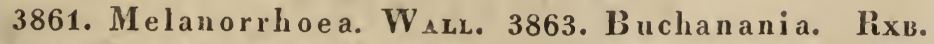
3862. Cambessedea. Kктн.

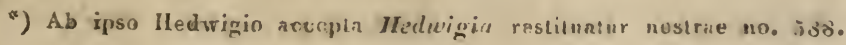


3864. Mangifera. L. 3865. Holigarna. Rxв. 3866. S emec a r pus. L. fil. Anacardium. Lam. 386\%. Anacardinm. Rotrb.
Acajou. T. Acajuba. G. Cassuvium. $\mathbf{L}$ a m. 3868. Rhinocarpus. Bert.

FORMATIO II.

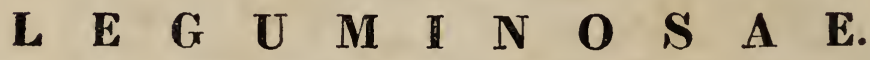
HUELSENFRUECHTIGE.

FAMILIA XC.

PAPILIONACEAE. SchmetTERLINGSBLÜThIGE.

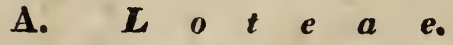

a. Trifolie a e.

3869. Trifolium. L.

a. Lagopus. Ser.

b. Phleastrum. Ser.

c. Eutriphyllum. Ser.

d. Trifoliastrum. Ser.

e. Vesicastrum. Ser.

f. Lupinaster. Much. Pentaphyllon. P.

g. Chronosemium. Eer. 3870. Melil otus. T.

a. Coelorutis. Ser.

b. Plagiorutis. Ser.

c. Campylorutis. Ser. 3871. Pocockia. DeC. 38\%2. Trigonella. L.

a. Grammocarpus, Ser.

b. Foenumgraecum. Ser. c. Buceras. Mnch, e. e.

d. Falcatula. Brot. 3873. Dorycnium. T. 3874. Lotus. L.

a. Krokeria. Mnch.

b. Lotea. Med.

c. Eulotus. Ser. 3875. Tetragonolobus. Scop. Scandalida. N eck. 38\%6. Acropodium. Dest. 38\%. Medicago. L.

a. Hymenocarpus. Sav.

b. Lupularia. Ser. s c. Spirocarpus. Ser.

3878. Cyamopsis. DeC.

\section{b. $A s t r a g a l$ e $a$ e.}

3879. Giildenstedtia. Fisch. 3882. Astragalus. L. e e. 3880. Phaca, L. 3881. Oxytropis. DeC. 3883 ? H a rpalyce. Fl. mex. 3884. Radiusia. Rснв. 
Pseudosophora. DC.

3885. Bis errula. L. 3886? Diploprion. Vir.

$$
\text { c. G a l e g } c \text { a } e \text {. }
$$

388\%. Petalostemum. Mснх.

a. Peialostemou. DC.

b. Kuhnistera. Lam. Cylopogon. Rafin.

3888. D al e a. I.

Parosella. Cav.

3889. Glycyrrhiza. T.

a. Glycyrrhiza. Minch.

b. Liquiritia. Mnch. 3890? Crafordia. Rafin. 3891. Gralega. T. 3892. Tephrosia. P.

Crucca. L. Zeyl.

Necdhamia. Scop. non al.

a. Mundulea. DC.

Robinia. Rxb. non $\mathbf{L}$.

b. Brissonia. Neck. Ercbinthus. Mitch.

c. Craccoides. DC.

d. Reineria. Mnch. 3893. Amorpha. L.

Bonafidis. Neck. 3894. Ey sen hard tia.K.K.B. 3895. N is solia. Jcę.

a. Nissolia. G.

b. Gomezinm. DC.

c. Machaerium. P. 3896. Miillera. L. fil. 389\%. Lo n chocarpus. K. H. B. 3898. Ro binia. L. e. e.

Pseuducacia. T. 3899. Poit ea. Vent.
Poitaea, DC.

3900. Sabinea. DC. 3901. Coursetia. DC. 3902. Sesbania. P. Sesban. Poir. Sesbana. P. Br.

3903. A gati. Rheed. 3904. Glottidium. Desr. 3905. P iscidia. I.

Piscipula. Löffl.

Ichthyomethia. P. Br. 3906. Daub ent onia. DC. 390\%. Coryella. DC. Corynites. Spr.

3908. Ca ragana. Larr. 3909. Halim od e ndron.

Fisch. Halodendron. DC. non A. P.

Th.

3910. Diphysa. Jce. 3911. Calophaca. Fisch. 3912. Colutea. L. e. e, 3913. Sphaerophysa. DC. 3914. Sutherlandia. RBr. Colutice. Mnch.

3915. Swainsonia. Stuss. Loxidium. Vent.

3916. Lessertia. DC.

Sulitra. MI dic.

3917. C armichä̈la. RBr.

B. F $a \quad b$ a c c e $a$ e.

a. Vicieac.

3918. Ervum. L.

a. Lens. $\boldsymbol{T}$. b. Ervilia. Lk. 3919. Vicia. L. 
150 CLASS, VIJ. CAL YC. ORD. 1. VARIFL. FAM. XC. PAPILION.

a. Wiggersia. G. Mr. S, Fl. 3923. L a th y r us. L.

b. Vicia, Fl. Wett.

$$
\text { Wett. a. Ochrus. P. }
$$

3920. Faba. T. *)

3921. Cic er. L.

Clymenum. DC.

3922. Pisum. L.

b. Lathyrus. Auct. Eulathyrus. Ser. 3924. O robas. L.

\section{b. $P h a s$ e o le a e.}

3925. Abrus. L.

3926. Sweetia. DC.

392\%. M a crant hus. Lour.

Murcanthus (erron.) Willd.

3928. Götzea. Rснв.

Rothia. P. non al.

3929. T e ramnus. RBr.

3930. Amplicarpaea. DC.

Amphicarpa. E11.

Savia. Rafin.

Frícata. $\mathrm{Gm}$.

3931. Amphodus. Lindu.

3932. Kennedya. Vent.

Cautinia. Mnch.

3933. IR h y chosia. Lour. Arcyphyllum. E11.

3934. Erios ema. DC. Desv. 3935. Fagelia. Neck. non

Schwк.

3936. Wisteria. Nutr.

Thyrsunthas. Ell.

Krauniia, Rafin.

3937. Apios. Вогкн.

Bradica. Ad.

3938. P h a s e olus. L.

a. Phaseolus. Much.

Euplaseolus. DC.

b. Phasellus. Mnch. 3939. So ja. Mrch. 3940. Dolichos. L. a. Eudolichos. DC.

b. Catiang. DC.

c. Ungnicularia. DC. 3941. Vig a. S. Sivr. 3942. Lablab. Adans. 3943. Cacara. A. P. Th. Pachyrrhizus. Rich. 3944. Parochetus. Hamirt. 3945. Dioclea. K. H. B. non Spr.

Hymenospron. S pr.

3946. P s o ph oc a r p us. Neck. Botos. Ad.

3947. Canavalia. DC.

Canavali. Ad. Malochia. Savi.

3948. Taeniocar pum. Drsv. 3949. Muсu иа. Á.

Hornera. N e ck. Stitzolobium. Pers. Negretia. Rz. Pav. Cilla, Lour.

Labradia. S wed. Carpopogon. Rxb. Macroceratides, Raddi?

a. Zoophthalmum. P. Br.

b. Stitzolobium. P. Br. 3950. Calopogon inm. Desv. 3951. Cruminium. Desv. 3952. Cajanus. DC. Cajan. Ad.

\footnotetext{
* Species el characterem cf. ap. Ronb, in Mossserar encheiridio 11. p.
} 
3953? Phyllolobium. Fisch. 395\%. Rudolphia. Fi. 3954. Lu pin us. L. 3955. Cylista. Art. 3958. Butea. Roxb. 3956. Erythrina. L. Coruliodendron. T. Mouricon. Ad. Plaso. Rhe ed. 3959? Sarcodum. Lova sarcodium. P.

c. Glycineac.

3960. Chatocalyx. DC. Boenninghausiu. Spr. 3961. Gly cine. L. e. e. 3962. Dumasia. DU: 3963. Pueraria. DC. 3964. Otoptera. DC. 3965. Coll a ea. DC. 3966. Gron a. Lour. 396\%. B arbieria. DC. 2968. Vilmorina. DC. 3969. Odonia. Bertol. 39\%0. Galactia. P. Br. 3971. Cologania. K. H. B. 3972. Martia. LEand.
Marliusia, Schuls. 3973. Neurocarpum, Desr. Rhombifolizm. Rich. 39\%4. Clitoria. I.

Ternutes, $\mathbf{T}$. Clitorius. Petiv. Vexillaria. IIffgg.

a. Ternatea, $\mathbf{T}$.

b. Euclitoria. DC.

c. Centrosema, DC.

d. Glycinopsis. DC. 3975. Indig ofera. L. 3976. P s oralea. L. Dorycnium, Mn $\mathbf{n}$ h. Ruterice. Mnch.

$$
\text { C. } H \text { e d y s a } r \text { e } a c \text {. }
$$$$
\text { a. Coronilleac. }
$$

397\%. S corpiurus. L. Scorpioides. T. Scorpius. Lo is. non M $\mathbf{n c h}$. 3878. Cor onill a. L. e. e. a. Coronilla, T,

b. Emerus, T. 3979. Arthrolobium. DEsv. em. Artrolobium. Desv.
Astrolobium, DC. 3980. Ornith opus. L. Ornithopodium. T. 3981. Hippocrepis. L. Ferrum equinum. $\boldsymbol{T}$. 3982. B on averia. Scop. Securidaca. 'T. Securilla. P. Securigera. nC.

$$
\text { b. Onobryclecae. }
$$

3983. Diphaca. Lour. 3984. Pictetia. DeC: 3985. Ormocarpum. P. B. 398\%. Poiretia. Vextonon al. 
Turpinia. $\mathbf{P}$.

3988. Plan arium. Desv. 3989. M yriadenus. Desv. 3990. Z o rnia. GM.

Anonyma. Walt. 3991. Stylosanthus. Sw. 3992. P a tagonium. Schrk. Adesmia. DC.

3993. Hete rol o m a. Desv. Patag. II. Chactotricha. DC. 3994. Aeschynomene. L. 3995. Sinithia. Art. Petaguanu. Gm.

3996. Flemingia. $\mathbf{R x B}$. IIitlingtonia. $\mathbf{R} \times \mathbf{b}$. msc. $a$. Flemingiastrum. DC.

b. Ostryodium. Desv. Lourea. J. St. Hil. Maghania. J. St. H. 399\%. L o urea. NEck. Christia. Mönch. 3998. Uraria, DEsv. Doodia. Rxb. non RBr. 3999. Nicolsonia. DC. Perrottetia. DC. 4000. Des modium. DEst. a. Eudesmodium. DC,

b. Pleurolobium. DC.

c. Chalarium. DC. 4001. D icerma. DC.

a. Phyllodium. Desv.

b. Aphyllodium. DC. 4002. Tavernaria. DC. 4003. He d y s a um. H. e. e.

a. Echinolobium. Desv.

b. Leiolobium. DC. 4004. Onobrychis. T.

a. Eubrychis. DC.

b. Hymenobrychis. DC.

c. Dendrobrychis." DC.

d? Echinobrychis. DC.

4005. Eleiotis. DC.

4006. Lespedeza. Mchx. 400\%. Eb enus. L.

4008. Alh agi. T.

Manna. Don.

4009. Alysicarpus. Neck. Hallia J. St. H. non Thub. Fabricia. Scop. non Gr.

4010. Bremontiera. DC. Ornithopodium. Burm.

$$
\text { c. } D \text { a } l \text { b ergie a e. }
$$

4011. D erris. Lour. 4012. End es perm um. Blum. 4013. Pongamia. Lam. Gatedupa. Lam.

4014. Dalbergia. Roxb. Solori. Ad.

4015? Viborquia. Ont. Varennea. DC. 4016. Pterocarpus. L.

a. Montouchia. Aubl. Griselinia. N eck.

b. Amphymenium. Knth.

c. Santalaria. DC.

d. Ateleia. Fl. mex.

4017? Weinreichia. Rens.
Pteroc. Echinodiscus. DC. non Zoolog.

4018. Drepanocarpus. W. MEY.

Nephrosis, Rich. msc. Orucaria. Juss. msc. 4019. Hecastophyll um. P. Br. ex em.

Ecastaphyllım. B. Pr. K. H. B. 4020. Amerimnum. P. Br. 4021. Brya. P. Br. Aldina, Ad.

4022. D eguelia. Aubr. Cylizoma. Neck. 
4023. D i p t e rix. Schreb.

a. Coumarouma. Aubl.

Cumaruma. Lam.

Baryosma. G.
Hcintzea. Scop.

b. Taralea. Aubl.

Bolducia. Neck.

4024. B rownea. JAce.

FAMILIA XCI.

CA S S I A E. CaSSIEEN.

A. $G e n i s t e a c$.

4025. Anthyllis. L.

a. Coruicina. DC.

b. Vulneraria. T.

c. Dorycuoides. DC.

d. Aspalathoides. DC.

e. Erinacea. Clus.

4026. Requienia. DC.

402\%. Ononis. L.

a. Lotononis. DC.

b. Euononis. DC.

c. Natrix. DC.

d. Natridium. DC.

e. Bugrana. DC.

4028. Adenocarpus. DeC.

4029. Cytisus. L, e. e.

a. Alburnoides. DC.

b. Laburnnm. DC.

c. Calycotome. Lk. Calycotomon. II!rgg.

d. Tubocytisus. DC. Viborgicl. Mnch, non Thnb.

e. Lotoides. DC.

f. Chronanthus, DC. 4030. Genista. Lam.

a. Salzwedelia. G. MT. S. FI. Wett.

b. Voglera, G. MT. S.

c. Genista. G. M. S.

4031. Śpartium. L. e. e;

Spartianthus. Lk.

4032. Stauracanthus. LK. 4033. Ulex. Lk.

4034. A spalathus. L. a. Eriocalyx. Neck.

b. Aspalathus. Neck.

4035. S a r c o p h y $11 \mathrm{um}$. Thre.

prod.

Sarcophyllus. Thnb. Fl. cap. 4036. Le beckia. Thins. 403\%. Dichilus. DeC. 4038. Loddiggesia. Sims. 4039. Viborgia. Spr. Wiborgia. Thnb. 4040. H y p o cal y p tus. ThNв. 4041. Clavulium. Desv. 4042. Crotal aria. L. 4043. Heylandia. DC. 4044. Hallia. ThNB. 4045. Priestleya. DC.

a. Eisothea. DC.

b. Aneisothea. DC. 4046. Liparia. L. e. e. 404\%. Achyronia. Wendr. 4048. Borbonia. L. 4049. V a s coa. DC. 4050. Ra fnia. ThNB. Oedmannia. Thnb. 4051. Templetonia. RBr. 4052. Scottia. RBr. Scottea. DC. 4053. Groodia. Salisb. 4054. Westonia. Spr. 4055. Bossieua. Vent, em. 
Bossinea. Vent, et auct. 4056. Platylobium. SM. Cheilococcu. Salisb. 405\%. H o vea. RBr.
Poiretia, Sm,

Physicarpos. Poir.

4058? Amphinomia. DC.

$$
\text { B. Sop bore a e. }
$$

4059. Mirbelia. SM. 4060. Daviesia. Sm. 4061. Pultena ea. SM.

a. Phyllota. DC.

b. Hymenota. DC. 4062. Euchilus. RBr. 4063. Gastrolobium, RBr.
4080. A m modend rou. Fisch. *)

4081. Cyclopia. Vent. Ibbetsonia. Sims. 4082. Dela ria. Desv. 4083. Baptisia. Vent. Crotalopsis. Mchx. 4064. Sclerothamnus. RBr. 4084. Thermopsis. RBr. 4065. Eutaxia. RBr. 4066. Dillwynia. Sm. 406\%. Xeropetalum, RBr. 4068. A otus. SM. Thermia. Nntt. 4085. Anagyris. T. 4086. Macrotropis. DC. 408\%. Virgilia. Lam. 4069. Sphaerolobium, SM. 4088. Ormosia. JAcks. 40\%. Viminaria $S_{M}$. 40\%1. Jacks onia. RBr. 4072. Burtonia. RBr. 4073. Gompholobium. Sr. 4074. Brachysema. RBr. 40\%5. Callis t a chys. Vent. Callissachys. Sm. 4076. Oxylobium. Andr. 407\%. Podol obium. RBr. 4078. Chorizema. LaB. 4079. Podalyria. Lam. Aphora. Neck. 4089. Edward sia. Ś StlisB. 4090. Sophora. RBr. 4091. My ros permum. JcQ. a. Myrospermum. K. H. B. Calusia. Bert, msc.

b. Toluifera. L. gen. Myroxylon. Mut. Liv.

4092? Exostyles. Schotт. 4093? Melanoxyl on. Schotт. 4094? A cos mi um. Sснотт. Sweetia. Spr. non DC. 4095? Lacara. Spr.

C. Cassieac ge $u$ uinue. a. G e off $\boldsymbol{r}$ o y e a e.

4096. Arachis. L. Arachidua. Plam. 409\%. Voandzeia.
Cryptolobus. Spr.

4098. P e raltea. K. H. B. A.P. Th. 4099. Bronguiartia.K.H.B.

*) Propter stamina libera quae video, hic inserendum, habilus Alhagearum, fructun non vidi. 
4100? Riveria. K. H. B. 4102. Gre offroya. Jeq. 4101. Andira. Lam. Acouroa. Aubl. Vouacapoua. Aubl. Drakensteinia. Neck.

\section{b. Ceratoniea .}

\section{(APETALAE.)}

4103. D iali u m. Burm.

Aruna. Aubl.

Cleyria. Neck.

4104. Ceratonia. L. Siliqua. T.

4105. Jonesia. Roxb.

Saraca. Burm.

4106. Hardwickia.

410\%. Copaifera. L.

Copaiva. Jcq. 4107b. Weigeltia. Rcns.

4108. Crudia. Schrsa.

Cyclas. Schreb.

Apalatoa. Aubl.

Touchiroa. Aubl.

Voutrana. Aubl.

- Parivora. Aubl.

rraldschnidtia. Neck.

Crudya. DeG.

4109. Geissois. Iob.

$$
\text { c. Ca esalpiniea } \text {. }
$$

4110. Codarium. Sol.

4111. Lab ichea. Gaudicir.

4112. Vouapa. Aubl.

Macrolobium. Schreb.

Krügeria. Neck.

4113. O u te a. Aubr.

4114. Anthonota. P. B.

4115. Intsia. A. P. Th.

-4116. Ta mariudus. L.

411\%. Heterostemon. Desf.

4118. II u in b old i a. V. Batschia. V.

4119. A'mherstia. WALl. 4120? V atairea. Aubl. 4121. Bowdichia. K. H. B. 4122. A maria. Mur. 4123. Aloexylon. Luor. 4124. Palove a Aubu. Giunania. Scop.

4125. Cercis. L.

Siliquastrum. T.

4126. Bauhinia. Puum. a. Banhinia. Kuth. Caulotropis. Rich.

b. Phasera. Lour.

c. Syinphyopoda. DC.

d. Pauletia. Cav.

e. Casparia. Knth.

4.12\%. Schnella. Radd.

4128. Hy m e naea. L. Courbaril. Plum.

4129. Parivoa. Aubl. Adlevia. Neck. Dimorpha. TV.

4130. E p e rua. AusL.

Rolmannia. Neck.

Panzera. W.

4131. Cynom etra. L.

Cynomorium. Rmph.

4132. Sch otia. JAcQ.

a. Schotia. Thub.

b. Schottiaria. DC.

c. Omphalobioides. DC.

4133. Afzelia. SM.

Poucovia. IV.

4134. Metrocyni a. A. P. Th. 
4135. Cassia. L.

๘. Chamaecrista. Breyn.

Grimaldiu. Schrk. non Radd.

b. Absus. DC.

c. Baseophyllum, DC.

d. Chamaeseuna. DC.

c. Senna. $\boldsymbol{T}$.

f. Herpetica. Rmph.

f. Chamaefistula. DC. Chamaecassia. Brejn.

h. Cathartocarpus. $\mathbf{P}$. Bactyrilobium. IV.

Fistula. DC.

4136. II o lde n ha u ra.

Schrad.

Dolichonema. Neow.

413\%. Ba ryx yl u m. Lour.

4138. T a chigalia. Aubr.

Cubaea. Schreb.

Valentynia. Neck.

Tassia. Rich. msc.

Tachia. P. non Aubl.

4139. Zuccagnia. CAv. non ThNB.

4140. Ca di a. Forsk.

Panciatica. Pàcciav.
4141. Parkinsonia. Puum. 4141. Ha e mat oxylon. L.

4142. Pomaria. Cav.

4144. Melanosticta. DG.

4145. Hof $\mathrm{m}$ anseg gia. CAv. 4146. Reichardia. Rтн. 414\%. Mezoneurum. Desf. 4148. Poinciana. L.

Poircia. Neck.

4149: C a esal pinia. Plum.

$a$ ? Libidibia. DC.

b. Campecia. Ad.

Sapponia. DC.

c? Brasilettia. DC.

d? Ticanto. Ad.

Nugaria. DeC.

4150. C oult eria. K. H. B. Adenocalyx. Bert. msc.

Tara. MTolin.

4151. G uilan dina. Juss.

Bonduc. Plum.

4152. A n oma. Lour.

4153. G y mnocladus. Lax. 4154. Gleditschia. I.

FAMILIA XCII.

MIMIOSEAE. Mrmoseen.

A. Detarieac.

4155. D e t a ri u m. Juss.

4156. Cordyla. Lour. Cordylia. P.

B. Swarzieae.

4157. S warzi a. Schreb.

Tounatea. Aubl.

Gynanthistrophe. Poit. msc.

4158. Zollernia. Mart.
4159. Baphia. Afzez. 4160. Possira. Aubl.

Rittera. Schreb.

Hoelzelia. Neck.

C. Mimoseac genuinae.

4161. Entada. Ad.

Gigalobium. P. Br. 
4162. Mimosa. An.

a. Agne. $\mathbf{R}$. Eumimosa. DC.

b. Habbasia. DC.

c. Cathara. R. Batuucaulon. DC.

4163. Gagnebina. Nrck.

a. Parkia. RBr.

b. Erythrophlaeum. RBr.

4164. Iuga. Prum. Amosa. Neck. 4165. Schrankia. W. 4166. Darlingtonia. DC.
416\%. Des manthus. W.

a. Neptunia. Lour.

b. Desmanthea. DC.

c. Dichrostachys. DC.

1168. Adenanthera. L.

$?$ Clypearia. Rmph.

4169. Dim or phandra.Schotr.

41\%0. Prosop is. L.

a. Adenopis. DC.

b. Algarobia. DC.

4171. Lagonychium. MB. 4172. Acacia. Neck. 


\section{ORDO II. \\ $\begin{array}{llllllll}C & \mathbf{O} & \mathbf{N} & \mathbf{F} & \mathbf{I} & \mathbf{N} & \mathbf{E} & \mathbf{S} \text {. }\end{array}$ \\ AEHNLICHBLUETIGE.}

FORMATIO 1.

\section{SE D I E L O R A E. SEDUMBLUETHIGE.}

FAMILIA XCIII.

CORNICULATAE. Gehörnterüchtige.

A. Crassulaceac.

a. Se de a e.

4173. Tilla eа. Мrсн.

4174. Bulliarda. DeC.

4175. Dasystemon. DeC.

4176. Septas. L.

417\%. Crassula. L.

Gomara. Ad.

a. Crassula. Haw.

b. Turgosea. HIaw.

4178. Globulea. Haw.

41\%9. Cyrtogyne. Haw.

4180. Grammanthes. DeC.

Vananthes. HI a w.

4181. Rochea. DeC.

\section{Larochea. $\mathbf{P}$.}

4182. Dietrichia. Tratr.

Kalosanthes. Haw, non Bl a me.

Franciscea. DeC. non Pohl.

Franciscuria. DeC.

4183. Calanch ö̈. Ad. em. P'.

Kalanchoë. Ad.

Kalenchoё. Ha w.

Vereia. Andr.

Verea. W.
4184. Bryophyllum. SALisB.

Crassouvia. C o mmers. Physocalycium. V est. 4185. Coty ledon. L. 4186. P is torin ia. DeC. 418\%. Umbilicus. DeC.

a. Rosularia. Dr.

b. Mucizonia. Ort.

c. Cotyle. DC.

d. Orostachys. Fisch. 4188. Echeveria. Del. 4189. S ed u m. L. Anacampseros. T. a. Rhodiola. $\mathbf{L}$.

b. Sedum. L.

4190. Sempervivum. L.

a. Monanthes. Haw.

b. Jovibarba. DC.

c. Chrouobium. DC.

4191? Goodallia. Bown. 4192? Cephalotus. LaB. 4192b? Frankoa. CAv.

Panke. Mol. 


\section{b. Penthoreac.}

4193. Diamorpha. Nutr. 4194. Penthorum. D.

$$
\text { B. Saxifrageac. }
$$

a. Genuinae.

4195. Adoxa. L.

Moschatellina. T.

4196. Donatia. Forst.

419\%. Chrysosplenium. L. 4198. H e u c h e ra. L. 4199. Astilbe. Hamint. 4200. Tiarella. L. 4201. Mitella. L. 4202. Tellima. RBr.
4203. Saxifraga. L.

a. Tridactylites.

b. Saxifragaria. (Aizoon.)

c. Leiogyne. Don.

d. Robertsonia. Haw. Gymnopera. Don.

c. Diptera. Brkh.

$f$. Micranthes. Haw. 4204. Bergenia. MNCH.

Geryonia. S c h r k, non al.

\section{b. Cunoniaçe $a$.}

4205? Codia. Forst. 4213 ? Plectronia. I. 4206. Callicoma. Rhr. -4214. Cyrilla. L. 420\%? Ceratop etal um. Sm. 4215. It ea. L. 4208? B uma ld a. Thunb. 4209. Bauera. ANDr. 4210. Weinmannia L. 4211. Cunonia. L. Osterdyckia. Burm.

4212. Vahlia. Thnв. Diconangia. Ad. 4216. Hydrangea. L. a. IIydrangea. Lam. b. Hortensia. Lam. 421\%. Forgesia. Juss. Defforgia. Poir.

$$
\text { c. } P \text { t } i l a d e l p l e a e \text {. }
$$

4218? Deutzia. Thnb. 42 19. Philadelphus. L. Syringa. T.
4220. Decumaria. L.

Forsythia. W a 1 t. non V.

\section{Bruniaceae.}

4221. Berzelia. Bangn. non 4223. Raspailia. Brgn. Makt: 4224. Staavia. This. 4222. Brunia. L. 4225. B e rardia. BRngN non Vill. 
160 CLASS. VII. CALYC. ORD. II. CONFIN. FAMT. XCV. RIBESIAC.

4225b L o n c hos to m a.

Wickstr. deleas 3000 .

4226. Linconia. L.

4227. Andouinia. Brgn.

4228. Mösslera. Rснв.
Tittmannia. B r g n. non

Rch b. 1824.

4229. Thamnea. Soland.

4230. Poranthera. Rudg.

FAMILIA XCIV.

LOASACEAE. LoAsAceen.

a. Lo a sea $e$.

4231. Gronovia. L.*)

4233. Bl um en bachia.Schrad.

4232. Loasa. Ad.

a. Ortign. Feuill. 4234. Bartonia. Sims.

b. Helicteroides. DC.

4235 Mentzelia. Plum.

4236. Klaprothia. K. H. B.

b. $T u r n \in r e a e$.

523\%. Turnera. Puum.

4238. Piriqueta. Aubl.
Burghartia. Neck.

Burcardia. Scop.

c. $F \circ u g u$ i $е$ rea $e$.

4239. Fouquiera. К. H. B. 4240. Bronnia. К. H. В.

FAMILIA XCV.

RIBESIACEAE. Ribesiaceen.

a. Cactea $e$.

4241. Mamillaria. Haw.

Cereaster. DC.

4242. Melocactus. C. BaUH. 4245. Opuntia. T.

4243. Echinocactus. SALM. D.

Tuna. Dill.

4244. Cactus. L. e. e.

4246. Pereskia. Puum.

a. Epiphyllum. Herm.

Phyllanthus. N eck.

b. Cereus. J.

4247. Rhipsalis. G̈̈RT.

Hariota. Ad.

*) Q, po loco genuino iam ante trienninm ennmeravi, serius singularem cotyledorum sitnm infractum descripsi, nbi Blumenbachiae germinationem illnstravi : Hort. Bot. Centur. Il. p. 121. 


\section{b. Grosec $\operatorname{lar} i e a c$.}

4247. Ribes. L.

a. Robsonia. Berland.

b. Grossularia. T.

c. Ribesia. Berland.
Ribes et

Botryocarpum. Rich.

d. Symphocalyx. Berld.

c. Escalloniea .

4248. Aristotelia l'Henrt. 4249. Escallonia. Mur. non Ad. nec L o u r. Stereoxylon. Rz. Pav. 4250. Anopterus, LaB.

\section{FORMATIO II. \\ $\begin{array}{lllllllllll}\text { R } & \mathbf{O} & \mathbf{S} & \mathbf{I} & \mathbf{F} & \mathbf{L} & \mathbf{O} & \mathbf{R} & \mathbf{A} & \mathbf{E} \text {. }\end{array}$} ROSENBLUETHIGE.

FAMILIA XCVI. portulacaceae. Portulacaceen.

A. Par o ny $c$ hi e a $e$. a. Sclerantheat.

4251? Litophila. Sw. 4252. Loefflingia. L. 4253. Minuarta. LöfrL. 4254. Queria. IöfFL.
4255. Mniarum. Fonsk.

Ditoca. Banks. G. 4256. Scleranthus. L. 425\%. Guilleminia. K. H. B.

$$
\text { b. Polycarpeae. }
$$

4258. Pollichia. Sox. non al. Neckeria. Gm. non al. Meerburgia. Mn ch.

4259. Ortegia. Löfru. Orlega. L. Juncaria. Clus. 4260. Poly carpon. Löfft. Trichlis. H a 11.
Anthyllis. e. e. Ad. 4261. Ca rdia. Moc. Sess. 4262. Stipulicida. Mchx. 4263. Polycarpaea. Lam. Hagaca. V ent. Mollia. W. non M a r t: Lahaya. R. S. Hyala. l'Her. msc. Authyllis. e. e. Ad. 
? Polia. Lour.

4264. Cardionema. DC.

Bivonaea. Fl. mex. non DC. nec Spr.

4265. Paronychia. T. J.

$a$ ? Acanthonychia. DC.

b. Eunychia. DC.

c. Chaetonychia. DC.
4266. Hllecebrum. L. e. e. 426\%. Anychia. Mchx.

Queria. G.

4268. G y mn ocarpum. Forsk. 4269. Herniaria. T. L.

4270? Sellowia. Rth.

Winterlia. Spr.

42\%1? P o ly ch r o a. Lour.

c. Spergu? e a e.

4272. Larbrea. A. St. Hil. 42\%6. Spergula. I. 42\%3. Drymaria. W. 4274. Holosteum. L. 42\%5. Spergularia. P. 427\%. Mollugo. L. 4278. Pharnaceum. L. Stipularia. Haw. non P. B. 4280 ? Ph y s. A. P. Th. Lepigonum. Fr.

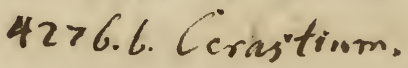

B. Pol y g

a. Genuinae.

4281. Koenigia. L.

4282. Oxygonum. Burch. 4283. Calligonum. L.

4284. Ox y ria. Hrla.

4285 Emex. Neck.

4286. Rumex. L.

a. Acetosa. T.

b. Lapathum. T. 428\%. Th tum. L. 4288. Polygonella. Mchx. 4289. Atraphaxis. L.
4290. Tragopyrum. M. B. 4291. Poplygon um. L. . $\left.{ }^{*}\right)$

a. Centinorlia. J. Bauh. Avicularia. Meisn. non

\section{Gresn.}

b. Amblyogonon. Meisn.

c. Bistorta.. T.

d. Aconogonum. Meisn.

e. Persicaria. T.

f. Tiniaria. Meisn.

- g. Fagopyrum. T.

\section{b. Begoniaceac.}

4293. Begonia. L.

*) Subgenera recepi inncta; sed quam multa Cruciferarum jugeremus e principiis iisdem! 
CLASS. VII. CALYC.ORD. II. CONFIN. FAM. XCVI. PORTUL. 162

$$
\text { c. Cac collob e a e. }
$$

4294. Podopteris. Hв. BpL. 429\%. Kydia. Roxb.

4295. Brünnichia G̈̈вт 4298. Blochmannia. WeIG. 4296. Coccoloba. L. 4299. Triplaris. $L$.

$$
\text { C. } P \cap r t u l a c e a c \text {. }
$$$$
\text { a. Tel e } p \text { hi e a e. }
$$

4300. Corrigiola. L. 4301. Telephium. T. L.

b. Montiniene.

4302. Montinia. L. 4303. Hаиуа. Moc. Sess.

$$
\text { c. } T a l i n e a e \text {. }
$$

4304. Leptrina. Rafin. 4305 . Mhantia, Le .... 4306. Crypta. Nutr. Cryptina. Rafin. 430\%. Claytonia. L. Limnia. $\mathbf{L}$. 4308. U 11 î c us. Loz.

4309. Aylmeria. Mant. 4310. Trianthema. SAUv. Zalega. B u r m. Rocama. Forsk. Papularia. Forsk.

4311. Cy pselea. Turp. Ractiana. Rafin.

4312. Portnlaca. T. L. Meridiana. $\mathbf{L}$.

Lemia. $\mathbf{V}$ aud. IIerida. Neck.

4.312.6. Thelyggenem K

FAMILIA XCVII.

A I Z O I D E A E. A I ZOIDEEN.

A. $l t r i p l i c e$ a $c$.

a. Che n o pode $e$ e.

4319. Salicornia. L. 4 4321. Caroxylon. Thne. 4320. Halocnemon. M. B. 4322. Anabasis. L. 
4323. Salsola. L. 4324. Corispermum. $L$. 4325. Poly cnemum. L. 4326. Camphorosina. L. Camphorata. T.

432\%. Halimocnemis. Led. 4328. Traga n um. DeL. 4329. Cornulaca. Dez. 4330. Sclerolaena. RBr. 4331. Anisacantha. $\mathrm{RBr}$. 4332. Pteranthus. Forsk. 4333. Ceratocarpus. L. 4334. Ha limus. Wallk. 4335. Diotis. SCHRÉr. 4336? Dysphania. RBr. 433\%. H e m ich roa. RBr. 4338. Kochia. Rтн. $/ 6$ / 4339. Suaeda. Forsk. 4340. Schanginia. LED.
4341. Schoberia. LED. 4342. Cochliospermum. LAG. 4343. Chenopodium. L. a. Orthospermum. RBr.

b. Chenopodium. 4344. Atriplex. L.

a. Atriplexum.

b. Obione. G.
4345. B lit um. L. ? 4346. Threlkeldia. RBr. 434\%. Crucita. LöffL. 4348. Axy ris. L. I, 4349. Acnida. L. 4350. Spinacia. L. 4351. B e ta. L. 4352. Rhagodia. RBr. 4353. Enchylaena. RBr.

\section{b. $A m a r a n t h e a c$. a. Gonphrencac.}

4354. T rom m s dorffi a. 4362. Cladostachys. Don.

4355. Iresine. W.

4356. Ros ea. MArt. 435\%. Philoxerus. RBr. 4358. Heb anthe. Mart. 4359. Gomphrena. L.

Schultesia. S chrad.

4360. Hoplotheca. Nutr. em?

Oplotheca. Nut t.

Frölichia. Mnch.

4361. Les tiboudesia. A. 43\%. Digera. Forsk. 436\%. Am a r anthus. L. 4368. Cham is soa. K. H. B. 4363. Celosia. L. 4364. Hermbstaed ti a. Rchb. Berzelic. MI a r tonon Brngn. 4365. Aërva. Forsk.

4366. Acroglochin. Schind. Lecanocarpus. N. v. E. Blitanthus. Rchb. 4369. Charpentiera. Gaudich. P. Th. 43\%1. Deeringia. $\mathrm{RBr}$.

\section{B. Achyrantheat.}

4372. Pupalia. Juss. 4374. Ach y ant hes. L. 4373. Desmochaeta. DeC. 4375. Nyssanthes. RBr. 
4376. Ptilotus. RBr. 4380. Brandesia. Mart. 43\%. Trichinium. RBr. 4381. Mogiphanes. MART. 43\%8. Alternanthera. Fonsk. 4383. Serturnera. Mant. 4379. Bucholzia. Mart. 4384? C y a thula. Lour.

$$
\text { भ. Petiverieac. }
$$

4385. Petiveria. L.

$$
\begin{aligned}
& \text { c. Phytolaccea } \\
& \text { a. Basellea }
\end{aligned}
$$

4386. Cuscut a. L. 438\%. Hablizia. MB. 4388. Anredera. Juss.
4389. Basella. L. 4390. Boussing aultia. K.

$$
\text { B. } R i v i n \in a e \text {. }
$$

4391. Microtea. Sw. 4394. B o s ea. I. 4392. Ancistrocarpus, H. B. Polamophila. Schrk. H. B. 4382. Pfaffia. Mart.

K.


4404. Miltus, Lour.

4405. Aizoon. L.

Vestingia. F a b r.

Ficoidea. Dill.

4406. S esuvium. L.

440\%. Tetragonia. L. a. Tetragonioides. DC.

b. Tetragonocarpis. Comm. 4408. Mesembryanthemum.

L.

Hymenogyne. II a w.

Ficoides. T.

\section{c. $\quad N$ e $u r a d e a c$.}

4409. Neurada. B. Juss. 4410. Grielum. L.

$$
\text { C. Tra ma } \boldsymbol{r} \text { i s c } \boldsymbol{e} \text { a e. }
$$

a. Nitrarieac.

4411. Adenogramma.Rchb. *) 4412. Nitraria. Scнов.

b. $T a m a r i s c e a c \quad g e n u i n a e$.
4413. T a m a rix. L.
c. Polyadenia. Ehrib.
a. Oligarlenia. Ehrnb. 4414. Myricaria. Desr.
b. Decadeuia. Ehrnb.

$$
\text { c. } R \text { e } a \text { u } m u r i e a c \text {. }
$$

4415. Holol achne. Ehrna. 4416. Reaumuria. Hassazequ. - 6. Theleggonem.

FAMILIA XCVIII.

\section{ROSACEAE. ROSAGE N.}

a. Cliffortie a e.

441\%. Chrys ospermum. 4419. Galopina. Tren.

Rснв. -4420. Cliff ortia. L. ${ }^{* *}$ )

4418. Anthospermum. L. a. Nenax. G.

") Cum descriptione analysique fnit edita plantnla elegans iam Febrnario mense a 1827. in Hort. bot. Cent. II, 109.

**) Inter species plnrimas auctornn, etian novas quasdan possideo, mirnm in modun e habitu Anthospermi in illum Fothergillae transientes, qnasdam forsan pro Anthospermis falso descriptas; C. betulifolia Schollii (cui Schollia dicata) folia fere Alni refert. 

b. MTorilandia. Neck.
4421. Xanthosia. Rudg. *)
c. Cliffortia. Neck.

b. $\boldsymbol{R} \circ \boldsymbol{s} e \boldsymbol{e} e$.

4422. Poterium: L. Pimpinella. Ad.

a. Leiopoterium. DC.

b. Rutidopoterium. DC. 4423. S angu is orba. L. sulb Pimpinella. T.

4424. Acaen a. V.

a. Acaena. L.

b. Ancistrum. Forst. 4425. Polylepis. Rz. Pat. 4426. Margyricarpus. Rz. 4431. Agrimonia. L.

442\%. Alchimilla. T. a. Aphanes. L.

b. Alchemilla. L. 4428. Cercocarpus, K. H. B. Bertolonia, MI o c. S e s s.

4429. Brayera. KNтr. 4430. Aremonia. Neck. Agrimonioides. T. Amonia. N e stl. Spallanzania. Poll.
4432. Rosa. L.

$$
\text { c. } \quad P \quad o \quad m \quad a r c c c c .
$$

\section{a. Potentillea}

4433. Sibbaldia. L. 4434. II o r kel i a. Cham.

4435. T ormentilla. L. 4436. Potentilla. L.

a. Fragariastrum. Ehrh.

b. Potentillastrum. Ser.

c. Trichothalamus. Lehm.

443\%. Com a r un. L. 4438. Fragaria. T, L.

a. Fragaria. Sm. 4442. Waldsteiuia. W. 4443. Si eversia. Wrudd. 4444. Bieb erst e in ia. Steph. 4445. Ge u m. L.

a. Buchhavea. R. Adamsia. Fisch. non M. B. Laxmannia. Fi s ch, non $\mathbf{R B r}$. Oreogeum et Sticlogeum voc. hybr. S er.

b. Caryophyllata. T.

c. Caryophyllastrum. S.

b. Duchesnea. Sm. non Cass. 4439. Dalibarda. L. 4440. C ow a n i a. Don. 4441. Comaropsis. Rich.

$$
\text { b. } S p \text { ir } r \text { e e } u e \text {. }
$$
44

a. Ulmaria.
a.

b. Aruncus.

c. Sorbaria. S.

*) Analysis a me instituta, figuram Rndgeanam esse malam docuit. 
168 CLASS, VII. CALYC. ORD. II. CONFIN, FAM. XCVIII. ROSAC.

d. Spiraria. 8. et Chamaedryon. $\mathrm{S}$.

e. Physocarpus. Camb. 4450. Kerria. DC. 4451. Gillenia. Mrch. 4452? Dicoryphe. 4453. Neillia. Dos. 4454. Purshia. DeC.

$$
\text { c. Pomaсва ве ge in a e. }
$$

4460. Crat a egus. L. 4461. Raphiolepis. Livdr. 4462. Ch amaemeles. Lindi. 4463. Photinia. Lindu. 4464. Eriobotrya Lindu. 4465. Cotoneaster. MEDIK. 4466? G la phyria. JАCK. 446\%. Amelanchier. MEdik. 4468. MI e s pilus. L. Mespilophora. N eck.
Tigarea. Prsh, non A ubl. Kunzia. S p r. 4455. K a gen eckia. Rz. PAv. 4456. Quillaja. J. Smegmadermos. Rz. Pav. 445\%. V a uquelinia. Corr. 4458. Lindley a. K. H. B. 4459? Euphronia. MLart.

4469. Os te omeles. Lindu. 44\%0. Pyrus. L.

a. Chamaemespilns.

b. Aronia. P. e. e.

c. Sorbus. $\mathbf{L}$.

d. Torminaria, DC.

e. Aria.

f. Malus. T.

g. Pyrophorum. Neck. 4471. Cydonia. T.

d. Rosaceae non satis cognitue.

4472. Amoreuxia. Moc. Sess. 4474. Trilepisium. A. P. Th. 4473. Lecostomon. Moc. 4475? Dichroa. Lour. Sess. 4476? Phoberos. Lour. 
ORDO III.

\section{$\begin{array}{lllllllllll}C & \mathbf{O} & \mathbf{N} & \mathbf{C} & \mathbf{I} & \mathbf{N} & \mathbf{N} & \text { A } & \text { E. }\end{array}$ GLEICHFOERMIGE.}

\section{FORMATIO $\mathbf{I}$. \\ $\begin{array}{llllllllllll} & \mathbf{N} & \mathbf{A} & \mathrm{G} & \boldsymbol{R} & \mathbf{I} & \mathbf{F} & \mathbf{L} & \mathbf{O} & \boldsymbol{R} & \mathbf{A} & \mathbf{E}\end{array}$ NACHTKERZENBLUETHIGE.}

FAMILIA XCIX.

halorageaE. Halorageen.

a. $\left.H y d r \quad \circ \quad b i a e^{*}\right)$.

447\%. Myriophyllum. VArlx. 4478. Proserpinaca. I. Pentapterophyllum. Dill. Trixis. Mitch. Gärt.

a. Pentapteris. Hall.

6. Ptilophyllum. Nutt.

Purstia. Rafin. non DeC. 4479. Trapa. L. Tribuloides. T.

\section{b. Cercodere.}

4480. Hippuris. L.

Limnopeuce. V aill.

Pinastella. Dill.
Gonatocarpus. W.

a. Apodogynus. DC.

b. Pterogynus. DC.

4481. Serpicula. L. non al. 4483. Meionectes. RBr. Laurembergia. B erg. 4484. Ha lo ragis. Forst. 4482. Goni ocarpus. Kön. 4485. Ce r codia. Murr. Gonocurpus. Thnb. Cercodea. La m.

$$
\text { c. Datiscea } e \text {. }
$$

4486. Tetrameles. RBr. 448\%. Datisca. L.

EAMILIA C.

,ONAGREAE. NACHTKERZEN.

$$
\text { A. Jus } \boldsymbol{J} \boldsymbol{e} u \boldsymbol{e} \text { a } \mathrm{e} \text {. }
$$

4488. Callitriche. L. Stellaria. Dill.

*) Hygrobiac iam Zoologis solemnes. 
4490. Isnardi a. L.

Dantia. A. P. Tli.
Jussieua. P.

4491. Ludwigia. L.

Ludw. et Isu. Ludwigiaria. DC. 4492. Prieurea. DeC. 4493. Jussiaea. L.
4494. Fuchsia. Plum. L.

a. Quelusia. Vaud. Dorvalia. Com mers. Nahusia. Schneev.

๖. Skinnera. Forst.

\section{B. Gaureac.}

4495. P leurostemon. Rafin. 4499. Clarkia, Prsh. Pleurandra. Rafin. 4500. Epilobium. L.

4496. Camiss onia. Lr. a. Chamaenerioll. T.

b. Lysimachion. Tsch. Ser.

\section{Oenoth. sect. Sphaerostigma.}

449\%. Oenothera. L.

a. Onagra. T. e. e.

b. Oenotherium. Ser. 4498. Onosuris. Rafin. **) 4504? C areya. Rxb.
Commers.

4502? Foetidia. 4503? Grias. L.

$$
\begin{aligned}
& \text { C. M } y x \circ b \text { a } l \text { a ne } a \text { e. } \\
& \text { a. Vochysie ce. }
\end{aligned}
$$

4505. Lopezia. Cav.

Pisaura. Bon at.
4510. Q u al ea. Aubr.

4511. Circaea. L.

4506. Callisthene. Mart. 4512? Rumphia. L.

450\%. Amphilochia. Mart. 4513. Erisma. Rudg.

4508. Vochysia. Juss.

Debraea. Ti. S.

Vochy. Aubl.

Vochya. V and.

Salmonia. Neck.

Cucullaria. Schreb.

4509. Salvertia. A. St. H.
4514? Lozania. Sen. Mauk. 4515? Agardhia. Spr. non Cabr. nec Grax. 4516? Schweiggeria. Spk.

-) Falso hnc ducitur in DeC. prodr. III. p. 64. genus Camissoniae Lk. quod ex Oenoth. dentata Rz. PAv. ab ill. anctore constitutum est, ut supra diximus. Pnod reliquum est, cl. Adurs. a Cuasisso praeter Chamissoam etian Cammissonia dignissimus! 


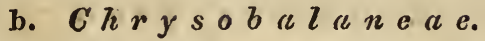

451\%. Stylobasium. Desp. 4518. Hirtella. L.

Causea. S cop.

Cosmibuena. Rz. Pav, prod. 4519. Thelyra. A. P. Th. 45\%0. Grangeria. Comm. 4521. Parinariu m. Juss. Parinari. A u bl. Dugortia. Neck. Petrocarya. Schreb. 4522. Acioa. Aubl.
Acics. Schreb.

4523. C o u e i ia. Aubr.

4524. Moquil ea. Aubr. 4525. Chrysobalaus. L. Icaco. Plum.

4526. Alangium. Lam. Angolam. Ad. Angolamia. Sc o p. 4527? Petal ot oma. DC. Diatoma. L o u r. non DC.

$$
\text { c. Combretuceae. }
$$$$
\text { c. Combreteate. }
$$

4528. Qu isqualis. Rмph. 4530. Combretum. Lörfr. 4529. Lumnitzera. Willd. 4531. Cacoucia. Aubl.

$$
\text { non JACQ. *) Schousbocu. W. }
$$

$$
\text { B. Terminalieal. }
$$

4532. Ceratostachys. Buvme. 4540. Chuncoa. Pav.

4533. Poivrea. Commers.

Cristuria. S o n.

G'onocarpus. II a mil t.

4534. Guiera. Juss.

4535 ? B о bu a. DeC.

Bobul. Ad.

Eugenioides. L.

4536? Bruguiera. A. P. Th. non Lam.

453\%. Laguncularia. Gärt. Sphenocarpus. Rich.

4538. Conocarpus, GärT.

Rudbeckia. Ad.

a. Anogeissus. DC.

b. Leiocarpus. DC.

c. Euconocarpus. DC.

4539. R amatuell a. K. H. B.
Gimbernatia. Rz. Pav.

4541. Getonia. Roxi. Culycopteris. Lam. 4542. Pentaptera. Roxb. 4543. Terminalia. L.

a. Catappa. G.

b. Myrobalanus. Lam. II. et Buclamia. G.

4544. Ag a this an the s. Buume.

4545. Bucida. L.

Buceras. P. Br.

Hudsonia. Ro b ins.

4546? Coupoui. Aubr.

4546b. Dirca. L.

4546c. Lagetta. Juss.

4546d. Causjera. Juss.

*) Lumnitzcram Jaç. ex errore supra no. 2S09. enumeratam Moschosma dicamus. 
172 CLASS, VII. CALYC, ORD.III. CONCIN. FAM. CI. LYTHRAR.

$$
\text { y. Gyrocarpeae. }
$$

454\%. Gyrocarpus. JACQ.

\section{FAMILIA CI.}

LYTHRARIAE. WEI DRTHE.

A. Hydropityeae.

4548. Hydropityon. Gärt. 4549. Honottia. Rchв.

Hottonia. Burm. non Boerh.

$$
\text { B. } \quad \begin{array}{llllllll}
\boldsymbol{L} & y & t & h & \boldsymbol{r} & \boldsymbol{e} & \boldsymbol{a} & \boldsymbol{e} \text {. }
\end{array}
$$

a. $S a l i c a r i e a c$.

4550. Suffren ia. Bell.

4551. Ameletia. DeC.

4552. Rotala. L.

4553. Elatine. L.

4554. Bergia. L.

4555. Peplis. L.

4556. Amm annia. Houst.

Cornetia. II a d.

455\%. Cryp totheca.

4558. L y th r u m. L.

a. Ammannioides. DC.

b. Hyssopifolia. C. B. Pythagorea. Rafin.

c. Salicaria. T.

4559. Cuphea. JAcQ.

Bankisia. $\mathrm{D}$ omb. non $\mathrm{L}$.

Melvilla. Anders.

Balsamona. V and.

a. Melanium. P. Br.

Parsonsia. P. Br.

b. Cuphea. P. Br.
4560. Acisanthera. P. Br. 4561. Fatioa. DeC. 4562. P e mphis. Fonst. 4563. Heimia. Lк. et Oтто. Chrysolyga. If ffgg. 4564. Dipl us o d o n. Poнt. Friedlandia. Cham. 4565. Phy soc aly mma. Poнг. 4566. D ecodon. Gm. Budme. 456\%. N e s a ea. Commers. 4568. Crene a. Aubr. 4569. Laws onia. L. 45\%0. Antherilium. Rohr. 4571. Dodecas. L. 4572. Ginoria. JAcQ. Ginora. $\mathbf{L}$. Gienoria. $\mathbf{P}$. 45\%3. Ad enaria. K. H, B. 4574. Grislea. LöfrL. 4575? Phy s opodi um. Desv. 4576? Sy mmetria. Buume.

b. Lagerstroemieae. 457\%. Lagerstroemia. L. a. Sibia. DC.

-) Buraranvi planta non specie tantum a praecedente, sed ino genere distincta: ,capsula subrotunda, unilocrlaris, semina condens plurima."6 
b. Münchhansia. L.

Banava. C a m.

c. Adambea, Lam.
4578. Lafoense a. VAND.

Calyplectus. Rz. Pav.

\section{c. $G r a n a t e a e$.}

4579. Punica. L.

\section{Melastomeac.}

a. Me lastomeac cochliosperma e.

$$
\text { a. } R h \text { e } x \text { i e a e. }
$$

4580. Appendicularia. DeC. 4588. Pachyloma. DeC.

4581. Comolia. DeC.

4582. Spennera. Mart.

4583. M i crolicia. Don.

4584. Ernestia. DeC.

4585. Siphanthera. Ponz. 4586. Rhexia. P. Br. L. 458\%. Heteronema. DeC.
4589. Oxyspora. DeC. 4590. Marcetia. DeC. 4591 Trembleya. DeC. a. Jacobia. DC.

b. Abrahamia. DC.

c. Erioleuca. DC.

4592. Adelobotrys. DeC.

\section{B. Osbeckieac.}

\section{L a s i andra. DeC.}

4594. Chatogastra. DeC.

a. Monacentra. DC.

b. Diotauthera. DC.

c. Bractearia. DC.

4595. Arthrostemma. Pav. IIelanium. Rich. ined.

a. Chaetopetalum. DC.

b. Brachyotum. DC.

c. Ladanopsis. DC.

d. Trifurcarium. DC.

e. Monochaetum. DC. a. Microlepis. DC.

b. Chaetolepis. DC.

c. Pterolepis. DC.

d. Osbeckiaria. DC.

459\%. Tibouchina. Auru. Savastenia. Neck. 4598. T rist e $\mathrm{m} \mathrm{m}$ a. Juss. 4599. Melastoma. Burm L. 4600. Pleroma. Don. e. e. 4601. Diplostegium. Don. 4602. Aciotis. Don. 4596. O s b e ckia. L.

b. I e lastomeacoospermae.

$$
\text { a. Merianie a } e_{\text {. }}
$$

4603. Meriania. Sw. 4604. Axinaea. Rz. Pav. Wrigtia. Sol. msc. non Br. 4605. Chastenaea. DeC. 
4606. Lavoisiera. DeC. 460\%. Davya. DeC. 4608. Graffenriedera. DeC. 4609. Centronia. Don. 4610. Truncaria. DeC. 4611. Rhynchanthera. DeC. Proboscidia. Rich. 4612. Macairea. DeC.
4613. Bucquetia. DeC. 4614. Cambessedesia. DeC. 4615. Chaetostem ma. DeC. 4616. Salpinga. Mart. Aulacidium. Rich. 461\%. Bertolonia. Radd. non al. Triblemma, RBr. 1618. Meisnera. DeC.

\section{B. Bl $\left.\quad k \in a \in e a c{ }^{*}\right)$}

4619. Rous seauxia. DeC. 4620. Le andra. RAdD. Bertolonia. S p r.

a. Leandraria. DC.

b. Leandroides. DC. 4621. Ts ch ud y a. DeC. 4622. Clidemia. Don. 4623. Myriaspora. DeC. 4624. Tococa. Aubr. 4625. Maieta. Aubr. 4626. Calophysa. DeC. 462\%. M e d in ell a. Gaudicr. 4628. Hub exia. DeC. 4629. Calycopteris. Rich. non LAM.

Calycogonium. DeC. 4630. Ossaea. DeC. 4631. Sagraea. DeC. 4632. Tetrazygia. Rich. 4633. Heterotrichum. DeC. 4634. Conostegia. Dox. Calycolomus. et Buquiera. Rich.

a. Eriostegia. DC.

b. Euconostegia. DC. 4635. Diplochita. DeC. Clitoniu. Don, non MI oc. Fothergilla. Aubl. non $\mathbf{L}$. 4636. Phyllopus. DeC. 463\%. Henrietia. DeC. 4638. Lo re ya. DeC. 4639. Miconia. Rz. Par.

a. Leiosphaera. DC.

b. Eriosphaera, DC. Hypoxanthus. Rich, hb. c. Eumiconia. DC. 4640. Oxymeris. DeC. 4641. Crem a ni um. Don. 4642. Blakea. L. Topobea. A ubl. Vuldesia. Rz. Pav. Bellucia. Neck. Drepancondrum. Neck. 4643. Sarcopyram is. WALL. 4644? Sonerila. Roxb.

\section{c. $C \hbar \pi r i a n t h e a e$.}

4645. Kibessia. DeC. 4646. Charianthus. Don.

\footnotetext{
*) Divisiones ab antiquo genere semper nominandas esse pntavi.
} 
Chacnanthera. Rich. hb. 4648. Astronia. Blume. 4647. Chatnopleura. Rich.

FORMATIO II.

\section{$\begin{array}{lllllllllll}M & \mathbf{Y} & \mathbf{R} & \mathbf{T} & \mathbf{I} & \mathbf{F} & \mathbf{L} & \mathbf{O} & \mathbf{R} & \mathbf{A} & \mathbf{E} .\end{array}$ MYRTENBLUETHIGE.}

FAMILIA CII.

MELAleuceae. Melaleuceen.

a. Is ecythidere.

4649. L e cythis. LöffL. 4650. Eschweilera. Mant. 4651. Bertholletia. HB. BPL.

4652. Couroupita. Aubl.

b. $B a r r i n g t o n i e a c$.

4654. Barringtonia. Fonst.

Butonica., L a m.

Commersona. Sonn.

Hultum. Ad.

Mitraria. Gm.

4655. Strav adium. Juss.
Pontoppidana. Scop. Elshollzia. Rich. non W. 4653. Couratari. Adbl. Lccythopsis. Schrk. Curupita. Gm.

$$
\text { c. Calothamneae. }
$$

465\%. Calothamnus. LaB.

a. Calothamnus. LaB.

b. Billotia. Colla.

Baudinia. Les ch.

c. Pextaphalanx. Rchb. 4658. La a m a rkea. Gaudich.
Stravadia. $\mathbf{P}$. Meteorns. L $\mathbf{0}$ u $\mathbf{r}$. Merichea. Lour. 4656. Gustavia. L. Pirigara. A ubl. Spallanzania. Neck.

*) Metrosid. capitata $S_{M}$. ericifolia $S_{\mathbf{M r}}$, et corifolia Vent, fortasse etiam M. ciliata $S_{M}$. a veris generis antiqui speciebus vel stigmate capitato vel habiln toto distinctie, iam ex influrescentia hac nec ad Leptospermeas referendae. Kunzeo Lipsiensi praeclarissimo amico dicatae. 
a. Chamaelaucieae.

4662. Pileanthus. LaB. 4666. Calythrix. LaB. 4663. Genetyllis. DeC. 4664. Ch a m a el a u ci u m.

DESF. e. e. 4665. Verticordia. DeC.

466\%. Eudesmia. RBr. 4668. Tristania. RBr.

\section{b. L e p tospermeat.}

4669. Bartlingia. Brngn. 46\%. B a e kea. L.

B. et Jungia. G.

B. et Imbricaria. $\mathbf{S} \mathrm{m}$. Mollia. G m. a. Euleptospermum. DC.

b. Agonis. DC. 4672. Fabricia. G̈̈нт. 46\%3.MeŁrosideros. Gäнт.e.e. 4674. Angophora, C.Ar.

46\%1. Leptospermum. Forst. 4675. Eu c a l y p t s l'Herit.

$$
\text { c. } M y r t e a e \text {. }
$$

46\%6. Caly ptrantes. Sw. 4683 ? Catinga. AubL.

Chytralia. Ad.

Chytraizlia. P. Br.

Suzygizum. P. Br.

Calyptranthus. Jus s. non A. P. Th, nec Blu me.

46\%\%. S y z y i um. G. Opa. L our.

4678. Caryop hyllus. T. L. 46\%9. Acmena. DeC. 4680? Crossostylis. Forst. 4681. Eugenia. Mrch.

plinia. L. $\mathbf{f}$

Greggia. G.

Olynthia. Lindl.

Guapurium. Juss.

4682. J a m b o s a. RMPн.

Iambos. $\mathbf{A}$.
4684. Myrcia. DeC. 4685. Myrtus. L.

a. Leucomyrtus. DC.

b. Rhodomyrtus. DC. 4686. J o s si inia. Commers. 468\%. Psidium. L.

Guariava. T. Ps. et Burcharda. Neck. 4685? Melan o psidi um. Colla. 4689. Campomanesia. Rz.

Pav.

4690. Nelitris. G̈̈RT. Decaspermum. Forst. 4691. Sonneratia. L. Aubletia. Gärt. non al. 4692? Myrrhinium. Schotт. 
FAMILIA CIF.

\section{AMYGDALACEAE. Amygdalceen.}

$$
\text { a. } S \text { a } m y d e \text { a } e \text {. }
$$

4693. S a m y da. L.

a. Samyda. L.

Eusamyda, DC.

b. Guidonia. Plum.

4694. Chaetocrater. Rz. PAV.

Crateria. P exs.

4695. Casearia JACQ.

a. Sareacia. R.

b. Iroucana. Aubl.
Casearia. J a c q.

Athenaea. Schreb.

c. Anavinga. Lam.

Pitumba. A ub $\mathbf{l}$.

Melistuurum. Forst.

d. Careacia. R.

Lindleya. Knth. non al.

loco.

4696? Crinodendron. Mours.

b. $A c o m e a r$.

469\%. Na pimoga. Aubl. 4698. A c o m a. Ad:

Racoubea. Aubl.

Homalium. J c q. non $Z_{0} 0010$ g 4699. Pineda. Rz. Pav. 4700. Blakwellia. Comm.
4705. Cerasus. Juss.

a. Cerasus. T. Cerasophora. Neck.

b. Laurocerasus. T. Padus. Mill. 4706. Prunus. T.
4701? Asteropeia. A.P. Th. 4702. Astranthus. Lour. non Asteranthus. D esv.

4703. $\mathrm{N}$ is a. A. P. Th. 4704. Myriantheia. A.P.Th.

c. Cerasea e. 



\section{consusts tith \\ THALA MANTHAE. STIELBLUETHIGE. \\ ORDO $\mathrm{I}$. \\ THYLACHOCAR PICAE. HOHLFRUECHTIGE.}

Formatio I.

CR U CIF L O RAE.

KREUZBLÜTHLER.

Fam. CV. Tetradynamae. Viermächtige.

Fann. CVI. Papaveraceae.

Papaveraceen.

Fam. CVII. Capparideae. Capparideen.
Formatio II.

C I S T I F L O R A E. CISTUSBLÜTHLER.

Fam. CVIII. Violaceae. Violaceen.

Fam. CIX. Cistineae. Cistineen.

Fam. CX. Bixaceae. Bixaceen.

\section{ORDO II. \\ SCHIZOCAR PICAE. SPALTFRUECHTIGE.}

Formatio I.

RANUNCULIFLORAE.

RANUNKELBLÜTHLER.

Fam. CXI. Ranunculaceae.

Fam. CXII. Rutaceae. Ranunculaceen. Rutaceen.

Fam. CXIII. Sapindaceae. Sapindaceen.
Formatio II. GERANIIFLORAE. STORCHSCHNABELBLÜTHLER. Fam. CXIV. Malvaceae. Malvaceen.

Fam. CXV. Geraniaceae. Geraniaceen.

Fam. CXVI. Oxalideae. Oxalideen.

\section{OR D O III. \\ I D I O C A R P I C A E. SAEULENFRUECHTIGE.}

Formatio I.

T I L I I L O R A E. LINDENBLÜTTHLER.

Fam. CXVII. Caryophylleae. Caryophylleen.

Fam.'CXVIII. Theaceae. Theaceen. Fan. CXIX. Tiliaceae. Tiliaceen.
Formatio II.

A URANTIIFL ORAE.

ORANGENBLÜTHLER.

Fam. CXX. Hypericineae. Hypericineen.

Fam. CXXI. Guttiferae. Guttiferen.

Fam. CXXII. Hesperideae. Hesperideen.

Vgl. Botanik. S. 485-526. nebst Tabelle. 
Plurima insuper naturae minus occultatae restigia passim impressa manent, observatorem non eludentia, caeteris persequendis ac totius progressioni manifestandae praeprimis utilia.

A. L. de Jussieu. 
CLASSIS VIII.

\title{
THALAMANTHAE.
} STIELBLUETHIGE.

\section{ORDO I. THYLACHOCAR PICAE. HOHLFRUECHTIGE.}

\author{
FORMATIO I.

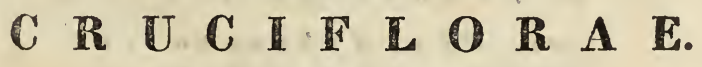 \\ KREUZBLUETHLER.
}

FAMILIA CV.

TETRADYNAMAE. VIERMächtige.

A. $\mathbb{L}$ o $e_{n} \boldsymbol{t} a \boldsymbol{c} e \boldsymbol{a}$.

a. $C$ a $l$ ille $a$ e. $0=$

4710. Euclidium. RBr. 4713. Cakile. T.

4711. Ochthodium. DeC. 4714. Cordylocarpus. Dess. 4712. P ugi o n i m. G̈̈̈r.

b. Anchionea e. 0))

4715. Goldbachia. DeC. 4717. Sterigmostemon.M.B.

4716. Anchionium. DeC. Arthrolobus. Steev. Sterigma. DC.

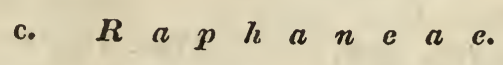

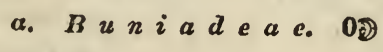

4718. Bunias. L.

Erucago. $\mathbf{T}$.
4719. Laelia. Pers.

4720. Erucaria. Gäht. 


\section{B. Zilleae. $0>>$}

4721. Calepina. And. 4723. Zilla. Forsk. 4722. Muricaria. Dest.

$$
\text { r. } R \text { apistreac. } 0>>
$$

4724. Crambe. T.

a. Sarcocrambe. DC.

b. Leptocrambe. DC.

c. Dendrocrambe. DC.

4725. Rapistrum. BовRH.
4726. Didesinus. Dest. 4727. Enarthrocarpus.

LaB. 4728. Raphanistrum. G̈̈RT. 4729. R aphanus. L.

$$
\begin{aligned}
& \text { B. } \quad \text { p t a t a e. } \\
& \text { AA. Siliculosa e. } \\
& \text { a. Transversiseptae. } \\
& \text { aa. Vix dehiscentes. } \\
& \text { a. Anastaticeae. } 0=
\end{aligned}
$$

4730. Anastatica. L. 4731. Morettia. DeC.

$$
\text { B. Is atidea e. (0)) }
$$

4732. Aphragmus. Andrz.

3. Glastum. Rupp.

4733. Tauscheria. Fisch. 4735. Myagrum. L. e. e. 4734. Is a tis. L. 4736. Sobelewskia. M. B.

c. Sameraria. DC.

$$
\text { y. Velleae. } 0>
$$

4737. Succovia. Med. 4739. Boleum. Desv. 4738. Carrichtera. DeC. 4740. Vell a. L.

$$
\begin{aligned}
& \text { bb. Dehiscentes. } \\
& \text { a. Thlaspidiea e. } 0=
\end{aligned}
$$

4741. Capsella. Vent. Rodschiedia. F1. Wett. 4742. Thlaspi. L.

a. Pachyphragma. DeC.

b. Carpoceros. DC.

c. Nomisma. DC.

d. Nemotropis. DC.

c. Pterotropis. DC.
4743. Teesdalia. RBr.

Hutchius. b. Nasturtiolum. DC.

4744. Iberis. L.

a. Iberidium. DC.

b. Iberidastrum, DC. 4745. Biscutella. L. a. Thlaspidium. Med. 
CLASS. VIII. THALAM. ORD. T. THYLACH. FAM. CV. TETRAD. 183

b. Ioudraba. Med. 4747. Cremol ob us. DeC. 4746. Megacarpaea. DeC. 4748. Menonvillea. DeC.

$$
\text { B. } I \text { epidie a e. 0)) }
$$

4749. Senebiera. Porr.

a. Nasturtiolum. DC.

b. Coronopus, Hall. Curara. Caesalp.

e. Cotyliscus. DC.

4750. Cardaria. Desv. Cardiolepis. Wallr. 4751. L e pidi n m. L.

a. Ellipsaria. DC.

6. Bradypiptou. DC.

c. Cardamon. DC.

d. Lepia. Desv.

Lasioptera. Andrz. e. Senkenbergia, Fl. Wett. Nasturtioides. Med. Dilcptium. Rafin.

f. Piperella, Lob.

g. Iberidella. DC. Noccuea. Mnch.

Hutchinsia. RBr. e. e. non $\mathbf{A g}$.

Hutch. Iberidella. DC. 4752. Bivonaea. DeC. 4753. Eunomia. DeC. 4754. Aethionema. RBr.

$$
\text { y. Ps } y \in k i n \in \text { a e. } 0>>\text { et } 0 \text { S }
$$

4755. Schouwia. DeC. 4757. Brachycarpaea. DeC. 4756. Psychine. DeC.

\section{b. Paralleliseptae.}

aa. Vix dehiscentes.

4758. Clypeola. G̈̈rt.

a. Jonthlaspi. T.

b. Orium. DC. c. Bergeretia. DC. 4759. Peltaria. L.

bb. D e his c e ntes.

a. Alyssinease. $0=$

4760. Subularia. L. (0乞)

4761. Draba. L.

a. Erophila. DC.

b. Drabella. DC.

c. Holarges. DC.

d. Leucodraba, DC.

e. Chrysodraba. DC.

f. Aizopsis. $\mathrm{nC}$. 4762. Petrocallis. RBr. 4763. Cochlearia. T. L. a. Ionopsidinm. DC. Ionopsis. DC.

b. Cochlearia. Lob.

c. Armoracia. Rupp. 4764. Meniocus. Dest. 4765. Alyssum. L.

a. Odontostemon. DC.

b. Adyseton. Scop. 4766. Lobularia. Desv. 476\%. Schiwereckia. Andrz. 
4\%68. Vesicaria. Lam.

a. Alyssoides. DC. Alyss. Anodontea. DC.

b. Vesicaria, Lam. 4769. Aubrietia. Ad. 47\%0. F a rsetia. Turr.

a. Berteroa, DC.

b. Fibigia. Med.

c. Cyclocarpaea. DC. d. Farsetiana, DC. 4791. Ricotia. L. 4772. Savignia. DC, 4793. Kon i ga. RBr. 4774. Lun aria. L. 47\%5. Selenia. Nutr.

4796 ? D is covium. Rapin.

$$
\text { B. Camelineace } 0 \text { ) }
$$

477\%. Neslia. Desv.

Vogelict. F1. Wett. non Lam.

49\%8. Kernera. Med.

4779. Eudema. RBr.

4780. Camelina. Cintz. a. Mönchia. Rth. Chamaelinum. DC.

b. Leiolobium. Rohb. Pseudolizum. DC. 4781. Stenopetalum. RBBr.

$$
\begin{gathered}
\text { BB. Siliquasae. } \\
\text { a. Arabideae. } 0=
\end{gathered}
$$

4782. Macrocerati $\mathrm{um}$. DeC. Notoceras Macrocerat. DC.

4783. Car d amine. L. 4784. Pteroneurum. DeC. 4785. Dentaria. L. 4786. Macropodium. L. 4\%8\%. Neuroloma. Andiz. 4788. Parrya. RBr. 4789. Oudneya. RBr. 4790. Arabis. L.

a. Abasicarpou. Andrz. Alomatium. DC.

b. Arabisa. R. *)

c. Lomatospora. DC. em. Lomaspora. DC. d. Turrita. TVallr.

4791. Stevenia. Ad.

4792. Streptanthus. Nurt. 4793. Turritis. L.

4794. Notoceras. DeC.

a. Diceratium. DC.

b. Tetraceratiun. DC. 4795. Cheiranthus. L.

a. Psilostylis. Andrz. Cheiroides. DC.

b. Cheiri. Dorst.

4796. Nasturtium. RBr.

a. Nasturtium. Trag. Cardaminum. DG.

b. Brachyolobus. All. Roripa. Scop. - B. Barbares $\searrow$.

*) Sectio iam diu a me in Srunmrr Fl, germ. indicata, male legitur ibi seminibus subulatis pro subalatis; snnt nempe fundo alata. In MorssteRraxo Enchiridio quod edidi, II. p. 1154. pariter sphalma occurrit, pro Arabisa iteram Arabis typotheta composuit. 
c? Clandestinaria. DC. 4\%9\%. Triceras. Andrz. a. Pinaria. DC.

b. Acinotum. DC.
4798. Matthiola. RBr.

a. Iuperia. DC.

b. Pachynotum. DC.

\section{b. $S i s y$ on $b r e a e .0)$ )}

4799. O r о bi u m. Reнв.

Oreas Cham. non Brid. nec

Zoolog.

4800. Eutrema. RBr. 4801. Platypetalum. RBr. 4802. Braya. Strne. Hpr. 4803. Leptaleum. DeC. 4804. Stanl e ya. Nutr. 4805. Andreoskia. DeC.

Hesperidopsis. DC.

Dontostemon. Andrz:

4806. Hesperis. L.

a. Hesperidium. DC.

b. Deilosma. Andrz.

480\%. Malcolmia. RBr. Malcomia (e sphalm.) H. Kew. -6. Alliaria $\vec{J}_{\text {ray et }}$ DeC.

4808. V elarum. Puin. a. Klukia. Bess.

b. Kibera. Ad. 4809? Redowskia. Cham. 4810. Sis ymbrium. L.

a. Arabidopsis. DC.

b. Norta. DC.

c. Psilostylium. DC. em. Psilostylum. DC.

d. Irio. Col.

4811. Leptocarpaea. DeC.

4812. Conringia. Heist. Brass. Coringia. P. 4813. Erysimum. L.

a. Syrenia. Audrz. Stylonema. DC.

b. Cuspidaria. DC.

c. Cheirinia. Lk. Erysimastrum. DC.

\section{c. Brassiceac. $0>>$}

4814. Eruca. T.

Euzomum. Lk.

4815. Ramphos pe $\mathrm{rmum}$.

Andr.

Sinap. Lcucosinapis." DC.

4816. Diplotaxis. DeC.

a. Anocarpum. DC.

b. Catocarpum. DC.

481\%. Brassica. L.

a. Micropodium. DC.

Günthera. Andrz, non al. b. Erucastrum. DC.

Finuca. Lam.

c. Brassica. Plin. 4818. Sin ap is. L.

a. Hirschfeldia. Mnch.

b. Kabera. R. Ceratosinupis, DC.

c. Melanosinapion. DC. em. IMelanosinapis. DC.

4819. Moricandia. DeC.

$$
\text { d. He liopli }
$$

4820. Chamira, ThNB. 4821. Heliophila. L. a. Carponema. DC.

b. Leptormus. DC. 
c. Ormisens. DC.

d. Selenocarpaea, DC.

e. Orthoselis. DC.

7. Pachystylium DC. em. g. Cephaloceras. Rchb. ")

h. Lanceolaria. DC.

i. Carpopodinm. DC. 4822. Schizopetalon. Srms. **)

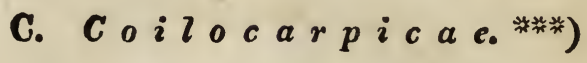

\section{RESEDEAE.}

4823. S e s a mella. Rchi. Sesamoides. T.

4824. R es eda. L.

a. Luteola. T. b. Reseda. T.

4825. Singana. Aưr. Sterbeckia. Schreb. 4826? Calispermum. Loun.

PAMILIA CVI.

\section{Papaveraceae. Papateraceen.}

a. Fumarieac.

482\%. Fumaria. T.

w. Sphaerocapnos. DC.

b. Platycapnos. DC.
4832. Dielytra. BRки. Diclytra. DC. Cucullaria, Rafin. 4828. D iscocapnos. Cham. 4833. Corydalis. Vent. 4829. Sarcocapnos. DC. a. Capnoides. Boerh. 4830. Adlumia. Rafin. b. Neckeria. Scop. 4831. Cysticapnos. Boenh.

c. Leonticoides. DC.

\section{b. Boc conie a e. $\dagger$ )}

4834. Impatiens. Rrv. 4836. Ep ime dium. L. $t_{\dagger}$ ) 4835. B als a mina. Riv. 483\%. Mahonia. Nutr.

-) cí. Ic exot. I. 55.

*) Cotyledones absque dubio sicnt in Meliophilis. cf. nostrain Ic. exot. I. 55. Quod reliquam est, hains generis in Resedaceas transitum, quem e plnribus abhinc annis ex omni parte demonstravi, non porro obscuram fore censeo.

***) Coilocarpium ab oinni fructunn genere diversnm.

j) Bocconiae forma tribam absolvit. Berberideae tantnm Fumariaceas repetendo eas praeparant.

if) Balsaminae quasi Peloria normalis. Tribns ceterum inseparabilis, generibrs quinque anterioribus folia inucronato-ciliata, reliquis continue unarginata. Singula examinavi. 
4838. Berberis. L. 4839. Nandina. ThNB. 4840. Le on tice. L. Lcontopetalum. T.
4844. Podophyllum. L. 4845. Sanguinaria. Drk. 4846. Bocconia. L. 484\%. Macleya. RBr. 4848. Eschholtzia. Chas. conf. B. Reg. 1168.

\section{c. $P a p a v e r e a c$.}

4849. Hypecoum. L.

4850. Chelidonium. L. 4851. Glaucium. T. 4852. Roemeria. MEd.
4853. Stylophorum. Nutr. 4854. Meconopsis. DeC. 4855. Argemone. T. 4856. Papaver. T.

FAMILIA CVII.

\section{capparideaE. Capparideen.}

a. Cle o m e a e.

485\%. Dactyla ena. Schrad. 4862. Polanisia. Rafiv. 4858. Cleomella. DeC. 4859. Peritoma. DeC. 4860. Gynandropsis. DeC. Podogyne. Hitgg. 4861. Cl e o me. I.

a. Siliquaria, Forsk, non Brugn. nec Lam.

b. Cleome. L.

Pedicellaria. DC. non Lour. nec Zoolog. a. Brachystylium. DC. em. Jacksonia. Rafin.

8. Macrostylium. R. Stylaria. DC. non Zoolog. 4863. P hy s os te m o n. Mart. 4864. Rorida. Forsk. et Roridula. Forsk. Siliguariae sp. DeC. non Forsk. nec Brug. nec Lam.

\section{b. $\quad C a p p a r e a e$.}

4865. Co rynandra. Schrad.*) 486\%. O thrys. Noronh. 4866. Crataeva. L.

Nicbuhria? DeC.

-) Inaginem elegantissimi generis cułn analysi edidi Maio mense anni 1827.

Ic. exot. tab. 147 . 
4868. Niebuhria. DC. 4869. Bos cia. Lam.non Then. 48\%0. C a d a ba. Forsk. 48\%1. Schepperia. Neck.

Macromerum. Burch. 4872. Stephania. W. 4873. Sodada. Forsk. 48\%4. Capparis. L.

a. Quadrella. DC.

b. Reyniba. R.

Breynia. Plum. non Forst.
Breyniastrum. DC.

c. Calanthea. DC.

d. Cynophalla. DC.

e. Capparidastrum. DC.

f. Capparis. Plum. Eucapparis. DC. 4875. Moriso nia. Prum. 4876. Thylachium. Lour. 4879? Hermupoa. LörfL. 4878? M a e rua. Fonsk.

\section{c. Flac ourtieac.}

48\%8b. Ochradenus. Der. 4882. Stigmarota. Luur. 4879. Rh amnopsis. Rchr.

Flac. sepiaria. $\mathbf{R x b}$. 4880. R o u m ea. Port. 4883. Kiggelaria. L. 4881. Flacourtia l'Henit. 4884. Hy d nocarpus. GäRT:

\section{FORMIATIO II.

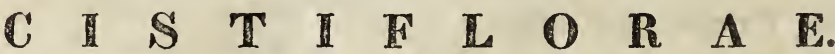 CISTUSBLUETHLER.}

FAMILIA CVIIT.

VIOLACEA E. VIOLACEEN.

a. Violere.

4585. Viola. T.

a. Leptidium. Ging.

b. Nomimium. Ging.

c. Dischidium. Ging.

d. Ionium. R. Chamatamelanium. Ging.

c. Grammeionium. Rchb. Iacea. Camer. non T. Vaill. Melanium. DeC. non Br. 4886. Solea. SPR.
488\%. Pombalia. VAND. 4888. I on id i um. Vent. 4889. Hybanthus. JAcQ. 4890. Corynostylis. Mart. Calypirion. Ging. 4891. No is ettia. K. H. B. 4892. A n c hiet ea. A. St. Hil. 4893. G lossarrhen. Mant. 


$$
\text { b. Alsodi } \text { a } a \text { e. . }
$$

4894. Conohoria. Aubl. Rinorca. Aubl. 4895. Als odeia. A. P. Th.

Riana. Aubl.

Piparca. Aubl.

Passoura. Aubl.

Ceranthera. P. B.

Passalia. Banks.

Cuspa. Humb, relat. 4896. Tachibota. Aubr.
Salmasia, Schreb. 4897. Spatellaria. A. St.

Hil. em. Spathularia. A. St. Hil. non Pers. nec Zoolog. Amphirrhox. Spr. 4898? Penta loba. Lour. 4899. Physiphora. Sox. 4 y00. Hy menan thera. Banks. 4901. Piparea. Aubl.

c. $S a u v a g$ ese a e.

a. Frankienieae.

4902. Frankenia. L. 4903. B eatsonia. Rxb.

$$
\text { b. Sa uvageseae genuinac. }
$$

4904. Sauragesia. JAcQ. 4906. Luxemburgia. A. St. Sauvagea. Neck. 4905. Lavradia. Velloz. Plectunthera. Mart.

Hil.

$$
\text { c. Erythroxylea e. }
$$

4906b. Erythroxylon. L. 4906c. Sethia. K. H. B.

\section{FAMILIA CIX.}

\section{CIST IN E A E. CISTINTEN.}

$$
\text { a. } \boldsymbol{P} a \boldsymbol{r} \text { a s s i e a e. }
$$

490\%. Parnassia. T.

$$
\text { b. Drosereat. }
$$

4908. Aldrovanda. Mont. 4909. B y bli S. Salisb. 4910. Drosera. L.

a. Rorella. Rupp. b. Eryaleium. DC. 4911. Roridula. L. 4911b. Drosophyllu m. LK.

4912. D i o n e a. Erx.
4913. Lechea. L. 4914. Hudsonia. L. 4915. II elian themum. T.

a. Helianthemum. Euhelianthemum. DC.

b. Pseudocistus. DC.

\section{c. Cisteat.}


190 CLASS. MII. THLLAN. ORD. I.THYLACH. FAM. CX. BIX AC.

4916. Cistns. T.

a. Ledonia. DC.

b. Erythrocistus. I.

FAMILIA CX.

B I X A C E A E. BIXAGEN.

a. Erythrospermeac.

4917. Melicytus. Fonsr. 4918. Erythrospermum.

LıM.

b. Patrisiene.

4919. Ryania. VihL.

4920. Patrisia. Rrch.

Ryanuca. DC.

c. Bixinea e.

4921. L $\mathrm{u}$ di a. LAM.

b. Prockiaria. DC.

4922. Kuhlia. K. H. B. Tiner. Spr, non Zool. 4923. Azara. Rz. PAv. 4924. Ban a ra. Aubr. 4925 ? X y lan deni us. Hamilt. 4926. Prockia. Brw.

a. Aphloia. DC. Lightfootia. Sw. non l'Herit. 4927. Laetia. L. 4928. Mayna. Aubr. 4929. Bixa. L. 4230. Echinocarpus. Blume. 4931. Trich os per mum. Blume. 


\section{ano on \\ S CH I Z O CA R I CA E. SPALTFRUECHTIGE.}

\section{FORMATIO I. \\ R A N U N C U L I F L O R A E. RANUNKELBLUETHLER.}

\section{FAMILIA CXI.}

RANUNCULACEAE. RANUNGUAGEN.

A. $R a n u n c u l$ e $a$ e.

a. Ranunculeaegenuinae.

4932. M yosurus. Dilc. 4938. Casalea. A. St. Hil.

4933. Cerat ocephalus. 4939. Batrachium. DC. Mönch. 4940. F i caria. Dile.

4934. A do $n$ is L.

a. Adonis. C. B.

b. Consiligo. Plin? DC. 4935. Ha m a d r y as. Commers. 4936. Hydrastis. L. 493\%. Knowltonia. SALrsb. Anamenia. Vent.
4941. Ranunculus. L.

a. Hecatonia. DC.

$$
\text { non Lour. }
$$

b. Ranunculastrum. DC.

c. Philonotis. R. Echinella. DC. non Ag.

4942. Aphanostemma. A.

St. Hil.

\section{b. $A n$ emon e a e.}

4943. Hepatica. Dilx. 1sopyrum. Ad. 4944. Anemone. L.

a. Anemonanthea. DC.

b. Asteranemia. R. Pulsatilloides. DC. 4045. Puls atilla. T.

a. Prennanthes. DC.

b. Pulsatilla. Bauh. 4946. Clematis. L. a. Flammula. Rupp. Clematitis. Mnch.

b. Viticella, Dill. 4947. Atragene. L.

a. Viorna. Pers. Mruralta. Ad. Cheiropsis. DC.

b. Atragene. 4948. Naravelia. DC. 


$$
\text { c. } H e l l \text { e borea }
$$

4949. Delphinium. T.

a. Calcatrippa. MIatth. Consolida. DC. non Rupp.

b. Delphinellum. DC.

c. Staphisagria. Riv. Delphinastrum. DC.

4950. Aconitum. T.

a. Anthora. Riv.

ъ. Napellus. Riv.

Calliparion.

Euchylodeon.

Cammurum.

Corythaeolon.

Napellus.

c. Lycoctonum. Diosc.

4951. Garidella. T. Nigellastrum. Magnol.

4952. Nigella. T. Melanthium. Diosc.

a. Nigellastrum. Siegesb.

b. Nigellaria. DC.

c. Nigella. Mnch. Erobatos. DC.

4953. Thalictrum. T.

a. Tripterium. DC.

b. Physocarpidium. R. Physocurpus. DC.

c. Thalictrum. Euthalictrum. DC. 4954. Lentop y rum. Rchв. Isol' fumarioides. $\mathbf{L}$.
4955. I s o p y r u m. Hall. Olfa. Ad.

4956. Cop tis. Salisi. Chrysa. Rafin.

495\%. Ci micifuga. L. 4958. Macrotys. Rakin.

4959. A c t a ea. L. Cl:ristophoriana. T.

4960. Xantorrhiza. MArsh. Zanthorrhiza. l'Herit.

4961. Caltha. RAJ.

a. Populago. T.

b. Psychrophila. DC. 4962. Trollius. L.

a. Gaissenia. Raf.

b. Trollius. Raf.

4963. Eranthis. SAlisb.

Kocllea. Bir. Robertia. Merat.

4964. Hell e borus. T.

a. Helleboraster. Lob. H. foetidus. $\mathbf{L}$.

b. Helleborus. Hippocr.

4965. Paeonia. L.

a. Paeonia. C. Bauh.

b. Moutan. Chinens.

\section{B. Dillenieac.}

$$
\text { a. Illicieae. }
$$

4966. Illicium. L.

496\%. T'e mus. Mor. 4968. Drymis. Forst.

a. Eudrymis. DC. b. Wintera. Murr. Winterania. Sol. 4969. Tasmannia. RBr. 
CLASS. VIII. THALAM. ORD.IT.SCHIZ. FAM. CXI.RANUNCUL. 193

\section{b. $D$ e $l i m e a e$.}

49\%0. T e tracera. L.

Tigarea. Aubl.

Rhinium. Schreb.

Euryandra. Forst.

Wahlbomia. Thnb.

49\%1. D a vill a. VAND.

4972. Doliocarpus. Rox. 4973. Empedoclea. A.St. Hil.
4974. Delima. L. 4975. Curatella. L. 4976. Trachytella. DeC. 497\%. Recchia. Sess. 4978. Burtonia. SAlisb. ${ }^{*}$ ) non $\mathbf{R B r}$.

4979? Othlis. Schotr. 4980? Dasynema. Schotr.

\section{c. $H i b b e r t i e a c$.}

4981. Pachynema. RBr. 4982. Hemistemma. Juss. 4983. P l e u r a ndra. LaB. 4984 Candollea. LaB. 4985. Adrastaea. DeC. 4986. Wormi a. Rotтв.
Lenidia. A. P. Th. 498\%. Hibbertia. ANDr. 4988. Colbertia. SAlisb. 4989. Dillenia. L. 4990. Capellia. Buume,

$$
\text { C. } \quad \text { a } a g \text { u }
$$

a. Magnolieae genuinae.

4991. Liriodendro n. L. 4993. Ta la uma. Juss.

4992. Magnolia. L.

a. Guillimia. Rottl.

b. Magnoliastrum. DC. 4994. A ro m a dendron. BuUme. 4995. Ma nglie ti a. Blume. 4996. Michelia. L.

b. Anonere.

499\%. Rollinia. A. St. Hil. 4999. Anona. Ad. 4998. K a d s u r a. Juss.

$$
\text { c. Un } \boldsymbol{U} \text { n e a e. }
$$

5000. Anaxagorea. A. St. 5002. Duguetia. A. St. Hil.

Hil. 5003. Bocage a. A. St. Hil. 5001. Guatteria. Rz. PAr. 5004. Xylopia. L.
5005. Artabotrys. RBr.

*) Quid ab Hibbertia imo a Dilleniaceis reris v. Ilibbertieis differat, iam a. 1824. in Ic. plant. cult. et colend. LXXIV. demonstravi. 
194 CLASS.VIII. THALAM. ORD. II. SCHIZOC. FAM. CXII. RUTAC.

5006. Unona. L.

a. Unona.

Marcutera. Noronh.

Oetania.

b. Desmos. Lour.

c. Melodorum. Lour.
500\%. Uvaria. L. 5008. Porcelia. Rz. Pav. 5009. A simina. Ad.

5010. Monodora. Dun.

FAMILIA CXII.

R U T A C E A E. R U T A C E E N.

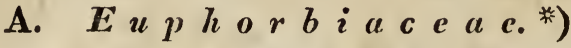

a. Tithymaleat.

5011. Pedilanthus. Neck.

5012. Euphorbia. L.

a. Euphorbia. Neck.

b. Keraselma. Neck.

c. Athymalus, Neck.

d. Tithymalus. Neck.

Euphorbia. Haw.

Trësia. Haw.

Dactylanthes. Haw.
Mredusea. Haw. Tithymulus. Haw.

Gularrhoeus. Haw.

Esula. Haw.

Anisophyllum. Haw.

5013. Hendecandra. Eschsch. 5014. An thostem ma. Ad. Juss. 5015 Dalechampia. Puum.

b. Hippomaneae.

5016? Hecatea. A. P. Th. 5024. Hippomane. L. $501 \%$ O mphalea. L. Omphalandria. RBr. Duchola. Ad.

5018. Maprounea. Aubr. Aegopricon. $\mathrm{L}$. fil. Mancanilla. Plnm. 5025. Homalanthus. An. Juss. em. 5019. Styloceras. Ad. Juss. 5020. Commia. Lour. 5021. Excoecaria. L. Gymnanthes. Sw. prod. 5022. Seb a stiania. Spr. 5023. Hura. L.

Omalunthus. Ad. Juss.

5026. Triadica. Lour.

5027. Stillingia. Garden. 5028. Sapium. JacQ. 5029. C n e mid os tach ys.

Mart. Microstachys. Ad. Juss.

*) Observantia unagis quam nrgente natura huc usque per longum intervallum a Rutaceis remotae, ex inde tamen ab embryone eiusqne evolntione usque ad fructum, unum eundemqne persequentes continun typun. 


$$
\text { c. Acalyplacal. }
$$

5030. Tragia. Puus.

Schorigeram. Ad.

5031. Pluknetia. Plum.

5032. Anabaena. Ad. Juss. 5033. Mercurialis. L. 5034. Acalypha. L.

Cupameni. Ad.

5035. Caturus. L.
Galurus. Spr.

5036. M a p a. Ad. Juss. 503\%. Macananga. A. P. Th.

Punopia. Noronh.

5038. Cla oxylon. Ad. Juss. 5039. Conceveibum. Rich. 5040. Alchornea. Sw.

Hermesia. Bonpl.

\section{d. $R i c i n e a$ e.}

5041. Argythamnia. P. Br. 5056. Ricinocarpus. Dess. 5042. Dita $x$ is. V. 5043. Caperonia. A. St. Hil. 5044. Crozophora. NECK.

Tournesolia. Scop.

5045. Croton. L.

Ricinoides. T.

Croton. Neck.

Aroton. Neck.

J.contia. Neck.

Cinogasum. Neck.

Tridesmus, Lour.

Cascurilla. Ad.

5046. Crotonopsis. Mchx.

Leptomon. Rafin.

Friesia. Spr.

- 5047. Adelia. L. non Mchx.

Bernardia. Houst.

5048. A cidoton. Sw.

5049. Adriana. Gaudich.

5050, Rottlera. RxB.

5051. Codia eum. Ruph.

Phylluurea. Lour.

5052. Gelonium. Roxb.

5053. H i s ingera. Hell.

5054. Mozinna. Orteg.

Loureira, Cav.

5055. A mperea. Ad. Juss.
Echinosphacra. Sieb.

Röperia. Spr.

505\%. R i c inus. L.

5058. Manihot. Ad.

Ianipha. K. H. B.

5059. Cnidoscolus. Pohr.

Iussicua. Houst.

Bivoncea. Rafin.

5060. Adenorhopium. Porr.

5061. I a tropha. L.

Castiglionea. Rz, Pav.

Curcas. Ad.

Bromifeldia. Neck.

5062. El a e oc occa. Comm.

Dryandra. Thnb. non RBr.

Vernicia. Lour.

5063. A l e u rites. Forst.

Ambinux. Commers.

Camirium. Rmph.

5064. Anda. Piso.

Joannesia, Vell.

5065. Siphonia. Rrch.

Hevca. Anb.

5066. Mabea. Aubu.

506\%. Hy a en a che. Lamb.

Tovicodendron, Thnb.

5068. G a r cia. Rohr. 


$$
\text { e. } P h y l a n t h e a e \text {. }
$$

5069. Epistylium. Sw. FI. 5079. Xylophylla. L. 50\%. Gynoon. Ad. Juss. 50\%1. Glochidion. Forst. Bradlcia. Gärt. Genisiphylla. l'Herit. 5080. M e n a rd a. Сомм. 5081. Micranthea. DESF. 5072. Anisonema. Ad. Juss. 5082. Agyneia. L. 5073. Leptonema. An. Juss. 5083. Andrachne. L. 5074 ? Tricaryum. Lour. 5075. Cicca. L. 5076. Em blic a. G̈̈rt. 507\%. Kirganelia. Juss. Ardinghelia. Comm. 50\%. Phyllanthus. L. Niruri. Ad. 5084. Briedelia. WiLLD

Conami. Aubl. 5085. Cluytia. Art. Clutia. Boerh. Altona. Ad. Cratochwilia. Neck. 5086 ? Stilago. L. 5087? Antidesma. L.

$$
\text { f. } B u x e a e \text {. }
$$

5088. Drypetes. VAHL. Crantzia. Sw. 5059. S a r c o c o c ca. Lrind. 5094. S e curin e ga. Juss. 5090. Thecacoris. Ad.Juss. 5095. Savia. Wilhd. 5091. Pachysandra. Mchx. 5096. Amanoa. Aubr. 5092, B uxus. L. 5093 ? Tricera. Schreb. 509\%. Richeria. VAHL. 5098. Flïggea. WiLn.

$$
\text { Euphorbiaceae minus cognitae. }
$$

5099. Margaritaria. L. fil. 510\%. Synzyganthera.. Rz. 5100. Suregada. Roxb. 5101. Hexadica. Lour. 5102. Hom ono ia. Lour. 5103. Clad odes. Lour. 5104. Echinus. Lour. non ZooLog.

Ulassium. Rmph? 5105. Colligu a ya. Moux. 5106. Lascadium. RafiN.

Didymandra. W. 5108? Mall otus. Loun. 5109? Meborea. Aubr. Rhopium. Schreb. Tephranthus. Neck. 5110? Co metes. Burm. 5111? P era. Mut. 5112? Peridium. Schotr. 5113? Pennantia. Forst.

\section{B. $\boldsymbol{R} u t a r i a r$.} a. $E m p e t r e a e$.

5114. Ceratiola. Rich. 5115. Corema. Dun. 
5116. Empetrum. L.

511\%. Cyminosma. G̈̈rt.

Jambolifera. $\mathbf{L}$.
5118. Cneorum. L.

Chamaclea. T.

b. $S t a c k h$ ous $i \in$ a $e$.

5119. Stackousia. SM.

$$
\text { c. } \mathbb{R} u t e a \text { e. }
$$

5120. Peganum. L. Harmalum. T. 5121. Aplophyllum. Ad. Juss. non H. Cass.
5122. $R$ ut a. L.

5123. Boenninghausenia.

Racris.

Ruta albifl. Hook. exot. F1.79.

\section{d. Moringe a e.}

5124. Moringa. LaM.

Hyperanthera. V.
Guilundina. Jcq. Alandina. Neck.

$$
\text { e. } Z a n t l b o x y l e a c \text {. }
$$

5125. Ptelea. L.

Bellucia. Ad.

5126. Vepris. Сомм.

512\%. D uncania. Richr.

Boscic. Thnb. non Lam. 5128. Blakburnia. Forst. 5129. T od d a li a. Juss.

Cranlzia. Schreb.

Scopolia. Sm. non L. fil. nec Forst.

5130? Philagonia. Buuge. 5131. Boymia. Ad. Juss. 5132. Zanthoxylum. L.

Xanthoxylum. Sm.

Ampacus. Rmph.

Fagura. L.

Ptcrota. Ad.
Ochroxylum. Schrel. Kampmannia. Rafiu. Langsdorfia. Leand. non Rich.

Pohlana. N. et M. Aubertia. B. St. V. 5133. L a b o r d i a. Gaudich. 5134? Thysamus. Lour. 5135. Brunellia. Pa. PAv. 5136. Brucea. MrLx.

Gonus. Lour.

513\%. Galvezia. Rz. PAv. non Domb.

5138. Dictyoloma. DeC. 5139? Triceros. Lour. 5140? Trattinickia. W. - b. Ailanthus $\mathscr{Y}$.

$$
\begin{aligned}
& \text { f. } \quad D i \text { o } s \quad m e \text { a } e \text {. } \\
& \text { aa. Cusparie ac. }
\end{aligned}
$$

5141. Moniera. Aunz. Aubletia. Rich. 
5143. Erythrochiton. N. et $M$.

5144. Diglottis. N. et M. 5145. Galipea. Avbr. Cusparia. Humb. Bonplandia. W. Angostura. R. S. Conchocarpus. Mik. Ravia. N. et Mr.
Lasiostemon, N, et M. Obentonia. Vell. Raputia. Aubl. Sciuris. Schreb. Pholidandra. Neck. 5146. Almeid ea. A. St. Hil. Aruba. N. et MT, non Aubl. 5147. Spiranthera. A. St. Hil. Terpnanthus. N. et M.

bb? Pilocarpeac.

5148. Choisya. K. H. B. 5153. Evodia. Forst. 5149. Hortia. VAND. non G̈̈̈RT.

5150. Pilocarpus. VAmL. 5154. Melicope. Forst. 5151. Metrodorea. A. St.Hil. Entoganum. Bnks. Gärt. 5152. Esenbeckia. K. H. B.

\section{cc. Diosmeac genuinae.}

a. Europacae.

5155. Dict a mnus. L. Fraxinella. T.

$$
\text { B. C a p e ns e s. }
$$

5156? Polembryum. Ad.Juss. 5162. Euchaetis. Barte. et 515\%. C a lo d e ndron. Thnb.

Wende.

Pallasia. Houtt.

5158? Barraldeia A. P. Th.

Barranltia. Spr.

5159. Ad enandra. W. En.

Gilandulifolia. Wendl.

Oclia. Dietr.

Okenia. Dietr.

5160. Coleonema. Bantu. et Wendl.

5161. D i o s ma. Berg.)

$$
\text { 7. Australasicac. }
$$

5166. Zieria. SM.

a. Boronia. R.

516\%. B oronia. SM.

c. Robonia. R. *)

*) Ic. exot. $73-74$. 
5168. Eriostemon. SM. *) 5172. Diplolaena. RBr. 5169. Crowea. SM. Ventenctum. Lesch, ined. 51\%0. Philotheca. Rudg. $\left.{ }^{* * * * *}\right)$ 5173. Correa. SM. 51\%1. Phebalium. Vent. Mazentoneuron. LaB.

$$
\text { C. Simar } u b \text { e a e. }
$$$$
\text { a. Quassiea e. }
$$

5174. Quasia. L.

51\%5. Sim a ruba. Aubr.

51\%. Simaba. Ausc.

Aruba. Aubl.

Zwingera. Schreb.

Phyllostemma. Neck.

51\%. Tapiria. Aubr.

Ioncquetia. Schreb.

51\%8. S a m a d er a. G̈̈rt.

Samandura. L. zeil.
Locandi. Ad.

Vitmannia. Vahl.

Niotu. Lam. non Ad.

Biporcia. A. P. Th.

ITanduyia. Comm. ined.

5179. Nima. Hamilt.

5180. Ebelingia. Rchs.

Harrisonia, RBr. non Ad. rec Hook.

\section{b. $C$ or $i a r i e a c$ :}

5181. Coriaria.' Niss.

5182? Pedicellia. Lour.

$$
\text { c. O c } h n \in a e \text {. }
$$

5183. Castela. Turp.

5184. Elvasia. DeC.

5185. Walkera. Śchreb.

Mcesia. Gärt.

5156. Diporidi un. Barte.

WENDL.
518\%. O chna. Schreb.

5188. Go mphia. Schreb.

Ouralea. Aubl.

Correia. Vell. Vand.

5189? Philomeda. Noronh.

5190. Brownlowia. RxB.

\section{FAMILIA CXIII.}

SAPINDACEAE. SAPINDACEEN.

$$
\begin{aligned}
& \text { A. Zygophylleac. } \\
& \text { a. } T r i b u l e a c .
\end{aligned}
$$

5191. Tribulus. T. 5193. Ehrenbergia. Mart. 5192? Kallströmia. Scop.

*) F. dolabratus n. sp. describitnr Ic. exot. II. p. 36.

**) Speciem securdam cum analysi cf. Ic, exot. 200. 
b. Gua iace $\boldsymbol{a} e$.

5194. Fagonia, T.

5195. S e e z en ia. RBr.

5196. Roepera. Ad. Juss.

519\%. Zy gophyllum. L.
5198. Larrea. Car. 5199. Porliera, Rz. Par. 5200. Gu a i a cum. Puum.

$$
\text { c. Hippocastanea e. }
$$

5201. Pavia. Boerh. 5202. A esculus. L. Hippocastanum. T.

\section{B. $\quad S \quad a \quad p \quad i \quad n \quad d \quad e r r$.}

\section{a. Staphylaceac.}

5203. Staphylea. L. Staphylodendron. T. Bumaida. Thnb.
5204. Turpinia. Vent. Dalrympelea. Rxb. Dalrymplea. Spr.

b. $C u p a n i e a c$.

5205? Vatentinia. Sw. 5206. Stadmannia. LAM. 520\%. Schleichera. WiLLd. 5208. Melicocca. Juss.

a. Melicocca. L. Oococca. DC.

b. Sphaerococca, DC. 5209. Hypelate. P. Brw. 5210. C os signi a. Commers. Cossinia. Spr.

5211. Tina. R. S. Gelonium. G. non al.

5212. Cupania. Plum.

a. Trigonis. Jcq.

b. Molinaea. Juss.

c? Odontaria. DC.

d? Guioa. Cav.

5213. Dimereza. LaB. Diplopetalum. Spr.

5214. Toulicia. Aubu. Ponacu. Schreb.
5215. Thou inia. Port. 5216. Euphoria. Сomm. Dimocarpus. . Lour.

Scytalia. G. Nephelium. L.

521\%. Schmidelia. L. Ornitrophe. Juss. Allophyllus. L. Toxicodendron. G.

5218. A poretica. Forst Pometia. Forst. Gemella. Lour.

5219? Matayba. Aurl. Ernstingia. Neck. Ephielis. Schreb. 5220. Talisia. Aubl. 5221. Blighia. KöN. Akeesia. Thnb. Bonunnia, Rafin. 3222. Sa pindus. L. 5222b. Loxostylis. Spr. *)

*) Nostr. Ic. exot. 205. ubi generis pulchri analysis editar completa. 


$$
\text { c. Dod o n a e r c e a e. }
$$

5223. Koelreuteria. Laxm. 5228. A lectryon. Gärr. 5224. A mirola, Pers, 5228b. Empleurum. Sor. Liaguroa. Rz. Pav. 5225. Ph a e carpus. Mart. 5229? Eustathes. Lour. 5226. Dodonaea. L. Valentinia. Räusch. Magonia. A. St. Hil. 5230? Racaria. Aubl. 5231? Ratonia. DeC. 5227? Heterodendron. Desf. 5232? H a rpullia. Roxb.

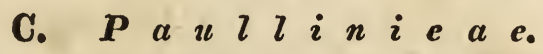

$$
\text { a. Meliantheac. }
$$

5233. Melianthus. T. 5234. Chitonia. Moc. Sess.

$$
\text { b. Cardiospermeae. }
$$

5235. Cardiospermum. L. Corindum. T. 5236. Urvillaea. K. H. B. 523\%. Serjania. Plum.
Scriana. Schum.

5238. P a ull in ia. Schum. Cururu. Plum. 5239. Enourea. Aubl.

$$
\text { c. Acerea e. }
$$

5240. N e gund o. Mönch. Negundium. Rafin. 5241. Acer. L. e. e.

Negraum, Rafmo

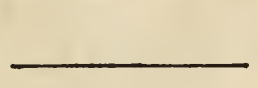

FORMATIO II.

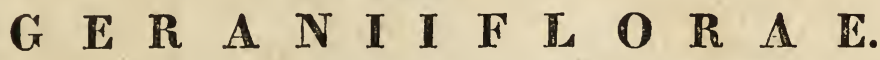 STORCHSCHNABELBLUETHIGE.}

FAMILIA CXIV.

$$
\begin{aligned}
& \text { I A L V A C E A E. Malvace en. } \\
& \text { A. } \quad \begin{array}{llllllll}
\boldsymbol{a} & \boldsymbol{a} & \boldsymbol{l} & \boldsymbol{o} & \boldsymbol{p} & \boldsymbol{e} & \boldsymbol{a} & \boldsymbol{c} \text {. }
\end{array} \\
& \text { (carpidiis aggregatis.) } \\
& \text { a. Nudiflorac. }
\end{aligned}
$$

5242. Palavia. CAv.

$$
\text { b. I } u \text { volucrutuc. }
$$




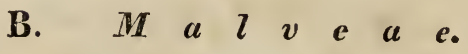
(carpidiis radiatim positis.)

a. Sidecue, nudiflorae.

5245. Gyrostemon. Desf. a. Napaea. L.

b. Sida. $\mathbf{L}$.

5246. Cristaria. Cur. 5249. Gауа. K. H. B.

5247. Anoda. Car. 5248. Sida. L.
5250. B astardia. K. H. B.

5251. Abutilon. Dill.e. e. 5252. Periptera. DeC.

b. Lavatereae, involucratae.

5153. Urena. L.

5254. M alva. L.

a. Malvastrum. DC.

b. Maluchia. DC.
5260? S en ra. CAv.

Serra. Gm.

Senraea. W.

Serraca. Spr.

5255. Sphaeralcea. A.St.Hil. 5261. Pavonia. Cav. MIalv. HI. Sphueroma. DC.

a. Typhalea. DC.

5256. H а у $\mathrm{n}$ е а. Rिснв. Modiola. Mnch. non Zoolog. $525 \%$ Althaea. L.

a. Althaeastrum. DC.

b. Alcea. $\mathbf{L}$.

c. Alphaea. DC. $525 \varepsilon$. Lavatera. L.

a. Stegia. Mnch.

b. Olbia. Mied.

c. Axolophia. DC. em. Axolopha. DC.

d. Anthema. Med. 5259. M a l a chra. L.

b. Malache. Trew.

c. Cancellia. R. Cancellaria. DC. non Zool. et Mrin.

5262. Thorntonia. Rens. Hibisc. II. Penlasp. *) DC. 5263. L opimia. Makt. 5264. Lebretonia. Schrk. 5265. Achania. Sw.

a. Mralvaviscus. Dill.

b. Anotea. DC.

5266. Astrapaea. Lindu.

\section{C. $\boldsymbol{H}$ i $b$ is ce a c. (capsula perfecta.)}

a. Ingunaeece, nudiflorae.

526\%. L a g u naea. CAv. Solandra. Murr.
5268? Ingenhouzia. Moc. SEsS.

b. Ketmieae, involucratae.

5269. Lagunaria. DC. Hibisc. XI. Lagunar. DC.
52\%. Fugosia, Juss.

Cienfuegosia. Cav.

*) Capsula non pentasperina cf. Ic, exot. 162. 
5271. Redoutea. Vent. 5272. Gross y pi um. L. $X y l o n$. T.

5273. Hibiscus. L.

a. Nanihot.

b. Ketmia.

c. Furcaria.

d. Abelmoschus. Med. c. Bombycella.

$f$. Trionum.

g. Sabdariffa. Azanza. Moc. Sess.

5274. Parita. Ścop.

Parilio Ad.

Paritium. A. St. Hil.

5275. Thespesia. Corr.

FAMILIA CXV.

G E R N I A C E E. Gerantacen.

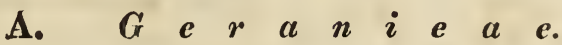

a. Trop $\quad$ r e o l e c e.

5276. Magallana. CAV. 52\%. Tropaeolum. L.

b. Erodieac.

5278. P elargoni um l'Herit.
a. Hoarea. Siveet.
b. Dimacria. Lindl.
c. Campylia. Sweet.
d Ityrrhidium. DC.
e. Jenkinsonia. Sweet.
$f$. Chorisma. Lindl.
g. Pelarģium. DeC.

Ciconiam. Sweet.

h. Cynosbata. DC.

i. Peristera. DC.

k. Otidia. Lindl.

7. Polyactium. DC.

$m$. Isopetalum. Sweet. 52\%9. Erodi u in I'Herit. 5280. Geranium. L. e. e.

\section{c. $M$ I n sonie $\operatorname{e}$.}

5281. Mons onia. L. fil.

6. Odontopetalum. DC.

a. Holopetalum. DC. em. 5282. Sarcocaulon. DeC.

Olopetalum. DC.

$$
\text { B. Sterculieue. }
$$

a. $R h y n c h o t h e c e a c$.

5283. Rhynchotheca. Rz. Par.

b. Sterculieae genuinae.

5284. Kleinhovia. L.

5285. Heritiera. AIT.
Balanopteris. G.

Sumandura. L.

5286. Triphaca. Lour.

528\%. Sterculia. L. 
c. Goetheaceac.

5288. GIuta. L.

5289. Eriolaena. DeC. 5290. Schillera. Rchв.
Wallichia. DeC. non Rxb. Jackiı. Spr. non Wall, nec Blume. 5291. Go ethea. N. et Mart.

$$
\begin{aligned}
& \text { C. Byttnerieae. } \\
& \text { a. Theobroméa }
\end{aligned}
$$

5292. A yenia. L.

5293. B yttueria. Lörfl.

Rulingia. RBr. 5295. Glossostemon. DESF. Cacuo. T."

5296. G и a z ma. Plum. Bubroma. Schreb.

5297. A broma. L. fil. 5294. Commers onia. Forst. 5298. The o b r oma. Juss.

5299? M aranthus. Buume.

\section{b. I as iopetal e a e.}

5300. Keraudrenia. Gay. 5301. Thomasia. GAY. 5302. Guichenotia. GAY.
5303. La siopetalum. Sm. 5304. S e ringia. Gax.non Spr. Gaya. Spr, non K. II. B.

\section{c. Dombeyacere.}

5305. Mahernia. L. 5306. Hermannia. L. Lophanthus. Forst.

a. Hermannella. DC.

b. Trionella. DC. 530\%. Altheria. A. P. Th. 5308. W altheria. L. 5309. Riedlea. Vrant.

Visena. Houtt. Mrougeotia. Knth. Riedleia. DC. 5310. Melochia. L. e. e.
5311. Pentapetes. L. Brotera. Cav.

5312. Assonia. Car.

6313. Dombeya. CAV. 5314. Ruizia. Cav. 5315. Melhania. Forsk. 5316. P t e ros p er m u m. Schreb.

a. Pterolaena. DC.

b. Velaga. G. 531\%. Trochetia. DeC. 5318. H u gon i a. L. *)

*) IIugoniae genus a cl. DeC. prod. I. 522, Chlaenaceis iunctum vel iisdem certe affine dictum, sed minns completum visum, ab iis mnltis numeris mihi videtur distinctum, et multo magis Dombeyaceis et ipsi quidem Pterospermo atque Trochetiae afline, fructu tamen omnino singulari hu- 
FAMILIA CXVI.

BOMBACEAE. B O B ACEEN.

a. $O x a l i d e a e$.

5319. Oxalis. L. oxys. T.

5320. Biophytum. DeC. Oxal. sect. A. St. Hil. *)
5321. Averihoa. L.

5322. Ledocarpon. Desf.

b. Bombacene genuinae.

5323. Helicteres. L.

a. Spirocarpaea. DC.

b. Orthocarpaea. DC.

5324. Cheir os temon. Hb. Bru. Cheiranthodendron. Larr.

5325. Chorisia. KNTH. 5326. M y ro di a. Schréb. 5327. Durio. L. 5328. O chroma. Sw. 5329. Matisia. Hв. BPL. 5330? Plagianthus. Forst.
5331. Pourretia. W. Cavanitlesia. Rz. Pav. 5332. Montezuma Moc. SEss. 5333. O phelus. Lour.

5334. Carolinea. L. Páchira. Aubl.

5335. B o m b ax. L.

5336. C eїba. Plum. Eriodendron. DC.
5337. Adansonia. L. Buobab. J. Bauh.

$$
\text { c. } R h i z 0 b \circ l e a e \text {. }
$$

5338. Caryocar. L. Pekea. Aubl.

Saouari. Aubl.
Rhizobolus. Gärt. 5339? Ge is s o is. LaB.

ius familiae maxime absoluto, propriam sectionem constituens. Chlaenacearnon specimina, ab ipso cl. A. Ркт. Тночаrs collecta, comparavi in Museo Parisiensi, Iugonias autem ipse possideo : serratam atque tomentosam, et hanc non miuns ac illain spinas gerere corniculatas attestor.

*) Nobis genus non quidem artificiale, sed eo magis naturale, vicinis intexunedinin. 


\section{ORDO III. \\ ID I O C A R I I A E. SAEULENFRUECHTIGE.}

\section{FORMATIO I. \\ T I L I I F $\mathbf{L}$ O LINDENBLUETHLER.}

FAMILIA CXVII.

Caryorhyllaceae. Caryophyluceen.

A. Alsine a e.

5340. Holosteum. L.

5341. Sagina. L.

5342. Mönchia. Енrн.

5343. Hymenella. Moc.

5344. B u f f on ia. SAuv.

5345. Möhringia. L.

5346. Physa. Noronh.

5347. Spergulastrum. Mcнх. Micropetalum. $\mathbf{P}$.

5348. Cherleria. Hahl.

a. Somerauera. Hpp.

b. Cherleria. I.
5349. Stellaria. L. 5350. Alsine. G̈̈вт. Lepigonum. Fr. Honclicnya. Ehrh. Halianthus. Fries. Adenarium. Rafin.

5351. G o uffeia.Rob. et Cast. *) 5352. S pergella. Rchb. $S_{i}$ ergalla exslip. Auct. 5353. Arenaria. L. 5354. Wilhelmsia. Roнв. Merlia. Fisch, non Borkh. - 6. Cerastiumx

$$
\text { B. Caryophylleac. }
$$

5355. Drypis. L.

5356. Brachystemina. Don.

535\%. Velezia. L.

5358. D i anthus. L.

5359. Gypsophila. L. a. Banffya. Baumg. *)

b. Struthium. Ser.

c. Rokejeka. Forsk.

d. Petrorhagia, Ser. 5360. S a ponaria. L. a. Proteina. Ser.

*) Conf. Ic. plant. crit. 585.

*) Conf. Ic. plant. crit. 586. 
b. Bolanthus. Ser,

c. Bootia. Neck.

d. Saponaria. Neck.

5361. Vaccaria. Dod.

5362. Lychnis. L.

a. Githago. Desf.

b. Agrostemma. L.

c. MIuscipula. Riv.

Coronaris. I.

d. Eulychnis. DC.

c. Viscaria. Riv.

5363. Silene. L.

a. Ocymastrum, Riv.
Corone. Hifgg.

Stachyomorpha. Otth.

Alocion. Otth.

b. Viscago. Hall.

Siplenomorpha. Otth.

Behenantha. Otth.

c. Otites. Tabern.

Nanosilene. Otth.

Rupifraga. Otth.

Otites. Otth.

5364. Cucubalus. L. e. e.

Scribaca. G. M. S.

Lychnanthus. Gm.

\section{Ma $\boldsymbol{l}_{\boldsymbol{p}} \mathrm{igh} \boldsymbol{i} \boldsymbol{e} \boldsymbol{a}$.}

a? Schiede e re.

5365. S chied ea. Cham.

$$
\text { b. } B \text { anisterieat. }
$$

5366. Hiraea. K. H. В. 536\%. M a s cagnia. Bert. 5368. Triopteris. L. 5369. Tetrapteris. Cav. 5370. Banisteria. L.

5371. Heteropteris. K. H. B.

a. Heteropteris. V.

b? Anomalopteris. DC.
5372. Hiptage. Gäntn.

Giürtncra. Schreb.

Molina. Cav.

5373. Tristellaria. A.P.Th.

Zymum. Nor.

5374. Thryallis. L.

5375. Gaudichaudia.K.H.B.

5376. C a m a rea. A. St. Hil.

$$
\text { c. Ga } \boldsymbol{C}_{\boldsymbol{p}} \boldsymbol{h} \text { i m i e a e. }
$$

537\%. Caucanthus. Forsk. 5380. Byrsonima. Rrch.

5378. G alphimia. Cav. 5381. Malpighia. L.

5379. Bunchos i a. Juss.

\section{FAMILIA CXVIII.}

TH E A C E A E. Theacen.

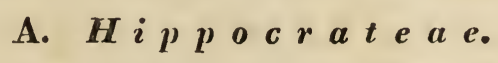

5382. Hippocratea. L. Coa. Plum.
Anthodus. Mart. 5384. Raddisia. Leand. 5383. Anthodon. Rz. PAv. 5385. Salacia. L. 
208 CLASS. VIII. THALAM. ORD. III.IDIOC. FAM. CXVIII. THEAC.

Tontelea. Aubl.

Tonsella. Schreb.

Calypso. A. P. Th.

Sicclium. Brw.
5386. Johnia. RxB.

538\%. Trigonia. Aubr. 5388. Lacepedea. K. H. B. Triceros. Lour.

B. Celastreac.

5389. Evon y us. T. 5390. Celastrus. L. Catha. Forst.

Evonymoides. Mnch.

5391. Ma y tenus. Feurl. 5392. Alzatea. Rz. PAv 5393. P oly cardia. Juss.
5394. Elaeodendron. Jcẹ. Rubentia. Commers. Schrebera. Rtz.

5395. Ptelidium. A. P. Th. Seringia. Spr. non Gay. 5396. T ralliana. Louk. 5397? Perottetia. K. H. B.

C. Ternströmieae.

5398? Microstemma. LaB. 5411: Reinwardta. BuUne.

5399. Thea. L.

5400. Caraipa. Aubl. 5401. Kielmeyera. Mart. 5402. Marila. Sw. e. e. 5403. Mahurea. Aubl.

Bonnetia. Schreb.

5404. Architaea. Mart. 5405. Bonnetia. Mart. non Schreb.

5406. Ventenatia. P. B. 540\%. C a mellia. L. 5408. Gordonia. ELx.

a. Lacathea. Salisb.

b. Lasianthus. DC. 5409. Schima. Rernw. 5410. L a p l a ce a. K. H. B. Haemocharis. Salisb.
Blumia. Spr.

5412. Malach odendron. CAv. 5413. Stewartia. CAv. 5414. Sauraja. W.

a. Saurauja. DC.

b. Apatelia. DC. Polava. Rz. Pav.

5415. Ge eria. Blume. 5416. Eurya. Thnв. 541\%. Lettsomia. Rz. Par. 5418. Freziera. Sw. 5419. Cl e yera. ThNB. 5420. Ternstroemia. Mur. Tonabo. Aubl.

5421. Cochlosperm um.K.H.B. ITaximilianea. Mart. Wittelsbuchia. Mart.

FAMILIA CXIX.

T I L I A C E A E. ThIACER.

a. $T r e m a n d r e a e$.

5422. Tetratheca. Sm. 5424. Godoya. Rz. PAv. 5423. Trem andra. RBr. 


\section{b. Elceocarpeae.}

5425. Friesia. DeC. 5426. Dicera. Fonst. 542\%. V all ea. Mut. 5428. Tri cuspidaria. Rz.P.Av. Tricuspis. P. 5429. Ageratium. DeC.
5430. Elaeocarpus. L. Lochneria. Scop.

5431. Acronodia. Blume. Acrozus. Spr.

5432. V ateria. L.

\section{c. Tilieae.}

5433. Triumfet $\mathrm{t}$ a. L.

a. Triumfetta. G.

b. Bartramia. G.

5434. Porp a. Blume.

5435. B I u m e a. Richв.

Esenbectia. Bl. non Brid.

5436. Hon ck e ny a. W. 543\%. Sparmannia. ThNe. 5438. Entelea. RBr.

5439. Hasseltia. K. H. B. 5440. A blania. Aubl.

Trichocurpus. Schreb.

5441. H o u miria. Aubr. em.

Houmiri. Aubl. cf. 3595.

Verniselia. Scop.

IIyrodendron. Schreb.

Humiria. DC.

5442? Abatia. Rz. Puv.

5443? Helio c a r pus. L.

Montia. Houst.

5444. Antichorus. L. fil.

5445. Corch orus. L.

a. Coreta. P. Br.

b. Coretoides. DC.

c. Ceratocoreta. DC.

d. Gauja. Rmph.

e. Guazumoides. DC.

5446. Grewia. L.
Mricrocos. $\mathbf{L}$.

5447? Dodecadia. Lova.

5448. Columbia. Pers.

Colona. Cav.

5449. Tilia. L.

a. Tilia. R.

b. Lindnera. $R$.

5450. D iplophractum. DESF.

5451. Muntingia. L.

5452. A peiba. Aubl.

Aubletia. Schreb.

Oxytandrum. Neck.

5453. S l o a nea. L.

a. Sloanea. Plum.

b. Gynostemon, DC.

c. Myriochaeta. DC.

d. Oxyandra. DC.

e. Foveolaria. DC. non Rz. Pav.

5454. Espera. Willd.

5455. Christiania. DeC.

5456. Lii he a. Wintd.

545\%. Alegria. Moc. Sess.

5458. Vatica. L.

5459. Wickströmia. Schrad.

5460. B er y a. Rxв.

5461. Trilix. L.

Jacquinia. Mut.

5462? Oncoba. Forsk. 
FORMATIO II.

\section{A U R A N T I I F L O RA E. ORANGENBLUETHLER.}

FAMILIA CXX.

HYPERICINEAE. HyPERICINEEN.

a. Lineae.

5463. Radiola. Drux.

5464. Linum. L.

b. $\boldsymbol{H} y \boldsymbol{p}$ er $\boldsymbol{i} \boldsymbol{c} \boldsymbol{e} \boldsymbol{a} e$.

a. Eucryphieac.

5465. Eucryphia. Car. 5466. Carpodontos. LaB.

\section{B. Ascyreae.}

5467. Sarothra. LAM.

5468. A s c y rum. L.

Hypericoides. Ad.

5469. Lancretia. DeL.

54\%. Cratoxylon. BuUme.

5471. Hypericum. L.

a. Brathys. Mut.

b. Hypericum.
Perforaria. Chois.

c. Tridesmos. Chois.

d. Ascyreia. Chois.

5472. Triaden ium. Rafin. Martica. Spr. non Leand. Elodea. Prsh.

5473. Androsaemum. Arr.

y. Vismieae.

5474. Vismia. VAND. 5475. Ha ronga. A. P. Th.

Harongana. Lam.

Arongana. $\mathbf{P}$.

Haemocurpus. Noronh.

c. Chlaenaceae.

5476. Rhodolaena. A.P Th. 5478. Leptolaena. A. P. Th. 647\%. Schizolaena. A. P. Th. 5479 Sarcolaena. A. P. Th.

FAMILIA CXXI.

\section{GUTTIFERAE. GuTtifEREN.}

a. Marcgravieae,

5480. Antholoma. LaB. 5482 Norantea. Aubr. 5481. Marcgravia. Plum. Ascyum. V. 5483, R u y s ch i a. JcQ. Souroubea. Aubl.

b. Clusiene.

5484. Haretia. К. Н. В. 5485. Arrudea. САмв. 
5486. Cl usia. L. Quopoya. Aubl. Xanthe. W.

5487. Verticillaria. Rz. P^v. Chloromyron. $\mathbf{P}$.
5488. Tovomita. Aubl. Marialva. Vand. Becuharnoisia. Rz. Pav. Ochrocarpus. A. P. Th. Micranthera. Chois.

$$
\begin{gathered}
\text { c. Garcinieae. } \\
\text { a. Calophylleae. }
\end{gathered}
$$

5489. M esua. L. 5490. Galophyll um. L.

B. Mangostaneac.

5491. Stalagmites, Murr. Xanthochymus. Rxb. Brindonia. A. P. Th. Oxycurpus. Lour.

5492. Garcinia. L. Cambogia. L. Mangostanu. G.
5493. Rheedia. L. 5494. Mammea. L. 5495? M a c a n e a. Juss. Macahanea. Aabl. 5496? Macoubea. Aubz. 5497? Ascra. Schoтr.

y. Chrysopieate.

5498. Chrysopia. A. P. Th. 5499. Moronobea. Aubr. Symphonia. I. fil.

FAMILIA CXXII.

hesperideaE. Hesperideen.

a. Leeaceac.

5500. L e a. L. 5502. Lasianthera, P. B. 5501? Touroulia. Aubx. 5503? Odontandra. K. H. B. Rotinsonict. Schreb.

$$
\begin{aligned}
& \text { b. } \quad M \text { e } l \text { i } e \text { a } \\
& \text { a. Strigilieae. }
\end{aligned}
$$

5504? Amoora. Roxb.

5505. Melia. L.

5506. S andoricum. CAv. 5507. Strigilia. Car. Foveolaria. Rz. Pav. Tremanthus. P.
5508. Quivisia. Cомm. Gilibertia. Gm. 5509, Turraea, L. 5510? G e ru m a. Forsk. 5511. Canella. BRw. Wintercena. L. 
212 CLASS. FIII,THALAM. ORD. II. IDIOC. FAM. CXXII. HESPER.

\section{B. Trichilicae.}

5512. Trichilia, L. Elcaja. Juss. Portesia. Juss.
5513. Ekebergia. Sparm, 5514. Gu a rea. L. 5515. Hеуnea. Roxв.

y. Cedreleae.

5516. C edrel a. L. Cedrus. Mill.

5517. Swietenia. L.
5520. Carapa. Aubl. Xylocarpus. Schreb. Persoonia. W. 5518. Chloroxylon. DeC. 5521. Macarisia, A. P. Th. 5519. Flindersia. RBr.

\section{c. Auranticuc.}

5522. Atalantia, Corr. 5523. Triph a ca, Lour, 5524. Limonia. L. 5525. Cookia, Sonn. Lansium. Rmph. Quinaria. Lour. 5526. Bergera. KöN. 552\%. Murraya. KöN.
MIarsana. Sonn. 5528. Aglaia. Lour. 5529. Clausena. Burm. 5530. Glycos mis. Corr. 5531. F e ronia. Corr. 5532. A egle. Corr. Belon. Ad. 5533. Citrus, L. 


\section{$\begin{array}{llllllll}\text { A } & \mathbf{D} & \mathbf{D} & \mathbf{E} & \mathbf{N} & \mathbf{D} & \mathbf{A} & *\end{array}$}

100, Medusula. Tode.

453 b. M i cromega, AG.

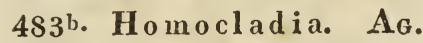
489b. Alisidium. Ag. 490 b. Tha um asia. Ag. 498b. Dasia. Ag. 498. S tichocarpus. AG. 508 b. D ictyo pt e r is. Lamx. Ncurocarpus. W. MI. 530d. Blasia. L. 679b. Cyst opt te r is. Benri. 794. Polyperse. Lour. 7941. Sagittaria. L. 939b. Monachne. P. B. 953\%. Limnas. Trin.

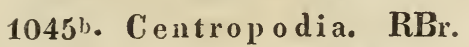
1082b. G ussonea. Prsc. 1085 b. Albikia. Prst. 1130?. Lilaea. Hв. BPL。 117\%l. Cruiks hankia.Mrers. 1184. Genosiris. LaB. 122\%b. P öp pigi a. Kz. chil. 1236" Clivia. Lindl. 1355'. Tulipa. L. 1381. Tulbaghia. L. reducatur post No. 135\%, nil 2452. Impia. N. v. E. enim aliud videtur nisi Gil- 2453. Xerotium. N. v. E. liesiacearum, quae incom- 2455. Achctriterium. N. v. E. plete suan seriem explent, 248\%i. Arnica. L. forma absoluta, Alliaceis on- 2538b ? Achyrosteph us. Kz. nino aliena.

* Difficillima fuit correctio qunin imprimeretur Lipsine libellus, Dresdae contra habitaren ego. 
2743b? Acharia. Thns. 2785. Moquinia. Spr. 3597a. G laux. L. Trientalianteponenda.

subscribatur (secundum insti- 36471, Embryopteris.GAert. tutum examen, cf. Ic. exot. Cavanillea. Lam.

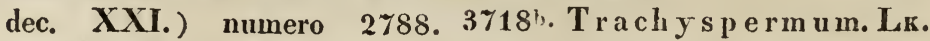
synonymon.

2791b. Strumpfia. L. 3053. Mylanche. Wallr.

Leptamnium. Rafin. $3931^{\text {l' }}$ ? Steganotropis. LEHM.

40\%2. W e ihe a. Rснв. Burtonia. RBr. non Salisb. 3054b. Con opholis. Wallk. 42s8. Lyonic. Rafin. non al. 3058. H a e modo ron. Wallr. 4332. Louichea. l'Herit. non S. 4335. Cerciospermum. Schreb. 3060". A noplon. Walle. Krascheninnikovia. Güldenst.

Orob. III. Anoplon. Willr. 4421". Praesepiu in. SPR. ante Alectram. 3126. Ge is someri a. Lindr. 3224. Streptocarpus. Lindl. 3381. Daboecia. Ray. 3503b. G a r d neria. WALL. 3588. Hunc numerum sequens adnotatio sic continucanda: $\mathbf{E x}$ Jussieur Rubiaceis p. 15. omnino quidem genus Paederiae analogon, sed capsulare. 5360? Hagenia. Minch.

4424') Ham a ri a. Kz. chil.

443\%. Pancovia. Heist. 4527\%. Marlea. Roxв. 5113". Gristinia. Fonst. non Necr.

Scopolia. Forst.

5310b. Juergensia. Spr. Medusa. Lour.

Medusula. Pers. non Tode nec Eschw. 


\section{VIX C O G N I T A,}

nondum vel fragmentarie descripta.

5534. Adolia. Rheed. 5535. Antelaea. G̈̈rt. 5536. Apactis. Tunb. 553\%. A patilia. Hamilt. 5538. B accaurea. Lour. 5539. Bait aria. Rz. PAv. 5540. B al an tium. Hamilt. 5541. Banjol ea. Bowd. 5542. B embix. Lour. 5543. Bigelovia. Spr. Entp. 5544. B rickellia. Rafin. Spogopsis. Mchx. 5545. C a m p y lus. Lour. 5546. Catonia. Brw. 5547. Corallophyll um. Hв. BPL. 5548. Chilmo ria. Hamilt. 5549. Di cal yx. Lour. 5550. Duraucellia. Bowd. 5551. Euthemis. Jack. 5552. Findlaya. Bowd. 5553. F lo sc opa. Lour.

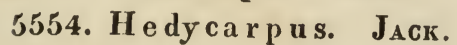
5555. Helicanthera. Lour. 5556. Heptaca. Lour. 5557. Hilbertia. Thour. 5558. H ornschuchia.N.v.E. 5581. Smeguaria. W.
5559. Hornschuchia. Sipr. Ento. 5560. Hymenodictyon. WALL.

5561. Keiria. Bowd. 5562. L a terradea. Anval. EURop. 5563. Luechea. L. 5565. Manoellia. Bowd. 5566. Moldenhauera. Spr. non Schkd. Cavanilla. Thnb. 556\%. Myrmecodia. JАск. 5568. N e m um. Hamilt. 5569. Neowedia. Schrad. 55\%0. Ottonia. Spr. 5571. Pancovia. W. 5572. Pedicellia. Lour. 5573. Physema. Nor. 5574. Pierardia. Rxb. 5575. Plegorrhiza. Mour. 5576. P terotum. Lour. 557\%. Puralia Hamilt. 55\%8. Pythagorea. Lour. 5579. Sassia. Mourn. 5580. S eguiera. L. 
5582. S poud y loc occus. W. 5590. Trichocladus. Pers. non Mrtch. Daklia. Thnb.

5583. St ephania. Lour. 5584. Stixis. Lour. 5585. Striga. Lovr. 5586. Syudesmis. Walz. $558 \%$. Sy mphorema. Roxi. 5588. T o binia. Hamir. 5591. 'Triphaca. Lour. 5592. Varonthe. Juss. 5593. Vils henica. Thov. 5594. Viviania. CAv. 5589. Trewia. L. 


\section{N D E G G L LICO-LATINUS.}

Obs. omissa sunt utriusque linguce homonyma.

Abalon: Helonias. Abandion: Bulbocodium. Abérème: Aberoma. Ablanie: Ablania. Abricotier: Armeniaca. Abricotier-Alberge. Acajou : Casuvium. Acanthe: Acanthus, Dilivaria. Acéna: Acaena. Acharie: Acharia. Achillée: Achillea. Achimènes : Achimenes. Achit : Cissus. Achmée: Aechmea. Acianthe: Acianthus. Acicarphe: Acicarpha. Acisanthère: Acisanthera. Acmelle: Acmella. Acnide: Acnida. Acomas : Acoma. Aconit : Aconitum. Acore: Acorus. Acouroa: Acuroa. Acrostique : Acrostichum. Actée : Actaea. Actinée: Actinea. Adlambé: Adambea. Adlelie: Adelia. Adène: Adema. Adenostème: Adenostemum. Adlianthe: Adianthum. Adole : Adolia. Adonide: Adonis. Aegérite: Aegerita.
Aegicère: Aegiceras.

Agalloche: Excoecaria. Agaric: Agaricus. Agathomeride: Agathomeris. Agave: Agave. Agérate: Ageratum. Agnanthe: Cornutia. Agripaume: Leonurus. Aïdia : Aïlia.

Aigremoine: Agrimonia. Ail : Allium. Airelle: Vaccinium. Aitchar: Atchar. Aitone: Aitonia. Ajonc: Vlex. Ajouvé: Ajovia. Akée: Akeesia. Albergine: Solan. Melongena. Alcée : Alcea. Alchemille: Alchemilla. Alcornée: Alchornea. Allée: Aldeaea. Aldine: Aldina. Aldrovande: Aldrovanda. Alectorolophos: Alectorolophus. Alectre: Alectra. Alétris : Aletris. Alévrite: Aleurites. Alihoufier: Styrax. Alkenge: Physalis Alkekengi. Allamande - Orélia: Allamanda. Allasie: Allasia. Alleluja : Oxalis. Allione: Allionia. 
Allophile: Allophyllus.

Allosore: Allosorus.

Aloës : Aloë.

Alpam: Apama.

Alpinie: Alpinia.

Alpiste: Phalaris.

Alsodée: Alsodeia.

Alstone: Alstonia.

Alternanthère: Alternanthera.

Alrarde: Lygeum.

Alypon: Globularia Alyp.

Alysse: Alyssum.

Alzatée : Alzatea.

Amandier: Amygdalns.

Amanite: Amanita.

Amanoier: Amanoa.

Ainansie: Ainansia.

Amaranthe: Amaranthus.

Amaranthine: Gomphrena.

Amarillis : Amaryllis.

Amasonie: Amasonia.

Ambararate: Cajanus.

Ambelanier: Ambelania.

Amblyoda: Amblyodum.

Ainbrette: Centaurea suaveolens.

Ambrerate: Cajanus.

Ambrome: Abroma.

Ambrosie: Ambrosia.

Ambrosinie: Ambrosinia.

Ambulie: Ambulia.

Amelle: Amellus.

Ainethystée: Amethystea.

Ammane: Ammania.

Amome: Amomum.

Amorphe: Amorpha.

Amphipogone: Amphipogon.

Aubase: Anabasis.

Anacarde: Anacardium.

Anacycle: Anacyclus.

Anagire : Anagyris.

Ananas: Anauassa, Bromelia.

Anarrhine: Anarrhiunn.

Anartria: Anarthria.

Anavingue: Anavinga.

Anazé, Anazue: Adans.dig.

Ancitre: Ancistrum.
Ancolie: Aquilegia.

Andarèse: Premna.

Andersone: Andersonia.

Andrachne: Andrachne.

Andrée: Audreaea.

Andreusie: Andrewsia.

Andromède : Andromeda.

Androsace: Audrosace.

Androsème: Androsaemum.

Andryale: Andryala.

Anemone: Anemone.

Aneth: Anethun.

Angelin : Andira.

Angélique: Angelica.

Angolan: Alangium.

Angourie: Anguria.

Angrec: Epidendrum, Cymbidium.

Anibe: Aniba.

Anictangie: Anoectangium.

Anigosanthe: Anoegosanthos.

Anisomèle: Anisomoles.

Anode: Anodia.

Allome : Anoma.

Anrèdra: Anredera.

Ansérine: Chenopodiun.

Antelée: Antelaea.

Anthérie: Anthericun.

Anthérilier: Antherylium.

Antherure: Antherura.

Anthistirie: Anthesteria.

Anthocère: Anthoceros.

Athocone: Anthoconun.

Antholome: Antholoma.

Antholise: Antholyza.

Anthonote: Anthonota.

Anthosperme: Anthospermum.

Anthyllide: Anthyllis.

Autichore: Antichorus.

Antidesme : Antidesmum.

Antofile: Anthophyllus.

Antrogynette: Stachygynandrunn.

Anvali: Phyllanthus Emblica.

Anychie: Anychia.

Aote: Aotus. 
Apactis : Apactis. Apalanche: Prinos. Apalatou: Apalatoa. Aporétic : Aporetica. Apargia: Apargia. Apeiba: Apeiba. Aphacé: A phaca. Aphytée: Aphytea. Aplude: Apluda. Apociu : A pocyuum. Apouget: A ponogeton. Aquart: Aquartia. Aquilice : Aquilicia. Arabette: Arabis, Arachide: Arachis. Aralie: Aralia. Arbute: Arbutus. Archangelique: Archangelica. Arctione: Arctiun. Arctoticle: Arctotis. Arec: Areca. Arethuse: Arethusa, Disperis. Arétie: Aretia. Argan : Sideroxylon. Argemone: Argemone. Argentaire: Argyreia. Argitame: Argythannia. Argolase: Argolasia. Argophylle: Argophyllum. Argoussier: Hippophaë. Aristicle: Aristida. Aristoloche: Aristolochia. Arjone: Arjona. Armoise: Abrotanum. Armoselle: Seriphium. Arnosère: Arnoseris. Arounier: Aruna. Arousse: Arachus. Arroche: Atriplex. Artédie: Artedia. Artichaut: Cynara. Arube: Aruba. Asaret: Asarum. Ascarine: Ascarina. Asclepiade: Asclepias. Ascophore: Ascophora.
Ascyre : Acyrum. Aspalat: Aspalathus. Aspérelle: Asperella. Asperge: Asparagus. Aspérule: Asperula. Asphodèle: Asphodelus. Aspilie : Aspilia. Assiminier, Corossolier : Asimina. Assone: Assonia. Astère: Aster. Asterelle: Asterelia. Astérisque: Asteriscus. Asteroide: Asteroides. Asterope: Asteropeia. Asteroptère: Asteropterus. Astragale: Astragalus. Astrance: Astrantia. Astranthe: Astranthus. Astroin: Astronium. Athamante: Athamantha. Athanasie: Athanasia. Athécie: Athecia. Atractobole: Atractobolus. Atraphace: Atraphaxis. Atrichie: Atrichou. Aubergine: Solanum Melongena. Aubertia, Ampacus: Aubertia. Aune: Alnus.

Avicenne: Avicennia. Avoine: Avena, Danthonia. Avoira: Elais. Axie: Axia. Axinée: Axinaea. Axiris : Axyris. Ayenue: Ayenia. Azalée : Azalea. Azédarac: Melia Azederach. Azime : Azima. Azolle: Azolla. Azorelle : Azorella.

Bacasie: Bacazia. Baccanthe: Baccharis. Bacie: Echinaria. Bacille : Crithmum. Bacope: Bacopa. Badamier: Terminalia. 
Bagassier: Bagassa.

Baguenaudier: Colutea.

Baillière: Baillieria.

Balanophore: Balanophora.

Balbisie : Ballisia.

Balétuvier: Bruguiera.

Balimba, Bolimba : Averr. Bil.

Ballotte : Ballota.

Balsamier : Amyris.

Balsamine: Balsamina, Impatieus.

Balsamite: Balsamita.

Bambage, Bambagia: Bombax.

Bambou: Bambos.

Bananier: Musa.

Banare: Banara.

Bancsie : Banksia.

Banistère: Banisteria.

Baquois: Pandanus, Athrodac-

Barbacène: Barbacenia. tylis.

Barbon: Andropogon.

Bardian : Illicium.

Barnadez: Barnadesia.

Bartone: Bartonia.

Barixyle: Baryxylum.

Baselle: Basella.

Basile: Basilaea.

Basilic: Ocymum.

Bassove: Bassovia.

Batarrée: Batarrea.

Batis: Batis.

Banhine: Bauhinia.

Beanharnoise: Beanharnoisia.

Beckmannie: Beckmannia.

Refare: Bejaria.

Bégone: Begonia.

Béholte : Caryophyllata.

Béjuco: Hippocratea.

Belladone: Atropa.

Bélnoite: Genm.

Bénoite: Caryophyllata.

Béole: Baea.

Bergie: Bergia.

Berle: Sium.

Bertière: Bertiera.
Beslere: Besleria.

Bétoine: Betonica.

Bette: Beta.

Bignone: Bignonia.

Bihai: Heliconia.

Blacoues : Blackwellia.

Blairie: Blairia.

Blasie: Blasia.

Blatti : Someratia.

Blegue : Blechum.

Blête: Blitum.

Bluet : Cyanus.

Boabab: Adansonia.

Boccone: Bocconia.

Boisivrant: Piscidia.

Bolet: Boletus.

Boltone: Boltonia.

Bonamie: Bonamia.

Bonduc: Guilandina.

Boopis: Boopis.

Boraquèbe : Barreria.

Bosé: Bosea.

Bossie: Bossicua.

Boucage : Pimpinella.

Bouleau: Betula.

Boulésie: Bowlesia.

Bourrache : Borrago.

Brabei : Brabeium.

Bradlée: Bradleia.

Bragantie: Bragantia.

Bratis: Brathys.

Bressillet : Caesalpinia.

Buidonier: Bridonia.

Brignole : Brignolia.

Brodie: Brodiaca.

Brome: Bromus.

Broualle: Browallia.

Brosime: Brosimum.

Brossé: Brossea.

Brotère : Brotera.

Bronne: Brownea.

Brucé : Brucea.

Brunelle: Prunella.

Brunie: Brunia.

Bruyere: Eria.

Bruguière: Bruguiera. 
Bry: Bryum.

Bryone: Bryonia.

Bubon: Bubon.

Buchuère: Buchnera.

Budlèje: Bulleja.

Bufone: Buffonia.

Bugle: Ajuga.

Buglosse: Auchusa.

Bugrane: Ononis.

Buis : Buxus.

Bulbocode: Bulbocodiun.

Bullaire: Bullaria.

Bumalde : Bumalda.

Bunion: Bunium.

Burmane: Burmannia.

Bursaire: Bursaria.

Buttuère: Biittuera.

Butome: Butomus.

Butonie: Butouica.

Buxbaume: Buxbaumia.

Cabrillet: Ehretia.

Cacalie: Cacalia.

Cacaoyer: Cacao.

Cacoucier: Cacoucia.

Cactier: Cactus.

Cactiers - Nopalées.

Cadaba: Cadaba.

Cadelari : Achyranthes.

Caffeyer: Coffea.

Caïnitier: Chrysophyllum.

Calaba: Calophyllun.

Calabure: Muntingia.

Calac: Arduina, Carissa Carandas.

Caladénie: Caladenia.

Calapitte: Calappa.

Calcéolaire: Calceolaria.

Calectasie: Calectasia.

Calebassier: Crescentia.

Caligni: Licania.

Calle: Calla.

Callicarpe: Callicarpa.

Calligon: Calligonun.

Callisperme: Callispermum.

Calliste: Callista.

Callitric: Callitriche.

Callixène: Enargea.
Callixène: Callixene.

Calodendron: Calodendrum.

Calomère: Calomeria.

Calostème: Calostemma.

Calpidie: Calpidia.

Calumet: Mabea.

Calycant: Calycauthus.

Calycoptère: Calycopteris.

Calyptranthe: Calyptranthes.

Calystegia : Calystegia.

Calytrice: Calythrix.

Calytriple: Calytriplex.

Camarine: Empetrum.

Camboge: Cambogia.

Camecerisiers: Chamaecerasus.

Camelée: Cneorum.

Cameline: Myagrum, Camelinə.

Camelli : Camellia.

Camiri : Camirium.

Camomille: Anthemis.

Campanule: Campanula.

Campêche: Haematoxylon.

Camphréc: Camphorosma.

Campyle: Campylus.

Canabine: Datisca.

Canamelle: Saccharum.

Canang: Uvaria, Apacare.

Canari: Canarium.

Canerine: Canarina.

Canche: Aira.

Candori : Adeuanthera.

Canelle: Canella.

Canéphore: Canephorá.

Canne: Canna.

Cansjan: Canscora.

Cansjava: Canuabis.

Capucine: Tropaeolum.

Carambolier: Averrhoa.

Cardère: Dipsacus.

Cargillie: Cargillia.

Carline : Carlina.

Carmantine: Justicia, Dicliptera.

Caroubier: Ceratonia.

Carpodet: Carpodetus.

Carpodonte: Carposlontos.

Carpolépide: Carpolepidum. 
Caryobole: Caryobolis.

Caryophyllées: Caryophylleae.

Caryote: Caryota.

Cassipourier: Cassipourea, Legnotis.

Cassyte: Cassyta.

Castillée: Castilleia.

Cassupe: Cassupa.

Catesbée: Catesbaea.

Cature: Caturus.

Caulerpe: Caulerpa.

Caulophylle: Caulophyllum.

Cebathe: Cebatha. Menisp. edule.

Cédrel: Cedrela.

Celachnée: Celachne.

Célastre: Celastrus.

Celsie : Celsia.

Cenarrhènes : Cenarrhenes.

Centaurée: Centaurea.

Centenille: Centunculus.

Centranthère: Centrauthera.

Céphalanthe: Cephalanthus.

Cephalophore: Cephalophora.

Céphaloxe: Cephaloxys.

Céraiste : Cerastium.

Ceratanthère: Ceratanthera.

Ceratiole: Ceratiola.

Ceratocarpe : Ceratocarpus.

Cératopétal : Ceratopetaluın.

Cératosperme: Ceratospermum.

Cératostemme: Ceratostemma.

Cercollée: Cercodea.

Cerfeuil: Authrisc. Cerefol.

Cerium: Cerium.

Ceropège : Ceropegia.

Ceroxylon: Ceroxylon.

Cervantèse: Cervantesia.

Cestreau: Cestrum.

Césulie: Caesulia.

Chalef: Elacagusus.

Chamire: Chamira.

Champac: Michelia.

Chancelague: Chancelagna, Gentiana peruv.

Chantarelle: Cantharellus.

Chanvre: Cannabis.
Charagne: Chara.

Cha racher: Charachera, Lant. vib.

Châtaignier: Castanea.

Chataire: Nepeta.

Chaussetrape: Calcitrapa.

Chayaver: Chaya, Oldenland. umbellata.

Chêne: Quercus.

Chélidone: Chelidonium.

Chenillette: Scorpiurus.

Cherlère: Cherleria.

Chevrefeuille : Louicera.

Chicorée: Cichorium.

Chicot: Gymnocladus.

Chigomier: Combretum.

Chionanthe: Chionanthus.

Chirone: Chironia.

Chloanthe: Chloauthes.

Chlore: Chlora.

Chlorophyte: Chlorophytum.

Choill: Schoenus.

Chorêtre: Choretrum.

Chorizandre : Chorizandra.

Chou : Brassica.

Chrisopie: Chrysopia.

Chunco: Chuncoa, Gimbernatiil.

Ciche: Cicer.

Cicutaire: Cicutaria.

Cienfuegosa: Fugosia.

Cigiie: Cicuta.

Cimicaire: Cimicifuga.

Cinéraire: Cineraria.

Ciocoque: Chiococca,

Cipon: Ciponima.

Cipure: Cipura.

Circée : Circaea.

Ciste: Cistus.

Clandestine: Amblatnum.

Clandestine: Lathraea.

Clathre: Clathrus.

Clavaire: Geoglossum.

Clavaire: Clavaria.

Claytone: Claytonia.

Clématite: Clematis, Atrajène.

Clethra : Clethra.

Cleyere: Cleyera. 
Clibale : Clibadiun.

Clifforte: Cliffortia.

Clinopode: Clinopodium.

Clitorie: Clitoria.

Clusier: Clusia.

Clutelle: Clutia.

Clypéole: Cly peola.

Cobéa : Cobaea.

Cobrésie: Cobresia.

Coccocipsile: Coccocy psilum.

Cocotier: Cocos.

Cocrête: Gymnandra.

Colchique: Colchicum.

Coldène: Coldenia.

Colletier: Colletia.

Collinsone : Colliusonia.

Colorinéc: Columnea.

Columbie: Columbia.

Columelle: Columella.

Colurellée: Columellea.

Comaret: Comarum.

Comète : Cometes.

Comméline: Commelina.

Commerson: Commersonia.

Comoclade : Comocladia.

Conanthère: Conanthera.

Conceveibe: Concereiba.

Concombre: Cucumis.

Condrille: Chondrilla.

Conferve: Conferva.

Connare: Connarus.

Contarde: Hydrolea.

Coprosine : Coprosina.

Copaier: Copaifera.

Coqueret: Physalis.

Coqueluchiole: Cornucopiae.

Cocjuemollier: Theophrasta.

Cordylie: Cordylia.

Corête: Corchorus.

Coriope : Coreopsis.

Corisperme: Corispermum.

Cornaret: Martynia.

Coruête: Bartsia.

Cornouiller: Cornus.

Corouille: Coronilla.

Corosol: Anona.
Corrée : Correa.

Corrigiole: Corrigiola.

Coryphe: Corypha.

Cosmélie: Cosmelia.

Cotelet: Citharexylon.

Cotonnier: Gossypium.

Cotule : Cotula.

Cotylet : Cotyledon.

Coublande: Coublandia.

Coulequin: Aınbaiba.

Coumarou: Coumarouna.

Comnier: Couma.

Coupi : Acioa.

Couratari : Couratari.

Courbaril : Hymenaea.

Courge: Cucurbita.

Couroupite: Couroupita.

Coussapier : Cosapoa.

Coussari: Coussarea.

Coutoubée : Contoubea.

Cranique: Cranichis.

Crauson: Cochlearia.

Crapandine : Sideritis.

Crassule : Crassula.

Crenée: Crenea.

Cresse: Cressa.

Cretelle: Cynosurus.

Cretelle: Dineba.

Crignon: Bucida.

Crinole: Crinum.

Crisite : Chrysithrix.

Crisocome : Chrysocoma.

Crisogone: Chrysogonum.

Crossostyle: Crossostylis.

Crotolaire : Crotolaria.

Crouée: Crowea.

Crucianelle: Crucianella.

Cruzite: Crucita.

Cryptocary : Cryptocarya.

Cucubale: Cucubalus.

Cuellaire: Cuellaria.

Cumin: Cuminum.

Cumpani : Cupania.

Cunile: Cunila.

Cunone: Cunonia.

Cupidone: Catananche. 
Curatelle : Curatella.

('urcuma: Curcuma.

Cussonie: Cussonia.

Cuvière: Cuviera.

Cyanelle: Cyanella.

Cyathée: Cyathea.

Cycas: Cycas.

Cyclame: Cyclamen.

Cylindrie: Cylindria.

Cyliste: Cylista.

Cymbalaire: Cymbalaria.

Cynoglosse: Cynoglossum.

Cynomètre: Cynometra.

Cynomoire: Cyuomorium.

Cyprès: Cypressus.

Cyrille: Cyrilla.

Cyrta: Cyrta.

Cyrtandre: Cyrtandra.

Cyrtanthe: Cyrtauthus.

Cyrtostylis: Cyrtostylis.

Cystance: Cystanche.

Dactyle: Dactylis.

Dalberg: Dalbergia.

Dalćchampe : Dalechampia.

Dampiere: Dampiera.

Danaé : Danaea.

Danaïle : Danaïs.

Danä̈le : Paederia.

Daphnot: Pontia.

Dattier : Phoenix.

Davallie: Davallia.

Daville : Davilla.

Décadie: Decadia.

Decumaire: Decumaria.

Defforge: Defforgia, Forgesia.

Déguele: Deguelia.

Deidamie: Deidamia.

Délime: Delime.

Delphinette: Delphinium.

Dentaire: Dentaria.

Dentelaire: Plumbago.

Dentelle: Dentella.

Dentidie: Dentidia.

Detar: Detarium.

Deutzie: Deutzia.

Diali: Dialium.
Dianelle : Dianella.

Diapenze: Diapensia.

Diaphore : Diaphorea.

Diatome : Diatoma.

Dicalice: Dicalyx.

Dichapetale: Dichapetalum.

Dichondre : Dichondra.

Dicksone: Dicksonia.

Dictame : Dictamnus.

Dictyoptère: Dictyopteris.

Dictyote: Dictyota.

Didelta : Didelta.

Didymandre : Didymandra.

Didymelée: Didymeles.

Digitale : Digitalis.

Dimérie: Dimeria.

Diode: Diodia.

Dionée: Dionaea.

Diosma : Diosma.

Diphillée : Diphylleia.

Diphise: Diphysa.

Diplacre: Diplacrum.

Diplarrène : Diplarrhena.

Diplazie: Diplaziun.

Dipode: Dipodium.

Disandre : Disandra.

Disodée: Disodea.

Dissolena: Dissolena.

Dober: Dobera.

Dodart : Dodartia.

Dodecade : Dodecadia.

Dodonée : Dodonaea.

Dolic: Dolichos.

Dombey : Araucaria.

Dombey : Dombeya.

Donatie : Donatia.

Doradille : Asplenium.

Dorine: Chrysoplenium.

Doronic: Doronicum.

Doronic: Arnica.

Dorstène: Dorstenia.

Dracocephale : Dracocephalum.

Draconte: Dracontium.

Dragonier: Dracanea.

Drapête : Drapetes.

Driandre : Dryandra. 
Drimis : Drymis.

Drupatris : Dupatris.

Druse : Drusa.

Dryade: Dryas.

Duboisie: Duboisia.

Durante: Duranta.

Durion: Durio.

Dysode: Dysodium.

Dysphanie: Dysphania. Echinier: Echinus.

Echinophore : Echinophora.

Echioïle : Echioïles.

Echionope: Echinops.

Echite: Echites.

Eclipte: Eclipta.

Ectrosie: Ectrosia.

Edère : Oedera.

Edounde: Edwardsia.

Egilope: Aegylops.

Ehrharte: Ehrharta.

Ekeberg: Ekebergia.

Elaterie : Elateriun.

Elatine: Elatine.

Elatostème : Elatostemma.

Eleocharis: Eleocharis.

Elephantusie: Elephantusia.

Eléphantope: Elephantopus.

Ellise: Ellisia.

Elyme: Elymus.

Elyue: Elyne.

Elytraire: Elytraria.

Embothrion: Embothrinm.

Emplèvre: Empleurum.

Encélie: Encelia.

Enchylène: Enchylaena.

Endrach : Humbertia.

Enourou: Enourea.

Enydre : Enydra.

Epacride: Epacris.

Kpéru: Eperua.

Epervière: Hieracium.

Ephemerine: Tradescantia.

Epibate: Epibatium.

Epicia : Epicea.

Epigée: Epigaea.

Epilobé: Epilobium.
Epimède: Epimedium.

Epinard: Spinacia.

Erable: Acer.

Eranthème: Eranthemum.

Erinace: Hydnum.

Erine : Erinus.

Erinéum: Erineum.

Eriocéphale: Eriocephalus.

Eriosperme : Eriospermum.

Erithal : Erithalis.

Ernodée: Ernodea.

Ers: Ervum.

Erysiphe: Erysiphe.

Erythrine: Erythrina.

Escalone: Escallonia.

Ethulie: Ethulia.

Euclée: Euclea.

Eucryphie : Eucryphia.

Eufraise: Euphrasia.

Eupare: Euparea.

Eupatoire: Eupatorium.

Euphorbe: Euphorbia.

Evé: Evea.

Exoacanthe : Exoacantha.

Exocarpe : Exocarpus.

Fagarier: Fagara.

Fagone: Fagouia.

Fagré: Fagraea.

Fallope: Fallopia.

Falkia : Falkia.

Faramier: Faramea.

Fernel: Fernelia.

Ferole: Ferolia.

Feronie: Feronia.

Ferrare : Ferraria.

Ferule: Ferula.

Fétidier: Foetidia.

Fétuque: Festuca, Koelera.

Févier: Gleditschia.

Ficoide: Mesembrianthemum.

Figuier: Ficus.

Filarea: Phillyrea.

Fissilier: Fissilia.

Flagellaire: Flagellaria.

Fléchièr: Sagittaria.

Fléole: Antitragus, Phleum. 
Floscope: Floscopa.

Flonve: Anthoxanthun.

Flugge : Fliiggea.

Flutteau: Alisma.

Fontinale: Fontinalis.

Forestière: Forestiera.

Forskale: Forskolea.

Forstère: Forstera.

Fothergil: Fothergilla.

Fragon: Ruscus.

Franchipanier: Plumeria.

Franklaudie: Franklandia.

Franqueune: Frankenia.

Fransérie : Franseria.

Frène: Fraxinus.

Frezière: Freziera.

Fritillaire: Fritillaria.

Fromage: Bombax.

Froment; Triticum.

Fuchsie: Fuchsia.

Fuirène: Fuirena.

Fumeterre: Fumaria.

Fusane: Fusanus.

Gaillet: Galium.

Gainier: Cercis.

Galactite : Galactites.

Galane: Chelone.

Galardienne: Galardia.

Galaxie : Galaxia.

Galé : Myrica.

Galéope : Galeopsis.

Galiène: Galenia.

Galipier: Galipea.

Gandasnl: Hedychinm.

Ganitre: Elaeocarpus.

Ganitre: Adenodns.

Garance: Rubia.

Gardène : Gardenia

Garidelle: Garidella.

Garo: Aquilaria.

Gaston: Gastonia.

Gatilier: Vitex Agnus.

Gayac: Guaiacnm.

Genarrhenes: Cenarrhenes.

Geniostome: Geniostoma.

Genipayer: Genipa.
Genosiris: Patersonia.

Geutiane: Gentiana.

Geranion: Geraniun.

Gerarde: Gerardia.

Gévin : Gevina.

Germaine: Plectrauthus.

Gesuère: Gesneria.

Gesse : Lathyrus.

Gethille: Gethyllis.

Gingo: Ginkgo.

Ginore: Genoria.

Ginsen : Panax.

Giroflée: Cheiranthus.

Giroflier: Caryophyllus.

Gisèque: Gisekia.

Glabrier: Glabraria.

Glauce: Glaux.

Glayeul : Gladiolus.

Glayuel: Aglaia.

Glinole : Glinus.

Globolée: Globba.

Globulaire: Globnlaria.

Gluttier: Sapium.

Gınelin : Gmelina.

Guaphale: Gnaphalium.

Gnavelle: Scleranthus.

Gnet: Gnetum.

Guidienne: Gnidia.

Gomosie : Gomosia.

Gomphie: Gomphia.

Gonocarpe: Gonocarpus.

Gonolobe : Gonolobus.

Gonzalea : Gonzalagunia.

Gordon: Gordonia.

Gortère: Gorteria.

Goseiller: Ribes.

Gouane : Gonania.

Gollaré: Gouarea.

Gunet: Arum.

Gouets : Aroideae.

Gonttifères: Guttiferae.

Goya: Psidinm,

Graınite : Grammitis.

Granger: Grangeria.

Grasette: Pinguicula.

Gratelier: Cnestis. 
Gratgal: Randia.

Gratiole: Gratiola.

Grémil : Lithospermum.

Grenadier: Punica.

Grenadille: Passiflora.

Greuvier: Grewia.

Griel: Grielum.

Grindélie: Grindelia.

Grislé: Grilsea.

Grone: Grona.

Grouove: Gronovia.

Grubbi : Grubbia.

Guadrette: Rhexia.

Guardiole: Guarliola.

Guettard: Guettarda.

Guier: Guiera.

Guimauve : Althaea.

Guimillie: Gumillea.

Gundèle: Gundelia.

Gunnère: Gunnera.

Guzmane: Guzmanuia.

Gymnauthère: Gymnanthera.

Gymnogyne: Gymnogynum.

Gymnostome: Gymnostomum.

Gymnostyle : Gymnostyles.

Gypsophile: Gypsophila.

Gyrocarpe: Gyrocarpus.

Gyroselle: Dodecatheon.

Haemodore: Haemodorum.

Halesier: Halesia.

Haller : Halleria.

Hamadriade: Hamadryas.

Hamel: Hamelia, Amajoua.

Hantol: Sandoricum.

Haricot : Phaseolus.

Hartoge: Hartogia.

Hastingie: Hastiugia.

Hébenstrête : Hebenstreitia.

Hécale: Hecatea.

Hedwigie: Hedwigia.

Hédycaire: Hedycarya.

Hédyosme: Hedyosmon.

Hédyote: Hedyotis.

Heister: Heisteria.

Hellebore: Helleborus.

Hélenie: Helenium.
Hélianthe: Helianthus.

Hélictère: Helicteres.

Héliocarpe: Heliocarpus.

Héliophile: Heliophila.

Héliotrope: Heliotropium.

Helvelle: Helvella.

Hémanthe : Hemanthus.

Hemérocalle: Heinerocallis.

Hémigène : Hemigenia.

Héminéride: Alonsoa, Hemimeris.

Hémionite: Hemionitis.

Heuchère : Heuchera.

Henné : Acronicha, Lawsonia.

Hépatique: Marchantia.

Hermane: Hermannia.

Hermésie: Herınesia.

Hernandier: Hernandia.

Herniaire: Herniaria.

Hétérosperme : Heterospermum.

Hetre: Fag'us.

Hévé : Hevea.

Hille: Hillia.

Hipociste: Cytinus.

Hippie: Hiplia.

Hippocrèpe : Hippocrepis.

Hirtelle: Hirtella.

Hispidelle: Hispilella.

Hodsone : Hudsonia.

Hoitzie : Hoitzia.

Holosté : Holosteum.

Hombac : Sodada.

Hopée : Hoppea.

Horminelle: Horminum.

Hornemane : Hornemannia.

Horsfielde : Horsfieldia.

Hortense: Hortensia.

Hottone : Hottonia.

Houblon: Humulus.

Houque : Holcus.

Houstone: Houstonia.

Houttuyne: Houttyinia.

Hou: Hex.

Hovène: Hovenia.

Huertée: Huertea.

Hugone: Hugonia. 
Hydnocarpe: Hydnocarpus. Hy drangelle: Hydrangea. Hydroctyle : id.

Hydrophylace: Hydrophylax. IIydrophylle: Hydrophyllum. Hydraste: Hydrastis. Hyoséride: Hyoseris. Hypécoon: Hy pecoum. Hypue: Hypnum. Hysope: Hyssopus. Ibéride : Iberis.

Icacore: Icacorea.

Iciquier: Icica.

Igname: Dioscorea.

Illipé : Bassia.

Immortelle: Xeranthemum.

Imperatoire : Imperatoria.

Incarville: Incarvillea.

Indel: Elate.

Indigotier: Indigofera.

Inocarpe: Juocarpus.

Inule: Inula.

Ipomopses : Ipomopsis.

Irésine : Id.

Iriartée: Iriartea.

Ironcan : Ironcalla.

Isaure; Isaura.

Ischème: Ischaemum.

Isote : Isoetes.

Ité : Itea.

Ivraie : Lolium.

Ixore: Ixora.

Jabrose: Jabrosa.

Jacinthe: Hyacinthis.

Jacquinier; Jacquinia.

Jambolier: Jambolifera.

Jambosier: Eugenia.

Jaquier: Artocarpus.

Jasmin : Jasminum.

Jeffersone: Jeffersonia. Jenevrier: Juniperus.

Jérose : Anastatica.

Joannie: Joannesia.

Jonc: Juncus.

Jouci:elle: Eriocaulon.
Jonciole: Aphyllanthes.

Jonge : Jungia.

Joséphine: Josephina.

Jugoline: Jugcoline.

Julieune: Hesperis.

Jungermane: Jungermannia.

Jusquiame: Hyoscyamus.

Jussiće: Jussieua.

Ketmie: Bamia, Hibiscus.

Killinge: Killinga.

Kleiuhove: Kleinhovia.

Kleinie: Kleinia.

Knautie: Knautia.

Knepier: Melicocca.

Knoxie: Knoxia.

Kramer: Krameria.

Kuhuie: Kuhnia.

Lachenale: Lachenalia, Phormium.

Lachnéé : Lachnaea.

Laet: Laetia.

Lagenule: Lagenula.

Lagerstrome: Lagerströmia.

Laget: Lagetta.

Lagocie : Lagoecia.

Lagunoa: Amirola.

Lagure: Lagurus.

Laiterou: Sonchus.

Laitue: Lacluca.

Lamier : Laminm.

Lampsane : Lampsana.

Lancisie: Lancisia.

Laudolphie: Landolfia.

Langit: Ailanthus.

Languette: Aizoon.

Lappuliue: Triumfetta.

Larmille: Coix.

Larrée : Larrea.

Laser: Laserpitium.

Lasianthère: Lasianthera.

Lasiopétale: Lasiopetalum.

Latanier: Latania.

Laugier: Langieria.

Laurélie: Laurelia.

Laurelle: Cansiera. 
Lauréole: Daphne.

Laurier: Laurus.

Laurose : Nerium.

Lavande: Lavandula.

Lavatère: Lavatera.

Lavenias : Adenostemma.

Lavenie: Lavenia.

Laxınan: Laxmannia.

Lechenaultie: Lechenaultia.

Lède: Ledum.

Lée: Leea.

Léersie: Leersia.

Leflinge: Loeflingea.

Leguée: Lechea.

Lemia: Lemanea.

Lénidie : Lénidia.

Lenticule: Lemna.

Lentisque : Lentiscus.

Léontice : Id.

Lépanthe: Lepanthes.

Lépidagathis : Id.

Lépironie: Lepironia.

Leptosperme: Leptospermum.

Lepture: Lepturus.

Leratone: Crotonopsis.

Lerquée: Lerchea.

Leschen : Antiaris.

Leucosceptre: Leucosceptrum.

Levenhookia: Id.

Leysère: Leysera.

Licuale: Licuala.

Lierre : Hedera.

Lilas : Lilac.

Liméole: Limeum.

Limodore: Limodorum.

Limonellier: Limonia.

Limoselle: Limosella.

Linaigrette: Eriophorum.

Linaire: Linaria.

Lincone: Linconia.

Linderne: Linderuia.

Lindsée : Lindsaea.

Linkie : Linkia.

Liondent : Leontodon.

Lippi: Lippia.

Lis : Lilium.
Lisérole : Evolvulus.

Lisimaque: Lysimachia.

Litchi : Dimócarpus.

Lithophile: Litophila.

Litsé : Litsea.

Littorelle: Littorella.

Livêche: Ligusticum.

Livistone: Livistonia.

Loase: Loasa.

Lobélie: Lobelia.

Loddligésie : Loddigesia.

Lodoïce: Lodoïcea.

Loesélie: Loeselia.

Lonchite: Lonchitis.

Lopèze : Lopezia.

Lophire: Lophira.

Loranthe: Lorauthus.

Lotier : Lotus.

Loureira: Mozinna.

Lubinie: Lubinia.

Ludwige: Ludwigia.

Ludier: Ludia.

Lunaire : Lunaria.

Lunettière : Biscutella.

Lupin : Lupinus.

Luzerne: Medicago.

Luziole: Luziola.

Lychnite: Agrostemma.

Lyciet : Lycium.

Lycope: Lycopus.

Lycopode: Lycopodium.

Lycopside: Lycopsis.

Lygiste: Lygisticum.

Lysianthe : Lisian thus.

Mabier: Mabea.

Maceron: Sinyrnium.

Macoucou: Macoucoua.

Macranthe: Macranthus.

Macrocnème : Macrocnemum.

Madi : Madia.

Magellane: Magellana.

Magnolier: Magnolia.

Maherne: Mahernia.

Mahogon: Swietenia.

Mahuri: Mahurea.

Maïne: Mayna. 
Maïs : Zea.

Malachre: Malachra.

Malani : Antirrhaea.

Malherbe: Malesherbia.

Mallote: Mallotus.

Mamei: Mammea.

Manebo: Manabea.

Mancenillier: Hippomane.

Miandragore: Mandragora.

Mangier: Mangifera.

Manglier: Conocarpus. Cerb.

Manisure: Manisuris. Mangh.

Mantisie: Mantisia.

Manulée : Manulea.

Mapane: Mapania.

Mapromier: Maprounea.

Maqui: Aristotelia.

Maquire: Maquira.

Marcgrewe: Marcgravia.

Margaritaire : Margaritaria.

Maripe : Maripa.

Marrube: Marrubium.

Marsile: Lemna.

Masette : Typha.

Massone: Massonia.

Mataybe: Mataïba.

Matelé: Matelea.

Matise: Matisia.

Matouri: Matourea.

Maurico: Mauritia.

Manve: Malva.

Mauvisque : Malvaviscus.

Mayague: Mayaca.

Mayepe : Mayepea.

Mayten: Maytenus.

Méborier: Meborea.

Médéole: Medeola.

Médicinier: Jatropha.

Méduse : Medusa.

Mélalenque: Melaleuca.

Mélampire: Melainpyruin.

Mélampode: Melampodium.

Mélanthe: Melanthinn.

Mélastôme: Melastoma.

Mélianthe: Melianthus.
Mélicite: Melycitus.

Mélier: Blakea.

Mélicone: Entagonium.

Mélilot: Melilotus.

Mélique: Melica.

Mélisse: Melissa.

Mélite: Melittis.

Mélochie : Melochia.

Mélodin: Melodinus.

Mélodore: Melodorum.

Mélongéne: Melongena.

Mélotrie: Melothria.

Ménisce: Meniscium.

Ménisperme: AbutaMenispermum.

Menthe : Mentha.

Mentzèle: Mentzelia.

Menzièse: Menziesia.

Mercuriale: Mercurialis.

Mérendère: Merendera.

Mériane: Meriania.

Mertense: Mertensia.

Mérua : Maerua.

Mérule: Merulius.

Mésa : Baeobotrys.

Météoride: Meteorus.

Methonique: Gloriosa.

Méyère: Meyera.

Michanxie: Michauxia.

Micocoulier: Celtis.

Micranthème: Micranthemum.

Microcarpe: Microcarpea.

Micrope: Micropus.

Microtea: Microtea.

Millérie : Milleria.

Millepertuis : Hypericum.

Millington: Millingtonia.

Mimule: Mimulus.

Mimusope: Mimusops.

Minuart: Minuarta.

Miriofle: Myriophyllum.

Mirosperme: Myrospermum.

Miroxile: Myroxylon.

Mirsine : Myrsina.

Mitchelle : Mitchella.

Mitella: Mitella.

Mitraire: Mitraria. 


\section{cusasss w. \\ A C R O B L A S T A E. SPITZKEIMER.}

\section{ORDO I. \\ RHIZO - ACROBLASTAE. \\ WURZEL - SPITZKEIMER.}

\section{FORMATIO I. \\ $\begin{array}{llllllllll}\mathbf{L} & \mathbf{I} & \mathbf{M} & \mathbf{N} & \mathbf{O} & \mathbf{B} & \mathbf{I} & \mathbf{A} & \mathbf{E} .\end{array}$ \\ TAUCHERGEWAECHSE.}

FAMILIA XXXII.

ISOETEAE. BrachsenkRaeUter.

753. Isoëtes. L.

FAMILIA XXXIV.

POTAMOGETONEAE. Fluthigraeuter.

A. Zostereac.

754. Z ostera. L.

755. Thalassia. Banks. Zost. sp. Forsk.

756? Phucagrostis. Carox. 75\%. Posidonia. Caul. et Koen.

B. Potamogeae.

760. Zannichellia. L. 761. Ruppia. L. 762. Graumiillera. Rohв. Rupp. unturct. LaB.
Kernera. W.

Caulinia. DC.

758. Halophila. A. P. Th. 759. Barkania. Ehrnb. 
44 CLASS.IV.ACROBL. ORD.I.RHIZO-ACR. FAM. XXXV.AROID.

FAMILIA XXXV.

AROIDEAE. AroIDEen.

A. Callacela

a. Lemneae.

764. L e mna。 L.

b. Staurogeton. Rchb.

a. Lenticula. $T$.

Lemn. trisulca. $\mathrm{L}$.

b. Pistiaceac.

765. Pistia. L.

\section{c. Calleae.}

766. Ambrosinia. L.

Ictodes. Rigel.

76\%. Cryptocoryne. Fisch. 791. C all a. L.

768. Aris arum. T.

769. Arum. L.

a. Arum. Auct.

b. Candarum. Rchb.

Arum Canda Rxb. non Canda.

Lamx. Zool.

792. Richardia. Knth. Calla. L.

Zantedeschia. Spr.

773. C a la di u m. Vent.

a. Caladium, Auct.

b. Culcasia. P. Beauv.

7y0. Symplocarpus. SAursB.

c. Baursea, Angl.

Spathyema. Rafin.

d. Pothoinac.

794. Dracontium. L. 795. Pothos. L. Quebitea. Aubl.

$$
\begin{aligned}
& \text { B. Ta c c rece a e. } \\
& \text { a. Gymnostachyeae. }
\end{aligned}
$$

7r6. Gymnostachys. RBr. 7\%. Acorus. L.

b. Tuceac.

798. Orontium. L. 799. Rohdea. Rth. 780." Tup istra. Ker. 781. Aspidistra. Ker. 782. Sanseviella. Rchb. Sansevicra. Andr.
783. Eriospermum. JucQ. 784. A taccia. Prsi. 785. Tacca. Forst.

786. Roxburghia. W. 
Mniare: Mniarum.

Mocanère: Visnea.

Moisissure : Mucor.

Mollé: Schinus.

Molène: Verbascum.

Mollugine: Mollugo.

Mollavi: Balanopteris.

Mombin: Spondia.

Momordique: Momordica.

Monarde : Monarda.

Monière: Moniera.

Monilie: Monilia.

Monjoli : Varronia.

Monotrope: Monotropa.

Monsone: Monsonia.

Montabier: Montabea.

Montie: Montia.

Montin : Montinia.

Montire : Montira.

Morée: Moraea.

Morelle: Solanum.

Morène: Hydrocharis.

Morgania : Morgania.

Morgeline: Alsine.

Morinde: Morinda.

Morine: Morina.

Mosambe: Cleome.

Moscaire: Moscaria.

Moscatelline: Adoxa.

Mouroucou: Mouroucoa.

Mourère: Mourera.

Mouriri : Mouriria.

Mouron: Anagallis.

Moutabée: Moutabea.

Moutouchi: Pterocarpus.

Muflier: Antirrhium.

Muguet: Convallaria.

Miihlenbergie: Mühlenbergia.

Miiller: Muillera.

Mûrier: Morus,

Murrai : Murraya.

Muscadier: Myristica.

Mussende : Mussaenda.

Mutise: Mutisia.

Myginde: Myginda.

Myonime: Myonima.
Myosote: Myosotis.

Myrianthe: Myrianthus.

Myriotheque: Myriotheca.

Myrosme: Myrosma.

Myrte: Myrtus.

Nacibe: Nacibea.

Nagas : Mesua.

Najade: Naias.

Nandine: Nandina.

Nandirobe: Fevillea.

Napée: Napaea.

Napimogal: Napimoga.

Napoleone: Napoleona.

Narcisse: Narcissus.

Nard : Nardus.

Nassauve: Nassauvia.

Nattier: Imbricaria.

Nauclèe: Nauclea.

Navarrête: Navarretia.

Néflier: Mespilus.

Négrétie: Negretia.

Nélitre: Nélitris.

Nélumbe: Nelumbium.

Némaspore: Nemaspora.

Némésie: Nemesia.

Néntaphar: Nymphaea.

Néottie: Neottia.

Népenthe: Nepenthes.

Néphèle: Nephelium.

Nerterie: Nerteria.

Neurade: Neurada.

Nicotiane: Nicotiana.

Nictage: Mirabilis.

Nictanthe: Nyctanthus.

Nidulaire: Nidularia.

Nidulaire: Cyathus.

Nierembergie: Nierembergia.

Nigelle : Nigella.

Nigrine: Nigrina.

Niote: Niota.

Nissole: Nissolia.

Nitraire: Nitraria.

Nivéole: Leucoïum.

Nolane: Nolana.

Noline: Nolina.

Norante: Norantea. 
Norantea: Ascinm.

Nuxier: Nuxia.

Nymphantes: Nymphanthus.

Nyssanthe: Nyssanthes.

Obolaire : Obolaria.

Obéliscaire: Obeliscotheca.

Ocote: Ocotea.

Octarille: Octarillum.

Octodicère: Octodiceras.

Oeillet: Dianthus.

Oldenlande : Oldenlandia.

Olivetier : Elaeodendron.

Olivier: Olea.

Olyre: Olyra.

Omphalier: Omphalea.

Omphalocarpe: Omphalocarpum.

Onagraire: Oenothera.

Onagres: Onagrae.

Oncine: Oncinus.

Onoclée: Onoclea.

Onoporde: Onopordon.

Onosmode: Onosmodimm.

Opégraphe: Opegrapha.

Opélie: Opelia.

Operculaire : Opercularia.

Ophèle : Ophelus.

Ophioglosse: Ophioglossum.

Ophiorhize: Ophiorrhiza.

Ophiose: Ophioxylon.

Ophire: Ophira.

Ophisperme: Ophispermum.

Ophrise: Ophrys.

Oranger: Citrus.

Orcanette: Onosma.

Orélia: Allamanda.

Oréobole : Oreobolus.

Oréocalle : Oreocallis.

Orge: Hordeum.

Origan: Origanum.

Orune: Ulmus.

Ormocarpe: Ormocarpum.

Ormosie : Ormosia.

Ornithogale: Ornithogalum.

Ornithope : Ornithopus.

Orobe: Orobus.

Orouce : Orontium.
Orpin: Sedum.

Ortegie: Ortegia.

Orthopyxe: Orthopyxis.

Orthotric: Orthotrichum.

Ortie: Urtica.

Osbeck : Osbeckia.

Osmite: Osmites.

Osmonde: Osmunda.

Osmonde: Anemia.

Osmonde: Aphyllocarpa.

Osteosperme: Osteospermum.

Othère: Othera.

Othoune: Othouna.

Ottélie: Ottelia.

Ourisie: Ourisia.

Oursine : Arctopus.

Outay: Outea.

Oviède: Ovieda.

Oxalide: Oxalis.

Oxycarpe: Oxycarpus.

Oxystelme: Oxystelma.

Pachirier: Pachira.

Pachysandre: Pachysandra.

Pacourier: Pacouria.

Pacourine: Pacourina.

Paederote: Paederota.

Paliure: Paliurus.

Palladie: Palladia.

Pallasie: Pallasia.

Palsivelours: Celosia.

Panais : Pastinaca.

Pancrais : Pancratitum.

Panic: Panicum.

Panicaut: Eryngium.

Papayer: Papaya.

Papirier: Papyrus.

Pappophore: Pappophorum.

Paquerette: Bellis.

Paquerolle: Bellium.

Paralé: Paralean

Pareire: Cissampelos.

Pariane: Pariana.

Pariétaire: Parietaria.

Parinari : Parinarium.

Parkinson: Parkinsonia.

Parnassie: Parnassia. 
Paronique: Paronychia. Paropsie: Paropsia. Parthène: Parthenium. Passerage: Lepidium. Passoure: Passoura. Pastel : Isatis. Patabé: Patabea. Patagne: Crinodendron. Patagone: Boerhaavia. Patagonule: Patagonula. Patarole: Patarola. Patersone: Patersonia. Patience: Rumex. Patime: Patima. Paturin: Poa. Pavie: Pavia. Pavon : Pavonia. Parot: Papaver. Payrole: Payrola. Pectis : Id.

Pedali: Pedalium. Pédicellie: Pedicellia. Pédiculaire: Pedicularis. Peigne: Scandix. Pek : Pekea. Pelargon: Pelargonium. Pélégrine: Alstroemeria. Peliosanthe: Peliosanthes. Pencovie: Pancovia. Pennante: Pennantia. Pentalobe: Pentaloba. Pentapète: Pentapetes. Penthore: Penthorum. Peplide : Peplis. Pepon: Pepo. Percepier: Aphanes. Pérézie: Perezia. Pergulaire: Pergularia. Perille : Perilla. Periploque: Periploca. Persil : Apium. Persoonie: Persoonia. Pesse: Hippuris. Petalome: Petaloma. Pétésie: Petesia. Pétitie: Petitia.
Petivère: Petiveria. Pétrée : Petrea.

Petune : Petunia. Peucedane: Peucedanum. Peumo: Peumus. Peuplier: Populus. Peziza : Peziza. Phacélie: Phacelia. Phaetuse: Phaëtusa. Phalangère: Phalauginu. Pharelle: Pharus. Pharnace: Pharnaceum. Phasque: Phascum. Phébalie: Phebalium. Phélipée: Phelipaea. Philèse: Philesia. Philydre: Philydrum. Phlomide: Phlomis. Phrynie: Phryuium. Phylique: Phylica. Phyllanthe: Phyllanthus. Phyllide: Phyllis. Picride: Picris. Picridie : Picridium. Picramnie: Picramuia. Pigamon: Thalictrum. Pilaisie : Pilaisaea. Piloselle: Hieracium Pilosella. Pilulaire : Pilularia. Pimprenelle: Poterium. Piparée: Piparea. Piratinier: Piratinera. Pirigare: Pirigara. Piripe : Piripea. Pisone: Pisonia. Pitcairne: Pitcarnia. Pittone: Tournefortia. Pittospore: Pittosporum. Pivoine: Paeonia. Plagianthe: Plagianthus. Plagueminaire: Diospyros. Planère: Planera. Plautain : Plantago. Plantane: PJatanus. Plectrone: Plectronia. Plegorrhize: Plegorrhiza. 
Plinie: Plinia.

Plocamier: Plocama.

Podalyre: Podaliria.

Podophylle: Podophyllumn.

Podosème: Podosaemum.

Podostème: Podostemon.

Pohlie: Pohlia.

Poincillade: Poinciana.

Poirier: Pyrus.

Poivrier : Peperomia.

Pois : Pisum.

Polémoine: Polemonium.

Pollie : Pollia.

Pollique : Pollichia.

Polyachyre: Polyachyrus.

Polycarde: Polycardia.

Polycarpe: Polycarpon.

Polycarpée: Polycarpaea.

Polycuème: Polycnemum.

Polymnie: Polymnia.

Polypode: Polypodium.

Polyprème: Polypremum.

Pomaire: Pomaria.

Porneruelle: Pomernella.

Pommier: Malus.

Poncelet: Ponceletia.

Pongati : Pongatium.

Pongelion : Aylanthus.

Pontédaire: Pontederia.

Populage: Caltha.

Porane: Porana.

Poranthère: Poranthera.

Poraquèbe: Poraqueba.

Porcélie: Porcelia.

Porcelle: Hypochaeris.

Posoquieri : Posoqueria.

Possire: Possira.

Potalie: Potalia.

Potamophile: Potamophila.

Potentille: Argentine, Potentilla.

Poupart: Poupartia.

Pourpier: Portulaca.

Poutérier: Pouteria.

Prasion: Prasium.

Prasophylle: Prasophyllum.
Prêle: Equisetum.

Proté : Protea.

Prunelle: Prunella.

Prunier: Prunus.

Psilote: Psilotum.

Psoralier: Psoralca.

Ptélidie : Ptelidium.

Ptéranthe: Pterauthus.

Ptérocarpe: Amerimnum.

Pterone: Pteronia.

Ptérosperme: Pterospermun.

Ptérote: Pterotum.

Ptéryogodie: Pterygodinn.

Ptychosperme: Ptychosperma.

Pulmonaire. Pulmonaria.

Pungamie: Pungamia.

Pnschkinie: Puschkinia.

Pychnauthème: Pycnanthemum.

Pycré: Pycreus.

Pyrèthre: Pyrethrum.

Pyrole: Pyrola.

Pyrostre: Pyrostria.

Pyrulaire: Pyrularia.

Pythagorée: Pythagorea.

Pyxidanthère: Pyxidanthera.

Quadrette: Rhexia.

Quadrie: Quadria.

Qualier: Qualea.

Quamoclit: Ipomaea.

Onapalier: Sloanea.

Quapoyer: Quapoya.

Quassier: Quassia.

Quatelé : Lecy this.

Québite: Quebitea.

Quenouillet: Atractylis.

Quenouillette: Acarna.

Queria: Queria.

Quil, Quirpele: Ophioxylon.

Quinquina: Pinknaya.

Quirivelia: Ichnocarpus.

Quisquale: Quisqualis.

Quivi: Quivisia.

Racarier: Racaria.

Racle: Cenchrus.

Radis : Raphanus. 
Raisinier: Coccoloba.

Raïana : Rajania.

Ramonda: Ramondia.

Ranoncule : Ranunculus.

Rapane: Rapanea.

Rapate: Rapatea.

Rapette: Asperugo.

Rapinie: Rapinia.

Raponcule: Phyteuma.

Raputier: Raputia

Ratoncule: Myosurus.

Ratteau: Biserrula.

Rauvolfe: Rauwolfia.

Raveusara: Agathophyllum.

Réaunure : Reaumuria.

Redoutea: Redoutea.

Réglisse: Glycyrrhiza.

Remire : Remirea.

Rénanthère: Renanthera.

Reneaulme: Renealınia.

Renoncule: Ranunculus.

Renouée: Polygonum.

Renouelle: Eriogonum.

Requerie: Riqueria.

Réséla: Reseda.

Restrépie: Restrepia.

Reticulaire: Reticularia.

Retzie: Retzia.

Rhantère: Rhanterium.

Raptostyle: Raptostylum.

Rhicophore: Rhizophora.

Rhodiole: Rhodiola.

Bhodore: Rhodora.

Rhopale : Rhopala.

Rhubarbe: Rheum.

Rhynchosie : Rhynchosia.

Riane: Riana.

Ribelier, Ribelia: Embelia.

Riccie: Riccia.

Riccin : Ricinus.

Richarde : Richardia.

nichérie: Richeria.

Ricinelle: Acalypha.

Ricomorphe: Rhizomorpha.

Ricotie: Ricotia.

Riédlée: Ricdlea.
Rimbot: Oncoba.

Rinore: Rinorea.

Ripogone: Ripogonum.

Riqueure: Riqueria.

Rivine: Riviua.

Rivulaire : Rivularia.

Riz: Oryza.

Rizoé: Rizoa.

Rizole: Oryopsis.

Robertie : Robertia.

Robinier: Robinia.

Roccelle: Roccella.

Rocheforte: Rochefortia.

Roelle : Roëlla.

Roconier: Bixa.

Rohria: Agriphyllum.

Rokéje: Rokejeca.

Romarin : Rosmarinus.

Ronabe: Ronabea.

Ronce: Rubus.

Roucinelle : Dalibarda.

Rondelier: Rondeletia.

Rondier: Borassus.

Rongo: Harungana.

Rapourea: Cama.

Ropourier: Ropourea.

Roridule: Roridula.

Roscoée : Roscoea.

Rosier: Rosa.

Rosinaire: Arundinaria.

Rossolis: Drosera.

Rotale: Rotala.

Rothe: Rothia.

Rottbolle: Rotthölla.

Rottlère : Rottlera.

Rotule: Rotula.

Rouhamon: Id.

Roupale: Roupala.

Rourelle: Rourea.

Rousseau: Roussea.

Roxburge: Roxburghia.

Royène: Royena.

Rubareau: Sparganium.

Rudbèque: Rudbekia.

Rudolphe: Rudolphia.

Rue: Ruta. 
Ruellée: Ruellia.

Ruizé : Ruizia.

Rumphe: Rumphia.

Rupinie : Rupinia.

Ruppie : Ruppia.

Ruysche: Ruyschia.

Ryanie: Ryania.

Sabice: Sabicea.

Sabine: Sabina.

Sablier: Hura.

Sabline: Arenaria.

Sagine: Sagina.

Sagone: Sagonea.

Sagouier: Sagus.

Sainfoin: Hedysarum.

Sajore: Pluknetia.

Salace: Salacia.

Salicaire: Lythrum.

Salicorne: Salicornia.

Saliquier: Cuphea.

Salic̣uier: Balsamina.

Salomone : Salomoniu.

Salpiglosse : Salpiglossis.

Salpianthe : Salpianthus.

Salsepareille: Smilacina.

Salsilis : Tragopogon.

Salsigrame : Geropogon.

Salvadore: Salvadora.

Salvinie : Salvinia.

Samare: Samara.

Samole: Samolus.

Samyde: Samyda.

Sanguinelle: Sanguinella.

Sanguinière: Sauguiuaria.

Sanguisorbe: Sanguisorba.

Sanicle: Sanicula.

Sanvitale: Sanvitalia.

Santalin: Sautalum.

Santoline: Santolina.

Sapin : Abies.

Saponaire: Saponaria.

Sapotillier: Achras.

Saraca: Saraca.

Saracène: Saracenia.

Saraquier: Saracha.

Sarcocolier: Penaea.
Sarcode: Sarcodum.

Sarcophylle: Sarcophyllum.

Sargasse: Sargassum.

Sarmiente: Sarmienta.

Sarothre: Sarothra.

Sarrette: Serratula.

Sarriole : Isanthus.

Saussurée: Saussurea.

Saturier: Psathura.

Satyre: Phallus.

Satyrion: Satyrium.

Saule: Salix.

Saurure: Saururus.

Sauvagèse: Sauragesia.

Savonier: Sapindus.

Saxifrage: Saxifraga.

Scabieuse: Scabiosa.

Scheuchzère: Scheuchzeria.

Schisandre: Schizandra.

Schizanthe: Schizanthus.

Schlechtendale: Schlechtendalia.

Schleichère: Schleichera.

Schoepfie: Schoepfia.

Schoualbe: Schwalbea.

Schouinque: Schwenkia.

Schradère: Schradera.

Schrebere: Schrebera.

Scille: Scilla.

Sciadaphylle, Actinophylle: Sciadophyllum.

Scirpe: Scirpus.

Sclérie : Scleria.

Sclérocarpe: Sclerocarpus.

Sclérote: Sclerotium.

Scolopier: Scolopia.

Scolosanthe: Scolosanthus.

Scolyme: Scolymus.

Scoparie: Scoparia.

Scopolier: Scopolia.

Scorzionère: Scorzonera.

Scottie : Schottia.

Scrophulaire: Scrophularia.

Scutule : Scutula.

Scyphophore: Scyphophorus.

Sechi : Sechium.

Securille: Securilla. 
Seguier: Seguiera.

Séhime: Sehima.

Seigle: Secale.

Selagine : Selago.

Selin : Seliuum.

Senacier : Senacia.

Senebière: Senebiera.

Seneçou : Senecio.

Senrée: Senra.

Septade: Septas.

Serbane : Agati.

Seriana: Paulinia, Serjania.

Seriole: Seriola.

Sérisse: Serissa.

Serisse: Dysoda.

Serpicule: Serpicula.

Serpolet: Serpillum.

Sesame: Sesamum.

Sesbaue: Sesbana.

Sésèli : Seseli.

Sessée: Sessea.

Sesslère: Sessleria.

Sétaire: Setaria.

Seymèrie: Seymeria.

Shultzie: Schultzia.

Sialite : Dillenia.

Sibbalde: Sibbaldia.

Sibthorpe: Sibthorpia.

Siciote: Sicyos.

Sigesbèque: Siegesbeckia.

Siléné: Silene.

Silphie: Silphium.

Simabe: Simaba.

Simbulète: Simbulata.

Simire : Simira.

Singane: Singana.

Siparounier: Siparuna.

Siphonanthe: Siphouanthus.

Sisyubre: Sisymbriun.

Skimmie: Skimmia.

Smithie: Smithia.

Solaudre: Solandra.

Soldanelle : Solılanella.

Soramie: Soramia.

Sorbier: Sorbus.

Sorgho: Sorghum.
Soude: Salsola.

Soucher: Cyperus.

Souci : Calendula.

Sparganophore: Sparganophorus.

Spargoutine: Spergula.

Spathélier: Spathelia.

Spathodée: Spathodea.

Spathulaire: Spathularia.

Spermodermie: Spermodermia.

Sphaegne: Sphagnum.

Sphaerolobe: Sphaerolobium.

Sphéranthe: Sphaeranthus.

Sphérie: Sphaeria.

Sphérophore: Sphaerophorus.

Spilanthe: Spilanthus.

Spilmane: Spielmannia.

Spinelle: Spinifera.

Spirée: Spiraea.

Splane: Splachnum.

Stachyde: Stachys.

Stadmane: Stadmannia,

Stapèle: Stapelia.

Staphylier: Staphylea.

Staticé : Statice.

Stauracanthe: Stauracanthus.

Stellaire: Stellaria.

Stellérine: Stellera.

Stématosperme: Stemmatos-

Stémodie: Stemodia. perma.

Stémone: Stemona.

Stémonite: Arcyria, Stemonitis.

Sténauthère: Stenanthera.

Sténoglosse: Stenoglossum.

Sterculier: Sterculia.

Stéréocaule: Stereocaulon.

Stéréoxlyou: Stereoxylon.

Stériphe: Steripha.

Sterubergie: Sternbergia.

Stewarte: Stewartia.

Stigmanthe: Stigmauthus.

Stigmarote: Stigmarota.

Stilbe: Stilbum.

Stilbospore: Stilbosporum.

Stillingue: Stillingia.

Stipe: Stipa. 
Stipulaire: Stipularia.

Stipulicide: Stipulicida.

Stobée: Stobaea.

Stramoine: Datura.

Stratiote: Stratiotes.

Stravadum: Stravadia.

Strèble: Streblus.

Strélitz: Strelitzia.

Streptostachyde: Streptostachys.

Streptope: Streptopus.

Strigilie: Strigilia.

Strophante: Strophanthus.

Strumaire: Strumaria.

Struthiole: Struthiola.

Stylocoryne: Stylocoryne.

Stylosanthe: Stylosanthes.

Styphélie: Styphelia.

Subulaire: Subularia.

Suerce: Swertia.

Suffrénie: Suffrenia.

Sumac: Rhus.

Symphonie: Symphonia.

Symphyonème: Symphyonema.

Symploque: Symplocos.

Synaphée: Synaphea.

Tabagelle: Zygophyllum.

Taberne: Amsonia.

Tabouret: Thlaspi.

Tachi: Tachia.

Tachibote: Tachiboto.

Tachigale: Tachigali.

Tacsone: Tacso.

Tagête: Tagetes.

Taligale: Taligalea.

Talin: Talinum.

Talisier: Talisia.

Tamarinier: Tamarindus.

Tamboul: Ambora.

Taminier: Tamus.

Tamonée: Tamonea.

Tanaisie: Tanacetum.

Tanghinie: Tanghinia.

Taniboucier: Tanibouca.

Tapier: Crataeva.

Tapogome: Tapogomea.

Tapure: Tapura.
Tarconante: Tarchonanthus.

Targione: Targionia.

Teck: Theka.

Télèphe: Telephium.

Temo : Temus.

Terebinte: Terebinthus.

Terrelle: Glechoma.

Tétracere: Tetracera.

Tétragonie: Tetragonia.

Tétranthe: Tetranthus.

Tetrapile: Tetrapilus.

Tetrapogone: Tetrapogon.

Thalassie: Thalassia.

Thalie: Thalia.

Thapsie: Thapsia.

Thé: Thea.

Thélébole: Thelebolus.

Théléphore: Telephora.

Theligone: Thelygonum.

Théméda: Themeda.

These: Securinega.

Thésion: Thesium.

Thilaque: Thylachium.

Thonarse: Thouarea.

Thrasye: Thrasya.

Thrinace: Thrinax.

Thryosperme: Trichospermum.

Thym: Thymus.

Thysanos: Thysanus.

Thysanothe: Thysanothus.

Tiarelle: Tiarella.

Ticorée: Ticorea.

Tigaré: Tigarea.

Tillande: Tillandsia.

Tilleul: Tilia.

Timmie: Timmia.

Tinelier: Anguillaria, Heberdenia, Ardisia.

Tinier: Tinns.

Tiongine: Beckea.

Tithonie: Tithonia.

Tocoyène: 'Tocoyena.

Toddali: Toddalia.

Todée: Todlea.

Tolut: Toluifera.

Tomé : Tomex. 
3794. Siebera. Rснв.

Fischera et Trachym. S p r. non Rudg.

37\%5. Mulinum. Pers.
377\%. A zorella. LAm. Chamitis. G.

3798. Pectophyllum, K.H.B. 3\%99? Fragosa, Rz, PAv.

3\%6. B o la X. Commers.

c. Araliacear grenuinae.

3780. Maralia, A, P. Th, 3\%84. Schefflera, Forst. 3781. Aralia, L. 3785. Polyscias. Forst. 3782. Sciadophyllum. RBr. 3786. Gilibertia, Rz. PAv. 3783? Actinophyllum, Rz. 378\%. Phytocrene. WaLt. Pav.

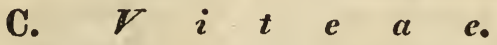

$$
\begin{aligned}
& \text { a. } H a g e n i a c e \text { a ? }
\end{aligned}
$$

3788? H a genia. LAM.

$$
\text { b. } C \text { is } s \text { e } a \text { e. }
$$

3789? Cayratia, Juss. 3791. Ampelopsis, Mchx. Columella. Lour. non Jeq. 3792, V itis, L. 3790. Cissus. L.

$$
\text { c. Hederacere. }
$$

3793. Hedera, L. 3795? Dicophe. Wart. 3794. Gastonia, Commers.

\section{FAMILIA LXXXVIII.}

R H A M N E E. KREUZDORNE.

a. Go $о$ a $n$ i $a c$ c $a$ e.

3\%96. Crumenaria, Mart, 3\%9\%. Gouania, L. Retinaria. G.

b. Ceanothe a e.

3798? Spixia, Leand, non 3801, Pomaderris, Lar.

\section{Schrk,}

3\%99. Ce an othus, L. e, e. Forrestia. Rafin.

Euceanothus (Sect.) DC. 3800. Nolt e a, Rchi。

Ceanothus. R afin. Willemetia. Brngu.non Neck.
Pomatoderris. Iffgg.

Pomatiderris. Knth. 3802. Cryptandra, SM. 3803. Trich ocephalus. Brngen. 3804. Phylica. L. e. e. 
3805. Soulangia. Bhnge. 3807. Colletia. K. H. B. 3806. Scutia. Сомm. msc. 3808. Hovenia. Thnb. Sentis. Comm. hb. 3809. Colubrina. Rrch.

$$
\text { c. Frangulaceac. }
$$

3810. Retanilla. Brngn. Mrolinuea. Com m. in sc. 3811. Paliurus. T. Dsf. 3812. Sageretia. Brngn. 3813? Eustathes. Lour. 3814. Olinia. ThNB. 3815. Goup ia. Aubl.

Giupia. I. St. II. Glossopetalum. Schreb. 3816? C a rpodetus. Forst. 3817. Zizyphus. T. Dsf. 381s. Condalia. Car. 3819. Berchemia. Neck. Oenoplea. Hog. Oenoplia. R. S. 3820. Ventilago, Gärt. 3821. Dulong ia. KNтн. 3822. Rh a m in us. L. e. e.

a. Alaternus, $\mathbf{T}$. Marcorella. N e ck. b. Crevispina. Dill. c. Frangula, T.

FAMILIA LXXXIX.

\section{TEREBINTHACEAE. Terebinthacen.}

$$
\text { A. Chailletiacea e. }
$$

2823. Chailletia. DC. Patrisia. Roh r. a. Mestotes. Soland.

b. Dichapetalum. DC, 3824. Plappertia. Rснв.
Leucosia. A. P. Th. non Zoolog.
3825. T a pura. Aubl. Rohria. Schreb.

\section{B. Connarace $a$ e.}

3826. Con na rus. L. Malbrancia. Ne ck. Omphalobium. G. 382\%. Rourea. Aubl. Robergia. Schreb.
3828. Eurycoina. JAck. 3829. Cnestis. Juss.

3830? T e tradium. Lour.

$$
\begin{gathered}
\text { C. Tereb. genuina e. } \\
\text { a. Anyrideac. } \\
\text { a. Iuglandeae. }
\end{gathered}
$$

3831. Juglans. L. 3832. Carya. Nutr.
3833. P terocarya. Nutr. 3834? Decostea. Rz. Pav. 
Tonine: Tonina.

Tontane: Tontanea.

Tontel: Tontelea.

Topobé: Topobaea.

Toque: Scutellaria.

Tordyle: Tordylium.

Torène: Torenia.

Tormentille: Tormentilla.

Tortule: Tortula.

Tounate: Tounatea.

Trachèle: Trachelium.

Trachis: Trachys.

Trachymène: Trachymene.

Tragie: Tragia.

Trèfle: Trifolium.

Tremelle: Tremella.

Trianthème: Trianthema.

Trianthère: Trianthera.

Trianthime: Gymnocarpus.

Tribule: Tribulus.

Tricarier : Tricarium.

Tricere: Tricera.

Tricerote: Triceros.

Trichie: Trichia.

Trichilie: Trichilia.

Trichoclade: Trichocladus.

Trichode: Trichodium.

Trichoderme: Trichoderma.

Trichomane: Trichomanes.

Trichostème: Trichostemma.

Tricorine: Tricoryne.

Tricrate: Tricratus.

Tricuspidaire: Tricuspidaria.

Tricycle: Tricycla.

Tridace: Tridax.

Tridènus: Tridesmus.

Triène: Triaena.

Trientale: Trientalis.

Triglochine: Triglochin.

Trigonelle: Trigonella.

Trigonier: Trigonia.

Triguère: Triguera.

Trilice: Trilix.

Trillie: Trillium.

Trinacte: Trinax.

Trioptère: Triopteris.
Trioste : Triosteum.

Triphacue: Triphaca.

Triphasier: Triphasia.

Triphylle: Triphyllus.

Tripinnaire: Tripinnaria.

Tripterelle: Tripterella.

Tripsac: Tripsacum.

Triptilione: Triptilion.

Trisanthe: Trisanthus.

Tristemme: Tristemma.

Tritonie: Tritonia.

Trixide: Proserpinaca.

Troène: Ligustrum.

Trolle: Trollius.

Trọmite: Tromita.

Tubéreuse: Polyauthes, Tuberosa.

Tuberculaire: Tubercularia.

Tubiflore: Tubiflora.

Tulbage: Tulbagia.

Tulype: Tulipa.

Tulipier: Liriodendron.

Tuniquée : 'Tunicata.

Turie: Turia.

Turnère: Turnera.

Turrée: Turraea.

Tussilage: Tussilago.

Ubion : Ubium, Dioscorea?

Vlassi : Ulassium.

Ulet: Ulete, Agulet.

Ulve: Ulva.

Unari : Geoffraea.

Uniole: Uniola.

Unjale: Unhala.

Unone: Unona.

Unone: Desmos.

Upas : Antiaris Toxicaria.

Upoderme: Hypoderma.

Uralier: Anthocercis

Uranote: Siloxerus:

Uraté : Ouratea.

Urcéolaire: Cyathodes.

Urédo : Uredo, Aecidium.

Urène: Urena.

Urotte: Anopterus.

Ursinie : Ursinia. 
Urticulaire: Urticularia.

Urule : Comesperma.

Ustérie: Usteria.

Usube: Schmidelia.

Uvette: Ephedra.

Uvulaire: Uvularia.

Vagère: Vohiria.

Vaginaire: Vaginaria.

Vaginelle: Lepidosperma.

Vagurelle: Actinotus.

Vahée: Vahea.

Vaillantie: Valantia.

Valeriane: Valeriana.

Valentine: Valentina.

Vallée: Vallea.

Vallesia: Id.

Vallisnère: Valisneria.

Vampi: Cookia.

Vandelle: Vaudellia.

Vanguier: Vangueria.

Vanelle: Stylidium.

Vanierie: Vanieria.

Vanille: Vanilla.

Vantane: Vautanea.

Varaire: Veratrum.

Varec: Fucus.

Varette: Adenanthos.

Velote: Dillwynia.

Vermiculaire: Vermicularia.

Veronique: Veronica.
Verrucaire: Verrucaria.

Vesce: Vicia.

Vésicaire: Vesicaria.

Vigne: Vitis.

Vilfea: Vilfa.

Villarsie: Yillarsia.

Violette: Viola.

Vipérine: Echium.

Vitmanue: Vitmannia.

Vogèle: Vogelia.

Volin: Pimela.

Volkamier: Volkameria.

Vulpin: Alopecurus.

Wallène: Wallenia.

Walthère: Waltheria.

Wébère: Webera.

Weissie: Weisia.

Witsène: Witsenia.

Wrightie: Wrigthia.

Xantosie: Xanthosia.

Xilophylle: Xylophylla, Genesiphylla.

Xinnénésie: Ximenesia.

Ximénie: Ximenia.

Xyphalier: Atherosperma.

Zannichellie: Zanichellia.

Zapane: Aloysia, Zapania.

Zélari: Gahnia.

Zerumbet: Zingib. Zerumb.

Zuccagne: Zuccagnia, 


\section{N D E X \\ GENERUM, SUBGENERUM, SYNONYMORUM.}

A bama All. 1383. Abasicarpon Audrz. 47!10a.

Abatia Rz. Pav. 5tt?. Abelia RBr. 19 is. Abelicea Sin. 1828. Abelmoschus Med.5273. Abies T. 1725.

Abiltgardia Vahl. 1091. Ablania Aubl. 5440.

Abolboda K. II. B. 1148. Abrahamia DC. $4591 \mathrm{~b}$. Albroma L. fil. 5?19\%. Abronia Juss. 18t?. Abrus L. 3925. Absinthinu Adıs. 2369. Absils D(. 41351). Abunon Ad. 1372. Abuta Aubl. $188 \%$. Abutilou Dill. 5251. Acacia Neck. 41 i?. Acaena V. 4424. Acajon 'T'. 3Stiz. Acajuba G. $386 \%$. Acalyplia L. 5034. Acanios Adiuns. 2324. Acanthoilum Del. 3118. Acinthollychia DC. 4265i.

Acaulhosperinum

Schrk. 2713. Acauthospora Spr. 1276. Acallius L. 31:0. Acarna 6. 230\%). Acaria Vill. 2330. Acer L. 5241.

Acerates 1ill. 3426 . Aceras RBr. 1456 . Acetibulum Lamk. 449. Acelosa T. 4 :8tia. Achania Swo 5?65. Acharia Thub. 2743b. ex alli. Achariterima N. v. E. 245is. ex anlis. Achetaria Cham. 3158.
Achillea Vaíll. $2399 . \quad$ Acosta Rz. Par. 3632. Achintilles P. Br. 3235. Acouroa Aubl. 4102. dchimenes Vahl. 3236. Acremoninn Lk. 134. Achlya N. v. E. 441. Acriopsis Reinw. 1583. Achianthes B.St.V. 446. Acrocomia Mart. 1623. Achuathermu P.B.915a. Acrodryon Spr. 1939. Achnotoudon P. B. 949. Arroglochin Schrad. Achras L. 364t. 43606 .

Achroanthesßafin.1576. Acronia Prsl. 1553. Achyranthes L. 4374. Acronodia Blnme. 5431. Achyrocoma H. Cass. Acropodium Desv. 3876. 2184. Achyronia Wendl 4047. Acrosuorium N Y E 114 Achyropappus K. H. B. Acrostichum L. 733. 2572.

Achyrophorus G. 2154. Achy rophorus Vaill. $2160 \mathrm{~b}$.

Acia Schreb. 4522.

Acianthus RBr. 1555 . Acicarpa Radd. 1061. Acicarpha Juss. 2739. Acidoton S'v. j048.

Acidodontium Schwg. 648.

Acilepis Don. 2241.

Acinophora Rafin. 197. Acinos P. 2820.

Acinotum DC. 4797b.

Aciunla Fr. 152.

Acioa Aubl. 45?2.

Aciotis Don. 4ti0?.

Acisanthera P. Br. 4560.

Acladium Lk. 100.

Aclisia E. Mey. 1169.

Acmadenia Bartl. et Weull. stij3.

dcmella Rich. 2623.

Acmenil Dec. 4679.

Acriila L. $\$ 349$.

Acoma All. 4698 .

Acouirun T. 4950 .

Aconowoum Meisn. 42911.

Acorus L. $59 \%$.

Acrothamnium N. v. E p. 15.

Acrotriche RBr. 3334.

Acroxis Trin. $905 \mathrm{~b}$.

Acrozin S Spr. 5431.

Actaea L. 4959.

Actidium Fr. 256.

Actinea Juss. 2573.

Actinocarpus RBr. 796 .

Actinochloa W. 871 .

Actinocladium Ehrnb. 125.

Actinodontium Schwg. 569.

Actinomyces Meyer. 280.

Actinonema P. p. 15.

Actinophyllum Rz. Pav. 3783.

Actinothyrium Kz. 236. Actinotus LaB. 3752.

Adlambea Lam. 4577 c. Adlamsia W. 1371.

Adlansia Fisch. 44t5a. Adlansonia L. $533 \%$.

Adelia L. 5047.

Adelmannia Rchb. 2648. Adelobotrys DeC. 459?. Adenandra W. En. 5159. Adenanthera L. 4168. didenanthosLa Bill.1735

Adenaria K. H. B. 4573 
Adenariom Rafin. 6350.| Aeschynomene L. 3994. Aitonia Forst. 519.

Alenium Ehrnb. 3540. Aesculus L. 5202.

Ailenocalyx Bert. 41j0. Aethalium Lk. 179.

Aitonia L. fil. 3228\%.

Aizooı L. 405.

Allenocarpus DeC. 4025. Aetheilema RBr. 3126. Aizopsis DC. 47616.

Adenogramma Rchb. 4411 .

AdenophoraFisch.2799.

Aclenophorus Gaudich. 732.

Adenophyllum P. 2352. Allenopis DC. 4170 a.

Allenorhopium Pohl. 5060 .

Adenosina RBr. 3130.

Allenostemum Pers. 1916b. ex add.

Adostemma Forst. 2235.

Ailenostyles H. Cass. 2245.

Adenotrichia Lizdl. 2219b. ex adil.

Adesmia DC. 3992.

Adianthum L. 689.

Adina Salisb. 1941.

Allleria Neck. 4129.

Adlumia Rafiu. 4830 .

Ailolia Rheed.add. 5534.

Adonis L. 4934.

Allonis C. B. 4934a.

Adoxa L. $\$ 195$.

Adrastaea DeC. 4985.

Adriaua Gaudich. $50 \$ 9$.

Adupla Bosc. 1089.

Adyseton Scop. 476.jb.

Aechmea Rz.Par. 1281.

Aecidiun Pers. 9.

Aegerita Pers. 41.

Aegralitis RBr. 1925.

Aegialitis Trin. 950.

Aegiceras L. 3605.

Aegiceras Green. 618.

Aegilops L. Sú.j.

Aeginetia L. 305\%.

Aeginetia Cav. 204t.

Aegiphila L. 2924.

Aegle Corr. 5.532.

Aegira Fr. 435.

Aegropogon P. B. 87\%.

Aegopricon L. fil. . 5018 .

Aegoseris R. 2143h.

Aeluropus Triu. 841 .

Aeollanthus Mart. 2812.

Aëranthes Lindl. 1608.

Aërides Lour. 1503.

A erophy tou Eschw.142.

Aërva Forsk. 436j.

Aeschynanthus Jack. 3227.
Aetheorrhiza H. Cass.
2162b.

Ajuga L. 284ti.

Akeesia 'Thuh. 5221.

Aethionema RBr. 4754. Alafia A. P. Th. 3il?.

Aethusa L. 3729.

Afzelia Sin. 4133.

Afzelia Ehrh. 605. 638.

Afzelia Gm. 3166.

Agapanthus l'Herit.

1372.

Agardlia Spr. 4515.

Agaricus L. 349 .

Agastachys RBr. 1748.

Agathaea H. Cass. 2:57.

Agathelepis Chois. $30 y$ s.

Agathis Salisb. 1729.

Agathisanthes Blume. 4544.

Agathomeris Delaun. 2370.

Agathophyllum Commers. 1916.

Agathosina W.Eu.5165.

Agati Rheed. 3903.

Agrave L. 1284.

Agave L. 128tc.

Agrdestis Moc. Sess. 1889.

Ageratium DeC. 5429.

Ageratum L. 2229.

Acreria Ad. 36261).

Aglaea Pers. 1188.

Aglaia Laur. Jjos.

Ague R. 416?a.

Agonis DC. 4671b.

Agoseris Rafiu. 2131b.

Agraulos P. B. 902c.

Agrricolaea Schrli. 2929.

Agrimonia L. 4431.

Agrimonioides $\mathrm{T}$. 44:30.

Agriphyllum Juss. 26S3.

Agropyrun Gärt. \$5.3.

Agrostemma L. 5362\}).

Asrosticula Radd. 1060.

A wrostis I. 902.

Agrostis P. B. 902a.

Agyneia L. 50\$2.

Ayyrium Fr. 28?.

Ahowai T. 3569 .

Aiax Saliśb. $1268 \mathrm{~d}$.

didelus Spr. 30133.

A idia Lour. 1981.

Aira L. 1036.

dirochloa Lk. 1019.

Airopsis Desv. 1034.
Alamannia LuLlav. 1500.

Alandiua Neck. 5124.

Alangin m I.am. 45'?ti.

Alaterus T. 352?:

Albertinia Sj). '2!26. adcl.

Albuca L. 136 S.

Albucea R. $136 \%$.

Alburnoirles VC. 402:.

Alcea L. 5257.

Ilchimilla T. 4427.

Alchornea Siv. 5040.

Alcina Cav. 26j9\%.

Arleaca Rz. Pav. 2981.

Aldina Ad. 4021.

Ildinia Rchl). 3469 c.

Ildrovandi Mout. $490 \mathrm{~S}$.

Alectoria Ach. 405. 409. 410.

Alectorolophus frall. $30 \%$.

Alectra Thub. 3060.

Alectryon Gäit. 5?2S.

Alepidea LaRoch. 3751.

tlepyrum IRBr. 1131.

tletris L. 1402.

Aleurites Forst. 5063.

Aleuritia Dub. 3361a.

Alfonsia K. H. B. 1692.

Alearobia DC! $41 \% 0 \mathrm{~b}$.

Alhagi 'T. 4008. add.

Alisina L. 795 .

Allamanda L. 3541 .

A'lantodia RBr. 700.

Allasia Lour. 2767.

Allionia L. 1841 .

Allium L. $137 \%$.

Allocarpus K. H. B3.25\%t.

Allophyllus L. 5217.

Allosorus Bermh. 705.

Almeidea A. St. Hil. 5146.

Almus T. 1791.

Aloe L. 1410.
Albikia Pisl. 1030 b. ex

Alchemilla L. $412 \%$.

Alegria Moc. Sess. 5437.

Alfrealia H. Cass. 2320.

Alisidium Ag. 4896. ex 
Aloexylon Lour. 4123. Amerimunm P. Br.4020. Ainyris L. 3849.

Alomatiun DC. 4790. Amethystea L. 2843. Anabaeua Al. Juss.

Alomia K. H. B. 2232. Amherstia Wall. 1419. 5032.

Alonsoa Rz. Pav. 3189. Amicia K. H. B. 39S6. Anabaena B. St. V. Alopecurus L. 945.

Aloysia Ort. 2202.

Alphaea DC. 52:3\%. Amirola Pers. 5224. 45.5a.

Alpinia L. 1591. Ammannia Koust. $\$ 556$. Anabasis L. $^{4} 432$.

Anmanioicles DC. Anabata W. 3543. 45058 .

Alphitomorpha Wallr. Ammi L. 3719. 149.

Alsëis Schott. 2042.

Alsine Gïrt. 5350 .

Alsine 'T. $4+03$.

Ainurobium RBr. 2424.

Anacalypla Röhl. 608.

Anacainpseros Sims.

Ammocharis Herb.1250. Anacampseros T. 4189.

Anmodendron Fisch. Anacamptis Ricb. 1463. 4080.

Alsorleia A. P. Th. 4895. Ammophila Host. 909.

Alsomitra Blume. 2772b.

Alsophila $\mathrm{RBr}$. 676.

Alstonia RBr. 3525.

Alströme:ia $L$. 1343.

Ammyrsine Prsh. 3402.

Ainomuin L. $15: 17$.

Amonia Nestl. 4430.

Amoora Roxb. 5.504.

Altenstemia Knth.1469. Amorenxia Moc. Sess.

Alternanthera Forsk. 4378.

Alteruarid N. v. E. 69.

Althaea L. 525\%.

Altineastrum DC.525\%a. Altheria A. P. 'Th. 5307.

Altingia Noronh. 1731.

Altona Ail. 505:.

Alypuin T. 3092b.

Alysicarpus Neck. 4009 .

Alysium Ag. 474.

Alyssoides DC. 4768a.

Alyssum L. $476 \%^{\circ}$

Alyxia Banks. 355.j.

Alzatea Rz. Par. 5392.

Amaioua Aubl. 2053.

Amanita P. $3499^{\circ}$.

Amanoa Aubl. 5096.

Amansia Lamx. 499.

Amaranthus L. $436 \%$.

Amaria Mut. 4122.

- Imaryllis L. 1247.

Amasonia L. 2934.

Ambelania Aubl. 3562.

Ambinux Commers. 50153.

Amblyodon P. B. 643. 663.

Amblyogonon Meisn. 42916.

A inbara Inss. 1823.

Ainbrosia L. $2 \%+1$.

Ambrosinia L. 766 .

Ambultigera R. 3317.

Amelauchies Medik. $446 \%$.

Ameletia Dec. 4551.

Ainellus L. 25:59.

Amellus Adus. 2551. 4472.

Amorpha L. 3893.

Amasa Neck. 4164.

Ampacus Rmph. 5132.

Aupelodesmos Lk. 10.9.

Ampelopsis Mchx.3791. Ananassa Lindl. 1278.

Amperea Ad. Juss. 505.5. Anandria Siegesb. 2264.

Ampherephis K. H. B. Ananthocyclus Vaill. 2198.

Amphicarpa Ell. 39.30. AnonthopusRafin.18j8.

Amphicarpaea D(.3030. Allarrhimm Desf.3171.

Amphidium N. v. E.626. Anarthria RBr. 1141.

Amphichorda Fr. 48. Anasser Juss. 3503.

Amphiconium N. v. E. Anastatica L. 4730. $6 \%$.

Amphilochia Mart. 4507.

Ainphiloma Fr. 402. Anaxetan Gärt. 2426.

Amphilophium K. H. Anchietea A. St. Hil. B. 3274 .

Amphinomia DC. 4058. Anchionium DeC.4716.

Amphiphytum Schwz. Anchusa L. 2963.

28t.

Amphirrhinum Green. 649.

Ainphirrhox Spr. 4897. Anda Piso. 5064.

Amphisporium Lk. 174. Andersonia IRBr. 3347.

Amphitrichum N. v. E. Andira Lann. 4101. 70.

dudoninia Bran. 422\%.

Amphîtrichum Spr. 269. Andrachne L. 5083.

Amphodus Lindi. 3931. Andraspis Duby. 3359 b.

Amphymenim Kuth. Andreciea Ehrh. 534. 4016b.

Amsonia Walt 353\% Alliteaskia DeC. 4505.

Anyo Ance 3484.

Amyidalophora Neck. Androcymbium W. 4709 .
1301. 
Andromeda L. 3383. Androphilax Wendl. 1875.

Audropogon L. 973.

Andropogon P. B. 973b.

Androsace L. 3359 .

Androsaemum Ail. 5473.

Andryala L. 2160.

Aniecio e. e. Neck.2496. Aneilema RBr. 1173.

Aneim:a Sw. 735 .

Aneisothea DC. 40951).

Anemagrostis Trin. $90 \%$.

Anemonanthea DC. 4944.

Anemone L. 4944.

Anemosperma Cominel. 2672.

Anemospermum Coinmel. 2670.

Aneslea Andr. 814.

Anethnin L. 3673.

Angelica L. 3698.

Angelonia Hb. Bpl.31s8. Angianthus Wendl. $2+46$.

Anmiopteris Hoffin. 7 $\$ 6$. Aligolam Ad. 4526.

Antrolamia Srop. 4526. Angophora Cav. 4674. Angostura R. S. 514.5. Angraecum A. P. 'Th. 1509.

Anumillaria RRr. 1297. Auguina Mich. 27iss. Alguloa Rz. Par. 1526. Alsuria L. 2765.

Aniba Anisl. 1914. Anictangiam Hook. Air 11.535.

AnigosanthusRed.12?2. Aluigozanthos LaB.1?2?. Anisacan!ha RBr. 4331 . Anisanthina R. 1167.

Anisoleris H. Cass. 2142 .

Anisorlontiun R. 2985a.

Anisodus Lk. 3309.

Anisomeles RBr. 2843.

Auisonema Ad. Juss. 5072.

Anisopetalun Hook. 15.29.

Anisophyllum Haw. 501 . Anisopogon RBr. 1042. Anthora Riv. 4930a.

Anixia Fr. 160.

Ammesorrhiza Cham, 3734.

Anocarpum DC. 4816a. Anthotiun RBr. 2781.

Anoda Cav. 5247. Anthoxanthum L. 1033.

Anodontera DC. 4769. Anthriscus Pers. 3659.

Anodontinm Brid. 584a. Anthrisens Riv. 365\%a.

Anoegosanthus Lab. ein. 1222.

Anoectangium Hdir.53\%. $566 \%$.

Anogeissus DC. 4538a.

Anoina Lour. 415?.

Anomalopteris DC. $5371 \mathrm{~b}$.

Alomatheca Ker. 1195. Anomoron llook et Tayl. 564.

Anona Ad. 4999.

dinonyma Walt. 3990.

duonymos Walt. 1210. Anopton Wallr. 3060a. ex add.

Anopterus LaR. 4250.

Anotea DC. 5215.51).

Anredera Jsss. 4388 .

Aitelaea Gärt. addl. 5535.

Antennaria Gärt. 2131. Antennaria Lk, 71 .

Antenunlaria Rchh. 71. Authactinia B. S.t. V. 3472 .

Anthema Mod. 52:58.

duthemis Blich. 23:3.

Anthenantia P. B. 931. Antephora Schreb. 830. Anthericum L. 1336j.

Anthericun Lam. 1334. Antherilium Rohr. 4570. Antherura four. 2031a. Authestiria L. 981.

Authina $\mathrm{Fr}_{0}, 50$.

Anthistiria Lam, 981.

Anthobolus RBr. 1701. Anthocercis LaB. 3?01. Anthoceros L. 525.

Authocleista Afzel. 5308.

Anthodendron Rchb. 3397.

Anthodon Rz.Pav.5\%s3. Anthodus Mart. 5383. Antholoma LaB. $5+80$. Antholyza L. 1193. Anthonota P. B. 4114. Authopogon Nutt. 918.
Anthospermun $I_{1} .4418$.

Anihostemma Adl. Juss. 5014.

Authrophyun Kaulf. 698.

Authyllis L. 4025.

Aitlifylis Ad. 4261. 4263.

Antiaris Lesch. 1816.

Antichorus L. fil. 5444.

Antidesma L. 5087.

Antirrhea Commers. 2023.

Antirrhinum L. 3175.

Antitrawıs Gärt. 943b.

Antitrichia Brid. 51j4.

Antoiria Radd. 53?b.

Antrocarpon Mey. 381.

Allychia Mchx. $426 \%$.

Aotus Sin. 4068.

Apactis Thul, add, $5 \% 36$.

Apalatoa Aisbl. 4108.

Apargia IV. 213\%.

Apatelia DC, 5\&14l).

Apatilia Ilamilt. add. 5537.

Apeiba Anbl. 5852.

Apera Allus. y07.

Aphanes L. 4427 a.

Ajhanostemma A. St. Hil. $4 ! 42$.

Aphelandra RBr. 3125.

Aphelia RBr. 1132.

Aphloia DĆ. 4926a.

Aphora Neck. $407 y$.

Aphraginus Andrz. 4732 .

Aphyllanthes L. 1150.

Aphyllocanlan LaG. $225 \%$.

Aphyllodium DC.

$$
40011 \text {. }
$$

Aphyteia L. 1663.

Apicra IV. 1406.

A pios Boerh. 3937.

Apium L. 3726.

ApTolon RBr. 601.

Aplophyllim Ad. Juss. 5121.

Aplophyllim H. Cass. 2269.

Apluda L. 978.

Apocynum L. 3522.
Apiosparium Kz. 157. 
Apodanthes Blume. 165.

Apoiloginyus DC. 4482a, A pouncetou Thub. 793. Aporefica Forst. 5218. A poseris Neck. 21'?.

Anpendicularia DeC. 4580.

Aptosimum Burch.3214. A prileja Gärt. 2683.

Apus 324. 327. 3381).

Aquarria Jarr. 3292.

Aquilolium T. 3624.

Ayuilaria Lam. 178 \$.

Arabiclopsis DC.

Arabis L. 4790.

Arabisa R. 4790 h.

Araciun Neck. 2150.

A rachidua Plum. 4096. Arachis L. 4096.

Arachuiou Schwz. 218.

Arachuospermum Berg. 21.53.

Aramoa K. H. B. 3199. Aralia I. 3781.

Arapabaca Pluin. 3551. Arauca ria Juss. 1730. Arauja Brot. 34:32.

Arluntus I. 3385.

Arceuthohim Yis.1960. Archangelica Hoffun. $36 ! 99$.

Archidium Bricl. 579. Architaea Mart. 5404. Arcrion Dalech. '23'5. Arctiun Laur. 2325. Arctium L. 2319.

Arctotheca Wenill.267. Arctopus L. 3754.

Arciostaphylos Adans. 3386.

Arctotis I. 2672.

Arcyphyllum Eil. 393.3.

Arryria Pers Pers. 187. Arrliughelia Comm. $50 \% \%$.

Arulisia Sw. 3599. Arduina L. 3.jtjt.

Areca 1. 1154?

Aremonia Nerk. $4 \$ 30$.

Arenaria L. 53.3.3.

Areñ. Lal). 11525.

A rethusa Sir. 1438.

Aretia L. 33.39 .

Aripenoue T. 485.5.

Arunolasia Juss. 1225.

Arophlillum Forsi. $337 ?$.
Argostemua WVall.2029.|Arthrozamia Rchb. 751. Aryylia Don. 3269.

Arigyroia Lour. 3014.

Ariyraulhes Neck 2426.

Areythamia P. Br. $50+1$.

Aria 4tz0e.

Alisarm T. 768.

Aristea L. 1206.

Aristella Trin. 920a.

Aristida L. 921.

Aristida P. B. 921a.

Aristolochia L. 185 fj.

Aristotela Adns. 2515.

A ristotelia l'Herit. 4248.

Armeniaca T. 4707.

Aruneria IV. 1923.

Armoracia Rupp.4j63c.

A ruica L. 2487.1.ex add.

Aruoldia II. Cass. 2731.

Arumọonou W. 2178.

Aruoseris Gärt. 212 t.

Aromatendron Blume. 4994.

A rongana P. 5475.

Aronia P. e. e. 4.170b.

Aronicun Neck. 2488.

Arotou Neck. 5045.

Arpophyllum LaLlav. $143 \%$.

Arrhenachne H. Cass. '204.

Arrhenatherun P. B. 1041.

Arrlienopterum HIJg. 6.5.3.

Arruilea Camb. 5495.

Artabotrys RBr. 5005.

Artedia L. 3694 .

Artemisia T. 2368.

Arthonaria Fr. 353.

Arthonia Ach. 356.

Arthratheruin $\mathbf{P}$. B. 921a.

Arthraxon P. B. 894.

Aithriuiun Kz. 105.

Arthritica Dub. 3361c.

Arthrolobium Desv. em. 3979.

Arfhrolobus Steer. $471 \%$.

Arthropodium RBr. 1385.

Arlhrostachya Lk. $10 ? 1$.

Arthrostemma Pav. 4515.

Arthrostylis RBr. 1095.
Artocarpus L. 1817.

Artrolohium Desv. 3979.

Aruba Aubl. 517t.

Aruba N. et M. 5146.

Aruin Auct. 769a.

Aruma Aubl. 4103.

Arumcus 4449b.

Arundinaria Mchx. 1051.

Arundinella Radd. 1062.

Arundo L. 1047.

Asaphes Spr. 2\%os.

Asariua 1'. 3175a.

Asarum L. $181 j 0$.

Ascaricida H. Cass.2186.

Ascariua Forst. 1788. 1954.

Ascilliophora Rchb. 92.

Asclepias L. 3425.

Ascobolus :ers. 315.

Ascophura Schwz. 92.

Ascophora Tod. 139b.

Ascra Schott. 5497.

Ascroë Labill. 225.

Ascospora Fr. $26 . j$.

Ascyreia Chois. 5471d.

Ascyrum L. 5468.

Ascyum V. 5452.

Asimina Ad. 5009.

Aspalathoides DC. 40'5ól.

Aspalaihus L. 403ł.

Aspalathus Neck.4034b.

Asjulragus L. 1319.

Aspelina H. Cass. 2491.

Aspergillus Mich. 11S.

Asperococcus Lanx. 507.

Asperugo L. 2971.

Asperula L. $198 \%$.

Asphodelus L. 1389.

Aspidalis Gärt. 2679.

Aspiclistra Ker. 781.

Aspidium Sw. 681.

Aspirlosperma Mart. $354 \%$.

Aspilia A. P. Th. 2600.

Asplenim L. 699.

Asprella Cav. 844.

Asprella Hst. 819.

Asjrella Schreb. 953.

Assonia Cav. 5.312.

Astelia Buks et Sol.1224.

Astelına RBr. 2429b.

ex add.
Aruili L. 769. 
Astephananthes B. St.|Atractium Ik. 37.

V. $3472 b$.

Atractobolits Toul. 215.

Astephauns RBr. 3451.

Aster 'T. $20 j 1$.

Atractylis L. 230\%.

Atragene L. 4947.

Asteranemia 12. 4944b. Atracene 4y47b.

Asteranthus Desv.3618. Atraphaxis L. 4299.

Asterias Brkh. 3499c. Atriplex L. 4344.

Asteripholis Pont. 2551. ATriplexmm L. 434ta.

Asterisca Mey. 391.

Asteriscium Cham. 3773.

Atropa L. 330i.

Attalea K. II. B. 1626. Bactyrilobium IV.

Aubertia R. St. Y.j132. 413ish.

Asterocephalus Vaill. Anbertia P. B. 538. 1933.

Asterolinum Lk. 3326.

Anbletia Gärt. 4691 .

Aubletia Rich. 51\$1.

Asteroma DeC. 230.

Aubletia Schreb. 54j2.

Asterophora Ditm. 170. Aubrietia Ad. 4769.

Asterothrix H. Cass. Anchemangiun Brid. 21;6b.

Astianthus Don. 3271.

Astilbe Hamilt. 4199.

Astragalins L. 3882.

Astranthus Lour. 4702.

Astrantia L. 3753.

Astrapaea Liull. 5266.

Astrocaryum W. Mey. 1622. 6011 .

Anchenangium Brid. Msc. 609.

Aucubá L. 1961.

Augea Thub. 4397.

Aulacomnion Schwr. 652.

Aulacilium Rich. 4616.

Aulax Berg. 1749.

Astrodontium Schwg. 563.

Aulaxanthis Ell. 931. Aulaxis Nutt. 431.

Astrolobium DC. 3976.

Astroloma RBr. 3341 .

Astronia Blume. 4648 .

Astronimu Jcy. 3858 .

Anrelia H. Cass. 2521.

Auricula T. 3361b.

Auricularia Bull. 324.

Avena I. 104.3.

Asteropeia A. P. Th. Avenaira R. 1043a. 4701 .

Astrophen DeC. $3 \pm 62$.

Astrosporium $\mathrm{Kz}$. $2 S$.

Astrothelium Eschw. 366.

Astrycum Rafin. p. 15. Avenaria R. 10\$3c.

Averihoa L. 5.3?1.

Avicennia L. 2942.

Avicennia A. P. Th. $29+2 b$.

Alacia Prsl. 784.

Avicularia Meisn. 4291 a.

Atalantia Corr. 55?2. Axia Lour. 18.50.

Ateleia Fl. mex. 4016 d. Axinaea R\%. Pav. 4604.

Aihalmum Neck. 2476. Axolopha DC. 5258.

Alhamanta L. 3710.

Athamı: Neck. 230j.

Athanasia L. 2386.

Afhelia P. p. 15.

Axolophia DC. em. $5258 \mathrm{c}$.

Axonopus P. P. 88\%b.

Axyris L. 4348.

Alhenaea Schreb. 4695.

Atheolaena H. Cass. 2793.

Athreropogon Mhlub. 873. -

A'hirisperma Lab. 18:\%.

Athroilactylis Forst. $1 ?: 0$.

Ayenia L. 5292.

Aylmeria Mart. 4309.

Azalea L. 3392.

Azalea Auct. 3397.

Azanza Moc. Sess. $5273:$.

Azara 1Rz. Pav. 4923.

Azima Lam. 3566 .

Azolla Lam. slti.

A1l, malıs Veck. 5012c. Azorella Lam. 377.

Aiocion Otth. 5363a. Azygites Mong. 131.

Ballarnia G. 45\$3.

Badliera DeC. 30:33.

Baea Commers. $31+1$.

Baikea L. 4670 .

Baeobotrys Forst.33ti6.

Baeoinyces Pers. 413.

Baetonica Lam. 46.54.

Bagalatta Rxh. 1580.

Bahia LaG. 2575 .

Bailleria Aubl. 2601.

Baiteria Rz. Pav. add. 5539.

Balanophora Forst. 1681.

Balamopteris G. 52Sj.

Balantium Hamilt. add. 5540.

Balautium Kanlf. 656.

Balbisia W. 2576.

Baldingera G. M. S. 952.

Baldingeria Neck. 2375.

Balduina Nutt. 257\%.

Balfuria RRr. 3j2S.

Ballota L. 2883.

Ba!samea Gled. 3839.

Balsamina Riv. 4835.

Balsaınita Vaili. 2376.

Balsamodendron $K$ inth. 3838.

Balsaınona Vand. 4559.

Baltimora L. $26+6$.

Bambos R!z. 105 ?

Bambusa Schrob. 1052.

Bambusella R. 936c.

Banara Aubl. 49?4.

Banava Cans. 4577.

Banffya Baume. 6359a.

Bancia Lgh. 400 .

Banisteria L. 5370.

Banjolea Bowd. adıl. 5i)t1.

Banksia L. fil. 1769.

Bauksia Doml). 45:59.

Baobab. J. Bauh. 5337.

Baphia Afzel. 4159.

Baptisia Vent. 4083. 
Barbacenfa Vand. 1228. Rarlieria DC. $396 \%$. Barbula Hdg. 635. Barclaya Will. 81\%. Barkania Elırub. 759. Barkhausia H. Cass. $21+2 b$.

Barkhnusia Much.2142.

Barleria L. 3128.

Barnadesia L. 2295.

Barnardia Lindl. 1366.

Barosma W. E11. 5164.

Barraldeia A. P. 'Th. siss.

Barreria Scop. 1899a. ex adl.

Barriugtonia Forst. 4 (1i.) 4 .

Bartholina RBr. 1479.

Bartlingia Brugn'n. 4669.

Bartonia Sims. 4234.

Bartonia W. 3484.

Bartramia G. 5433b.

Bartramia Hdg. 654 .

Bartsia L. 3084.

Baryosina G. 4023a.

Baryosma R. S. 5164."

Bary xyluin Lour. 4137.

Basclla L. 4389.

Baseophyllum DC. 4135c.

Basilaea Laın. 1360.

Passia Köı. 365.5.

Bassovia Aubl. 3298.

Bastardia K. H. B. 5250.

Basteria All. 1832.

Basteria Hontt. 2(j83.

Batarrea Pers. 22\%.

Pataucanlon DC. 4162c.

Batis L. 1692.

Batrachium DC. 4939.

Batrachosperma B. St. V. $43 \%$.

Batrachospermum Rth. $43 \%$.

Batschia Much. 2230.

Batschia 'Thub. 1888.

Batschia V. 4118.

Bandinia Lesch. $465 \%$.

Banera Audr. 4209.

Bauhinia Kilth. 4126a.

Bauhiuia Plum. 4126.

Baumea Gaudich. 1100.

Banmortenia Spr. 1154.

Baumgartia Much. 1851 .

Baursea Angl. 773c.
Baxtera Rchb. 3454. Bertolonia Spr. 1620.

Beatsonia Rxh. 4903. Berula Hoffin. 3736.

Beauharnoisia Rz. Pav. Berzelia Brngn. 4221. 5488.

Beaunontia Wall. 3516. Besleria L. 3230.

Beckeria Bruh. 1014. Bessera Schult. 2947.

Beckmamia Host. 870. Beta L. 4351.

Beera P. B. 1125. Betonica L. 2884.

Beesha Rheed. 1056. Betilla L. 1790.

Befaria Mnt. 33\%5. Benteria Jcq. 2984.

Begonia L. 4293. Beurreria Ehr. 1832.

Behenautha Otth."5363b. Beyrichia Chan. 3159.

Belemcanda Mönch. Biatora Fr. 412. 1208.

Belilla Rheed. 2061.

Belis Salisb. 1728.

Bellardia Schreb. 2094. Bifora M. B. 3702.

Bellendena $\mathrm{RBr}$. 175t. Biloris Spr. 3702.

Bellevalia Lap. 1363. Bigelovia Spr. 1998.

Bellidiastrum Michel. Bigelovia Spr. Entd. $25 \% 1$.

Bellidioides Vaill. 2351. Bign onia L. 3259.

Bellinginia Radd. 552a. Biliarderia V. 2020.

Bellinia R. S. 3304.

Bellis T. L. 2569.

Belliim L. 2570.

Bellncia Ad. 5125.

Bellucia Neck. 4642.

Belon Ad. 5532.

Belonia L. 2060.

Belvisia Desv. 3619.

Bembix Lour. add.5542, Benillcasa Sav. 2752.

Berardia Vill. '2325.

Berardia Brugu. 4225.

Berberis L. 4838.

Berchemia Neck. 3819.

Bergensia Much. 4204.

Bergera Kön. 5526.

Bergeretia DC. $4758 \mathrm{c}$.

Berghias Soun. 2111.

Bergia L. 4554.

Berinia Brig'l. 2145.

Berkheya Ehrh. 2683.

Berinuliaua Gärt. 117\%.

Beruardia Houst. 504\%.

Beruhardia W. 1678.

Berrya Rxb. 5460 .

Berteroa DC. 4770a.

Bertholletia Hb. Bpl. 4651.

Bertiera Aubl. 2086.

Bertolonia DeC. 2278.

Bertolonia Radi. 4617. Blechoria

Bertolonia Del Spig. Blepharidium DC. 3046.

Bertolonia Moc. Sess. Blepharis Juss. 3121.

4428.
Billbergia Thub. 1280.

Billotia Colla. $465 \%$ b.

Binectaria Forsk. 3639.

Biophytum DeC. 5320.

Biotia H. Cass. 2698.

Biporeia A.P. Th.5178. Biscutella L. 4745 .

Biserrula L. 3885.

Bistorta T. 4291c.

Bivonaea DeC. 4752.

Bivonaea Rafin. 5059.

Bivonaea Moc. Sess. 4264.

Bixa L. 4929.

Blandfordia Sin. 1404.

Blandfordia Andr. 3373.

Blandovia W. 526.

Blainvillea H. Cass. 2624.

Blairia L. $337 \%$.

Blakburnia Forst. 5128.

Blakea L. 4642.

Blakuvellia Comm.4700.

Blankana Ad. 622b.

Blasia L. 532. add.

Blaxium H. Cass. 2729.

Blechnum L. 711.

Blechum Juss. 3123. 3029d. 


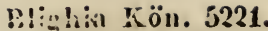
Bitialllius Rchb. 4366. Blifum L. 4.345.

แs!or!nuannia Weig. $4 ! ! 48$.

I3lusnea Mchb. 5435.

Blutirenbachia Schrad. 42.33 .

Blamenbachia Koel. USt.

Blismia Spr. st11.

Blyxa Aub. B. Tii. 806.

Bobartia L. 11 is.

Bobea Gaudich. 2099.

Bobu Ail. 4535.

Bobua DeC. 4535.

Bocawea A. St. Hil. 600.3 .

Bocconia J. $48 \pm 6$.

Boclumeria Jcy. I801.

Bornuinthansenia

lachb. J12:.

Bocuninghausia Spr. $391 ; 0$.

Boerhavia L. 1839.

Bolanthus Ser. 5360b.

Bolax Commers. 3775.

Bolilea Juss. 1838.

Bolilua Cav. 1846.

Bolducia Neck. 4023b.

Boletus Dill. 339.

Boleum Desv. 4739.

Bolivaria Cham. 3549 .

Boltonia l'Herit. 2silit.

Bomarea Mirb. 1343.

Bombax L. 5335.

Bombycella 5273e.

Bonalidia Neck. 3893.

Bonamia A. P. 'T'h. 3009.

Bonannia Rafin. 5221.

Bonapartea liz. Pav. 1276.

Bomapartea W. Lk.1281.

Bollatea WV. 1473.

Bonaveria Scop. 3982.

Bomellia Brot. 3643.

Bonplandia Cav. 3100.

Bonplandia W. $514 J$.

Bonduc PJum. 4151.

Bonnaya Lk. 3133.

Bonnemaisonia Ag. 498.

Bonnelia Mart. 5405.

Bnunetia Schreb. 5403.

Buntia L. 3045 .

Buopis Juss. 2738.

Inotui Nieck. 5360c.

Borassus Sonner. 1662.

Borassus L. 1563.
Borbonia L. 4048.

Borkhausia Lk. 2142.

Boronia Sin. 5167.

Borrago L. 2973.

Borreria Mey nou Ach 1998.

Borya Lab. non IT 1154.

Bory "a Gaillo:1. 483h. Boscia Lain. $4819 \%$.

Boscia Thub. 512\%.

Bosea L. 4394.

Bossiaen Vent. 4055.

Bossiena Vent, e:n. $\{0 j 5$

Bostrychia Fr. 247.

Bosivellia Rxb. 836 .

Botos Ad. 39tio.

Botrycarpum Rich. $4: 17$.

Bofryceras Wr. 3tj2.).

Botrychium Sw. 749 .

Botrydimm Wallr. 4i2a.

Butryocarpum Kz. $42+7 c$.

Bofryopteris Prsl. 748.

Bofrytis Mich. Ilj.

Botrytis N. V. E. e.p. $115 \mathrm{~d}$.

Boussingaulita K. II. B. 4390 .

Bonteloua Lag. 871.

Bonvardia Salisl). 2046.

Bovista Pers. 201.

Bowvilichia K. H. B. 4 '21.

Bowiea Haw. 1408.

Boymia Ad. Juss. 5131.

Brabeium L. 1753.

Bracliycarpaea DeC. $475 \%$.

Brachycome If. Cass. 250 s.

Brachyderea H. Cass. 2146.

Brachyelytrum P., B. 915 .

Brachyglottis Forst. 2518.

Brachylaena FiBr. 2203.

Brachymeniun Hook. 645.

Brachyolobus All. 47961 .

Brachyotum DC. 4505l).

Brarliypetalum DC. $491 \%$.

Brachypodium P.B. 852. Bromus Bess. 102?).
Brachypodium Brial. non P. B. 619.

Brachy ris Nutt. 2530.

Brachyscome H. Cass. $200^{\circ}$ í.

Brachysema RBr. 407.

Brachysteleun Rchb. 619.

Brachystemma Don.

b3üt.

Brachystylium DC. em. 4813 ?.

Brachytrichum Röhl. 6?21

Brachytropis DeC. 3030.

Braciearia DC. 4594c.

Braclearia Pöpl. 206l.

Bricteogiuma bec. 3469.

Brallea All. 39.37.

Bradleia Gär. 5071.

Bralypipton DC. 47516.

Bragintia Lour. 1857.

Brandesia Mart. 4350.

Brandonia Rchb. 3319.

Brasenia Prsb. E1?.

Brasilettia DC. $41 \$ 9 \mathrm{c}$.

Brassa vola $13 \mathrm{Br} .1 \mathrm{j} 43$.

Brassia $\mathrm{RBr}$. 1521.

Brassica L. 4817.

Brassica Pliu. 4817c.

Brathys Mut. 5t7la.

Braya S(rul). Hpp. 4802.

Brainea W. 181i!)

Bravoa IIerl). 1232.

Brayera Kuth. 4429.

Breilemeyera IV. 3039.

Bretenillia Buchoz. 21380.

Brevin Norouh. 3630.

Breyuia Plım. 4574l.

Brei uiastrum DC. 4874.

Brickellia Rafin. add. sist4.

Briedelia Villa. 5084.

Bricnolia Bertol. 3711.

Brillantitisia P. B. 3113.

Brimlonia A. P. 'Th. 5) 491 .

Brissonia Neck, 389?b.

Briza L. 1032.

Brizopyrum Lk. 849.

Brodiaea Sm. 1358.

Bromelia L. 1279.

Bromfeldia Neck. 5061.

Bromus L. 10??.
Breweria RBr. 3008. 
Bronguiartia K. H. B.|Buffonia Sauv. 5344. |Cacoucia Anbl. 4531. $40 y y$.

Bronnia K. H. B. 4240.

Brosimum Sw. 1804.

Brossaea Plum. 3389.

Brotera Spr. 2699.

Brotera Civ. 5311.

Brotera IV. 2297.

Brotightouia IiBr. 1548.

Broussonetia Vent. 1814.

Browallia L. 3108.

Brownea Jacq. 4024.

Buginvillea Coinm.185?

Bugrana DC. 4027 e.

Bujula T. Juss. 2846a.

Bulbine W. 1384.

Bulbocastanum $\mathbf{T}$. $3718 b$.

Bubochaete Ag. 468.

Bulbocodium L. 1308.

Buthopbyllum A. P.

'Th. 1572.

Bubostylis Stev. 1118. Bulgaria Fr. 314.

Brownetera Kich. 1696. Bullaria DeC. 17.

Browulowia $\mathrm{R} \times \mathrm{b}$ ). 5190.

Brucea Mill. 5135.

Bruchia Schwg. 582.

Brînuchia Gärt. 4295.

Brugimansia P. 3286.

Bruguiera A. P. Th. 4536.

Bruguiera Lam. 1968.

Brunellia T'. 's13.

Brumellia Rz. Pav. 5135.

Brunfelsia L. gen. 3217 .

Brunia L. $422 \%$.

Brunouia Sm. 1922.

Bruusfelsia L. sp. 3217.

Bruusvigia Ker. 1246.

Brucuiera Rich. 4634.

Brya P. Br. 4021 .

Bryocladium Kz. 333 .

Bryonia L. $27+9$.

Bryophyllum Salisb. 4184.

Bryopsis Lamx. 473.

Bryum L. 612.

Brymu Dill. 642b.

Bubon L. $367 \%$.

Bubou Spr. 37isb.

Bubroma Sichreb. 5295.

Bucco Wendl. 5165.

Buceras Much. 3872c.

Buceras P. Br. 4545.

Buchanauia Rxb. 3863.

Buchliavea R. 44tia.

Buchia K. H. B. 2918.

Buchuera. L. 3104.

Bucholzia Mart. 4379.

Buchozia l'Her. 2096.

Bucida L. 4545.

Bucquetia DeC. 4613.

Buddle:1 Spr. 3204.

Buillleia L. 3204.

Buena Cav. 2115.

Buena Pohl. 2066.

Biichnera Scop. $323 j$.

Bittneria Duh. 1832.

Bulliarla DeC. 4174.

Bumalia Thuub. 4208.

Bumalla Thub. 5203.

Bumelia Sw. 3636.

Bunchosia Juss. 5379.

Bunias L. 4718.

Binium L. 3718 b.

Biphone Herb. 1249.

Bupleurum L. 3740.

Bupleurum Hoffin. $3 \% 401$ b.

Buphthalıum L. 2475. Burasaia A. P.Th. $186 \%$.

Burcarlia Scop. 4238.

Burcharda Neck. 4687.

Burchardia RBr. 1299.

Burchellia RBr. 2117c.

Burghartia Neck. 4238.

Burunanuia L. 1213.

Burmannia Lour. 1212.

Bursaria Cav. 3048.

Burscra Jacc. 3841.

Burtonia RBr. 4072.

Burtonia Salish). 4978.

Bustia Adaus. 2475.

Butea Roxb. 3958.

Butouns L. 1306.

Buxus L. 5092.

Byblis Salish. 4909.

Byrsonima Rich. 5380.

By ssus L. 67.

Bystropogon l'Herit. 2863.

Byıtueria Löm. 5293.

Caballeria Rz. Pav. 3641.

Caboinba Aubl. 811.

Cabrera Lag. 862.

Cabritta 12. 3205a.

Cacalia L. 2509.

Cacao T. 5298.

Cacara A. P. Th. 3943.

Cachrys T. L. 3748.

Cactus L. 4244.
Cadaba Forsk. $48 \%$.

Cadauba Soll. 2107.

Cadia Forsk. 4140.

Caeuotus Nutt. 2546.

Caeoma Lk. diss. Ya.

Caesalpinia Plum. 4149.

Caesia RBr. 1391.

Caesulia Rxb. $2 \% 00$.

Cagou Ad. 3952.

Cainito Plnm. 3640.

Cajanus DC. 3952.

Cajn- puti Ad. 4659.

Cakile T. 4713.

Calarlenia RB̉. 1447.

Caladium Auct. 7\%3a.

Caladium Yent. $7 \% 3$.

Calaena RBr. 1451.

Calamagrost is Rth. 908.

Calamilia P. B. 982 .

Calamintha Lk. 2821.

Calamochloë Rchb. 983.

Calamus L. 1616.

Calanchoë Ad. 4183.

Calandrinia K. II. B. 4315.

Calauthe RBr. 1485.

Calanthea DC. 4874c.

Calathea W. Mey.1607.

Calcatrippa Natth.

4949a.

Calccolaria L. 3142.

Calcenlus 157\%a.

Calcilrapa P. $2688 a$.

Calcitrapa Vaill. 2688.

Caldasia WV. 3100.

Calea RBr. 2578.

Caleacte RBr. 2579.

Calectasia $\mathrm{RBr}$. 1314.

Calendula L. 2728.

Calepina Ard. 4721.

Caliciun Ach. 360a.

Caliciuin Pers. 360.

Caliunx Rafin. 1711.

Calispermum Lour. 4826.

Calla L. 771.

Callanthus R. $1192 b$.

Callicarpa L. 2920.

Callicocca Brot. 2119c.

Callicoma RBr. 4206.

Callicomia Burm. 2408.

Calligonmm L. 4283.

Calliopsis Rchb. 2605.

Callisace Fisch. 367 .

Cacosmia K. H. B. 2191. Callisia L. 1172. 
Callistachya Rafin. |Calyplectus Rz. Par./Canahia Spr. 3436. 4064. 4378.

Canaria 3845a.

Callistachya Sm. 4075. Caly pogeia Radl. 532g. Canarina L. 2802.

Callistachys Vent. $407 \%$.

Callistemma H. Cass. 2563.

Callistemon RBr. 4660.

Cullistephus H. Cass. 2563.

Calypso Salisb. 1567. Canarium L. 3845.

Calypso A. P. Th. 5385. ('anavali Ad. 3947.

Calyptrantes Sw. 4676 . Canavalia DC. $344 \%$.

Calyptranthus Juss. Caurellaria DC. 5\%61. $46 \% 6$.

Cancellia R. 5?61c.

Callisthene Mart. 4506.

Callithamnion Lgb. 483.

Callitriche L. $4+88$.

Callitris Yent. 1721.

Callixene Juss. $13+6$.

Callopisma Mart. 3494 .

C:lluna Salisb. 3380.

Calocephalus RBr. 2447.

Calocera Fr. $29 \%$.

Calochilus RBr. 1432.

Calochortus Prsh. 1295.

Calodendron Thub. $515 \%$.

Calodíy um Desv. 3396.

Calogyne RRr. "2776.

Calomeria Vent. 23\%0.

Calophaca Fisch. 3911.

Calophyllum L. 5490.

Calophysa DeC. 4626.

Calopogon RBr. 140.

Calopogoniun Desv. 3950.

Calyptrion Ging. 4890.

Candarum Rchb. 769b.

Calystegia RBr. 301'. Candollea LiB. 1919.

Calythrix LaB. 4665 . 4984.

Calytriplex Rz. P. 3\%1. Candollea Radıl. 5321.

Calyxhymenia Cav. 1840.

Canella Brw. 5511.

Camarea A.St. Hil.j376.

Camaridium Lindl. 1492.

Camax Schreb. 2976.

Canephora Juss. 2117 .

Canna L. 1608.

Cannabis L. 1820.

Canscora RBr. 3487.

Cambderia Knth. 1230. Cantharellus All. 312.

Cambessedea Knth. 3832.

Cambessedesia DeC. 4614.

Cambogia L. 5492.

Camelina Crutz. 4780.

Camellia L. 5 \pm 07.

Cameraria L. 3536.

Camiriun Rmph. 5063.

Camissonia L!. 4496.

Cammarum 12. $40 \mathrm{O} 0 \mathrm{~b}$.

Campanula L. 2800.

Caloptiliun LaG. 2291.

Calostemma MBr. 1266.

Calostoma Desv. p.15.

Calotham inss LaB. 4657.

Calothrea Desv. 1007.

Calothric Ais. 450 .

Calotropis RBr. 3432.

Calpidia A. P. Th. 1833.

Coltha Raj. 4961.

Caltha T. 2728.

Calusia Bert. 4091a.

Calycanthus L. 1832.

Cilycera Cav. 2737.

Cilycobolus. W. 2998.

Calycocorsus Schm. 2161.

Calycogoninm DeC. 4629.

Campecia Ad. 4149b.

Campelia Kich. 1170.

Campella Lk. 1036.

Camphorata T. 4326.

Camphorosma L. 4326.

CampomanesiaRz.Pav. $468 \%$.

Campsotrichum Ehrub. 126.

Cainptosporium Lk. 122.

Camptoum Lk. 10 \&.

Campuleia Honk. 3088.

Campuloa P. B. 867.

Campulosus Desv. 863.

Campylauthus Rih. 3099.

Calycopteris Rich. $\mathbf{4 6 2 9 .}$

Calycopteris Lam. 4541.

Caly cotome Lk. 4029c.

Calycotomon Hffge. 4029.

Calycotomus Rich. 4634.

Calyilermos LaG. 2580.

Caınpyleia A. P. T'h. 3058.

Cantharifera Rumph. 788.

Camthium Lam. 2035.

Cantua Juss. 3027.

Capellia Blume. 4990.

Caperonia A. St. Hil. 5043.

Capillaria P. p. 15.

Capitularia Flk. 41 th.

Capnoides Boerh. 4833i.

Ca| nopliyllum Gärt. $36 \%$.

Capparidastrum DC. 4874 .

Capparis L. 4374.

Capparis plum. 48\%4f.

Capraria L. 3205.

Caprifolium 's. 1198.

Capriola A.1. 86?.

Capsella Vent. 4741.

Capsicum L. 3299.

Capura L. 1783c.

Caquepiria Gm. 2111.

Caragana Lain. 3908.

Caraguata Piso. 1274.

Caraipa Aubl. 5400.

Carallia Roxb. 1969.

Carallumn RBr. 3413.

Carapa Aubl. J́s20.

Campylia Sweet. 5278c.

Campy lopus Brid. 597.

Campylopus P. B. 591.

Campylorutis Ser. $38 \% 0 \mathrm{c}$.

Campylus Lour. adi. 50็.j.

Carapichea $A$ ubl.21193.

Carara Cacsalp. 4749.

Cardanine L. 4783.

Carlaminum DC. 4796.

Cardamon DC. $4731 \mathrm{c}$.

Carlaria Desv. 4750.

Carderina H. Cass. 2494.

Cardia Moc. Sess. 4261.

Carliaca T. 2881 .

Calymperes Sw. 612. Cainpynema LaB. 1216. Cardiacastrum R.2881b. 
Cardiolepis Wallr. 4750. Casalea A. St. Hil. 4938.|Caulophylium Mchx.

Cardioneına DC 4264. Cascarilla Ad. 5045.

Cardiolochia R. 1856d. Casearia Jacq. 4695. $48+1$.

Cardiospernum L. 5235.

Cardispermum Trant. 2730 .

Casparia Knth. 4126e.

Caunotropis Rich. 4120 a.

Cassebeera Kaulf 691. Causea Scop. 4.318.

Cassia L. 4135.

Carlopatium Juss. 2297.

Carduncellus Lob. 2308.

Carduncellus Adus. 2690.

Carduus T. 2338.

Careacia R. $4695 \mathrm{~d}$.

Carex L. 1071.

Careya Rxb. 4504.

Cargillia $1 \mathrm{BBr} .3648$.

Carica L. 2773.

Carissa L. 3560 .

Carliua L. 2306.

Casselia N. v. E. 2906

Cassida 1'. 2830.

Cassine L. 3620 .

Cassinia RBr. Bot. Reg. Cavanillesia Rz. Pav. $2+22$.

Cassinia RBr. H. Kew. Caviniun A. P. Th. 2446.

Cavanilla Thub. ad. 5566.

Cavanillea Lam. $3647 \mathrm{~b}$. ex add.

Cassipourea Anbl. 1970. Cavonillea Borkh. 605.

Cassupa Hb. Bpl. 2012. Cayratia Juss. 3789.

Cassuvium Lam. 3867. Ceanothus L. 3799.

Cassytha L. 1904. Cebatha Forsk. 1872.

Carlowitzia Much.2305.

Carluclovica Rz. Pav. 1614.

Castalis H. Cass. 2732.

Castanea T. 1797.

Castela Turp. 5183.

Castiglionea Rz. Pav.

Carmichaëla Rßr. 3917.

Carolinea L. 5334.

Caroxylon Thnb. 4321.

Carpesium L. 2465.

Carpha RBr. 1110.

Carphalea Juss. 2047.

Carpiuns I. 1792. 5061 .

Castilleia Mut. 3075.

Casuarina L. 1690.

Catabrosa P. B. 1029a.

Catagyna RBr. $106 \%$.

Catalpa Juss. 3255.

Catanance T. 2133.

Carpoceros DC. 47.12b. Catananche L. 2133.

Carporletus Forst. 3816. Catappa G. 4543a.

Carpodontos LaB. 5466. Catapodium Lk. 848 .

Carpolepis P. B. 531 . 532a.

Cataria T. 2876a.

Catasetum Rich. $152 \%$.

Carponema DC. 4821a. Catesbaea Gron. 2091.

Carpopodium DC. 4821i. Catha Forst. 5390.

Carpopogon Rxb. 3949. Cathara R. 4162c.

Carrichtera DeC. 4738. Catharinea Ehrh. 669.

Carthamoides Vaill. 230 s.

Carthainus T. 2309.

Cartolium Sol. 2449.

Cathartocarpus P. 113jh.

Catiang DC. 3940h.

Catimbium Juss. $15,90$.

Cartonema RBr. $115 \%$.

Carum L. 3718.

Caruncularia Haw. $3+11$ h.

Carvi T. 3718a.

Carya Nutt. 3832.

Caryocar L. 5338.

Caryochloa Sipr. 954.

Caryolobis Gärt. 191\%،.

Caryophyllaster Rmph. 2031.

Caryophyllastrum $S$. 444 ic.

Cary ophyllata T.445J).

Caryophyllus T. L. 4 ij78.

Caryota L $16+8$.

Catoca rpum DC. 4816 b.

Catonia Mnch. 2147.

Catonia Brw. adl. 55 46.

Catoptridium Brid. 419.

Catoscopium Bricl. 607.

Cattleya Liudl. 1547.

Cattutella Rheed. 2027.

Caturus L. 5035.

C'aucalis L. 3665.

Caucanthus Forsk. 537\%.

Caulerpa Lamx. 180.

Caulinia DC. $75 \%$

Caulinia WV $166 \%$

Canliuia Much. 3932.

Cauloglossum Grev. 206.
Cecalyphum P. B. $5 \mathbb{1}$.

Cecropia L. 1819.

Ceirela L. 5516.

Cedrota Schreb. 1914. ex add.

Cedrus Mili. 5516.

Cedrus T. 1727.

Ceïba Plum. 5336.

Celastrus L. 5390.

Celinisia H. Cass. 2248.

Celosia L. 4363.

Celsia L. 3190 .

Celtis L. $18: 30$.

Cenangium Fr. 260.

Cenarrhenes LaB. 1751.

Cenchrus L. 829 .

Cenia Comm. 237.

Cenococcum Moug. 161.

Cenomyce Ach. 41\%.

Centaurea L. 2691.

Centaurella Mchx.3484.

Centaurium Adns. 265\%.

Centinodia J. Banh. $4291 \mathrm{a}$.

Centipeda Lour. 2481.

Centotheca Desv. 1006.

Centrachena Sichott. 2382.

Centranthera P. Br. 3162.

Centranthus DeC. 1945.

Centrapalus H. Cass. 2192.

Centratherum H. Cass. 2193.

Ceutrolepis LaP.1130.

Centronia Don. 460?.

Centrophoram Trin. 971.

Centropodia R Br.1045\%. ex add. 
Centroseuna DC. 3974c.|Ceratostemma Juss,

Centrosperinum Knili. 2701.

Centrospermum Spr. 2382.

Centunculus L. 3320 .

Cephaëlis Swv. 2119.

Cephalacladimm R.115. Cercodia Mnr. 4485.

Cephalaeodiım Kz. ol. 35b.

Cephalanthus L. 1938.

Cephalaphora Car. 2581.

Cephalaria Schrad. 1934.

Cephalenros Kz. 143.

Cephaloceras Rchb. $4821 \mathrm{~g}$.

1973.

Cerbera L. 3569.

Cercis L. 4125.

Cercocarpus K. H. B. Chaetotricha DC. 3993. 4428.

Cercodea Lam. 4485.

Cerlana Rz. Pav. 2987.

Cereaster DC. 4244.

Cerefolium IIall. 3659 b.

Ceresia P. 887e.

Cereus J. 4244h.

Cerimthe I. $295 \%$.

C'erionanthe R. 2957a.

Cerionanthus Schott. 1934.

Cephalotus LaB. 4192.

Cephalotrichum Lk. 49.

Cephaloxys Desv. 1285.

Ceraia Lour. 1587.

Ceraminm Blume. 1859.

Ceraminm Bonnem. 483a.

Ceramium Pth. 484.

Cerania Ach. $\$ 14 f$.

Ceratanthera Horn. 1589.

Coratfen Cerauthera P. B. 4895

"raffor" Cerasophora Neck. 4705́a.

Cerasus Juss. $470 う$.

Cerasus T. 4705 .

Ceratiola Rich. 5114.

Ceratiun Alb. Schwz. 45.

Ceratocarpus L. 4333.

Ceratocephalus Mönch. 4933.

Ceratochloa P. P. 1003.

Ceratocoreta DC. $5445 \mathrm{c}$.

Ceratodon Brid. 618.

Ceratolepis H. Cass. $2 ? 86$.

Ceratonema P. p. 15.

Ceratonia L. 4104.

Ceratopetalum:Sm.420\%.

Ceratophyllum L. 1666.

Ceratopteris Gaudich. 709.

Ceratosanthes Juss. 2758.

Ceratosinapis DC. $4918^{\circ}$

CeratospermunSchreb 4335. ex add.

Ceratostachys Blume. 4532 .

Ceriscus Gärt. 2080.

Cerium Lour. 3218.

Ceropegia I. 3403.

Cerophora Rafin. p. 15.

Ceruana Forsk. 2473.

Cervantesia Rz. Par. 1706.

Cervaria G. $36 \%$ tc.

Cervia Rodrigr. 0002.

Cervicina Delil. 2796.

Cestrinus H. Cass. 2310.

Cestrum L. 3288.

Ceterach TV: 725.

Cetraria Ach. 405.

Cevallia LaG. 2743.

Ceuthospora Fr. 245.

Chabraea DeC. 2278.

Chaenanthera Rich. 4646.

Chaenarrhinum DeC. 3173.

Chaenocarpus Neck. 1997c.

Chaenopleura Rich. 4647.

Chaerophyllum L. 3663.

Chaetauthera Rz. Pav. 2267.

Chaetanthus RBr. 1144.

Chaetaria P. B. 921c.

Chaetocalyx DC. 3960.

Chaetocrater Rz. Pav. 4694.

Chaetogastra DeC. 4594.

Chaetolepis DC. 45065 .

Chaetomium Kz, 156.

Chaetony chia DC.4265c.

Chaetopetalum DC: 4595.

Chaetophora Schrk.424.

Chaetophora Brid. 515.
Chaetopsis Grer. 127.

Chaetospora RBr.1111.

Chaetostenuma DeC. 4615.

Chaefurus Lk. 899.

Chaiturus Ehrh. 2882.

Chailletia DC. 2823.

Chaixia Lap. 3278.

Chalariun DC. 4000 c.

Chamaebuxus DC. $3029 \mathrm{c}$.

Chamaecassia Breyn. $4135 \mathrm{~g}$.

Chamaecrista Breyn. 4135 a.

Chamaedaphue Mitch. 2004.

Chamaedorea TV. 1649.

Chamaedryon S. 44491 .

Chamaedrys T. 2851 a.

Chamaefistula DC. 4135.

Chanaelaucium Desf. 4664 .

Chamaelea 'T. 5118.

Chamaeledon I/k. 3392.

Chamaeliumm DC.4780.

Chamaelirium W.1294a.

Chamaenelanimn Ging. 4385.

Chamaemeles Lindl. 4462.

Chamaemelum T. 2383. 2394.

Chamaemespilus 4470a.

Chamaeneriou T'. 4500a.

Chamaepytis T. 284fib.

Chamaepence $\mathbf{P}$. $\mathrm{Al}_{\mathrm{p}}$. 2328.

Chamaerepes Spr. 1475.

Chamaeriphes Ponted. $165 f j$.

Chamaerops L. 1656.

Chamaerhaphis RBr. 939.

Chamaesenna DC. 4135 d.

Chama siclericlis 2879 b. Chamagrostis Borkh. 882.

Chamira Thub. 4820.

Chamissoa K. H. B. 4368.

Chamitis G. 3797.

Chamorchis Rich. 1475.

Champia Ag. 487.

Chantrausia DeC. 469. 
Chaos B. St. Y. p. 15. Chlaenobolus H. Cass. Chaptalia Vent. 2259. Chara L. 1665.

Chardinia Dsf. 2303.

Charianthus Don. 4646.

Charicis H. Cass. 2558.

Charpentiera Gaudich. 4369 .

Chasallia Comm. 2033.

Chasmanthiın Lk. 1010.

Chasine Kngt. 1745.

Chastenaea DeC. 4605.

Chatiakella H. Cass. 2647.

Chevreulia H. Cass. 2411.

Cheilanthes Sw. 690.

Cheilococca Salisb. 4056.

Cheiranthodendron

Larr. 5324.

Cheiranthus L. 4795.

Cheiri Dorst. 4793b.

Cheirinia Lk. 4813c.

Cheiropsis DC. 4947a.

Cheiroides DC. 4795.

Cheirospora Moug. 27.

Cheirostemon Hb. Bpl. 5324.

Chelidonium L. 4850.

Chelone L. 3178.

Chenopodium I. 4343.

Cherina H. Cass. 2266.

Cherleria Hall. 5348.

Cherleria L. 5348b.

Chilianthus Purch.2926.

Chiliotrichum H. Cass. 2556.

Chilochlna P. B. 9481).

Chilmoria Hamilt. add. 5.548.

Chilodia RB Br. 2831.

Chiloglottis RBr. 1448.

Chilopsis Don. 3270.

Chimaphila Prsh. 3371.

Chimophila Prsh. em. $33 \%$.

Chimarrhis Jcr. am. 2040.

Chimnonthus Lindl. 1833.

Chiococca J. Br. 2030.

Chiodecton Ach. 3634 .

Chionanthus I. 3582 .

Chirita Hamilt. 3164.

Chironia L. $349 \%$.

Chitonia Don. 4635.

Chitonia Moc.Sess.5?34. 2210.

Chlamysporum Salisb. 1397.

Chlidant'sus Herb. 11581 .

ChlidanthusLindl. 1158. Chloanthes RBr. 2904.

Chlora L. 3491.

Chloraea Lindl. 1425.

Chloranthus Siv. 1955. Chlorilium Lk. 124.

Chloris Dsv. 866a.

Chloris Sw. 866.

Chlorococcum Fr. 418. Chrysopogon Trin.

ChloromyronPers.5487. $9 \% 4$.

Chloronitum Gaillon. $46.7 \mathrm{~b}$.

Chlorophytum Ker. 1385.

Chloroxylom DeC. 5518. ChrysospleniumL.4107. Chnoophora Kautf. 677. Christia Mönch. 3997. Choisya K. H. 3. 5148. Chrysurus P. 99\%.

Chomelia Jcy. am. 2010. Chthonia H. Cass. 2353. Chomelia Scop. 2022.

Chondodendron Rz. Pav. 1371.

Cliondria Ag. 493.

Chondrachne RBr.1073.

Chondrilla L. 2165.

Chondrocarpus Nutt. 3764.

Choindrosium Desv. 871.

Chondrus Lamx. 497a.

Chorila Leb. 505.

Chordaria Lk. 430. $50 j$.

Chordostylium Tod. 145.

Chnretrum RBr. 1710.

Chorisia Knth. 5325.

Chorisma Lindl. 5278f.

Choristea 'Thub. 2680.

Chorizandra RBB. 107t.

Chorizema LaB. 4073.

Christiania J)C. 5455.

ChristophorianaT.4959.

Chronantlums DC. $4029 f$ f

Chronolsium DC. 4190c.

Chrousemium Ser. $38600 \ldots$.

Cliroolepus Am. 67.

Chrysa Rafin. 4956.

Chrysanthellina H. Cass. 2503.

Chrysanthellım Rich. 2604.

Chrysanthemuin T, $L$ 2381.
Chrysithrix Cass. 209.3.

Chry sobalanus L. 4525.

Chrysocouna L. 2537.

Chrysodraba.DC.4761e.

Chrysogonmm L. 2702.

Chrysolyga Hffge. 4563.

Chrysophyllum I.3640.

Chrysophiala Lamb.

1262:

Chrysopia A. P. Th. 5348

Chrysopsis Nutt. $25 ? 8$. 2550.

Chrysospermun Rchb. 4417.

Chinncoa Pav. 4540.

Chucuiraga Juss. 2302.

Chusquea Kuth. 105t.

Chytrainlia P. Br.46\%o.

Chytralia Ast. 4676.

Cibotium Kaulf. 687.

Cicca L. 5075.

Cicendia Ad. $347 \%$.

Cicer L. 3921.

Cicerbita Walle. 2168. 2171.

Cichorium T. 2133.

Ciconium Sweet.5278g.

Cicuta T. 3729.

Cicuta L. 3730.

Cicutaria Riv. 3730 .

Cieca Me!lic. $3 \div 72 b$.

Cienfuemosia Cav. 0370.

Cilicia Fr. 88.

Gimicifiga L. 4957.

Ciminalis Prkh. 31991).

Cinchona I. 2065.

Cincinalis Desv. 720 .

Cincliclium Sw. 643.

Cinclidofus P. B. 595.

Cineraria L. 251\%.

Cimmamomum N. V. E. 1912.

Cinogasum Neck. 5045.

Cipura Aubl. 1176.

Circaea L. 4511.

Circinotrichim N. v. k. 121.

Cirrhaea Lindl. 1535. Cry somallmm A. P. 
Cirrhopetalum Lindl. 1579.

Cirrolus Mart. 182.

Cirsellium G. 230\%a.

Cirsium T. 2334.

Cissus L. 3790.

Cissanpelos L. 1884.

Cistanche Lk. Hiffgg. 3058.

Cistus T. 4916.

Citharexylon L. 2899.

Citrosma Rz. Pav. 1834.

Citrullus Neck. 2762.

Citrus L. 5533.

Citta Lour. 3949.

Cladauthus H. Cass. 2397.

Cladium P. Brivne. 1102.

Cladobotryon N. v. E. 115b.

Cladodes Lour. 5103.

Cladodian Brid. 641. Cladonia Hoffin. 414. Cladonia Ach. 41 te.

Cladosporium Lk. 100. Cladostachys Dou.436?. Cladostephus Ag. 503. Claudestiula T. 3054 .

Clandestinaria DC. $4796 \mathrm{c}$.

Claoxylon Ad. Juss. 5035.

Clarionea LaG. 2283.

Clarisia Rz. Pav. 1698.

Clarkia Prsh. 4499.

ClasterisporiumSchwz. 83.

Clathrus Mich. 224.

Claudea Liunx. 502.

Clausena Burm. 55?9.

Clavaria Vaill. 300.

Clavija Rz. Pav. 3600.

Clavulium Desv. 4041.

Claytonia L. $430 \%$.

Cleistostoma Bril.610a.

Clematis L. 4946 .

Clematitis Mirh. 1940a.

Cleome L..4261.

Cleome L. 4sbilb.

Cleomella Dec. 4858.

Cleomena P. B. 913.

Cleonia L. 2817.

Cleophora Gärt. 1661.

Clernontia Gandich. 2788.

Cleroriendron L. 2929.

Clethra L. 340s.
Clethroa I. Br. 224b.

Cleyera Thub. 5419.

Cley ria Neck. 4103.

Clibadinn Allem. 1809.

Clidèmia Don. 4622.

Cliffortia L. 4420 .

Clif́ortia Neck. 4420c. Codouphora Lindl.

Cliftonia Banks. 3405. 3243.

Climacium W. M. 557. Codonoprasum Rchb.

Clinauthus Herb. 1158a. 1379.

Clinclinia DC. 3029c. Coelachne RBr. 1016.

Clinopodium L. 2828.

Cliostomum Fr. 255.

Clithris Fr. 260c.

Clitocybe Fr. 349. H. e.

Clitopilus Fr. 349. G. d.

Clitoria L. 3974.

clitorins Petiv. 3974.

Clivia Lindl. 1236. ex adli.

Clomenocoma H. Cass. $23 ; 9$.

Clomiun Adans. 2338.

Clusia L. 5486.

Clutia Boerh. 5085.

Cluy tia Ait. 5085.

Clymenum DG. 3923a.

Clypea Blimme. 188.5.

Clypearia Risph. 4168 .

Clypeola Gärt. 4758.

Cuestis Juss. 3829.

Cnemidostachys Mart. 5029.

Cneorum L. 5118.

Cinicus Neck. 2333.

Cuicus Hoffin. W. 2334.

Cnicus Vaill. 2689.

Cuidium Cuss. 3713.

Cnidoscolus Pohl.5059.

Coa Plum. 5382.

Cobaea Cav. 3275.

Coccocypselum J. Br. 2093.

Coccochloris Spr. 418.

Coccoderma Kuz. 375.

Coccoloba L. 4296.

Coccotrichum Lk. 130.

Cocculus C. Bauh. 1870.

Cochlearia T. L. 4763.

Cochlearia Loh. 47633).

Cuchlidiosperma Rchb. 306 ?.

Cochlidium Kanlf. 724. Colpodium 'T'rin. 912a.

Cochliospermun LaG. Colquhomuia Wall.28!j2. 4342.

Cochlospermun K. H. B. 5421 .

Cocos L. 1634.
Coelestina H.Cass.2231.

Coelogyne Lindl. 1561.

Coelorutis Ser. 3870a.

Coenocarpus Rębent. 95.

Coenogoniun Ehrnb. 87.

Coenolophim Koch. 3717.

Colfea L. 2034.

Cogswellia Spr. 3760.

Coilanthe Brkh. 3499b.

Coix L. 940 .

Colbertia Salisb. 4988.

Colchicum L. 1311.

Collenia L. 295 .

Coleanthus Seidl. 911.

Colebroukia $\mathbf{R x b} .2853$.

Co!ebrookia Doll. 1589.

Coleonema Bartl. et Wendl. 5160.

Collacystis Kz. $155 \mathrm{~b}$.

Colladoa Gav. S32.

Colladonia Spr. 1983.

Collaea DC. 3965.

Collaca Sipr. 2604.

Collema Hoffm. 397.

Colletia K. II. B. $380 \%$.

Colliguaya Molin. 5105.

Colliusia Nutt. 3192.

Collinsonia L. 2808.

Collybia Fr. 349. H. c.

Collomia Nutt. 3023.

Colobachne P. B. 947.

Coloryuthis T' 2700.

colopania K. H. B.3971.

Colona Cav. 5448.

Colonnaria Rafin. 224.

Colophouia Commers. 3844

Colsmamuia Lehon.2950.

Colubrina Rich. 3809.

Columbia Pers. 5448.

Columella Lour. 3789.
Colletosporium Lk. 81. 
Columellea Jcr. 2467. Conostomum Sw. 659. Conreia Vell. Vand.

Columellia Rz.P. 3229. Columuea L. 3232.

Colutea L. 3912.

Colntia Much. 3914.

Coly mbea Salisb. 1730.

Comandra Nitt. 1708b.

Comaropsis Rich. 441.

Comarim L. 4437.

Combretum Löfll. 4530 .

Comesjerma LaB. 3031.

Cometes Burm. 5110.

Commelina L. 1167.

Commely ua Hffgg.1167.

Commersona Sonn. 4634.

Coinocladia P. Br. 3857.

Commersonia Forst. 5294.

Comunia Lour. 5020.

Comolia DeC. 4581.

Compsoa Don. $121 \%$.

Compsanthus Spr. $121 \%$.

Comptonia Banlss. 1699.

Conami Aubl. 5078.

Conaugium Fr. 382.

Conanthera Rz. Par. 1395.

Concereibum Rich. 5039.

Conchium Sm. 1755.

ConchocarpusMik.514j.

Condalia Cav. 3818.

Condylocarpus Hoifm. 3685.

Conferva L. 4 (j)7.

Congen $\mathrm{Rxb}$. 2911.

Conianthus P. B. 53?b.

Coniocarpon DeC. 351.

Coniocybe Ach. 361 .

Coniolóma FI. 390.

Coniophora DeC. 326.

Conioselinum Hoffin. $37 ? 1$.

Coniosporimn Lk. 15j. Conjugata Vanch. 466.

Conjugata Lk. 466c.

Coniuin L. 3743.

Commaris L. 3826 .

Conobea Aubl. 3180.

Conocarpus Gärt. 45.38.

Conohoria Aibl. 4894.

Counpholis Wallr. 30541). ex actl.

Cowoplea Pers. 106.

Connporlinm Koch.3737.

Conospermum Sur.1733.

Conostegia" Dun. 4ij34. Correa Sm. 5173.

Cora Fr. 89. add. 5547. 1454.

Cordia L. 2987.

Corlylia P. $41: 6$. 4714.

Corion Lk. $3 \% 02$.

Coris L. 3101. $409 \mathrm{c}$. $410 \mathrm{c}$.

Coruus L. 1944.
5188.

Conringia Heist. 4812. Corrigiola L. 4300.

Consiligo Plin. 493\$b. Corsinia Raddi. 523.

Consolida DC. 4949a. Cortesia Cav. 3004.

Conuleuin Rich. 1718. Corthusa L. 3362.

Convallaria L. 1321.

Convolvulus I. 3011.

Corthısina 3361ı.

Cortinarins Fr. 349F.

Conyza H. Cass. 245\%. Corvisartia Merat. 2458.

Conyzella Dill. 2546. Corycarpus Zea. 1009.

Cookia Sonn. 5525. - Corycinm Sw. 1484.

Copaifera L. $410 \%$.

Copaira Jcq. $410 \%$.

Coprinarius $\mathrm{P} .349 \mathrm{~A}$.

Coprinus Pers. 448.

Coprosina Forst. 3572.

Coptis Salisb. 4956.

Corpolletes Herb.1262b.

Corallodendron T.3956.

Corallophyllum H. B.

Corallorrhiza Hall.

Corchorus L. 5445.

Cordiopsis Hamilt.2989.

Cordyceps Fr. 279d.

Corlly la Lour. 4156.

Corly line Comm. 1394.

Corly-locarpus Desf.

Corema Don. 5115.

Coremimu Lk. 120.

(oreopsis L. 2696.

Corcta P. Br. 5445́a.

Coretoides DC. 5445b.

Coriandrum T. L. 3701 .

Coriaria Niss. 5181.

Coriulum T. 5235.

Corispermum L. 4324.

Cornelia Hard. 4556.

Cornicina BC. 402:a.

Cornicularia Ach. 205 c.

Cormucopiae L. 941 .

Cornulaca Del. 4329.

Cornitia L. 2916.

Coronaria L. 5362.

Corone Hiffyg. 5363a.

Coronilla L. 3878 .

Coronilla T. 3878a.

Corylalis Vent. 4833.

Corylus L. 1795.

Corymbium L. 2183.

Coryuandra Schrad. 4865.

Corynelia Fr. 271.

Coryuella DC. 3907.

Corynephora Ag: 422.

Corynephorus P. B.

1036a.

Corymem N. v. E. 30 .

Coryuites Spr. 3907.

Corynodesmium Wallr. 3 bे).

Coryuostylis Mart. 4890.

Corypha L. 1658.

Corysanthes RBr. 1450.

Corythaeolon R. 4950b.

Corypha Rxb. 1659.

Coscinium Colebr. 1893.

Coscinodnu Spr. 608.

Cosmea W. 2607.

Cosmelia RBr. 3346.

('osinia Domb) 4315.

Cosmibnena Rz. Par. 2066. 4518.

Cosmos Cav. 2607.

Cossiguia Comm. 5210.

Cossinia Spr. 5210.

Costus L. 1594.

Cotimns '1'. 3850a.

Cotoneaster Medik. 4465 ,

Cotnla L. 2375.

Cotyle DC. $418 \%$

Colyledon L. 4185.

Cotyliscus DC. 4749c.

Conepia Aubl. 4523.

Coulteria K. H. B. 4150

Couma Aubl. 3577.

Coumiromina Aubl. $40 \div 3$ a.

Couponi Aubl. 4546.

Couratari Aubl. 4653.

Comrbaril P'Imn. 412S.

Comroupita Aubl. 415\%". 
Coussarea Aubl. 2021.

Contarea Aubl. 20 sy.

Contoubea Aubl. 348 ?

Covalia Neck. 1997b.

Cowania Doll. 440.

Cracca L. Zeyl. 3892.

Craccoides DC. 384.?c.

Crafordia Rafiu. 3540.

Crambe 'T. 4724.

Cranichis Swr. 1424.

Craniolaria L. 3239.

Craniospermum Lehır. 2951.

Craniotoine Rchb. 2849.

Crautzia Nutt. 37t5.

Crautzia Schreb. 5129.

Crantzia Sw. 5093.

Craspedia Forst. 2449 .

Craspedou Fee. 366c.

Crassocephalum $\mathbf{H}$. Cass. 2505.

Crassouvia Commers. 4184.

Crassula L. $41 \%$.

Crassula Haw. 417\%a.

Crataegus L. 4460.

Craterellus P. 342.

C: ateria Pers. 4694.

Craterimm Trentep.191.

Crataeva L. 4866.

Craterostegia R. 1171a.

Crafochwilia Neck. 5085.

Cratoxylon Blume. $5: 0$.

Cremanimn Don. 4611.

Cremocephalum $\mathbf{H}$. Cass. '2505.

Cremolobus DeC. $47 \pm$.

Crenea Anbl. 4568.

Crenias Spr. $16 \% 0$.

Crepidium Tsch. 2141.

Crepidotus Fr. $349 d$.

C'repinia Rchl). 21Jj.

Crepis L. '2145.

Crescentia L. 3219.

Cressa L. 2996.

Crevispina Dill. 382'b.

Cribraria Schrad. 183.

Criuita Mrech. 2535.

Criuitaria H. Cass. 2535.

Crinorlendron Molin. 46960.

Criumin L. 1252.

Ciristaria Cav. 5246.

Cristaria Soll. 4533.

Crithmmm T. 3716.
Crocod:lodes Ädans.

2683.

Crocodylium Vaill.2686.

Crocus L. 1200.

Crossandra Salish. 3124.

Crossopetalum P. Br. 362.3.

Crossosiy lis Forst. 4680.

Crotalaria L. $40+2$.

Crotalopsis Mch x. 4083.

Croton 1. 5045.

Croton Neck. 5C45.

Crotonopsis Mchx. 5046.

Crowea Sin. 5169.

Crozophora Neck. 5044. Crucianella L. 1958.

Crucita Löfll. 4347.

Crudia Schreb. 4108.

Crulya DeC. 4108.

Cruikshankia Miers. $1179 \mathrm{~b}$. ex add.

Crumenaria Mart. 3796.

Cimminimu Desv. 3951.

Crupina l'ers. 2685.

Cryphaea Web. 550.

Cryphaea Hamilt. isjob. ex add.

Cryphia RBr. 2833.

Cryphiospermm P.B. 2705.

Crypsis Ait. 943.

Crypta Nutt. 4306 .

Cryptandra Sin. 3802.

Cry prarhena IBBr. 1498.

Cryplima Rafin. 4306.

Cryplocarpa Kuth. 3842.

Cryptocarpha H. Cass. 2739.

Cryptocarpus K. H. B. 439.3.

Cryptocarya RBr. 1911.

CryptochilusWall.1584.

Cryptocoryne Fisch. $76 \%$.

CryptogrammaRBr.705.

Cryptolepis $\mathrm{RBr} .3523$.

Cryptolobus Spr. 4098.

Crypropetalon H. Cass. 2350.

Cryptopodium Brid. $65 \%$.

Cryptopus Linll. 1510.

Cryptosphaeria Grev. $2+7$.
Cryptostylis RBr.1421.

Cryptosporium Kz. 23.

Cryptostemuna $13 B r$. 2670.

C'ryptostomum Schreb. 3631.

Cryptotheca Blume. $405 \%$

Ctenim Pz. 867.

Cubaea Schreb. 4138.

Cucifera Bul. 16tio.

Cucubalus L. 5364.

Cucullaria Rafin. 4832.

Cucullaria Schreb. 4508.

Cucumis I.. 2760.

Cucnmis T. 2\%60.

Cucurbita L. 2762.

Cuellaria Rz. Pav. $340 \%$.

Cnitlanzina LaLlav. 1525.

Cnlcasia P. Beanr.7\%3b.

Culcitium Boupl. 2489.

Cullumia RBr. 2́is2.

C'umaruma Lam. 4023a.

C'mninum L. 3669.

Cinucea Hamilt. 1996.

Cumila L. 283\%.

Cinuinghamia Rich. 1728.

Cumninghamia Schreb. 2022.

Cinnonia L. 3211.

Cupameni Ad. 50:34.

C'upania Plun. 5?2.

Cuphaea Jacr. 4559.

Cinplea P. Br. 4559b.

Cupressus L. $172 ?$.

Curanga Juss. 3065.

Cinrare IImub. 8568.

Cinratella L. 4975.

Curculigo Gärt. 1218.

Cinrcas Ad. 5061.

Curcuuna L. 1599.

Curupita Gm. 4653.

Curtia Cham. 3478.

Curtisia Ait. 3622.

Curtopogon P. B. 921b.

Cururu Plum. 5238.

Ciscuita L. 5386.

Cispidaria DC. $4813 \mathrm{~b}$.

Cuspa $H u m b .4895 ́$.

Cusparia Humb. 5145.

Cuspidia Gärt. 2679.

Cinssouia Thub. 3759.

Cintubea Mart. 3482.

Cinviera DeC. 2102.

Cuviera Koel. 842a. 
Cyamopsis DeC. 3378.|Cypselea Turp. 4311.

Cyamus Salish). 818.

Cyanella L. 1396.

Cyauopsis H. Cass.2694.

Cyanotis Don. 1171b.

Cyanins DeC. 2691.

Cyathodes Lab. 3338.

Cyathophoriun P. B. 537.

Cyathea Sin. 679.

Cyathula Lour. 4384.

Cybbanthera Hamilt. 3161.

Cybele Sal. 1763.

Cybelion Spr. 1512.

Cycas L. 752.

Cyclainen L. 3356 .

Cyclauthus Poit. 1613.

Cyclas Schreb. 410 s.

Cyclocarpaea DC.47\%0c.

Cyclomyces Kz. 332.

Cyclopia Vent. 4081.

Cyclopogon Prsl. 1434.

Cydonia T. 4471.

Cylactis Rafin. 444.

Cylindrachue H. Cass. 2471.

Cylindria Lonr. 1769b. Cylindrosporinm Gr.22. Cylista Ait. 3955.

Cylizoma Neck. 4022.

Cylopogon Rafin.388\%). Cymation Spr. 1300.

Cymbidimm Sw. 1513. Cymbachne Retz. 861. Cymbaria L. 3181.

Cymbopogon Spr. 972. Cyminosina Gärt. 511\%. Cymopterus Rafin.3700. Cynanchum L. 3440. Cyuara T. 2323.

Cynoctonum Gin. 3552 . Cynodon Rich. 86?. Cynodon Prid. 638. Cynodontium Hdg. 638 . Cynoglossum L. 2966 . Cyuometra L. 4131. Cynomorium Ruph. 4131.

Cynomoriun L. 1679. Cynophalla DC. 48741. Cyuosbata DC. 5278h. Cyาiosurus L. 839. Cypiella Hook. 1181. Cyperns L. 1088.

Cypheliuin Ach. 360b. Cyphella Fr. 293. Cypripediun L. $157 \%$.
Cy rilla L. 4214.

Cy rilla l'Herit. 3235.

Cyrta Lour. 3596.

Cyrtandra Forst. 3223.

Cyrtanthus Schreb. 2074.

Cyrianthus Ait. 1233.

Cyrtocinon Lk. 247).

Cyrtochilum Kuth. 1524.

Cyrtogyne Haw. 4179. Cyrlopodia Röhl. 567b.

Cyrtopodium LBBr. 1523.

Cyrtopus Brid. 567.

Cyrtostylis RBr. 1449.

Cystanthe RBr. 3343.

Cystapophysium R. $602 \mathrm{~b}$.

Cysticapnos Boerh. 4831.

Cystopteris Bruh.679b. ex adil.

Cystoseira Ag. 514a.

Cytheraea DC. 3854 .

Cytispora Ehrub. 24\%.

Cytiuus L. 168\%.

Cy tisus L. 4029.

Czackia Bess. 1375.

Czernya Prsl. 1048.

Daboecia Ray. 3381. ex add.

Dacrina Fr. 43.

Dacry dium Banks.169J.

Dacry oinyces N. V. E. 286.

Dactylaena Schrad. $485 \%$.

Dactylanthes Haw. 5012.

Dactylis L. 999.

Dactyloctenium W. 869.

Daedlalea Pers. $33 \%$.

Dahlia Cav. 2609.

Dahlia Thnb. add. 5590.

Dais L. $17 \%$.

Dalbergia Roxb. 4014.

Dalea L. 3888.

Dalechanpia Plum. 5015.

Dalibarda L. 4439.

Dalry inpelea Rxb.5204.

Dalrymplea Spr. 5?04.

Dalionia Hook. et 'Tayl. $5 \% 0$.

Damasonium Juss. 596.
Dahlia Thub. 1902.
Damasonium Sclireb. 808.

Damatrias H. Cáss. 2673.

Dammara Gärt. 3843 .

Dammara Mirb. 1729.

Dammacanthus Gärt. f. 2035.

Dampiera RBr. 2784.

Dana All. 3745.

Danaea Sw. 745.

Damais Commers. 2053.

Danthonia RBr. 1045.

Dantia A. P. Th. 4490 .

Daphue L. 1791.

Daphnitis Spr. 1783.

Darea W. 701.

Dargeria Chan. 3165.

Darlingtonia DC. 4166.

Dartus Lour. 3290.

Darwinia Rudg. $1 \%$.

Dasia Ag. 494 .

Dasynema Schott. 4980.

Dasystemon DeC. A1\%.

Dasystephana Brhh. $3 \pm 39 h$.

Dasypogen RPr. 1313. Datisca L. $48 \%$.

Datura L. 3285.

Danbentonia DC. 3906.

Daucus G. 3670a.

Daucus I. $36 \% 0$.

Davallia Sin. 682.

Daviesia Sin. 4060.

Davilla Vand. $49 \% 1$.

Davya DeC. $460 \%$.

Dawsonia Lamx. 501.

Dawsonia RBr. 666.

Dazus Lour. 2120c. ex adl.

Debrapa R. S. 4513.

Decadenia Ehrub.4.113h.

Decadia Lonr. $3653 \mathrm{~b}$.

Decaloba DeC. $347 \%$ c.

Decaria DeC. 3470 b.

Decasperinum Forst. 4690.

Decaspora RBr. 3332.

Derlieuxia K. H. H. 2013.

Decorlon Gin. 4566.

Decostea Rz. Pav. 3834.

Decumaria L. 4220 .

Deerisgia liBr. 4.371.

Defforgia Poir. 421\%.

Deguelia Aubl. 402?.

Deinira Cinall. 3494. 
Deillamia A. P. Th. Diachea Fr. $186 . \quad$ Dicraeia A. P. Th. 1673. 3665.

Dialesta K. H. B. 2196. Dicranum IId 617.

Deilosina Audrz. 4806b.

Delaria Desv. 4082.

Delesseria Lamx. 501.

Delilia Spr. 2727.

Delima L. 4974.

Delisea Lam. 498.

Delissea Gaudich. 2786.

Deloderium H. Cass. 2152.

Delostoma Don. 3272.

Delphinastrum DC. 4949c.

Delphinellum DC. 4949b.

Delphinium T. 4949.

Dematium Pers. 100.

Demetria LaG. $25 \% 0$.

Dendrina Fr. 108.

Dendrium Desv. 3402.

Deudrobium Sw. 1556.

Dendrobrychis DC. $4004 r$.

Dentrocrambe DC. $4724 \mathrm{c}$.

Denekia Thub. 2466.

Dentaria L. 4785 .

Dentella Forst. 2052.

Depazea Fr. 229.

Dermatocarpon Eschw. $37 !$.

Dermea Fr. 261.

Deriniuus 3490.

Dermocybe $3+9$ F.a.

Dermosporium Li. 42.

Derris Lour. 4011.

Deschainpsia P. B. $1036 \mathrm{l}$.

Dialium Burm. 4103.

Dictammus L. J15i.

Diamorpha Nutt. 4193. Dictydium Sichrad. 184.

Diamphora Mart. 141. Dictyoloma DeC. 51:34.

Dianella Lam. 1393. Dictyonema Ag.post 48.

Dianthera L. 311?a.

Dianthus L. 5358 .

Diapensia L. 3394.

Diapenzia Whlnh.3394.

Diaphora Lour. 1068.

Diarina Rafin. 1009.

Diarrhena Rafin. 1009.

Diascia Lk. 3149 .

Diasia DeC. 1188.

Diaspasis RBr. 2 \%3.

Diastella Sal. 1743.

Diatoma DeC. 450.

Diatoma Lour. $45 \% \%$.

Diatrypa Fr. 278l.

Diciyopeplos v. Hass. $2 ? 3$.

Dictyopteris Lamx. j081). add.

Dictyota Lamx. 509 .

Didelta l'Herit. $21 j 80$.

Diderına Lk. $19 \pm$

Didlesmus Desv. 4\%?6.

Didymandra W. 510\%.

Didymeles A. P. 'Ph. 1831.

Didymium Lk. 194.

Didymium Schrad. i!4.

Didimocarpus Jack.

Dicaeoma N. v. E. 10.

Dicaly x Lour. add. 5úf9.

Dicarphus Rafiı. p. 15.

Dicaryum W. hb. 3571.

Dicera Forst. $54^{\prime} 36$.

Diceratiun DC. 4794a.

Dicerma DC. 4001.

Dichaena Fr. 270 .

Dichautium Willem. yร3a.

Dichapetalum DC: 28231 .

Dichilus DeC. $403 \%$.

Dichounla Forst. 299?.

Dichonema N. v. E. 87.

Dichorisandra Mik. $11 \% 5$.

3224.

Dilfmochlaena Desv. rot.

Dillymocrater Mart. 140.

Dillymodon Hily. 629.

Didymodon schreb. 6.38.

Didymomeles Spr.1831.

Dillymosporimu N. v. F. 25.

Diectomis K. H. B. $96 \%$.

Dielytra Brkh. 4832.

Diena Lindl. 1565.

- Dierbachia Spr. 3297.

Diervilla T. 1989.

Dietrichia Tratt. 418 ?

Dichosporium N. v. E. Dimenca Ag. 481. 166. $\quad$ Digera Forsk. 4370.

Desfontainia Rz. Par. Dichostyles P.'B. 1114, 3312.

Desmanthea DC. 4 (67).

Desmanthus W. $416 \%$.

Desmarestia Lamx.jofi.

Desmatodon B:-idl. 63\%.

Desmia Lg'b. 506.

Desınidium Ag. 453.

Desmidochus Ehrub. $3+12$.

Dichroa Lour. 417\%.

Dichroma Cav. $311 \mathrm{j}$.

Dichromena Rich. 1115.

Dichrostachys DC. $4167 \mathrm{c}$.

Dicksouia Ehrh. ¿39.

Dicksonia Herit. 68\%.

Diclidauthera Mart. 3594 .

Desmochacta DeC. 4373. Dicliptera Juss. 3114.

Desmodium Desv. 4000. Diclytra DC. 483?.

Desmoncus Mart. 1630.

Desmos Lour. 500ijl.

Detarium Juss. 4155.

Detris Allus. 25j\%.

Desvauxia RBr. 1130.

Dentzia Thub. 4?18.

Deyeuxia Clar. 9081.

Dicnemon Schwgr. 56?.

Dicoma H. Ciass. 2:99.

Diconongia Ad. 421j.

Dicophe Wall. 3795.
Dicoryphe A. P. Th. Dillwy nia Sm. 4066."

Diuntalis L. 3198 .

Digitalis Lindl. $3198 a$.

Divitaria Hall. 892.

Digiraria Schrad. Sis.

Diglossus I1. Cass. 2351.

Diglottis N. et M. J144.

Digraphis Triu. 952.

Dilatris L. 1223.

Dileptinm Ralin. 4751.

Dilepyrm ilchx. "116.

Dilivaria Juns. 3119.

Dilubeia A. P' 'Th. 173:3.

Dillenia L. tisg.

1901. 4452.

Dillwynella B. St. $Y$. 45.50.

Dimacria Liudl. 597Sb.

Dimera Fr. $11 \%$

DictyophoraDesv.2281,. Dimereza Laß. 5213. 
Dimeria RBr. 976. Diplophractum Desf. Dimerostemma H.Cass. 5450. 2583.

Dimia Spr. 3444. Diplophyllum Lehm. $3062 \mathrm{~b}$.

Dimocarpus Lour. 5216. Diplopogon RBr. 924.

Dimorpha WV. 4129. Diploprion Viv. 3856.

Dimorphandra Schott. Dip!osporium Lk. 117. A16?.

Dimorphantes H. Cass. ?i) 43.

Dincha Del. 864 .

J)inema Lindl. 1550.

Dioclea K. H. B. 3945. Diplotaxis DeC. 4816.

Bioclea Spr. 2us?.

J) Jodia Gron. 2001.

Diunedea H. Cass. 2648. Diplusodon Poht. 4564.

Jionaea Ell. 4912.

Dioscorea L. 1333.

Diosina Berg. j161.

Diospyros L. 3647.

Jiotauthera DC. $4594 \mathrm{~b}$.

Diotis Dsf. 2338.

Jiotis Schreb. 4335.

Diaphyllum Holfu. $3740 \mathrm{c}$.

Dipera Spr. $14 \$ 2$.

Diphaca Lour. 3983.

Diplıryllum Rafiu. 1586.

Diphtherium Ehrub. 19\%.

Dipliylleia Mchx. 4842.

Dipliysa Jcq. 3910.

Diphysciun W. M. 665.

Diplachise P. B. 1002 .

Diplachy rium $\Lambda$. v. E. ist.

Diplacrum RBr. $107 \%$.

Biplanthera A. P. Th. 1669.

Diplauthera RPr. 3197.

Diplarrhena Laß.1189.

Diplasia Rich. 1126.

Diplazimu Sw. 703.

Diplocalymma Spr. 3013.

Diplochita DeC. 4635.

Diplocoea Rafin. 1015.

Diplocomiun W. $\mathrm{W}$. 6654.

Diploderma Lk. 207.

Diplogon Poir. 924.

Diplogon Rafin. 2528.

Diplolaena RBr. $51 \% 2$.

Diplolepis R $\mathrm{Br}$. 3437.

Diplomeris Doll. 146\%.

Diplopippus H. Cass. 2528.

Diplopetalum Spr. 5213. Disy uanthes Ralin.2386 $1676 \mathrm{c}$.

Diplost eginun Don.ti01.

Diplostephium K. H. B. 2550

Diplothemium Mart. 1631.

Dipodium RBr. 1518.

Dipogonia P. B. 924 .

Diporidimn Bartl. Wendl. 5186.

Dipsacus L. 1936.

Diptera Brkh. 4:03c.

Dipteris Reinw. 723a.

Dipterix Schrel. 40?3.

Dipterocarpus Gärt. f. 1917a.

Dirca L. 4546b.

Dirina Fr. 401.

Disa Berh. 1470.

Disandra L. 3069.

Disarrhenum LaB. 1038.

Discapophysium R. 602c.

Discelinm Brid. 606.

Dischilia RBr. 3420.

Diso!!ea Poir. 2039.

Disparago Gärt. 2440.

Disporum Salisb. 1348.

Disperis Sw. 1482.

Dissodon Grew. et Arn. $601 a$.

Dissolena Lour. $356 \%$.

Distephana Juss. 3469b.

Distephanus H. Cass. 2195.

Distephia Salisb. 3469J.

Distichia Bridl. 546.
¡Ditassa RBr. 3443 .

Ditaxis V. 5012.

Ditiola Fr. 310.

Ditmaria Spr. 4513.

Ditoca Banks. G. $425 \%$

Ditrichum II. Cass.2625.

Ditrichum Timm. 629.

Diplnstachyum P. B. Diuris S!n. 1419.

Dischillium Ging. 4885 c. Dorobaea H. Cass. 2492.

Dischimia Chois. 309\%. Doronicum L. 2487.

DiscocapnosCham.4828. Dorstenia L. 1811.

Discovium Rafin. 47\%6. Dorvalia Commers. 4494 a.

Doryanthes RPr. 1283.

Dorycnium Mnch. 3!976.

Dorycnoides DC. $4025 \mathrm{c}$.

Dorycnium T. 3873.

Dothidea Fr. 266.

Donglassia Lindl. $336 j 0$.

Dovera Ehrib. 1910.

Draba L. 4761.

Drabella DC; 476 lb.

Dracaena L. 1412.

Dracaenella R. I3!?a.

Dracocephalum L. 2814.

Distomaea Spenn. 1427. Dracnutium L. 774.

Distreptus H.Cass. 21:44. Dracophyllum LaB.

Distylis Gaudich. 27i9. 3345.

Disynathus Rafil. Dracopis H. Cass. 2649.

Drakenstemia Neck. 4102. 
Draparnaldia B. St. V.'Duperreyà Gaudich. 438.

Drapetes Lam. 1773.

Drepanandrum Neck. 4612.

Drepania Juss. 2151.

Drepanocarpus W. Mey. 4018.

Drepanophyllum Rich. 540.

Drepanophyllum Wib. $3 \% 20$.

Drimia Jcq. 1365.

Drosera I. 4910.

Drosophyllum Lk. $4911 \mathrm{~b}$.

Drozia H. Cass. 2285.

Drusa BeC. 3770.

Dryandra RBr. 1768.

Dryandra Thub. 5062.

Diyas L. 4446.

Drymaria B. St. V. \% 23 b.

Drymaria W. 4273.

Drymis Forst. 4968.

Drymophila RBr. 1318.

Dryobalanops Gärt. f. $1917 \mathrm{c}$.

Dryopoeia A. P. Th. 1478.

Drypetes Vahl. 5088.

Diypis L. 5355.

Dryptodon Brid. 590. 591.

Duboisia RBr. 3202.

Duchesuia H. Cass. 24100.

Duchesnea Sim. 4438b.

Duchola Arl. 50:7.

Dufonrea Ach. 406.

Dirfourea in. H, B. 2998.

Dufourea W. 1674.

Dugortia Neck. 4521.

3302.

Dupontia RBr. $103 \%$.

Duranta L. 2:109.

Durio L. 5327.

Duroia L. f. '007.3.

Duvalia Haiv. $3411 \mathrm{c}$.

Duralia N. v. E. 52Sa.

Duvaua Kuth. 38.j?.

Duvaucellia Bowd. add. 5550.

Dysoila Lour. 2096.

Dysosinia DeC. 3472f.

Dysorlium Rich. 2703.

Dysphania IRBr. 4336.

Dyssodia Cav. 235\%.

Dysticia Rafin. p. 15. Ebetingia Rchb. 5180.

Ebenus L. 4007.

Ebeums Co:nuners. 3647.

Ecastaphyllum P. Br. 4019.

Ecbalium Rich. 2753.

Eccilia 349 G. c.

Eccremocarpus Rz. Pav. 3264.

Echentais H. Cass. 2321.

Echeandia Ort. 1395.

Echeveria DeC. 4188.

Echinacea Much. 2650.

Echinalysium T'rin. $\$ 40$.

Fchinanthus Neck.2340.

Echinaria Dsf. 992.

Echinella Ag. 448.

Echinella DC. 4941c.

Echinobrychis DC. 40041 .

Echinocactus Salm. D. 4243.

Echinocarpus Blame. 4230.

Échinochloa P. B. 891.

Echinochloa P. B. 8911 .

Echinodiscus DC. 4017.

Echinolaeua K. H. B.

Jounuetia A. St. Hil. 890. 6002.

Duhamelia Domb. 3641.

Duhnamelia P. 2109.

Dulichium Rich, 1108.

Du!numia Knth. 3821.

Dumsesa DC. 39ti?.

i) unerilia LaG. 2275.

Bumortiera N. v. E. $5 ? 8$ i).

Sumalia K. H. B. 3297.

Bitnalia Spr. 2015.

Daucania Rrchb. 512\%.
Echinolobium Desv. $4003 a$.

Echinolytrum Desr. 1114.

Echinophora L. $3 \%$ ? Eckinopogon P. B. 946 . Echinops L. 2349.

Fchitiopis PIill. 2349. Echinospermum Sw. 2969.

Echinosphaera Sicb. 5056.
Echinus Lour. 5!04.

Echinchilon Desf. 2944 .

Echites L. 3513.

Echitim L. 2943.

Eclipta L. $26 \% 1$.

Eiclopes G. 2406.

Ectocarpus Lob. 470.

Eclosperma Vauch. 472.

Ertostroma Fr. 231.

Ectrosia IRBr. 933 .

Eriechi Löfl. 2106.

Elluondia H.Cass.2427.

Ellvarisia Salisb. 1089.

Egletes II. Cass. 2479.

Ehrenbergia Spr. 3646.

Ehrenbergia Mart.5193.

Ehrenbergia Spr. 2083.

Ehretia L. 2985.

Ehrharta Thub. 960.

Eisothea DC. $4045 a$.

Ekebergia Sparm. 5513.

Eklonia Hornem. $511 \mathrm{~b}$.

Elaeagnas L. 1715.

Elaeis Jcr. 1624.

Elaeocarpus L. 5430.

Elaeococca Comm. $306 ?$.

Elaeodendron .Jc\%. 5304.

Elaphomyces N. V. E. 211.

Elaphryum Jacr. 3835.

Elate Ait. 1635.

Elaterium Much. 2753.

Elaterium L. 2748.

Elatiue L. 4553.

Elcaja Juss. 5512.

Electra Pz. 1013.

Elevia Thub. 1133.

Eleiotis DC. 4005.

Eleocharis RBr. 1118.

Elephantusia IV. 1271.

Elephantopus L. 2344.

Elephas T. $30 \% 6$.

Elettaria Maton. 1591.

Eleusine Lam. 868.

Flentheranthera Poit. 2717.

Elcutheria P. B. 567.

Elichrysum W. 24?9.

Elionurus TV. 964.

Ellean!hus Prsl. 155?.

Bllebocarpis Kaulf. $70 \%$.

Ellintia Miihlub. 3404 .

Ellipsaria DC. 4751 a.

Ellisia L. 2980.

Elmigera Rchb. 3177b.

Elodea Mchx. 80.3.

Elodea Prsh. 547?. 
Elphegea H. C'ass. 2022. Entosthymenium Brid.|Eriocephalus Dill. 2399. Disholzia W. 2868.

Eisho!tzia Rich. 4652. Bivasia DeC. 5181. iilvira II. Cass. 2701. fivmins L. 842 .

il ymus Koe!. 8㣙.

lijyua Schrad. 106 s. $^{\circ}$

Eifnanthus P. B. 1094. Biyouras W. 964 .

Silytraria Mchx. 3110.

Wiytrigia Desv. 853a.

Jilytropappus H. Cass. i 442 .

Plytrophorus P. B. 840. Eiubelia Burm. 31003.

Jinblica Gïrt. 5076.

Embolhrinm Forst. 1760.

Eimbryopteris Gärtu. 364 \%̆b. ex adil.

Emericia R. S. 3519.

limerus '9. 3S78h.

Ninex Neck. 4285.

Emilia I1. Cass. 251?.

Einproclociea A. St. Hil. 4 45.3.

Empetram L. 5116.

Eumpleurum Sol. 5228b.

Finpusa Lindl. 13560.

Enydra Lour. 2705.

Epacris Forst. 335?.

Eperna An!s. 4130.

Ephebe Fr. $396 j$.

Ephedia L. 1691.

Sphemenu 33:7d.

Ephielis Suhreb. 5219.

Epibaterium Forst. 1879.

Epiblema RBr. 1420.

Epichysizm Tol. 44.

Epicnccum Lk. 39.

Epidendrum L. 1544.

Epigatea L. 3395.

Epilatoria Comm. 2522.

Epilobiam L. 4500.

spimedium L. 4831 .

Epipactis Sw. 1453.

Epiphegus Nutt. 3053.

Epiphericae Fr. 276a.

Epiphyllum Herm. t? 4 ta.

Epiphystis Trin. 880.

Epipogin!n RBr. 1456. Epistephium Knth. 1459.

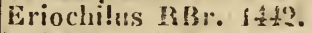

Eriochloa K. U. 15. 865.

Evioclerysis ?. S. USta.

Lriocline H. Cass. 2736.

liriocoila lirkh. 3499a.

Eriocoma Nitt. 920ع.

Eriocoma K. II. B. 2806.

Eriodendron DC. 5336.

Eriogonam Mrhx. 49y2.

Eriolaena DeC. 528\%.

Eriolepis H. Cilss. 2332.

Eriolenca D(.. 45.511 c.

Eriophorum he 1123.

Srophyllim LaG. 2584.

Eriosema Dr: 3934.

Eriosperma liaín. p.15.

Eriospermn!n Jacy. 783.

Eriosphacra DC. 4639 b.

Eriostegia DC. $1634 a$.

Eriostemon S: 5168.

Eriostomum Hffgg. Lk. $28 \% 9 \mathrm{c}$.

Erinthrix H. Cass. 2490.

Eriphia P. Br. 3240.

Erisma Rudg. 4513 .

Epistylium Sw. Fl. Erithalis J. Pr. 2100.

Empusa pia Rchb. 15fj6.

Enalcilia II. Cass. 2355.

Enatizrocarpus LaB. 4727.

Fucalypta Inlg. 637.

Eucelia Arlus. 260?

Euchylatua RBr. 4353.

Fincoelium Ag. 50\%.

Bincyclia Hook. 1 j\$f.

Fincyanthus Lomr. 3398.

Endesperinmm Blım. 401 ?

Endiandra RBr. 190ذ.

Endocarpon Heliv.3s0.

Jindogone Lk. $2 ? 0$.

Endolenca H. Cass. 2435.

$506 \mathrm{9}$.

Epochuium Lk. 110.

EyuiseinII L. 1689.

Ernestia DeC. 4534.

Errodea Sw. 2003.

Ernstingia Neck. 5219.

Brapostis P. B. 1031. Erobatos DC. 4!62c.

iragrostis P. B. 1031a. Erodendron Sal. 1741.

Eranthemum RBr.3116. Erodimn l'Herit. 5279.

Eranthis Salish. 4963. Erophila DC. tifia.

Enobinthus Mitch. Erporim Brid. 547. 38!!:b.

Erechtites Rafin. 2511.

Fruca 'T. 4814 .

Eremorton Brid. 601a.

Eremophila RBr. 3047.

Eremurus MB. 1390.

Eria Lindl. 1554.

Erianthus Mchx. 9\&8.

Erica L. $33 \% 9$.

Erigenia Nutt. 3766.

isrigeron L. 2546 .

Endrachium Juss. 3007. Eriulacea Lamx. 497f.

Fukianthus Lour. 338s. Frinacea Cilus. 4025e.

Enneaponon Desv. 930. Hrineum Pers. 59.

Enodium Gaul. 10?3h. Firims L. 3103.

Firica Lam. 4817.

Erucago T. 4718.

Erucaria Gïrt. 4720.

Erucastrum DC. 481\%b.

Eruptoria 386a.

Ervilia Lk. 3918b.

Ervum L. 3918.

Eryaleinm DC. 4910h.

Erycibe $\mathbb{R x b} 3003$.

Eryngimm L. 3750.

Erysibe Rebut. 149.

Erysimastrum DC. $4813 \mathrm{c}$.

Enourea Aubl. 523\%.

Eisslinia Rutt. 3128.

Jirlarla Ail 4161 .

Enteridium Ehrub. 180.

Euthostodon Schwg. 6 fij.

Eriolionrya Limil. 4464.

Erincalia Sim. 375?.

Briocalyx Neck. $4034 a$.

Eriocarpum DC. 49151 .

Eriocaulon L. $114 \%$.

Eriocephalus Vaill. 2332. 2336.

Erysimum L. 4813.

Erysiphe Ildg. 149.

villhraen lich. 3496.

Exythrina L. 3956.

Eivthrochiton N. et $M$. i) 13.

Iirythrocistus L. 4916 b. 
Erythrodamum A. P.|Eudorus H. Cass. 2507. îh. 2005.

Erythronium L. 1350.

Frythropalıun Bi:ım. 2751.

Erythrophlaeum RBr. +163b.

Ervthrorrhiza Mchx. 3373.

Erythrospermum Lam. +4y18.

Erythrostictus

Schlecht. 1302.

Eryth roxylou L. 4906b.

Fiscallonia Mut. 4249.

Eschentachia Mönch. 2543.

Eschscholtzia Cham. 4348.

Esch ireilera Mart 4650.

Escoledia Rz. Par. 3186 .

Esenbeckia K. H. B. $51: 32$.

Esenbeckia Brid. 549.

Escubeckin Blume.5435.

Espeletia Boupl. 2608.

Espera IV. 5454 .

Jisterhazya Mik. 3209.

Esula Haw. 5012.

Ethulia L. 2187.

Eubrychis DC. 4004a.

Eucalyptus l'Herit. $4(j 7:)$.

Eucapparis DC. 4874 .

Enceanothus (Sect.)DC. $37(M)$.

Eurhaetis Bartl. et Wendl. 5lti?.

Euchilus Bilr 4062.

Eucliylouran R. 4950 b.

Eucluoma Nutt. 3085.

Euclea L. $31 j 45$.

Euclidium RBr. 4710.

Euclinia Salish. 2084.

Euclitoria DC. 3974b.

Eucnemis Bricl. 562.

Eucomis lHerit. 1360.

Eizronocarpus DC. $453 \mathrm{Sc}$.

FuconostegiaDC.4634b.

Eucrosia Ker. 1242.

Eu ryphia Car. 5465.

Eudema RBr. 4779.

Eutlesmia $R B R$. $466 \%$.

Eudesınodium DC. 4000 a.
Eudrymis DC. 49ij8a.

Engenia Mich. 4681.

Filgenioides L. 4535.

Euhelianthemnm DC. 4915.

Eulathyrus Ser. 3923b.

Euleptospermun DC. 4671 .

Eulirion R. 1355b.

Eulophia RBr. 1528.

Eulotus Ser. 3874c.

Eulychuis DC. 5362d.

Eumiconia DC. 4639c.

Eumimosa DC. 4 t62a.

Ennomia DeC. 4753.

Eunychia DC. $4265 \mathrm{~b}$.

Eunouis DC. 4027b.

Emparea Banks. 3323.

Eupatorium L. 2227.

Euphorbia L. 5012.

Enphorbia Haw. 5012.

Euphorbia Neck.5012a.

Euphoria Comm. 5216.

Euphrasia L. 3080.

Euphronia Mart. 4459.

Euplassa Sal. 1761.

Eurotiun Lk. $13 \%$.

Eurya Thub. $5+16$.

Euryale Salisb. 814.

Euryandra Forst. 4970.

Eury hia H. Cass. 25:32.

Eurycoma Jack. 38?8.

Enryops H. Cass. 2514.

Euryspermum Sal.1745.

Eurythalia Rrkh. 3499a.

Ensany da DC. 4693.

Eustachys Dsv. 866l).

Eustathes Lour. 3813. 5229.

Eustemia RBr. 3448.

Eusiephia Cav. 1261.

Eustichia Bricl. 542b.

Eustrephus RBr. 1327.

Eutacsonia DC. $346 ! 9$ (1.

Eutassa Salisb. 1730.

Entaxia RBr. 4065.

Eutelaea RBr. 5438.

Enterpe Gärt. 1644.

Euthales RBr. 2797.

Enthalictrum DC. $4953 \mathrm{c}$.

Euthamia Nutt. 2526.

Enthemis Jack. add. 5.51.

Eutoca RBr. 2979.

Eutrenna RBr. 4800.
Euphaseolus DC. 3938a.
Eutriphyllun Ser. $3869 \mathrm{c}$.

Euxenia Cham. 2707.

Euzomun I.k. 4814.

Evaudra RRr. $110 j$.

Evax 6.2451.

Evea Aubl. 2118.

Evernia Ach. 409.

Evorlia Gärt. 1!) 16.

Evodia Forst. 51:3.

Evolvulus L. 2995.

Evollymoides Mnch. 5.390.

Evoly yuus T'. 5389.

Evopis II. Cass. 2684.

Evosina Audr. 3502.

Evosinia Hb. Bpl. 2108.

Evosuus Nutt. 1908.

Exacum L. 3477.

Exarrhena RBr. 2972.

Excipula Fr. 250.

Excoecaria L. 5021.

Exidia Fr. 289.

Exoacantha LaB. 3703.

Exocarpus LaB. $170 \%$.

Exosporimm Lk. 34.

Exostemma Hb. Bpl. $206 \%$.

Exrostyles Schott. 4092.

Eysenharllia K. H. B. 3894.

Faba T. 3920 .

Fabiana Rz. Pav. 3200.

Fabricia Gïrt. 4672.

Fabricia Scop. 4009.

Fabricia Thub. 1215.

Fabronia Rac!li. 571.

Facelis H. Cass. $2+13$.

Fagara L. 5132.

Fagelia Neck. 3935.

Fagonia T. 5194.

Fagopyrum T. $4291 \ldots$.

Fagraea Thub. 3.j0y.

Fag'us L. 1796.

Falcara Gm. 3930 .

Falcatula Brot. $38 \pi 2 d$.

Falimiria bess. 1021.

Falkia L. 2993.

Faramea Aubl. 2008.

Farfara DeC. 2249.

Farsetiana DC. $4790 \mathrm{~d}$.

Farsetia Turr. $47 \% 0$.

Fatioa DeC. 4.j(j1.

Faujasia II. Cass. 2503.

Fansiula H. Cass. 2416.

Favolus P. B. 345.

Favonium Gärt. 2681.

Feaea Spr. 2002. 
Fedia Much. 1947. Feea B. St. V. f\%rlia. Fequatella Raddi. 528c. Felicia II. Cass. 25150. Felicia H. Cass. 2547. Ferdinauda LaG. :2653. Fereira Vand. 3510. Fermandezia Rz. Pav. 1532.

Fernelia Comm. 2092. Feronia Corr. 5531. Ferraria L. 1182.

Ferreola Rxb. 3589.

Ferrum equinum $T$. 3981.

Ferula L. 3678.

Ferulago Koch. $36 \% 9$.

Festucia L. 1001.

Fenillea P. 2771.

Fevillea L. 2771.

Fibiyia Koel. 862.

Fibigia Med. 4770b.

Fibrillaria P. p. 15.

Ficaria Dill. 4940.

Ficoidea Dill. 4405.

Ficoilles T. 4408.

Ficus L. 181 .

Fieldia Cunningh. 3247. Fieraurea Lonr. 1876.

Filago W. 241.

Fimbriaria N. v. E. 527.

Fimbrillaria H. Cass. $25+2$.

Fimbristyles Rich. 1113.

Fimbristylis Vahl.1113. Findlaya Bowd. add. $55 \%$ ?.

Fischera DeC. 3439 .

Fischera Sw. 3402.

Fischera Spr. 3774.

Fissideus Hdg. 543.

Fissilia Commers. 3612.

Fistula DC. 413.jh.

Fisfuliua Bull. 334.

Flabellaria Lamk. 471. Flacourtia l'Herit. 4881.

Flagellaria L. 1163.

Flammella 349 D. e.

Flaumula Rupp. 4946a.

Flaveria Juss. 2708.

Flemingia Rxb. 3996 . Flemingiastrum DC. 3996ja.

Fliurlersia RBr. 5519. Floerkea Spr. 2799.

Floerkea Willd. 813.

Florestina H. Cass. 2585.
Floscopa Lour. 1341.

Flotovia Spr. 2274.

Fliigurea Rich. 1324.

Fliigunea Willd. 5098.

Fluvialis P. 1667.

Foeniculutn 'T. 3704.

Foenumbraecuin Ser. 38721).

Foetidia Cornmers. 4502.

Fontanesia LaB. 4489.

Fontinalis L. 553.

Forgesia Juss. $421 \%$.

Fornicinm H. Cass. 2313.

Forskolea L. $180 \mathrm{~s}$.

Forstera L. 19?0.

Forsylhia Vahl. 3548.

Forsythia Walt. $42: 0$.

Fossombronia Radd. 5.32f.

Fothergilla Aubl. 4635.

Fotherwilla L. 1903.

Fongeria Much. 2654.

Foncuiera K. H. B. 42:39.

Fonrcroya Vent. 128佔.

Foveolaria Rz. Pav. 5507.

Foveolaria DC 5453

Fragaria Sin. 4438a.

Fragaria T. L. 4438.

Fragariastrum Ehrh. 44:30.

Fragerial Del. 2278.

Fragilaria Logh. $4 j 1$.

Fragosa R\%. Pav. 3\%7s. Galarhoeus Haw.

Franciscaria DeC. 4182. Galatella H. Cass. 2533.

Franciscea Pohı. 3109. Galax L. 3373.

Franciscea DeC. 4182. Galaxia L. 1199.

Frangula T. 3822c.

Frankenia L. 490?.

Franklandia RBr. 1750

Frankoa Cav. 41921$)$.

Franseria Cav. 2742.

Frasera Walt. 3485.

Fraxinella T. 5155.

Fraxinus L. 3580 .

Frenela Mirl). 1721.

Freyrinetia Gandich. 1272.

Freylinia Colla. 3205c.

Freziera W. 5418.

Friclericia Mart. 3258.

Fricullaudia Chau.4564.

Friesia DeC. 54\%5.

Friesia Spr. 5046.

Frisea R. 1\%08b.
Fritillaria L. 1353.

Froelichia Much. 4360.

Froelichia V. 2020.

Frullaria Radd. 532c.

Frusiulia An. 444.

Fuchsia Pluin. 4494.

Fucus L. 513.

Fugosia Juss. 5270.

Fuirena Rottb. 1127.

Finumia DC, 4915c. .

Fumaria T'. 48\%7.

Funaria Hdy. 6tj2.

Funkia Spr. 13\%6.

Furcaria DC. 5273c.

Furcellaria Laux. 51 .

Fusanus L. 170t.

Finsariıu Lk. 36.

Fusisporim Lk. 109.

Grigea Ker. 136jo.

Gagea Raddi. 6?(j.

Gïrnera Laın. 3506.

Gärtuera Schreb. 5372.

Gagnebina Neck. H163.

Gahuia Forst. 1101

Gaillardia Funger. 2iss(j.

Gaillonella B. St. V. 451.

Gaillonia Bonnem. 4S.3.

Gajauus Rmph. $36 . j 1$.

Galactia P. Br. $39 \% 0$.

Galactites Much. 2336.

Galactodeudron Kuth. 1805.

Galednpa Lam. 3013.

Galecka \%. 3891.

Galena 349. D. c.

Galenia L. 4401.

Galeobdolon Sin. 2872.

Galeola Lour. 1582.

Galeopsis L. 287?!.

Galeopsis T. 28!.j.

Galinsoga Cav. 2.857.

Galipea Aubl. 5145.

Galium L. 1986.

Galliu:m 'T'. 1986.

Galopina Thub. $4+19$.

Galorrhoens Fr. 347.

Galphimia Cav. 5378.

Galurus Spr. 5035.

Galvania Vell. 'Yaud. 2032. 30+81). 
Galvezia Rz. Par. 1906. Genista Lam. 4030. 5137.

Garcia Rohr. 5068.

Garciana Lour. $11 j 6$.

Garcinia L. 5392.

Garlenia L. f. 2033 .

Gardenia Ellis. 2030.

Garlneria Wall. 3503!). ex add.

Gardoquia Rz. P. 2824.

Garidella T. 4931.

Garuga lixb. 38t8.

Garuleum H. Cass. 2734.

Gasteria Duv. 1409.

Gastonia Commers. 3794.

Gastridium P. B. 901.

Gastrochilus Don. 151\%.

Gistrodial JiBr. 1450.

Gastrolobiam $\mathrm{RBr}$. 4063.

Gastronema Sins. 1231.

Gattenhoffia Neck. 2730.

Gatyona hi. Cirss. 2111.

Gandichaudia K. H. B. $53 \% 5$.

Gaudinia P. B. 850.

Gauja Ruph. 54t5d.

Ganltheria L. 3390.

Gaura L. 4501.

Gausapia Fr. 85.

Gavila Feuill. 1425.

Gaya Gaud. 3708a.

Gaya K. I1. 13. 5?49.

Gily a Spl. 5304.

Gaylussacia K. H. B. i976.

Gazania Gärt. 267\%.

Geastrum Desv. :02a.

Geastrum Mich. 202.

Geria Blume. 5t!j.

Geissois La B. $410 ! .5339$.

Geissomeria Liudl. $312 b j$. ex add.

Geissorrhiza ker. 1203.

Gela Lomi. 3614.

Gelasia H. Cass. $217 \%$.

Gelidium Lamx. 49\%).

Gelonimin G. 5?11.

Gelonium Roxb. 5052.

Gelsemiın Juss. 32166.

Gemella Lour. 521S.

Gemunularia Ráin. I63.

Genetyllis DeC. 46 tiz.

Geniosioma Forst. 3503.

Gen

Geni 氵iphyjla l'Herit. 5079 .
Genista G. M. S. 4030 c Gealisia Rchb. 1207.

Genoria P. 45i2.

Genosiris Laß. $118 \%$. ex add.

Gentiana J. 3499.

Gentiana Schm. 3499c.

Gentianella Brkh. $3494 a$.

Geochorda Cham. 3154. Geodoruin Jacks. 1515.

Geoffioy: Jcy. 4102.

Geoglossum Pers. 302.

Geonema IV. 1639.

Geophila Bou. 2036.

Gecroia Spr. 21iog.

Georina V. 2609.

Geraciun Rchb. 2149.

Gerauiun L. 52830.

Gerarlia Cham. 3i6ja.

Gerardia L. 3165 .

Gerbera Grou. '2257.

Gerberia H. C'ass. 2257.

Germal:ea Lisiı. 2811.

Geropogon L. 2180.

Gerumi Forsk. 5010.

Geryonia Sihrk. $4 \% 04$.

Gesncra L. 3242.

Gethy!lis L. 1214.

Getonia Roxh. $45+1$.

Geam L. 4445.

Gemsia Moc. Sess. $431 j$.

Ghinia IV. 2899.

Gibharia H. Cass. 2733.

Gibbera Fr. 27?.

Gifola H. Cirss. 2452.

Gigalobium P. Br. 4161.

Gisartiua Lowb. $400 \mathrm{~b}$.

Gigarlina Lamx. 498.

Gigartina Lamx. sp. tive.

Gilia Rz. P. 30 ?.

Gilibertia Gm. 5508 .

Gilibertia R3\%. Pav.3786.

Gillenia Much. 4451.

Gilliesia Lindl. 1356.

Gimbernatia Rz. Pav. 4540 .

Gingillium Forst. 3708c.

Ginginsia D\{: 4279.

Giukgo 'Thub. $169 \%$.

Ginora L. 4572.

Ginoria Jacy. 4572.

Gisekia L. 4400.

Gissonia Sal. 1745.
Githngo Desf. 5362ส.

Giuninia Scop. 412\%.

Glabraria L. 1909.

Gladiolus L. 1190. 1100 a.

Glauciularia Gm. 2894.

Glandulifolia Vrendi. 5159.

Glaphyria Jack. 4460.

Glastuin Riup). $473 \%$ L.

Glauciun T. $\$ 851$.

Glaux L. 359\%a. ex adl.

Glechoma L. 2875.

Glechon Spr. $28 \% 0$.

Gleditschia L. 4154.

Gleichenia Sw. 741.

Glinola DC. 4t03a.

Giliuus I. $4,103$.

Gliotrichum Eschw. 73.

Globla L. 1550.

Globularia L. $309 ?$.

Globuluria T. 3092a.

Globulea Haw. 4178.

Globulina Lk. 466:.

Glochidion Forst.5071.

Gloicnema As. 428.

Glonimm Miihlub. 258.

Gloriosa L. 1351.

Glossarihen Mart.4503.

Glossaspis Sipr. 146?.

GIossocardia H. Cass. 2610.

Glossocomia Don. 2750.

Glossodia RBr. 1444.

Glossoma Schreb. 4.318.

Glossopetalum Schreb. 3815 .

Glossostemon Desf. 5295.

Glossost y lis Chaın.3160.

Glossula Lindl. 1462.

Glottidium Desv. 3.104.

Gloxinia l'Herit. 3237.

Gluta L. 5288.

Glutinaria Commn. 2522.

Glyceria Nutt. 3764 .

Glyceria RBr. $102 \%$.

Glyciue L. 3961 .

Glycinopsis DC. 3974 .

Glycyrrhiza Much. 38 šma.

Glycyrrhiza T. 3889.

Glyphia H. Cass. 2360.

Glyphis Ach. 392.

Glyphocarpa RBr. 656.

Glyphocarpus Brid.6j6.

Glyphomitrium Bricl. 620. 
Gmelina L. 2935. $\quad$ Goodenia Sm. 2774. Graphalimm L. RBr. $2+18$.

Griephosis H.Cass.2445. Guetum Forsk. $195 \%$.

Gnidia L. $177 \%$.

Godinella Lestib. $3327 a$. Godoya Iiz. Pav. 5424. Goethea N.et Mart.5291. Goetzea Rach). 3923 . Goldbachia DeC. 4715. Goldbachia Trin. 983.

Gomara Ad. $41 \%$. Gomara liz. Pav. 3210. Gomaria Spr. 3210. Gomeza Libr. 15:34. Gomezia Nut. 2005. Gomeziun DC. 3895i). Gomortega Rz. Pav. 1916b. ex adll. Gomozia Schreb. 2005. Gomphia Schreb. 5188. Gomphocarpus RiBr. $34 \% 7$.

Gompholobium S 4073.

Gomphonema Ag. 445. Gomphoplorus Brid. $66 \%$.

Goısphora Fr. $30 \%$.

Gomphrena L. 4359.

Gomphus P. 301.

Goinphus 349 [s. d.

Gomutus Rimpf. 1625.

Gonatocarpus iv. 48 ?

Gongora liz. Pav, 1540.

Gouiocarpus Kön. 48?.

Goniocaulon II. Cass. 2635.

Goniodium Kz.' $17 \% 1$.

Goniosporium Lk. 103. Gomocarpus Hanilt. 4533.

Gouocarpus Thn!.1482. Gonolobus $\mathrm{RBr} .3423$. Gonostemon Hawr. 3411 .

Gonotheca Rafiu. 2622. Gonus Lour. 5136.

Gonyanthes Bhun.1684. Gony clarlon Lk. 461.

Gonytrichum N. v. E. 76 .

Gonzalagunia Rz. Pav. 2115.

Gonzalea P. 2115.

Goodallia Bowd. 4191.
Goodia Salisb. 4053.

Goodyera RBr. 1415.

Gordonia Ell. 5408.

Gorteria L. 2675.

Gorteria Lam. 2683.

Gossypinm L. 5272.

Gothofreda Vent. 2435.

Gouania L. 3797.

Gonffeia Rob. et Cast. 5351.

Goupia Aubl. 3815.

Graemia Hook. 2582.

Graffenriedera DeC. 4608.

Grahamia Spr. 2582.

Grammanthes DeC. 4180.

Grammarthron H. Cass. 2488.

Grammeionium Rchb. $4885 \mathrm{e}$.

Grammita Bonnem.484.

Grammitis Sw. 728.

Grammocarpus Ser. $3372 \mathrm{a}$.

Granadilla DeC. 3472d.

Grangुea Adns. 2480.

Grangeria Comm. 4520.

Graphephorum Dsv. $1017 \mathrm{a}$.

Graphiola Poit. 252.

Graphis Ach. 387.

Grateloupia Ag: 496.

Grateloupia Bonnem. $483 \mathrm{c}$.

Gratiola L. $314 \%$.

Graumiillera Rchb. 762.

Greggia G. 4681.

Gregoria Duby. 3359b

Grevillea RBr. 1756.

Grewia L. 5446 .

Grias L. 4503.

Grielum L. 4410.

Griflinia Ker. 1243.

Griffithia RBr. $6 ? 0$.

Griffitsia Am. 486.

Grimaldia kadll. 520.

Grimaldia Schrk. 4135a.

Grimmia Hilg. 589.

Grindelia W. 2520 .

Griunla Fr. 296.

Griselinia Forst. 5113b. ex acld.

G riselinia Neck. 4016a.

Grislea Lïm. 4574.

Grona Lonr. 3950 .
Gronovia L. 4231.

Grossularia $\mathrm{T}$. $4247 \mathrm{~b}$.

Grubbia Berg. 1700c.

Gruhlmaunia Neck. 1997a.

Guadua Knth: 1053.

Guaiacana T. $364 \%$.

Guaiacum Plum. 5200.

Guaiava T. 4687.

Guapurium Juss. 4681.

Gnardiola Bonpl. 2611.

Guarea L. $551 \%$.

Guariruma H. Cass. 2268.

Guatteria Rz. Pav. 5001.

Guazuma Plum. 5296.

Guaznmoides DeC. $5445 \mathrm{e}$.

Giildenstedtia Fisch. 3879.

Giinthera Trev. 523.

Giinthera Andrz. 481\%.

Giintheria Spr. 2213.

Guepinia Fr. 292.

Finettarda 4. $210 \%$

Guevina Molin. 1746.

Guichenotia Gay. 5302.

Guidonia Plum. 46931.

Guiera Juss. 4.534.

Guilandina Juss. 4151.

Guilaudina Jct. 5124.

Gailielma Mart. 1628.

Gnilleminia K. H. B. 4257.

Guillimia Rottl. 4992a.

Guioa Cav. 5212d.

Gumillaea. Rz. Pav. 3511.

Gumteolis Hamilt. 3162.

Gundelia L. 2348.

Gunnera I. 1818.

Gupia I. St. H. 3815 .

Gussonea Prsl. 108\%i). ex a dill.

Gustavia L. 4656.

Gutierrezia LaG. 2531.

Guzmannia Rz. Pav. 1273.

Gyalecta Ach. 399.

Gymnadenia RBr. 1472.

Gymnandra Pall. $308 \%$.

Gymnanthemum $H$. Cass. '2212.

Gymuanthera $\mathbf{R B r}$. 34.58.

Gymnanthes Sw. prod. 5021. 
Gymmarrhena Desf. 2483.

Gymnena RBr. 3421.

Gymizocarpum Forsk. 4268.

GymuocephalusSchwg. 052.

Gymmocladus Lam. 4153.

Gymnocline H. Cass. 2379.

Gymnogramma Desv. 730.

Gyinnogynun P. B.

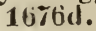

Gymnolomia K. H. B. 2655.

Gymnopera Doll. 4203.

Gymnopogon P. B.918.

Gymnosporangium

DeC. 33.

Gyinnostachys RBr.776.

Gymnostichum Schreb. 844.

GymnostomumHdg.j84. Gymnostyles Juss.?23ł?

Gymuothrix P. B. 926b.

Gynandropsis DeC. 4860.

Gynanthistrophe Poit. $415 \%$.

Gynerinm K. B. H.1050.

Gynestum Poit. 1639.

Gynoon Ad. Juss. 5070.

Gynoplenra Cav. 3473.

Gynopogon Forst. 3555 .

Gynostemma Blume. 1883.

Gynostemon DC. 5453b. Gynox is H. Cass. 2500. Gyunra H: Class. 2506. Gy psophila L. 5359.

Gyptis H. Cass. 223.1. Gyrinops Gärt. 1786.

Gyrocarpus Jack. 4547. Gy rolophinm Kz. 328. Gyrophora Ach. 394.

Gyrostemon Desf. 5245. Gyrostomum Fr. 368.

Gyrotrichum Spr. 121. Habbasia DC. 4162 b.

Habenaria W. 1471.

Hablizia MB. $438 \%$.

Haemadictyon Lindl. 3514.

Haemanthus L. 1248.

Haemaria Lindl. 1417. Haematoxylon L. 4141 .
Haemocarpus Noronh.|Hardwickia 4106. 5475.

Haemocharis Salisb. 5410 .

Haemodoron IVallr. 3058. ex adel.

Haemodorum Sim. 1219.

Haenkea Salisb. 1316. Harpalyce Moc. Sess. Haenselera LaG. 3745.

Hagaea Vent. 4263.

Hagenbachia N. v. E. 1161.

Hagenia Lam. 3789.

Hariota All. 4247 .

Harualum T. 5120.

Haronga A. P. 'Th. 5+75.

Harougana Lam. 5 $\$ 75$.

Harpalium H. Cass. 2628. 3883.

Harpullia Roxb. 5?32.

Harrachia Jacy. $31^{\prime 24}$

Harrisonia Adans. 541. 588.

Hagenia Mnch. 5360a. Harrisonia Hook. 3454. ex ald.

Hakea Schrad. 1755.

Halenia Brkh. 3501.

Halesia L. 3593.

Halesia P. Br. 2107. Hasselquistia L. $368 \%$.

Halianthus Fries. 5350. Hasselia K. H. B. 5\$39.

Halidrys Lgh. 313b.

Halimiun ifC. 4415i.

Halimocnemis Led. 4327.

Haliuodendron Fisch. 3909.

Halimus Wallr. 4334.

Haliseris Ag. 5086.

Hallia I. St. H. 4009.

Hallia 'Thub. 4044.

Halleria L. 3196

Harrisonia RBr. 5180 .

Hartogia Thub. 3621.

Hartogia Berg. 5164. 5165.

Hastingia Sin. Kön. 2913.

Hanya Moc. Sess. 4303.

Havetia K. H. B. 5484.

Haworthia Duv. 1407.

Hayuea Rchl. 5256.

Haynea IV. 2200.

Hebanthe Mart. 4358.

Hebe Juss. 3063c.

Hebea Pers. 1190b.

HebeaudraBonpl.3038a.

Halocnemon MB. 4320. Hebelia Gm. 1293.

Halodendron DC. 3909. Hebeloina 349 D.f.

Halodendron A. P. Th. Hebenstreitia L. 3094. 2942a.

Halophila A. P. 'Th. 758.

Haloragis Forst. 4484 .

Halyuenia Ag. 500.

Hanadryas Commers. 4935.

Hamanelis L. 189 ?

Hamaria Kz. 4424 b. ex Hedycarya Forst. 1836. acid.

Hanelia Jcy. am. 2109. Hedyotis L. 2043.

Hamiltonia W. 1711. Hodyos mum Sw. 1956.

Hamiltonia Dou. 1330. Hedypnois T. 2130.

Hainulium H. Cass. Hedysarum L. 4003.

$26 ? 7$.

Hareornia Gomez.3565. Heimia Lk. et Otto.

Hapilaithus Jcq. ein. 4563. 11 \%?.

Hapalostephim Don. $21 \leq 7$.

Haplaria Lk. $115 h$.

Fieintzea Scop. 4023a.

Heintzelmannia Neck. 3156.

Heisteria Berg. 3030.

Haplotrichum Lk. 115i. Heisteria L. 3613.

Haplotrichuin Eschw. Helenim L. 2588. 72.

HeleocharisLestib.1118. 
Heleochloa Host. 943a. Hemimeris L. 3189.

Heleogiton Lestib. 1119. I Iemionitis L. 731.

Heleophila P. B. 1119. Hemiphragma Wall.

Helia Mart. 3489.

Helianthemnu T. 4915. $4915 a$.

Helianthus L. 2629.

Helicanthera Lour.adl. 530\%.

Helichiysum Vaill.2129.

Helicia Lomr. 17 tj.

Heliconia L. 1609.

Helicophyllmm Brid. $53 \%$.

Helicosporium N. v. E. 77.

Helicta H. Cass. 26.36.

Helicteres L. 5323.

Helicteroides DC. $4232 \mathrm{~b}$.

Heliocarpus L. 5443.

Heliophila L. 4821.

Heliophthalmun Rafin. $265 \%$.

Heliopsis Pers. 26.38.

Heliotropium 2. 2958.

Helleboraster Lob. $4964 a$.

Helleborns Hippocr. $4964 b$.

Helleborus T. 4264.

Hellenia W. 1593.

HellmanniaRrh!).3472c.

Heluinthia Juss. 213S.

II elıniuthora Fr. 432 .

Helminthostachys Kaulf. 748.

Helmisporimm Lk. 78.

Felonias L. 1294.

Helonias IV. 129.h.

Helopodia Ach. 414il.

Helopis Trin. 886.

Helosciadiun Koch. 3735.

Helosis Rich. 1682.

Helospora Jack. 2017.

Helotium Torl. 294.

He:Ivella L. 320 .

Helxine Rer. 180\%.

Hemarthria RBr. 8?1.

Hemerocallis L. 1374.

Heiniaudra RBr. $280 \%$.

Hemianthus Nutt. 3134. dele: 3324.

Hemichlaeua Schrad. 1092.

Hemichroa RBr. $433 \%$.

Hemidesmus RBr. 3456 .

Hemistemina Ehrub. 2887. 640.

Hemitelia Br. 678. 5013.

Heukelia Spr. 3224.

Heurietia DeC. 4637.

Hepatica Dill. 4943.

Heraclenm Hoffin. 368 b.

Heraclemn L. 368 ?.

Hercospora Fr. 240.

Hericium Fr. 306.

Heritiera Ait. 5285.

Hermanuia I. 53306.

Hermas L. 3761. 4364.

Hermodactylos PBr. 1307. Heriandia L. 1864.

Herpestes G. $315 \%$.

Herreria Ad. 2100.

Hertia Neck. 2j15.

Hesperis L. 4806.

Hessea Berg. 1240. 2:38. 3102.
Heterodendron Desf. 5227.

Heterolepis H. Cass. 2669.

Hemistemmn Juss. 4982.

Hemisynapsiun Brid.

Hendecandra Eschsch.

Henricia H. Cass. 2561.

Hepataria Rafin. p. 15.

Heptaca Lour. adil. 5556.

Heritiera Schrk. 1293.

Hermannella DC. 5306a.

Hermbstaedtia lichb.

Hermesio Bonpl. 5040.

Hermione Salisb. 1?68a.

Hermupoa Löfl. 4377.

Heruiaria T. L. 4269.

Herpetica Rmph. 4135f.

Herpiciun H. Cass. 2674.

Herpotrichum Fr. 35.

Herreria Rz. Pav. 1334.

HerschelliaBowvi.3301.

Herminimm RBr. 1476.

Hesperantha Ker. 1205.

Hesperidium DC. 4806a.

Hesperidopsis DC. 4805.

Heteranthemis Schott.

Heterauthera P. B.1165.

Heteranthia N. v. E.

Heteranthus Bupl.2284.
Heterocoma DeC. 2213.

Heteroloma Desv. 3993.

Heteromorpha Cham.

3741.

Ifeteromorpha H. Cass. 2669.

Heteronema DeC. $458 \%$.

Heteropogon P. 969.

Heteropteris V. 5371 .

Heteropteris K. H. B. 5371.

Heterospermum Cav. 2 li12.

Heterosphaeria Grev. 259.

Heterostega Desv. 872.

Heterostemon Desf. 4117.

Heterostomu in Fr. 277a.

Heterotax is Liull.1445.

Heterotheca H. Cass. 2529.

Heterotrichum DeC. 4633.

Heteryta Rafin. 3026.

Heuchera L. 4198.

Heuchera Nurr. 2044.

Heuruia Spr. 3409.

Hevea Aubl. 5065.

Hewenia Haw. 3411 .

Hexadica Lour. 5101.

Hexanthus Lour. 1909.

Heylandia DC. 4043.

Hey massoli Aubl. 3614.

Hey uca Roxb. 5.515.

Hibbertia Andr. $498 \%$.

Hiliscus L. 5273.

Hieracium L. 2150.

Hierochloa Gm. 1039.

II ieginsia P. 2005.

Milacium P. B. 2031 .

Milaria K. H. F. $83 \%$

Hilbertia Thon. add. $5 \% \%$.

Hillia Jcq. am. 2070.

Hilspubergia Tsch. 2932.

Himanthalia Syb. 513a.

Himanlia ?. ?. 15.

Himantoglossuma Syr. 1466.

Hingstha Roxb. 250.5.

Hingstonia RnÂ.

2724.

Horthia Necl. 2391a. 
Hippeastrum Herb. $125 \%$.

Hippia L. 2372.

Hippion Spr. 3492.

Hippion Schın. 3499a.

Hiplocistannm 'T'.5202.

Hip pocentanrea Schnlt. $3+7 \%$.

Hippocratea L. 5382.

Hippocrepis L. 3981.

Hippomane L. 5024.

Fippomarathrum Lk. 374.

Hippophaё L. 1716.

Hipposeris II. Cass. $2: 73$.

Hippotis Rz. Pav. 2088.

Iippuris $\mathrm{L} .4480$.

Hiptage Gärtn. 5372.

Yiraea K. H. B. 5366.

Mirnellia H. Cass. 2444.

Hirneola Fr. 291.

Hirschfeldia Mnch. 4818 .

Hirtella L. 4518.

Hisingera Hell. 5053.

Hispiclella Lam. 2125.

Hoarea Sweet. 52\%

Hoelzelia Neck. 4160 .

Inoffinannia Str. 2048.

Holfinanseggia Cav. 4145.

Hohenwartha Vest. 2339.

Hoilzia Jiss. 3022.

Holanthus R. 1962a.

Holarges DC. $4761 \mathrm{c}$.

Molarthena RBr. $351 \%$.

Holboellia Wall. 1890.

Holocheilns H. Cass. 2281.

Holcus L. 1040.

Holigarna Pixb. 3865.

Holmskioldia I? $1 z$. 2913.

Hololachne Ehrnb. 4415.

Hololepis DeC. 2214.

Holopetalum DC. em. 5281a.

Holostemma RBr. 3439 .

HolosteumL.te!e: ${ }^{427}$. rectims 5.340 .

Holothrix Rich. $14 \%$.

Homalauthus Ad. Juss. em. 5025.

Homalium Jcq. 4693.

Homalocenchrus Misg. 953.
Homalocline II. Cass. Huberia DeC. 4628. $2 ! 48$.

Hubertia B. St. V. 2502.

Homanthis K. H.B.2285. Hudsonia I. 4914.

Homanthus K. H. B. Hudsonia liobins. 4545. 2233.

Homeria Vent. 1185.

Huernia RBr. 340 .

Nuertea Rz. Pav. 3850.

Inigelia Rchb. 3760c.

ex adt.

Iomogyne H. Cass. 22.14 .

Homoianthus Bonpl. 2284.

Homonema Ag. ex add.

Hugonia I. 5.318.

Huinbertia Comm. 3007.

Humboldia V. 4118.

Humea Sin. $23 \%$.

Humiria DeC. 5441.

Humulus L. 1810.

Hanteria Roxb. 3637.

Homonoia Lour. 5102.

Homoplitis Mrin. 968.

Honckenya Ehrh. 5350.

Honckenya IV. 5436.

Honottia incib. 4549.

Hookeria Sin. 545.

Hookeria Schl. 604.

Hookia Neck. 2320.2311.

Hopea Roxb. 3654 .

Hopkirlia Spr. $263 \%$

Hoplotheca Nitt. cm. 4360 .

Hoppea Rachb. 2252.

Hordeum I. 845 .

Horleum P. B. 845h.

Horkelia Cham. 4434.

Horminum E. 2823.

Horminum T. 2805a.

Hura L. 5023.

Intchinsia Ag. 484.

Hutchinsia RBr. 4751.

Huttum Ad. 4654.

Hyacinthus L. 1362.

Hyaenanche Lamb. 5067.

Hyala l'Her. 4263.

Hybanthus Jacq. 4889.

Hybridella H. Cass. 2709.

HydnocarpusGärt.488t.

Hyduum L. 333.

Hydrangea Lain. 4216a.

Hydrangea L. 4216.

Hydran thelium $\mathbf{K}$. I. B. 3148 .

Hornemannia W. 31\%0. Hy lrastis L. 4936.

Hornera Nerk. 3949.

flornschuchia N. v. E. add. 5558.

Hornschnchia Spr. add. 5559.

Mornstedtia Retz. 1596.

Horteusia Lam. 42i6i).

Hortia Vaud. 5149.

Hoslundia $\mathbb{L}$. 28.45.

Hosta Jec. 2910.

Hosta Tratt. 1376.

Mostia Mnch. 21.2a.

IIottonia L. 3325.

Hottonia Burm. 45.49.

Howniri Anbl. 3545. 5.111.

Houmiria Juss. 3505.

Inomirim Rirh. 3595.

Houstonia L. 3183.

Honttuynia 'Phub. 794.

Houttuynia Hontt. 1192.

Hovea RBr. 4057.

Hovenia 'Thmb. 3808.

Hoya RBr. $3 \frac{4}{2} 16$.

Huanaca Car. 3732.
Hydrilla Rich. $80 ?$.

Hydrocharis L. 810 .

Hydrochloa $\mathrm{RBr} .958$.

Hydrochloa Hartm. 1029.

Hydrocleis Rich. $79 \%$.

Hydrocoryne Schwab. 422.

Hydrocotlye L. 3763.

Hydrodictyon Rih. 463 .

Hy drogeton Pers. 800.

Hylroolossum WV. 737.

Hydrolea L. 3020 .

IIydromyces Rafin.

p. 15.

II dromy stria IV. Mey. 798.

Hydronema Carus. 128.

Hydropeltis Michx.81?.

Hydrophoma rod. $13 !$ c.

Rydrophylax \&. f. 2002.

Eydrophyllum L. 29\%.

Hydrophyinin Jack. 2018.

Hydropityon Gärt.4543. 
Hydropogon Brid. 590. Hypericum L. 5471. Hydropyxis Rafin.4:317. Myperrhiza Spr. 210. Hydrostachys A. P. Th. 1675.

Hydrurus Ag. 436.

Hygrocrocis $\mathrm{Ag} . \$ 16$.

Hyoropila RBr. 3129 . Hylogyne Sal. 1767.

Hymenachue P. P. 859 . Hymenaea L. 4128.

Hymenanthera Banks. 49.0 .

Hymenatherum H.Cass. 2361.

Irymentla Moc. Sess. 5.343.

Hymenobrychis DC. $400+1)$.

flymenocallis Iferb. 1263.

IIymenocarpus Sav. $38 \%$ \%.

Hymenochaete P. B. 1121.

Hymenodistyon Wall. adll. 55bo.

Hy menogye Haw. 4408.

Hymenolepis Kaulf. 707.

Hy monema I. Cass. 2132.

Hymenophallus $N$. v. E. 228.

Hymenophyllum Sm. 674.

Hymenopappus l'Her. 2589.

Hymenopogon Wall. 2054.

Hy inemopogon P. P.665.

Jy menospronspr.3945.

Hymenostachys 6761).

Hyinenostylium Brid. ¿85.

Hymenota DC. 4061 .

Hymenotheciun LaG. 878.

Mymenula Fr. 281.

Hyobanche L. 3059.

Myominila Brid. JStc.

IIyoseyamus \&. 2279.

Hfyoseris Juss. 2128.

11yosparhe Mart. 1638. Hypecoum L. $48 \div 9$.

Hypelate P.Brw. 5200.

Hyperanthera V. 5124.

Hypericoides Ad. 5468.
II y pha Pers. j3.

Iyphaene Gärt. 1660.

Hyphelia Fr. $1 \% 5$.

Hypholema 349B. c.

Hyphy dra Schreb. 1146.

Hy priea Lamx. $49 \%$.

fly puan L. $57 \%$.

Hypocalyptus Thnb. $40+0$.

Hy pochaena Fr. 169.

Hypochaeris Gärt. 2133.

Hypochuus Ehrub. 82.

Hypocrea F. 279. 279c.

Hy porlema R. 1577 b.

Hy podermim Lk. diss. 91).

HypolerminmLk.sp.21.

Ilypoëlyptum Vahl. 1085.

Hypoëlytrum Rich. 1085

Hypoëstes Sol. 3115.

Hypolaena RBr. 1143.

Hypolepia Rafin. p.15.

Hypolepis P. B. 1096 i.

Hypolepis Pers. 1682b. ex add.

Hypolyssus P. 318.

Hypomyce Fr. 279b.

Hypopterygium Brid. 536.545.

Hyporhodius $3 \mathbf{4 9 G}$.

Hypospila Fr. 274.

Hypoxanthus Rich. 4639 b.

Hypoxis I. 1215.

Hypoxylon Bull. 278.

Hy poxylon Fr. 2\%Sc.

Hypt is Jarc. 2877.

Hy ssopifolia C. B. 45585 b.

Hyssopus L. 2867.

Hysteria Reinw. 1458.

IIysterina Ach. 386ıl.

Hysterionica IV. 2593.

Hysterium Fr. 251.

Hystrix Much. 844 .

Ibbetsonia Sims. 4081.

Iberillastrum DC. 1744 b.

Iheridella DC. $4751 \mathrm{~g}$.

Iheridium DC. 474ta.

iberis L. 4744 .

Icacina A. Juss. 3617.

Ícaco PInm. 4525.

Ichuanthus F. B. 1026.

Iclenocarpus RBr. 3.515.
Ichthyomethia P. Br. 390.5.

Ichthyosma Schlecht. $168 i$.

Ícica Anbl. 3839.

Ictimus Ul. Cass. 2676.

Ictodes Bigel. 90 .

Idiciun Nêk. 2263.

llloga H. Cass. 24:0.

Ignatia L. 3579 .

Ionatiana Lotir. 3397.

llea Fr. 478.

llex L. 3624.

llicioides Dum. Cours. 3627.

Illecebrum I. 4266.

IIIicium L. 4966.

Illosporium Nart. 154.

Ina!ophyllum Hook. 1?36!).

Imbricaria Commers. 3638.

Imbricaria IIoffin. $400 \mathrm{c}$. $402 \%$. $411 \mathrm{~d}$.

Imbricaria Sm. 4670.

Imhofia Herb. 1245.

Impatiens Riv. 4834.

Ininerata Cyr. $98 \%$.

Imperatoria T. 3675.

Impia N. v. E. $2 \times 52$. ex add.

Incarvillea Juss. 3265.

Incillaria Fr. 354 .

Indigofera L. $39 \%$.

Inga Plum. 41 fi4.

Ingenhouzia Mloc. Sess. 5268.

Inocarpus Forst. 3651.

Inoconia A. Lib. p. 15 .

Inocybe $349 \mathrm{E}$.

Inoloma 349. F. b.

Institiale Fr. 172.

Intsia A. P. Th. 4115.

Intybellia H. Cass. $215 \%$.

fnula Gärt. $245 \%$.

Involncraria Ser. 2763.

Iphiona II. Cass. 2169.

fiomea d. 3015.

Ipomeria Nutt. 3024.

Ipomopsis Mchx. 3024.

Iresine IV. 4355.

Iria Rich. 1091.

Iriartea Rz. Par. $16 t f$.

Iridiogalva P. B. 1293.

Irio Col. $4810 \mathrm{~d}$.

Iris L. 1187.

Irlbachia Mart. $3 \$ 90$. 
Iroucana Aubl. 4695b. Irpex Fr. 330.

Isachne RBr. 937. lsanthina R. 116 ib. Isanthus Mchx. 2861. Isaria P. 51 .

Isatis I.. 4734.

Ischaemım L. 831. Isertia Schreb. 211 ? Jschmanthus R.S. 1026. Isidium Ach. 359. Isnarilia L. 4490. Isinene Herb. 1269. Isocarpha $\mathrm{RBr}$. 2630. Isochilus $\mathrm{RBr}$. 1549. Isoetes L. 753. Isolepis RBr. 1117. Isonema H. Cass. 2215. Isonema $1 \mathrm{Br}$. 3518 . Isopetalum Sweet. $5278 \mathrm{~m}$.

Isophyllum Hoffin. 374011.

Isoplexis Lindl. 3190b. Isopogon RBr. 1741. Isopyrun Ad. 4943. Isopy rum Hall. 4955. Isothecium Brid. 576. Isotoma $\mathbf{R B r}$. 2789. Isotria Rafin. 1585. Isotypus K. H. B. 2254. Itea L. 4215.

Iva $L .27 t 0$.

Jxeris H. Cass. 2162c. Ixia L. 1204.

Ixiolirion Herb. 1209. Ixodia RBr. 2423. Jxora L. 2011.

Jaborosa Juss. 3311. Jacaranda Juss. 3261. Jacea Camer. 4895. Jacea Neck. 2687. Jackia Blume. 3034. Jackia Wall. 2028. Jackia Spr. 5290. Jacksonia Rafin. 48 ij2. Jacksonia RBr. 4071. Jacobaea T. 2497. Jacobia DC. 4591a. Jacquinia L. 3606. Jacquinia Mut. 5461. Jaegeria Kuth. 2710. Jambolifera L. $511 \%$. Jambos Adl. 4682. Jambosa Rmph. 4682. Janipha K. H. B. 5058. Jarava Rz. Pav. 920l). Jasione L. 2792.
Jasminum L. $3544 . \quad$ Kallias H. Cass. 2659. Jasonia H. Cass. 2463. Kallströ̀nia Scop. 5192. Jatropha L. 5061. Jeffersonia Bart. 4843. Kalosanthes Haw.418?. Jenkinsonia Sweet. $5278 \mathrm{e}$.

Joachimia Ten. 870. Joannesia P. 2:302. Joannesia Vell. 5064.

Jodes Blume. 1892.

Johannia W. 2302. Johnia Rxb. 5386. Johusonia RRr. 1153. Joliffia Boj. 2770.

Joncquetia Schreb. 5177. Joudraba Med. 4745b. Jonesia Roxb. 4105. Jonidium Vent. 4888. Joninm R. 4885d. Jonopsidinn DC. 4763a. Jonopsis DC. 4763. Jonopsis Knth. 1512. Jonthlaspi T. 4758a. Josephia Sal. 1768

Josephinia Vent. 3253. Jossinia Commers.4686. Jovellana Rz. P. 3143. Jovibarba DC. 4190b. Juanulloa Rz. Pav. 3303. Jubaea K. H. B. 1633.

Juglaus L. 3831.

Juncaria Clus. 4259.

Juncis Desv. 128\%c.

Juncus L. 1287.

Jungermannia Mich. 532.

Jungermannia Radd. $532 \mathrm{e}$.

Jumghansia Gı. syst. 3622.

Jiirgensia Spr. 5310b. Jungia Gärt. 4670. ex atli.

Jungia L. f. 2276. Juniperus L. 1719.

Jurinea H. Cass. 2315. Jussiaea L. 4493.

Jussiena P. 4493.

Jussieua Honst. 5059.

Justicia L. 3112. 3112b. Kabera R. $4818 \mathrm{~h}$.

Kadlsura Juss. 4998.

Käınfferiat L. 1600.

Kageneckia Rz. Pav. 4455.

Kalanchoë Ad. 4183.

Kalenchoë Haw. 4183. Kalimeris H. Cass.2562.
Kalmia L. 3403.
Kalosanthes Haw.415?.

Kämpfera Houst. 2898 . Kampmannia Rafin. 5132.

Kanahia RBr. 3436.

Kanlfussia N. v. E.2558.

Keiria Bowdl. adll.5́561.

Kennedya Vent. 3932.

Kentrophyllum Neck. 2690.

Keraselına Neck. 5012b

Kerandrenia Gay. 53c0.

Kernera Med. 4788.

Kernera W. $75 \%$.

Kerneria Much. 2613.

Kerria DC. 4450 .

Ketmia 5273b.

Kibera Ad. 4808b.

Kibessia DeC. 4645.

Kielıneyera Mart. 5401.

Kiggrelaria L. 4883.

Kingia RBr. 1315.

Kinkina A 1 . 2065.

Kirganelia Juss. 5077.

Kitaibelia Willd. 524t.

Klaprothia K. H. B. 4236.

Klasea H. Cass. 2316.

Kleinhovia L. 5284.

Kleinia Juss. 2362.

Kleinia L. 2509.

Klukia Bess. 4808a.

Kuappia Sw. 882.

Knautia L. 1931.

Knema Lour. 1863.

Knightia PBr. 1\%6?.

Knoxia L. 1993.

Knowltonia Salisb. $493 \%$.

Kobresia W. 1066.

Kochia Rth. 4338.

Koeleria Pers. 1020.

Koellea Bir. 4963.

Koelpinia Pall. 2123.

Knelreuteria Laxm. 5223.

Koenigia I. 4281.

Kolbea P. B. 2769.

Kolbea Schlecht. 1304.

Kolowratia Prsl. 1598. Koniga RBr. 4773.

Krameria Löff. 3042 .

Kraschenimikovia

Giildenst.4335.ex add. 
Kramhia Rafin. 3936. Lagoseris MB. 2156. Krigia Schreb. 2127. Krokeria Much. 3874a. Krubera Hoffin. 3684. Krïgeria Neck. 4112. Kuhlia K. H. B. 4922. Kuhnia L. f. 2238. Kuhnistera Lam. 3887b. Kunthia Humb. $16+1$. Kumzea Rchb. 4661. Kunzia Spr. 4454. Kyberia Neck. 2569. Kydia Roxb. 4297. Kylliuga Rottb. 1082. K yllingia L. f, 1082. Labatia Sw. 3590.

Labichea Gaudich. 4111. Labillardiera R. S. $30 \pm y$. Lablals Allaus. 3942.

Labordia Gaudich.5133. Labradia Swed. 3949. Labrella Fr. 238.

Labrella Desinaz. 242. Laburuum DC. 4029b. Lacara Spr. 4095.

Lacathea Salish. 5408a. Lacepedea K.H.B.5388. Lachenalia Jcฯ. 1369.

Lachnaea L. 1776.

Lachnagrostis'Trin.903. Lachuanthes EII. 1227. Lachuobolus Fr. 176. Lachnospermun W. 2455.

Lachnostoma K. H. B. 3433.

Lacis Schreh. 1671.

Lacistema Sw. 1826.

Lactuca L. 2167.

Ladanousis DC. 4595c.

Laenuecia H. Cass. 2544.

Laelia Pers. 4719.

Laetia L. 4927.

Lafoensea Vand. 4578. Lafientea LaG. 3089. Lagascea Cav. 2343. Layenaria Ser. 2761. Lagenifera H. Cass. 2568.

Lagenophora H. Cass. 2568.

Lageunla Lour. 1783b. Lagerstroemia L. 4577. Lagetta Juss. 4j\$fc. Lagoecia L. 374!. Lagonychim MB. 417. Lagopus Ser. 3869a. Lag'oseris Lk. 2143.

Lagoseris R. 2143a.

Lağotis Gärt. $308 \%$.

Lagumaea Cav. $526 \%$.

Lagunaria DC. 5269.

Lagurus L. 898.

Lahaya R. S. 4263.

Lamarkea Gaudich. 4658.

Lambertia Sin. 1758.

Laminm L. 2874.

Lamium Rth. '2874h. 3083.

Lampsana T. 2121.

Lanaria Ait. 1225.

Lancisia Gärt. 2374.

Lancisia P. 2384.

Lancretia Del. 5469.

Laudia Comm. 2063.

Lang'sdorfia Leand. 5132.

Lanosa Fr. 54.

Lantana L. 2940.

Lapageria Rz. Pav. 1340.

Lapathum T. 4286b.

Lapeyronsia Pourr. 1195.

Lapsana L. 212t. 1865.

Larix T. 1726.

Larochea P. 4181.

Larrea Cav. 5198.

Lasia Brid. 548.

Lasia P. B. 561.
Lag'unculariaGärt. 4337.

Lamarkea Poir. 3289.

Lamarkia Mnch. 997.

Lamiuaria Laux. 509.

Lampocarya R Br. 1099.

Lamouronxia K. H. B.

Lainyra H. Cass. $232 \%$.

Lanceolaria DC. $4821 \mathrm{~h}$.

Lancisia Ponted. 2375.

Laudolphia P. B. 3564.

Langsilorffia Rich. 1680.

Lanipila Burch. 2392.

Lansinm Rmph. 5525.

Laplacea K. H. B. 5410. Lappa T. 2319.4.yo s.y

Larbrea A. St. Hil. 4272.

Lardizabala Rz. Pav.

Lascadium Rafin. 5106. Laserpitiun L. 3690.

Lasiandra DeC. 4593.

Lasiobotrys Kz. 147.

Lasiopera Lk. Hffgr. 3086.

Lasiopetalum Sm. 5303.

Lasiopogon H. Cass. 2419

Lasioptera Andrz. 4751.

Lasiopus H. Cass. 2258.

Lasiorrhiza LaG. 2278.

Lasiosperunum Fisch. 2176.

Lasiospertnum LaG. 2390.

Lasiospermum Trev. 2392.

Lasiospora H. Cass. 2176.

Lasiosiemon $N$, et $M$. 5145.

Lasiostoma Schreb. 3568.

Lastrea B. St. V. 723c.

Latania Com. 1661.

Laternea Turp. '224a.

Laterradea Annal. eur. add. 5562.

Lathraea L. 3054.

Lathyrus Auct. 3923b.

Lathyrus L. 3923.

Laugeria Jcq. am. 2106.

Laugeria Sw. 2025.

Launaya H. Cass. 2173b.

Laurelia Juss. 1825.

Laurembergia Berg. 4481.

Laurocerasus T. 4705b.

Lauropyllus Thunb. 1714b.

Laurus L. 1907.

Lauzan Buch. 3863.

Lavaudula L. 2856 .

Lavandula T. 2856a.

Lavatera L. 5258.

Lavenia Solaud. 2235.

Lavoisiera DeC. 4606.

Lavradia Velloz. 4905.

Lawsonia L. 4569.

Laxmamia Fisch. $445 \mathrm{a}$.

Laxmannia RBr. 1151.

Leaeba Forsk. 1873.

Leaudra Radd. 4620.

Leandraria DC. 4620a.

Leand roides DC. 4620 b.

Loangium Lk. 192.

Lasianthera P. B. 5502. Leathesia Gray. 422.

Lasianthus Jack. 2016. Lebeckia Thnb. 4036.

Lasianthus DC. 5408b. Lebetina H. Cass. 2363. 
Lebretonia Schrk. 5264. Leonticoiles DC. 4833c. Leptolaena A. P. Th. Lecanauthus Jack. 2055. Leontorlon Juss. 2135.

Lecanocarias N. V. E. Leontodon Schreb.2162. Leptomeria RBr. 1709. -4366 .

Leontopetalum T. 4840 .

Lecanopteris Reinw. Lenutophthalmum IV. 638. (688i). 2590.

Lecanotis lischw. 385 .

$\begin{array}{ll}\text { Lechea L. 4913. } & \text { Leonurus L. 2881. } \\ \text { Lechenanitia RBr.2780. } & \text { Leopoldinia Mart. 163\%. }\end{array}$

Leontopodium P. 2450.

Lecheoides IDC. 4915h.

Lecidea Ach. 411.

Lecostomon Hoc. Sess. 4173 .

Lecy this Lom. 4649 .

Lecy thopsisschrk. 4653 .

Leda B. St. Y. 464 .

Ledlebonria Lk. 3738c.

Ledchouria Rth. 1323.

Lellocarpon Desf. 5322.

Lelonia DC. \$!)16a.

Ledlum L. 3401.

Leea L. $5,500$.

Leersia Siv. 953.

Leeuwenhoolia $\mathbf{R B r}$. 1918.

Legnotis Sw. $19 \%$.

Legonsia Durand. 2801.

Lehmamia spr. 3281.

Leibnitzia II. Cass.2264.

Leighia H. Cass. 2631 .

Leiocarpus DC. 4538b.

Leiogyne Don. 4203c.

Leiolobium DC. 40031 .

Leiolobium Rchb. 4780b.

Leiophyllum Pers.340?.

Leinpoteriun DC.442?a.

Leiosphaera DC. 4634 a.

Leiotheca Brid. 623.

Lejeunia A. Lib. 531.

Lemalis Fr. 290.

Lemanea B. St. V. 461.

Lemia Vand. 4312.

Leinna. L. 764.

Lemniscia Schreb. 3616.

Lemonia Pour. 1190c.

Lenidia A. P. Th. 4986.

Lens 'T'. 3918a.

Lentago Rafin. 1952.

Lentilunaria Gesu. 3317 .

Lenticula T. 764.

Lentims Fr. 344.

Dentiscus T." 3859 b.

teonia Rz. Par. 3!j09.

Eeonotis $\mathrm{RBr} .2889$.

Leontia Neck. 5045.

Leontice L. 4840.

Leotia Hill. 3
Leotia P. 318.

Lepechinia WV. 2826.

Lepeocercis Trin. 967.

Leperiza IIerb. 1262a.

Lepia Desv. $4751 d$.

Lepidayathis IV. 3127.

Lepiclaploa H. Cass. 2216.

Lepiciium L. $\$ 751$.

Lepidocary um Mart. $16 ! 9$.

Lepiilophorum Neck. 2402.

Lepiclophyllum Ir. Cass. 2532.

Lepiclopilınn Brid. 552.

Lepicloseris R. 21 $13 \mathrm{c}$.

Lepidosperma LaB.

1128.

Lepidotis P. B. 16\%6e.

Lepiedra LaG. 1253.

Lepigonum Fr. 4275.

Lepiota 349 H.h.

Lepirgnia Rich. $10 \% 2$.

depiscline H. Cass.2425.

Lepra Hall. Mey. 350.

Lepraria Ach. 61. 350.

Lepia Lour. 3629.

Leptadenia RBr. 3415.

Leptaleum DeC. 4803.

Leptammium Rafin. 3053. ex adid.

Leptandra Nutt. 3064. Lepranthus Michx. 1164.

Leptaspis $13 B \mathrm{r}$. (691.

LepteranthusDeC.2691.

Leptidium Gins. 4885a.

Leptinella H. Cass. 2373.

Leptocarpaea DeC.4811. Leptocarpus RBr. 1138.

Leptocarpus VV. 2898.

Leptochilus Kaulf. 708.

Leptochloa P. B. 865 .

Leptocramise DC.4724b.

Leptodermis Wall.1330.

Leptodon Web. 548.

Leptogium Fr. 398.
Leptomitus As. 443.

Leptomon Rafin. 5046 .

Leptonena Ad. Juss. 5073.

Leptonia $3 \pm 9$ G. b.

Leptonina Rafin. 4304.

Leptophytum H. Cass. 2409.

Leptoporla Nutt. 2591.

Leptopy rumiRchb.4954.

Leptorinus DC. 48:21b.

Leptosolena Prsi. 159?.

Leptospermum Forst. $46 \%$.

Leptostachys Mey. $\$ 65$.

Leptostegia Don. 695.

Leptostomum RBr. 6 tit.

Leptostroma Fr. 235.

LeptostromaRafu.p.15.

Leptotheca Schwo. 640 .

Lepturus $\mathbf{R B r}$. 8\%ं.

Lepuropetalon EII.3374.

Lepyroulia RBr. 1134 .

Lerchea L. adl. $5 j 63$.

Lerchia H. Cass. 2614.

Leria DeC. 2262.

Lerouxia Merat. 3327a.

Leskea Hedw. 575.

Leskia Brid. 575́a.

Lespedeza Richx. 4006.

Lessertia DC. 3916.

Lestibodea Neck. 2730.

cestibondesia A. P. 'Th. 4361.

Lettsomia Rxb. 3014.

Lettsomia Rz. Pav. $\$ 17$.

Lencadendroin Boerh.

$1 \% 45$.

Lencaeria DeC. 2279.

Lencanthemum Adns. 2381.

Lencas RBr. 2888.

Leucheria LaG. 2279.

Lencodon Schwogr. 560.

Lencodraba DC. 47611.

Lencogramma Mey.388.

Leucoilum L. 12 l.

Lencoloma Brid. 633.

Lencomeris Bon. 2242.

Leucomyrtus. DC. 4 isja.

Leuconotis Jack. 3563.

Lencophanes Brid. 614.

Lencophyllum K. H.B. 3215 .

Leucophyta RBr. 24tS. 
Leucopogon RBr. 3336.|Limodorum Tourn. Leucosceptrum Sm. 2855.

Lencosia A. P. Th. 3824. Limosella L. 3137.

Leucosinapis DC. 4815. Liuaria T'. 3174.

Leucospermum RBr. 1743.

Lencosporus $349 \mathrm{H}$.

Leuzea DeG. 2312.

Leveuhookia RBr.1918.

Levisticun Koch. 3696.

Lewisia Prsh. 801.

Leycestera Wall. 2110.

Leysera L. 2408.

Liabum Adaus. 221\%.

Liagora Ag. 431.

Liagora Lamx. 49 ?.

Liaguroa Rz. Pav. 5224.

Liatris Schrel. 2240.

Libanotis Hoffm. 3715c.

Libertia Lej. 1004.

Libertia Spr. 1179.

Libidibia DC. 4149a.

I icea Schrad. 189.

Lichina Ag. 90.

Lichtensteinia Cham. 3762.

Lichtensteinia W. 1300.

Licuala Rmpf. 16.3.

Lidbeckia Berg. 2384.

Lieberkiihna H. Cass. 2261.

Lightfootia Sw. 49 ?6.

Lightfootia l'Her. 2797.

LightfootiaSchreb.2050.

Lignidiun Lk. $17 \%$.

Ligusticum Spr. 3708a.

Ligusticun T. 3708.

Ligustrum L. 3546 .

Lilac T'. 3547.

Lilaea Hb. Bpl. 1130a. ex add.

Lilium L. 1355.

Limacia Lour. $187 \%$.

Limacium 349 H. $\cong$.

Limbarla Adns. 2459.

Limboria Ach. 367\%.

Limnas Trill. 953b. ex add.

Limnetis Rich. 85Sa.

Limnia L. 4307.

Limniris Tsch. 1187c.

Limnobinn Rich. 809.

Limuocharis IIb. Bpl. 799.

Limnochloa P. B. 1120.

Limnopeuce Vaill. 4480.

Limnophila RBr. 3157.
Linconia L. 4226.

Lindera Thub. add. 5564 .

Lindernia L. 3138.

Lindleya K. H. B. 4458. Lomatinn Rafin. 3760.

Lindleya Knth. 4695.

Lindnera R. 5449b.

Liudsaya Dry. 6992.

Linkia Cav. 1752.

Liukia Lgb. 423.

Linkia Pers. 3312.

Linnaea Gron. $197 \%$.

Linociera Sm. 3583.

Linosyris H. Cass. 2536.

Linum L. 5464.

Lioy dia Neck.2484.2555.

Liparia L. 4046.

Liparis Rich. 1564.

Lipotriche RBr. 2632.

Lippia L. 2939.

Liquidambar L. 2745.

Licquiritia Mnch. 3889b.

Liriodendron L. 4991.

Liriope Herb. $126 \%$.

Liriope Lour. 1403.

Liriopsis Rchb. $126 \%$.

Lisianthus L. 3488 .

Lissanthe 13ß3r. 3337.

Lissochilus RBr. 1514.

Listera RBr. 1428.

Lita Schreb. 3495.

Lithachue P. B. 9/9a. Lophiris Tsch. 1187b.

Lithagrostis Gärt. 940. Lophium Fr. 25\%.

Lithocarpidia R. 1078h. Lophospermum Don.

Lithos permum L. 2946.

Litophila Sw. 4251.

Litsea Lam. 1909.

Littaea 'Tagliab. 1284a.

Liltorella L. 3353.

Livistona RBr. $165 \%$.

Loasa Ad. 4232.

Lobadium Rafin. 3850e.

Lobaria Hoffin. 402h.

Lobelia L. 2787.

Lobularia Desv. 4766. Locaudi Acl. 5it8.

Lockhartia Hook. 1531.

Lochnera Rehb. 3533h.

Lochueria Scop. 5430.

Loddiggesia Sims. 4038.

Lodicularia P. B. 860.

Lodoicea LaB. 1662.

Loefflingia L. 4252.
Loeselia L. 3028.

Logania RBr. 3502.

Logfia H. Cass. 245.3.

Loiseleria Desv. 3392.

Lolium L. 828.

Lomaria W. 710.

Loinandra LaB. 1312c.

Lomaspora DC. 4790.

Lomatia RBr. 1764.

Lomatolepis H. Cass. 2163.

Lomatophyllun W. 1411.

Lomatospora DC. em. 4790 c.

Lonas Adns. $238 \%$.

Lonchitis L. 696.

Lonchocarpus K. H. B. 3897.

Louchostoma Wickst. 4225b.

Longchampsia Willd. 2410.

Lonicera L. 1984. 1985.

Lonicera Plum. 1962.

Lontarus Rimpf. 1663.

Lopezia Cav. 4505.

Lophanthus Forst. 5306.

Lophia Hamilt. 3245.

Lophia Banks. 1917b. ex add.

Lophidimm Rich. 736.

Lophiola Ker. 1226.

Lophiolepis H. Cass. 2331.

$317 \%$.

Lopimia Mart. 5263.

Loranthus L. 1962.

Loreya DeC. 4638.

Loroglossunn Rich.1466.

Loropetalum $\mathrm{RBr} .1900$.

Lotea Med. 3874 b.

Lotoides DC. 4029e.

Lotononis DC. 4027a.

Lotus L. 3874 .

Louichea l'Herit. 4332. ex adl.

Lourea J. St. Hil. 3996 .

Lourea Neck. '3997.

Loureira Cav. 5054.

Loxidium Vent. 3915.

Loxocarya RBr. $113 \%$.

Loxodon H. Cass. 2260.

Loxonia Jack. 3225. 
Loxostylis Spr. 5222b. Lozania Seb. 4514. Lubinia Vent. 3328. Lucilia H. Cass. 2412. Lucuna Jisss. 3642.

Lurlia Laun. 4921.

Ludolfia Willd. 1051.

Ludovia Poit. 1614.

Lud wigia L. 4491.

Ludwigiaria DC. 4491.

Luihea Willd. 5456.

Luffa Cav. 2759.

Luida Adans. 613. 591.

Lumnitzera JacG. 2809.

Lunnitzera Willd.4529.

Linaria L. 4774.

Libularia Mich. 529.

Luperia DC. 4798a.

Lupinaster Mnch.3869f.

Lupinus L. 3954.

Lupsia Neck. 2336.

Lupularia Ser. 3877b.

Lussacia Spr. 1976.

Luteola T. 4824a.

Luxemburgia A. St. Hil. 4906 .

Luziola Juss. 963.

Luzula Desv. 1286.

Luzuriaga Rz.Pav.1328.

Lychnanthus Gm.5364.

Lychnis L. 5362.

Lychnophora Mart. 2218.

Lусіии L. 3313.

Lycoctoutim Diosc. $4950 \mathrm{c}$.

Lycogala Mich. 195.

Lycoperdon Mich. 200.

Lycopersicon T. 3296.

Lycopodium L. 1676.

Lycopsis L. 2948.

Ly copus L. 2844.

Lycoris Herb. 1244.

Ly coseris H. Cass. 2272.

Lycurıs K. H. B. 928.

Lyellia RBr. $66 \%$.

Lygeuin L. 1058 ,

Lyginia RRr. 1142.

Lygistuin J. Br. 2089.

Ly godisodea Rz, Pav. 2039.

Lygodium Sw, $73 \%$.

Lynghya Ag, 4.44.

Lyonia Nuit, 3384.

Lyonia Rafin. 4288, ex add.

Lyouia Ell. 3438.

Lyonsia RBR. 3521.
Lysanthe Sal. 1756.
Lysimachia L. 3327.

Lysimachion Tsch. $4500 \mathrm{~b}$.

Lysiuema $\mathrm{RBr}$. 3351 .

Lysionotus Don. 3226.

Ly sipoma K. H. B. 2791.

Lysurus Fr, 226.

Lythrum L. 4558.

Maasa R. S. 3366.

Maba Forst. 3589.

Mabea Aubl. 5066.

Maburnia A.P.Th.1212.

Macahanea Aubl. 5495 .

Macairea DeC. 4612.

Macananga A. P. Th. $50.3 \%$.

Macanea Juss. 5495.

Macarisia A.P.Th.5521.

Machaerina Rich. 1112.

Machaerium P. 3895́c.

Machaonia Hb. Bpl. 2041.

Macleya $\mathbf{R B r}$. 4847.

Maclura Nutt. 1814b. ex add.

Mocoubea Aubl. 5496.

Macradenia RBr. 1520.

Macranthus Lour. $392 \%$.

Macrauchenium Brid. 647.

Macroceratides Raddi. 3949.

Macroceratium DeC. 4782.

Macrocuernum J. Br. 2064.

Macrocystis Ag. 510.

Macrolobium Schreb. 4112.

Macromerum Burch. 4871.

Macromitrium Brid. 624.

Macropodinm L. 4786. Macroscepis K. H. B. 3434.

Macrosporiun Fr. 122.

Macrosiylium R. $4362 \mathrm{~b}$.

Macrothecinm Brid. 648.

Macrotrichum Grev. 116.

Macrotropis DC. 4086 .

Macrotys Rafin. 4058.

Macularia DC. 4915l.

Madia Molin. 2711.
Maerua Forsk. 4878.

Maesa Forsk. 3366.

Mazallana Cav. 5?76.

Maghania J. St. H. 3996.

Magnolia L. 4992.

Magnoliastrun DC. $4992 b$.

Magonia A. St.Hil.j226.

Mahernia L. 5305.

Mahonia Nutt. 4837.

Mahurea Aubl. 540\%.

Maianthemum Wigg. 1324.

Maieta Aubl. 4625.

Mairania Neck. 3386.

Maireria Scop. 3016.

Malabaila Hoifin.3681b.

Malache Trew. 5261b.

Malachodendron Cav. 5412.

Malachra L. 5259 .

Malanea Aubl. 2022.

Malaxis L. 1562.

Malbrancia Neck. 3826.

Malcolmia RBr. 4807.

MialcomiaH.K ew.4807.

Malesherbia Rz. Pav. 3473.

Mallotus Lour. 5108.

Malochia Savi. 3947.

Malope L. 5243.

Malpighia L. 5381.

Maluchia DC. 5254b.

Malus T. 4470f.

Malva L. 5254.

Malvastrum DC. 5254a.

Malvaviscus Dill. 5265.

Mamillaria Haw. 4241.

Mamınea L. 5494.

Manabea Aubl. 2925.

Mancanilla Plum. 5024.

Mandragora 'T. $330 \%$.

Manduy ta Comm. 5́178.

Manellia Mnt. 20.49.

Maugifera L. 3864.

Manglietia Blume. 4995.

Mauglilla Juss. 3641 .

Mangostana G. 5492.

Manicaria Gärt. 1621.

Mąnihot Ad.5058.5?73a.

Mailna Doll. 4008.

Mancellia Bowd. add. 5565 .

Mautisia Curt. 1588.

Maunlea L. 3105.

Mapania Aubl. 1086.

Maponria Aubl. 2031c. Mappa Ad. Juss. 5036. 
Maprounea Aubl. 5018. Massonia L. 1359.

Maracanga A. P. Th. 34151.

Maralia A. P. Th. 3780.

Maranta L. 1606.

Marauthus Blume.5299.

Marathrum Humb. $\mathbf{B p l}$. $16 \% 2$.

Marattia Str. 744.

Marcanthus Willd. 3927.

Marcetia DeC. 4590.

Marcgravia Plum. 5481.

Marchantia Mich. 528.

Marcorella Neck. 3822a.

Marcutera Noronli. 500ija.

Mirgaritaria L. fil.5099. Maruinaria 722.

MarifyricarpusRz.Pav. 44210.

Mirialva Vand. 5488.

Marica Sclireb. 1176.

Mariguia Commers. $38+.5$.

Marila Sw. 5402.

Maripa Aubl. 3570.

Mariscus Hall. 1089.

Markia Rich. 3289.

Marlea Roxb. 4527 b. ex add.

Marrubiastrum 2885b. Marrubiun L. 2885.

Marsana Somu. 5327.

Marsdenia RBr. 3418.

Marsea Adus. 2042.

MarshalliaSchreb.2592.

Marsilea L. 673.

Marsipjosperium Desv. 1287\%.

Marsupianthes Mart. 2878.

Marlagon Cam. 1355a.

Martia Leand. 3972.

Martia Spr. 5472.

Martinezia Kz. Pav. 1627.

Martiusia Schult. 3972.

Martrasia LaG. $227 \%$.

Martyuia L. 3238.

Maruta H. Cass. 2395.

Mascaguia Bert. 5367.

Maschalanthus Holg.

fil. 572.

Maschalocarpus Spr. 572.

Masilevallia Rz. Paŕ. 1539. 2290. 2318.

ILatixa Spr. 2392.

Matelea Aubl. 3424 .

Matrella P. 881 .

Matthiola RBr. 4798.

Matthissonia Radl. 2120.

Mattia Schult. 2967.

Mattuschkea Schreb. 2938.

Mauhlia Dahl. 1372.

Mauritia L. 1620.

Maxillaria Rz. Par. 1489.

Maximilianea Mart. 1632. 5421 .

Mayaca Aubl. 1158.

Mayepea Aubl. 3588.

Mayua Aubl. 4928. 5173.

Mayus Lour. 3183.

Meborea Aubl. 5109.

Merleola L. 1326.

Medicago L. 3877.

Medinella Gandich.

4627. ex add. add.

Meesia Holy. 663.

Meesia Gärt. 5185.

Megraclinium Lindl. $153 \%$.

Negastachya P. B. $1031 \mathrm{~b}$.
Iastigophorus H. Cáss.

Mastrucium H. Cass̀.

Hitayba Aubl. 5219.

Matisia Hb. Bpl. 5329.

Matourea Aubl. 3150.

Matricaria Vaill. 2383.

Matthiola Plum. 2107.

Maurandia Jacy. 3176.

Mauria K. H. B. 3851.

Maytenus Fenill. 5391

Mazeutoneuron LaB.

Meconopsis DeC. 485 4 .

Hedicusia Much. 2137.

Medusa Lour. 5310b. ex add.

Medusea Matv. 5012. Melichrus RBŕ. 3339.

Medusula lischw. 391. Melicocca Juss. 5208.

Medusula Pers. 5310b. Melicocca L. 5208a.

Medusula Torle. 100. ex

Meerburaia Mnch.4258.

Megacarpaea DeC.4746.

Melicope Forst. 5154.

Melicytus Forst. $491 \%$.

Melidium Eschw. 94.

Jelilotus T. 3870.

Melinis P. B. 935.

Ueliola Fr. 269.

Melissa L. 282?.

Melistanium forst. 4695.

Melittis L. 2829.

Melo 'T'. 2760.

Meionectes RBr, 4483. MelocactusC. Banh. 1? 1? 
Melocauna Trin. 1056. Meteorus Lour. 4655. Melochia L. $5310 . \quad$ Methonica Herin. 1351. Melodinus Forst. 3573. Melodorum Lour.5006c. Melopepo T. 2762.

Meloseira $A_{3} .452$.

Melothria L. 2757.

Melvilla Auders. 4559. Memecylon L. 1964.

Menais L. 3005.

Menirala Comm. 5080.

Mendoncia Vellz. Vand. 2936.

Mendoni Rheed. 1351.

Mendozia Pz. Pav.2936.

Meniocus Desv. $\$ 764$ :

Menisciun Śchreb. 729

Meniscotia Blume. 1891 .

Menispermum 'T. 1886.

Menispora Lk. 122.

Menoceras $\mathrm{RBr}, 27 \% 8 \mathrm{a}$.

Menodora Hb. Bpl. $3544 \mathrm{~b}$.

Menonvillea DeC. $4 \% 48$.

Nentha L. $28 \% 1$.

Mentzelia Plum. 4235.

Menyanthes L. 3174.

Menziesia Sin. 3381.

Meoschinm P. B. 966.

Meratia H. Cass. 2714.

Hercurialis L. 5033 .

Merendera MB. 1309.

Meriania Sw. 4603.

Merichea Lour. 4655.

Merida Neck. 4.312.

1.ierilliana L. 4312.

Merislium Ag. 447.

Merisma 338e. 327c.

Merki, Borkh. 530.

Merkia Fisnh. 53.54.

Mertensia W. $7 \$ 2$ cf. app.

Merulius P. 342.

Merulius Hall. 336.

Mesembryanthemum L. 4408 .

Mesogloia A $\mathrm{s}$. 434.

Mesopus324 4.327 e.338e.

Mespilophora Neck. 4408.

Mespilus L. 4468.

Messers chmidia L. 2983.

Mestotes Soland.2823a.

Mesua L. 5489.

Metalasia RBr. 24.34.

Metaplexis $\mathbf{R B r}$. $3 \frac{142}{2}$.

Mletastelma RBr. 3449. Microstachys Ad. Juss.

IVIeteorina H. Cass.2730.
Metopiun 3850h.

Metorium Brid. $551 \mathrm{~b}$.

Metrocynia A. P. Th. 4134.

Metrodorea A. St. Hil. 5151.

MetrosiderosG ïrt.4673.

Metrosideros Hortul. 4660.

MetroxylonRottb.161\%.

Metternichia Mik. 3316.

Metzgeria Radd. 530c.

Meuin T. 3705.

Meyera Schreb. 2705.

Mezonemrun Desf.4147.

Mibora All. 88 ?.

II icarea Fr. 395.

Michanxia l'Her. 2803.

Michauxia Neck. 2406.

Micheluria Dumort. 1004.

Michelia L. 4996.

Iiconia Rz. Pav. 4639.

BicrampelisRafin.?750.

Wicranthea Desf. 5081.

Micranthemum Mchx. 3322.

MicrantheraBess.1022n.

MicrantheraC'hois.jł 43.

Micranthes IIaw.4203f.

Micranthus P. 1192a.

Micranthus IVendl. 3126.

Microcale Hríg. Lk. 3477.

Microcarpaea RBr.3135.

Microchilus Prsl. 1435.

Microchloa RBr. 85t.

Microcorys RBr. 3841 .

Microcos L. 5446.

Microdon Chois. 3095.

Nicrolaena RBr. 956.

Microlepis DC. 4596a.

Microcoleus Demaz.455.

Microlicia Don. 4583.

Microloına $\mathrm{RBr}$. 3450.

Micromega Ag. $453 \mathrm{~b}$. ex adil.

Micropetalum P. 5347.

Microplenra LaG. 3771.

Micropodiun DC.4817a.

Micropns L. 245t.

Iicrospermum $\mathbf{L a G}$. 2364. 5029.
MicrostemmaRBr.3414.

Microstemma LaB. 3398 .

Microstylis Nutt. 1563.

Microtea Sw. 4391.

Microtis RBr. 1452.

Microthonarea A. P.

Th. 895.

Millotis Fr. 322.

Miegia Pers. 1051.

Miegra Neck. 2150.

Miegia Schreb. $108 \%$.

Mieria Lk. 2351.

Miersia Lindl. 1357.

Mikania W. 2228.

Miliariun Mnch. 914.

Milium L. 914.

Miliun Much 936a.

Milla Cav. 1380.

Milleria L. 2715.

Millingtouia Rxb. 3617 b. 3996.

Miltus Lour. 4404.

Mimetes Sal. 1742.

Mimosa All. 4162.

Mimulus L. 3182.

Mimusops L. 3639.

Minuarta Lö月l. 4253.

Mirabilis L. 1843.

Mirbelia Sin. 4059.

Mitchella L. 2004.

Mitella L. $\$ 201$.

Mithracarpus Zacc. 1999.

Mithridatea Commers. 1822.

Mitra Houst. 3550.

Mitraria Cav. 3246.

Mitraria Gm. 4654.

Mitrenyces N. v. E. 205.

Mitreola L. Rich. 3550.

Mitrula Fr. 303.

Mnasium Schreb. 1145.

Mnesiteon Rafiu. 2615.

Muiarum Forsk. 4255.

Muiopsis Mart. 1670.

Mnium Dill. 6j1.

Mccanera Jisss. 3656.

Mocinna La G. 2594.

Mocronax Rafin. 1051.

Modecca Rheed. $346 \%$.

Modiola MInch. 5250.

Moehnia Neck. 267\%.

Möhringia L. 53f5.

Mönchia Ehrh. 5342.

Mönchia Rih. 4780a.

Mösslera Rchb. 4228 .

Mogiphanes Mart. 4381.

Mogorium Juss. 3545 . 
Mohria Sw. 738.

Moldenhamera Schrad. 4136.

MIoldenhanera Spr.add.

Molinaea Juss. 5212b. Moquilea Aubl. 4524.

Molinaea Comm. 3810. Moquinia Spr. 1783.

Aolinia Schrk. 1029.

Molle T. 3853.

Mollia Mart. 5431b. ex app.

Mollia Schrk. 63j.

Mollia Gm. $46 \% 0$.

Mollia W. 4263.

MollinediaRz.Pav.1835.

Mollıgo L. $427 \%$.

Molopospermum Koch. 3712.

Molpadia H. Cass. 2472.

Molikia Lehm. 2954.

Boluccella L. 2890.

Mombin Plum. 3854.

Momordica L. 2754.

Monacentra DC. $4594 a$.

Mouachue P. B. 939b. ex add.

Monactiueirma B. St. V. $3472 b$.

Monactis K. H. B. 2716.

Monauthes Havv. 4190a.

Monarda L. 2839.

Mouarlheus H. Cass. 2207.

Monathera Rafin. 867.

Monenteles LaB. 2209.

Monerma P. B. 825.

Monetia l'Herit. 3566.

Moniera Aubl. 5141.

Monilia Pers. 68.

Monilifera Vaill. 2735.

Monimia A. P. Th. 1824.

Monuina Rz. Pav.3038.

Monocaryum RBr.1310.

Monocera Ell. $86 \%$.

Mounchaetun DC. $4595 \mathrm{e}$.

Monochonia Prsl. 1387. Miihlenbergia Schreb.

Monoclea Hook. 5?4.

Monodora Duir. 5010 .

MoungrammaSchk.697.

Monotoca RBr. 333.3.

Monotropa L. 3364 .

Monotropsis Schwuit\%. 3368.

Mnnsonia L. fil. 5281 . Moutbretia DeC. 1191. Montezuma Moc. Sess. 5332.
Montia Houst. 5443.

Montia L. 14305.

Montinia L. 4302.

ex adl.

Moraea L. 1185.

Morchella Dill. 321.

Iorella Lour. 1788.

Iorenia Rz. Pav. 1654.

Morettia DeC. 4731.

Morgania RBr. 3184.

Moricandia DeC. 4819.

Iorilandia Neck.4420 b.

Morina Dou. 2908.

Moriua L. 2893.

Moriula Vaill. 1942.

Moriuga Lam. 5124.

Morisonia Plum. 4875.

Moronobea Aubl. 5499.

Morus L. 1815.

Moscaria Rz. Pav. 2126.

Moscharia Forsk. $23 \pm 7$.

Moschatellina T. 4195.

Moschifera Mol. 2126.

Moschosma Rchb. 2809.

ex pag. 171.

Mosigia Spr. 2126.

Mongeantia Ag. 465.

Mongeotia Kuth. 5309.

Mouricon Ad. 3956.

Mouriri Aubl. $196 j$.

Mouriria Juss. 1966.

Mouroncoa Aubl. 3016.

Montabea Aubl. 3631.

Moutan Chinens. 496:s.

Ioutoubea Aubl. 3631 .

Mozima Orteg. 5054.

Incizonia Ort. 418\%.

Mincor Mich. 139.

Mucor Tod. 139d.

Mucuira Ad. 3949.

Mulgedium H. Cass. 2171.

904.

Miillera L. fil. 3896.

Niiuchhausia L. 4577b.

Mulium Pers. 3776.

MIImdia K. H. B. $303 \%$.

Mundulea DC. 3892a.

Munuickia Blume. 1859.

Munuozia Rz.Pav.2219.

Inutingia L. 5451.

Munychia H. Cass.2547. IIuralta Ad. 494\%a.
Muraltia Neck. 3036 .

Muricaria Dest. 4722 .

Muricia Lour. 2764.

Murraya Kön. 5527.

Murucuia T' 3470 .

Musa L. 1611.

Muscari Desf. 1361.

Muscipula Riv. 5362c.

Mussaenda Herm. 2061.

Mussinia W. $267 \%$.

Mustelia Spr. 2237.

IIItisia L. f. 2270.

Myagrum L. 4735.

Mycastrum Rafiu. p.15.

Mycena 349 H. b.

Mycelis H. Cass. 2168.

Mycinema Fr. 62.

Mycoderina P. p. 15.

Mycogone Lk. 133.

Mycomater Fries. 1.

Iy conia Lap. 3278.

My conia Neck. 2380.

Mycoporum Mey. 365.

Hygalurus I.k. 1000.

Myginda Jc(. am. 31523.

My lanche Wallr. 3053.

ex add.

Mylitta Fr. 162.

Mylocaryum W. 3405.

Myouima Com. 2105.

Myoporum Banks.3046.

Myoschilos Rz. Par.

1714.

Myoseris M. B. 2157.

Myosotis L. 2962.

My osurus Dill. 4932.

Myrcia DeC. 4684.

Myriadenus H. Cass.

24 fit.

Myriadenus Desv. 3989.

My riantheia A. P. Th. 4704.

Myrianthus P. B. 2768 .

Myriaspora DeC. 4623.

Myrica L. 1700.

Myricaria Desv. 4414.

Myriochaeta DC. $5453 \mathrm{c}$.

Myriococcum Fr. $21 \%$.

Myriodactylon Desv. 424.

My rmecodia Jack. adı. 5567.

Myrodendron Schreb. 5441.

Myrodia Schreb. 5326.

Myrrhidium DC.5?781.

Myriophyllum Vaill. 4477. 
Myriostoma Desv. 202b. Nasturtiolum DC. 4749a. |Nephrosis Rich.

My ristica L. 1862.

Myrobalauus Lain. 45431.

Myrodendroin Schreb. 3595.

Myrosma L. fil. 1603.

Myrospermum Jcq. 4091 .

Myrosperinum $\mathrm{K}$. H. B. 4091 .

My rothecium Tod. 165.

Myroxylon Mut. Liv. 40911 .

Myrrhinimu Schott. 4692 .

Myrrhis Scop. 3664.

Myrsidium Rä́n. 503.

Myrsine L. 3600.

Myrsiphyllinin W. 1329.

Myrstiphyllum J. Br. 2031 .

Myrtus L. 4685.

Myscolus H. Cass. 2182.

MyXouema Fr. 439.

Myxosporium Lk. 19. Negundimm Rafin. 5240.

Myxotheciun Kz. 268. Neg̈undo Möuch. 5240.

Myxotrichum Kz. 80. Neillia Don. 4453.

Nabalıs H. Cass. 2170. Nelitris Gärt. 4690.

Nacibea Aub1.2049,2095. Nelumbium Juss. 818.

Naemaspora Pers. 19. Nelumbo 'T. 818.

Naematelia Fr. $28 \%$.

Naevia Fr. 6. 353.

Nageia Gärt. 1700b.

Nahusia Schneev.449ta.

Naias L. 16668.

Nama L. 3018.

Nandina Thub. 4839.

Nanosilene Otth. 5363e.

Napaea L. 5248a.

Napellus Riv. 4950 b.

Napimoga Aubl. 4697.

Napoleona P. B. 3619 .

Naravelia DC. 4948.

Narcissus L. 1268.

Narcissus Salisb.1268b.

Nardosmia H.Cass. 2250.

Nardus L. 1057.

Nartheciun Mohr.1383.

Narvaliua H.Cass.2616.

Nasiny thia Huds. 1147a.

Nassauvia Commers. 2289.

Nassawia LaG. 2289.

Nassovia P. 2289.

Násturtioides Med. 4751.
$47 \$ 3$.

Nasturtium RBr, 4796. Neptunia Lour. 4167a.

Nasturlium Trag. 4796a. astus Juss. 1050.

Natrix DC. 4027 c.

Nauclea L. 1940.

Naucoria 3490 . d.

Nauplims H. Cass. 247\%. Neslia Desv. $47 \%$.

3017. Nestlera spr. 2467.

Neaea Rz. P. 1851.

Neckera Hilg. 5157.

Neckeria Scop. 4833 .

Neckeriin Gim. 4258.

Neclatudra Berg. 1774.

1778.

Nectria Fr. 279a.

Neellhamia RBr. 3330.

NeedhamiaH.Cass.2616.

Needhamia Scop. 3842.

Negretia Rz. Pav. 3949. Nhaudiroba Plum. 2771.

Nelsonia RBr. 3111.

Nematora Fee. 366a.

Nematospermum 1Rich. Nierembergia liz. P. 1826.

Nemauchenes H. Cass. 2140.

Nemesia Vent. 3172.

Nemopanthes Rafiu. 36 ?\%.

Nemophila Bart. 2978. Nigrina Thub. 1955.

Nemotropis DC. 4742d. Nigrina L. 3165.

Nemun Hamilt. add. Nigritella Rich. 1464. 5568.

Nenax G. 4420a.

Nima Hanilt. 5179.

Niota Lain. 5178.

Nenuphar Hayue. 815. Nipa 'Thub. 1618.

Neoceis H. Cass. 2504.

Neottia L. 1427.

Neowedia Schrad. add. 5569.

Nepeta L. 2876.

Nepenthes L. 788.

Nephelimm L. 5216.

Nephroulium Mch x . 681.

Nephroia Lour. 1878.

Nephroma Ach. $404 d$.
Niphobolus Kanlf. 71S.

Nirmri Ad. 5078.

Nisa A. P. Th. 4703.

Nissolia G. 3895a.

Nissolia Jcq. 3895.

Nitella Aщ.. 1664.

Nitraria Schol). 4412.

Nivenia Vent. 120\%.

Nivenia RBr. 17.38.

Nocca Cav. 2.543.

Noccaea Jc५. 2343. 
Noccaea Mnch. 4751. Nodularia Mert. 4ti1. Noiseltia K. H. B. 4891. Nolana L. 3276.

Nolanea 349G.a. Nolina Mchx. $129 ?$.

Nolletia H. Cass. 2538. Noltea Richb. 3800 .

Nomimiun Ging. 48855 b. Nomisma DC. $4742 \mathrm{e}$. Nomochloa P. B. 1107. Nonatelia Aubl. 2097. Nonea DeC. 2919. Norantea Aubl. 5482. Norna Wahlub. 1567. Norouhia A. P. Th.3587. Noria DC. 4810b. Nortenia A. P. Th. 3152. Nosophloea Fr. 2. Nostoc Adans. 420. Notelaea Vent. 3584. Nothites H. Cass. 2247. Nothochlaena RBr. 720. Notobasis H. Cass. 2329. Notoceras DeC. 4794. Notylia Lindl. 1536. Nugaria DeC. 41491. NumezhariaRz.P.1649. Nunnezia W. 1649. Nuphar Sm. 815. Nuxia Commers. 2923. Nyctagella R. 3283a. Nyctago Juss. 1843. Nyctalis Fr. 341 .

Nycterium Vent. 3293. Nymphaea L. 816.

Nymphoides T. 3475. Nyssa L. 1712.

Nyssanthes RBr. 4375. ObaejacaH.Cass.2496. ObeliscariaH Cass.2660 Obeliscotheca Adus. 2476.

Ohentonia Vell. 5145. Obesia Hav. 34l1d. Obione G. 434th. Obolaria L. 3051. Ocellularia Mey. 370. Ochna Schreb. 5187. Ochradenus Del. 4878b. Ochrocarpus A. P. Th. 5488.

Ochroma Siv. 5.328. Ochrosia Juss. 3553. Ochroxylum Schreb. 5132.

Ochris P. 3923a. Ochthodium DeC. 4711.] OliganthesH.Cass.2221. Opercularia Gärt. 1929.

Oligarrhena RBr. 3329. Oligocarpha H. Cass. Ockia Dietr. 5159,
Ocotea K. H. B. 1913. Oligoca. 2203.

Octarillum Lour. 1713. Oligosporus H. Cass. Octoblepharis Schreb. 2367. b13. Octoblepharum Hdg. Olisbea DeC. $196 \%$ 613.

Octodiceras Brid. 541. Olmedia Rz. Pav. 1813. Octomeria RBr. 1486.0 Olomitrium Brid. 593. Ocymastrum Riv.2363a. Olopetalum DC. 528la. Odonia Bertol. 396!. Olyunhia Lindl. 4681. Odontandra K. H. B. Olyra L. 919. 5503.

Odointaria DC. 5212c. Oinalanthus Ad. Juss. Odonthalia J.g.b. $490 \mathrm{~b}$. 5025 . Odontites Pers. Stev. Omalia Brid. 575b. 3081.

Odontites Spr. 3740a.

Odontoglossum Knth. 1522.

Odontoloma K. H. B. 2190.

odontopetalum DC. $5282 b$.

Odoutostemon DC. $4765 \mathrm{a}$.

Oetlemium Lk. 75.

Oedera L. 2441.

Oedipachne Lk. 886.

OmaloclineH.Cass.2148.

Omphalandria RBr. 5017.

Omphalea L. $501 \%$.

Omphalia $349 \mathrm{H}$.d.

Oimphálobioides DC. 4132c.

Omphalobium G. 3826.

Omphalocarpis $P$. B. 3657.

Omphalodes T. 2968.

Oinphalospora Bess. 3062a.

OedipodiumSchwg.599. Onagra T. 4497a.

Oedmannia Thub. 4050. Oncidium Sw. 1519.

Oedogonium Lk. 462. Oncoba Forsk. 5462.

Oenauthe L. 3733.

Oncoina Spr. 2914.

Oenocarpus Mart. 1643. Oncophorns Brid. 615.

Oenoplea Hdg. 3819. Oncorrhiza Pers. 1339.

Oenoplia R. S. 3819. Oncus Lour. 1339.

Oenothera L. 4497. Oneillia Ag. 502.

Oenotherium Ser.4497b. Onobroma Gärt. 2308.

Oeonia Lindl. 1511. 2690.

Oetania 5006a.

Ogiera H. Cáss. 2717.

Oolifa H. Cass. 2455.

O-Higginsia Rz. Par. 2095 .

Oidlum Lk. 64.

Okenia Dietr. 5159.

Olax L. 3610

Olbia Med. 5258b.

OldenlandiaPlum.2044. Onosuris Rafin. 4498.

Olea L. 3586.

Olearia Möuch. $25 \%$ t.

olfa Ad. 4955.

Olfersia Radd. 734.

Oligactis H. Cass. 2220. oligadenia Ehrnb. ti13a.

Onobrychis T. 4004.

Onoclea L. 715.

Ononis L. $402 \%$.

Onopix Ratin. 2340.

Onopordon Vaill. 2324.

Onoseris W.' 2253.

Onosina L. 2955 .

Onosmodium Mchx. 2945.

Onotrophe H.Cass. 2333.

Onychium Kaulf: 706.

Onychimm Rein w.688a.

Onygena Pers. 168.

Oococca DC. 5208a.

Opa Lour. $467 \%$.

Opegrapha Pers. 386. 
Opetiola Gärtn. 1076. Ophelus Lour. 5333.

Ophioglossum L. 747. Ophiopogon Ker. 1324. Ophiopteris Reiuw. $681 b$.

Ophorrhiza Forsk.2049. Ophioscorodon Wallr. $137 \%$.

Ophiospermum Lour. 1785.

Ophiotrichmm Kz. S0l). Ophioxylon Burm. 3559 .

Ophioxylon P. 3553.

Ophira L. 1700d.

Ophiurus Gärt. 824.

Ophiys L. 1467.

Opilia Rxb. 3601 .

Oplismems P. B. 889.

Oplotheca Nutt. 4360.

Opopanax Koch. 3680.

Oporanthus Herb. 1238.

Opulus Rafill. 1951.

Opuntia T. 4245.

Orbea Haw. 3411e.

Orchidium Sw. 1567.

Orchis L. $1 \$ 61$.

Oreas Bricl. 609.

Oreas Cham. 4799.

Oreobolus RBr. 1129.

Oreocallis $\mathrm{RBr}$. 1765.

Oreochloa Lk. 84 ij.

Oreogeun Ser. 4445a.

Oreoselinmm T. 3674c.

Oribasia Schreb 2097a.

Origanum L. 28:59.

Orites $\mathrm{RBr} .1760$.

Orinn DC. 47581).

Orlaya Hoffm. 36\%?.

Ormenis H. Cass. 2396.

Ormiscus DC. 4821 .

Ormocarpum P. B. 3985.

Ormosia Jacks. 4088.

OrnithidiumSalisb.1493.

Oruithocephalus Hook. $149 \%$.

Ornithogalum L. 1367.

Ornithoglossum Salisb. 1300.

Oruithopodium Burm. 4010.

Ornithoporlium 'T. 3980.

Ornithopus L. 3980.

Ornitrophe Juss. 5217.

Ornns P. 3581.

Orobanche L. 3055.

Orobium Rchb. 4799.
Orobus L. 3924.

Orontium L. 779.

Orontium P. 3175l,

Oropetimm Trin. 82?.

OrostachysFisch.4187d Ortega L. 4259.

Ortegia Löff. 4259.

Orthocarpaea DC.5.3231.

Orthocarpus Nutt. 3078.

Orthocentron H. Cass. 2335.

Orthoceras RBr. 1422.

Orihoclada P. B. 1011 .

Orthodon B. St. V. 100.

Orthodontium Schwg: blis.

Orthopognn $\mathrm{RBr}$. 889 .

Orthopyxis P. B. 644 . 650.

Orthoselis DC. $4821 \mathrm{c}$.

Orthospermum $\mathrm{RBr}$. $4343 a$.

Orthotheca Rrid. 594.

Orthotrichum Hilg. 1)21.

Orthotrichum Rohl. 621b.

Ortiga Feuill. 4232a.

Orucaria Jiss. 4018.

Orvala L. 2873.

Orygia Forsk. 4402.

Oryza L. 962.

Oryzopsis Mchx. 917.

Osbeckia L. 45y6.

Osbeckiaria DC. 4596 l.

Oscillatoria Vauch. 455.

Osinites L. 2401.

Osmitopsis H.Cass. 2400.

Osmodium Raf. 2945.

Osmuinda L. 740.

Osmundaria Lam x. 511.

Osproleon Wallr. 3055́a.

Ossaea DeC, 4630.

Osteo ineles Lindl. 4469.

Osteosperinum L. 273 j.

Osterdamia Neck. \$\$1.

Osterlyckia Buru.4211.

Ostericum Hoffin. 3697.

Ostracoderma Fr. 173.

Ostropa Fr. 273.

Ostrya Mich. 1793.

Ostryodium Desv. $3946 b$.

Osyris L. 1702.

Otauthus Lk. 2388. ex add.

Othera Thnb. 3604.

Othlis Schott. 4979.

Othonna L. 2515.
Othrys Noronh. 4867.

Otidia Lindl. 5278k.

Otites Otth. 5363c.

Otites Tabern. 5363c.

Otoptera DC. 3964.

Ottelia Pers. 808.

Otton K. H. B. 3731 .

Ottonia Spr. add. 5ij70.

Ondneya RBr. 4789.

Ouratea Aubl. jiss.

Ourisia Commers. 3212.

Ontea Aub!. 4113.

Ouvirandra A. P. Th. 800.

Ovieda L. 2928.

Ovieda Spr. 1194.

Oxalis L. 5319.

Oxera LaB. 2914.

Oxyandrá DC. 54531.

Oxyanthus Del. 2078.

Oxybaphus l'Her. 1840.

Oxycarpus Lour. 5491.

Oxycoccus Pers. 1971.

Oxylenia Nutt. S65a.

Oxygon!m Burch. 428?.

Oxylobinu Andr. $40 \% 6$.

Oxymeris DeC. 4640.

Oxypetalum RBr. 3435 .

Oxy ria Hill. 4284.

Oxys T. 5319.

Oxysphaeria Fr. $23 \% 0$.

Oxyspora DeC. 4589.

Oxystelma RBr. 3429 .

Oxystoma Fr. 386c.

Oxy tandrum Neck.545?.

Oxytropis DeC. 3851.

Ozonimm Pers. 84.

Ozophyllım Schreb. 5142.

Ozothainnus RBr. 2432. Pachira Aubl. 5.334.

Pachyloma DeC. 4588.

Pachyma Fr. 163.

Pachynema RBr. 4981.

Pachy uotum DC. 479\$b.

Pachyphragma DeC. 474'2a.

Pachyphyllum Knth. $155 \%$.

Pachypleurum Ledeb. 3707.

PachyrrhizusRich.3943.

Pachysaudra Mchx. 5091.

Pachystylium DC. em: 4821f.

Pacouria Aub1. $35 \%$.

Pacourina Aubl. 2200. 
Paconrinopsis H. Cass.|Pariana Anbl. 835. 2201.

Padina Lamx. 508a.

Padus Mill. 4705b.

Paederia L. 3575.

Paederota L. 3066.

Paeonia C. Bauh. 4965a.

Paeonia L. 4965.

Pagainea Aubl. 3505.

Palafoxia LaG. 2246.

Palavia Cav. 5242.

Paleolaria H. Cass.2246.

Palicourea Aubl. 2032.

Palisota Rchb. 1174.

Palimbia Bess. 3688.

Paliurus T. Dsf. 3811.

Pallasia Houtt. 5157.

Pallenis H. Cass. 2476.

Palinella Lob. 427.

Palinstruckia Rtz. fil. 3107.

Palovea Aubl. 4124.

Paludella Ehrh. 650.

Pamphalea DeC. 2286.

Panargyrum DeC. 2292. Panargyrus LaG. 2292.

Panax L. 3758.

Panciatica Picciav.4140.

Pancovia W. 4133.

Pancovia Heist. 4437. ex add.

Paucratiun L. 1264.

Paudauns L. f. 1270.

Panicastrella Much. 992.

Panicum L. 936.

Pauke Mol. 4192.

Pauopia Noronh. 5037.

Pauphalea LaG. 2586.

Panzera W. 4130.

Panzeria Mnch. 2881c.

Papaver 'T. 4856.

Papaya T. 2773.

Pappophorum Schreb. 930.

Papularia Forsk. 4310.

Papularia Fr. 16.

Papyrus Bruce. 1090.

Paquerina H. Cass.2566.

Paracfaenum P. 13.9361).

Paragnathis Spr. 1465.

Paranomus Sal. 1738.

Parapetalifera WendI. 5164.

Pardanthus Ker. 1208.

Pardisium N. L. Burm. 2255.

Parentucellia Viv. 3079.
Parietaria L. $180 \%$.

Parietaria Req. 180\%b.

Pariuari Aubl. 4521.

Pariuarium Juss. 4521.

Paris L. 1316.

Parita Scop. 5274.

Pariti Ad. 5274.

Paritiun A.St.Hil.5274. Parivoa Aubl.4129.4108.

Parkeria Hook. 709.

Parkia RBr. 4163a.

Parkinsonia Plum.4141.

Parmelia Ach. 402.

Parmentaria Fee. 372.

Parnassia T. 4907.

Parochetus Hamilt.3944.

Paronychia T.J. 4265.

Paropsia Noronh. 3461.

Parosella Cav. 3888.

Parrya RBr. 4\%8.

Porsousia P. Br. 4559a.

Parsousia RBr. 3520.

Parthenium L. 2618.

Pascalia Ort. 2661.

Paschanthus Burch. 3468.

Paspalum L. 887.

Paspalus Flg.' $887 a$.

Passalia Banks. 4895.

Passerina L. 1780.

Passiflora R.St.V.3472c.

Passiflora L. 3472.

Passoura Aubl. 4845.

Pastiuaca Hoffin. 3681 .

Pastiuaca L. 3681.

Patabea Aubl. 2117b.

PatagoniumSchrk.3992.

Patagonula L. 2986.

Patellaria Fr. 263.

Patellaria Hoffin. 412.

Patersonia RBr. 1184.

Patima Aubl. 21 I6.

Patrinia Juss. 1948.

Patrisia Rich. 4920.

Patrisia Rohr. 2823.

Pauletia Cav. $4126 i$.

Paullinia Schum. 5238.

Pavetta Rheed. 2012.

Pavia Boerh. 5201.

Pavonia Cav. 5261.

Pavonia R. P. 1825.

Pecheya Scop. 2021.

Pectophyllım K. H B. 3779.

Pectis L. 2356.

Pedalium L. 3252.

Pedicellaria DC. 4861.
Pedicellia Lour. 5182.

Pedicularis L. $30 y 0$.

Pedilauthus Neck. 5011.

Pedilea Lindl. 1573.

Pegauum L. 5120.

Pegia Colebr. $383 \%$.

Pekea Aubl. 5338.

Pelargium DeC. 52\% $8 \mathrm{~g}$.

Pelargonium l'Herit. 5278.

Pelexia Poit. 1414.

Peliosanthes Audr. 787.

Pellia Radd. 530b.

Peltanthera Rth. 3519.

Peltaria L. 4759.

Peltidea Ach. 404c.

Peltidiuin Zollik. 2161.

Peltigera Hoffm. 404.

Peltodon Pohl. 2864.

Peltopsis Rafin. 763a.

Pemphis Forst. 4562.

Penaea L. 3041.

Peuicillaria W. 927.

Penicilliun Lk. 119.

Penuantia Forst. 5113.

Penuisetum P. B. 926a.

Peunisetum P. 926.

Peluisetum RBr. 925.

Pentacalia H. Cass. 2510.

PentachondraRBr.3331.

Pentaloba Lour. 4898.

Pentaneris P. B. 1046.

Pentauema H. Cass. 2468.

Pentapetes L. 5311.

Peutaphalanx Rchb. $465 \%$ c.

Pentaphragina Zucc. 3447.

Pentaphyllon P. 3869f.

Pentapogon RBr. 923.

Pentapteris Hall. 4477a.

Pentapterophyllum

Dill. 4477.

Pentaria DeC. 470a.

Pentarrhaphia Lindl. 3244.

Pentarrhaphis K. H. B. 874.

Pentaptera Roxb. 4542.

Pentastemon l'Herit. 3179.

Penthorum L. 4194.

Pentzia Thub. 237\%.

Peperidia Rchb. 1855 b. ex add. 
PeperomiaRz.Pav.1854.|Petrea L. 2907.

Peplidium DeC. 3136.

Peplis L. 4500 J.

Pepo T. 276?.

Pera Mit. jll1.

Peraltea K. H. B. 4098.

Perama Aubl. 2938.

Peramibus Rafiu. 2619.

Peranema Don. 683.

Perdicium L. 2263.

Pereskia Plum. 4246.

Perezia LaG. 2282.

Perforaria Chois.j 471 b.

Pergularia L. $3+19$.

Periballia Triu. 1035.

Pericalia H. Cass. 2508.

Perichaena Fr. 190.

Periconia Tod. 91.

Peridium Schott. 5112.

Perigrapha Fr. 2iic.

Perilla IV. 28út.

Perilomia K. H. B. 2834.

Periola Fr. 153.

Periphragmos Rz. Pav. $302 \%$.

Periploca L. $345 \%$.

Periptera DeC. 5252.

Perisperma Ratiu. p. 15.

Perisporium Fr. 146.

Peristera DC. 5?\%8i.

Peritoma DeC. $\$ \$ 59$.

Pernettia Gaulici.338\%.

Perona P. 2yt.

Peronia DC. 1605.

Perotis Ait. 950 .

Perotriche H.Cass.2437.

Perrottetia DC. 3999.

Perottetia K.H.B. $539 \%$.

Persea Gärt. 1912.

Persica T. 4708.

Persicaria T. 4291e.

Personaria Lam. 2675.

Persoonia Mchx. 2592.

Persoonia Sin. 175\%.

Persoonia W. 5520 .

Petaguia Guss. 3756.

Petaguana Gm. 3995.

Petalauthus R. 1962b.

PetalolepisH.Cass.2433.

Petaloma Sw. 1966.

Petalostemon DC..3587a.

Petalostemum Mchx. $388 \%$.

Petalotoma DC. $452 \%$.

Petasites CB. 2251.

Petesia J.Br. 208.4.

Petilium L. H.Cliff.1354.

Petitia Jcr. 291\%.
Philuxerus RBr. $435 \%$

Petrobium RBr. 2634. Philyllrum Bauks. 115tj.

Petrocallis RBr. 476\%. Phijisia Trin. 91?a.

PetrocaryaSchreb. 4521 . Phlaeoseoria WVallr.275.

Petrophila RBr. 17t0. Phleastrum Ser. 3869b.

Petroseliuum Hoffiu. Phlebia Fr. $32 j$. 3725.

Petunia Juss. 3282.

Petiveria L. 4385.

Petrorhagia Ser. 53jod.

Peucedanum L. $36 \%$. 36 ita.

Peumus P. 1838.

Peziza Dill. 316.

Pfaffia Mart. $\$ 382$.

Phaca L. 3880.

Phacelia Mchx. 2981.

Phacidium Fr. 253.

Phaecasium H. Cass. 2144.

Phacosperma Haw. 431 j.

Phaeocarpus Mart.5225.

Phaeotus 349 C.

Phaetusa Gärt. 2718.

Phagualou H.Cass.2417.

Phalocramesus H. Caas. 2208.

Phalaugium Lam. 1386.

Phalaris L. 9j1.

Phallus Mich. 22S. 228a.

Phanera Lour. 4126b.

Pharnaceum L. $\$ 278$.

Pharus L. 990.

Phascuin L. 580 .

Phasellus Mnch. 3938b.

Phaseolus L. 3938 .

Phaseolus Much. 3938a.

Phaylopsis IV. 3126.

Phebalium Vent. 5171.

Pheboanthe Tsch. 2852.

Phelipaea T. 3056.

Phellandriun T. L. 3733b.

Phelline LaB. 3591.

Phelypaea Thub.1682b. ex add.

Phemeranthus Rafin. 4314c.

Philadelphus L. 4219.

Philagonia Blume.5130.

Philibertia K.H. B.344j.

Phillyrea L. 3585.

Philoneda Noronh. 5189.

Philonotis Brid. 65j.

Philonotis R. $49+1 c$.

Phlebocarya RBr. 1220.

Phlebomorpha P.p. 15.

Phlegmacium 349 F. $c$.

Phlegunatiun Fr. 60.

Phleum L. 948.

Phleum F. B. 948a.

Phloeoconis Fr. 3.

Phlomis L. 2856. 2886a.

Phlomitis R. 2886b.

Phlox L. 3021.

Phoberos Lour. 44\%.

Phoenix Cav. ic. 1656.

Phoenix L. 1615.

Phoenixopus H. Cass. 2166.

Pholidlandra Neck.j145.

Pholidia RBr. 3044.

Fholiclota Lindl. 1494.

Pholiota 349 D. g.

Pholiurus Trin. 826.

Phoma Fr. 24t.

Phormium L. 1398.

Photinia Lindl. 4463.

Phragmidium Lk. 12.

Phragmites Triu. 1048.

Phragunotrichum Kz.31.

Phryma L. 2816.

Phryluium W. 1604.

Phrissotrichia Brid.604.

Phryma Forsk. 2895.

Phucagrostis Cavol.756.

Phycella Lindl. 1255.

Phycomater Fr. 417.

Phycouryces Kz. 93.

Phyla Lour. $17 \%$.

Phylacteria 32\%a.

Philesia Juss. 1344.

Phylica L. 3804.

Phyllachue Forst. 1921.

Phylla mphoraLour. 788.

Phyllanthus Neck. $+24 \pm a$.

Phyllanthus L. 5078.

Phyllaurea Lour. 5051. Phyllerium Fr. 59.

Plyyllis L. 1992.

Phyllocharis Fee. 366 h.

Phyllocladus Rich.1696.

Phyllodiun Desv. 4001.

Phyllodoce Salisb. 3382.

Philotheca Rudg. 5170. Phylloedium Fr. 15. 
Phyllogonium Brid. $5+2.542 a$.

Phyllolobinm Fisch. 3953.

Phylloma Lk. 479b.

Phyllopta Fr. 283.

Phyllopus DeC. 4636.

Phyllostegia R. 1171b.

Phyllostemma Neck. 5176.

Phyllota DC. 4001a.

Physa Noronh. 5346 .

Physa A. P. Th. 4280.

Physalis L. 3300 .

Physarum Pers. 193.

Physcia Ach. $410 \mathrm{~b}$. 409b. 402f. 405b.

Physcomitrimn Brid. 584 '.

Physedium Brid. 581.

Physema Nor.add.5573.

Physianthus Mart.3453. Physicarpos Poir. 405\%. Physiphora Sol. 4899. Physkium Lour. 805.

Physocalycimn Vest. 4184.

Physocalymma Pohl. 4565 .

Physocalyx Pohl. 3206. Physocarpidium R. $4953 \mathrm{~b}$.

Physocarpus Cawib. 4449 .

Physocarpus DC. 4953b.

Physopodium Desv. 4575 .

Physospermam Cuss. 3745.

PhysostemonMart.4863. Physurus Rich. 1+16.

Phyt elephas Rz. P.1271. Phytemna L. 2793.

Phytocrene Wall. 3787.

Phytolacea T. L. 4398.

Phytoxys Molin. 2858.

Piaranthus RBr. $3 \$ 10$.

Picnemon Dalech. 2330.

Picotia Schult. 2968.

Picramnia Sw. 3856 .

Picria Lour. 3222.

Picridinun Desf: 2172.

Picrinm Schreb. 348?.

Picris L. 213ti.

Picteria DeC. 3984.

PierardiaR xb.add.5574.

Piesmyces Rafin. p.15.

Pilacre Fr. $16 \%$.
Pilea Lindl. 1799.

Pileanthus LaB. 4662.

Pilidium Kz. 237.

Pilobolns Tod. 212.

Pílocarpus Vahl. 5150.

Pilophora Jcq. 1621.

Pilopogon Brid. 630.

Pilotrichum P. B. 546. 550. 551. 551 .

Pilularia L. 672.

Pimela Lour. 3845b.

Pimelea Forst. 1772b.

Pimpinella Ad. 4422.

Pimpinella L. 3738.

Pimpinella Spr. 3738a.

Pimpinella T. 4423.

Pinalia Lindl. 1488.

Pinardia Neck. 2551.

Pingnicula L. 3318.

Pinaria DC. 4797a.

Pinastella Dill. $\$ 480$.

Pinckneya Mchx. 2062.

Pineda Rz. Pav. 4699.

Pinonia Gandich. 68\%.

Pinus L. 1724.

Piparea Aubl.4901.4895.

Piper L. 1855.

Piperella Lob. 4i51f.

Piperatla Lob.

Piptatherum P. B.915b. Platylobimn Sm. 40.56.

Piptocarpha RBr. 2421. Platypetalum PBr.4801.

Piptocoma H.Cass.2222.

Piptopogon H. Cass. 2159.

Piqueria Cav. 2234.

Pirigara Aubl. 4650.

Piringa Juss. 2111.

Piripea Anbl. 3106.

Piriqueta Aubl. 4238.

Pisaura Bonat. 4505.

Piscidia L. 3905.

Piscipula Löfll. 3905.

Pisocarpiun Lk. 209.

Pisolithus Alb. Schw.

209.

Pisomyces Fr. 209.

Pisonia L. $18+8$.

Pisonia Rottb. 3589.

Pistacia L. 3859 .

Pistia L. 766.

Pistillaria Fr. 295.

Pistolochia R. 18úfic.

Pistorinia DeC. 4186.

Pisum L. 3922.

Pitcairnia l'Herit.1282.

Pithosillum H. Cass. 2513.

Pittocarpinm Lk. 178.

Pittonia Plum. 2982.
Pittosporum Buks.3050.

Pitumba Aubl. 4695.

Pityrodia RBr. 2919.

Placodium Hoffun. 402. 411c. 412c.

Placoma L. Gm. 1994.

Placus Lour. 2543.

Pladera Roxb. $348 \%$.

Plagianthus Forst. 5330.

Plagiopus Brid. 658.

Plagiorutis Ser. 3870b.

Plananthus P. B. 1676a.

Planarinm Desv. 3988.

Planera Mchx. 1829.

Plantago L. 3354.

Plappertia Rehb. 3824.

Plaso Rheed. 3958.

Platanthera Rich. 1474.

Platamis L. 1794.

Platonia Rafin. 2897.

Platunium Juss. 2913.

Ptatycapnos DC. 4827\%.

Platycarpunn Hb. Bpl. $325 \%$.

Platycheilus H. Cass. 2281.

Platyoramma Mey. 389.

Platyraphium H. Cass. 2326.

PlatypterisK.II.B.2635. $36 \% 1$.

Platy zoma RBr. 743.

Plaubelia Britl. 631.

Plazia Rz. P. 2294.

Plecostoma Desv. 20?c.

Plectaneia A.P.'Th. 3539 .

Plectanthera Mart. 4906.

Plectranthus l'Her.2811.

Piectronia L. 4213.

Pleea Mchx. 1305.

Plegorrhiza Molin. add. $55 \% 5$.

Pleione Don. 1545.

Plenkia Rafin. 4403\%.

Pleomeles Salisb. 1403.

Pleopeltis K. H. B. 719.

Pleroma Don. 4600.

Pleurandra Rafin. 4495.

Pleurandra LaB. 4983.

Pleuraphis Torr. 878.

Pleuridinm Brid. 555.

Pleuroblepharon Kz.

1470b. ex add.
Platyspermun Hoffu. 
PleurolohiumDC.4000b. Pohlana N. et M. 5132. Polymnia L. 2719.

Pleuroplitis Trin. 934. Pohlia Hdg. 649.

Pleuropogon RBr. 1028. Poincia Neck. 4148.

Pleuropus 324c. 327d. Poinciana L. 4148. 338d.

Pleurospermum Hoffm. 3746.

Pleurostemon Rafin. 4495.

Pleurothallis RBr. 1568.

Pleurotus 349 H.a.

Plinia L. f. 4681 .

Plocaina Ait1994.

Plocamium Lamx. 497d.

Plocaria N.・ v. E. 496.

Pluchẹ H. Cass. 2211.

Pluknetia Plum. 5031.

Plumbago L. 1926.

Plumeria L. 3538.

Pneumonanthe Schm. $3499 \mathrm{~b}$.

Poa L. 1030.

Poarion Rchb. 950.

Poarium Hamilt. 3074.

Pocockia DeC. 3871 .

Pocophorumieck.3850c.

Porlalyria Lam. 4079.

Podanthe Haw. 3411h.

Podanthus LaG. 2662.

Podaxis Desv. 199.

Pollisoma Lk. 32.

Podocaryus l'Herit. 1694.

PodocomaII. Cass. 2548.

Podogyne Hifing. 4860.

Podolepis LaB. 2430.

Podolobium $\mathrm{RBr}$. $407 \%$.

Podophyllum L. 4S44.

Podopteris Hb. Bpl. 4294.

Pollosaemum Desv. 906.

Podosperma LaB. 2414.

Podospermum DeC. 2174.

Podosphaeria Kz. 148.

Podostemon Mchx. 1673.

Podostigna Ell. 3431.

Podostrombium Kz.158.

Podotheca H. Cass.2414.

Pöppigia Kz. 1227b. ex add.

Pogonatherum P.B.968.

Pogonatum P. B. 670.

Pogonia Andr. 3046.

Pogonia Juss. 1441.

Pogonirion R. 1187a.

Poiretia Cav. 3349.

Poiretia Gin. 3483.

Poiretia Sm. 4057.

Poiretia Vent. 3987.

Poitaea DC. 3899.

Poitea Vent. 3899.

Poivrea Commers. 4533.

Polameia A. P. Th.191 5.

Polanisia Rafin. 4862.

Polava Rz. Pav. 5414b.

Polemannia Berg. 1370.

Polembryum Ad. Juss. 5156.

Poleinonium L. 3025.

Polia Lour. 4263.

Polianthes L. 1399.

Polium T. 2851b.

Polla Adans. 642c.

Pollalesta K. H. B. 2197.

Pollia Thunb. 1162.

Pollichia Medic. 2961.

Pollichia Rth. 2874a.

Pollichia Sol. 4255.

Pollinia Spr. 974.

Polyacantha Vaill.2329. $232 \%$.

Polyachurus DeC. 2293. Polyachyrus LaG. 2293.

Polyact is Lk. 115. 115f.

Polyactiun DC. 52781.

Polyadenia Ehrnb. 4413c.

Polyangium Lk. 116.

Polyanihea DeC. 3472a.

Polyanthes W. 1399.

Poly botrya K.H.B.734.

Polycardía Juss. 5393.

Polycarpaea Isam. 4263.

Polycarpon Löfh. 4260.

Polycenia Chois. 3096.

Polycephalus Fork. 2482.

Polychaetia Tsch.2151b. Polychroa Lour. 4271.

Polycnemum L. 4325.

Polygala L. 3029.

Polygalon Dr. 3029e.

Polygaster Fr. 221.

PolygonatumDesf.1322.

Polygonella Mchx.4288.

Polygonum L. 4291.

Polyides Ag. 433.

Polylepis Rz. Pav.4425.

Pogostemon Desf. 286\%. Polymeria RBr. 2994.
Polymniastrum Lam. 2720.

Polyodon K. H. B. 875 .

Poly osus Lour. 2007.

Polyozus Lour. 2120b. ex add.

Polypara Lour. 794. ex add.

Polypera P. 209.

Polyphacum Ag. 511.

Polyphema Lour. 1817.

Polyphragmon Dsf. 2113.

Poly podium L. 723.

Polypogon Desv. 900.

Poly porus Mich. 338.

Polypremum L. $\mathbf{3 0 4 5 .}$

Polypteris Nutt. 2595.

Polysaccum DeC. 209.

Polyscias Forst. 3785.

Polysiphonia Grev. 48í.

Polystachya Hook.14y1.

Polystegia R. 1248a.

Polysticta 338f.

Polystigma DeC. 266.

Polythrincium Kz. 101.

Polytrichum L. 671.

Poinaderris LaB. 3801.

Pomaria Cav. 4142.

Poinatiderris Kuth. 3801.

Pomatiun Gärt. 207\%.

Poniatoderris Hffyg. 3801.

Poinax Soland. 1930.

Poinbalia Vand. $488 \%$.

Pometia Forst. 5218.

Pominereulla L. 942.

Ponaea Schreb. 5214.

PonceletiaA.P.Th.858b.

Ponceletia RBr. 3348.

Pongamia Lain. 4013.

Ponyati Rheed. 4399.

Pongatiun Juss. 4399.

Pontederia L. 1166.

Ponteleria Rafin.1166a.

Ponthieva RBr. 1426.

Pontoppidana Scop. 4652 .

Popodothion Fr. 375.

Poppya Neck. 2754.

Populago T. 4961a.

Populus L. 1789.

Porana Burun. 3010.

Poranthera Rudg. 4230.

Poraqueiba Aubl.1899a. ex add. 
Porcelia Rz. Par. 5008.|Preslea Spr. 2960.

Porcellites H.Cass.2154.

Porella Dill. 532h.

Purina Ach. 363.

Porliera Rz. Pav. 5199.

Porouia IV. 278b.

PorophyllumVaill.2357.

Porophora Mey. 363.

Porotheliun Eschw. 365.

Porothelinun Fr. 335.

Porotrichum Brid.55\% b.

Porpa Blume. 5434.

Porphyra Ag. 479a.

Porpliyra Loirr. 2921.

Porruin T. 1378.

Portesia Juss. 5512.

Portlandia J. Br. 2068.

Portulaca T. L. 431 .

Portulacaria Jcq. 4316.

Posillonia Canl. 757.

Posoqueria Aubl. 2074.

Possira Aubl. 4160.

Potalia Aubl. 3507.

Potamogeton L. 763.

Potamogetou Rafin. $763 \mathrm{~b}$.

Potanophila RBr. 957.

Potamophila Schrk. 4392.

Potentilla L. 4436.

Poteutillastrum Ser. $4436 b$.

Poterium L. 4422.

Pothos L. 7\%6.

Potima P. 2019.

Pottia Ehrh. 584a.

Poupartia Connmers. 3855.

Pourretia Rz. P. $127 \%$.

Ponrretia W. 5331.

Pouteria Aubl. 3590.

Pozoa LaG. 3772.

Praesepium Spr. 4421b. ex adl.

Prangos Liudl. 3691.

Prasiola Lk. 479il.

Prasium L. 281 j.

Prasophyllum RBr. 1423.

Pratellarins 349 C. a.

Pratellins 34913.

Premua L. 2910.

\begin{tabular}{l|l} 
Prenanthes L. 2169. & 2885c. \\
Preouanthes DC. 4945a. & Pseudolimm DC. 4780.
\end{tabular}

Prepusa Mart. 3480.

Prescotia Lindl. 1457.

Preslaea Mart. 2960.
Prestonia RBr. 3527.

Pretrea Gay. 3251.

Prevostea Chois. 2998.

Priapus Rafin. p. 15.

Priestleya DC. 4045.

Priestleya Meyen. 454.

Prieurea DeC. 4492.

Primula L. 3361.

Prinoides DC. $3626 a$.

Prinos L. 3626.

Printzia H. Cass. 2555.

PrionanthesSchrk.2170.

Prionoschoenus R. $1287 d$.

Prionotes RBr. 3350.

Prismatocarpus l'Her. 2801.

Priva Adaus. 2895.

Probnscidia Rich. 4611.

Prockia Brw. 4926.

Prockiaria DC. 49:6b.

Procris Coinıners. 1802.

Proiphys Herb. 1265.

Prolifera Vanch. 462.

Proserpinaca L. 4478.

Prosopia Fechb. 3091.

Prosopis L. 4170.

Prostanthera LaB.2832.

Prostheminn Kz. 239.

Protea L. 1744.

Proteina Ser. 5360a.

Protin:n Burun. 3840.

Protorema Ag. 45.

Protococcus Ag. 418.

Proustia LaG. 2265.

Prunella L. 2813.

Prnnophora Neck. 4706.

Prumus T. 4\%06.

Psalliota 349 B. c.

Psamina P. B. 909.

Psau acetum Neck. 2376.

Psathura Comin. 2100. 2101.

Psathyra 349 B. a.

Pseliuin Lour. 1882.

Pseulacacia T. 3898.

Psendaleia A. P. Th. 3615.

Psendaleioides A. P. 'Th. 3616.

Psourlocistus DC. 4915b.

Pseurlo - Dictainnus 2883́c.

Psendosophora DC. 3884.

Psiadia Jc4. 2524.
Psidium L. 4687.

Psiguria Neck. 2765.

Psilanthus DeC. 3469a.

Psilathera Lk. 995.

Psilobium Jack. 2056.

Psilocybe 349 B. b.

Psilouia Fr. 107.

Psilopilum Bridl. 668.

Psilostylis Andrz.4795a.

Psilostylium DC. 4810c.

Psilotum Sw. 1678.

Psilostylum DC. 4810c.

Psilurus Triu. 819.

Psittacoglossum

LaLlav. 1499.

Psophocarpus Neck. 3946.

Psoralea L. 3976.

Psorima Ach. 402b. 411b. 412b.

Psychanthus Rafiu. 3029 .

Psychine DeC. 4756.

Psychoiria L. 20:31.

Psychotrophum J. Br. 2031.

Psychrophila DC.4961b.

Psyllium T. Juss. 335.5.

Psyllocarpus Mart.2014.

Pielea L. 5125.

Ptelidiun A.P.Th.5395.

Pterauthus Forsk. 4332.

Pterigium Corr. $191 \%$.

Pterigyuandrum Holg. 572.

Pteris L. 693.

Pterium Desv. 838.

Pterocarpus L. 4016.

Pterucarya DeC. $303 \mathrm{sh}$.

Pterocarya Nutt. 3833.

Pterocephalus Vaill. 1932.

Pterogoninm Sw. 572.

Pterogyus DC. 4482b.

Pternix Rafiu. 2341.

Pterolaena DC. 5316a.

Pterolepis DC. 4596ic.

Pteroueuriu DeC.4794.

Pteronia Printz. 25.33.

Pterophora Neck. 25.33:

Plerophorus Vaill. 25.33.

Pterophyllum Brid.571.

Pterophyton H. Cass. 2636.

Pterospermum Schreb. 5.316.

Pterospora Nutt. 3369.

Pterostylis IRBr. 1443. 
Pterota Ad. 5132. |Pyrola L. 3370.

Pterotheca H.C'ass.2156. Py rolirion Herb. 1235. Pterotropis DC. 4it2e. Py:ophorum Neck.

Pterofum Lour. add. 5576.

\begin{tabular}{l|l} 
Pterula Fr. 298. & Py rostria Comm. 2104. \\
Pterygodiun Sw. 1481. & Pyrularia Mchx. 1711.
\end{tabular} $4470 \mathrm{~g}$.

Pyrostoma Mey. 2931. Ranunculus L. 4941.

Ptery'gophyllum Brid. $5+5$.

Pyrus L. 4470.

Ptileris Rafin. 2504.

Ptilocnema Don. $15 \% 0$.

Ptilophyllum Nitt. $4477 \mathrm{~h}$.

Ptilostemon H. Cass. 2328.

Ptilostephinm K. H. B. 2596.

Ptilota A $A$. 491.

Ptilotus IRBr. 4376.

PtychospermaLaB.1640.

Piychostomum Hornsch. 639.

Ptychotis Koch. 3723.

Pinccinia Pers. 14.

Pueraria DC. 3963.

Pugioniun Gärt. 4712.

Pulicaria Gärt. 2461.

Pulmouaria L. 2947.

Pulsatilla Banh. 4944b.

Pulsatilla T. 4045.

PulsatilloidesDC.4944b.

Pultenaea Sin. 4061.

Pulveraria Ach.Flk.350.

Punica L. 4579.

Pupalia Juss. 4372.

Puralia Hamilt. add. $557 \%$.

Purshia Rafin. 44jzb.

Purshia DeC. 4454.

Purshia Spr. 2945.

Puschkinia All. 1371.

Pycuauthemum Mchx. 2860.

Pycnapophysium R. $602 a$.

Pycnostachys Hook. 2869.

Pythagorealiafin.45j8b

Pyihagorea Lour. add. 5578.

Pythiun N. V. E. 440. Pyxidauthera MIchx. 3343.

Pyxirlauthera Schreb. 3374.

Pyxine Fr. 393.

Quarrella DC. 4874a.

Quadria Rz. Pav. 1746.

Unalea Aubl. 4510.

Uuamoclit T. 3015.

(Yuassia L. 5174.

Yuebitea Aubl. 774.

Uueltia Salisb. 1268c.

Uuelusia Vand. 4494a.

Uutercus L. 1798.

Queria Löff. 4254.

Uneria G. $426 \%$.

Quillaja J. 4456.

Quinaria Lour. 5525.

Quinchamali Fenill.

1705.

Quiuchamalium Juss. 1705.

Quisqualis Rmph. 4528.

Guivisia Comm. 5508 . Uunpoya Aubl. 5486.

Unoya Gaudich. 3233. Racaria Aubl. 5230.

Racodium Pers. 79.

Racomitrinu Brid.592.

Racopilum P. B. 5.38.

Racoubea Aubl. 4698.

Raddisia Leand. 5384. Rademachia Thub.1817. Radia A. Rich. 1230. Radiana Rafin. 4311. Pycuothelia Ach. 414a. Radiola Dill. 5463.

Pycreus P. B. 1081.

Pylaisaea Desv. 574.

Radiusia Rchb. 3884.

.

Py ramiclium Brid. 586. Rafflesia RBr. 1685.

Pyramidula Brid. 586. Rafuia Thub. 4050.

Pyrenastrum Eschw. 372.

Pyreniun Tod. 285.

Pyreuthea Fr. 371.

Pyrenula Ach. 373a.

Rajania L. 1337.

Ramalina Ach. 408.

RamatuellaK.H.B.4539.

Ramoulia Rich. 3278.

RamphosperinumAndr.

Pyrethrum Hall. 2380. 4815 .

Ramspeckia Scop. 2074.

Raudia Houst. 2084.

Ranumculastrum DC. $4941 \mathrm{~b}$.

Ranunculus L. 4941.
Rapanea AubI. 3634.

Rapatea Aubl. 1145.

Raphanistrum Gärt. $+728$.

Raphanus L. 4729.

Raphia P. B. 161\%.

Raphiolepis LindI. 4461.

Rapinia Lour.3344.4399.

Rapistrum Boerh. 4725.

Rapourea Aubl. 2976.

Raputia Aubl.2915.3145.

Raspailia Brgu. 4223.

Ratonia DeC. 5231.

Rauwolfia L. $35 j 5$ t.

Ravapa Rheel. $210 \%$.

Ravenala ddans. 1612.

Ravensara Sonn. 1916.

Ravia N. et M. 5145.

Razumovia Spr. 3234. 2370.

Reaumuria Hasselyu. $4+16$.

Rebouillia Raddi. 528d. Recchia Sess. 497\%.

Redontea Veut. 5271.

Redowslaia Cham. 4809.

Reichardia Rih. 4146.

Reichelia Schreb. 3001.

Reichenbachia Spr.1844.

Reimaria Flgg. 879 .

Reineria Much. 3892d.

Reinwardta Spr. 2998.

Reinwardta Blume. 5411.

Relhania l'Her. 2406.

Relhania Gm.sy st.3622.

Remirea Aubl.y10.108\%.

Renauthera Lour. $15+1$.

Renealmia RBr. 1179.

Renealunia L. f. 1590.

Repandra Lindl. 1480.

Requienia DC. 4026.

Reseda I. 4824.

Reseda T. 4824 b.

Restio L. 1136.

Restrepia Kuth. 1560.

Resupinatus 324a.333a. 338a.

Retanilla Brngn. 3810.

Reticularia Bull. 177.

Retinaria G. 3797.

Retiniphyllum Hb. Bpl. 2097b. 
Rettbergia Radd. 1064. IRhynchosia Lour. 3933.|Rochefortia Sw. 3498. Retzia Thub. 3006.

Reyniba R. 4874 b.

Rhabdia Mart. 2990.

Rhabdochloa P. B. 865b)

Rhablocriuun R:hb. 1352.

Rhablotheca H. Cass. 2164.

Rhacoma Adns. 2311. 2313.

Rhacoina L. 3623.

Rhagolla RBr. 4352.

Rhagodiolns T. 2122.

Rhynchospora Vahl. 1109.

Rliyuchotheca Rz. Pav. 5?83.

Rhytachne Hamilt. 833.

Rhytiphioea Ag.. 489.

Rhytisma Fr. 25t.

Riana Aubl. 4895.

Ribes L. 4247.

Ribesia Berland. 424\%c.

Riccia Mich. 515.

Richaeia A. P. Th. 1970

Richardia L. 1995.

Rhamnopsis Rchb. 4879. Richardia Kuth. 773.

Rhanterinm Dest. 2470. Richardsonia K. H. B.

Rhapis Ait. 165́́.

Rhaphis Lour. 971.

Rhaponticum Vaill. 2311.

Rhamuus L. 3822.

Rheedia L. 5493.

Rhegmatodon Briel.558.

Rhein L. 4287:

Rhexia P. Br. L. 4586 .

Rhinactina W. 2278 .

Rhinanthus L. 3076.

Rhiuimn Schreb. 4970.

Rhinocarpus Bert. 3868.

Rhipidodendrum W. 1110.

Rhipsalis Gärt. 4247.

Rhizina Fr. 317.

Rhizoholıs Gärt. 5338.

Rhizoctonia DeC. 159 .

Rhizogoninm Brid.544.

Rhizowm Burch. 3268.

Whizomorpha Rth. 98.

Rhizophora L. 1968.

Phizopogon Fr. 222.

Rhizopus Ehrub. 139a.

Rhodiola L. 4189a.

Rhododendron L. 3399.

Rhodolaena A. P. Th.

5476.

Rhodlomela Ag. 490.

RhodoinyrtusDC.4685b.

Thodora L. 339 .

Rhorlothamuus Rchb. 3400.

Rhombifolium Rich. 3974.

Rhopala $\Lambda$ ubl. 1761.

Rhopinum Schreb. J109.

Rhus L. 3850.

Rhus T. 3850c.

Rhyuchauthera DeC. 4611. 1995.

Richea LaB. 2449.

Richea RBr. 3344.

Richeria Vahl. 5097.

Richnophora F. 225.

Ricinocarpus Desf.5056.

Ricinoides T. 5045.

Ricinus L. $505 \%$.

Ricotia L. 4771.

Riedlea Vent.' 5309.

Riedleia DC. 5309.

RiencurtiaH.Cass.2721.

Rigocarpus Neck. 2760.

Rimella Rafin. 196.

Rindera Pall. 2953.

Rinodina Fr. 400a.402a. 411b. $412 a$.

Rinorea Anbl. 4894.

Ripidium Trin. 988.

Ripogonmm Forst. 1332.

Rittera Schreb. 4160.

Riveria K. H. B. 4100.

Rivina L. 4395.

Rivularia Rth. 423.

Rizoa Cav. 2891.

Robergia Schreb. 3827.

Roberitia Merat. 4963.

Robertia DeC. 2160b.

Robertsonia Haw. 4203d.

Robinia L. 3898.

Robinia Rxb. $3892 a$.

RobinsoniaSchreb.5501.

Robignetia Gaudich. $150 \%$.

Robonia R. 5167c.

Robsonia Berland. 4247 .

Rocaına Forsk. 4310.

Roccarlia Neck. 2415.

Roccella DeC. 407.

Rochea DeC. 4181.
Rochelia Rchb. 2970.

Rochelia R. S. 2969.

Rodigia Spr. 2158.

Rodriwnezia Rz. Pav. 1533.

Rodschiedia Fl. Welt. 4741.

Roëlla L. 2795.

Rueineria Radl. 530a.

Roemeria Med. 4852.

Roemeria T'hul). 3602.

Roeperia Spr. 5056.

Roepera Acl.Juss. 5196.

Roestelia Lk. 9c.

Rogeria Gay. 3250.

Rohilea Rth. 780.

Rohria Vahl. 2683.

Rohria Schreb. 3216. 3825.

Rokejeka Forsk. 5359c.

Rolaudra Rottb. 2347.

Rollinia A. St. Hil.4997.

Rolofa A(l. 4403\}).

Romanzoffia Cham.30\%1.

Romulea Maratt. 1202.

Roulabea Anbl.' 2038.

Rondeletia Plum. 2050.

Rorain Ad. 992.

Rorella Rujj). 4910a.

Rorida Forsk. 4864.

Roridnla Forsk. 4864.

Roridula J. 4911.

Roripa Scop. 4796.

Rosa L. 4432.

Roscoea Silı. 1601.

Rosea Mart. 4356.

Roseuia Thub. 2407.

Rosmarius L. 2806.

Rosularia DC. 4187a.

Rostkovia Desv. 1287a.

Rostraria Trill. 993.

Rotala L. 45.5?.

Rothia P. 3y's8.

Rothia Schrel). 2160c.

Rothınania Thb. 2081.

Rotmammia Neck. 4130.

Rottboella L. fil. 820 .

Rottlera V. 3224.

Rolllera Rxb. 5050.

Rotileria Brid. $584 c$.

Rotula Lour. 2975.

Rouhamon Aubl. 3568.

Rollmea Poit. 4880.

Rourea Aubl. 3827.

Roussea Sın. 3633.

Rousseauxia DeC. 4619 .

Royena L. 3652. 
Roxburghia W. 786. Roylsia Roxb. 1786b. Rubia T. 1990.

Rubentia Commers. . 5394.

Rubus L. 4448.

Rulbeckia L.. 2663.

Rudbeckia Ad. tỏ3s.

Rudgea Salisb. 203\%.

Rulolphia W. $395 \%$.

Ruellia L. 3122.

Ruizia Cav. 5314.

Rulingia Haw. 4313.

Rulingia RBr. 5293.

Runnex L. 4286.

Rumia Hoffı. 3728.

Rıınia Lk. 3676.

Ruınohra Radll. 6S1c.

Ruizia Rz. Pav. 1838.

Rumphia L. 4512.

Rupifraga Otth. 5363e.

Ruppia L. 761.

Rupinia L. 319.

IRnscus L. 1331.

Russelia L. 3194.

Russula Pers. 346.

Ruta L. 51?'?.

Ruteria Mnch. 3976.

Rutidea DeC. 2026.

Ruidopoterinu DC. $4+22 b$.

Ruyschia Jcq. 5483.

Ryania Vahl. 4919.

Ryallapa DC. 4919. Sabal Ail. 1652.

Sabbatia Ad. 3493.

Sabbatia Much. 2862.

Sabilariffa 5273.

Sabia Colehr. 3427.

Sabicea Aubl.309.5.2114.

Sabinea DC. 3900 .

Saccellium K.H.B.2991.

Saccharum L. 989.

Saccharum P. B. 989b.

Saccolina R. 404a.

Saccoloma Kaulf. 684.

Sacidlum F. N. v. E.234.

Salleria Kanlf. 712.

Sagedia Ach. 378.

Sageretia Brugu. 3812.

Sayina L. 5341.

Sagittaria L. 7\$9b. ex aild.

Sagonea Aubl. 3001.

Sayraea DeC. 4631.

Samuerus Rmpf. 1625.

Sagus Rimpf. $161 \%$.

Sahlbergia Neck. 208?.
Salacia L. 5385.

Salaxis Salisl. 3378.

Salicaria T. $4538 \mathrm{c}$.

Salicornia L. 4319.

Salisburia Sin. 1697.

Salix L. $178 \%$.

Salınacis B.St. V.

Salınasia Schreb. 4896.

Salınea Cav. 1403.

Salmea DeC. $263 \%$.

Salınia WV. 1614.

Salınonia Neck. 4508.

Saloınonia Lour. 3032.

Salpianthus Hb. Bpl. 1845.

Salpiglossis Rz. P. 3145. Salpinga Mart. 4616.

Salsola L. 4323.

Salvadora L. 4396.

Salvertia A. St. H. 4509.

Salvia L. 2805.

Salvia T. 2805́c.

Salvinia Guett. $51 \%$.

Salzwedelia G. M. S. 4030a.

Samalera Gärt. 517S.

Samandura L..j1 is.j28j.

Samara L. 3634 .

Sambucus L. 1949.

Sameraria DC. 4734a.

Samolıs L. 336.3.

Samy la L. 4693. 4693a.

Sanchezia Rz. P. 311\%.

Saudoricum Cav. 5506.

Sanyuinaria Dill. 4845.

Salıguisorba L. $\$ 223$.

Sanicula L. 375.́.

Sanseviella Rchb. 783. Saurania WV. Stit.

Sanseviera Thub. 1400. Saurauja DC. J414a.

Sanseviera Andr. 783. Saururus L. 792.

Sautalaria DC. 4016c.

Santalum L. 1703.

Santia Sav. 900.

Santolina T. 2389.

Sanvitalia Lam. 2638.

Saonari Aubl. 5338.

Sapincius L. 5222.

Sapium Jacr. 5028.

Saponaria Neck. ‘360d.

Sapouaria L. 5360.

Sapota Pliun. 364t.

Sappania DC. 4it9b.

Saprolegnia N. v. E.442.

Saproma Brid. 58?.

Saraca Burm. 4105.

Saracha Rz. P. 330t.

Sarcauthemum H. Cass. 2523.
Sarcanthus LindI. 1506.

Sarcocapnos DC. 48?9.

Sarcocarpon Blume. 1898.

Sarcocaulon DeC. 5282.

Sarcochilus RBr. 15is.

Sarcococca Lindll. 5089.

Sarcocrambe DC.4724a.

Sarcodium P. 3959.

Sarcodiun Lour. 3959.

Sarcoglottis Prsl. 1+3\%.

Sarcolaena A. P. 'Th. 5479 .

Sarcolobils RBr. 3422. Sarcophyllum Thub. t03.

Sarcophyllns Thub. 4035.

Sarcophytum Sparm. 1686.

Sarcopodiun Ehrub.52.

Sarcopyramis Wall. 4643.

Sarcostemina RBr.3446.

Sarea Fr. 312.

Sareacia R. 4695a.

Sargassum R. 514.

Sargassimn Ag. 51 tb.

Sarissus Gärt. 200?.

Sarmienta Rz. P. 3?2S.

Sarolhra Lam. $546 \%$.

Sarracenia L. 789.

Sassafras Siebli. 1911. ex arld.

Sassia Molin. a dd. 5579.

Satureia L. 2862.

Satyrium Sw. 1483.

Sanssurea DeC. 2304.

Sallvagea Neck. 4904.

Sanragesia Jacq. 4904.

Savastaná Schrk. 1039.

Savastenia Neck. 4597.

Savia Rafiı. 3930.

Savia Willd. 5095.

Savinnia DC. 4772.

Saxifragaria (Aizoon.) 4203b.

Saxifraga L. 4203.

Scabiosa L. Vaill: 1935.

Scaerola L. 2782.

Scandalida Neck. $38 \% 5$.

Scaudix 'T. L. 3660.

Scaphis $386 \mathrm{~b}$.

Scepinia Neck. 2534.

Schasmaria Ach, 414c. 
Schanginia Led. 4340. Schedonorus P. B. 1021. Schefilera Forst. 3784. Schelhammera RBr. 1347.

Schelhammerianönch. 1083.

Schepperia Neck. 4871.

Scheuchzeria L. 1290.

Schiellea Cham. 5365.

Schillera Rchb. 5290.

Schima Reiuv. 5409.

Schima Forsk. 831.

Schinus L. 3853.

Schismoceras Prsl.1551.

Schismus P. B. 1013.

Schistidium Brid. 588. dele: 535 .

Schistostewa TV. M. 539.

Schisvereckia Andrz. $476 \%$.

Schizaca Sw. 736.

Schizandra Mchx.1896.

Schizauthus Rz. P. 314t.

Schizoderma Kz. 21.

Schizolaena A. P. Th. $547 \%$.

Schizoloma Gaudich. 69 ?.

Schizonema Ag. 425.

Schizopetalou Sims. 4822 .

Schizophyllum Fr. 340. Scilla L. 1364.

Schizopogen Rchb. 970.

Schizoxylon Pers. 240.

Schkuhria Rıh. 259\%.

Schlechtendalia Willd.

2352.add.Spr.-Mollia.

Schleichera IVilld.5207.

Schlotheimia Bricl. 625.

Schmalzia Desv. 3850e.

Schmidelia L. 5215.

Schınidtia Tratt. 911.

Schmidtia Mnch. 2151b.

Śchnella Radd. $412 \%$.

Schoberia Led. 4341.

Schoenobiblus Mart. 1782.

Schoenocaulon R.1147b.

Schoenodorus Auct. 1024.

Schoenopsis Lestib. 1098.

Schoenorchis Reinw. 1575.

Schoenus L. 1097.

Schöpfia Sirheb. 1979.

Schollera lith. 1971.
Schollia Jcq. 3416.
Schorigeram Ad. 5030.

Sichotia Jacc. 4132.

Schotia Thub. 4132a. Schotliaria DC. 41:2b.

Schousboea W. 4531.

Schouwia DeC. 4755.

Schradera V. 1943.

Schrankia W. 4165

Schrebera Roxb. 3254 .

Schrebera Riz. 5349.

Schubertia Mart. 3440b.

Schubertia Mirb. 1723.

Schiiblera Mart. 3478.

Schultesia Mart. 3481.

Schultesia Spr. 866h.

Schultesia Schrad. 4359.

Schultzia Rafin. 3052.

Schultzia Spr. 3661.

Schwalbea L. 3169.

Schwaechrigenia Spr. 1222.

SchwaechrigeniaRchb. 3846.

Schweiggeria Spr.4516. Schweinitzia Ell. 3368. Schwenkfeldia Schreb. 2114.

Schwenckia L. 3146.

Schweykerta Gin. 3475. Sciadophyllum RBr. $3 \% 82$.

Scinaia Bivon. $47 \%$.

Scirpus L. 1121.

Sciuris Schreb. 2915. lele : 5145.

Scimis N. et M. 5142.

Sclarea T. 2805b.

Scleranthus L. 4256 .

Sclerella R. 1078a.

Scleria Bero. 1078.

SclerobasisH.Cass.2498.

Sclorocarpus Jcy. 2722.

Sclerochloa P. B. 998.

Sclerococcum Fr. 40.

Scleroderma Pers. 208.

Scleroderis Fr. 260a.

Sclerodontium Schwg. 559.

Sclerolaena RBr. 4330.

SclerolepisH.Cass.2236.

Sclerophyton Eschw. 384.

Sclerostemma Schott. 1933 a.

Sclerothamnus $\mathbf{R B}$. 4064 .
Sclerotium Tod. 150h.

Scleroxylon W. $36 \pm 1$.

Scolicotrichum Kz. 123.

Scolochloa M. K. 1047.

Scolopeudrium Sm.702.

Scolosanthus V. 2024.

Scolymis L. 2181.

Scoparia L. 3139.

Scopolia Jcq. 3280.

Scopolia Sin. 5129.

Scopolia Forst. 5113b. ex add.

Scopoliua R. S. 3280 .

Scoras Fr. 46.

Scorodonia Much. 2850.

Scorpioides T. $397 \%$.

Scorpiurus L. $397 \%$.

Scorpius Lois 3977.

Scorzonera Vaill. 2175.

Scottea DC. 405?.

Scottia RBr. 4052.

Scribaea G. M. S. 5364.

Scrobicaria H. Cass. 2501.

Scrophularia L. $318 \%$.

Scutellaria L. 2830.

Scutia Comm. 3806.

Scutula Lour. 1965.

Scyphiphora Gärt. 2002.

Scyphophora Ach.414b.

Scytalia G. 5216.

Scythymenia Ag. 429.

Scytonema Ag. 456 .

Scytosiphon Äg. 505.

Seaforthia $\mathrm{RBr}$. 1645.

Sebaea RBr. 3479.

Sebastiania Bertol.?604.

Sebastiania Spr. 5022.

Sebifera Lour. 1909.

Secale L. 856.

Secamone RBr. 345.5.

Sechium P. Br. 2746.

Securidaea L. 3040.

Securidaea T. 3982.

Securigera DC. 3982.

Securilla P. 3982.

Securinega Juss. 5094.

Sedgwickia Bnwd. 521.

Sedium L. 4189.

4189b.

Seezenia RBr. 5195.

Segestria Fr. 374.

Seguiera L. add. 5580.

Seiridium N. v. E. 29.

Sekra Adans. 595.

Selaginella P. B. 16\%6f.

Selago L. 3093.

Selenia Nutt. 4795. 
Selenocarpaea DC. $4821 d$.

Seliıum L. 3695.

Selliera Cav. $27 \% 5$.

Selliguea B. St. V. 727. Selloa K. H. B. 2652. Sellowia Rth. 4270.

Semecarpus L. fil. 3866.

Sempervivum L. 4190.

Senebiera Poir. 4749.

Senecio L. 2495.

Senega DC. 3029b.

Senkenbergia Fl. Wett. 4751 e.

Senna T. 4135e.

Senra Cav. 5260.

Senraea W. 5260.

Sentis Comm. 3806.

Sepedonium Lk. 111.

Septaria Fr. 18.

Septas L. 4176.

Septas Lour. 3132.

Septoria Fr. 18.

Serapias L. 1468.

Sergilus Gärt. 2539.

Seriana Schim. $523 \%$.

Seridia P. 2688b.

Seringia Gay. 5.304.

Seringia Sor. 5395.

Seriola L. 2160b.

Seriphium L. 2438.

Seris W. 2254.

Seriania Plum. 5237.

Serissa Comm. 2096.

Serpentaria R. 1856a.

Serpicula L. 4481 .

Serpicula L. Rxb. 802.

Serpyllum T. 2819b.

Serra Gin. 5260.

Serraea Spr. 5260.

Serratia Bergan. p. 15.

Serratula L. $231 \%$.

Serruria Sal. 1739.

Sersalisia RBr. 3639.

Serturuera Mart. 4383.

Sesamella Rchb. 4823.

Sesamoides T. 4823.

Sesamum L. 3249.

Sesban Poir. 3902.

Sesbana P. Br. 3902.

Sesbania P. 3902.

Seseli L. 3715.

Seseli Spr. 3715a.

Sesleria Hard. 996.

Sessea Rz. Pav. 3248.

Sesuvium L. 4406.

Setaria P. B. 925.

Sethia K. H. B. 4906 c. Sisymbrim L. 4810.

Shavia Forst. 2436.

Sherardia L. 1991.

Shorea Buks. 1917\%.

Sibbaldia L. 4433.

Sibthorpia L. 3068 .

Sickingia IV. 3256.

Sicyoides T. 2747.

Sicyos L. 2747.

Sida L. 5248. 5248b.

Sideritis L. 285\%. 2006. 2006.

Sideroxylon L. 3638 .

Siebera Rchb. 3774.

Siegesbeckia L. 2723.

Silaus Bess. 3709.

Silene L. 5363.

Siler Scop. 3689.

Siliqua T. 4104.

Siliquaria DeC. 4864.

Silphium L. 2620.

Siloxerus LaB. 2443.

Silybum Vaill. 2322.

Simaba Aubl. 5176.

Simaruba Aubl. 5175.

Siubuleta L. 3171 .

Simira Aubl. 2031b.

Simsia Pers. 2639.

Simsia RBr. 1734.

Simapis L. 4818.

Singana Aubl. 4825.

Sipanea Aubl. 2058.

Siphonia Rich. 5065. 5363i).

Siphusla Fr. 406.

Sirilium Spr. 29.

Sísaruan T. 3736.

Sison L. 3722 .

Sistotrema Pers. 331.
Sisyrinchium L. 1177.

Sitia DC. $457 \%$ a.

Sitodium Gärt. 1817.

Sium L. 3736.

Sheffieldia Forst. 3364. Skimmia Thub. 3628.

Shepherdia Nutt. 1717.

Silu uratia A. P. Th.3366.

SiceliunJ.Br.2094.5385.

Siderodendron Schreb.

Sideroxyloides Jcq.am.

Sieglingia Bruh. 1017b.

Sieversia Willd. 4443.

Siliquaria Forsk. 4861a.

Siliquastrum 'T. 4125.

Sinningia N. v. E. 3241.

Siphanthera Pohl. 4585.

Siphonanthus L. '29?8.

Siphonolochia R.1856b.

Siphonomorpha Otth.

Skimnera Forst. 4494 b.

Skitophyllum LaPyl. $5+1.543$.

Slateria Desv. 1324.

Slevogtia Rchb. 3492.

Sloanea L. 5453.

Smeathmannia Banks. 3460.

Smegimadermos Rz. Pav. 4456.

Smegimaria W. add. 5581 .

Sinilacina Dsf. 1325.

Sinilax L. 1333.

Smi:hia Ait. 3995.

Smyrnium L. 3744.

Sobelewskia M. B.4736.

Sobralia Rz. Pav. 1516.

Sodada Forsk. 4873.

Sogalgina H. Cass. '2598.

Soja Much. 3939.

Solanandra Vent. 3373.

Solandra L. 3315.

Solandra Murr. 5267.

Solanoides T. 4395.

Solanum L. 3294.

So!danella L. 3358.

Soldevilla LaG. 2125.

Solea Spr. 4886.

Solena Lour. 2749.

Solena W. 2074.

Solenanthus Ledeb. 2965.

Solenarimm Spr. 258.

Solenia Ag. 478.

Solenia Hoifm. 308.

Solenostemma Hayue. $3+41$.

Solidago L. 2527.

Soliva Rz. P. 2342.

Solori Ad. 4014.

Solorina Ach. 40tb.

Sonchus Vaill. 2173.

Sonerila Roxb. 3391. dele: 1211 et 4644.

Sonneratia L. 4691 .

Somninia Rchb. $3 \pm 37$.

Sophora RBr. 4090.

Sophronia Liull. 1490.

Sopubia Doll. 3163.

Soranthe Sal. 1737.

Sorauthus Ledeb. 3714.

Sorbaria S. 4\$49c. 
Sorbus L. 4470c.

Sorginum P. 984 .

Sorindeia A. P.'Th.3847.

Sorocea A. St. H. 1821. Sphaerocarpus Mich. Sorocephalus RBr.1737. 522.

Soulamea Lam. 3035. Sphaerocephalns LaG. Soulangia Brugu. 3805. 2291.

Souroubea Aubl. 5433. SphaerococcaDC.5?08b. Sowerlatea Sm. 115?. Sphaerococcus Stackh. Spailauzania Poll. 4430. 497.

SpallanzaniaNeck. 4656 . Sphaerolobium Sm. Spanauthe Jcy. 3768. Sparassis Fr. 305.

Sparaxis Ker. 1197.

Sparganiun L. 791.

Sparganophorus Gärt. 2183.

SparmamniaThnb.5437. Spartianthus Lk. 4031. Spartina Schreb. 858.

Spartiun L. 4031.

Spatalla $\mathrm{RBr} .1736$.

Spatellaria A.St.H.4897.

Spathandra R. 1312l. SphaerostigmaSer.4496.

Spathodea P. B. 326?. Suhaerotele Prsl. 1251.

Spathula Tsch. 1187e. Sphaerotheca Desv. 8b.

Spathularia A. St. Hil. Sphaerotheca Cham.

4897.

Spathularia Pers. 304. Sphagium L. 533.

Sivathulea Fr. 304.

Spathyema Rafin. 771.

Spatiun Lomr. 793.

Spenuera Mart. 4582.

Spergella Rchb. 5352.

Spergula L. 4276.

Spergularia P. 4275.

Spergulastrum Mchx. 5.347.

Spermacoce Dill. 1997. Spermadyction Rxb. 2059.

Sperinatura Rchb. 3662.

Spermaxyrum LaB. 3611.

Sperinndon P. B. 1079. Spermoedia Fr. 5.

Spermophylla Neck. 2672.

Sphacelaria Lgh. 504.

Sphacelia Lev. 5.

Sphaeranthos Vaill. 2482.

Sphaeralcea A. St. H. 5255.

Sphaeranthus L. 2482. Sphaerella Sommerf. 418.

Sphaeria Hall. 276.
Sphaeroma DC. 5255.

Sphaeromphale Rchb. 374.

Sphaeroplea Ag. 4j\%.

Sphaerophoron Pers.

362.

Sphaerophysa DC. 3913. Sporidesmimn Lk. 35.

Sphaerostemma Blume. Sporisorium Lk. 99.

Sporobolus RBr. $912 \mathrm{~b}$.

Sporochmus Ag. 506.

Sporocybe Fr. 47.

Sporophloeum Lk. 102.

Sporotrichum Lk. 113.

Sprekelia Her\$. 1255.

Sprengelia Sm. 3349.

Spumaria Pers. 181.

Sphenocarpus Rich. 4537.

SpheuocaryaWall.3635.

Sphenoclea Gärt. 4399.

Sphenogyne RBr. 2403.

Sphenopus Trin. 1012.

Sphinctosporium $\mathbf{K z}$. 243.

Sphondylium T. 3682c.

Spicularia P. ex p. 115. 11 J́i.

Spielmannia L. 2905.

Spigelia L. 3551.

Spilanthes Jcr. 2640.

Spilocoea Fr. $\%$

Spiloma Ach. 357.

Spinacia I. 4350.

Sphinctrina Fr. 241.

Spinifex L. 843.

Spiracantha K. H. B. 2315.

Spiraea L. 4449.

Spiranthera A. St. H. 5147.

Spiranthes Rich. 1429.

Spiraria S. 4449d.

Spiridens N. v. E. 554.

Spirocarpaea DC.5323a.

Spirocarpus Ser.387\%c.
Squamaria Hoff. 405 a.

Staavia Thub. 4224.

Stachyandra R. 1312e. Stachygyuandrum $\mathbf{P}$.

3. $16 \% 6 \mathrm{~h}$.

Stachylidium Lk. 136.

Stachylidium N. v. E. 11 se.

Stachyomorpha Otth. 53633 .

Stachys L. 2879.

Stachyura Rchb. 2876!.

Stachytarpheta V.2896.

Stackhousia Sin. 5119.

Staehulina Hall. 3084.

Staehelina L. 2301.

Staëlia Cham. 2000.

Stalaginites Murr.5491.

Stanleya Nutt. 4804 .

Stapelia Have. $3411 k$.

Stapelia L. 3411.

Staphisagria Riv. $4949 \mathrm{c}$.

Staphylea L. \$203.

Staphylodendron 'T. 5203.

Starbia A. P. Th. 3082.

Statice L. 1924.
Stadmannia Lam. 5206. 
Statice RBr. 19247.

Stauntouia DC. 1866.

Stauracauthus Lk. 403?.

Staurogetou Rehb. 5 tb.

Staurophora WV. 529.

Steganotropis Lehm. $3931 \mathrm{~b}$. ex add.

Stegia Much. 5258a.

Stegia Fr. 264.

Siegilla Rchb. 264.

Stegosia Lour. \$?0.

Stelis Sw. 1569.

Stellaria Dill. HiSS.

Stellaria L. 5349.

Stellaris Much. 136\%).

Stellera L. 17\%9.

Stemastrum Rafiu. 204.

Stem macantha H. Cass.

2314.

Stemınatospermum F. B. 1055 .

Stemmodontia H. Cass. $266 \div$.

Stemodia L. 3193.

Stemonitis Gled. $18 j$.

Stemonurus Blume. 1708c.

Stenactis H. Cass. $25+9$.

Stenanthera RBr. 3340.

Stenarhena Don. 2827.

Stenocarpus RBr.1763.

Stenochilus RBr. $30+3$.

Stenocladium Trin 905a.

Stenoglossum Knth. 1558.

Stenolepis H. C. 2338.

Stenolobium Don. 3273.

Stenomesson Herb. 126?c.

StenopetalumRBr.4781.

Stenoptera Prs!. 1436.

Stenorrhynchus Rich. 1431 .

Stenostomnm Gärt. f. 2025.

Stenofaphrum Trin.857.

Stephania W. 4872.

Stephania Lour. add. j5s3.

Stephananthus Lehın. $223 s b$. ex arld.

Stephanium Schreb. 2032.

Stephanotis A. P. Th. 340 Sb.

Stercuia L. 528\%.

Stereocaulon Schreb. 413.
Stereodon Bril. 578.

Stereım Lk. 323.

Sierigma DC. 4717.

Steriminostemon M. B. $471 \%$.

Steripha Gärt. 2992.

Sternbergia W.K.123\%.

SterneberwiaHerb.123\%.

Sterrebeckia Lk. 203.

Sierbeckia Schreb.452j.

Stereoxylon Rz.P.424y.

Sterenia Ad. 4791.

Sterensia Poit. $20 \% 1$.

Steria Car. 223\%.

Stewartia Car. j\$13.

Stichocarpus Ag. 490 c. ex add.

Sticta Schreb. 403.

Stictis Pers. 309.

Stictogeum Ser. 444 J.

Stiginanthus Lour.2076.

Stigmarota Lour. 488\%.

Stigmatidiun Mey. 366.

Stigonema Ag. fjz.

Stilago L. j0S6.

Stilhospora Pers. 26.

Stilbun Tod. 144.

Stilliugia Garden 502\%.

Stipa L. 920.

Stipularia Haw. 4275.

Stipulicida Mchx. 4262.

Stitzolobium P. Br.

3949 b.

Stitzolobiu!n Pers. 3949.

Stixis Lour. add. 5584.

Stobaea Thub. 2298.

Stoebe L. 2439 .

Stoechas T. 2856b.

Stokesia l'Her. 2?02.

Stomatechimm Lehm. 2964.

Stratiotes L. 807.

Stravadia P. 4650 .

Straradium Juss. 4655.

Streblotrichum P.B.63j.

Strelitzia Ait. 1610.

Streptachue K.H.B.92'.

Streptanthus Nutt. 4792.

Streptium Rxb. 2912.

Streptocarpus Liudl. 3224. ex add.

Streptogyna P. B.101s.

Streptopus Mchx. 1320.

Streptostachys Desr. $8 S 8$.

Striga Lour. adu. 5585.

Strigilia Car. 550\%.

Strigula Fr. $2 \% 0$.
Strongylium Ditın. 17\%.

Sirophanthus DeC.3531.

Struchinun P. Br. 2'?24.

Strumaria Jacฯ. 123j.

Strumella Fr. $t$.

Strumplia L. 2\%ylb. ex acide.

Struthia Roy. 17\%t.

Strithiola L. 17\%s.

Struthiopteris W. I16.

Struthium Ser. 5359!.

Sturuia Gärt. f. 2025.

Sturmia Hpp. S\&?.

Sturmia Rchl). 1564.

Strychnos L. 357s.

Stylaria DC. \$86?.

Stylidium Sw. 1919.

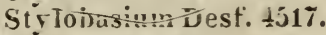

Siyloceras Ad. Juss. j019.

Stylocomium Brid. 66f5.

Siy ocoryna Cav. 2085.

Siyloncerus Spr. 2\$2.

Siylouema DC. $1 \$ 13 a$.

Stylophorum Nitt. $18 j 3$.

Stylosanthus Sw. 3991.

Stylurus Sal. 1706.

Stypandra RBr. 1392.

Styphelia Sin. 3342.

Styrax L. $359 ?$.

Suaeda Forsk. 4339.

Sinardia Schrk. 935.

Subinuatae Fr. 276b.

Subularia L. $\$ 760$.

Succisa Vaill. 1934.

Succovia Med. 473\%.

Suffrenia Bell. $45 j 0$.

Sulitra Medic. 3916.

Sunipia Lindl. 1495.

Suregada Roxb. 5100.

Sutherlandia RBr.3914.

Suzygium P. Br. 4676 .

SwaiusoniaSalisb.3915.

Swarzia Holg. 63.

Srrarzia Schreb. $\$ 15 \%$.

Sweeria DC. 39?6.

Sweetia Spr. $\$ 094$.

Swertia Ludw. 2151.

Swertia L. 3500.

Swietenia L. 5j517.

Syagrus Mart. 1636.

Syena Sochreb. 11 8.

Symmetria Blume. $45 \% 6$.

Symphocalyx Berld. $+247 \mathrm{~d}$.

Symphonia L. fil. J $\$ 99$.

Symphorema Roxb. add. $508 \%$. 
Symphoria Prsh. 1980.|Talinastrun DC. 4314b.|Tepesia Gärt. 2115.

Symphoricarpus K. H. T'ilinellun DC. 4314a. Tephranthus Neck.5109

B. 1980.

Symphyonema $\mathrm{RBr}$. 1747.

SyinphyopodaDC.4126c.

Symphysia Prsl. 1975.

Symphytum L. 2974.

Sympieza Lichtst. 3376.

Symplocarpus Salisb. 771.

Symplocos L. 3658.

Symalissa Fr. 96.

Syuandra Nutt. 2835.

Symaphea $\mathrm{RBr}$. 1732.

Synarthrum IH. Cass. 22499.

Synassa Lindl. 1433.

Syncarpha Ded. 2415.

Synconis Fr. 171.

Syndesmis TVall. add. 5586.

Synedrella Gärt. 2621.

Syntherisma Walt. 892.

Syutrichia Mohr. 636.

SynzygutheraRz.Pav. 5107.

Syorrhynchium Hffog. $117 \%$.

Syrenia Anirz. 4813a.

Syringa L. 3547.

Syringosma Mart. 3526.

Syrrhoporlon Schwg.

610. 610b.

Systrepha Burch. 3530.

Systylium Hornsch.603.

Syzygites Ehrub. 132.

Syzygimm G. $467 \%$.

Tabacina R. 3283c.

Tabacum R. 3283b.

Tabernaemontana L. 3534.

Tacca Forst. 785.

Tachia Aubl. 3436.

Tarhia P. 1138.

'Tachibnta Aubl. 4896.

Tachigalia Aubl. 4138.

Tacsonia Juss. 3469.

Tacsonioides DC. $3472 \mathrm{e}$.

Taeniocarpum Desv. 3943.

Tanuilis Sw. 721.

Tafalla Pz. Pav. 1956.

Tinetes T. 2366.

Talauma Juss. 4993.

Talea Gärt. 3095.

Taliera Mart. 1659.

Taligalea Aubl. 2900.
Talinum Ad. 4314.

Talisia Anbl. 5220.

Tamarindus L. 4116.

Tamarix L. 4413.

Tambourissa Somn.

1323.

Tammus Juss. 1335.

lamonea Anbl. 2898.

Tamus L. 1333.

Tauacetum 'T. L. 2378.

Tanaёcium Sw. 32?0.

Tangaraca Ad, $210 \%$

Tanghinia A.P.'Th.3553.

grapeinia Juss. 1201.

Tapelia R. 400a.

Taphria Fr. $5 \%$

Taphrina Fr. jo.

Tapinia 349 D.b.

Tapiria Aubl. $517 \%$.

TapogomeaAubl.2119a.

Tapura Aubl.3216.3825.

Tara Molin. 4150.

Taralea Aubl. 4023 b.

Taraxacum Hall. 2162.

Tarchonanthus L. 2205.

Targionia Mich. 518.

Tariri Anbl. 3856.

Tasmanuia RBr. 4969.

Tassia Rich. 4138.

Tauscheria Fisch. 4733.

Tavernaria DC. 4002 .

Taxanthema、 RBr. $192\lfloor$ b.

Taxodium Rich. 1723.

Taxus L. 1693.

Tayloria Hook. 604.

Tecoina Juss. 3260.

Tectoua L. 2927.

Teedia Rud. 3203.

Teesdalia RBr. 4743 .

Tegularia Reinw. 704.

Telamonia $340 \mathrm{~F}$. d.

Telekia Bammg. 2472.

Teleozouna RBr. 709.

Telephiastrum Dill. 4313.

Telephimm T. L. 4301.

Telfairia IIook. 2770.

Telipogon Kuth. 1496.

Tellima RBr. 4202.

Telopea RBr. 1767.

Templetonia RBr. 4051 .

Temus Mol. 4967.

'Tenorea Berter. 228.

Tenoria Spr. 3740e.

TentarideaB.St.V.466h.
Tephrosia P. 3892.

T'erainuus IRBr. 3929.

Terebinthus T. 3839 .

Terminalia L. 4543.

Termatea T.3974.3974a.

Terustroemia Mut.54?0.

Terpuanthus $N$. et $M$. 5147.

Tessaria Rz. Pav. 2206.

Testudinaria Salisb.

1336.

Tetracera L. 4970.

Tetraceratium DC. 479 b.

Tetracinis Prid. 596b.

Tetracolium Kz. 63.

Tetradinm Lour. 3830.

Tetrigastris G. $38+6$.

Tetragonia L. $440 \%$.

Tetringonioides DC. 4407 a.

Tetragnonocarpus

Comm. 440\%).

Tetragonolobus Scop. 3875.

Tetragonotheca Dill. 2622.

Tetrameles RBr. 4486.

Tetramerium Gärt. f. 2019.

Tetranthera Jacc. 1909.

Tetranthus Sw. 2358.

Tetrapathaea DeC.:3463.

Tetraphis Halg. 596.

Tetrapogon Dsf. Sij6a.

Tetrapteris Cav. 53159.

Tetraria P. B. $110 \sharp$.

Tetrarrhena RBr. !95̃.

Tetraspora Lk. $479 \mathrm{r}$.

Tetratheca Sm. 54?".

Tetrazygia Rich. 4ij32.

Tetrodontium Schwg. $596 a$.

Tetroncium WV. 1289.

Tencrium L. 2851.

Thalamia Spr. 1696.

Thalissia Banks. 755.

Thalia L. 1605.

Thalictrum 'T'. 4953. $4953 \mathrm{c}$.

Thannnea Soland. 4229.

Thamnidium Lk. 138.

Thammium Vent. 409a. 410a. 415.

Thamnochortus Berg. 1139. 
Thamnomyces Ehrnb.|Thuarea P. 895 . $9 \%$.

Thainu ophora Aщ. 495. Thammus Lk. 1.335.

Thapsia L. 369?.

Thapsium Nutt. 3727.

Thammasia Ag. 400!). ex add.

Thea L. 5399 .

Theracoris Ad. Juss. 5090.

Thecamthus Vickstr. $17 \%$.

Theka Rheed. "2927.

Thela Lour. 1928.

Thelebolıs Tosl. 214.

Thelephora Ehrh. 327.

'Theiepogon Rth. 975.

Thelotrema Ach. 370.

Thelymitra Forst. 1418.

6. Thely ra A.P.'Th. 4519.

Thelythamnos Spr. $256 \%$.

Themeda Forsk. 982.

Thenardia K.H.P.3524.

Theobroma Juss. 52.18.

Theophrasta L. $360 \%$.

Thernia Nutt. 4084.

Thermopsis RBr. 4084.

Thermutis Fr. 86.

Thesiosyris R. 1708c.

Thesium L. 1708.

Thesiun R. 1708a.

Thespesia Corr. 5275.

Thevetia Juss. 3557.

Thezera DC. 38501.

Thibandia Rz. P. 1974.

Thlaspi L. 474 .

Thisaspidium Mel. $475 a$.

Thoa Aubl. 1958.

Thomasia Gay. 5301.

Phompsonia BBr. 3ítifi.

Thorea B. St. V. $131 j$.

Thorntonia Rchl). 526?

Thottea Rot!b. 18 ij!.

Thoninia Poit. 5215.

rhrasya K. H. B. 893.

Threlieldia RBr. 4341 .

ThrichotheciunLk.11?.

Thriuax L. fil. 1651.

'Trincia Rth. 2134.

'Thrixspermum Lour. $1 ; 81$.

Thryallis L. 5374.

Thryocephalus Forst. 1082.
Thuia L. 1720.

Tinnbergia L. 3131 .

Thunberoia Mont.2111.

Thy lachium Lonr. $\$ 8 \% 6$.

Thymbra L. 28"5.

Thymeliua IIrgy. 1795.

Thymmophylla faG. $2360^{\circ}$.

Thymus L. 2819.

Thyrsanthema Neck. 2262.

Thy rsanthus Ell. 3936.

Thy rsamthus Schrk. 332\%).

Thysainus Loir. 5134.

Thysanomitrium

Schwg. 598.

Thysanotus RBr. 1397.

Thysselinum T. $367+b$.

Tiarella L. 4200.

Tiardiun Lehm. 2959. Tibouchina Aul:1. $459 \%$.

Ticanto Ad. 4149il.

ricorea Aubl. 5142.

Tigarea Prsh. 4454.

Tigarea Aubl. 4970.

Tigridia Juss. 1180.

Tilesia Mey. 2660.

Tilia L. 5449.

Tiliacora Colebr. 1895. Tillaea Mich. 4173.

Tillandsia I. 1275.

Tiunmia Hog. 660.

Timuia Gin. syst. 1374

Timutia DC. 3029a.

Tina R. S. 5?11.

T'inea Spr. 4926b.

Tiniaria Meisu. $4291 \mathrm{f}$.

Timus L. 3406.

Tipularia Mutt. 1501.

Tiresias B. St. V. 462.

ritil Srop. 1!170.

Tithonia Desf. 2666.

Tilhymalus haw. 5012.

Tiıly malusNerk.j0121

Tittuannia Bron. 4228.

Tittmannia Rehb. 3153.

Tmesipteris Berul. $167 \%$.

Tobinia Hamilt. add. 5588.

Tococa Aubl. 4624.

Tocoyena Aubl. 2075.

Todilalia Juss. 5129.

Todea W. 739.

Tofieldia Huds. 1293.

Tofielda P. 1293.
Tolasium Spr. 823.

Tolpis Adans. 2151.

Tohinfera L. $4091 \mathrm{~b}$.

Tomex Thub. 1909.

Tonabo Aubl. 5420.

Tonina Anbl. 114 ij.

Tonsella Schreb. 5.355.

Tontanea Aubl. 2094.

Toutelea Aißbl. 5355.

Tophora Fr. 56.

Topobea Anb!. 4642.

Torly ium L. 3686.

Torenia RBr. 3151.

Toresia Paz. Pav. 1038.

Torilis All. 3667.

Tormentilla L. 4435.

Torminaria DC. 4ti0l.

Torreya Spr. 1847.

Tortula Rxb. 2395.

Tortula Schwo. 636.

Torula Pers. 6.3b.

Torulinium Hamilt. 1106.

Tosagris P. P. 905b.

Touchiroa Aubl. 4108.

Tonlicia Aubl. 5214.

Toumatea Aubl. $415 \%$.

Touruefortia L. 2982.

Toumesolia Scop. 5044 .

Touroulia Aubl. 5501.

Tourretia Domb. $326 \%$.

Tovomita Aubl. 5488.

Toxicollendron ' $\mathrm{T}$. $38.50 \mathrm{c}$.

Toxicodendron G.5217.

Toxicodendron Thulb. 5067.

Tozzeltia Sav. 944 .

Tozria I. 3072.

Trache!ium L. 2794.

Trachylia Fr. 352.

Trachyloma Brid. 556.

Trachymene Rudg. 37601 .

Trachymitrinm Brid. 611.

Trachynia Lk. 851.

Trachynotea Mchx. $8 j 8 a$.

Trachyozus Rchb. 836 .

Trachypleurum Rchb. 3739.

Trachypodium Brid. 552.

Trachys Pers. 836.

Trachyspermum Lk. 3718b. ex ads.

Trachytella DeC. 4976. 
Traclescantia L. 11\%. |Trichocline H. Cass. Traganum Bel. 4328. Trasia Plum. 5030.

Trayiun Sipr. 3738s.

Tragoceros:.H.B.2941.

Tragopogon T. 2179.

Trayopyrum M.B.4290. Tragoselinum T. 3738.

Tralliana Lour. 5396.

Trapa L. 4479.

Trapaulos Rafin. 1953. Trasi P. B. 1093.

Trattinickia IV. 5140.

Treisia Haw. 5012.

Tremandra RBr. 5423.

Tremanthus P. $550 \%$.

Trematodon Brid. fil6.

Trembleya DeC. 4591.

Tremella Dill. 288.

Trentepohlia Mart. 6j.

Trevirania Wr. 3235.

Trewia L. adl. 5 j89.

Triachne H. Cass. 2288.

Trialeniun Rafiı.5472.

Trialica Lour. 5026.

Triaena K. I. В. $8 \% 6$.

Triauthema Sauv. 4310.

Trinthera Desv. 873 .

Triblemina RBr. 4ijl7.

Triblidium Rebent. 2(60).

Tribrachia Lindl. 1571.

Tribuloides T. $4 \$ 99$.

Tribulus '. 5191.

Tricaryum Lour. j074.

Tricera Schreb. 5093.

Triceras Aullz. 4797.

Triceros Lour. 5139. 5388.

Trichaeta P. B. 994.

Trichandrum Neck. 2429.

Trichelosty lis Lestib. 1116.

Trichera Schrad. 1935.

Trichia Hall. 188.

Trichilia L. 5512.

Trichinium RBr. $437 \%$.

Trichlis Hall. $\$ 260$.

Trichoa P. 1885.

Trichocarpus Schreb. 5440.

Trichocephaius Brugn. 3803.

Trichoceros hislle. 1538.

Trichocisloa DC. 905.

Trichocladus Pers. add. 5590.
23 'sf.

Trichoderma Pers. 164.

Tricho:lesma RBr. 2961.

Trichodium Mchx.90\%b.

Tricholoma 349 H. f.

Trichomaues L. 675.

Trichomitrium Brid. 565.

Trichonema Ker. 1202.

Trichophorum Rich. $112 \%$.

Trichophyllım Neck. 2599.

Trichopteris Prsl. $67 \%$.

'Trichointhes L. 27.58.

'Trichospernamblume. 4931.

Trichospira K. H. B. 2346.

Trichosporum Don. 3227.

Trichosporum Fr. 74.

Trichostemma L. 2818.

Truchostommm Hdy. 628.

Trichothalamus Lehm. $4436 \mathrm{c}$

Tricondylus Sal. 1764.

Tricoryue RBr. 1352.

Toicratus l'Herit. 1842.

Tricuspidaria Rz. Pav. $54 ? 8$.

Tricuspis P. B. 1025.

Tricuspis P. $\mathbf{3 4 2 8 .}$

Tricycla Cav. 1849.

Tridactylites 4203a.

Tridax L. 2576.

Tridens R. S. $102 j$.

Trislentea Haw. $3+11$ g.

Tridesmos Chois. 5471c.

Tridesinus Lour. 5045.

Trien!olis L. 3597.

Trifoliastrum Ser. 38699.

Trifolium L. 3869 .

TrifurcariunDC.4595d. Triglochin L. 1288.

Triglossum Fisch. 10j1.

Trigonella L. 3872.

Trignnia Auhl. $538 \%$.

Trigonis Jcy. 5?12a.

Triguera Cav. $327 \%$.

Trilepisium A. P. Th. 4474.

Trilisa H. Cass. 2?39.

Trilix L.54ti1.

Trillium L. $131 \%$.
Trimorphall. Ca is.25 5 .

Trimorpluaea 1f. Cass. $25 \pm 5$.

Trinacte Giirt. 2276.

Trinia Holfm. $372 \frac{1}{4}$

Triodex Rafin. 1071b.

Triodia P. B. 1045.

Triodia 13Br. 1017.

Trionella DC. 5306b.

Trioum 5273f.

Trionychon Wallr. 3050 l.

Triopteris L. 53658.

Triosteum L. 1937.

Triphaca Lour. 5:86.

Triphasia Lour. 5j523.

Triphocoma LaP y 1.666.

Tripiuna Lour. 3221.

Tripimaria P. 3221.

Triphragmium Lk. 11.

Triplaris L. 4299 .

Triplasis P. B. 1005.

Triplima Rafin. $1071 a$.

Tripogon Rth. 1023.

Tripsacum L. 834 .

Tripterella Mchx. 1210.

Tripterium DC. 4953a.

'riptilion Rz. P. 2287.

Trirrhaphis RBr. 932.

Trisanthus Lour. 3767.

Triselaria Forsk. 1044.

Triselu: P. 1044.

Tristania RBr. 4 ljtis.

Tristegia R. 12481).

Tristegis N. v. E. 935.

Pristellaria A. P. Th. 5373.

Tristemma Juss. 4568.

Tristicha A. P.Th.11jit.

Tristichis Ehrh. 66-4.

Triticum L. Sjł.

Tritoma Ker. 1405.

Tritomanthe Hiffog. 1405.

Tritonia Ker. 1196.

Triunfetta L. 5\$33.

Trixago Much. 2S79a.

Trixago Stev. 30isi.

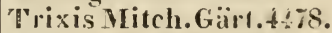

grixis P. Br. 2?(6).

Trizenxis Lindl. 1530.

Trochera Rich. !nit.

Trocletia DeC. 533\%.

Prochiscanthes Koc!. 3706.

Trochocarpa RBr. 3.3.3.

Trollius L. $496 \%$.

'Trollius Raf. \$46:?b. 
Tromms:lorffia Mart. 4354.

Tromotriche Haw. 3411 f:

Troparolum L. $52 \%$.

Trophis L. 1806.

Troximoı Gärt. 2131.

Truncaria DeC. 4610.

Trypethelimm Spr. $37 \%$.

Tschulya DeC. 4621.

Tuber Nich. 223.

Tuberaria DC. 4915 .

Tubercularia Tod. 88.

Tubilimm II. Cass. 2462.

Tubocytisus DC. 4029d.

Tula Ad. 2051.

Tulbaghia L. 1381b. ex adcl.

Tulipa L. 1355́b. ex add.

Tulostoma Pers. 198.

Tuma Dill. 4245.

Tupeia Cham. 1963.

Tupistra Ker. 781.

Turaria Mol. 3653.

Turgenia Holfin. 3666 .

Turgosea Haw. 417\%b.

Turia Forsk. 2750.

Turuem Plum. 523\%.

Turpinia Vent. 5?04.

Turpinia K. H. B. 2189.

Turpinia P. 3987.

Torpinia Rafiu. $3850 \mathrm{e}$.

Turriea L. 5509.

Turrita Wallr. 47901.

Turitis L. 4793.

Tursenia H. Cass. 2541.

Tussacia Rrhb. 3231.

T'ussilago 'T. 22:49.

Tylophora RBr. 3417.

Ty mpan is Toil. 262.

Typha L. 790.

Typhalea DC. 5261a.

Typhoiles Mnch. 952.

Typhula Fr. 299.

Tyrimuus H. Cass.?337.

Ucriana W. 2075.

Uilora Nutt. 802.

Ulassimm Rimph. 5104.

Ulex Lk. 4033.

UlIoa P. 3303.

Ullucus Loz. 4308.

Ulmaria 4449i.

Ulmus L. 1827.

Ulospermum Lk. 368t.

Ulota IV. M. 622.

Ulva L. 479.

Umsema Rafin. 1166h.

Uncinia Pers. 1069.

Undina Fr. 421.

Unguicularia DC. 3940c.

Uniola L. 1008.

Junua L. s006.

Unxia L. fil. 2725.

Uperrhiza Bosc. 210.

Urachme P. B. 915.

Uralepis Nutt. 1015.

Ura!epsis Nutt. 1015.

Urauia Schreb. 1612.

Uraria Desv. 3998.

Uraspermun Nult. 3662.

Urceola Vand. 353j.

Urceola Rxb. 3574 .

Urceolaria Ach. 369.

Urceolaria Gin. 1943.

Urceolaria Herb. 12:4.

Urceolina Richb. 1234.

Uredo Pers. 8

Urenta L. 5153.

Urochloa P. B. 891a.

Uropetalum Ker. 1370.

Urospermum Srop.2178

Ursinia Gärt. $240 t$.

Uríca L. 1800.

Urvillaea K. H. B. 5236.

Usnen Dill. 410.

Ustalia Fr. 383.

Usteria Lam. 3504.

Usteria Cav. 3176.

Ustiiagno I.k. 8a.

Utricularia L. $331 \%$.

Uvaria L. $500 \%$.

Uvedalia RBr. 31850.

Uvularia L. 1349.

Vaccaria Dorl. 5361.

Vaccillium L. 1972.

Vaginaria B. St. V.455b.

Vahea Jam. 3535.

Vahlia T'hub. 4?12.

Valantia T. 1989.

Valilesia Rz. Pav. 4642.

Valentinia Sw. 520j.

Valeutiuiaräusch.j229.

Valentynia Neck. $\mathbf{4 1 3 8 .}$

Valeriaua L. 19\$6.

Valerianella T. 1947.

Vallaris RBr. 3519.

Vallea Mut. 5427.

Vallesia Rz. Pav. 3556.

Vallisueria L. 86\%.

Vallota Herb. 1236.

Valonia Giun. 476.

Vandellia P. Br. 3150.

Vaucueria Juss. 2103.

Vanilla Sw. 1460.

Vantanea Aubl. 3616b.

Vareca Gärı. 3464 .

Varenuea DC. 4015.

Variolaria Pers. 358.

VarontheJuss.add.5J92.

Varronia L. 2988.

Vascoa DC. 4049.

Vatairea Aubl. 4120.

Vateria L. 5432.

Vatica L. 5458.

Vaucheria DeC. 472b.

Vanquelinia Corr. 4457.

Vavaina V. 2103.

Velaga G. 5316b.

Velarum Pliı. 4508. .

Velezia L. 5357.

Vella L. 4740.

Velleia Sil. 2778.

Velleia $\mathrm{RBr}$. 27\%8b.

Vellosia Vaud. 1230.

Yeltheimia Gled. 1401.

Venana Lam. 3630.

Ventenata Koel. 1043b.

Ventenatia P. B. 5406.

Ventenatia Sin. 1919.

Venlenatia Cav. 3341.

VentenatumLesch.5172.

Veltilağo Gärt. 3820 .

Vepris Comm. 5126.

Veratium L. 1291.

Verbascum L. 3191.

Verbena L. 28:4.

Verbesiun L. 2642.

Verea IV. 4183.

Vereia Auslr. 4183.

Vermicularia Torl. 267.

Veruicia Lour. 5062.

Verniselia Scop. 54t1.

Veruonia Schreb. 2185.

Veronica L. 3063.

Verouicastrum Mnch. 3064.

Veronicastrum Vet. $3063 \mathrm{~b}$.

Verpa Sw. 319.

Verticillium N. v.E.135.

Verticordia DeC 4665.

Vermearia Ach. 373b.

Verrucaria Pers. 373.

Verticillaria Rz. P.5487.

Valsa Fr. 27\%. 27\%). Vesicastrum Ser. 3869e.

Umbilicaria Hoffm. 394. Vananthes Haw. 4180. Vestia WV. $328 \%$.

Umbilicus DeC. 4187. $\mid$ Vanda RBr. 1504. $\mid$ Yestingia Fabr. 4405. 
Vexillaria Hffgg. 3974. Voigtia Spr. 2189.

Viborgia Spr. 4039.

Vihorgia Mnch. $4029 \mathrm{~d}$.

Viborquia Ort. 4015.

Vibrissea Fr. 313.

Viburuum L. 1950.

Vicia L. 3919.

Vicia Fl. Wett. 3919b.

Vieusseuxia LaRoch. 1186.

Vigna Savi. 3941.

Vignea P. B. 1070.

Viguiera K. H. B. 2643.

Vilfa Adans. 912.

Villanova LaG. 2726.

Villaria Guett. 2325.

Villarsia Vent. 3475.

Vilmorina DC. 2968.

Vilshenica Thou. add. 5593.

Viminaria Sm. 4070.

Viuca L. 3533.

Vincetoxicum P. 3440a.

Viola T. 4885.

Viorna Pers. 4947a.

Virecta L. f. $205 \%$.

Virga aurea Vaill. $252 \%$

Virgaria N. v. E. 115́c. Virgilia Lam. 4087.

Virgularia Rz.Pav.3208.

Viscago Hall. 5363b.

Viscraria Riv. 5362e.

Viscoides Jcq. am.2031e.

Viscum L. 1959.

Visena Houtt. 5309.

Vismia Vaut. 5474.

Visnaga G. 3670b.

Visnea L. 3656 .

Vitex L. 2903.

Viticella Dill. 4946b.

Vitis L. 3792.

Vitmannia Turr. 1840. Weigelia Thub. 3195.

Vitmannia Vahl. 5178. Weigeltia Rchb. 410\%).

Vittaria Sm. 69.t.

Viviania Cav. add. 5594.

Vleckia Rafin. 2866.

Voa Flacourt., 1916. ex add.

Voacanga A.P.Th.3558.

Voandzeia A.P.Th.4097.

Vochy Aubl. 4508.

Vochya Vand. 4508.

Vochysia Juss. 4508.

Yogelia Lam. 192\%.

Vogelia Gın. 1210.

Yogelia Fl. Wett. $477 \%$.

Voolera G. M. S. 4030h.

Vohiria Lam. 3495.

ex add.
Voitia Horusch. 583.

Volkamera L. 2933.

Volkameria P. Br. 3406.

Volkınamia Jcq. 2929. Wigandia K.H. P. 3019.

Volubilaria Lamx.490c. Wigandia Neck. 2440.

Volutaria H. Coss. 2696. Wiggersia G. M. S.

Volutella Tod. 311.

Volvaria $349 \mathrm{~B}$. $\mathrm{f}$.

Votomita Aubl. 4318.

Vonacapoua Aubl.4101.

Vouapa Aubl. 4112.

Vouarana Aubl. 4108.

Voyra Aubl. 3495.

Vrolikia Spr. 3102.

Vulneraria T. $4025 \mathrm{~b}$.

Vulpia Gm. 1000.

Wachendorfia L.1160.

Wahlbomia Thub.4970.

Wahlenbergia Schrad. 2798.

Waitzia Rchb. 1196.

Waldschmidtia Neck. 410 s.

Waldschmidtia Wigg. 3475.

Waldsteinia W. 4442.

Walkera Schreb. 5185.

Wallenia Sw. 2922.

Wallichia Rxb. $164 \%$.

Wallichia DeC. 5290.

Wallrothia Rth. 2930.

Wallrothia Spr. 3708b.

Waltheria L. 5308.

Wangenheimia Trin. $84 \%$.

Watsonia Ker. 1192.

Webera Gärt. f. 2090.

Webera Hdg. 642a.

Webera Schreb. 2035.

Vedelia Jcq. 2667.

Veihea Spr. 1970.

Weinmantia L. 4210.

Wein reichia Rchb.4017.

Weissia Hdlg. 60\%.

Wendia Hoffin. 368?a.

Wendlandia W. 1874.

Wermeria K. II. B. 2514.

Westonia Spr. 4054.

Westringia Sin. 28 \$0.

Wibelia G. M1.S. 2142b).

Wibelia Ipp. 2145.

Wibelia liobl. 2161.

Wibelia Pers. add.5595.
Wiborgia Thnb. 4039.

WickströmiaSpr. 2199.

Wickströmia Schrad. 5459.

$3919 a$.

Wilhelınsia Rchb. 5354.

Willdenowia Thunb.

1140.

Willemetia Brngn.3800.

Willemetia Neck. 2161.

Willichia Mut. 3148.

Wilsonia RBr. 299\%.

Willugbeia Scop. 3509.

Windsoria Nutt. 1025.

Winterania Sol. 4908.

Winterania L. 5511.

Winterlia Much. 3626c.

Winterlia Spr. 4270.

Wintera Murr. 4908b.

Wisteria Nutt. 3936.

Witharia Paucuy. 3306.

Witheringia l'Herit. 3295.

Witsenia L. 1183.

Wittelgbachia Mart. $5 \frac{121}{2}$.

Wolfia Spr. addl. 5596.

Woodsia PBr. 717.

Woodwardia Sm. 713.

Wormia Rollb. 4986.

Wormskioldia Spr. 501.

Wrangelia Ag. 482.

Wrightia RBr. 3532.

Wrigtia Sol. 4603.

Wulfenia Jacq. 3067.

Wulffia Neck. 2668.

Wurmbea Thub. 1296.

Xanthium L. 274t.

Xanthe W. 5486.

Xauthocephalum W. 2225.

Xanthochyıus Rxb. 5491.

Xanthocoma K. H. B. 2519.

Xanthoria Fr. $402 e$.

Xantorrhiza Marsh. 4960.

Xantorrhoea Sm. $115 j$. Xanthosia Rudg. 1421. Xauthoxylum Sim.j́132.

Xenodochus Schlecht. 13.

Xerantheınun L. Gärt. 2300. 
Xerochloa RBr. 9\%.

Xeropetalum RBr. 4067.

Xerophyta Juss. 1229.

Xenopoma W. 3213.

Xerotella R. $1312 b$.

Terotes RBr. 1312.

Nerotes Fr. 343.

Xerotinus Richb. 343.

Xerotium N. v. E. 2453. ex add.

Ximenia Plum. 3614.

Ximenesia Car. 2644.

Xiphidium Löff. 1159.

Xiphium Vill. 1187f.

Xiphopteris Kaulf. 726.

Xuaresia Rz. P. 320\%.

Xylandenius Hamilt. 4925.

Xylanthema Neck. 2327. 2332.

X ylaria Pers. 278a.

Xylissus Rafin. p. 15 .

Xylobium Lindl. 1529.

Xylocarpus Schreb. 5520.

Xyloma Pers. 232.

Xylomelium Sm. 1759.

Xylomycon P. 336.

Xylon T. 5272.

Xylopia L. 5004.

Xylophylla L. 5079.

Xylosteun T. 1984.

Xylostroma Tod.p. 15.

Xyridion Tsch. 118\%d.

Xyris L. 1149.

XyrisA. P. Th. 1149a. Zizania L. 959. $3 \$ 30$. Zamia L. 750 . $\$ 460$. Eea L. 1059.

Zeora Fr. 400. 1254 .
XyroidesA.P.Th.11491. [Zizia Koch. 3727. ysinalobium $\mathrm{RBr}$.

Xystidium Trin. 985. Yucca L. $1+13$. Zacyutha T. 2139. Zalega Burm. 4310.

Zaluzania Comm. 2087. Zaluzania P-Anthemis.

Zanonia Blume. 2772a. Zanonia L. 27\%2.

Zanonia Plum. $11 \%$.

Zannichellia L. 760 .

Zantedeschia Spr. 773. Zanthorrhiza l'Herit.

Zanthoxylum L. 5132. Zapania Scop. 2937.

Zarabellia Neck. 2683.

Zeocriton P. B. 845a.

Zephyranthes Herb.

Zeugites Schrel. 979.

Zeuxine Lindl. 1430.

Zeyheria Spr. 2405.

Zeyheria Mart. 3263.

Zieria Sm. 5166.

Zietenia Gled. 2880.

Zigadenus Mchx. 1298.

Zilla Forsk. 4723.

Zingiber Gärt. 1595.

Zinnia L. 2645.
Ziziphora L. 2838.

Zizyphus T. Dsf. 3817.

Zoegea L. 2692.

Zollernia Mart. 4158.

ZollikofferaN.v.E.2161.

Zonaria Drap. 508a.

Zunaria Rouss. 327.

Zoophthalıum P. Br. 3949a.

Zornia Gm. 3990.

Znsimia Hoffin. 3683.

Zostera L. 754.

Zosterospermum P. B. 1080.

Zoydia W. 881 .

Zoysia WV. 881 .

Zucca Commers. 2766.

Zuccaguia Cav. 4139.

Zuccagnia Spr. 2028.

Zwingera Schreb. 5176.

Zygis P. 2819a.

Zygnema $A$ g. 4 f 6.

Zyodon Hook.et Tayl. 626.

Zygoglossum Reinw. $15 \%$.

Zyomeris Fl. Mex. 3986.

Zygopetalon Hook. 150 .).

Zygophyllum L. $519 \%$. Zy gotrichia Brid. 634. $557 \mathrm{a}$.

Zy mum Nor. 5373.

Zythia Fr. 249.

\section{$\begin{array}{llllllll} & p & p & e & n & d & i & \end{array}$}

48\%. Mertensia. Thnb.

943. Pallasia. Scop.

1020b. Eriachne. RBr.

1830b. Momisia. Dietr.

Mertensia. K. H. B.

2133. Tege: Airachnopogon. Berg.
2626. Prollasia. 1'Heril.

2947. Mertensia. Rth.

3590. Labatia. Sw.

propr. gen. teste Mrartio.

4283. Pterococcus. Pall.

Pallasia. L. 


\section{E m e n d a n d a.}

ante 360. lege: Staubkelchflechten.

pag. 24. - bis : F is cher

- 588. - Hellwigia. Ehrh.

- 626. - Gragea pro Gayea.

- 638. - Cynodontium. Hdg. Cynodon Brid. non Rich.

- 643. - Cinclidium. Sw.

- 883. deleatur.

ante 955. lege: Tetrandrac.

no. 1135. delentur.

- 1136. lege: Restio.

- 1155. - Xanthorrhoea.

- 1188. - D e C. pro Del.

post 1260. - Narcissincie.

no. 1288. - Triglochin.

post 1291. - Heloniene.

no. 1293. lin. 5. lege: Iridiogalva.

- 1294. lin. 2. - Chamcelirium.

- 1324. lin. 4. - Maicnthemum referatur sub n. 1325.

- 1340. deleatur.

- 1373. deleatur.

- 1428. lin. 2. Distomrea referatur sub n. 1427.

- 157\%. lin. 3. lege . Cypr. venustum. Wall.

- 1650? ante synonymon deleatur.

- 2039. lege: Lygodisodea.

- 2102. - DeC. pro De 1.

- 2107. - Matthiola. Plum. pro Blum.

- 2336. lin. 2. lege: Eriocephatus.

- 2454. lege Micropus.

- 2571. - Bellidiastrum.

- 2804. deleatur.

- 3000. cum nomine suo ad $1.4225 \mathrm{~b}$. rectius relato, deleatur.

- 3388. lin 2. lege : Enkicunthus.

- 3511. lege: Giunillaea.

- 3631. - Moutoubea. Aubl. Moutraber Aubl. tab.

- 3638. - Co m mers.

post 3648. in adnotatione prima lege : Mag.; in altera lege : medio.

no. $3653 \mathrm{~b}$ ? lege : Decarlia.

- 3773. - Asteriscium, fiat 3760d. post institutum examen.

post 3863. in adnotatione lege: Hedwigia. Ehrh.

no. 4075. lin. 2. lege: Crullistuchya.

- 4133. lege: Pancovia. W.

post 4247. in ridnotatione lege: cotyledo.

- 4293. in adnotatione lege: jungeremus.

no. 4848. lege: Eschscholzia.

- 5523. Triphasia. Lour.

Quaedam alia per indicem inrenies emeudata. 



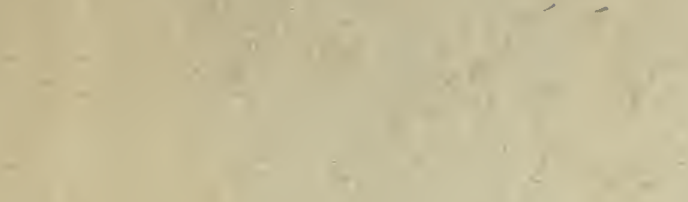

$-\infty$

.

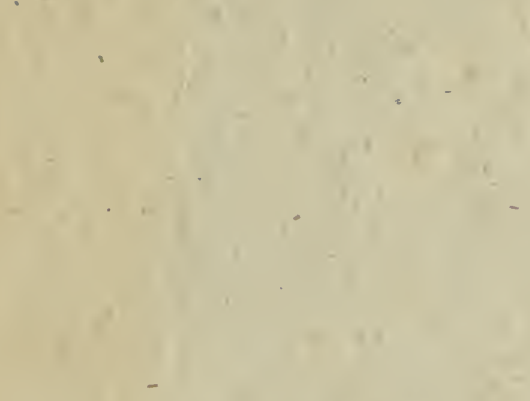

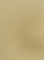

5 


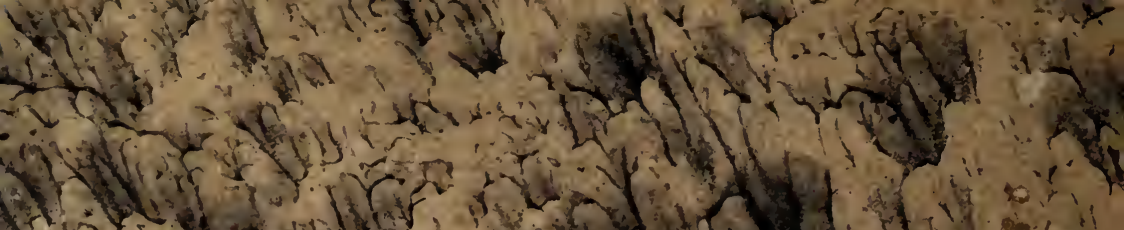

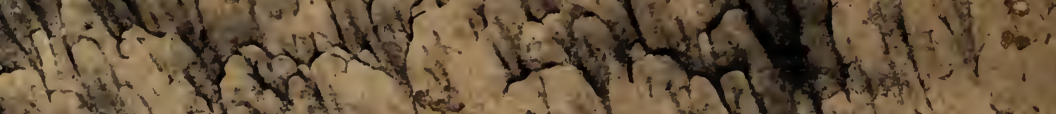

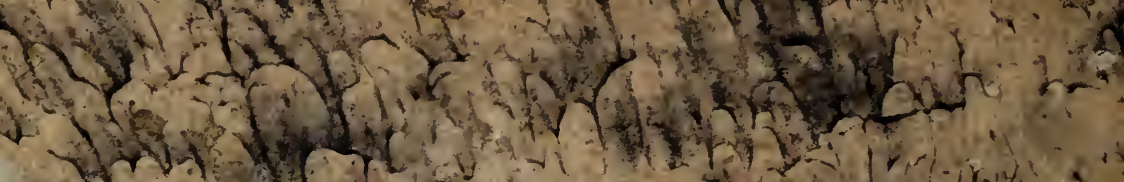

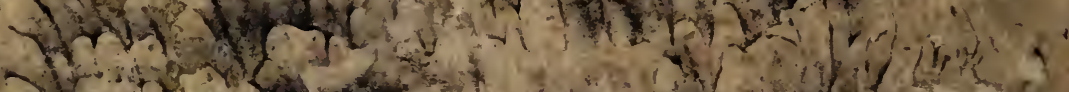

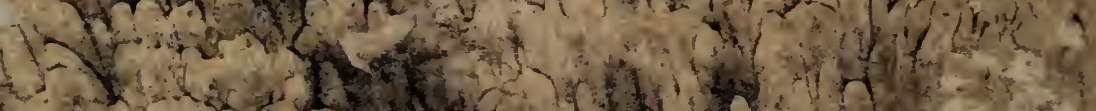

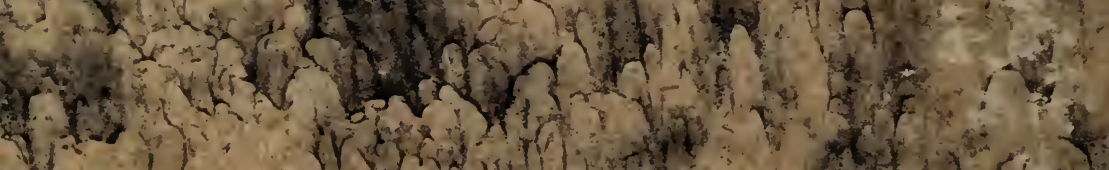

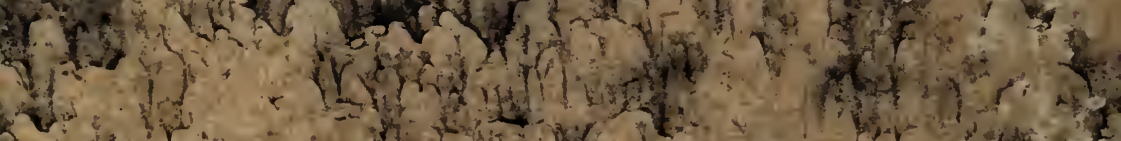
If (1.)

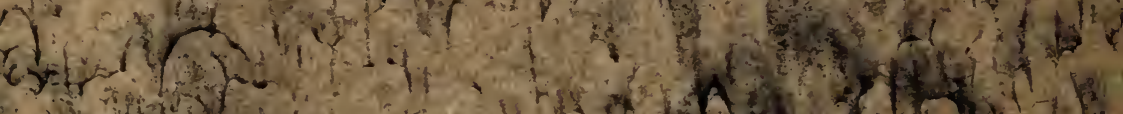

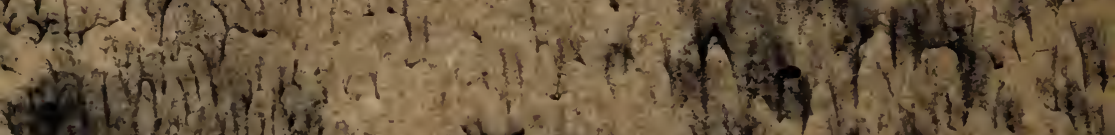
(1)

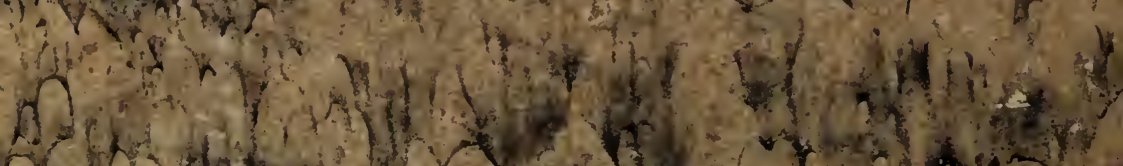

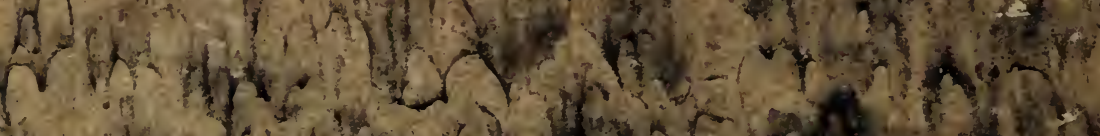

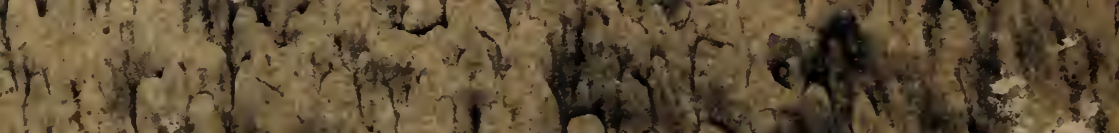

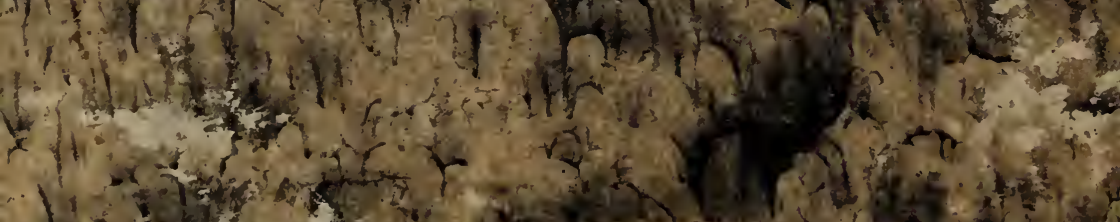

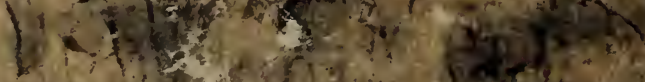

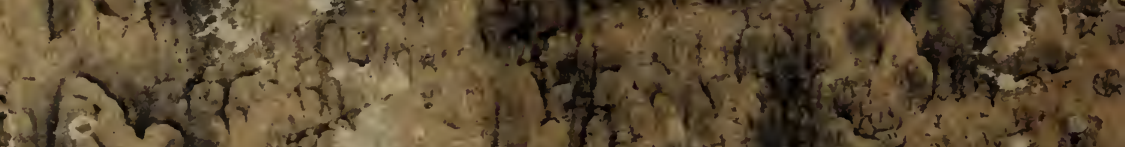
H.

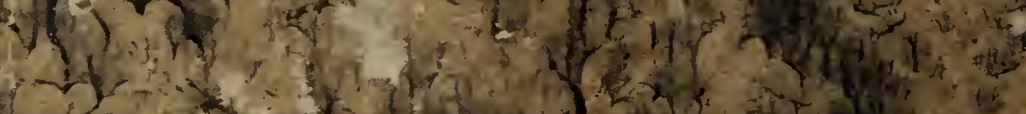

